\title{
Fundamental Aspects of Nuclear Reactor Fuel Elements
}

\section{Solutions to Problems}

\section{Donald R. Olander}

Department of Nuclear Engineering University of California, Berkeley

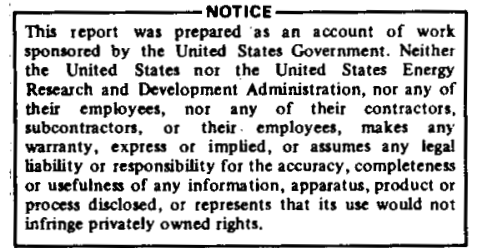

Published by

Technical Information Center, Office of Public Affairs Energy Research and Development Administration 


\section{DISCLAIMER}

This report was prepared as an account of work sponsored by an agency of the United States Government. Neither the United States Government nor any agency Thereof, nor any of their employees, makes any warranty, express or implied, or assumes any legal liability or responsibility for the accuracy, completeness, or usefulness of any information, apparatus, product, or process disclosed, or represents that its use would not infringe privately owned rights. Reference herein to any specific commercial product, process, or service by trade name, trademark, manufacturer, or otherwise does not necessarily constitute or imply its endorsement, recommendation, or favoring by the United States Government or any agency thereof. The views and opinions of authors expressed herein do not necessarily state or reflect those of the United States Government or any agency thereof. 


\section{DISCLAIMER}

Portions of this document may be illegible in electronic image products. Images are produced from the best available original document. 
This book was prepared from the best available copy.

Available as TID-26711-P2 for $\$ 13.50$ (foreign, $\$ 16.00$ )

National Technical Information Service

U.S. Department of Commerce

Springfield, Virginia 22161

\section{ERDA Distribution Category UC-79b}

Library of Congress Catalog Card Number 76-6485

$\rightarrow$ ISBN 0-87079-031-5 (v.2).

Printed in the United States of America

ERDA Technical Information Center, Oak Ridge, Tennessee

April 1976 
Index to Problem Solutions

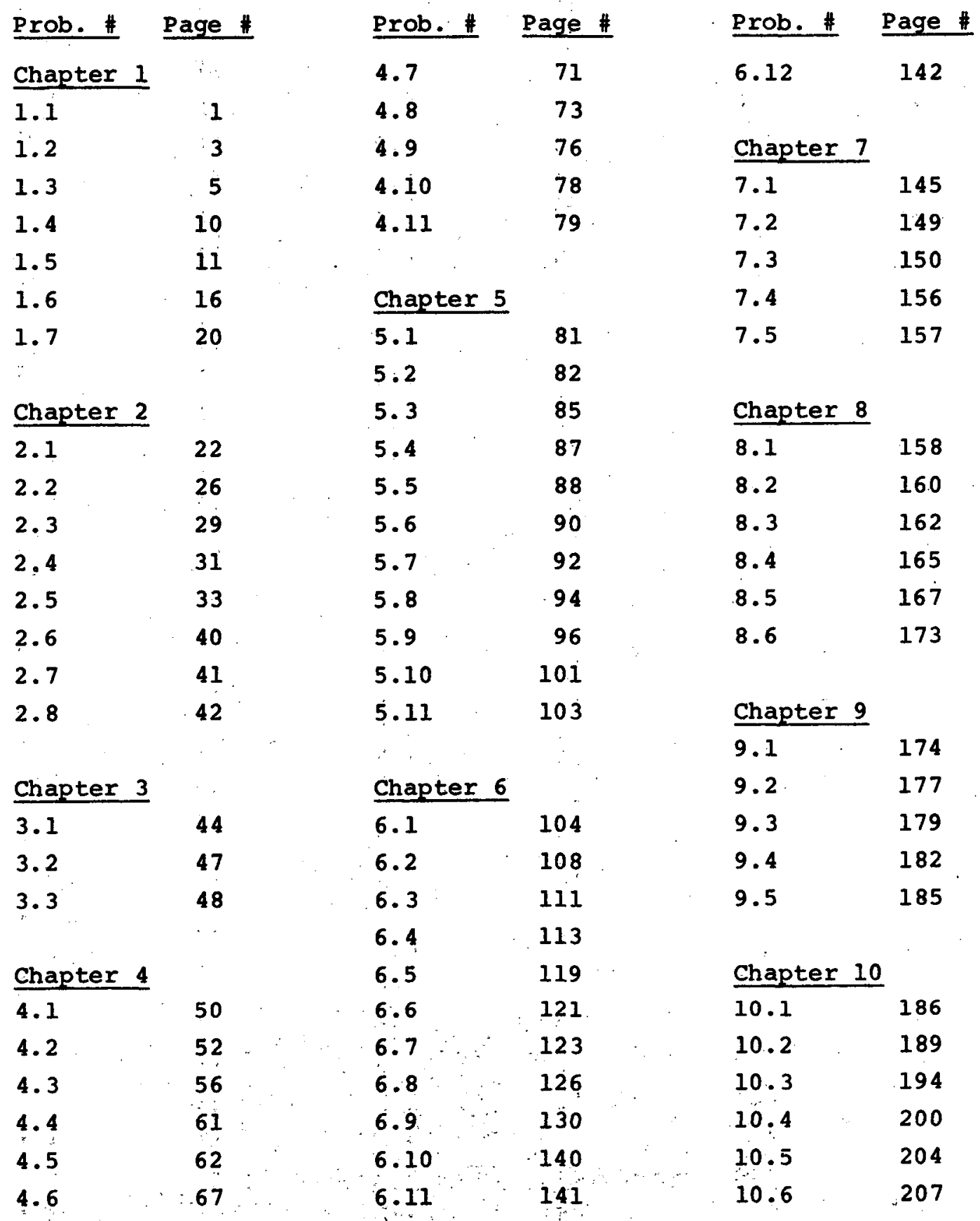

iii 
Index to Problem Solutions

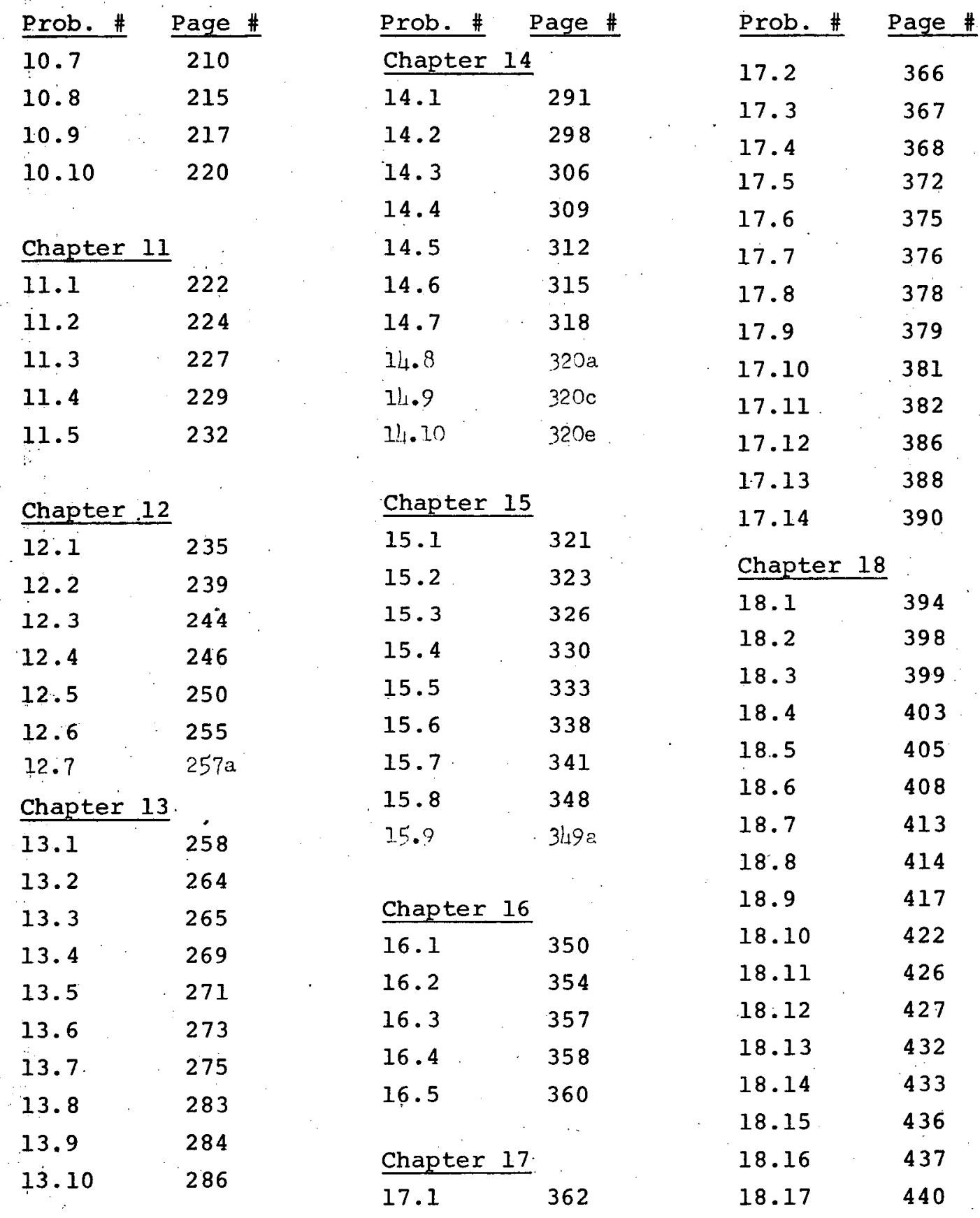


Index to Problem Solutions

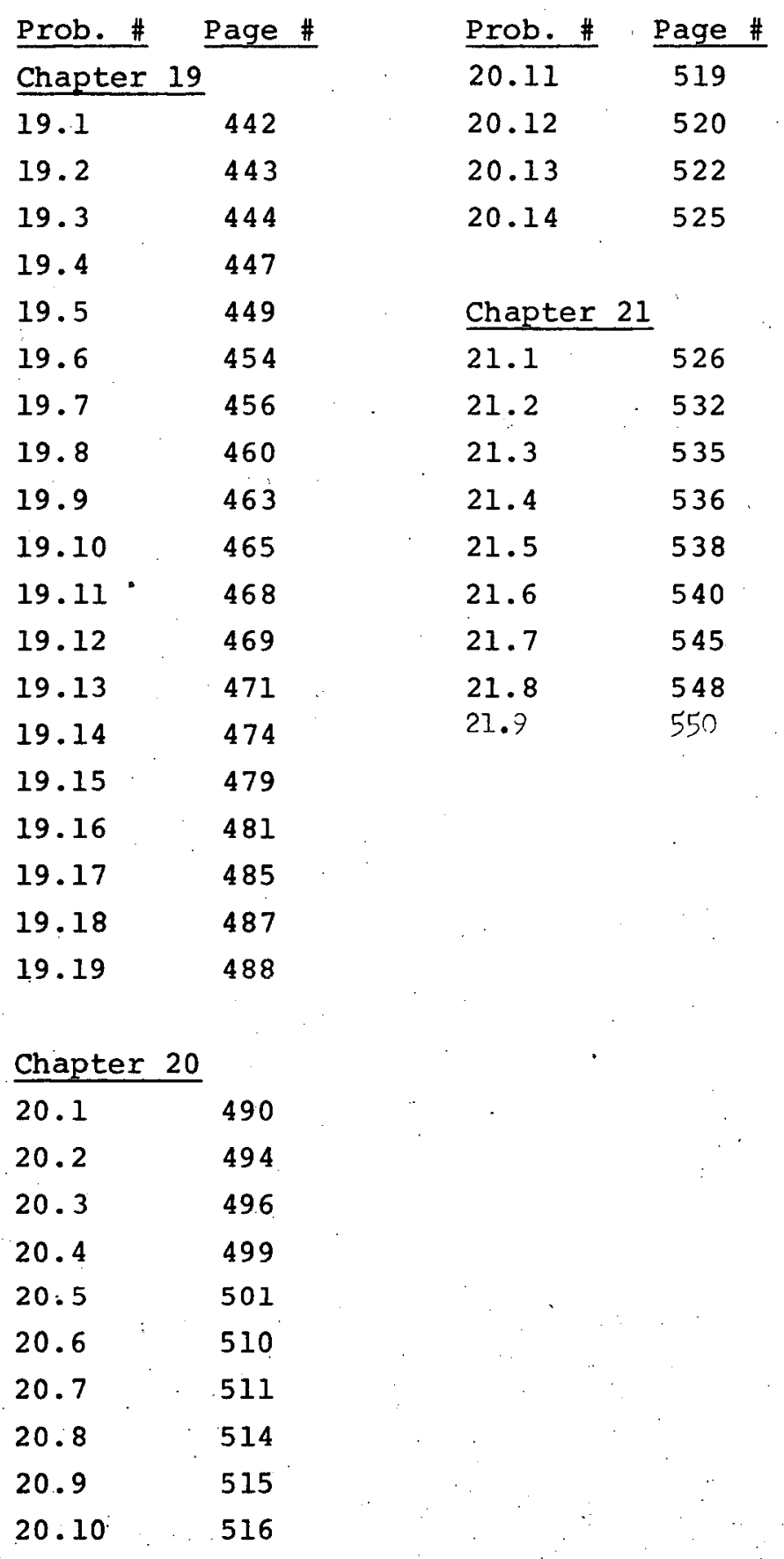


CHAPTER 1

Problem 1.1

The canonical partition function of an ideal gas of particles obeying Fermi-Dirac statistics is

$$
\ln Z=-\mu \frac{\mu N}{k T}+\sum_{j} \ln \left[1+\exp \left(-\frac{\epsilon_{j}-\mu}{k T}\right)\right]
$$

from Eq. (1.51).

However from Eq.(1.15), average energy per particle is

$$
\begin{aligned}
& U_{F}=\frac{k T^{2}}{N}\left(\frac{\partial \ln Z}{\partial T}\right)_{V}=-\frac{k}{N}\left(\frac{\partial \ln Z}{\partial(1 / T)}\right)_{V} \\
& \therefore\left(\frac{\partial \ln Z}{\partial(1 / T)}\right)_{V}=-\left(\frac{\mu N}{k}+\frac{N}{k T}\left(\frac{\partial \mu}{\partial(1 / T)}\right)_{V}\right) \\
& +\sum_{j} \frac{\partial}{\partial(1 / T)}\left\{\ln \left[1+\exp \left(-\frac{\varepsilon_{i}-\mu}{k T}\right)\right]\right\} \\
& \frac{\partial}{\partial(1 / T)}\left\{\ln \left[1+\exp \left(-\frac{\epsilon_{j}-\mu}{k T}\right)\right]\right\}=\frac{-\frac{\partial}{\partial(1 / T)}\left\{\left(\epsilon_{j}-\mu\right) / k T\right\} \cdot \exp \left(-\frac{\epsilon_{j}-\mu}{k T}\right)}{1+\exp \left(-\frac{\epsilon_{j}-\mu}{k T}\right)}
\end{aligned}
$$

But from Eq. (1.53)

$$
\bar{n}_{j}=\frac{\exp \left(-\frac{\epsilon_{j}-\mu}{k T}\right)}{1+\exp \left(-\frac{\epsilon_{j-\mu}}{k T}\right)}
$$

and $\frac{\partial}{\partial\left({ }^{\prime} / T\right)}\left\{\frac{\epsilon_{j}-\mu}{k T}\right\}=\frac{\epsilon_{j}-\mu}{k}-\frac{1}{k T}\left(\frac{\partial \mu}{\partial\left(I_{T}\right)}\right)_{V}$ 
2

Thus

$$
\begin{aligned}
& \sum_{j} \frac{\partial}{\partial\left({ }^{\prime} / T\right)}\left\{\ln \left[1+\exp \left(-\frac{\epsilon_{j}-\mu}{k T}\right)\right]\right\} \\
& \quad=-\sum_{j}\left\{\frac{\epsilon_{j}-\mu}{k}-\frac{1}{k T}\left(\frac{\partial \mu}{\partial\left(I^{\prime} / T\right)}\right)_{V}\right\} \overline{n_{j}} \\
& \quad=-\frac{1}{k} \sum_{j} \epsilon_{j} \overline{n_{j}}+\frac{\mu}{k} \sum_{j} \overline{n_{j}}+\frac{1}{k T}\left(\frac{\partial \mu}{\partial\left({ }^{\prime} / T\right)}\right)_{v} \sum_{j} \overline{n_{j}}
\end{aligned}
$$

But

$$
\begin{aligned}
& \therefore \sum_{j} \frac{\partial}{\partial(1 / T)}\left\{\ln \left[1+\exp \left(-\frac{\epsilon_{j}-\mu}{k T}\right)\right]\right\}=-\frac{1}{k} \sum_{j} \epsilon_{j} \bar{n}_{j}+\frac{\mu}{k} N \\
& +\frac{N}{k T}\left(\frac{\partial \mu}{\partial(1 / T)}\right)_{V} \\
& \therefore\left[\frac{\partial \ln z}{\partial(1 / T)}\right]_{V}=-\frac{\mu N^{\prime}}{k}-\frac{N}{k T}\left(\frac{\partial \mu}{\partial\left({ }^{\prime} / T\right)}\right)_{V}-\frac{1}{k} \sum_{j} \epsilon_{j} \dot{n}_{j} \\
& +\frac{\mu N^{\prime}}{k}+\frac{N}{k T}\left(\frac{\partial \mu}{\partial(1 / T)}\right)_{V} \\
& =-\frac{1}{k} \sum_{j} \epsilon_{j} \bar{n}_{j}
\end{aligned}
$$

Substituting this into Eq. (1.15)' we get,

$$
\therefore U_{F}=-\frac{k}{N}\left[-\frac{1}{k} \sum_{j} \epsilon_{j} \bar{n}_{j}\right]=\frac{1}{N} \sum_{j} \epsilon_{j} \bar{n}_{j}
$$

Approximating the summation to an integral

$$
U_{F} \cong \frac{1}{N} \int_{0}^{\infty} \varepsilon \bar{n}(\varepsilon) D(\varepsilon) d \varepsilon
$$


3

Problem 1.2

(a) Assuming that the substance is heated at constant pressure

$$
S=\int_{0}^{T} c_{p} \frac{d T}{T}
$$

Since phase transformations take place at constant temperatures, the corresponding entropy changes are given simply by

$$
\begin{gathered}
\Delta S=\frac{\Delta H}{T} \\
\therefore \quad S=\int_{0}^{T_{f}} C_{p s}(T) \frac{d T}{T}+\frac{\Delta H_{f}}{T_{f}}+\int_{T_{f}}^{T_{b}} C_{p l}(T) \frac{d T}{T}+\frac{\Delta H_{v}}{T_{b}} \\
+\int_{T_{b}}^{T} C_{p g} \frac{d T}{T}
\end{gathered}
$$

(where $C_{p g}=5 / 2 R$ for ideal gas)

(b)

$$
S=k\left[\ln Z+T\left(\frac{\partial \ln z}{\partial T}\right)_{V}\right]
$$

For ideal gas, $Z=Z_{t r}=\frac{z_{t r}^{N}}{N !}$

$$
\begin{aligned}
z_{t r} & =V\left(\frac{2 \pi m k T}{h^{2}}\right)^{3 / 2} \\
\therefore \ln z & =N \ln z_{t r}-\ln N ! \\
\text { then }\left(\frac{\partial \ln z}{\partial T}\right)_{V} & =N\left(\frac{\partial \ln z_{t r}}{\partial T}\right)_{V}
\end{aligned}
$$


4

$$
\begin{aligned}
\therefore\left(\frac{\partial \ln Z}{\partial T}\right)_{V} & =N \frac{\partial}{\partial T}\left[\ln V+\frac{3}{2} \ln \left(\frac{2 \pi m k}{h^{2}}\right)+\frac{3}{2} \ln T\right] \\
& =\frac{3}{2} N \frac{\partial \ln T}{\partial T}=\frac{3}{2} \frac{N}{T} \\
\therefore \quad S & =k\left[\ln Z+\frac{3}{2} N\right] \\
& =k\left[N \ln 3+x-\ln N !+\frac{3}{2} N\right] \\
& =k\left[N \ln \left\{V\left(\frac{2 \pi m k T}{h^{2}}\right)^{3 / 2}\right\}-\ln N !+\frac{3}{2} N\right]
\end{aligned}
$$

but $p V=N k T$ for ideal gas

and $\ln N ! \cong N \ln N-N$ (Stirling's approximation)

$$
\begin{aligned}
& \therefore \dot{S}=k\left[N \ln \left\{\frac{N k T}{P}\left(\frac{2 \pi m k T}{h^{2}}\right)^{3 / 2}\right\}-N \ln N+N\right. \\
& \therefore S=k N\left[\ln \left\{\frac{k T}{p}\left(\frac{2 \pi m k T}{h^{2}}\right)^{3 / 2}\right\}+5 / 2\right] .
\end{aligned}
$$


5

Problem 1.3

From Fermi-Dirac statistics, the average occupation number of fermions is

$$
\begin{aligned}
\bar{\eta}_{j}=\frac{1}{e^{\left(\alpha+\epsilon_{j} / k T\right)}+1} & =e^{-\left(\alpha+\epsilon_{j} / k T\right)}\left\{1+e^{-\left(\alpha+\epsilon_{j} / k T\right)}\right\}^{-1} \\
& =e^{-\left(\alpha+\epsilon_{j} / k T\right)}\left\{1-e^{-\left(\alpha+\epsilon_{j} / k T\right)}+e^{-2\left(\alpha+\epsilon_{j} / k T\right)}\right\}
\end{aligned}
$$

First order approximation gives Maxwell- Boltzmann statistics. Taking $2^{\text {nd }}$ order approximation we get,

$$
\begin{aligned}
\bar{n}_{j} & \doteq e^{-\left(\alpha+\epsilon_{j / k T}\right)}\left\{1-\epsilon^{-\left(\alpha+\epsilon_{j} / k T\right)}\right\} \\
& =e^{-\alpha} e^{-\epsilon_{j / k T}}-e^{-2 \alpha} e^{-2 \epsilon_{j} / k T}
\end{aligned}
$$

Energy distribution $n\left(\epsilon_{j}\right)=$ number of particles with energy $\epsilon_{j}=$ number of states available with energy $\epsilon_{j}$ multiplied by the probability of occupation of state $\epsilon_{j}$.

$$
\text { ie. } n\left(\epsilon_{j}\right)=D(j) \bar{n}_{j}
$$

Assume classical limit, ie energy continuum.

$$
\begin{aligned}
\therefore n(\epsilon) & =D(\epsilon) f(\epsilon) \\
& =2 \times \frac{V}{4 \pi^{2}}\left(\frac{2 m}{\hbar^{2}}\right)^{3 / 2} \sqrt{\epsilon}\left\{e^{-\alpha} e^{-\epsilon / k T}-e^{-2 \alpha} e^{-2 \epsilon / k T}\right\}
\end{aligned}
$$


6

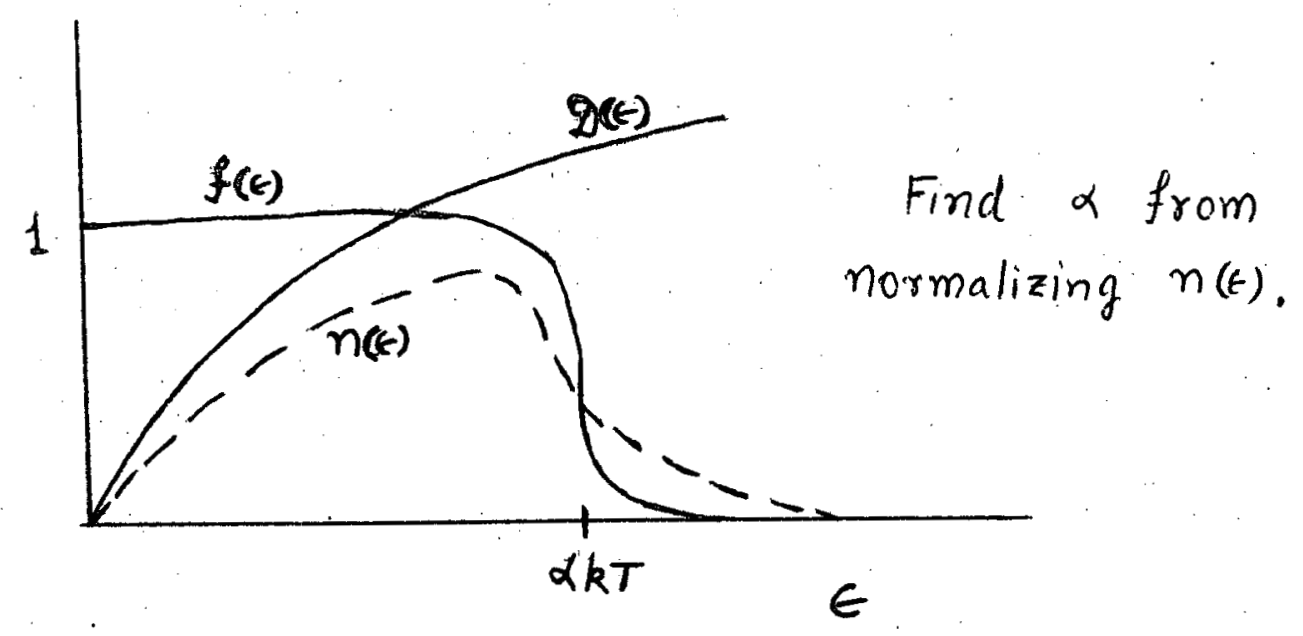

To justify classical limit it could be shown that $\alpha k T$ is far above discrete quantum levels.

Normalizing:

$$
\begin{gathered}
N=V n_{\text {tot }}=\int_{0}^{\infty} n(t) d \epsilon \\
N=\frac{V}{2 \pi^{2}}\left(\frac{2 m}{\hbar^{2}}\right)^{3 / 2}\left\{e^{-\alpha} \int_{0}^{\infty} \sqrt{\epsilon} e^{-\epsilon / k T} d t-e^{-2 \alpha} \int_{0}^{\infty} \sqrt{\epsilon} e^{-2 \epsilon / k T} d \epsilon\right\} \\
N=\frac{V}{2 \pi^{2}}\left(\frac{2 m}{\hbar^{2}}\right)^{3 / 2}\left\{\frac{e^{-\alpha} \sqrt{\pi}(k T)^{3 / 2}}{2}-\frac{e^{-2 \alpha} \sqrt{\pi}(k T)^{3 / 2}}{2^{5 / 2}}\right\} \\
N=Z\left(e^{-\alpha}-\frac{e^{-2 \alpha}}{2^{3 / 2}}\right)
\end{gathered}
$$

where $\quad Z=2 V\left(\frac{2 \pi m k T}{h^{2}}\right)^{3 / 2}=$ partition function of fermions 
7

Solving for $e^{-\alpha}:\left(e^{-\alpha}\right)^{2}-2^{3 / 2}\left(e^{-\alpha}\right)+\frac{2^{3 / 2} N}{z}=0$

$$
e^{-\alpha}=\sqrt{2} \pm \sqrt{2-\frac{2^{3 / 2} N}{z}}
$$

Since we want $\alpha>0$, we choose negative sign Numerical estimate shows $Z>2^{1 / 2} N$, so the root is real.

Expand the root to and order

$$
\begin{aligned}
& e^{-\alpha}=\sqrt{2}\left(1-\sqrt{1-\frac{\sqrt{2} N}{Z}}\right) \\
& \approx \sqrt{2} \cdot\left(\frac{N}{\sqrt{2} z}+\frac{N^{2}}{4 Z^{2}}\right) \\
& =\frac{N}{Z}\left(1+\frac{1}{2 \sqrt{2}} \frac{N}{Z}\right) \\
& =\underbrace{\frac{n_{\text {tot }}}{2}\left(\frac{h^{2}}{2 \pi m k T}\right)^{3 / 2}}\left(1+\frac{n_{\text {tot }}}{2^{5 / 2}}\left(\frac{h^{2}}{2 \pi m k T}\right)^{3 / 2}\right) \\
& \text { value for } M-B \text { statistics } \equiv A \\
& \therefore e^{-\alpha}=A\left(1+\frac{A}{2 \sqrt{2}}\right)
\end{aligned}
$$

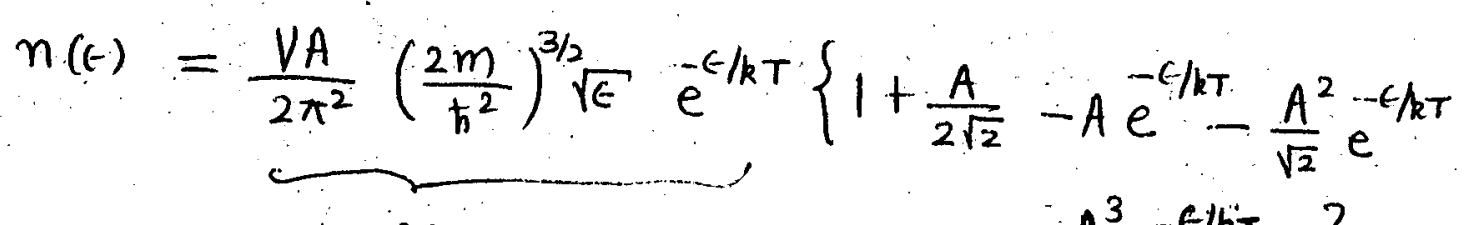

$$
\begin{aligned}
& \left.-\frac{A^{3}}{8} e^{-\epsilon / k T}\right\}
\end{aligned}
$$

the last four terms are Correction terms in decreasing
order of size 
8

$$
n(\epsilon)=n_{m-B}(\epsilon)\left\{\left(1+\frac{A}{2 \sqrt{2}}\right)-\left(A+\frac{A^{2}}{\sqrt{2}}+\frac{A^{3}}{8}\right) e^{-\epsilon / k T}\right\}
$$

(a) Average energy is

$$
\begin{aligned}
& E=\int_{0}^{\infty} \epsilon n(t) d \epsilon \\
& =\frac{1}{V \eta_{0 t}} \frac{V A}{2 \pi^{2}}\left(\frac{2 m}{\hbar^{2}}\right)^{3 / 2}\left\{\left(1+\frac{A}{2 \sqrt{2}}\right) \int_{0}^{\infty} \epsilon^{3 / 2} e^{-\epsilon / k T} d \epsilon\right. \\
& \left.-\left(A+\frac{A^{2}}{\sqrt{2}}+\frac{A^{3}}{8}\right) \int_{0}^{\infty} \epsilon^{3 / 2} e^{-2 C / k T} d \epsilon\right\} \\
& =\frac{2}{\sqrt{\pi}(k T)^{3 / 2}}\left\{\left(1+\frac{A}{2 \sqrt{2}}\right) \frac{3}{4} \sqrt{\pi}(k T)^{5 / 2}-\left(A+\frac{A^{2}}{\sqrt{2}}+\frac{A^{3}}{8}\right) \frac{3}{4} \sqrt{\pi} \cdot\left(\frac{k T}{2}\right)^{5 / 6}\right\} \\
& =\frac{3}{2} k T\left\{1+\frac{A}{2 \sqrt{2}}-\frac{A}{4 \sqrt{2}}-\frac{A^{2}}{8}-\frac{A^{3}}{32 \sqrt{2}}\right\} \\
& \text { ignore } \\
& =\frac{3}{2} k T\left(1+\frac{A}{4 \sqrt{2}}\right), \quad A=\frac{n_{t o t}}{2}\left(\frac{h^{2}}{2 \pi m k T}\right)^{3 / 2} \\
& =\frac{(N / N)}{2} \lambda^{3}
\end{aligned}
$$


9

The upper limit on $e^{-\alpha}$ is approximately the maximum value of $A$ : for neutrons,

$$
\begin{aligned}
A_{n}=\frac{n_{\text {tot }}}{2} & \left(\frac{h^{2}}{2 \pi m k T}\right)^{3 / 2}=\frac{n_{\text {tot }}}{2}\left(\frac{h^{2} c^{2}}{2 \pi m c^{2}}\right)^{3 / 2}\left(\frac{1}{k T}\right)^{3 / 2} \\
& \cong \frac{n_{\text {tet }}}{2}\left(\frac{10^{-42} \mathrm{meV}^{2} \times 10^{17} \mathrm{~cm}^{2}}{10 \times 10^{3} \mathrm{mlev}}\right)^{3 / 2}\left(\frac{10^{6}}{k T(\mathrm{kV})}\right)^{3 / 2} \\
& \approx n_{\text {tot }}\left(\mathrm{cm}^{-3}\right)(\mathrm{kT}(\mathrm{ev}))^{-3 / 2} \times 10^{-35}
\end{aligned}
$$

for $n_{\text {tot }}=10^{10} \mathrm{~cm}^{-3}, \quad A_{n} \cong(k T(\mathrm{ev}))^{-3 / 2} \times 10^{-25}$

$$
\therefore \text { for } T=1 \times K \sim 10^{-4} \mathrm{eV}, A_{n} \approx 10^{-19}
$$

Thus the expression of the F-D $\overline{n_{j}}$. must converge very quickly and the correction to the M-B value is very small.

(b) It is stated in Spitz, Modern Theory of Solids (c hl) that the approximation is good for most metals below their melting point. $\left(k T \ll \epsilon_{f}\right)$ 
10

Problem 1.4

The method derived in the text for calculating $Z$ is nit applicable here because of the small number of particle. Instar, we must count states of the system. For fermiDire purines with spin 1/2, there cere venery state $\times 2$ sid states $=6$ single-garticte states. The first piorticte con be in in y at the 6 states, the second can be placed in in y of the 5 remaining states. Therefore, the number if states of the system is $6 \times 5 / 2=15$, whose the denomin. altar 2 accounts for the inilistinguistablity of the twi particles. The distinct stem of the system are shown below. with the total energy of the state initiated.
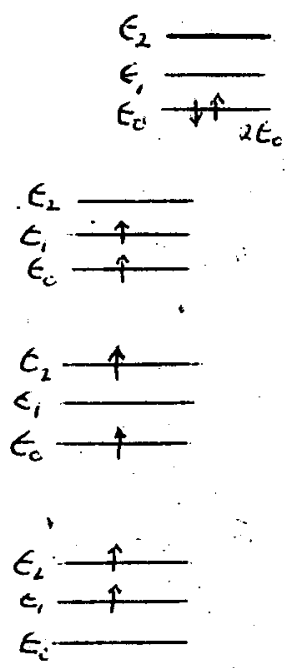
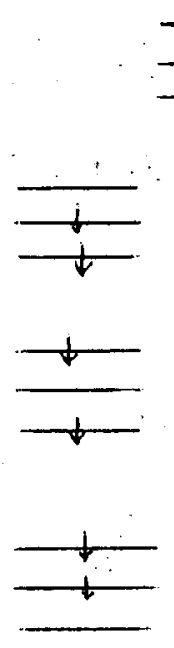
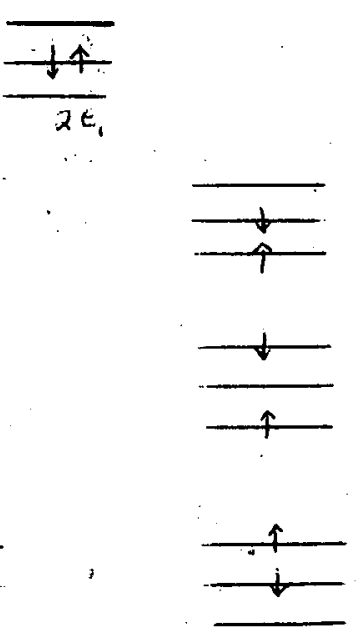
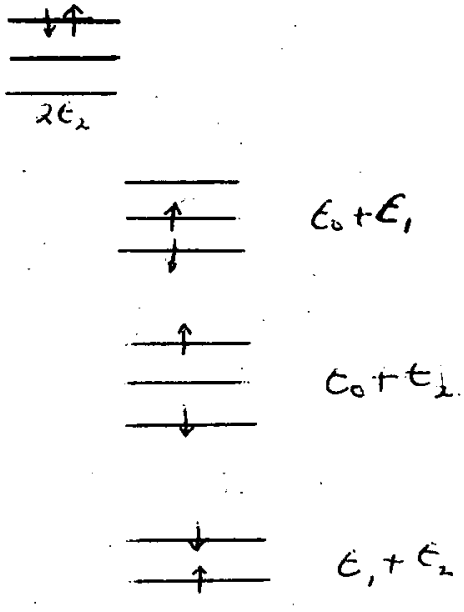

The partition function is:

$$
\begin{aligned}
Z= & e^{-\lambda \epsilon_{c} / k T}+e^{-\lambda \epsilon_{1} / k T}+e^{.2 \epsilon_{2} / k T}+4 e^{-\left(\epsilon_{c}+\epsilon_{1}\right) / k T}+4 e^{-\left(\epsilon_{c}+\epsilon_{2}\right) / k T} \\
& +4 e^{-\left(\epsilon_{1}+\epsilon_{2}\right) / k T}
\end{aligned}
$$


11

Problem 1.5

(a) Consider an equation it state of the form $p(V, T)$. The differential of $\beta$ is:

$$
d f=\left(\frac{\partial \rho}{\partial T}\right)_{V} d T+\left(\frac{\partial \rho}{\partial V}\right)_{\tau} d V
$$

or, at constant pressure.

$$
O=\left(\frac{\partial \rho}{\partial T}\right)_{V}+\left(\frac{\partial \beta}{\partial V}\right)_{T}\left(\frac{\partial V}{\partial T}\right)_{\beta}
$$

or $\left(\frac{\partial v}{\partial T}\right)_{p}=-\frac{\left(\frac{\partial p}{\partial r}\right)_{v}}{\left(\frac{\partial p}{\partial v}\right)_{T}}$

By definition $\alpha \equiv \frac{1}{V}\left(\frac{\partial V}{\partial T}\right)_{p}=-\frac{1}{V} \frac{\left.\frac{\partial p}{\partial T}\right)_{V}}{\left(\frac{\partial p}{\partial V}\right)_{T}}[$ frem(2)]

and $\beta \equiv-\frac{1}{V}\left(\frac{\partial V}{\partial \beta}\right)_{T}=-\frac{1}{V} \frac{1}{\left(\frac{\partial p}{\partial v}\right)_{T}}$

From $E_{q}(1.28) \quad p=k T\left(\frac{\ln z}{\partial V}\right)_{T}$

so

$$
\left(\frac{\partial p}{\partial V}\right)_{T}=k T\left(\frac{\partial^{2} \ln z}{\partial V^{2}}\right)_{T}
$$


12

and $\left(\frac{\partial p}{\partial T}\right)_{V}=k T \cdot \frac{\partial}{\partial T}\left[\left(\frac{\partial \ln z}{\partial V}\right)_{T}\right]_{V}+k\left(\frac{\partial \ln Z}{\partial V}\right)_{T}$

$\therefore$ Substituting (5) and (6) into (3) and (5) into (4)

$$
\alpha=-\frac{1}{V T}\left[\frac{T \frac{\partial^{2} \ln z}{\partial T \partial V}+\left(\frac{\partial \ln z}{\partial V}\right)_{T}}{\left(\frac{\partial^{2} \ln z}{\partial V^{2}}\right)_{T}}\right]
$$

and

$$
\beta=-\frac{1}{k T V} \frac{1}{\left(\frac{\partial^{2} \ln z}{\partial V^{2}}\right)_{T}}
$$

(b) From eq. (2) and the definitions of $\alpha$ and $\beta$

$$
\left(\frac{\partial p}{\partial T}\right)_{V}=-\frac{\left(\frac{\partial V}{\partial T}\right)_{\beta}}{\left(\frac{\partial V}{\partial p}\right)_{T}}=-\left(\frac{V \alpha}{-V \beta}\right)=\frac{\alpha}{\beta}
$$

Integrating this $\Delta p=\frac{\alpha}{\beta} \Delta T$ at constant volume.

$$
\therefore \Delta p=\frac{15 \times 10^{-5}}{5.4 \times 10^{-11}} \times 1=\frac{3.33 \times 10^{6} \mathrm{~N}^{\prime} \mathrm{m}^{2}}{(33 \mathrm{~atm})}
$$

(c) By definition

$$
\begin{aligned}
& C_{p}=\left(\frac{\partial H}{\partial T}\right)_{p}, \quad C_{V}=\left(\frac{\partial U}{\partial T}\right)_{V} \quad(10) \\
\therefore \quad & C_{p}-C_{V}=\left(\frac{\partial H}{\partial T}\right)_{p}-\left(\frac{\partial U}{\partial T}\right)_{V} \\
\therefore \quad & =\left(\frac{\partial U}{\partial T}\right)_{p}+p\left(\frac{\partial V}{\partial T}\right)_{p}-\left(\frac{\partial U}{\partial T}\right)_{V} \quad \begin{array}{c}
\text { (since } \\
U+p V=H)
\end{array}
\end{aligned}
$$

But $\left(\frac{\partial U}{\partial T}\right)_{p}=\left(\frac{\partial U}{\partial T}\right)_{V}+\left(\frac{\partial U}{\partial V}\right)_{T}\left(\frac{\partial V}{\partial T}\right)_{p}$

substituting this into the above

$$
C_{p}-C_{V}=\left(\frac{\partial U}{\partial V}\right)_{T}\left(\frac{\partial V}{\partial T}\right)_{p}+p\left(\frac{\partial V}{\partial T}\right)_{p}
$$


13

Using of (3) we get,

$$
C_{p}-C_{V}=\left(\frac{\partial U}{\partial V}\right)_{T} V_{\alpha}+p V_{\alpha}
$$

From part (d), $\varepsilon_{q}(z z)$ :

$$
\begin{aligned}
& \left(\frac{\partial U}{\partial V}\right)_{T}=\frac{\alpha}{\beta} T-p \\
\therefore \quad & C_{p}-C_{V}=\left(\frac{\alpha}{\beta} T-p\right) V \alpha+p V \alpha=\frac{\alpha^{2}}{\beta} V T
\end{aligned}
$$

for an ideal gas, $\beta V=R T$ and

$$
\begin{aligned}
\alpha & =\frac{1}{V} \frac{R}{\gamma}=\frac{1}{T} ; \quad \beta=\frac{1}{V} \frac{R T}{\gamma^{2}}=\frac{V}{R T} . \\
: \frac{c_{p}-c_{V}}{c_{p}} & =\frac{\left(\alpha^{2} / \beta\right) V T}{\frac{5}{2} R}=\frac{1}{T} \frac{R T}{V} V T / \frac{s}{2} R=0.4
\end{aligned}
$$

for mercury:

$$
\begin{aligned}
& \frac{c_{p}-C_{p}}{C_{p}}=\frac{\left(\alpha^{2} / \beta\right) V T}{c_{i}} \text {; at } 0^{\circ} \mathrm{C} ; \quad V=0.074 \mathrm{~cm}^{3} / \mathrm{gm}_{0}, C_{p}=0.14 \frac{\mathrm{J}}{\mathrm{gnq}} \\
& =\frac{\left(18 \times 10^{-5}\right)^{2}}{5.4 \times 10^{-11}} \frac{(0.074)(273)}{(0.14) \times 10^{6}}=0.087
\end{aligned}
$$

(d) The tat law of thermolnamics states

$$
d v=T d s-p d v
$$

or

$$
\begin{aligned}
\text { or } \quad\left(\frac{\partial U}{\partial V}\right)_{T} & =T\left(\frac{\partial S}{\partial V}\right)_{T}-\beta \\
\text { consider } \quad d F & =-g d V-S d T=\left(\frac{\partial F}{\partial V}\right)_{T} d V+\left(\frac{\partial F}{\partial T}\right)_{V} d T
\end{aligned}
$$

identifying coefficients of $d V$ and $d T$ in $E_{g}(18)$ :

$$
\left(\frac{\partial F}{\partial V}\right)_{T}=-\rho \quad \text { and }\left(\frac{\partial F}{\partial T}\right)_{V}=-S
$$


14

By forming mixed second derivatives:

$$
-\frac{\partial^{2} F}{\partial V \partial T}=\left(\frac{\partial \beta}{\partial T}\right)_{V}=\left(\frac{\partial S}{\partial V}\right)_{T} \quad \text { (Maxwell relation) }
$$

Substituting. Eg (20) into Eg (17):

$$
d U=\left(\frac{\partial U}{\partial T}\right)_{V} d T+\left(\frac{\partial U}{\partial V}\right)_{T} d V
$$

so by comparison between (20) \& (21)

$$
\left(\frac{\partial U}{\partial V}\right)_{T}=T\left(\frac{\partial p}{\partial T}\right)_{V}-p
$$

From part (a) eq. (9)

$$
\left(\frac{\partial U}{\partial V}\right)_{T}=\frac{\alpha}{\beta} T-p
$$

Now differentiating w.r.t. $V$ with constant $T$

$$
\left(\frac{\partial^{2} U}{\partial V^{2}}\right)_{T}=T\left(\frac{\partial(\alpha / \beta)}{\partial V}\right)_{T}-\left(\frac{\partial p}{\partial V}\right)_{T}
$$

Using eq. (4) in part (a), ie: $\left(\frac{\partial p}{\partial v}\right)_{T}=-\frac{1}{\beta V}$

$$
\left(\frac{\partial^{2} U}{\partial V^{2}}\right)_{T}=\frac{T}{\beta}\left(\frac{\partial \alpha}{\partial V}\right)_{T}-T \frac{\alpha}{\beta^{2}}\left(\frac{\partial \beta}{\partial V}\right)_{T}+\frac{1}{\beta V}
$$

$$
\text { but }\left(\frac{\partial \beta}{\partial V}\right)_{T}=\left(\frac{\partial \beta}{\partial p}\right)_{T}\left(\frac{\partial p}{\partial V}\right)_{T}=-\left(\frac{\partial \beta}{\partial p}\right)_{T} \frac{1}{\beta V} \quad \text { [From eq. (24)] }
$$

Thus $\left(\frac{\partial^{2} U}{\partial V^{2}}\right)_{T}=\frac{1}{\beta V}+\frac{T \alpha}{\beta^{3} V}\left(\frac{\partial \beta}{\partial p}\right)_{T}+\frac{T}{\beta}\left(\frac{\partial \alpha}{\partial V}\right)_{T}$

but. $\left(\frac{\partial \alpha}{\partial V}\right)_{T}=\left(\frac{\partial \alpha}{\partial p}\right)_{T}\left(\frac{\partial p}{\partial V}\right)_{T}=-\frac{1}{\beta V}\left(\frac{\partial \alpha}{\partial p}\right)_{T} \quad$ [from (21)]

From the definition of $\alpha, \beta$

$$
\begin{aligned}
& \left(\frac{\partial \alpha}{\partial p}\right)_{T}=\frac{\partial}{\partial p}\left[\frac{1}{V}\left(\frac{\partial V}{\partial T}\right)_{p}\right]_{T}=-\frac{1}{V^{2}}\left(\frac{\partial V}{\partial p}\right)_{T}\left(\frac{\partial V}{\partial T}\right)_{p} \cdot 10 \approx \\
& +\frac{1}{v} \frac{\partial}{\partial p}\left[\left(\frac{\partial r}{\partial r}\right)_{p}\right]_{T}
\end{aligned}
$$


15

$$
\left(\frac{\partial \beta}{\partial T}\right)_{p}=\frac{\partial}{\partial T}\left[-\frac{1}{V}\left(\frac{\partial V}{\partial p}\right)_{T}\right]_{p}=\frac{1}{V^{2}}\left(\frac{\partial V}{\partial T}\right)_{p}\left(\frac{\partial V}{\partial p}\right)_{T}-\frac{1}{V} \frac{\partial}{\partial T}\left[\left(\frac{\partial V}{\partial p}\right)_{T}\right]_{p}
$$

Thus $\left(\frac{\partial \alpha}{\partial p}\right)_{T}{ }^{\cdot}=-\left(\frac{\partial \beta}{\partial T}\right)_{p}$

(28) [rom the previous
two eq? s.]

$$
\begin{aligned}
\therefore\left(\frac{\partial \alpha}{\partial V}\right)_{T} & =-\frac{1}{\beta V}\left(\frac{\partial \alpha}{\partial p}\right)_{T}[\operatorname{frcm}(27)] \\
& =\frac{1}{\beta V}\left(\frac{\partial \beta}{\partial T}\right)_{p} \quad[\text { from (29)] }
\end{aligned}
$$

Substituting (29) into (26) we get,

$$
\left(\frac{\partial^{2} U}{\partial V^{2}}\right)_{T}=\frac{1}{\beta V}\left[1+T \frac{\alpha}{\beta^{2}}\left(\frac{\partial \beta}{\partial p}\right)_{T}+\frac{T}{\beta}\left(\frac{\partial \beta}{\partial T}\right)_{p}\right]
$$

(30) 
16

Problem 1.6

For an ideal gas

$$
\begin{aligned}
& Z=\frac{z_{t r}^{N}}{N !} \\
& z_{t r}=V\left(\frac{2 \pi m k T}{h^{2}}\right)^{3 / 2} \\
& \therefore \ln Z=N \ln z_{t r}-N \ln N+N \quad\left(\begin{array}{c}
\text { (using Stirling's } \\
\text { approximation) }
\end{array}\right.
\end{aligned}
$$

(a)

$$
\begin{aligned}
& \left.p=k T\left(\frac{\partial \ln z}{\partial V}\right)_{T} \quad[\text { From Eq. } 11.2 s)\right] \\
& \left(\frac{\partial \ln z}{\partial V}\right)_{T}=\frac{N}{z_{t r}}\left(\frac{\partial z_{t r}}{\partial V}\right)_{T}=\frac{N}{z_{t r}} \cdot \frac{z_{t r}}{V}=\frac{N}{V} \\
& \therefore \quad p=\frac{k T N}{V} \\
& \text { or } p V=N k T
\end{aligned}
$$

(b)

$$
\begin{aligned}
& S=k \ln z+k T\left(\frac{\partial \ln z}{\partial T}\right)_{V} \\
&\left(\frac{\partial \ln z}{\partial T}\right)_{V}=\frac{N}{z_{t r}}\left(\frac{\partial z_{r r}}{\partial T}\right)_{V}=\frac{N}{z_{t r}} V\left(\frac{2 \pi m k}{h^{2}}\right)^{3 / 2} \frac{3}{2} T^{1 / 2} \\
&=\frac{3}{2} \cdot \frac{N}{T}
\end{aligned}
$$

$$
\therefore \quad S=k N \ln \left[V\left(\frac{2 \pi m k T}{h^{2}}\right)^{3 / 2}\right]-k N \ln N+k N+\frac{3}{2} k N
$$




$$
\therefore \quad S=k N \ln \left[\frac{V}{N}\left(\frac{2 \pi m k T}{h^{2}}\right)^{3 / 2}\right]+\frac{5}{2} k N
$$

or $S=R \ln \left[\frac{P}{k T}\left(\frac{2 \pi m k T}{h^{2}}\right)^{3 / 2}\right]+\frac{5}{2} R$

[Using $R=k N$ and $P V=N k T$ ]

(c) From Prob. 1.5 e for ideal gas, $c_{p}=c_{v}+R, \mathrm{~m}$ :

$$
\begin{aligned}
& c_{p}=\left(\frac{\partial U}{\partial T}\right)_{V}+R=\frac{\partial}{\partial T}\left\{k T^{2}\left(\frac{\partial \ln z}{\partial T}\right)_{V}\right\}_{V}+R \\
& c_{p}=R\left\{2 T\left(\frac{\partial \ln z}{\partial T}\right)_{V}+T^{2}\left(\frac{\partial^{2} \ln z}{i T^{2}}\right)_{V}\right\}+R \\
& (\partial \ln z / \partial T)_{V}=3 N / 2 T \text { from fart }(f) \\
& \therefore c_{p}=k\left(3 N-\frac{3}{2} N\right)+R=\frac{3}{2} N R+R=\frac{5}{2} R
\end{aligned}
$$

(d)

$$
\begin{aligned}
Z & =\left(z_{t r} \cdot z_{\text {int }}\right)^{N} / N ! \\
\ln z & =N \ln \left(z_{t r} \cdot z_{\text {int }}\right)-N \ln N+N \\
z_{\text {int }} & =1+e^{-\Delta \varepsilon / k T} \\
\operatorname{let} z & =z_{t r \cdot} \cdot z_{\text {int }} \\
\frac{\partial z}{\partial V} & =\left(1+e^{-4 \varepsilon / k T}\right) \frac{\partial z_{t r}}{\partial V}=\frac{3}{V} \\
\left(\frac{\partial \ln Z}{\partial V}\right) & =\frac{N}{V} \quad \text { (unchanged) }
\end{aligned}
$$


18

Hence (a) part is unchanged, ie. $P V=N k T$.

Now

$$
\begin{aligned}
\left(\frac{\partial \ln z}{\partial T}\right)_{V} & =N\left(\frac{\partial \ln z+r .}{\partial T}\right)_{V}+N\left(\frac{\partial \ln f_{\text {int } t}}{\partial T}\right)_{V} \\
& =\frac{3}{2} N / T+N\left[\frac{\partial \ln \left(1+\bar{e}^{\Delta E / k T}\right)}{\partial T}\right]_{V}
\end{aligned}
$$

using $e^{-\Delta \varepsilon / k T} \ll 1$,

$$
\begin{aligned}
& \ln \left[1+e^{-\Delta \varepsilon / k T}\right] \cong e^{-\Delta \varepsilon / k T} \\
& \therefore \frac{\partial e^{-\Delta \varepsilon / k T}}{\partial T}=\frac{\Delta \varepsilon}{k T^{2}} e^{-\Delta \varepsilon / k T} \\
& \therefore\left(\frac{\partial \ln Z}{\partial T}\right)_{V}=\frac{3}{2} \frac{N}{T}+\frac{N}{T}\left(\frac{\Delta \varepsilon}{k T}\right) e^{-\Delta \varepsilon / k T} \\
& S=k N \ln \left[V\left(\frac{2 \pi m k T}{h^{2}}\right)^{3 / 2}\right]+k N \ln \left(1+e^{-\Delta \varepsilon / k T}\right)-k N \ln N \\
& \quad+k N+\frac{3}{2} k N+k N\left(\frac{\Delta \varepsilon}{k T}\right) e^{-\Delta \varepsilon / k T} \\
& \therefore \quad k \cong \ln \left[V\left(\frac{2 \pi m k T}{h^{2}}\right)^{3 / 2}\right]+k N e^{-\Delta \varepsilon / k T}-k N \ln N \\
& \quad+\frac{5}{2} k N+k N\left(\frac{\Delta \varepsilon}{k T}\right) e^{-A \varepsilon / k T}
\end{aligned}
$$

or $\quad S=k N \ln \left[\frac{V}{N}\left(\frac{2 \pi m k T}{h^{2}}\right)^{3 / 2}\right]+\frac{5}{2} k N+k N e^{-\Delta \varepsilon / k T}\left(1+\frac{\Delta \varepsilon}{k T}\right)$

additional term 
for the hat capacity, the furmulatroin part $(c)$ :

19

$$
c_{p}=k\left\{2 T\left(\frac{\partial \operatorname{La}_{a} z}{\partial \tau}\right)_{\nu}+T^{2}\left(\frac{\partial^{2} l_{n} z}{\partial T^{2}}\right)_{r}\right\}+R
$$

still holds because the electronic excitation does mut change the fuck that the gas is id bal. However, with electronic excitation,

$$
\left(\frac{\partial \ln Z}{\partial T}\right)_{V}=\frac{3}{2} \frac{N}{T}+\frac{N}{T}\left(\frac{\Delta \varepsilon}{x T}\right) e^{-\Delta t / R T}
$$

substituting in $c_{p}$ equation yields.

$$
c_{p}=\frac{5}{2} R+R\left(\frac{\Delta \varepsilon}{k T}\right)^{2} e^{-\Delta \varepsilon / k T}
$$

(additional term) 
20

Problem 1.7

$$
\text { let } e^{-\left(\epsilon_{k}-\mu\right) / k T}=a_{k}
$$

From Eq. (1.47)

From $E_{i} \cdot(1.48)$

$$
\bar{I}=\sum_{N=0}^{\infty}\left[\sum_{\left\{n_{0}, n_{1}\right\}} a_{0}^{n_{0}} a_{1}^{n_{1}}\right]=\left(\sum_{\left.n_{0}+n_{1}=N\right)}^{\infty} a_{0}^{n_{0}}\right)\left(\sum_{n_{1}=0}^{\infty} a_{1}^{n_{1}}\right)
$$

(to be shown equal)

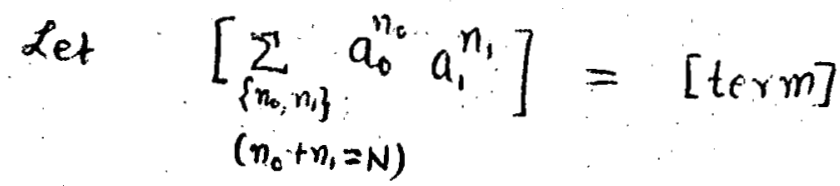

$$
\begin{aligned}
& N=0 \\
& n_{0}=n_{1}=0 \\
& \text { term }=1 \\
& N=1 \\
& n_{0}=1, \quad n_{1}=0 \quad \text { or } n_{0}=0, n_{1}=1 \\
& \text { term }=a_{0}+a_{1} \\
& N=2 \\
& \left.\left.\begin{array}{c}
n_{0}=2 \\
n_{1}=0 \\
a_{0}^{2}
\end{array}\right\} \text { or } \begin{array}{l}
n_{0}=1 \\
n_{0}=1
\end{array}\right\} \text { or } \begin{array}{l}
n_{0}=0 \\
n_{1}=2 \\
a_{1}^{2}
\end{array} \\
& \text { term }=a_{0}^{2}+a_{0} a_{1}+a_{1}^{2} \\
& N=3
\end{aligned}
$$

$$
\text { term }=a_{0}^{3}+a_{0}^{2} a_{1}+a_{0} a_{1}^{2}+a_{1}^{3}
$$


21

$N=4$

$$
\text { term }=a_{0}^{4}+a_{0}^{3} a_{1}+a_{0}^{2} a_{1}^{2}+a_{0} a_{1}^{3}+a_{1}^{4}
$$

Then RHS. of Eq. (1.47) gives,

$$
\begin{aligned}
\div= & 1+a_{0}+a_{1}+a_{0}^{2}+a_{0} a_{1}+a_{1}^{2}+a_{0}^{3}+a_{0}^{2} a_{1}+a_{0} a_{1}^{2} \\
& +a_{1}^{3}+a_{0}^{4}+a_{0}^{3} a_{1}+a_{0}^{2} a_{1}^{2}+a_{0} a_{1}^{3}+a_{1}^{4}+\ldots
\end{aligned}
$$

RHS of Eq. (1.48) gives.

$$
\begin{aligned}
\square= & \left(1+a_{0}+a_{0}^{2}+a_{0}^{3}+a_{0}^{4}+\cdots\right)\left(1+a_{1}+a_{1}^{2}+a_{1}^{3}+a_{1}^{4}+\cdots\right) \\
= & 1+a_{0}+a_{1}+a_{0}^{2}+a_{0} a_{1}+a_{1}^{2}+a_{0}^{3}+a_{0}^{2} a_{1}+a_{0} a_{1}^{2}+ \\
& a_{1}^{3}+a_{0}^{4}+a_{0}^{3} a_{1}+a_{0}^{2} a_{1}^{2}+a_{0} a_{1}^{3}+a_{1}^{4}+\cdots
\end{aligned}
$$

Thus both the series are identical up to the order 4 as seen above. Complete identity can be established by going to any arbitrary higher order. Therefore $E_{q}(1,47)$ and $E_{q}(1.48)$ give indention formulation 
22

CHAPTER 2

Problem 2:1

$$
\varepsilon_{i}=h \nu_{i}\left[\left(j_{i}+\frac{1}{2}\right)-x_{e}\left(j_{i}+\frac{1}{2}\right)^{2}\right]
$$

Since $\varepsilon_{i}$ is a function of $\left(j_{i}+\frac{1}{2}\right)$ and it is corresponding to some quantum number $j_{i}^{*}$.

Moreover for $j_{i}>j_{i}^{*} \varepsilon_{i}$ will decrease and these energy levels are meaningless. Hence wo have to consider only $0 \leq j_{i} \leq j_{i}^{*}$

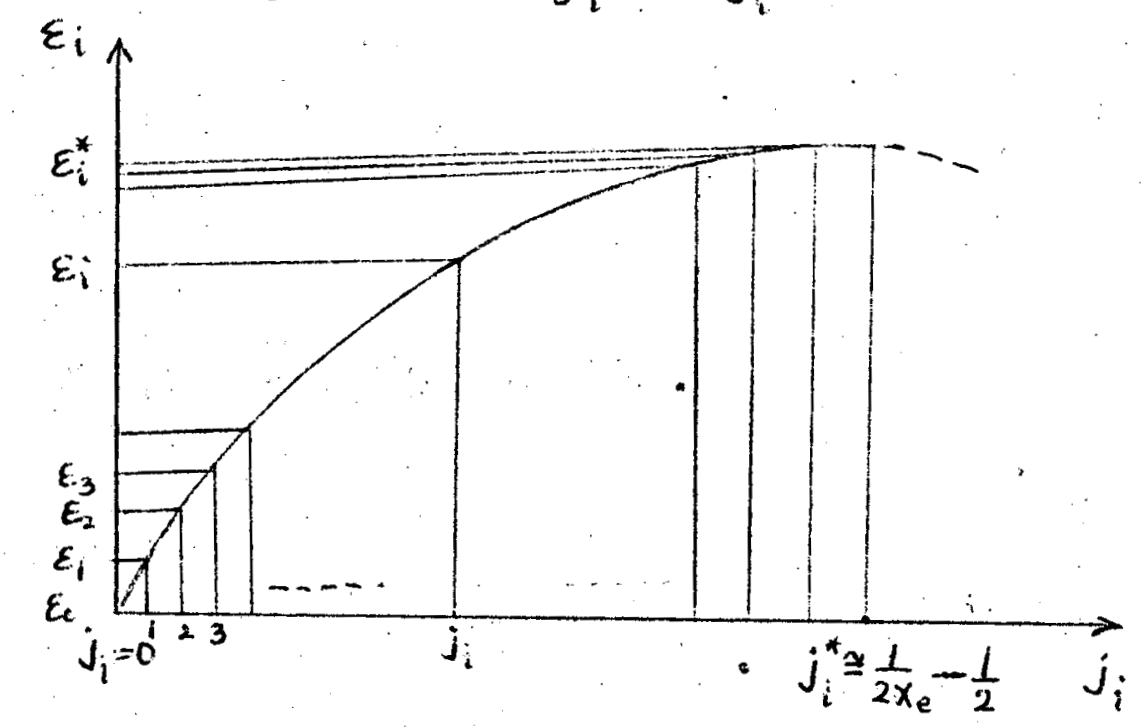

An approximate maximum quantum number is obtained by

$$
\left(\frac{d \varepsilon_{i}}{d j_{i}}\right)_{j_{i}^{*}}=0
$$

then $j_{i}^{*} \cong \frac{1-x_{e}}{2 x_{c}}$

and this is independent of modes. 
Since (1) can be wistten in the form

$$
\varepsilon_{i}=\frac{1}{2} h \nu_{i}\left(1-\frac{x_{i}}{2}\right)+h \nu_{i} j_{i}\left[1-x_{e}\left(j_{i}+1\right)\right]
$$

defining

$$
E_{c}=E_{c c h}+\sum_{i=1}^{3 N} \frac{1}{2} h \nu_{i}\left(1-\frac{x_{e}}{2}\right)
$$

the energy of a microstate of the system is

$$
E\left(j_{1}, j_{2}, j_{3} \ldots . . j_{3 N}\right)=E_{c}+\sum_{i=1}^{3 N} h \nu_{i} j_{i}\left[1-x_{e}\left(j_{i}+1\right)\right]
$$

Hence, the partition function for the crystal from Eq. (1.12) becomes

$$
Z=e^{-E_{0} / k T} \sum_{\left\{j_{1}, j_{2}, \ldots j_{i}, \ldots j_{3 N}\right\}} \exp \left\{-\frac{1}{k T} \sum_{i=1}^{3 N} h \nu_{i} j_{i}\left[i-x_{e}\left(j_{i}+1\right)\right]\right\}
$$

Now, in evaluating the sum. over microstates. since each of the quantum numbers $j_{i}$ may have any value from 0 to $j^{*} \cong\left(\frac{1}{2 x_{e}}-\frac{1}{2}\right)$, eq (2) may be
written as:

$$
z=e^{-E_{c} / k T} \prod_{i=1}^{3 N}\left\{\sum_{j=0}^{j^{*}} \exp \left\{-\frac{1}{k T} h \nu_{i} j_{i}\left[i-x_{e}\left(j_{i}+1\right)\right]\right\}\right\}
$$

Now define $z_{i}=\sum_{j=0}^{j} \exp \left\{-\frac{L}{k T} h \nu_{i} j_{i}\left[1-x_{e}\left(j_{i}+1\right)\right]\right\}$

then

$$
z_{i}=\sum_{j=0}^{j} \exp \left(-\frac{h \nu_{i}}{k T} j_{i}\right) \cdot \exp \left(\frac{h \nu_{i} x_{e}}{k T}\left(j_{i}^{2}+j_{i}\right)\right)
$$

Using the assumption that $X_{e}$ is so small that 
24

any exponential function which has $x_{e}$ in the argument can be approximated by a two-term Taylor series expansion.

$$
\exp \left\{\frac{h \nu_{i} x_{e}}{k T}\left(j_{i}^{2}+j_{i}\right)\right\}=1+\frac{h \nu_{i}}{k T} x_{e}\left(j_{i}^{2}+j_{i}\right)
$$

then (4) becomes,

$$
z_{i}=\sum_{j=0}^{k}\left[\exp \left(-\frac{h \nu_{i}}{k T} j_{i}\right)\right]\left(1+\frac{h \nu_{i}}{k T} x_{E}\left(j_{i}^{2}+j_{i}\right)\right)
$$

providing that $j_{i}^{2} \ll \frac{k T}{h \nu_{i} x_{l}}$

However, for $j_{i} \cong j^{*} \cong \frac{1}{2 x_{e}}-\frac{1}{2}$

$$
\begin{aligned}
& \exp \left\{-\frac{h \nu_{i}}{k T} j_{i}\left[1-x_{e}\left(j_{i}+1\right)\right]\right\}=\exp \left\{-\frac{h \nu_{i}}{k T} \frac{1}{2}\left(\frac{1}{x_{e}}-1\right) \frac{i}{2}\left(x_{e+1}\right)\right\} \\
& \cong \exp \left\{-\frac{h \nu_{i}}{k T} \frac{1}{4 x_{e}}\right\}
\end{aligned}
$$

This means that even if $j_{i}^{2} \ll \frac{k T}{h v_{i} x_{e}}$ is not satisfied for $j_{i} \cong j^{*} \exp \left\{-\frac{h \nu_{i}}{k T} j_{i}\right\}$ term is so small that the approximation in eq.(e) may, be valid for all $j_{i}$ as $\exp \left(-\frac{h \nu_{i}}{k T} j_{i}\right)$ is the multiplying factor. Moreover, the finite sum over $j i$ from $O$ to $j^{*}$ can be replaced by infinite sum and it then converges.

$$
Z_{i} \cong \sum_{j=0}^{\infty}\left[1+\frac{h \nu_{i}}{k T} x_{e}\left(j_{i}^{2}+j_{i}\right)\right] e^{-\frac{h \nu_{i}}{k T} j_{i}}
$$

for simplicity let $\frac{h \nu_{i}}{k T}=u_{i}$

then $z_{i}=\sum_{j_{i}=0}^{\infty} e^{-u_{i} j_{i}}+u_{i} x_{e} \sum_{j_{i}=0}^{\infty}\left(j_{i}{ }^{2}+j_{i}\right) e^{-u_{i} j_{i}}$ 
25

We know that $\sum_{j_{i}=0}^{\infty} e^{-u_{i} j_{i}}=\frac{1}{1-e^{u_{i}}}$

and $\sum_{j_{i}=0}^{\infty}\left(j_{i}^{2}+j_{i}\right) e^{-u_{i} j_{i}}=\frac{\partial^{2}}{\partial u_{i}^{2}} \sum_{j_{i}=0}^{\infty} e^{-u_{i} j_{i}}-\frac{\partial}{\partial u_{i}} \sum_{j_{i}=0}^{\infty} e^{-u_{i} j_{i}}$

$$
=\frac{\partial^{2}}{\partial u_{i}^{2}}\left(\frac{1}{1-e^{u_{i}}}\right)-\frac{\partial}{\partial u_{i}}\left(\frac{1}{1-e^{u_{i}}}\right)
$$

$$
\begin{aligned}
& =\frac{\partial}{\partial u_{i}}\left[\frac{-e^{-u_{i}}}{\left(i-e^{-u_{i}}\right)^{2}}\right]+\frac{e^{-u_{i}}}{\left(1-e^{\left.-u_{i}\right)^{2}}\right.} \\
& =\frac{e^{-u_{i}}}{\left(1-e^{\left.-u_{i}\right)^{2}}\right.}+\frac{2 e^{-i u_{i}} e^{-u_{i}}}{\left(1-e^{-u_{i}}\right)^{3}}+\frac{e^{-u_{i}}}{\left(1-e^{-u_{i}}\right)^{2}} \\
& =\frac{2 e^{-u_{i}}}{\left(1-e^{-u_{i}}\right)^{2}}+\frac{2 e^{2 u_{i}}}{\left(1-e^{-u_{i}}\right)^{3}} \\
& =\frac{2 e^{-u_{i}}}{\left(1-\bar{e}^{u_{i}}\right)^{3}}
\end{aligned}
$$

Substituting this result into (s) we get,

$$
z_{i}=\frac{1}{1-e^{-n \nu_{i} / k r}}+2 x_{e} \frac{h \nu_{i}}{k r} \frac{e^{-\frac{h i}{k T}}}{\left(1-e^{-\frac{h_{k}}{k T}}\right)^{3}}
$$

Then frow eq. (3) the total partition fiction becomes.

$$
Z=e^{-E_{0} / k T} \prod_{i=1}^{3 N}\left[\frac{1}{\left(1-e^{-\frac{h \nu_{i}}{k T}}\right)}+2 x_{i}\left(\frac{h \nu_{i}}{k T}\right) \frac{e^{-\frac{h \nu_{i}}{k T}}}{\left(1-e^{-\frac{h V_{i}}{k T}}\right)^{3}}\right]
$$

(ii) 
26

Problem 2.2

(a)

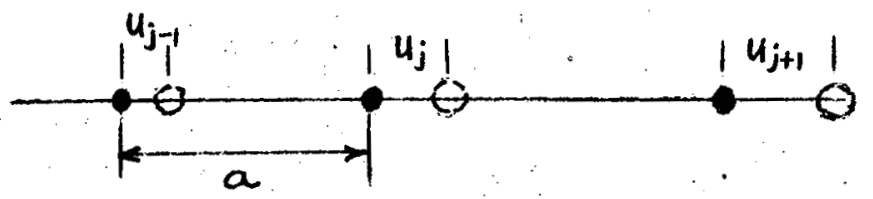

Force $a t j$ is given by

$$
\begin{aligned}
F_{j} & =k\left(u_{j+1}-u_{j}\right)-k\left(u_{j}-u_{j-1}\right) \\
& =m \frac{d^{2} u_{j}}{d t^{2}}=k\left(u_{j+1}-2 u_{j}+u_{j-1}\right)
\end{aligned}
$$

(b)

$$
\begin{aligned}
u_{j} & =A \sin (j a k) e^{-i \omega t} \\
\frac{d u_{j}}{d t} & =-i \omega A \sin (j a k) e^{-i \omega t} \\
\frac{d^{2} u_{j}}{d t^{2}} & =-\omega^{2} A \sin (j u k) e^{-i \omega t}
\end{aligned}
$$

Substituting for $u_{j}$ in eq.(1) we get,

$$
\begin{aligned}
-\omega^{2} m A \sin (j a k) e^{-i \omega t}= & k[A \sin [j+1) a k] e^{-i \omega t} \\
& \left.\left.-2 A \sin (j a k) e^{-i \omega t}+A \sin (j-1) a k\right] c\right] \\
\therefore-\frac{m \omega^{2}}{k} \sin (j a k)= & \sin (j a k) \cos (n k)+\cos (j a k) \sin (a k) \\
& -2 \sin (j a k)+\sin (j a k) \cos (a k) \\
& -\cos (j a k) \sin (a k) \\
\text { or } \frac{m \omega^{2}}{k}= & 2(1-\cos (a k))
\end{aligned}
$$

or $\omega=\left(\frac{4 k}{m}\right)^{1 / 2}\left|\sin \left(\frac{k a}{2}\right)\right|$ 
(c) Assume central differences

$$
\begin{aligned}
\frac{d u}{d x} \cong \frac{\delta u_{j}}{\Delta x} & =\frac{u_{j+1 / 2}-u_{j-j_{2}}}{\Delta x} \\
\frac{d^{2} u}{d x^{2}}=\frac{\delta^{2} u_{j}}{(\Delta x)^{2}} & =\frac{d u_{j+z_{2}}-d u_{j-1 / 2}}{(\Delta x)^{2}} \\
& =\frac{u_{j+1}-2 u_{j}+u_{j-1}}{(\Delta x)^{2}} \\
\therefore \frac{d^{2} u}{d x^{2}} & =\frac{u_{j+1}-2 u_{j}+u_{j-1}}{(\Delta x)^{2}} ; \Delta x=a \\
& =\frac{u_{j+1}-2 u_{j}+u_{j-1}}{a^{2}}
\end{aligned}
$$

Therefore from eq.(1) in part (a)

$$
\frac{m}{k a^{2}} \frac{d^{2} u}{d t^{2}} \cong \frac{d^{2} u}{d x^{2}}
$$

(d)

Generalizing the resilt of partec to three dimensions

$$
\frac{1}{v^{2}} \frac{\partial^{2} u}{\partial t^{2}}=\frac{\partial^{2} u}{\partial x^{2}}+\frac{\partial^{2} u}{\partial y^{2}}+\frac{\partial^{2} u}{\partial z^{2}}
$$

or in the finite difference form

$$
\begin{aligned}
\frac{1}{v^{2}} \frac{\partial^{2} u}{\partial t^{2}}= & \frac{\left(u_{j+1, k, \ell}-2 u_{j, k, \ell}+u_{j-1, k, \ell}\right)}{a^{2}}+\frac{\left(u_{j, k+1, \ell}-2 u_{j, k,}+u_{j, k+1}\right)}{a^{2}} \\
& +\frac{\left(u_{j, k, \ell+1}-2 u_{j, k, \ell}+u_{j, k, l-1}\right)}{a^{2}}
\end{aligned}
$$


28

for $\quad u_{j, k, l}=A \sin \left(j a \bar{k}_{x}\right) \sin \left(j a \bar{k}_{y_{j}}\right) \sin \left(j a \bar{k}_{z}\right) e^{-i a t}$

$$
\begin{aligned}
a_{s} \text { in part (b) } & \\
\therefore \frac{m \omega^{2}}{k}=2\left\{\left(1-\cos \left(a \bar{k}_{x}\right)\right)\right. & +\left(1-\cos \left(a \bar{k}_{y}\right)\right) \\
& \left.+\left(1-\cos \left(a F_{z}\right)\right)\right\}
\end{aligned}
$$

Note that this dispersion relation do rs not yield w as a function of $k=\sqrt{k_{x}^{2}+k_{y}^{2}+k_{z}^{2}}$ and so the density of states $Q$ (a) can not be evaluated by the arguments wised for the Debye model.

(e) For long colvelingthe $a \bar{b}_{x}, a \bar{k}_{y}, a \bar{k}_{z} \ll 1$ and $\cos (a k) \rightarrow 1-\frac{(a k)^{2}}{2}$

Then

$$
\begin{array}{r}
\frac{m \omega^{2}}{k} \rightarrow a^{2}\left(k_{\alpha}^{2}+k_{y}^{2}+k_{z}^{2}\right)=a^{2} k^{2} \\
{[\text { from eq u in part (d)] }}
\end{array}
$$

or $w=\sqrt{\frac{k a^{2}}{m}}$

or $a=c k$

and $c=\sqrt{\frac{k a^{2}}{m}}=$ Velocity of sound in the medium 
29

Problem 2.3

within the context of the Einstein model, graphite has two: characteristic vibration frequencies instead of one. The $N$ vibration modes representing vibrations perpendicular to the basal plane in Fig. 4.5 have a single frequency $\omega_{\perp}$ on an Einstein temperature:

$$
\theta_{1}=\frac{\hbar \omega_{1}}{k}<300 \% k
$$

Similarly, the $2 N$ modes for vibration garbled to the bios planes have a common frojuency $\omega_{11}$ or an Einstein temperature.

$$
\theta_{11}=\frac{\hbar \omega_{11}}{R}>300^{\circ} \mathrm{K}
$$

The frequency spectrum dor graphite in this model is:

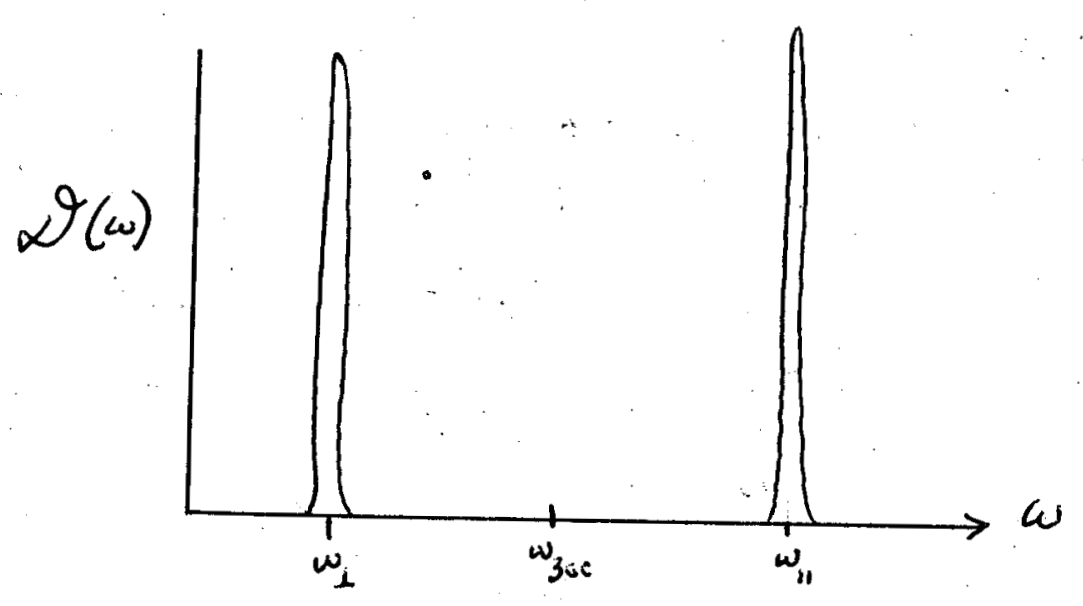

which are widely separated and fall on. either side of the frequency for an Einstein temperature at $300 \mathrm{ck}\left(\omega_{300}=\hbar / 300 k\right)$ The Frequency spectrum is:

$$
\theta(\omega)=N \delta\left(\omega-\omega_{1}\right)+2 N \delta\left(\omega-\omega_{11}\right)
$$


30

using $\sum_{f}(1)$ in $q(2.21)$ of the text and then determining 0 . by $\xi_{f}(1.15)$ and $c_{v}$ frown $(\partial V / \partial T)_{v}$ yields:

$$
c_{v}=R E\left(\frac{\theta_{1}}{T}\right)+2 R E\left(\frac{\theta_{11}}{T}\right)
$$

(2)

where $E(\theta / T)$ is the Einstein function:

$$
E(\theta / T)=\left(\frac{\theta}{T}\right)^{2} \frac{e^{\theta / T}}{\left[e^{\theta / T}-1\right]^{2}}
$$

(a) for $T=300^{\circ} K$,

$\frac{\theta_{1}}{306}<1$ and $E\left(\frac{\theta_{2}}{300}\right) \simeq 1$

$$
\frac{\theta_{11}}{3 c 0}>1 \text { and } E\left(\frac{\theta_{11}}{3 i c}\right) \simeq 0
$$

$\therefore u \operatorname{sing} \&(2)$

$$
C_{v}\left(3 \omega^{\circ} k\right)=R
$$

ie. at sou ck, only the $\perp$ modes od vibration are excited and they have attained the classical limit.

(b) In general, the variation of $C_{v}$ with $T$ for groghte es

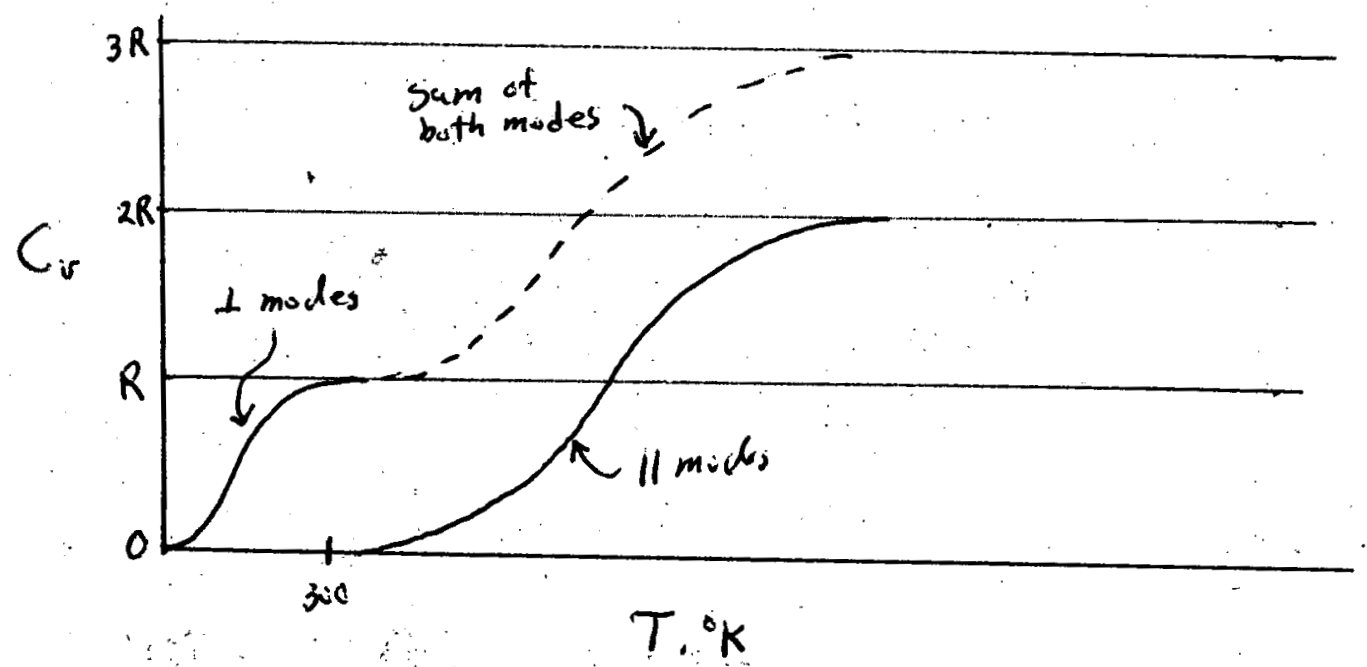


31

Problem 2.4

(a)

$$
\left.\begin{array}{rl}
D(\omega) & =3 N\left[\frac{3 \omega^{2}}{\omega_{0}^{3}}+\eta \delta\left(\omega \cdots \omega_{k}\right)\right] \\
& \text { for } 0<\omega<\omega_{4} \\
=0 & \text { for } \omega>\omega_{4}
\end{array}\right\}
$$

$D(\omega)$ must satisfy the normalization condition

$$
\int_{0}^{\omega_{4}} g(\omega) d w=3 N
$$

therefore $\quad 3 N \int_{0}^{\cos }\left[\frac{3 \omega^{2}}{\omega_{v}^{3}}+\eta \delta\left(\omega-\omega_{c}\right)\right] d \omega=3 N$. (3)

since $0<\omega_{B}<\omega_{G}$ and $0<\omega_{E}<\omega_{G}$ eq. (3) becomes

$$
\frac{\omega_{a}^{3}}{\omega_{b}^{3}}+\eta=1
$$

(b) From Eq.(2.21)

$$
\begin{aligned}
& \ln Z=-\frac{E_{c}}{k T}-\int_{c}^{\infty} D(\omega) \ln \left(1-e^{-\hbar \omega / k T}\right) d \omega \\
& =-\frac{E_{E}}{k T}-\int_{0}^{\omega_{G}} 3 N\left[\frac{3 \omega^{2}}{\omega_{0}^{3}}+\eta d\left(\omega-\omega_{E}\right)\right] \ln \left(1-e^{-h \omega T_{k T}}\right) d \omega \\
& =-\frac{E_{c}}{k T}-\frac{9 N}{\omega_{t}^{3}} \int_{c}^{\omega / \omega_{1}} \omega^{2} \ln \left(1-e^{-k \omega)_{k T}}\right) d \omega \\
& -3 N \eta \int_{0}^{\omega_{u}} \delta\left(\omega-\omega_{r}\right) \ln \left(1-e^{-\hbar \omega / k T}\right) d \omega \\
& \therefore \ln z=-\frac{E_{0}}{k T}-\frac{9 N}{\omega_{0}^{3}} \int_{0}^{\omega_{1}} \omega^{2} \ln \left(1-e^{-x \omega / k T}\right) d \omega \\
& -3 N \eta \ln \left(1-e^{-\frac{\hbar \omega_{E}}{k T}}\right)
\end{aligned}
$$


32

Since $c_{V}=\left(\frac{\partial U}{\partial T}\right)_{V}$ and $U=k T^{2}\left(\frac{\partial \ln z}{\partial T}\right)_{V}$

$$
\begin{aligned}
& U=E_{c}-k T^{2} \frac{a N}{\omega_{D}^{3}} \int_{\theta}^{\omega_{G}} \frac{\omega^{2}\left(-\frac{\hbar \omega}{k T^{2}}\right) e^{-\frac{k \omega}{k T}}}{\left(1-e^{-\frac{k \omega}{k T}}\right)} d \omega \\
& +3 N \eta \frac{k T^{2}\left(-\frac{h \dot{\omega}_{C}}{k T^{2}}\right) e^{-\frac{\hbar \omega_{E}}{k T}}}{\left(1-e^{-\frac{\hbar \omega_{i}}{k T}}\right)} \\
& =E+\frac{9 N \hbar}{\omega_{D}^{3}} \int_{0}^{\omega_{1}} \frac{\omega^{3}}{\left(e^{+h \omega}-1\right)} d \omega+\frac{3 N \eta \hbar \omega_{E}}{\left(e^{\frac{h t}{k T}}-1\right)} \\
& \therefore\left(\frac{\partial U}{\partial T}\right)_{v}=c_{r}=\frac{q N \hbar}{\omega_{D}^{3}} \int_{c}^{\omega_{\epsilon_{r}}} \frac{\left(-\frac{\hbar \omega}{k T^{2}}\right)\left(-e^{\frac{\hbar \omega}{k T}}\right)}{\left(e^{\frac{\hbar \omega}{k T}}-1\right)^{2}} \cdot \omega^{3} d \omega \\
& +\frac{3 N \eta \hbar \omega_{E}\left(-\frac{\hbar \omega_{k}}{k T^{2}}\right)}{\left(e^{\frac{\hbar \omega_{E}}{k T}}-1\right)^{2}}\left(-e^{\frac{\hbar \omega_{E}}{k T}}\right)
\end{aligned}
$$

Now define $\theta_{D}=\frac{\hbar \omega_{\nu}}{k}, \theta_{E}=\frac{\hbar \omega_{E}}{k}$ and $\theta_{i}=\frac{\hbar \omega_{G}}{k}$ let $x=\frac{\hbar \omega}{k T}$, then using the normalization condition (4) and $N_{k}=R$, we get,

$$
\begin{array}{r}
c_{V}=\left(\frac{\partial U}{\partial T}\right)_{V}=3 R\left\{3\left(\frac{T}{\theta_{G}}\right)^{3}(1-\eta) \int_{c}^{\theta_{B} / T^{x}} \frac{e^{4}}{\left(e^{x}-1\right)^{2}} d x\right. \\
\left.+\eta \frac{\left(\theta_{E / T}\right)^{2} e^{\theta_{E / T}}}{\left(e^{\theta_{E} / T}-1\right)^{2}}\right\}
\end{array}
$$

where $\left(\frac{T}{\theta_{D}}\right)^{3}=\left(\frac{T}{\theta_{h}}\right)^{3}\left(\frac{\theta_{G}}{\theta_{D}}\right)^{3}=\left(\frac{T}{\theta_{G}}\right)^{3}\left(\frac{\omega_{h}}{\sigma_{D}}\right)^{3}=\left(\frac{I}{\theta_{L}}\right)^{3}(1-\eta)$

$$
\therefore C_{V}=3 R\left\{\therefore(1-\eta) H\left(\theta_{\alpha} / T\right)+\eta E\left(\theta_{E / T}\right)\right\}
$$

was used. 
33

Problem 2.5

Consider a case of two atoms vibration in one dimension.
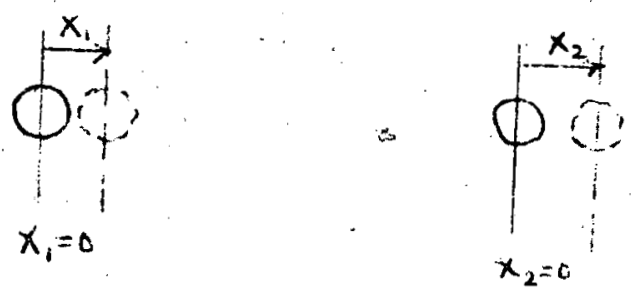

Total kinetic energy of the system is

$$
E_{k i n}=\frac{1}{2} m \dot{x}_{1}^{2}+\frac{1}{2} m \dot{x}_{2}^{2}
$$

and potential energy of the system is

$$
\begin{gathered}
E_{p i t}\left(x_{1}, x_{2}\right)=E_{p i t}(0,0)+\left(\frac{\partial E_{p i t}}{\partial x_{i}}\right)_{\substack{x_{1}=0 \\
x_{2}=0}}+\left(\frac{\partial E_{p i t}}{\partial x_{2}}\right) x_{2} \\
+\frac{1}{2} \sum_{i=c}^{2} \sum_{j=1}^{2} \frac{\partial^{2} E_{p i t}}{\partial x_{i} \partial x_{j}} x_{i} x_{j}
\end{gathered}
$$

Since at equilibrium $E_{p \rightarrow i}(0,0)=E_{i}$ and

$$
\begin{aligned}
& \frac{\partial E_{p r t}}{\partial x_{1}}=\frac{\partial E_{p r i}}{\partial x_{2}}=0, \quad d_{c} / \text { ming } \frac{\partial^{2} E_{p i}}{\partial x_{i} \partial x_{j}}=b_{i j} \\
& E_{p_{i e}}\left(x_{1}, x_{2}\right)=E_{0}+1\left(b_{1} x_{1}^{2}+2 b_{2} x_{1} x_{2}+b_{1}\right.
\end{aligned}
$$

$\left(b_{11} x_{1}^{2}+2 b_{12} x_{1} x_{2}+b_{22} x_{2}^{2}\right)$

$$
E=E_{c}+\frac{1}{2}\left[m\left(\dot{x}_{1}^{2}+x_{2}^{2}\right)+\left(b_{11} x_{1}^{2}+2 b_{12} x_{1} x_{2}+b_{22} x_{2}^{2}\right)\right]
$$

(A) 
34

(a) Vibration frequencies

Equation of motion of two atoms

$$
\begin{aligned}
& F_{1}=-\left(\frac{\partial E_{p i t}}{\partial x_{1}}\right)_{x_{2}}=-\left(b_{11} x_{1}+b_{12} x_{2}\right)=m \frac{d^{2} x_{1}}{d t^{2}} \\
& F_{2}=-\left(\frac{\partial E_{p t}}{\partial x_{2}}\right)_{x_{1}}=-\left(b_{12} x_{1}+b_{2,2} x_{2}\right)=m \frac{d^{2} x_{2}}{d t^{2}}
\end{aligned}
$$

Assuming the solutions in form of simple harmonic motion,

$$
x_{1}=c_{1} e^{-2 \pi i \nu t} \quad, x_{2}=c_{2} \epsilon^{-2 \pi i \nu t}
$$

and substituting them into (1) and (2), we get,

$$
\left.b_{12} c_{1}+b_{22} c_{2}=4 \pi^{2} 2^{2} m c_{2}\right\}
$$

or

$$
\left.\begin{array}{l}
\left(b_{11}-4 \pi^{2} \nu^{2} m\right) c_{1}+b_{12} c_{2}=0 \\
b_{12} c_{1}+\left(b_{22}-4 \pi^{2} \nu^{2} m\right) c_{2}=0
\end{array}\right\}
$$

For non trivial solution of eq. (4), the determinant of. coefficient matrix must vanish.

lie.

$$
\left|\begin{array}{cc}
b_{i 1}-4 \pi^{2} \nu^{2} m & b_{12} \\
b_{i 2} & b_{i 2}-4 \pi^{2} \nu^{2} m
\end{array}\right|=0
$$

er $\quad\left(b_{n}-4 \pi^{2} \nu^{2} m\right)\left(b_{i 2}-4 \pi^{2} \nu m\right)-b_{i 2}^{2}=0$ 
35

let $4 \pi^{2} 2^{2} m=a$

then $a^{2}-\left(b_{11}+b_{22}\right) a-\left(b_{12}^{2}-b_{11} b_{22}\right)=0$

solving for $a$

$$
\begin{aligned}
& a_{1}=\frac{1}{2}\left\{\left(b_{11}+b_{22}\right)-\sqrt{\left(b_{11}+b_{22}\right)^{2}+4\left(b_{12}^{2}-b_{11} b_{22}\right)}\right\} \\
& a_{2}=\frac{1}{2}\left\{\left(b_{11}+b_{22}\right)+\sqrt{\left(b_{11}+b_{22}\right)^{2}+4\left(b_{12}^{2}-b_{11} b_{22}\right)}\right\}
\end{aligned}
$$

for $a>0 ; \quad b_{12}^{2}<b_{11} b_{22}$ mist be held.

(b) Normal Mode Analysis:-

Now define new coordinates $q_{i}^{\prime} s$ as in Eq .(2.7)

$$
\begin{aligned}
& q_{1}=c_{11} x_{1}+c_{21} x_{2} \\
& q_{2}=c_{12} x_{1}+c_{22} x_{2}
\end{aligned}
$$

If the coefficients $c_{i j}$, are chosen according to the prescription Eq. (2.3), then

ie. for $i=1, l=1$

$$
\left.\begin{array}{ll}
i=1, l=2 & c_{11} c_{12}+c_{21} c_{22}=0 \\
i=2, l=1 & c_{12} c_{11}+c_{22} c_{21}=0
\end{array}\right\} \rightarrow c_{12} c_{11}+c_{22} c_{21}=0
$$

from $(10) \quad C_{11}^{2}=1-c_{21}^{2}$

from (12) $\quad c_{22}^{2}=1-c_{12}^{2}$ 
36

Substituting $C_{11}, C_{22}$ into (II) we get,

$$
\begin{aligned}
c_{12}^{2}\left(1-c_{21}^{2}\right) & =c_{21}^{2}\left(1-c_{12}^{2}\right) \\
\therefore c_{12}^{2} & =c_{21}^{2} \text { ie. } c_{12}= \pm c_{21}
\end{aligned}
$$

hence $\quad c_{11}^{2}=c_{2}^{2}=1-c_{i 2}^{2}$

so if $c_{12}=c_{21}$, from (11) $\quad c_{17}=-c_{22}$

if $\quad c_{12}=-c_{21}$, then $c_{11}=c_{22}$

Then with these coordinates, the original coordinates can be expressed as

$$
\begin{aligned}
& x_{1}=c_{11} q_{1}+c_{12} q_{2} \\
& x_{2}=c_{21} q_{1}+c_{22} q_{2}
\end{aligned}
$$

(c) Total Energy in Normal Mode Cocritinates

Substituting eq .(15) into eq. (A)

$$
\begin{aligned}
E=E_{0}+\frac{1}{2}\left[m\left(c_{11} \dot{q}_{1}+c_{12} \dot{q}_{2}\right)^{2}+m\left(c_{21} \dot{q}_{1}+c_{22} \dot{q}_{2}\right)^{2}\right. \\
+b_{11}\left(c_{11} q_{1}+c_{12} q_{2}\right)^{2}+2 b_{12}\left(c_{11} q_{1}+c_{12} q_{2}\right)\left(c_{21} q_{1}+c_{22} q_{2}\right) \\
\left.+b_{22}\left(c_{21} q_{1}+c_{22} q_{2}\right)^{2}\right] \ldots(16) \\
+E_{1}+\frac{1}{2} m\left[\left(c_{11}^{2}+c_{21}^{2}\right) \dot{q}_{1}^{2}+\left(c_{12}^{2}+c_{22}^{2}\right) \dot{q}_{2}^{2}+2\left(c_{11} c_{12}+c_{21} c_{22}\right) \dot{q}_{1} q_{2}\right] \\
+\frac{1}{2}\left[\left(b_{11} c_{11}^{2}+2 b_{12} c_{11} c_{21}+b_{22} c_{21}^{2}\right) q_{1}^{2}+2\left(b_{11} c_{11} c_{12}+b_{12} c_{22} c_{21}\right.\right. \\
+ \\
\left.+b_{12} c_{11} c_{22}+b_{22} c_{21} c_{22}\right) q_{12} q_{2} \\
\left.+\left(b_{11} c_{12}^{2}+2 b_{12} c_{12} c_{22}+b_{22} c_{22}^{2}\right) q_{2}^{2}\right]
\end{aligned}
$$


Now using (10), (11) and (12)

$E=E_{0}+\frac{1}{2} m\left[\dot{q}_{1}^{2}+\dot{q}_{2}^{2}\right]+\frac{1}{2}$ [same as in previous eq]

From the seiond term on the right we see that the

kinetic energy form cheiks with Eq. (2.10).

Then we claim that terms in the second bracket on the RHS of eq.(17), i.t. the last term on RHS of (17) is of the form:

$$
E_{p o t}-E_{0}=\frac{1}{2} 4 \pi m \cdot \nu_{1}^{2} q_{1}^{2}+\frac{1}{2} 4 \pi m \nu_{2}^{2} q_{2}^{2}
$$

Using $q_{1}$ and $q_{2}$ as functions of $x_{1}$ and $x_{2}$, we have.

RHS of

eq. (1s)

$$
\begin{aligned}
=\frac{1}{2} 4 \pi m\left[\nu _ { 1 } ^ { 2 } \left(c_{11}^{2} x_{i}^{2}\right.\right. & \left.+2 c_{11} c_{21} x_{1} x_{2}+c_{21}^{2} x_{2}^{2}\right) \\
& \left.+\nu_{2}^{2}\left(c_{12}^{2} x_{1}^{2}+2 c_{12} c_{22} x_{1} x_{2}+c_{22}^{2} x_{2}^{2}\right)\right]
\end{aligned}
$$

Identifying coefficients of $x_{1}^{2}, x_{1} x_{2}, x_{2}^{2}$ in eq. (A)

$$
\begin{aligned}
& b_{11}=\left[\nu_{1}^{2} c_{11}^{2}+\nu_{2}^{2} c_{12}^{2}\right] 4 \pi^{2} m \\
& b_{12}=\left[\nu_{1}^{2} c_{11} c_{21}+\nu_{2}^{2} c_{12} c_{22}\right] 4 \pi^{2} m \\
& b_{22}=\left[\nu_{1}^{2} c_{21}^{2}+\nu_{2}^{2} c_{22}^{2}\right] 4 \pi^{2} m
\end{aligned}
$$

defining as in eq (s)

$$
4 \pi^{2} m \nu_{1}^{2}=a_{1}^{2} \text { and } 4 \pi^{2} m \nu_{2}^{2}=a_{2}^{2}
$$

then

$$
\begin{aligned}
& b_{11}=a_{1} c_{11}^{2}+a_{2} c_{12}^{2} \\
& b_{12}=a_{1} c_{11} c_{21}+a_{2} c_{12} c_{22} \\
& b_{22}=a_{1} c_{21}^{2}+a_{2}^{2} c_{22}
\end{aligned}
$$

using

(13), $\quad c_{11}^{2}=c_{22}^{2}=1-c_{12}^{2}$ in 
38

$$
b_{11}=a_{1}\left(1-c_{12}^{2}\right)+a_{2} c_{12}^{2}
$$

or

$$
b_{11}-a_{1}=\left(a_{2}-a_{1}\right) c_{12}^{2}
$$

similarly

$$
\begin{aligned}
& b_{22}=a_{1} c_{12}^{2}+a_{2}\left(1-c_{12}^{2}\right) \\
\therefore & b_{22}-a_{2}=-\left(a_{2}-a_{1}\right) c_{12}^{2}
\end{aligned}
$$

from (22) \& (23)

$$
b_{11}-a_{1}=-\left(b_{22}-a_{2}\right)
$$

using (7)

$$
\begin{aligned}
& b_{11}-a_{1}=\frac{1}{2}\left\{\left(b_{11}-b_{22}\right)+\sqrt{\left(b_{11}-b_{22}\right)^{2}+4 b_{12}^{2}}\right\} \\
& b_{22}-a_{2}=\frac{1}{2}\left\{-\left(b_{11}-b_{22}\right)-\sqrt{\left(b_{11}-b_{22}\right)^{2}+4 b_{12}^{2}}\right\}
\end{aligned}
$$

so relation (24) is satisfied.

Now we use eqs.(13) and (14) in eq.(20), i.e.

$$
c_{12}=C_{21} \text { and } c_{11}=-C_{22}
$$

or $\quad c_{12}=-c_{21}$ and $c_{11}=c_{22}$

$$
\begin{aligned}
b_{12} & =a_{1} c_{11} c_{21}-a_{2} c_{21} c_{11} \\
& =\left(a_{1}-a_{2}\right) c_{11} c_{21} \\
\therefore b_{12}^{2} & =\left(a_{1}-a_{2}\right)^{2} c_{11}^{2} c_{21}^{2}
\end{aligned}
$$

using $(10)$, i.e. $c_{i 1}{ }^{2}=1-c_{21}^{2}$, we get

$$
b_{12}^{2}=\left(a_{1}-a_{2}\right)^{2} c_{21}^{2}\left(1-c_{21}^{2}\right)
$$

or using $c_{21}^{2}=c_{12}^{2}$

$$
b_{12}^{2}=\left(a_{1}-a_{2}\right)^{2} c_{12}^{2}\left(1-c_{12}^{2}\right)
$$


from (22)

$$
c_{12}^{2}=\frac{b_{11}-a_{1}}{a_{2}-a_{1}}
$$

from (23)

$$
\begin{gathered}
c_{i 2}^{2}=-\frac{b_{22}-a_{2}}{a_{2}-a_{1}} \\
c_{11}^{2}=1-c_{12}^{2}=1+\frac{b_{22}-a_{2}}{a_{2}-a_{1}}=\frac{b_{22}-a_{1}}{a_{2}-a_{1}}
\end{gathered}
$$

from (27), (28) and (29)

$$
\begin{aligned}
b_{12}^{2} & =\frac{\left(b_{11}-a_{1}\right)}{\left(a_{2}-a_{1}\right)} \cdot \frac{\left(b_{22}-a_{1}\right)}{\left(a_{2}-a_{1}\right)} \cdot\left(a_{1}-a_{2}\right)^{2} \\
& =\left(b_{11}-a_{1}\right)\left(b_{22}-a_{1}\right)
\end{aligned}
$$

i.e.

$$
b_{12}^{2}=\left(b_{11}-a_{1}\right)\left(b_{22}-a_{1}\right)
$$

This is the same as eq. (6).

Therefore, the potential energy term is that given by $E_{q} \cdot(2.10)$

Finally, we can detine the coefficients

$$
\text { from (28) } \quad c_{12}^{2}=\frac{b_{11}-a_{1}}{a_{2}-a_{1}}
$$

using (7) for $a_{1}$ and $a_{2}-a_{1}$, we get

$$
C_{12}^{2}=\frac{\left(b_{11}-b_{22}\right)+\sqrt{\left(b_{11}-b_{22}\right)^{2}+4 b_{12}^{2}}}{\sqrt{\left(b_{11}-b_{22}\right)^{2}+4 b_{12}^{2}}} \ldots \text { (30) }
$$

and from (B) and (14) we evaluate $c_{11}, C_{21}$ and $C_{22}$ If $b_{11}>b_{22}, c_{12}^{2}>1$ and $c_{11}$ and $c_{22}$ are complex. If $b_{11}<b_{22}, c_{12}^{2}<1$ and $c_{11} \& c_{22}$ are real. 
Problem 2.6

The energy of vibration of the $i^{\text {th }}$ mode is given by

$$
\varepsilon_{i}=\left(j_{i}+\frac{1}{2}\right) h \nu_{i}
$$

At $\mathrm{O}^{\circ} \mathrm{K}$, if the cohesive energy is chosen as the reference energy state, the residual vibrational energy, when all atoms of the solid are in the lowest quantum state, i.e. $j_{i}=0$ (for all $\left.i\right)$ is the zero point energy, and is given by

$$
\begin{gathered}
E_{z . p .}=\frac{1}{2} \sum_{i=1}^{3 N} h \nu_{i}=\frac{1}{2} \int_{0}^{\omega_{p}}(\hbar \omega) g(\omega) d \omega \\
\text { from } E_{q .}(2.34) \quad D(\omega)=\left\{\begin{array}{c}
9 N \omega^{2} / \omega_{D}^{3} \quad \text { for } 0 \leqslant \omega \leqslant \omega_{D} \\
0 \quad \text { for } \omega>\omega_{D}
\end{array}\right. \\
\therefore E_{z . p .}=\frac{9 N \hbar}{2 \omega_{D}^{3}} \int_{0}^{\omega_{p}} \omega^{3} d \omega \\
=\frac{9 N \hbar}{2 \omega_{D}^{3}} \frac{\omega_{D}^{4}}{4}=\frac{9}{8} N k\left(\frac{\hbar \omega_{D}}{k}\right) \\
\text { using } \quad \theta_{D}=\frac{\hbar \omega_{D}}{k}, N k=R \\
E_{z . p .}=\frac{9}{8} R \theta_{D}
\end{gathered}
$$


41

Problem 2.7

$$
\begin{gathered}
S=k \ln z+k T\left(\frac{\partial \ln z}{\partial T}\right)_{v} \quad E_{q \cdot}(1.17) \\
\ln z=-\frac{E_{c}(v)}{k T}-\int_{0}^{\infty} g(\nu) \ln \left(1-e^{-h / k T}\right) d \nu \quad E_{q_{0}}(2.21)
\end{gathered}
$$

Assume Einstein model: $\quad g(\nu)=3 N \delta\left(\nu-\nu_{1}\right)$ then

$$
\begin{aligned}
& \ln Z=-\frac{E_{0}}{k T}-3 N \ln \left(1-e^{-h \nu_{E} / k T}\right) \\
& \left.\frac{\partial \ln z}{\partial T}\right|_{V}=\frac{E_{B}}{k T^{2}}+3 N \frac{e^{-h \nu_{E / k T}}\left(+\frac{h \nu_{k}}{k T^{2}}\right)}{1-e^{-h \nu_{k} / k T}} \\
& \therefore S=k\left[-\frac{E_{C}}{k T}-3 N \ln \left(1-e^{-b \gamma_{E} / k T}\right)\right] \\
& +k T\left[\frac{E_{b}}{k T^{2}}+3 N \frac{e^{-h \nu_{E} / k T}\left(\frac{b \nu_{E}}{k T^{2}}\right)}{1-\epsilon^{-h \nu_{E} / k T}}\right] \\
& =-3 N k \ln \frac{h \nu_{E}}{k T}+3 N k\left(1-\frac{h k_{E}}{k T}\right) \\
& \left(u \operatorname{sing} e^{\frac{h \nu_{k}}{k T}} \cong 1-\frac{h \nu_{E}}{k T}\right) \\
& \therefore S \cong 3 N k\left(1-\ln \frac{h \nu_{E}}{k T}\right)
\end{aligned}
$$


42

Problem 2.8

From Eq. (2.33), $\quad \theta_{D}=\frac{\hbar \omega_{D}}{k}=\frac{\hbar}{k}\left(\frac{6 \pi^{2}}{v}\right)^{1 / 3} \bar{C}$

Omitting constants,

$$
\bar{c} \sim \frac{1}{\sqrt{\beta S}}
$$

or $\quad \theta_{D} \sim \frac{1}{v^{1 / 3} \sqrt{\beta S}}, \rho \sim 1 / v$

then $\theta_{D} \sim \frac{\gamma^{k}}{\beta^{k}}$.

$$
\begin{aligned}
\ln \theta_{D} & =\frac{1}{6} \ln v-\frac{1}{2} \ln \beta+\text { cost. } \\
\therefore \gamma & =-\frac{1}{6}+\frac{1}{2} \frac{d \ln \beta}{d \ln v} \\
\text { but } \beta & =-\frac{1}{v}\left(\frac{\partial v}{\partial p}\right)_{T}=\frac{1}{v}\left[\frac{1}{-\left(\frac{\partial p}{\partial v}\right)_{T}}\right] \\
\ln \beta & =-\ln v-\ln \left[-\left(\frac{\partial p}{\partial v}\right)_{T}\right] \\
\therefore \frac{d \ln \beta}{d \ln v} & =-1-\frac{d \ln \left[-\left(\frac{\partial p}{\partial v}\right)_{T}\right]}{d \ln v} \\
& =-1-\left\{\frac{v}{-\left(\frac{\partial p}{\partial v}\right)_{T}}\left(-\frac{\partial^{2} p}{\partial v^{2}}\right)_{T}\right\} \\
& =-1-\frac{v\left(\frac{\partial p}{\partial v}\right)_{T}}{\left(\frac{\partial p}{\partial v}\right)_{T}}
\end{aligned}
$$




$$
\begin{aligned}
& \therefore \gamma=-\frac{1}{6}+\frac{1}{2}\left[-1-v \frac{\left(\frac{\partial^{2} p}{\partial v^{2}}\right)_{T}}{\left(\frac{\partial p}{\partial v}\right)_{r}}\right] \\
& \text { or } \gamma=-\frac{2}{3}-\frac{1}{2} \frac{v\left(\frac{\partial^{2} p}{\partial v^{2}}\right)_{T}}{\left(\frac{\partial p}{\partial v}\right)_{T}}
\end{aligned}
$$


44

CHAPTER 3

Problem 3.1

(a) The unit cell shown in the sketch accompanying the problem statement is a rectangular parullepipal with the edges $(a, b$, and $i)$ of unequal length. Aacroling to Fig, 36 ; the letticetipe is orthorhombic. The corners of the unit cell do nit contain atoms. However two atoms are ogneidistant from the corners of the unit cell. In addition, the $a-b$ faces of the unit cell share a pair of atoms at the center of these faces. According to P.J. 3.6, this corresponds to the ecotype orthorhombic space lattice.

Therefore, the fill description of the $x$-uranium crystal structure is:

Face -centered orthorhombic space lattice with a basis of 2. Atoms per unit cell: Each corner has 2 atoms shaved among 8 contiguous unit cells. For 8 corners this contributes 2 atoms to the unit cell. The twi o alb faces have 2 atoms at Maid. centers these are divided between 2 umitcells. A faces control in te 2 atoms. So total atoms per ait cell $=4$.

(b) Let: $V=$ volume of unit cell.

$n=$ number of atoms in init cell

$m=$ moss of atom

then

$$
\text { density }=l=\frac{n \text { atins/unitcell }}{V \mathrm{~cm}^{3} / \text { unit cell }} \times \mathrm{m} \text { gpor/atom }
$$


45

$$
\begin{aligned}
& \text { but } \quad A=m \text { Nav. } \\
& \therefore \quad \rho=\frac{n A}{V N_{a v}}
\end{aligned}
$$

Nav, : Avogadro's \#

A : atomic mass

(1) UC

$$
\begin{aligned}
a=4.961 A^{\circ}, V & =\frac{1}{4} a^{3} \text { cc/ molecule } \\
& =\frac{1}{4}(4.961)^{3} \times 10^{-24}=3.06 \times 10^{-23} \mathrm{cc} / \text { molecule }
\end{aligned}
$$

( $\because$ For Nail structure 4 molecules/unitcell)

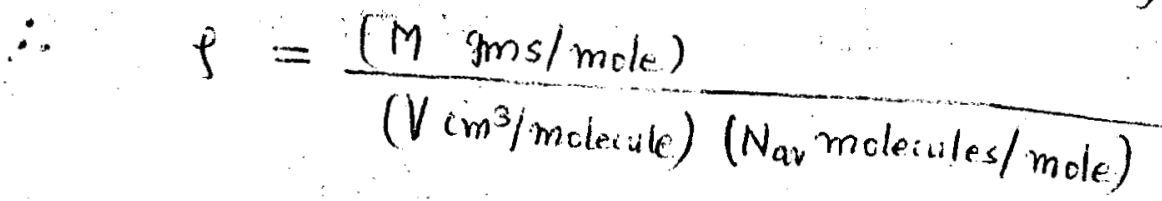

$$
\begin{aligned}
& =\frac{(238+12)}{\left(3.06 \times 10^{-23}\right)\left(6.023 \times 10^{23}\right)}=13.54 \mathrm{gm} / \mathrm{kc} . \\
& N_{U}=\frac{1}{3.06 \times 10^{-23}}=3.28 \times 10^{22} \quad U \text {-atoms } / c c .
\end{aligned}
$$

(2) $\mathrm{UO}_{2}$

$$
\begin{aligned}
& a=5.470 \mathrm{~A}^{\circ} \text { Fluorite structure - } \\
& 4 \text { molecules } \mathrm{VO}_{2} / \text { unit cell } \\
& V=\frac{1}{4} a^{3}=\frac{1}{4}(5.47)^{3} \times 10^{-24}=4.08 \times 10^{-2.3} \mathrm{cc} / \text { molecule } \\
& \rho=\frac{(238+16 \times 2)}{\left(4.08 \times 10^{-23}\right)\left(6.023 \times 10^{23}\right)}=10.97 \mathrm{gm} / \mathrm{cc} . \\
& N_{u}=\frac{1}{4.08 \times 10^{-23}}=2.44 \times 10^{22} \quad \text { atoms } / c c .
\end{aligned}
$$


(3)

$$
\begin{aligned}
& \underline{\alpha-U} \text { for } F C \text { structure 4-atoms/unit cell } \\
& V=\frac{1}{4} a b c=\frac{1}{4}\left(8.3 \times 10^{-23}\right) \\
& =2.075 \times 10^{-23} \mathrm{cc} / \text { atom } \\
& S=\frac{238}{\left(2.075 \times 10^{-23}\right)\left(6.023 \times 10^{23}\right)}=19.04 \mathrm{gm} / \mathrm{cc} .
\end{aligned}
$$

\begin{tabular}{|c|c|c|c|c|}
\hline Fuel & $\begin{array}{l}\text { Coystal } \\
\text { Structure }\end{array}$ & $\begin{array}{l}\text { Lattice } \\
\text { constant }\end{array}$ & $\begin{array}{l}\text { Theoretical } \\
\text { density } \\
g m \cdot k c .\end{array}$ & $\begin{array}{l}\text { Theoretical } \\
\text { Uranium } \\
\text { density } \\
\text { (itoms }(c) \times 10^{-22}\end{array}$ \\
\hline $\mathrm{UO}_{2}$ & Fluorite & $5.47 \mathrm{~A}^{\circ}$ & 10.97 & 2.44 \\
\hline UC & $\mathrm{NaCl}$ & $4.961 A^{\circ}$ & 13.54 & 3.28 \\
\hline$\alpha-v$ & $\begin{array}{l}\text { Fice Centered } \\
\text { orthortimbic. }\end{array}$ & $\begin{array}{l}a=2.854 \mathrm{~A}^{\circ} \\
b=5.87 \mathrm{~A}^{\circ} \\
c=4.956 \mathrm{~A}^{\circ}\end{array}$ & 19.04 & 4.82 \\
\hline
\end{tabular}

$N_{u}=\frac{1}{2.075 \times 10^{-23}}=4.82 \times 10^{22}$ atom-u/cc. 
47

Problem 3.2
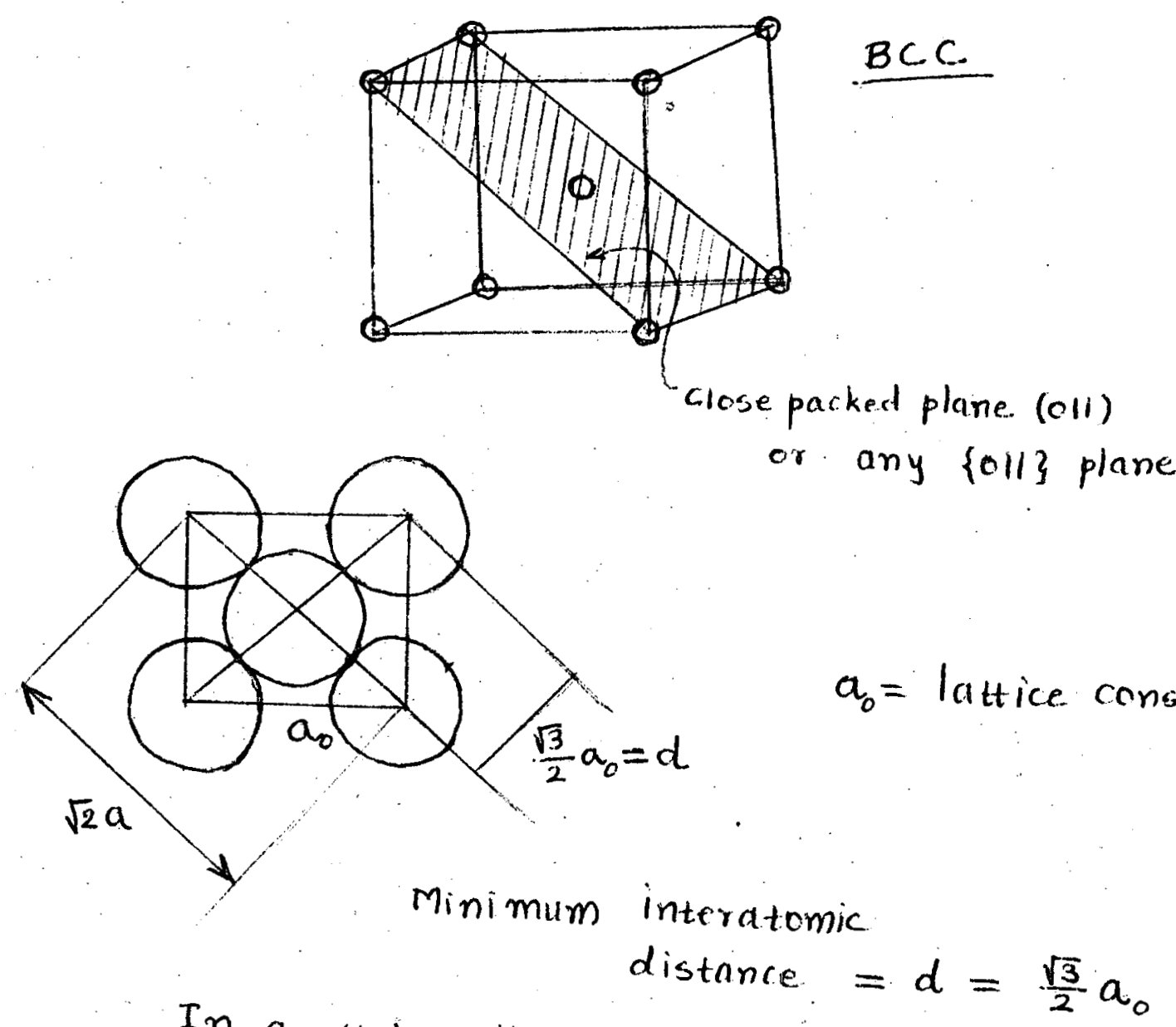

In a unit cell there are 2 atoms

$$
\begin{array}{r}
\text { taking up space }=2 \times \frac{4}{3} \pi\left(\frac{d}{2}\right)^{3}=\frac{\pi}{3} d^{3} \\
\text { Volume of } a \text { unit cell }=a_{0}^{3}\left(\frac{\sqrt{3}}{2}\right)^{3} a_{0}^{3}=\frac{\pi \sqrt{3}}{8} a_{0}^{3}
\end{array}
$$

Therefore fraction of space occupied by

$$
\text { hard sphere atoms }=\frac{\pi \sqrt{3}}{8}=0.68
$$

Therefore void fraction $=32 \%$ by comparison, the fac lattice has a vide d fraction of $26 \%$. 
48

Problem 3.3

Let $a_{0}^{i}$ be the $b c c$ edge length: and $a_{0}^{\prime}$ be the $f(c)$ edge length.

If $r_{0}$ is the atomic radius, then for touching nearest -neighbors structure.

$$
\begin{aligned}
\quad 4 r_{0} & =\sqrt{3} a_{0} \\
4 r_{0} & =\sqrt{2} a_{0}^{\prime} \\
\therefore \quad & \frac{a_{0}^{\prime}}{a_{0}}=\frac{\sqrt{3}}{\sqrt{2}}=\sqrt{\frac{3}{2}}=1.225
\end{aligned}
$$

In $f c c$ there are 4 atoms /uni tel

$$
\therefore \text { solid density }=\frac{4 m}{a_{0}^{13}}=f_{f c c}
$$

In bee there are 2 atems/unitcell

$$
\begin{aligned}
\therefore f_{\text {bc }} & =\frac{2 m}{a_{0}^{3}} \\
\therefore \frac{f_{\text {fcc }}}{s_{\text {bic }}}=\left(\frac{a_{0}}{a_{0}^{\prime}}\right)^{3} \times 2 & =\frac{2}{3} \times \sqrt{\frac{2}{3}} \times 2 \\
& =\frac{4}{3} \times \sqrt{0.667}=1.089
\end{aligned}
$$

For percentage change in volume, from bee to $\mathrm{fcc}$ of the same mass we have 
49

$$
\begin{aligned}
& \dot{s}_{f c c}=\frac{m}{V_{f c c}}, \quad \rho_{b c c}=\frac{m}{V_{b c c}} \\
& \therefore \frac{V_{f c c}}{V_{b c c}}=\frac{\rho_{b c c}}{f_{f c c}}=\frac{1}{1.089}
\end{aligned}
$$

$\therefore$ percentage change in Volume

$$
\begin{aligned}
& =\frac{V_{f c c}-V_{b c c}}{V_{b r c c}} \\
& =\frac{V_{f c c}}{V_{b c c}}-1 \\
& =-\frac{.089}{1.089} \\
& =-.082 \\
& =-8.2 \%
\end{aligned}
$$


50

CHAPTER 4

Problem 4.1

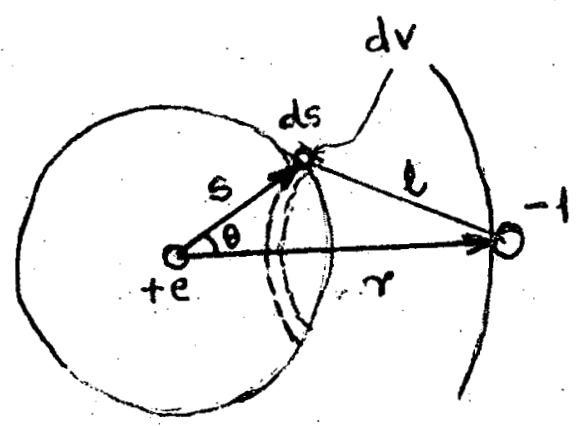

I) Coulomb energy between unit negative charge on the sphere and te at the center of the sphere.

$$
=\frac{(-1) \cdot e}{\gamma}=-\frac{e}{\gamma}
$$

I) For coulomb energy between unit negative charge and charge inside the sphere, consider a volume. element $d v$

$$
\begin{aligned}
d v & =s^{2} \sin \theta d \theta d \phi d s \\
\therefore \text { charge in } d v & =-\frac{e}{\frac{4}{3} \pi r_{e}^{3}} s^{2} \sin \theta d \theta d \phi d s
\end{aligned}
$$

$\therefore$ coulomb energy between negative unit charge - and charge in dy

$$
=(-1) \cdot \frac{-e}{\frac{4}{3} \pi r_{0}^{3}}\left(s^{2} \sin \theta d \theta d \theta d s\right) \times \frac{1}{l}
$$

Then the total coulomb energy between the unit - negative charge and negative charge inside the sphere is given by 
51

$$
\begin{array}{r}
\Phi=\int_{s=0}^{r} \int_{\theta=0}^{\pi} \int_{\phi=0}^{2 \pi} \frac{e}{\frac{4}{3} \pi r_{0}^{3}} \cdot \frac{s^{2} \sin \theta}{\left[r^{2}+s^{2}-2 r \cos \theta\right]^{\frac{1}{2}} d \phi d \theta d s} \\
\quad \text { (where } t^{2}=r^{2}+s^{2}-2 r \cos \theta \text { wars used) }
\end{array}
$$

lit $\quad r^{2}+s^{2}-2 r s \cos \theta=f$

then for constant $s, 2 r s \sin \theta d e=d s$

$$
\begin{aligned}
& \therefore \Phi=\frac{3 e}{2 r_{c}^{3}} \int_{s=0}^{r} s^{2}\left[\int_{i=0}^{\pi} \frac{\sin \theta d \theta}{\left[r^{2}+s^{2}-2 r s \cos \theta\right]^{i / 2}}\right] d s \\
& =\frac{3 c}{2 r^{3}} \int_{s=c}^{r} \frac{s^{2}}{2 r s} \int_{(r-s)^{2}}^{(r+s)^{2}} \frac{d f}{s^{4 / 2}}=\frac{3 e}{2 r^{3}} \int_{0}^{r} \frac{s}{2 r}\left[2 s^{b}\right]_{(r-s)^{2}}^{(r+s)^{2}} d s \\
& =\frac{3 e}{2 r_{c}^{3}}\left[\int_{0}^{r} \frac{2 \sigma^{2}}{\gamma} d s\right]=\frac{3 e}{\gamma \cdot r_{0}^{3}}\left[\frac{s^{3}}{3}\right]_{0}^{\gamma}=\frac{e r^{2}}{\gamma_{0}^{3}}
\end{aligned}
$$

Then the total coulomb energy is the sum of that due to positive charge and that due to negative charge inside the sphere, that is, the sum of I) and II)

$$
\therefore \text { Total coulomb energy } \begin{aligned}
\Phi_{c} & =-\frac{e}{\gamma}+\frac{e r^{2}}{r^{3}} \\
& =-\frac{e}{\gamma}\left[1-\left(\frac{r}{r_{0}}\right)^{3}\right]
\end{aligned}
$$


52

Problem 4.2

(a)

$$
\rho(\gamma)=\left[\frac{t}{\frac{4}{3} \pi r_{L}^{3}}\right] A e^{-\left(\gamma / r_{0}\right)}
$$

Consider a unit negative charge on the sphere. of radius $r$. By the same reason as in problem 4.1 the coulomb energy between a unit negative charge. and charge in a volume element $d v$ is

$$
d \Phi=-\frac{3 A e(-1)}{4 \pi r_{0}^{3}} e^{-s / r_{c}} \cdot \frac{s^{2} \sin e d e d \phi d s}{\left(r^{2}+s^{2}-2 \operatorname{sic}(\sin )^{1 / 2}\right.}
$$

$\therefore$ Total coulomb energy between unit negative charge and charge inside the sphere is

$$
\begin{aligned}
& \Phi=\int_{s=0}^{r} \int_{\theta=0}^{\pi} \int_{i=c}^{2 \pi} d \Phi \\
& =\frac{3 A E(2 \pi)}{4 \pi r_{0}^{3}} \int_{s=0}^{r} s^{2} \epsilon^{-s / r_{0}}\left[\int_{0}^{\pi \sin \theta d \theta}\left[r^{2}+s^{2}-2 r \operatorname{sis} \theta\right]^{2}\right] d s \\
& =\frac{3 A E}{2 r_{0}^{3}} \int_{s=0}^{r} \frac{2 s^{2}}{r} \cdot e^{-s / r_{c}} d s \\
& =\frac{3 A C}{x_{c}^{3} r}\left[\left.s^{2} \epsilon \cdot r_{r_{c}}\left(-r_{c}\right)\right|_{s=r} ^{r}+2 \cdot r_{c} \int_{0}^{r} s e^{s / r_{c}} d s\right]
\end{aligned}
$$

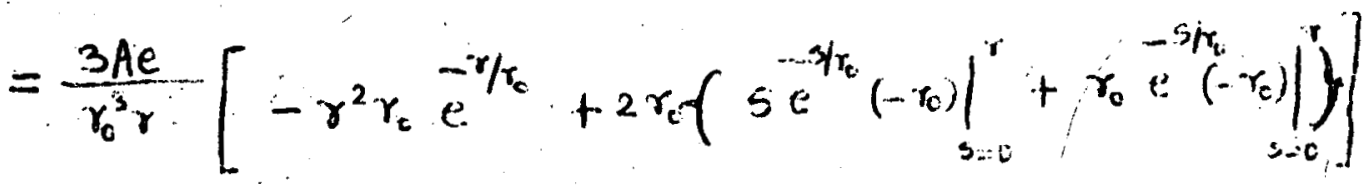

$$
\begin{aligned}
& =\frac{3 A e}{\gamma_{0}^{3} \gamma}\left[-\gamma^{2} \gamma_{c} e^{-\gamma / \gamma_{0}}-2 \gamma_{c}^{2} r e^{-\gamma / \gamma_{t}}-2 \gamma_{0}^{3} e^{-\gamma / r_{c}}+2 \gamma_{c}^{3}\right]
\end{aligned}
$$


53

$$
\therefore \Phi=\frac{3 A e}{r}\left|2-e^{-r / \gamma_{0}}\left\{\left(\frac{r}{\gamma_{0}}\right)^{2}+2\left(\frac{r}{\gamma_{0}}\right)+2\right\}\right|
$$

$\therefore$ Total coulomb energy between a unit charge on the sphere of radius $r$ and charge inside the sphere is

$$
\therefore \quad \Phi_{c}=-\frac{\epsilon}{r}+\frac{3 A e}{r}\left[2-e^{-r / r_{i}}\left\{\left(\frac{r}{r_{c}}\right)^{2}+2\left(\frac{r}{r_{c}}\right)+2\right\}\right]
$$

Therefore the coulomb energy between the sphere of radius $r$ and a spherion shell of thick uss drat r is

$$
d U_{c}=4 \pi r^{2} \frac{\epsilon}{\frac{4}{3} \pi r_{t}^{3}} A e^{-r / r_{c}} d r \Phi_{c}(r)
$$

Hence the total coulomb energy for this model is

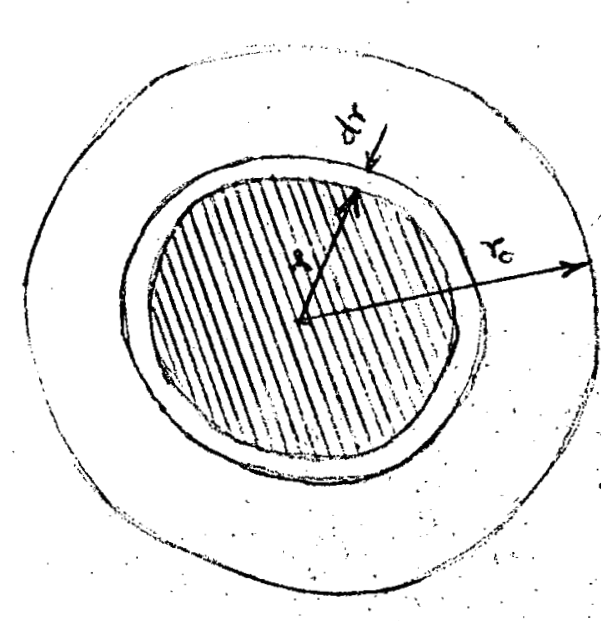

Using integration by prints, we get

$$
\begin{aligned}
& u_{c}=\int_{0}^{\gamma_{0}} d u_{c} \\
& =\frac{3 e^{2} A}{\gamma_{0}^{3}} d d r r^{2} e^{-\gamma / r_{0}} \cdot \frac{e}{r}\{-1 \\
& +3 A\left[2-e^{-r / r_{0} C}\left(\left(\frac{\gamma}{x_{c}}\right)^{2}+2\left(\frac{\gamma}{x_{c}}\right)+2\right]\right] \\
& U_{c}=\frac{3 e^{2} A}{\gamma_{0}^{3}} \int_{0}^{\gamma_{0}}\left[(6 A-1) r e^{-\gamma / r_{0}}-\frac{3 A}{\gamma_{0}^{2}} \gamma^{3} e^{-2 \gamma / r_{c}}\right. \\
& -\frac{G A}{r_{0}} \gamma^{2} e^{2 r / r_{0}}-6 A r \epsilon^{-2 r / r_{0}} \int d r
\end{aligned}
$$


54

$$
\begin{gathered}
U_{c}=\frac{3 e_{0}^{2} A}{r_{0}^{3}}\left[(6 A-1) r_{c}^{2}\left(1-\frac{2}{\epsilon}\right)-\frac{3 A r_{0}^{2}}{16}\left(6-\frac{38}{e^{2}}\right)-\frac{3 A r_{0}^{2}}{4}\left(2-\frac{10}{e^{2}}\right)\right. \\
\left.-\frac{3 A r_{c}^{2}}{2}\left(1-\frac{3}{e^{2}}\right)\right]
\end{gathered}
$$

(Here charge e is denoted by $e_{c}$ to avoid confusion with Napierian $e=2.71828 \ldots$ )

Value of $A$

Since the total negative charge inside the sphere of radius $r_{i}$ is unity, then

$$
\int_{e}^{\gamma_{0}} 4 \pi r^{2} f(r) d r=e_{r}
$$

and $\quad f(r)=\frac{e_{c}}{\frac{4}{3} \pi r_{c}^{3}} A e^{-r / r_{c}}$

$$
\begin{aligned}
\therefore \int_{0}^{r_{c}} 4 \pi r^{2} \cdot \frac{e_{c} A}{\frac{4}{3} \pi r_{c}^{3}} e^{-r / r_{c}} d r & =e_{c} \\
\therefore A & =\frac{r_{c}^{3}}{3 \int_{0}^{r_{c}} r^{2} e^{r / r_{0}} d r}=\frac{1}{3\left(2-5 c^{1}\right)} \\
& =\frac{\epsilon}{3(2 e-5)} \\
U_{c} & =\frac{e_{c}^{2}}{r_{c}} 3 A\left[(6 A-1)\left(i-\frac{2}{e}\right)-\frac{3 A}{5}\left(11-\frac{5 i}{e^{2}}\right)\right] \\
& =\frac{e_{c}^{2}}{r_{0}} \frac{e^{2}}{(2 e--5)^{2}}\left[\frac{5}{e}\left(1-\frac{2}{E}\right)-\frac{1}{3}\left(11-\frac{5 i}{e^{2}}\right)\right]
\end{aligned}
$$




$$
\begin{aligned}
& t=2.71828 \\
& U_{c}=-\frac{e_{c}^{2}}{r_{i}} \cdot 1.015 \\
& \text { or } U_{c}=-1.015 \frac{e^{2}}{r_{c}} \text { (where } e \text { is electronic } \\
& \text { Compare this with the result from constant } \\
& \text { charge density } \\
& U_{c}=-\frac{q}{\pi} \frac{e^{2}}{r_{c}}
\end{aligned}
$$

(b)

$$
\begin{aligned}
& U\left(x_{c}\right)=U\left(x_{c}\right)+U(\text { kineiic }) \\
& E_{\text {coll }}=U\left(r_{\text {cong }}\right) \\
& \text { let } U_{c}=-\frac{c_{1}}{r_{c}}, U_{\text {kinetic }}=\frac{c_{2}}{r_{c}^{2}}
\end{aligned}
$$

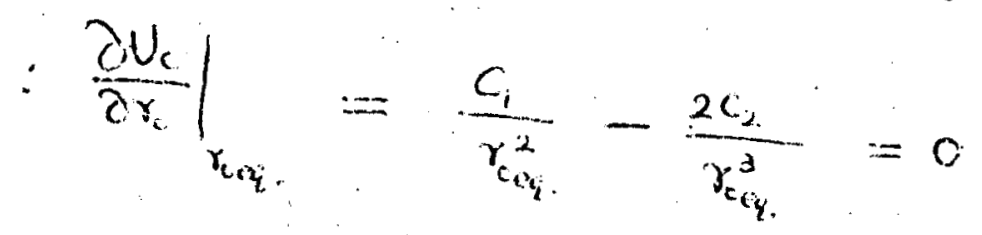

$$
\begin{aligned}
& \therefore \gamma^{c_{\text {cqq. }}}=\frac{2 C_{2}}{C_{1}} \text {. }
\end{aligned}
$$

Hence for larger $c_{i}$, rey becomes smaller, and

$$
\begin{aligned}
E_{c h_{1}} & =-\frac{C_{1}}{T_{c o}}+\frac{c_{2}}{r_{c e_{1}}^{2}} \\
& =-\frac{C_{1}^{2}}{4 C_{2}}
\end{aligned}
$$

Clearly, for larger $C_{1}$, the $E_{\text {cen }}$ is larger in magnitude. Hence for non-uniform charge distribution model $E_{c c h}$ is larger and reag. is smaller than those for Uniform charge density. 
56

Problem 4.3

(a) From Eq. (1.55)

$$
p=-\left(\frac{\partial U}{\partial r}\right)_{T=0}
$$

From $E_{i}(4-16)$ and (4.14), the diverge energy of: an electron at $0^{\circ} \mathrm{K}$ is $U_{F}$, where

$$
U_{F}:=\frac{3}{5} \epsilon_{F} \text { and } \epsilon_{F}=\left(\frac{k^{2}}{2 m}\right)\left(3 \pi^{2} \frac{N}{V}\right)^{4 \cdot 3}
$$

Hence,

for Fermi gas at ok containing $N$ electrons,

$$
U=\frac{3}{5} N \epsilon_{F}=\frac{3}{10} \frac{\hbar^{2}}{m} N\left(3 \pi^{2} \frac{N}{V}\right)^{2 / 3}
$$

ie. $U=A V^{-2 / 3}$, where $A=\frac{3}{10} \frac{h^{2}}{m} N\left(3 \pi^{2} N\right)^{2 / 3}$.

Substituting (3) into (1) we get,

$$
p=-\left(\frac{\partial U}{\partial V}\right)_{r=0}=\frac{2}{3} A V^{-5 / 3}=\frac{2}{3} \frac{U}{V}
$$

From the definition of Compressibility at $0^{2} k$.

$$
\begin{aligned}
\frac{1}{\beta_{0}}=-v\left(\frac{\partial p}{\partial v}\right)_{T=0} & =-v \frac{2}{3} A \frac{d}{d v} v^{-5 / 3} \\
& =\frac{10}{7} A v^{-5 / 3} \\
& =\frac{10}{9} \cdot \frac{3}{10} \cdot \frac{\hbar^{2}}{m} N\left(3 \pi^{2} N\right)^{2 / 3} V^{-5 / 3} \\
\therefore \quad \frac{1}{\beta_{i}} & =\frac{\hbar^{2}}{3 m}\left(3 \pi^{2}\right)^{2 / 3} \cdot\left(\frac{N}{v}\right)^{5 / 3}
\end{aligned}
$$


57

(b) In general we have to go back to $E_{2} \cdot(1,28)$

$$
p=k T\left(\frac{\partial \ln z}{\partial V}\right)_{T}
$$

Since free electrons constitute an ideal gas of particles obeying. Fermi-Dirac statistics, from Eq. $(1.51)$

$$
\ln Z=-\frac{\mu N}{k T}+\sum_{k} \ln \left[1+\exp \left(-\frac{\epsilon_{k}-\mu}{k T}\right)\right]
$$

Now, replacing sum over $k$ with density of state integral

$$
\ln Z=-\frac{\mu N}{k T}+\int_{0}^{\infty} D(t) \ln \left[1+\exp \left(-\frac{\epsilon-\mu}{k T}\right)\right] d t
$$

For Fermi electrons in volume $V$ from Eq. (4.8)

$$
D(\epsilon)=\frac{V}{4 \pi^{2}}\left(\frac{2 m}{\hbar^{2}}\right)^{3 / 2} \sqrt{\epsilon}
$$

and chemical potential $\mu$ is given in terms of $N$ by $E_{q} .(1.52)$

$$
N=\sum_{k}\left[\exp \left(\frac{\epsilon_{k}-\mu}{k T}\right)+1\right]^{-1}
$$

or

$$
N=\int_{i}^{\infty} 2(\epsilon)\left[\exp \left(\frac{\epsilon-\mu}{k r}\right)+1\right]^{-1} d t
$$

Using eq (4) and (c) we get

$$
\frac{N}{V}=\frac{1}{4 \pi^{2}}\left(\frac{2 m}{\hbar^{2}}\right)^{3 / 2} \int_{0}^{\infty} \sqrt{\epsilon}\left[\exp \left(\frac{t-\mu}{k q}\right)+1\right]^{-1} d t
$$


58

From this relation between $T, N / v$ and $\mu$, determine $\mu$ as a function of $T$ and $N / V$

$$
\text { ie. } \mu=\mu(T . N / N)
$$

Now substituting eq. (4) into eq. (3) we get

$$
\ln Z=N\left\{-\frac{\mu(T, N / N)}{k T}+\frac{1}{(N / V)} \cdot \frac{1}{4 \pi^{2}}\left(\frac{2 m}{\hbar^{2}}\right)_{c}^{3 / 2} \int_{c}^{\infty}\left[1+\exp \left(-\frac{\epsilon-\mu(\pi, N)}{k T}\right)\right]\right.
$$

(8)

Since $\mu$ is a function of $T$ and $N / N$, the bracketed term in eq .(8) is a function of the same. two variables, or

$$
\ln Z=N G(T, N / N)
$$

Substituting eq (9) into eq. (1), we get,

$$
p=k T N\left(\frac{\partial G}{\partial V}\right)_{T}
$$

Since $N$ is constant

$$
\begin{aligned}
& N \frac{\partial}{\partial v}=\frac{\partial}{\partial(v / N)}=-\left(\frac{N}{V}\right)^{2} \frac{\partial}{\partial(N / v)} \\
& \text { or } \quad p=-k T\left(\frac{N}{V}\right)^{2}\left[\frac{\partial G(T, N / v)}{\partial(N / v)}\right]_{T}
\end{aligned}
$$

(c) Telium model,

$$
E_{\text {con }}=-\frac{A_{\text {ceil }}}{r_{5}}+\frac{A_{f}}{r_{s}^{2}}
$$


59

$$
\begin{aligned}
& \frac{d E_{c b}}{d r_{s}}=\frac{A_{c c u}}{r_{s}^{2}}-\frac{2 A_{f}}{r_{s}^{3}}=0 \text { at equilibrium. } \\
& \frac{d^{2} E_{c c t}}{d r_{s}^{2}}=-2 \frac{A c o u l}{r_{s}^{3}}+\frac{6 A_{f}}{r_{s}^{4}} \\
& V=r_{s}{ }^{3} \ldots, r_{s}=1.30 \mathrm{~A}^{n} \\
& \frac{d^{2} E_{c c h}}{d v^{2}}=\frac{1}{9 r_{s}^{4}} \frac{d^{2} E_{c c h}}{d r_{s}^{2}}=\frac{1}{\beta r_{s}^{3}} \quad \text { (since } \frac{d E_{c c h}}{d r_{s}}=c \\
& \text { at equilibrium) } \\
& \therefore \frac{1}{\beta}=\frac{1}{9 r} \frac{d^{2} E_{c i n}}{d r_{s}^{2}}=\frac{1}{9 r_{s}}\left[-\frac{2 A_{c c i l}}{r_{s}^{3}}+\frac{6 A_{f}}{r_{s}^{4}}\right] . \\
& =\frac{2}{9 r_{s}^{3}}\left(\frac{A_{\text {call }}}{r_{s}}\right)\left[3 \frac{A_{f} / r_{\text {sci. }}}{A_{\text {ceil }}}-1\right]
\end{aligned}
$$

From $r_{\text {sig }}$ enloulation, ie. eq .(2)

$$
\begin{aligned}
& \frac{A_{c c u l}}{r_{\text {seq. }}}=\frac{9}{10} \frac{e^{2}}{r_{\text {sig }}} \quad \text { (since } U_{c i}=-\frac{q}{10} \frac{e^{2}}{r_{c}} E_{i .}(4.7) \\
& \left.=\frac{-A(c u)}{r_{u}}\right) \\
& =1.6 \times 10^{-18} \mathrm{nt} . \mathrm{m} \\
& \text { and } \frac{A_{f}}{A_{\text {ene }}}=\frac{1}{2} r_{\text {seq }} \\
& \therefore \frac{1}{\beta}=\frac{1}{9 V} \frac{A_{c e u l}}{\tau_{\text {sol }}}=\frac{1}{9} \times \frac{1.6 \times 10^{-18} \mathrm{rtm}}{(1.3)^{3} \times 10^{-24} \mathrm{~cm}^{3}}
\end{aligned}
$$


60

$$
\therefore \beta=1.2 \times 10^{11} \mathrm{~m}^{2} / \mathrm{mi} \text {. }
$$

- Form eq. (4) it is clear that, because of coulomb attraction the value of $1 / \beta$ is smaller, ie. coulomb attraction gives a negative contribution to $1 / \beta$. Therefore the value of $\beta$ will be larger due to the coulomb attraction. Thus the value of $\beta$ in part (c) will be larger than that in part (a) 
61

Problem 4.4

Born -Haber cycle

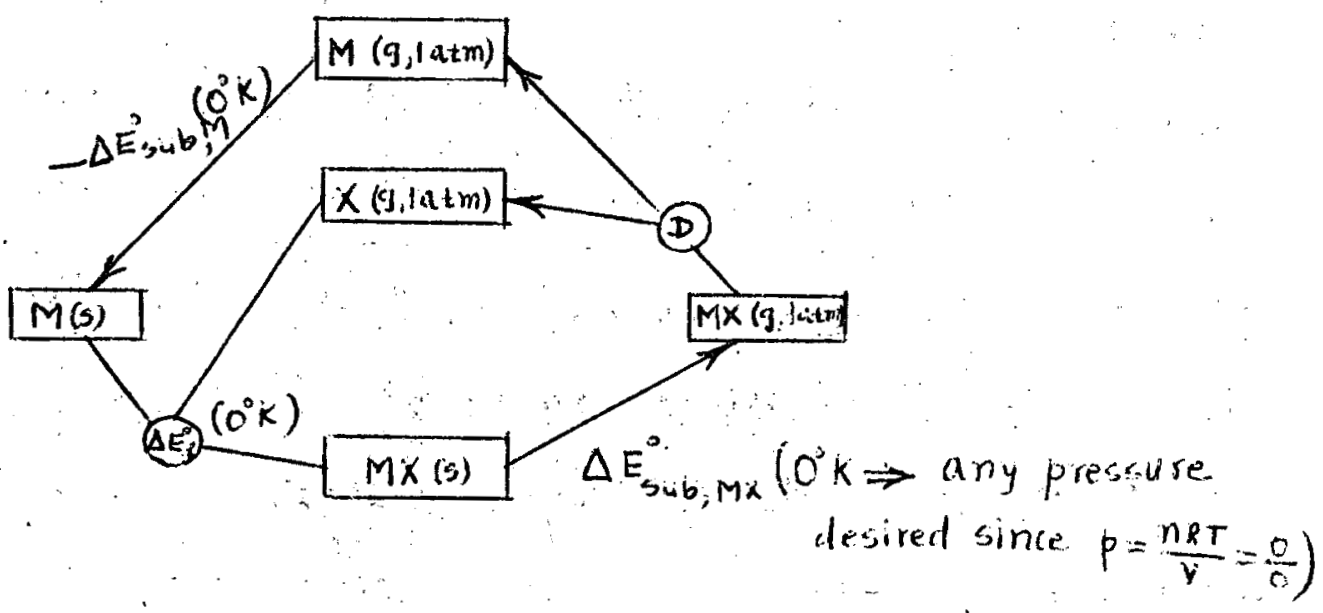

We want standard free energy of formation $\Delta E_{f}^{\circ}\left(0^{\circ} \mathrm{k}.\right)$

Quantity missing in problem statement is $\Delta E_{\text {SUb, M }}\left(\mathrm{O}^{\circ} \mathrm{K}\right.$.).

Energy balance on cycle gives

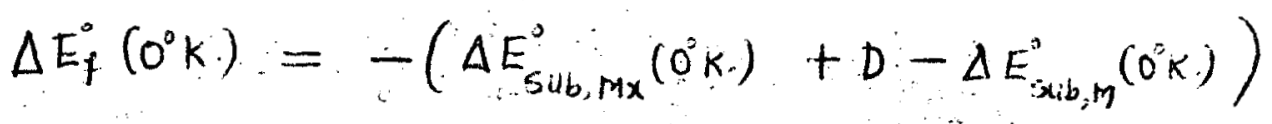


62

Problem 4.5

Col structure
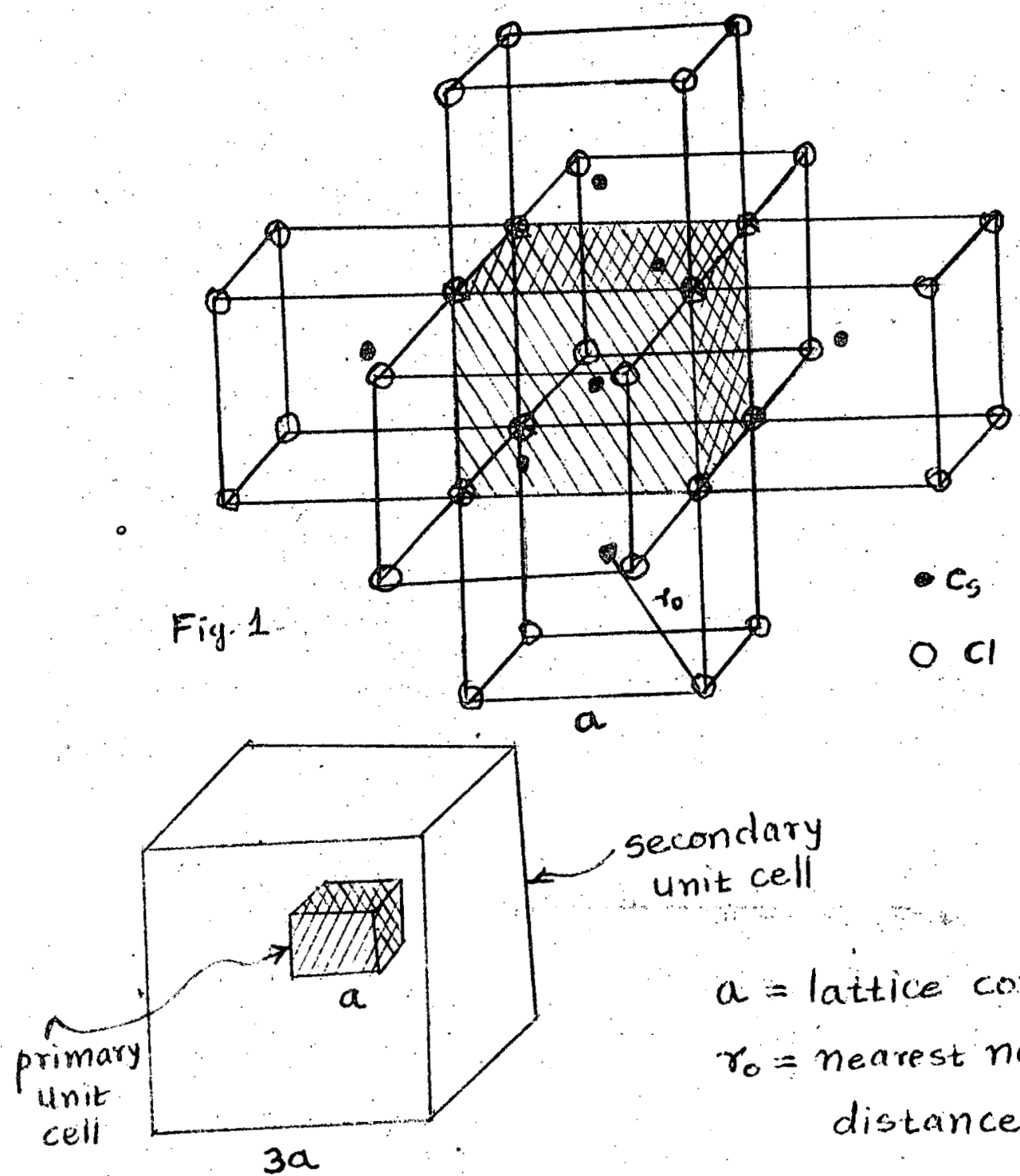

Fig. 2

$$
\begin{gathered}
a=\text { lattice constant } \\
r_{0}=\text { nearest neighbor } \\
\text { distance }
\end{gathered}
$$

$r_{0}=\frac{\sqrt{3}}{2} a$ 
63

$\left(C_{s}\right)_{0}=$ Central ion $=C_{s}$ in primary unit cell $1^{\text {st }}$ term

The $8 \mathrm{cl}$ ions on the corners of the unit cell are each shared by 8 adjacent unit cells.

$$
\therefore \text { inst term }=\frac{1}{8} \times 8=1 \quad \text { ( } \frac{e^{2}}{\gamma_{0}} \text { omitted) }
$$

The $2^{\text {nd }}$ term consists interactions for all ions in a cube of side $3 a$ surrounding the central ion. This shell, consists, 26 unit cells -6 attached to the faces of the primary unit cell (shown in Fig. 1 above) - 12 connected to the edges and 8 attached to the corners, plus the fraction of the $\mathrm{Cl}$ ions on the corners of the primary unit cell which are in the secondary cell.

Face cells (6) face cell

$(C s)_{0}$

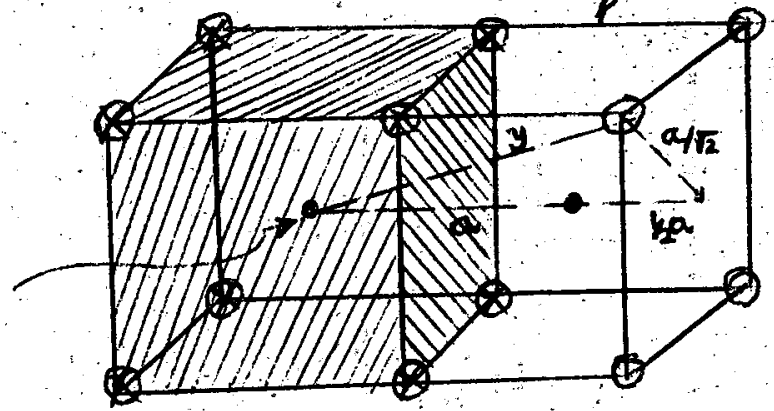


$64:$

$\left(c_{s}\right)_{0}-c_{s}$ separation $=a=\frac{2}{\sqrt{3}} \gamma_{0}$

interaction $=-\frac{\sqrt{3}}{2} \times 1=-\frac{\sqrt{3}}{2} \quad$ (minus sign because like. charges)

$$
\begin{array}{r}
y=\left(c_{5}\right)_{0}-c 1 \text { separation }=a \sqrt{\left(\frac{3}{2}\right)^{2}+\left(\frac{1}{\sqrt{2}}\right)^{2}} \\
=\frac{2}{\sqrt{3}} r_{c} \sqrt{\frac{\pi}{4}}=r_{0} \sqrt{\frac{11}{3}}
\end{array}
$$

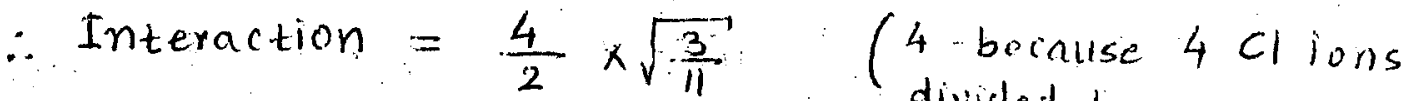
divicleil by 2 -because. each is only half in the secondary shell)

$\therefore$ Total interaction of 6 face cells $=6\left(-\frac{\sqrt{3}}{2}+2 \sqrt{\frac{3}{11}}\right)$

Edge: cells (12)

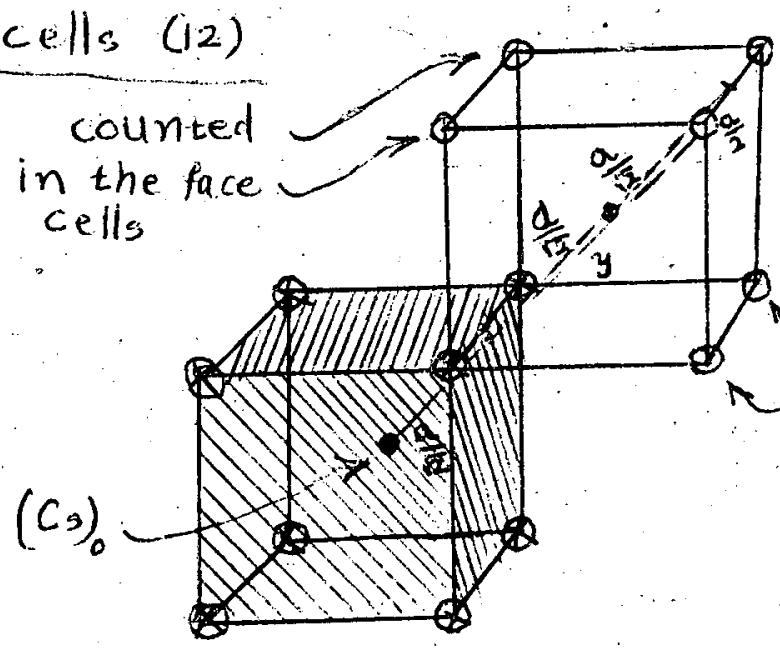

these have been counted in the face cells

$$
\begin{aligned}
& \left(C_{3}\right)_{0}-c_{5} \text { separation }=2 \frac{a}{\sqrt{2}}=\sqrt{2} a=\sqrt{2} \frac{2}{\sqrt{3}} \cdot \gamma_{0} \\
& \therefore\left(c_{3}\right)_{0}-c_{3} \text { interaction }=-\frac{\sqrt{3}}{2 \sqrt{2}} \times 1=-\frac{\sqrt{3}}{2 \sqrt{2}}
\end{aligned}
$$


65

$$
\begin{aligned}
& y=\left(c_{s}\right)_{c}-c l \text { separation }=\sqrt{\left(\frac{3 a}{\sqrt{2}}\right)^{2}+\left(\frac{a}{2}\right)^{2}} \\
& =a \cdot \sqrt{\left(\frac{3}{\sqrt{2}}\right)^{2}+\left(\frac{1}{2}\right)^{2}} \\
& =a \cdot \sqrt{\frac{19}{4}}=\frac{2}{\sqrt{3}} \frac{\sqrt{19}}{2} r_{0}=\sqrt{\frac{79}{3}} r_{0} \\
& \therefore \text { Interaction }=\frac{2}{4} \times \sqrt{\frac{3}{19}} \quad\left(2-n_{\text {umber of }} a\right. \text { ions in } \\
& \text { secondary shall } \\
& \therefore 4 \text { - each only } \frac{1}{4} \text { in the shell) }
\end{aligned}
$$

$\therefore$ Total interaction of edge cells $=12\left(-\frac{\sqrt{3}}{2 \sqrt{2}}+\frac{1}{2} \sqrt{\frac{3}{19}}\right)$

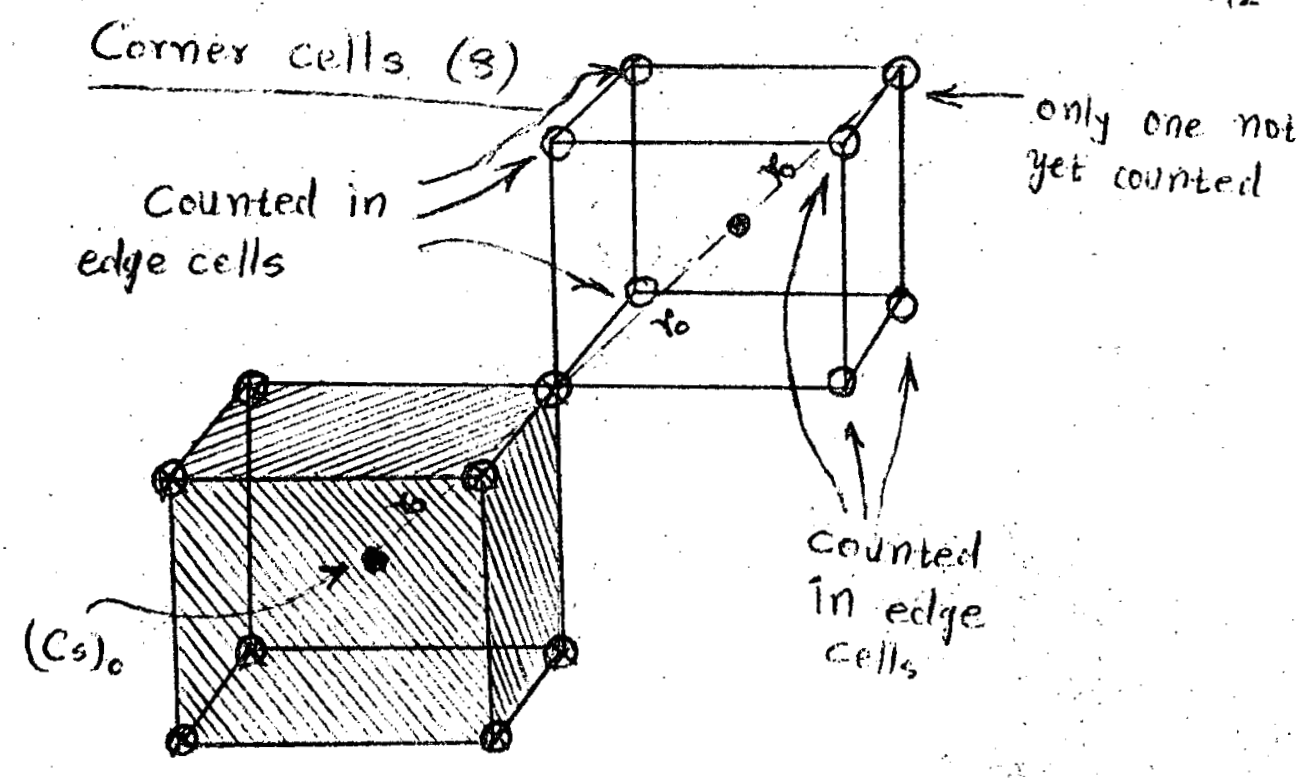

$$
\begin{aligned}
\left(C_{s}\right)_{0}-C \text { s separation } & =2 r_{c} \\
\therefore \text { Interaction }=-\frac{1}{2} \times 1 & =-\frac{1}{2} \\
\left(C_{s}\right)_{0}-C l \text { separation }=3 r_{0} & =\sqrt{\frac{27}{3}} r_{0}
\end{aligned}
$$


66

$$
\therefore \text { Interaction }=\frac{1}{8} \times \sqrt{\frac{3}{27}}
$$

$(1-\#$ of $C)$ ions in secondary shell 8 - only $\frac{1}{8}$ in the shell)

$\therefore$ Total interaction of 8 corner

$$
\text { shells }=8\left(-\frac{1}{2}+\frac{1}{8} \sqrt{\frac{3}{27}}\right)
$$

Interaction due to remainder $\mathrm{cl}$ at the corners of primary cell $=\frac{7}{8} \times 8$

$\therefore$ Second term in Madelung constant die to secondary shell is

$$
\begin{aligned}
&=6\left(-\frac{\sqrt{3}}{2}\right.\left.+2 \sqrt{\frac{3}{11}}\right)+12\left(-\frac{\sqrt{3}}{2 \sqrt{2}}+\frac{1}{2} \sqrt{\frac{3}{17}}\right) \\
&+8\left(-\frac{1}{2}+\frac{1}{8} \sqrt{\frac{3}{27}}\right)+\frac{1}{8} \times 8 \\
&=1.0706-4.964-3.667+7.000=-0.56
\end{aligned}
$$

Thus we see that the series for Madtlung constant

$$
M=1-0.56+\ldots
$$

does not converge easily. 
67

Problem 4.6

(a)

$$
\begin{aligned}
E_{\text {coil } / \text { vo }_{2} \text { molecule }}= & -\frac{z^{2} M e^{2}}{a} \\
M & =11.6365 \quad \ldots \text { (1) } \\
z & =2 \text { (oxygen charge) }
\end{aligned}
$$

Repulsive potentials

$$
\begin{aligned}
& 0^{-2}-0^{-2} \text { interaction } \phi_{c c}(r)=b_{00} e^{-r / s_{00}} \\
& U^{+4}-U^{+4} \text { interaction } \quad \phi_{u u}(r)=b_{u u} e^{-r / 5 u 4} \\
& u^{+4}-0^{-2} \text { interaction } \phi_{40}(\gamma)=b_{u 0} e^{-\gamma / s_{u 0}} \\
& b_{i j}=627.32\left(n_{i} n_{j}\right)^{1 / 2} g_{i} g_{j} \\
& f_{i j}=\frac{a_{c}}{\frac{1}{2}\left(g_{i}+g_{j}\right)} ; n_{i}=\begin{array}{c}
\text { total number of electrons } \\
\text { in ion }
\end{array} \\
& a_{0}=\text { Bohr radius for } H=.52 \% \\
& g_{0}=1.06, \quad g_{u}=1.62
\end{aligned}
$$

Lattice Sum for Repulsive Potentials

$$
E_{3 e p}^{0 \cdots 0}=\frac{1}{2} N_{0}\left[6 \phi_{c c}\left(r_{0 c}\right)\right]
$$

(since there are 6 nearest neighbour atoms as oxygen is in sc (attica)

The factor $\frac{1}{2}$ avoids counting bonds twice when sum over lattice is taken. 
68

$$
\begin{aligned}
& N_{0}=2 \mathrm{NuO}_{2} \text { and } \gamma_{0 \mathrm{oc}}=\frac{1}{2} a \quad(a-\text { entice constant } \\
& \text { of fluorite structure) } \\
& \frac{E_{\text {rep }}^{0.0}}{N_{4 a_{2}}}=6 b_{00} e^{-(a / 2) / \rho_{c o}} \\
& E_{\text {rep }}^{u-u}=\frac{1}{2} N_{u}\left[12 \phi_{u u}\left(\gamma_{u u}\right)\right] \text { (since there are } 12 \text { nearest } \\
& \text { neighbours, as uranium } \\
& \text { atoms are in fou entice) } \\
& N_{u}=N_{\mathrm{HO}_{2}} \text { and } r_{u u}=a / \sqrt{2} \\
& \therefore \frac{E_{\text {rep }}^{u-w}}{N_{4 O_{2}}}=6 b_{u 4} e^{-(a / \sqrt{2}) / s_{u u}} \\
& E_{\text {rep }}^{H-O}=N_{u}\left[8 \phi_{40}\left(r_{i c}\right)\right]
\end{aligned}
$$

(since each $U^{4}$ atom has $s$ nearest neighbour $0^{-2}$ atoms at a distance of $\gamma_{u_{0}}=\frac{\sqrt{3} a}{4}$.)

Also there is no need for the factor $\frac{1}{2}$ as sum is over different species.

$$
\therefore \frac{E_{\text {rep }}^{u-0}}{N_{u_{2}}}=8 b_{u c} e^{-(\sqrt{3} a / 4) / s_{u c}}
$$

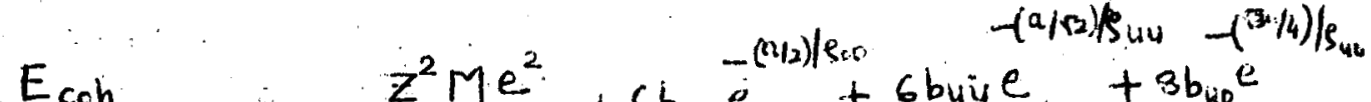

$$
\begin{aligned}
& \frac{E}{\mathrm{NuO}_{2}}=-\frac{z^{2} \mathrm{Me}}{a}+6 b_{\mathrm{bc}} \mathrm{e}+6 \mathrm{buve}
\end{aligned}
$$


69

(b) $\frac{d^{2}\left(E_{c b p} / N_{\left(\mathrm{CO}_{2}\right.}\right)}{d v^{2}}=\frac{1}{\beta \cdot V}$

$V=$ volume per $\mathrm{VO}_{2}$ molecule

$V=\frac{1}{4} a^{3}$. (Since 4 vo molecules per cube of length, see Fig. 3.12 (b) )

$$
\begin{aligned}
& \frac{d}{d v}=\frac{d a}{d v} \frac{d}{d a}=\frac{1}{\frac{3}{4} a^{2}} \frac{d}{d a} \\
& \frac{d^{2}}{d v^{2}}=\frac{d}{d a}\left(\frac{1}{\frac{3}{4} a^{2}} \frac{d}{d a}\right) \frac{d a}{d v}=\frac{1}{\frac{3}{4} a^{2}}\left[\frac{1}{\frac{3}{4} a^{2}} \frac{d^{2}}{d a^{2}}-\frac{8}{3 a^{3}} \frac{d}{d a}\right] \\
&=\frac{16}{9 a^{4}}\left[\frac{d^{2}}{d a^{2}}-\frac{2}{a} \frac{d}{d a}\right]
\end{aligned}
$$

But $\frac{d E_{c o n}}{d a}=0$ (since at equilibrium this (s) condition must be satisfied)

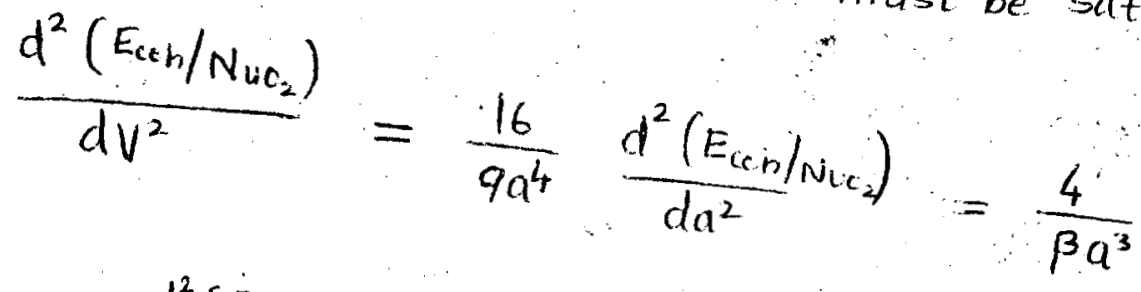

$$
\begin{aligned}
& \therefore \frac{d^{2}\left[\dot{E}_{\left.(i n) / N_{u c}\right]}\right]}{d a^{2}}=\frac{9 a}{4 \beta}=-2 \frac{z^{2} m \epsilon^{2}}{a^{3}}+6\left(\frac{1}{2 s_{c c}}\right)^{2} b_{c c c} e^{-a / 2 s_{c c}} \\
& +6\left(\frac{1}{\sqrt{25} s_{u u}}\right)^{2} b_{u v} e^{-i)^{2} \rho_{u v}} \\
& +8\left(\frac{\sqrt{3}}{4840}\right)^{2} b_{40} e^{-\sqrt{3} a / 4 s_{10}}
\end{aligned}
$$


70

$$
\frac{1}{\beta}=\frac{4}{9 a} \frac{d^{2}\left[E_{(\alpha)} / N_{u_{2}}\right]}{d a^{2}} \quad \text { from } E q \text { (6) }
$$

(c) From eq. (4) the cohesive energy per molecule of $\mathrm{NO}_{2}$ is

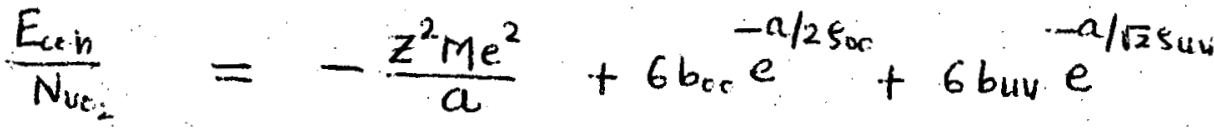

$$
\begin{aligned}
& +s b_{u c} e^{-\sqrt{3} m / 4 s_{u 0}}
\end{aligned}
$$

At equilibrium from (5)

$$
\frac{d E_{c i b}}{d a}=0
$$

or $\frac{z^{2} m e^{2}}{a^{2}}+6 b_{c c}\left(-\frac{1}{25_{e c}}\right) e^{-a / 2 \rho_{0 v}}+6 b_{u u}\left(-\frac{1}{\sqrt{2} s_{u v}}\right) e^{-a / v \rho_{u v}}$

$$
+8 b_{u c}\left(-\frac{\sqrt{3}}{4 \varphi_{40}}\right) e^{-\sqrt{34} / 4 s_{40}}=0
$$

We can substitute for bi from eq. (3) and $\rho_{i j}$ from eq. (4) into eq.(8) and solve for ' $a$ ' by trial.

and error. Resulting value of $a=a_{0}$ can be substituted into eq. (7) to obtain $E_{i c i n} / N_{u_{2}}$ and into eq. (6) to obtain $\beta$. 
71

Problem 4.7

(a) Repilsive potentials:

$$
\begin{aligned}
& U_{x x}^{\text {sep }}=12 \phi_{x x}^{x}\left(\sqrt{2} r_{0}\right) \\
& U_{x+1}^{r e p}=6 \phi_{x M}^{\gamma}\left(r_{c}\right) \\
& U_{m p}^{\text {rep. }} \cong 0 \\
& E_{c \text { cin }}=\frac{U_{\text {tct }}}{N}=\frac{1}{2} \cdot 12 \cdot \Phi_{x x}^{r}\left(\sqrt{2} r_{0}\right)+6 \Phi_{x / 1}^{r}\left(r_{0}\right)-\frac{M e^{2}}{r_{0}} \\
& \therefore E_{c s h} \quad=6 \phi_{x x}^{r}\left(\sqrt{2} r_{c}\right)+6 \phi_{x h}^{\gamma}\left(r_{0}\right)^{\prime}-\frac{M e^{2}}{r_{0}}
\end{aligned}
$$

(b)

$$
\begin{aligned}
& \phi_{x x}^{\gamma}(r)=b e^{2 R_{x} / s} e^{-\gamma / s} \\
& \phi_{m_{x}(\gamma)}^{\gamma}=b e^{\left(R_{x}+R_{m}\right) / s} e^{-\gamma / s}
\end{aligned}
$$

Neglecting anion-anion repulsive potential

$$
\begin{aligned}
& A=b e^{\left(R_{x}+R_{M}\right) / s} \\
& b e^{2 R_{x} / \mathrm{s}}=b e^{\left(R_{x}+R_{m}\right) / \mathrm{s}} e^{\left(R_{x}-R_{M}\right) / \mathrm{s}}=A f \\
& \text { where } f=e^{\left(R_{x}-R_{r_{1}}\right) / \mathrm{s}} \\
& E_{\text {coh }}=-179 \mathrm{kcal} / \mathrm{mole}=-749.44 \mathrm{kjoules} / \mathrm{mole} \\
& A=2.5 \times 10^{4} \mathrm{kcal} / \mathrm{mcle}=10.47 \text { kjoules } / \mathrm{mole}
\end{aligned}
$$


72

$$
\begin{aligned}
& \gamma_{\text {oe }}=0.93+1.48 .1=2.79 \mathrm{~A}^{\circ} \\
& \rho=0.321 \mathrm{~A}^{\circ}
\end{aligned}
$$

If anion-anion repulsion is included

$$
\begin{aligned}
& U=6 A f e^{-\sqrt{2} r_{0} / S}+6 A e^{-\gamma_{c} / S}-\frac{M e^{2}}{r_{0}} \\
& f=e^{(1.31-0.75) / 0.321}=e^{2.59}=13.3 \\
& e^{-\sqrt{2} r e q / 9}=e^{-(1.414)(2.79) / 0.321}=e^{-12.41}=4.1 \times 10^{-6} \\
& \text { correction factor }=(6)(2.5)(13.3)(4.1) \times 10^{-2} \\
& =8.18 \quad \mathrm{kcal} / \mathrm{mole} \\
& =34.24 \text { kjoile/mele. }
\end{aligned}
$$$$
\therefore \text { error }=\frac{8.18}{179} \times 100
$$$$
=4.6 \%
$$ 
73

Problem 4.8

(a)

$$
\begin{aligned}
& \left.\left.\phi(r)=\phi\left(r_{0}\right)+\frac{d \phi}{d r}\right)_{r_{0}}\left(r-r_{c}\right)+\frac{1}{2} \frac{d^{2} \phi}{d r^{2}}\right)_{\gamma_{0}}\left(r-x_{0}\right)^{2} \\
& \left.\frac{d \phi}{d r}\right)_{r_{0}}=\text { at equilibrium } \\
& \phi\left(x_{2}\right)=-D \\
& \frac{d \phi}{d r}=D(2 \alpha)\left[-\exp \left[-2 \alpha\left(r-r_{0}\right)\right]+\exp \left[-\alpha\left(r-r_{c}\right)\right]\right\} \\
& \frac{d^{2} \phi}{d r^{2}}=2 \alpha D\left\{2 \alpha \exp \left[-2 \alpha\left(r-\gamma_{0}\right)\right]-\alpha \exp \left[-\alpha\left(\gamma-r_{0}\right)\right]\right\} \\
& \left.\therefore \quad \frac{d^{2} \phi}{d r^{2}}\right|_{r_{c}}=K=2 \alpha^{2} D \\
& \therefore \phi(\gamma)=-D+\alpha^{2} D\left(\gamma-\gamma_{c}\right)^{2}
\end{aligned}
$$

(b) Lattice sum considering only nearest neighbour interactions is

$$
\begin{aligned}
& E_{\text {cen }}=\frac{1}{2} N \cdot \sigma-\phi(\gamma) \\
& \text { at } \gamma=r_{0}, \phi(\gamma)=-D \\
& \therefore E_{\text {cen }}=-3 N \cdot D
\end{aligned}
$$


$74^{\circ}$

(c) $\quad \frac{1}{\beta}=V \frac{d^{2} E_{0}}{d V^{2}}$

$E_{0}=$ Energy of lattice at $0^{\circ} \mathrm{K}$. for arbitrary nearest neighbour distance $r$;

$$
E_{c h}=E_{0}\left(\gamma_{c}\right)
$$

Lattice sum : (nearest neighbours only)

$$
E_{c}(\gamma)=\frac{1}{2} N \cdot 6 \cdot \phi(\gamma)
$$

sc lattice $V=N r^{3} \quad \frac{d v}{d r}=3 N r^{2}$

$$
\begin{aligned}
& \frac{d^{2}}{d v^{2}}=\frac{d r}{d v} \frac{d}{d r}\left(\frac{d r}{d v} \frac{d}{d r}\right)=\frac{1}{9 N^{2} r^{4}} \frac{d^{2}}{d r^{2}} \\
& \left(a s \frac{d}{d r} E_{0}=0 \quad \text { at } r=\gamma_{0}\right) \\
& \therefore \quad \frac{1}{\beta_{0}}=\left.N r_{0}^{3} \frac{\frac{1}{2} N \cdot 6}{9 N^{2} \gamma_{0}^{4}} \frac{d^{2} \phi}{d r^{2}}\right|_{\gamma_{0}} \\
& =\frac{\pi}{3 \gamma_{0}} \\
& \therefore \beta_{0}=\frac{3 \gamma_{0}}{K}=\frac{3 \gamma_{0}}{2 \alpha^{2} D} \text { from eq. (2) }
\end{aligned}
$$

(d) From Problem 2.2 of chapter 2, planes interact by force constant $k$. 
75

Equilibrium separation at $0^{\circ} \mathrm{K}=\gamma_{0}$

Velocity of sound $=\mu_{s}=\sqrt{\frac{\kappa r_{0}^{2}}{m}}$

simple cubic lattice, $\frac{V}{N}=r_{0}^{3}=$ volumelatom

Debye frequency $\quad \omega_{D}=C_{S}\left(6 \pi^{2} \frac{N}{V}\right)^{1 / 3}$

$$
\begin{aligned}
\omega_{D} & =\sqrt{\frac{k r^{2}}{m}}\left(\frac{6 \pi^{2}}{\gamma_{0}^{3}}\right)^{1 / 3} \\
& =\sqrt{\frac{\pi}{m}}\left(6 \pi^{2}\right)^{1 / 3}
\end{aligned}
$$

Gruneisen constant $\gamma=-\frac{v}{\theta_{D}} \frac{d \theta_{D}}{d v}$

$$
=\frac{d \omega_{D}}{d r_{0}}=0
$$


76

Problem 4.9

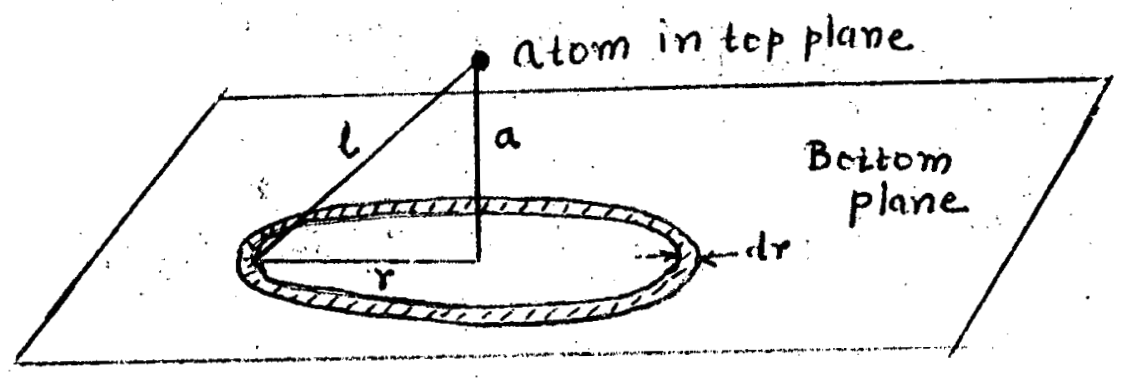

atom density $N$ per unit area

Pair potential $\phi$ is

$$
\Phi=-\frac{c}{r^{6}}+A e^{-\gamma / e}
$$

\# of atoms in differential ring $=2 \pi \operatorname{rdr} \mathrm{N}$

Then, potential (t. e-energy) between top atom and the differential ring, $d \Phi$

$$
d \Phi=2 \pi r d r N \Phi(\ell)
$$

where $l=\sqrt{a^{2}+r^{2}}$

So potential between an atom and the plane is given by

$$
\begin{aligned}
& \Phi=2 \pi N \int_{0}^{\infty} r\left[-\frac{c}{l^{6}}+A e^{-l / \rho}\right] d r \\
&=2 \pi N \int_{0}^{\infty}\left\{\frac{-c r}{\left(a^{2}+r^{2}\right)^{3}}+A r e^{-\sqrt{a^{2}+r} / \rho}\right\} d r \\
& \text { Now } \quad \int_{0}^{\infty} A r e^{-\sqrt{a^{2}+r^{2}} / s} d r=A \int_{a}^{\infty} u e^{-l / \rho} d u
\end{aligned}
$$


77

$$
\begin{aligned}
\therefore \int_{0}^{\infty} A r e^{-\sqrt{a^{2}+r^{2}} / \rho} d r & =-\left.A \rho u e^{-u / s}\right|_{a} ^{\infty}-\left.\rho^{2} A e^{-u / s}\right|_{a} ^{\infty} \\
& =+A \rho a e^{-a / s}+\rho^{2} A e^{-a / s} .
\end{aligned}
$$

and

$$
\begin{aligned}
& -\int_{0}^{\infty} \frac{c r d r}{\left(a^{2}+r^{2}\right)^{3}}=\left.\frac{c}{4\left(a^{2}+r^{2}\right)^{2}}\right|_{0} ^{\infty}=-\frac{c}{4 a^{4}} \\
& \therefore \Phi=\left\{-\frac{c}{4 a^{4}}+A \rho a e^{-a / s}+A s^{2} e^{-a / s}\right\} 2 \pi N \\
& \text { or } \Phi=2 \pi N\left\{-\frac{c}{4 a^{4}}+\rho^{2} A\left(\frac{a}{\rho}+1\right) e^{-a / s}\right\}
\end{aligned}
$$

At equilibrium, $\frac{\partial \Phi}{\partial a}=0$, gives.

$$
\frac{c}{s^{6} A}=\left(\frac{d}{s}\right)^{6} e^{-a / s}
$$
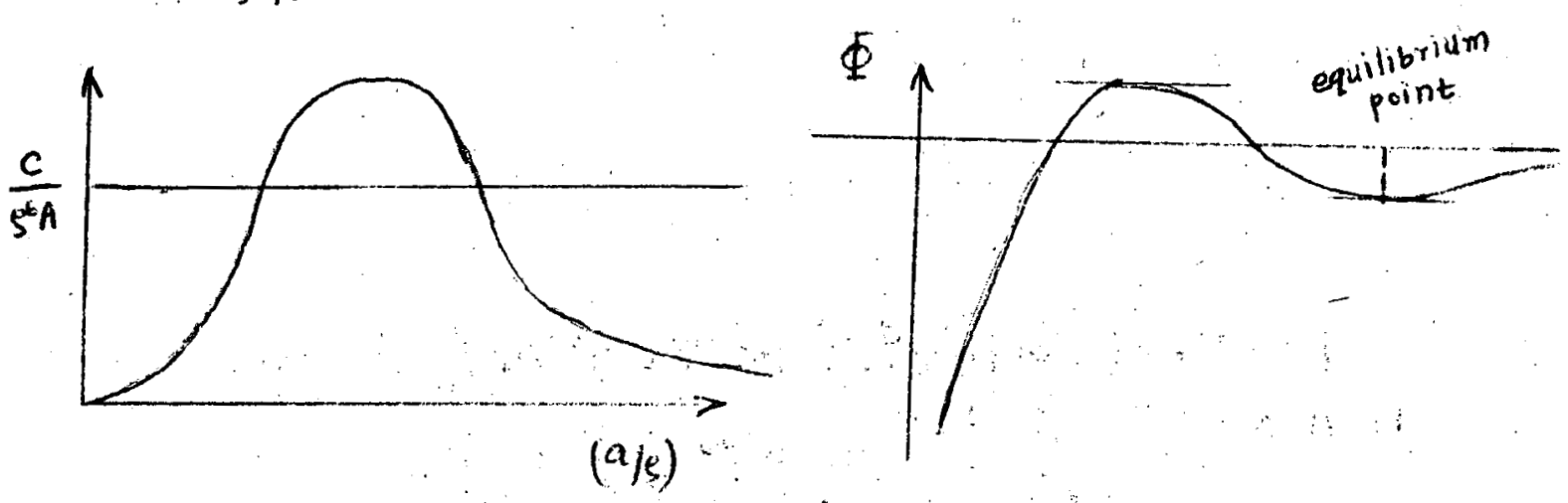

For given constants $c / 5^{6} A$, there are two solutions but only larger one is the correct answer. 
78

Problem 4.10

$$
\dot{\theta} \dot{r}_{0}^{\oplus} r_{r_{0}}^{\Theta}{ }_{r_{0}}^{\oplus} r_{r_{0}}^{\Theta} r_{0}^{\oplus}
$$

Look at the potential energy of the o ion.

The contribution due to 2 nearest $\theta$ ions is

$$
2 A e^{-r_{0} / s}-2 \frac{q^{2}}{r_{0}}
$$

2 next nearest (1) ions contribute $2 \frac{q^{2}}{2 \gamma_{0}}$

2 third nearest $\theta$ ions contribute $-2 \frac{q^{2}}{3 \gamma_{c}}$

So $u\left(r_{0}\right)=2 A e^{-r_{0} / s}-\frac{2 q^{2}}{r_{0}}\left(1-\frac{1}{2}+\frac{1}{3}-\frac{1}{4}+\cdots\right)$

$$
=2 A e^{-r_{0} / s}-\frac{2(\ln 2) q^{2}}{r_{0}}
$$

To find the equilibrium radius

$$
\begin{gathered}
\text { let } \frac{\partial u\left(r_{0}\right)}{\partial r_{0}}=0 \\
-A \frac{1}{5} e^{-6 / s}+(\ln 2) q^{2} \frac{1}{r_{0}^{2}}=0 \\
\text { or } A e^{-r_{0} / s}=\frac{\rho}{r_{0}^{2}}(\ln 2) q^{2}
\end{gathered}
$$

So $U_{\text {con }}=N U\left(r_{0}\right)=N\left[\frac{2 \rho}{r_{0}^{2}}(\ln 2) q^{2}-2(\ln 2) \frac{q}{r_{0}}\right]$ $=\frac{2 N}{\gamma_{0}}(\ln 2) q^{2}\left(\frac{\rho}{\gamma_{0}}-1\right)$ 
79

Problem 4.11

(a) Consider the De-Broglie wave length of the particle $\lambda=\frac{h}{p}$

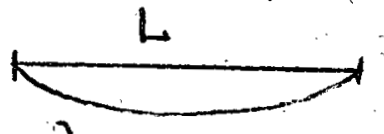

$$
\frac{\lambda}{2}=L
$$

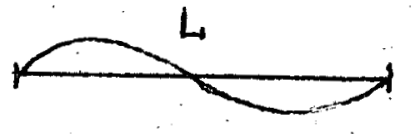

$2 \cdot \frac{\lambda}{2}=L$

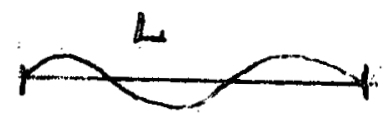

$3 \frac{\lambda}{2}=L$

Allowable quantized particle energy is

$$
n_{\lambda} \frac{\lambda}{2}=L \quad \Rightarrow n_{\lambda}=\frac{2 L}{\lambda} \text {. }
$$

or $n_{p}=\frac{p 2 L}{h}$ or $n_{\varepsilon}=\frac{2 L}{h} \sqrt{2 m \varepsilon}$

So $D(\varepsilon) d \varepsilon=d n_{\varepsilon}=\frac{2 L}{h} \sqrt{2 m} \frac{1}{2 \sqrt{\varepsilon}} d \varepsilon=\frac{L \sqrt{m}}{h \sqrt{\varepsilon}} \sqrt{2} d \varepsilon$.

For each state there are 2 electrons, and the electrons follow Fermi-Dirac distribution.

Thus

$$
N=\int_{0}^{\infty} \frac{2 D(\varepsilon) d \varepsilon}{1+e^{(\mu) / k T}}
$$

at $T=0$

$$
\begin{aligned}
\frac{1}{1+e^{(-\mu) / k r}} & =1 \text { for } \varepsilon \leqslant \mu \\
& =0 \text { for } \varepsilon>\mu
\end{aligned}
$$

So

$$
\begin{aligned}
N & =\int_{0}^{\mu} 2 g(\varepsilon) d \varepsilon=\int_{0}^{\mu} \frac{2 L}{h} \sqrt{\frac{2 m}{\varepsilon}} d \varepsilon \\
& =\frac{2 L}{h} \sqrt{2 m}-2 \cdot \sqrt{\mu} \quad
\end{aligned}
$$


80

$$
\begin{aligned}
\therefore \mu^{\frac{1}{2}} & =\frac{N h}{4 \sqrt{2 m} L} \\
\mu & =\left(\frac{N h}{4 \sqrt{2 m} L}\right)^{2}=\frac{N^{2} h^{2}}{32 m L^{2}}
\end{aligned}
$$

(b)

$$
\begin{aligned}
& \quad \bar{\varepsilon}=\frac{1}{N} \int_{0}^{\infty} \frac{2 \varepsilon D(\varepsilon) d \varepsilon}{1+e^{(\varepsilon-\mu) / k T}} \\
& =\frac{L}{N h} \sqrt{2 m} \int_{0}^{\infty} \frac{2 \sqrt{\varepsilon}}{1+e^{(\varepsilon-\mu) / k T}} d \varepsilon \\
& \text { At } 0^{\circ} k \quad \frac{1}{1+e^{(\varepsilon / \mu) / k T}}=1 \text { for } \varepsilon \leqslant \mu \\
& =0 \text { for } \varepsilon>\mu
\end{aligned}
$$

so $\bar{\varepsilon}=\frac{2 L}{N h} \sqrt{2 m} \int_{0}^{\mu} \sqrt{\varepsilon} d \varepsilon$

$$
\begin{aligned}
=\frac{2 L \sqrt{2 m}}{N h} & \frac{\mu^{3 / 2}}{3 / 2}=\frac{4}{3} \frac{L \sqrt{2 m}}{N h}\left(\frac{N h}{\sqrt{32 m} L}\right)^{3} \\
& =\frac{4}{3} \sqrt{2 m} \frac{N^{2} h^{2}}{32 m \sqrt{32 m} L^{2}} \\
& =\frac{N^{2} h^{2}}{96 m L^{2}}
\end{aligned}
$$


81

CHAPTER 5

Problem 5.1

With multicomponent systems, define $\mu$ such that

$$
d E=T d s-p d v+\sum_{i} \mu_{i} d N_{i} ;
$$

(a) Set $d E=d v=d N_{j}=0$, giving

$$
\begin{aligned}
& -T d S=\left.\mu_{i} d N_{i}\right|_{E, V, N_{j}} \\
& \text { or } \mu_{i}=-T\left(\frac{\partial S}{\partial N_{i}}\right)_{E, V_{,} N_{j}}
\end{aligned}
$$

(b)

$$
\begin{aligned}
& \text { Now } \quad F=E-T S, \text { and from eq.(1) } \\
& \begin{aligned}
d F=d E-T d s-s d T \\
=-S d T-p d V+\sum_{i} \mu_{i} d N_{i}
\end{aligned}
\end{aligned}
$$

Set $\quad d T=d v=d N_{j}=0$, giving

$d F=\left.\mu_{i} d N_{i}\right|_{T, V, N_{j}}$ or $\mu_{i}=\left(\frac{\partial F}{\partial N_{i}}\right)_{r, V, N_{j}}$

(c)

$$
\begin{aligned}
& \text { Also } G=E-T S+p V, \text { and from eq (i) } \\
& \begin{aligned}
d G=d E-T d s-s d T+p d V+V d p \\
=-s d T+V d p+\sum_{i} \mu_{i} d N_{i}
\end{aligned} \\
& \begin{array}{l}
d_{T}=d_{p}=d N_{j}
\end{array}
\end{aligned}
$$

Set $d_{T}=d_{p}=d_{N_{j}=0}$, giving

$$
d G=\left.\mu_{i} d N_{i}\right|_{r, p, N_{j}} \text { or } \mu_{i}=\left(\frac{\partial G}{\partial N_{i}}\right)_{T, P, N_{j}}
$$


82

Problem 5.2

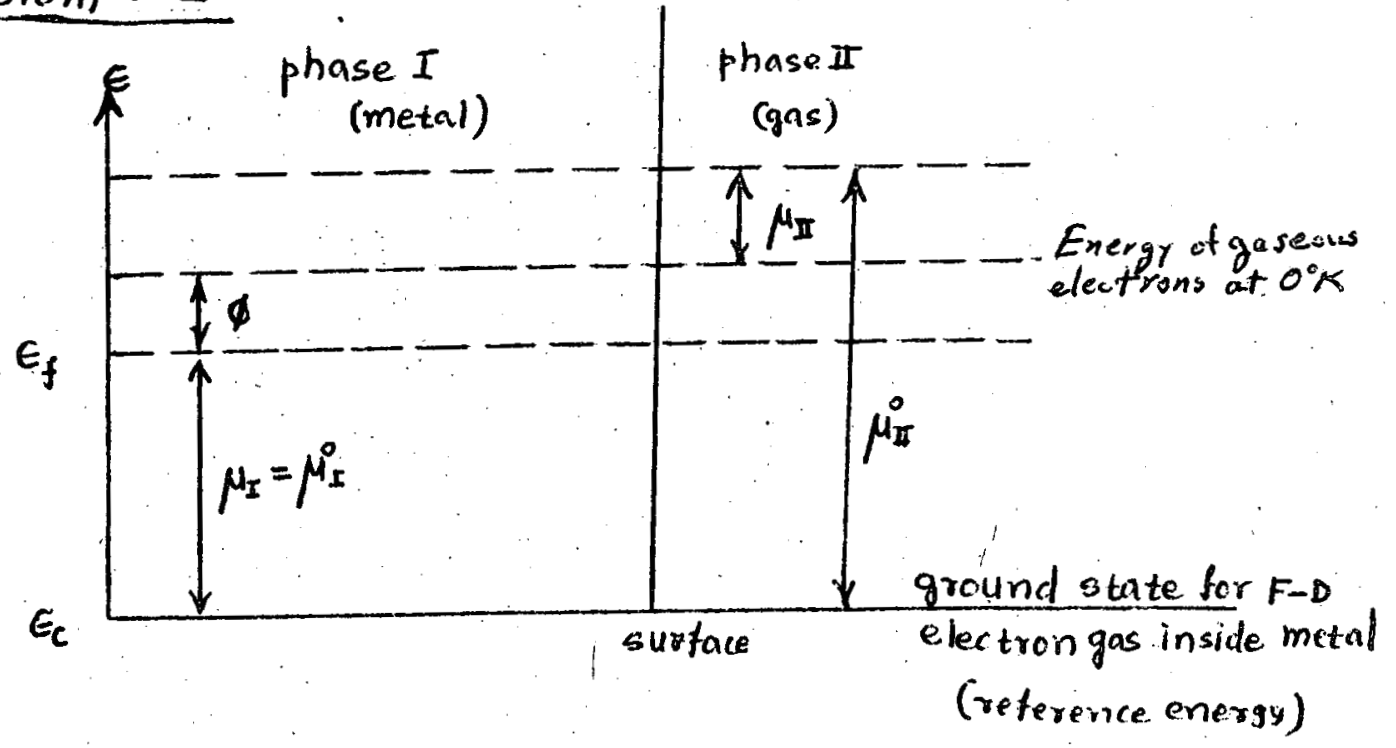

(a) Consider the electrons inside the metal as phase I and the electrons outside as phase II.

At equilibrium $\quad \mu_{I}^{0}=\mu_{\text {II }}^{0}$

Where the superscript o indicates the same reference point)

Phase II is low density, so treat electrons (II) as non -interacting particles (no collisions, no space -charge)

Therefore from classical statistics $Z=\frac{z^{N}}{N !}$

$$
\begin{aligned}
\frac{\partial \ln Z}{\partial N} & =\frac{\partial}{\partial N}(N \ln z-(N \ln N-N)) \\
& =\ln (z / N) \quad(\text { where } \ln N ! \cong N \ln N-N
\end{aligned}
$$
is Stirling's approximation) 
83

$$
\therefore \mu_{I}=-k T\left(\frac{\partial \ln Z}{\partial N}\right)_{V, T}=-k T \cdot \ln (z / N) \text {. }
$$

where $z=z_{\text {tr }}$ for ideal gas of fermions:

$$
\begin{array}{r}
z_{t r}=2 V\left(\frac{2 \pi m k T}{h^{2}}\right)^{3 / 2} \\
\therefore \mu_{I I}=-k T \ln \left\{\frac{2 V_{I}}{N_{I I}}\left(\frac{2 \pi m k T}{h^{2}}\right)^{3 / 2}\right\}
\end{array}
$$

Suppose for common energy reference we choose $\epsilon_{c}$.

$$
\therefore \mu_{I}^{0}=\mu_{I}
$$

Then $\mu_{\mathrm{I}}^{0}=\mu_{\mathrm{r}}^{0}+\phi+\mu_{\text {II }}$

$$
\therefore \mu_{\text {II }}=-\phi
$$

Also $\frac{V_{\text {II }}}{N_{\text {II }}}=\frac{1}{n_{\text {II }}}$

$$
\therefore n_{\pi}=2\left(\frac{2 \pi m k T}{h^{2}}\right)^{3 / 2} e^{-\phi / k T}
$$

(b) The rate at which gaseous electrons strike surface $=\frac{1}{4} n \bar{v}$ where $\bar{v}=\sqrt{\frac{8 k T}{\pi m}}=$ mean velocity of Boltzmann distribution

The surface impingement rate $=j_{x}=n \sqrt{\frac{k T}{2 \pi m}}$ setting $n=n_{\text {III }}, m=m_{e}$ 
84

then electrons in phase $\mathbb{I}$ incident on surface is

$$
\begin{aligned}
j_{I x} & =n_{\mathbb{H} \sqrt{\frac{k T}{2 \pi m_{e}}}} \\
& =\left(\frac{4 \pi m_{e} k^{2}}{h^{3}}\right) T^{2} e^{-\phi / k T \quad \text { per unit time per }} \text { unit area }
\end{aligned}
$$

If no electrons are reflected from the surface, then at equilibrium, the same current is emitted. by the metal; using the principle of detailed. balance:

$$
\therefore \quad i=\left(\frac{4 \pi \cdot m_{e} e k^{2}}{h^{3}}\right) T^{2} e^{-\phi / k T}
$$

charge per unit time per unit area

This treatment does not show the validity of the answer in non-equilibrium conditions. 
85

Problem 5.3

$$
C_{s}(g)=C_{s}^{+}(g)+\bar{e}(g)
$$

a)

$$
\begin{aligned}
& K=\frac{n_{e} n_{c_{s}}}{n_{c_{s}}}=\frac{z_{e} z_{c_{s}}}{z_{c s}} e^{-r / k T}\left(\begin{array}{c}
\text { from law of } \\
\text { mass action }
\end{array}\right) \\
& z_{C_{s}}=\left(\frac{2 \pi m_{c_{s} k T}}{h^{2}}\right)^{3 / 2} \\
& z_{c_{s}}=2\left(\frac{2 \pi m_{c_{s} k T}}{h^{2}}\right)^{3 / 2} \quad\left(\begin{array}{c}
\text { two spin states of } \\
1 \text { s electron }
\end{array}\right) \\
& \frac{n_{c_{s}}}{n_{c_{s}}}=\frac{z_{e}}{n_{e}}\left(\frac{z_{c_{s}}}{z_{c_{s}}}\right) e^{-I / k T}=\frac{1}{2} \frac{z_{e}}{n_{e}} e^{-5 / k T}
\end{aligned}
$$

b)

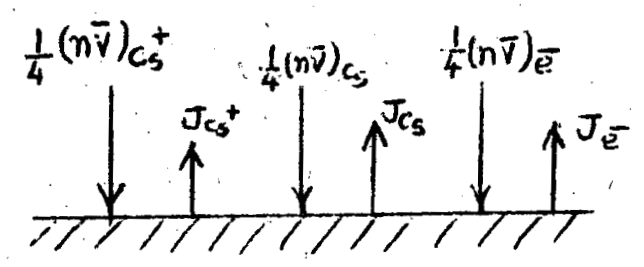

metal

Detailed balance:

$$
\begin{aligned}
& J_{C_{s}}=\frac{1}{4}(n \bar{v})_{C_{s}} \\
& J_{C_{s}}=\frac{1}{4}(n \bar{v})_{C_{s}} \\
& \therefore \frac{J_{C_{s}}}{J_{C_{s}}}=\frac{n_{c_{s}}}{n_{C_{s}}}=\frac{k}{n_{c}}=\frac{1}{2} \frac{\xi_{e}}{n_{e}} e^{-I / k T}
\end{aligned}
$$


86

$$
z_{e}=2\left(\frac{2 \pi m_{e} k T}{h^{2}}\right)^{3 / 2}
$$

From the previous problem

$$
\begin{aligned}
& n_{e}=2\left(\frac{2 \pi m_{e} k T}{h^{2}}\right)^{3 / k} e^{-\phi / k T}=z_{\bar{e}} e^{-\phi / k T} \\
& \therefore \frac{n_{e}}{z_{e}}=e^{-\phi / k T} \\
& \therefore \frac{J_{C_{s}}}{J_{c_{s}}}=\frac{1}{2} e^{-(I-\phi) / k T}
\end{aligned}
$$

(Saha-Langmuir equation) 
87

Problem 5.4

$E_{q .}(1.51)$ is $\ln Z=-\frac{\mu N}{k T}+\sum_{k} \ln \left[1+e^{-\left(\epsilon_{k}-\mu\right) / k T}\right]$

Eq. (1.52) is $\quad N=\sum_{k}\left[1+e^{\left(\sigma_{k}-\mu\right) / k r}\right]^{-1}$

$E_{q}$ (5.15) is $\mu_{i}=-k T\left(\frac{\partial \ln Z}{\partial N_{i}}\right)_{T, V, N_{j}}$

For a single component system $\mu=\mu(\tau, N N)$

and $\left(\frac{\partial}{\partial N}\right)_{V, T}=\frac{1}{V}\left[\frac{\partial}{\partial(N / V)}\right]_{T, V}$

$$
\begin{gathered}
\therefore\left[\frac{\partial \ln z}{\partial N}\right]_{T, V}=-\frac{\mu}{k T}-\frac{N / V}{k T}\left[\frac{\partial \mu}{\partial(N / V)}\right]_{T, V} \\
+\frac{1}{V} \sum_{k} \frac{\partial}{\partial(N / T)} \ln \left[1+e^{-\left(\sigma_{k}-\mu\right) / k T}\right] \\
\frac{1}{V} \sum_{k} \frac{\partial}{\partial(N / V)} \ln \left[1+e^{-\left(\epsilon_{k}-\mu\right) / k T}\right]=\frac{1}{V k T}\left[\frac{\partial \mu}{\partial(N / V)}\right]_{T, V} \sum_{k}\left[1+e^{\left(\epsilon_{k}-\mu\right) / k T}\right]^{-1}
\end{gathered}
$$

Substituting eq .(1.52) into the above, we get

$$
\begin{aligned}
{\left[\frac{\partial \ln z}{\partial N}\right]_{T, V} } & =-\frac{\mu}{k T}-\frac{N / N}{k T}\left[\frac{\partial \mu}{\partial(N / N)}\right]_{T, V}+\frac{N N}{k T}\left[\frac{\partial \mu}{\partial(N / N)}\right]_{T, V} \\
& =-\frac{\mu}{k T} \\
\therefore\left[\frac{\partial \ln z}{\partial N}\right]_{T, V} & =-\frac{\mu}{k T}
\end{aligned}
$$

This is Eq. (s.15) for a single component system. 
88

Problem 5.5

$$
H_{2}(g) \rightleftharpoons 2 H(g)
$$

a) Following the same steps as discussed in Chap. 5 from Eq. (5.29), writing

$$
\begin{aligned}
& P_{c}=P_{H} ; P_{A}=P_{H_{2}} \text { and using } a=1, b=0, c=2 \\
& K_{P}=\left(\frac{2 \pi}{h^{2}}\right)^{\frac{3}{2}(2-1)}(k T)^{\frac{5}{2}(2-1)}\left(\frac{m_{H}^{2}}{m_{H_{2}}}\right) \frac{1}{\left(z_{2}\right)_{\text {int }}} e^{-\Delta \epsilon_{0} / k T}
\end{aligned}
$$

Since $\frac{m_{H^{2}}^{2}}{m_{H_{2}}}=\frac{1}{2}$, and $\Delta \epsilon_{0}=D$

$$
\therefore \quad K_{p}=\left(\frac{\pi}{h^{2}}\right)^{3 / 2}(k T)^{5 / 2} \frac{e^{-D / k T}}{\left(z_{2}\right)_{i n t}} .
$$

b) Let $\alpha$ be the dissociation coefficient Then at equilibrium,

$$
\begin{aligned}
& H_{2} \rightleftharpoons 2 H \quad((1-\alpha) \text { and } 2 \alpha \text { are } \\
& (1-\alpha) \\
& \left.2 \alpha \quad \text { no. of moles } H_{2} \text { and } H\right)
\end{aligned}
$$

From Perfect Gas law $p V=n R T$ ( $n$ is no. of

$$
\begin{aligned}
& \frac{P_{H_{2}}}{1-\alpha}=\frac{P_{H}}{2 \alpha}=\frac{P}{1+\alpha} \quad(P \text { is total pressure) } \\
\therefore & P_{H_{2}}=\frac{1-\alpha}{1+\alpha} P \text { and } P_{H}=\frac{2 \alpha}{1+\alpha} \cdot P \\
\therefore & K_{P}=\frac{P_{H}^{2}}{P_{H_{2}}}=\frac{4 \alpha^{2} P^{2}}{(1+\alpha)^{2}} \cdot \frac{1+\alpha}{(1-\alpha) P}=\left(\frac{4 \alpha^{2}}{1-\alpha^{2}}\right) P
\end{aligned}
$$


89

$$
\begin{aligned}
\therefore \quad & x^{2}\left(4 P+K_{P}\right)=K_{P} \\
& \therefore \quad x=\sqrt{\frac{K_{P}}{4 P+K_{P}}}
\end{aligned}
$$


90

Problem 5.6

$$
\begin{aligned}
& \Delta S^{0}=c S_{c}^{0}-a S_{A}^{0}-b S_{B}^{0} \\
& \Delta\left(H^{0}-H_{0}^{0}\right)=c\left(H_{c}^{0}-H_{c_{0}}^{0}\right)-b\left(H_{B}^{0}-H_{B_{0}}^{0}\right)-a\left(H_{A}^{0}-H_{A_{0}}^{0}\right)
\end{aligned}
$$

Eq. (1.17) gives

$$
S_{i}^{0}=k \ln Z_{i}^{0}+k T\left(\frac{\partial \ln z_{i}^{\circ}}{\partial T}\right)_{V}
$$

Eq. (1.31) gives

$$
\begin{aligned}
& H_{i}^{0}-H_{i 0}^{0}=k T\left[T\left(\frac{\partial \ln Z_{i}^{c}}{\partial T}\right)_{V}+V\left(\frac{\partial \ln Z_{i}^{0}}{\partial V}\right)_{T}\right] \\
& \left.\frac{S_{i}^{0}}{R}-\frac{H_{i}^{0}-H_{i 0}^{0}}{R T}=\frac{\ln Z_{i}^{0}-V\left(\frac{\partial \ln Z_{i}^{0}}{\partial V}\right)_{T}}{N} \quad \text { (as } R=N k\right)
\end{aligned}
$$

$z_{i}^{0}=$ partition function above ground state of $i$ Eq. (5.18) and (5.19) give

$$
\begin{aligned}
& Z_{i}^{0}=\frac{V^{N_{i}}}{N_{i} !}\left(\frac{2 \pi m_{i} k T}{h^{2}}\right)^{\frac{3}{2} N_{i}}\left(3_{i}^{0}\right)_{i n t .}^{N_{i}} \\
& \ln Z_{i}^{0}=N_{i} \ln V+\frac{3}{2} N_{i} \ln \left(\frac{2 \pi m_{i} k T}{h^{2}}\right)+N_{i} \ln \left(3_{i}^{0}\right)_{\text {int. }} \\
& -N_{i} \ln N_{i}+N_{i} \\
\therefore & V\left(\frac{\partial \ln Z_{i}^{0}}{\partial V}\right)_{T}=N_{i} \\
\therefore & {\left[\frac{S_{i}^{0}}{R}-\frac{H_{i}^{0}-H_{i 0}^{0}}{R T}\right]=\ln \left[\left(\frac{2 \pi m_{i} k T}{h^{2}}\right)^{3 / 2}\left(\dot{Z}_{i}^{0}\right)_{\text {int. }}\left(\frac{V}{N_{i}}\right)\right] }
\end{aligned}
$$


98

$$
\begin{aligned}
\therefore \exp \left[\frac{\Delta s^{0}}{R}-\frac{\Delta\left(H^{0}-H_{0}^{0}\right)}{R T}\right]= & \frac{\left(\frac{2 \pi m_{c} k T}{i^{2}}\right)^{3 / 2 c}}{\left(\frac{2 \pi m_{B} k T}{h^{2}}\right)^{3 / 2 b}\left(\frac{2 \pi m_{A k T}}{h^{2}}\right)^{3 / 2 a}} \\
& \frac{\left(z_{C}^{0}\right)_{i n t}^{c}}{\left(z_{B}^{0}\right)_{i n t .}^{b}\left(z_{A}^{0}\right)_{i n t}^{a}} \cdot\left(\frac{V}{N}\right)^{(c-a-b)}
\end{aligned}
$$

But $\frac{N}{V}=n=\frac{P_{c}}{k T}$ molecules/cc

Note as $P_{0}, V$ and $T$ are same for all three species, $N_{A}=N_{B}=N_{C}=N$

$$
\begin{gathered}
\text { With } P_{0}=1 \mathrm{~atm}=10^{6} d_{\text {ynes }} /\left(\mathrm{cm}^{2}\right) \\
\exp \left[\frac{\Delta s^{0}}{R}-\frac{\Delta\left(H^{0}-H_{0}^{0}\right)}{R T}\right]=\left(\frac{2 \pi}{h^{2}}\right)^{\frac{3}{2}(c-a-b)} \frac{(k T)^{\frac{5}{2}(c-a-b)}}{\left(10^{6}\right)^{(c-a-b)}} \\
\cdot\left(\frac{m_{c}^{c}}{m_{A}^{a} m_{B}^{b}}\right)^{\frac{3}{2}} \cdot \frac{\left(z_{c}^{0}\right)_{\text {int. }}^{c}}{\left(z_{A}^{0}\right)_{\text {int. }}^{a}\left(z_{B}^{c}\right)_{\text {int. }}^{b}}
\end{gathered}
$$

agrees with Eq. $(5.29)$ with additional factor of $\left(10^{6}\right)^{(c-a-b)}$ to convert partial pressures from dynes/ cm. in Eq. (5.29) to atmospheres in Eq. (5.59) 
92

Problem 5.7

(a) The total partition function of the system is

$$
Z=\frac{\gamma_{e}^{N_{e}}}{N_{e} !} \cdot \frac{z_{h}^{N_{h}}}{N_{h} !} \cdot \gamma_{b}^{N_{b}}
$$

(b) The criterion for equilibrium is

$$
\mu_{b}=\mu_{e}+\mu_{h}
$$

(c)

$$
\begin{aligned}
& \mu_{b}=-k T \frac{\partial \ln z}{\partial N_{b}}=-k T \ln z_{b} \\
& \mu_{e}=-k T \ln \left(z_{e} / N_{e}\right) \\
& \mu_{h}=-k T \ln \left(z_{h} / N_{n}\right)
\end{aligned}
$$

(d) From eqs.(2)-(s) we have

$$
z_{b}=\frac{z_{e} z_{h}}{N_{e} N_{h}} \quad \therefore N_{e} N_{h}=\frac{z_{e} z_{h}}{z_{b}}
$$

Referring to the common ground state

$$
\frac{z_{e} z_{h}}{z_{b}}=\frac{z_{e}^{0} z_{h}^{0}}{z_{b}} e^{-\Delta \epsilon_{c} / k T}
$$

Since conduction electrons and holes are to be treated as ideal gas components with purely translational energy and possessing two spin states, 
93

then

$$
\begin{aligned}
& z_{e}^{0}=2 V\left(\frac{2 \pi m_{e k T}}{h^{2}}\right)^{3 / 2} \\
& z_{h}^{0}=2 V\left(\frac{2 \pi m_{h k} T}{h^{2}}\right)^{3 / 2}
\end{aligned}
$$

Bound electrons are treated as $3 D$ oscillators with very large $\nu$

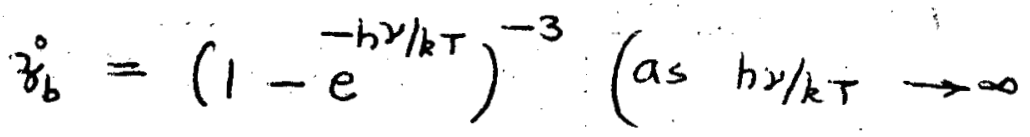

$$
\begin{aligned}
& \left.z_{b}^{3} \rightarrow 1\right) \\
& n_{e}=\frac{N_{e}}{V} ; \quad n_{h}=\frac{N_{h}}{V} \quad \text { (concentration) } \\
& \therefore n_{e} n_{b}=4\left(\frac{2 \pi m_{e} k T}{h^{2}}\right)^{3} \cdot e^{-\Delta \epsilon_{c} / k T} \\
& \left(m_{e}=m_{h}\right)
\end{aligned}
$$

This is the law of mass action for the mixture. 
Problem 5.8

$$
M(s)+O_{2}(g)=M O_{2}(s)
$$

(a)
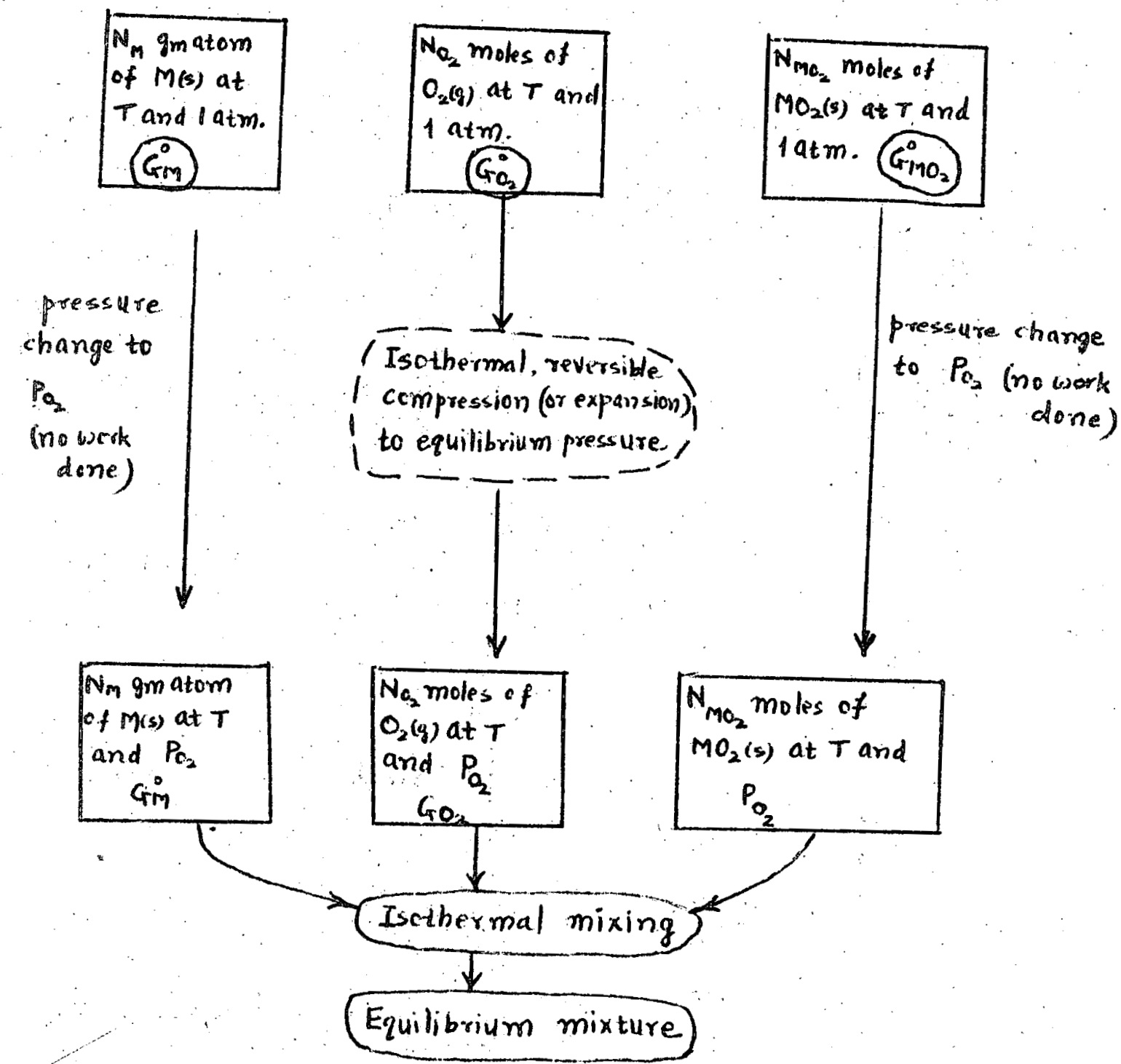

Phase Rule: $F=C-P+2 \Rightarrow C=$ cimponents $=2$ (Me and o)

$P=$ phoses $=3$ (metal, oxide, gias)

$F=$ degrees of freetom $=1\left(T\right.$ or $\left.\beta_{a_{2}}\right)$ 
95

Since the free energy change occurs only in the oxygen compression step, (it is given by Eq. (5.52)) the free energy of the equilibrium mixture $G$ is given by

$$
G=N_{M} G_{M}^{0}+N_{M_{2}} G_{M Q_{2}}^{0}+N_{O_{2}}^{0}\left[G_{O_{2}}^{0}+R T \ln P_{\mathrm{O}_{2}}\right]
$$

Criterion of chemical equilibrium is

$$
\begin{aligned}
& \mu_{m}+\mu_{\mathrm{O}_{2}}=\mu_{M O_{2}} \\
& \mu_{m}=\left(\frac{\partial G}{\partial N_{m}}\right)_{T_{1} P_{O_{2}}, N_{O_{2}}, N_{M_{2}}}=G_{m}^{0}
\end{aligned}
$$

Similarly

$$
\mu_{\mathrm{MO}_{2}}=G_{\mathrm{MO}_{2}}^{0}
$$

By Eq. (5.54)

$$
\begin{aligned}
\text { Eq. (5.54) } \mu_{\mathrm{O}_{2}}=G_{\mathrm{O}_{2}}^{\circ}+R T \ln P_{\mathrm{O}_{2}} \\
\therefore \quad G_{m}^{\circ}+G_{\mathrm{O}_{2}}^{0}-G_{\mathrm{MO}_{2}}^{\circ}=-R T \ln P_{\mathrm{O}_{2}}
\end{aligned}
$$

$\Delta G^{\circ}=$ standard free energy of reaction

$$
\begin{aligned}
& =G_{M O_{2}}^{0}-G_{O_{2}}^{0}-G_{M}^{0} \\
\therefore \Delta G^{0} & =R T \ln P_{O_{2}}
\end{aligned}
$$

(b) If $\mathrm{PO}_{2}$ is reduced be loco the equilibrium value, the $\mathrm{MO}_{2}(\mathrm{~s})$ phase changes into $M(s)$ and $\mathrm{O}_{2}(\mathrm{~g})$. This process continues until the oxide phase has completely disappeared. 
96

Problem 5.9

(a) Helium

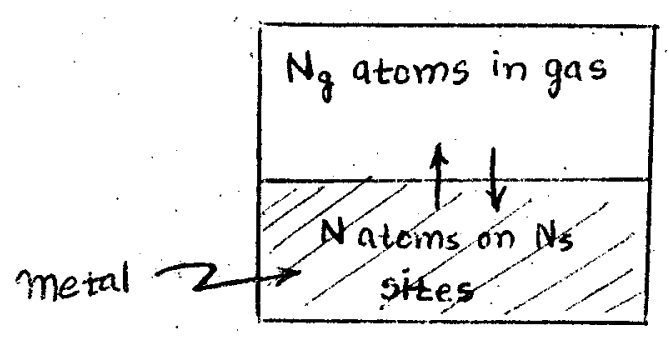

Criterion of Equilibrium:-

$$
\mu_{g}=\mu_{s} \quad(g \text {-gas } \quad(\text { s- solid })
$$

Reference energy: Isolated gas atom with zero kinetic energy $\left(0^{\circ} \mathrm{K}\right.$.)

Gas phase He:

$$
\mu_{g}=-k T \frac{\partial \ln Z_{g}}{\partial N_{g}}
$$

From Eq. (5.16) $\quad Z_{g}=\frac{3_{g}^{N_{g}}}{N_{g} !}$

$$
\therefore \quad \mu_{g}=-k T \ln \left(z g / N_{g}\right)
$$

From Eq. (5.18)

$$
z_{g}=\frac{V_{g}}{\lambda^{3}}
$$

where $\lambda=\frac{h}{\sqrt{2 \pi m k r}}=$ thermal - wavelength

$$
\left(E_{q} \cdot(1.73)\right)
$$


He in solid

Einstein oscillation in 3 dimensions (Eq. (2.19))

$$
Z_{s}=\left\{\frac{N_{s} !}{N !\left(N_{s}-N\right) !}\right\}\left[1-e^{-h \nu / k T}\right]^{-3 N} e^{-N E_{g} / k T}
$$

where the exponential term accounts for the difference between the reference energy and the zero point energy of He atoms in the solid.

$$
\begin{aligned}
& \epsilon_{q} \cong \text { heat of selution of the in meinl } \\
& \therefore \ln Z_{s}=\ln \left[\frac{N !}{N !(N,-N) !}\right]+3 N \ln z_{s}^{\circ}-\frac{N \epsilon_{g}}{k T}
\end{aligned}
$$

where

$$
\begin{aligned}
z_{s}^{0}=\left[1-e^{-i \nu / k r}\right]^{-1} \cong \frac{k T}{h \nu} & =\frac{\text { single particle }}{\text { partition function of }} \\
& \text { dissolved atom inensured } \\
& \text { from graind vibrational } \\
& \text { state }
\end{aligned}
$$

Using Stirling's appreximation and taking deriuntive of $\ln Z_{s}$ (cf. Eqs (5.42) and (5.31)) 
98

$$
\mu_{s}=-k T\left(\frac{\partial \ln Z_{s}}{\partial N}\right)=-k T\left[-\ln N+\ln \left(N_{s}-N\right)+3 \ln Z_{s}^{\circ}-\frac{\epsilon_{s}}{k T}\right]
$$

Using criterion of Chemical Equilibrium:

$$
\ln \left(\frac{V_{g}}{\lambda^{N} N_{g}}\right)=-\ln \left(\frac{N}{N_{s}-N}\right)+\ln \left(z_{s}^{\circ}\right)^{3}-\frac{\epsilon_{g}}{k T}
$$

or

$$
\frac{1}{\lambda^{3}} \frac{V_{g}}{N_{g}}\left(\frac{N}{N_{s}-N}\right) \frac{1}{\left(f_{s}^{0}\right)^{3}}=e^{-\epsilon_{g} / k T}
$$

Ideal gas law:

$$
\frac{N_{g}}{V_{g}}=\frac{P}{k T}
$$

Dilute Solution:

$$
N_{s} \rightarrow N
$$

$$
\frac{N}{N_{s}}=x \text {, atom fraction of dissolved } \mathrm{He}_{\mathrm{e}}
$$

$$
\therefore \frac{p}{x}=k T\left(\frac{h \nu}{k T}\right)^{3}\left(\frac{2 \pi m k T}{h^{2}}\right)^{3 / 2} e^{\epsilon_{g / k T}}
$$


(b)
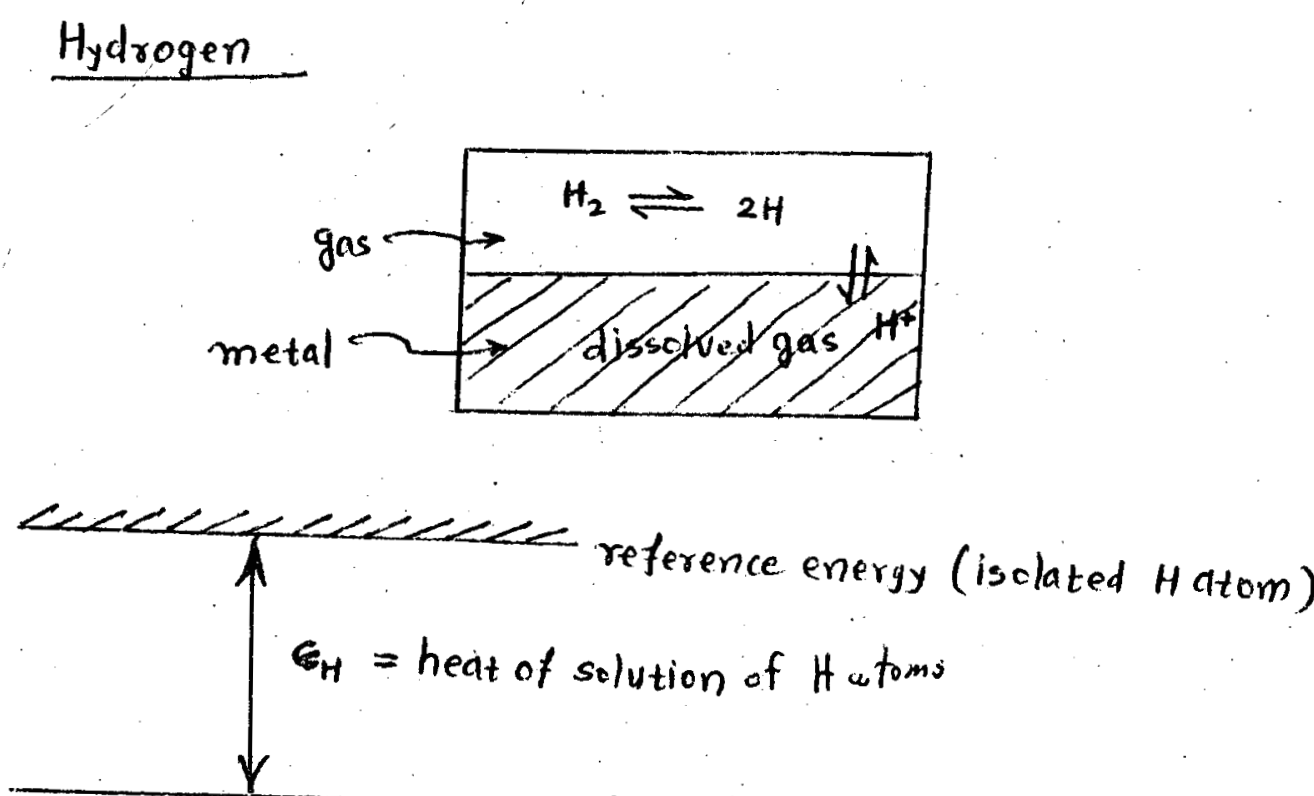

ground state of $\mathrm{H}^{+}$in solid

In considering the equilibrium

$H(g) \rightleftharpoons H$ (solid),

part (a) is applicable except that $z_{g}$ must be multiplied by 2 to account for the 2 spin states; of the 15 electron in the $H$ atom. This factor does not appear for the dissolved $H$ atom, which has been stripped of its electron. In addition the sign of the beat of solution term in $\mu_{s}$ is changed, since it takes energy to force He into a metal, whereas energy is required to force $H$ atoms out of a metal.

Also, we must use $N_{I}$ instend of $N_{s}$ for the. hydrogen analysis. We set $N_{I} / N_{S}=\alpha$, a constant for $a$ given lattice and hydrogen dissolution site.

The $H$-atom pressure $P_{1}$ in equilibrium with 
100

an atom fraction $x$ of hydrogen in the metal is therefore obtained by the methods of part (a) as:

$$
\begin{gathered}
\frac{P_{1}}{x}=\frac{2 k T}{\alpha}\left(\frac{h \nu}{k T}\right)^{3}\left(\frac{2 \pi m k T}{h^{2}}\right)^{3 / 2} e^{-\epsilon_{H} / k T} \\
m=\text { mass of } H \text { atom }
\end{gathered}
$$

For the gas phase equilibrium

$$
\mathrm{H}_{2}(\mathrm{~g}) \rightleftharpoons 2 \mathrm{H}(\mathrm{g})
$$

$K_{p}=\frac{P_{1}^{2}}{P_{2}}$ is given from the solution to problem 5.5

So

$$
P_{1}=\sqrt{P_{2}} \sqrt{k_{p}}=\sqrt{P_{2}}\left(\frac{\pi}{h^{2}}\right)^{3 / 4}(k T)^{5 / 4} \frac{e^{-\frac{1}{2} D / k T}}{\sqrt{\left(3_{2}\right)_{\text {int. }}}}
$$

where $D=$ dissociation energy of $H_{2} \cong 103 \mathrm{kcnl} /$ male $\left(z_{2}\right)_{\text {int. }}=$ internal partition function of $\mathrm{H}_{2}$

$$
\therefore \frac{\sqrt{P_{2}}}{x}=\frac{2}{\alpha}\left(\frac{\pi}{h^{2}}\right)^{-3 / 4}(k T)^{-\frac{1}{4}} \sqrt{\left(z_{2}\right)_{\text {int }}}\left(\frac{h \nu}{k T}\right)^{3}\left(\frac{2 \pi m k T}{h^{2}}\right)^{3 / 2} e^{-\left(\epsilon_{t}-\frac{1}{2} D\right) / k T}
$$

Fowler-Smithells equation 
101

Problem 5.10

(a) Equilibrium of a single component.

$$
\begin{gathered}
\mu^{I}=\mu^{I} \\
\mu^{I}=\left.\frac{\partial G}{\partial N^{I}}\right|_{T, P, N^{I}}=\mu^{I I}=\left.\frac{\partial G}{\partial N^{I}}\right|_{T, P, N^{I}} \\
G=N^{I} g^{I}+N^{I} \cdot g^{I} \\
\therefore g^{I}=g^{I I} \quad \text { True. }
\end{gathered}
$$

(b) For equilibrium $\left.d G\right|_{T, P}=0$.

$$
\begin{aligned}
& \text { So } d G^{I}=-d G^{I I} \\
& G^{I}=N_{A}^{I} g_{A}^{I}+N_{B}^{I} g_{B}^{I}, \quad G^{I I}=N_{A}^{I I} g_{A}^{I}+N_{B}^{I} g_{B}^{I I} \\
& g^{I}=g_{A}^{I}+g_{B}^{I}, g^{I}=g_{A}^{I I}+g_{B}^{I I} \\
& d G^{I}=d N_{A}^{I} g_{A}^{I}+d N_{B}^{I} g_{B}^{I}=d N_{A}^{I} g^{I}+\left(d N_{B}^{I}-d N_{A}^{I}\right) g_{B}^{I} \\
& d G^{I}=d N_{A}^{I} g_{A}^{I}+d N_{B}^{I} g_{B}^{I I}=d N_{A}^{I I} g^{I}+\left(d N_{B}^{I}-d N_{A}^{I}\right) g_{B}^{I I}
\end{aligned}
$$

So $\quad d N_{A}^{I} g^{I}+\left(d N_{B}^{I}-d N_{A}^{I}\right) g_{B}^{I}=-\left[d N_{A}^{I} g^{I I}+\left(d N_{B}^{I I}-d N_{A}^{I I}\right) g_{B}^{I I}\right]$

$$
\text { But } \begin{aligned}
d N_{A}^{I} & =-d N_{A}^{I I}, d N_{B}^{I}=-d N_{B}^{I I}, g_{B}^{I}=g_{B}^{\text {II }} \text { (from part } C \text { ) } \\
\therefore & d N_{A}^{I} g^{I}=-d N_{A}^{I I} g^{I I} \\
& \therefore g=g^{I I} \quad \text { True. }
\end{aligned}
$$


102

(c) For equilibrium, $d G=0$

$$
\begin{aligned}
& G= N_{A}^{I} g_{A}^{I}+N_{A}^{I I} g_{A}^{I}+N_{B}^{I} g_{B}^{I}+N_{B}^{I} g_{B}^{I} \\
& d G= d N_{n}^{I} g_{A}^{I}+d N_{A}^{I} g_{A}^{I}+d N_{B}^{I} g_{B}^{I}+d N_{B}^{I I} g_{B}^{I}=0 \\
& d N_{A}^{I}=-d N_{A}^{I}=d N_{A} \\
& d N_{B}^{I}=-d N_{B}^{I}=d N_{B} \\
& \text { So } \quad d G=d N_{A}\left(g_{A}^{I}-g_{A}^{I}\right)+d N_{B}\left(g_{B}^{I}-g_{B}^{I I}\right)=0
\end{aligned}
$$

Since $d N_{A}$ and $d N_{B}$ are independent

$$
g_{A}^{I}=g_{A}^{I} \quad \text { and } \quad g_{B}^{I}=g_{B}^{I}
$$

True. 
103

Problem 5.11

From Eq. (5.26) and $M^{\circ} \rightleftharpoons M^{++}+2 e^{-}$

$$
\frac{n_{e}^{2} n_{m^{H}}}{n_{m^{0}}}=\frac{2\left(\frac{2 \pi m_{e} k T}{h^{2}}\right)^{3 / 2^{2}}\left(\frac{2 \pi m_{m^{+} k T}}{h^{2}}\right)^{3 / 2}}{\left[\frac{2 \pi m_{r^{2} k T}}{h^{2}}\right]^{3 / 2}} e^{-I / k T}
$$

(factor 2 in the numerator is for the 2 spin states)

$$
\therefore \frac{n_{e}^{2} n_{m^{H}}}{n_{m^{0}}}=4\left(\frac{2 \pi m_{e k T}}{h^{2}}\right)^{3} e^{-1 / k T}
$$

From the result of problem 5.2 ,

we have

$$
n_{e}^{2}=2^{2}\left(\frac{2 \pi m_{e k} T}{h^{2}}\right)^{3} e^{-2 \phi / k T}
$$

while. $n_{p p}=\frac{p}{k T}=\frac{h}{k T} e^{-\Delta H_{s} / k T}$ (using clapeyron Eq)

Then $n_{m^{+}}=\frac{A}{k T} e^{\left(2 \phi-I-\Delta H_{0}\right) / k T}$ 
Problem 6.1

(a)

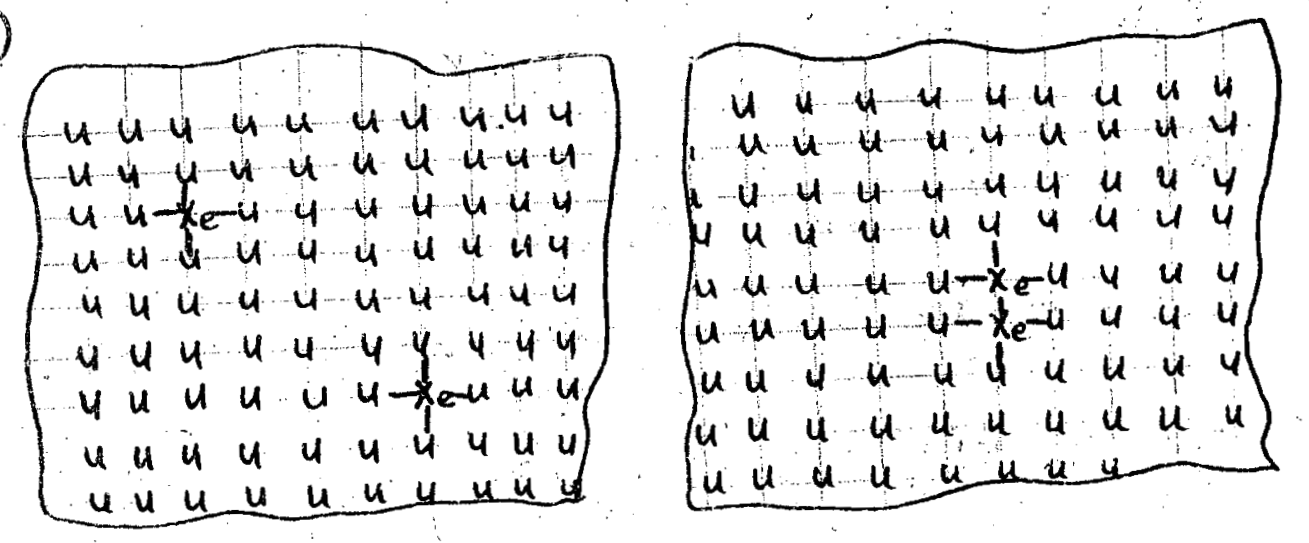

Crystal [1]

Crystal [2]

( $N u$ atoms of $U+2$ atoms of $X_{e}$ in each crystal)

The binding energy of $x_{e}-x_{e}$ in uranium medium is $\phi_{x_{e}-x_{e}}=\Phi_{[1]}-\Phi_{[2]}$, where $\Phi$ is the total energy of the crystal.

Qualitatively: The bond between $x_{e}$ atoms is very weak (Lennard-Jones potential)

In addition, the $X e$ atom is much bigger in size than the $U$ atom and when two $X_{e}$ atoms are placed side by side at $a$ distance of the $U$ atom lattice constant they repel each other. Therefore the energy of the crystal [2] is higher than the energy of crystal [1], or $\Phi_{[1]}<\Phi_{[2]} \quad \therefore \phi_{x_{e}-x_{e}}$ is negative. 
105

(b)

$$
E_{B E}=\left[b_{x_{e}-v} \phi_{x_{e-v}}+\underset{x_{e}-x_{e}}{\phi_{x_{e}-x_{e}}}+\underset{v v-v}{b_{v-v}}\right]
$$

1) $\quad E_{B E}=2 \phi_{x_{e}-V}=1.2 \mathrm{eV}$

2)

$$
\begin{aligned}
E_{B E} & =\left[2 \phi_{x_{e}-V}+\phi_{x_{B}-x_{e}}\right] \\
& =1.2-0.7=0.5 \mathrm{eV}
\end{aligned}
$$

3) $E_{B E}=4 \phi_{x_{e-V}}=2.4 \mathrm{eV}$

4)

$$
\begin{aligned}
E_{B E} & =8 \phi_{X_{e}-V}+4 \phi_{V-V} \\
& =4.8+0.4=5.2 \mathrm{eV}
\end{aligned}
$$

(c)

$$
\nu_{v} a+\nu_{x_{e}} x_{e} \rightleftharpoons D
$$

Law of Mass Action: at equilibrium

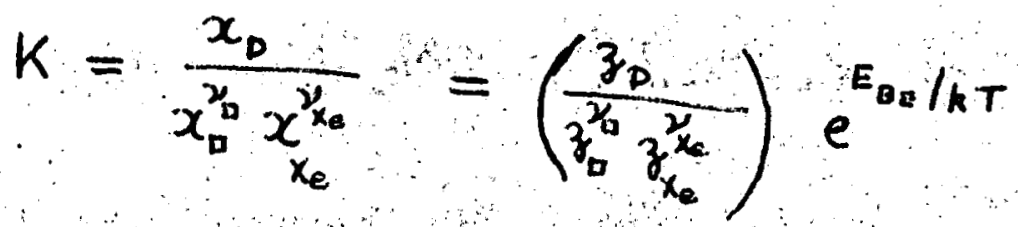

$$
\begin{aligned}
& \left(\frac{z_{D}}{z_{a}^{y} z_{x_{0}}^{y_{x_{0}}}}\right) \approx 1 \text { if excess entropy of mixing } \approx 0 \\
& x \text { is the molar fraction, ie } x_{i}=\frac{N_{i}}{\Sigma N_{i}}
\end{aligned}
$$


106

$$
\therefore\left(N_{v}+N_{x_{e}}+N_{D}\right)^{\nu+\nu_{x_{e}}-1}\left(\frac{N_{D}}{N_{v}^{2 y} N_{x_{e}}^{k_{k_{B}}}}\right)=e^{E_{B E} / k T}
$$

This holds for each defect process. For 4 processes in equilibrium simultaneously,

$$
\begin{aligned}
& N_{x_{e}}^{0}=N_{x_{e}}+\sum_{i=1}^{4} \nu_{x_{e_{i}}} N_{D_{i}} \\
& N_{v}^{0}=N_{v}+\sum_{i=1}^{4} 2_{v_{i}} N_{D_{i}}
\end{aligned}
$$

For (1) $\quad \nu_{v_{1}}=1, y_{x_{a_{1}}}=2$;

(2) $\nu_{v_{2}}=1, \nu_{x_{e_{i}}}=2 ;$

(3) $\quad \nu_{v_{3}}=2 ; \quad \nu_{x_{3}}=2$;

(4) $\nu_{v_{4}}=4, \nu_{x_{4}}=2$

$$
\begin{aligned}
\therefore N_{x_{e}} & =N_{x_{e}}^{0}-2 \sum_{i} N_{D_{i}} \\
& =N_{x_{e}}^{0}-2\left(N_{D_{1}}+N_{D_{2}}+N_{D_{3}}+N_{D_{4}}\right) \\
N_{V} & =N_{V}^{0}-\left(N_{D_{1}}+N_{D_{2}}+2 N_{B_{3}}+4 N_{D_{4}}\right)
\end{aligned}
$$

From (1),

$$
\begin{gathered}
\frac{N_{D_{1}}\left(N_{V}^{0}+N_{D_{e}}^{0}-2 N_{D_{1}}-3 N_{D_{2}}-4 N_{D_{3}}-6 N_{D_{4}}\right)^{2}}{\left[N_{V}^{0}-N_{D_{1}}-N_{D_{2}}-2 N_{D_{3}}-4 N_{D_{4}}\right]\left[N_{X_{e}}^{0}-2 N_{D_{1}}-2 N_{D_{2}}-2 N_{D_{3}}-2 N_{D_{4}}\right]^{2}} \\
=e^{\frac{1.2 \mathrm{eV}}{k T}}
\end{gathered}
$$


107

From (2),

$$
\frac{N_{D_{2}}\left(N_{V}^{0}+N_{C_{e}}^{0}-3 N_{D_{1}}-2 N_{D_{2}}-4 N_{D_{3}}-6 N_{D_{4}}\right)^{2}}{\left[N_{V}^{0}-N_{D_{1}}-N_{D_{2}}-2 N_{D_{3}}-4 N_{D_{4}}\right]\left[N_{X_{e}}^{0}-2 N_{D_{1}}-2 N_{D_{2}}-2 N_{D_{3}}-2 N_{D_{4}}\right]^{2}}=e^{\frac{0.5 \mathrm{eV}}{k T}}
$$

From (3),

$$
\frac{N_{D_{3}}\left(N_{V}^{0}+N_{X_{e}}^{0}-3 N_{D_{1}}-3 N_{D_{2}}-3 N_{D_{3}}-6 N_{D_{4}}\right)^{3}}{\left[N_{V}^{0}-N_{D_{1}}-N_{D_{2}}-2 N_{D_{3}}-4 N_{D_{4}}\right]^{2}\left[N_{X_{e}}^{0}-2 N_{D_{1}}-2 N_{D_{2}}-2 N_{D_{3}}-2 N_{D_{4}}\right]^{2}}=e^{\frac{2-4}{k T}}
$$

From (4),

$$
\frac{N_{D_{4}}\left(N_{1}^{2}+N_{K_{e}}^{0}-3 N_{D_{1}}-3 N_{D_{2}}-4 N_{D_{3}}-5 N_{D_{4}}\right)^{5}}{\left[N_{V}^{0}-N_{D_{1}}-N_{D_{2}}-2 N_{D_{3}}-4 N_{D_{4}}\right]^{4}\left[N_{R_{e}}^{0}-2 N_{D_{1}}-2 N_{D_{2}}-2 N_{D_{3}}-2 N_{D_{4}}\right]^{2}}=e^{\frac{5.2 \mathrm{eV}}{k T}}
$$

These 4 equations must be solved simultaneously for $N_{D_{1}}, N_{D_{2}}, N_{D_{3}}$ and $N_{D_{4}}$. 
108

Problem 6.2

(a)

From problem 1.5 of chapter 1, we knoll that

$$
c_{p}-c_{v}=\left(\frac{\alpha^{2} V}{\beta}\right) T
$$

where $\alpha=$ coefficient of thermal expansion

$$
\begin{aligned}
& \beta=\text { coefficient of compressibility } \\
& V=\text { Volume / mole of } \mathrm{UO}_{2}
\end{aligned}
$$

For an ideal crystal well above its Debye

Temperature

$$
\begin{aligned}
& C_{v}=3 R \frac{J}{g m-a t o m-{ }^{\circ} \mathrm{K}} \\
& \text { since } C_{y}=3 R H\left(\frac{\theta_{p}}{T}\right) \text { and } \\
& H\left(\frac{\theta_{p}}{T}\right) \rightarrow 1 \text { as } \frac{\theta_{p}}{T} \rightarrow 0
\end{aligned}
$$

but since there are 3 atoms per molecule of $\mathrm{NO}_{2}$

$$
c_{V}=9 R \frac{\mathrm{J}}{\text { mole } \mathrm{UO}_{2}-{ }^{\circ \mathrm{K}}}
$$

Then

$$
C_{p}=9 R+\left(\frac{\alpha^{2} V}{\beta}\right) T=b+c T
$$

or $b=9 R$ and $c=\frac{\alpha^{2} V}{\beta}$

(b) Frankel defects on Oxygen sublattice of $\mathrm{UO}_{2}$.

Crystal Structure $-M X_{2}$ (Fluorite) 
$N_{M}=$ number of $U^{+4}$ ions on normal lattice sites $N_{x}=$ number of $\mathrm{O}^{-2}$ ions on normal lattice sizes $N_{S M}=$ number of $U^{+4}$ lattice sites in $M X_{2}$ $N_{s x}=$ number of $\bar{O}^{-2}$ lattice sites in $\mathrm{Mx}_{2}$ $N_{S I}=$ interstitial sites in Fluorite structure $N_{X I}=$ number of $0^{-2}$ ions on interstitial sites $N_{x V}=$ number of anion vacancies

For the fluorite structure

$$
\begin{aligned}
& N_{s x}=2 N_{s M}
\end{aligned}
$$

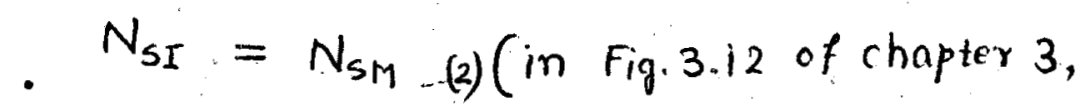

Electrical neutrality gives,

$$
4 N_{m}=2 N_{x}+2 N_{x I}
$$
which are unoccupied by $\mathrm{c}^{+4}$ ions are interstitial sites)

Since the cation sublattice is perfect

$$
\begin{aligned}
& N_{M}=N_{S M} \\
\text { and } \quad N_{s x} & =N_{x}+N_{x V}
\end{aligned}
$$

From (1), (3), (4) and (5), eliminating $N_{s x}, N_{S M}, N_{m}$ we get $N_{x y}=N_{X I}$

Law of Mass action gives.

$$
\text { (3) }
$$

$$
\frac{N_{X I}}{N_{S I}} \cdot \frac{N_{X Y}}{N_{S X}}=K_{F X}
$$


using (1), (2) and (6)

$$
\begin{aligned}
& \frac{N_{X I}}{N_{S M}} \cdot \frac{N_{X I}}{2 N_{S M}}=K_{F X} \\
& \therefore \frac{N_{X I}}{N_{S M}}=\sqrt{2 K_{F X}}
\end{aligned}
$$

$\frac{N_{x I}}{N_{s M}}=\frac{\text { interstitial Oxygen atoms }}{\text { Uranium atom }}=\frac{\text { moles of interstitial Oxygen }}{\text { mole of } \mathrm{VO}_{2}}$

$H=E+p V \rightarrow \Delta H=\Delta E+p \Delta V$ (at constant

- So excess enthalpy Hex (Joules) pressure) formation of $\left(\frac{\mathrm{mol}_{\mathrm{e}}-\mathrm{UO}_{2}}{)}\right)$ and energy of formation of Frenkal defects $E_{F}\left(\frac{J_{\text {oules }}}{\text { mole of } N_{\mathrm{XI}}}\right)$ have a

$$
H_{e_{X}} \cong \frac{N_{X I}}{N_{S M}} E_{F}
$$

$$
\begin{aligned}
& \text { (neglecting pav term since it is very } \\
& \text { Since } k_{F X}=e^{\Delta S_{F X} / R} e^{-E_{F / R T}} \\
& \text {.... (10) } \\
& H_{t X X}=\sqrt{2} E_{F} e^{\Delta S_{F X} / 2 R} \cdot e^{-E_{F} / 2 R T}
\end{aligned}
$$


Problem 6.3

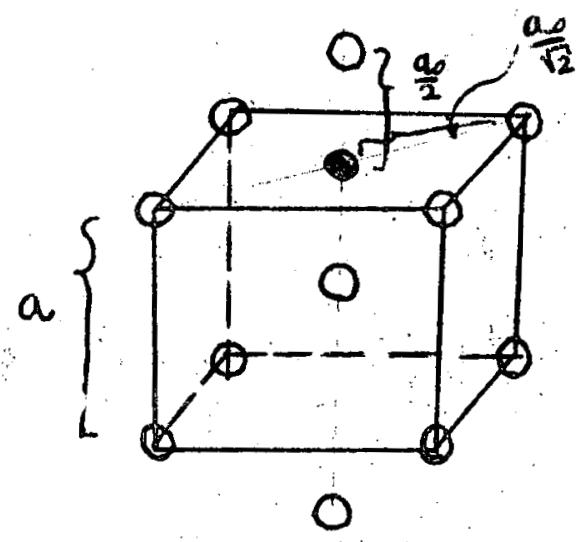

(a) Octahedral Interstitial

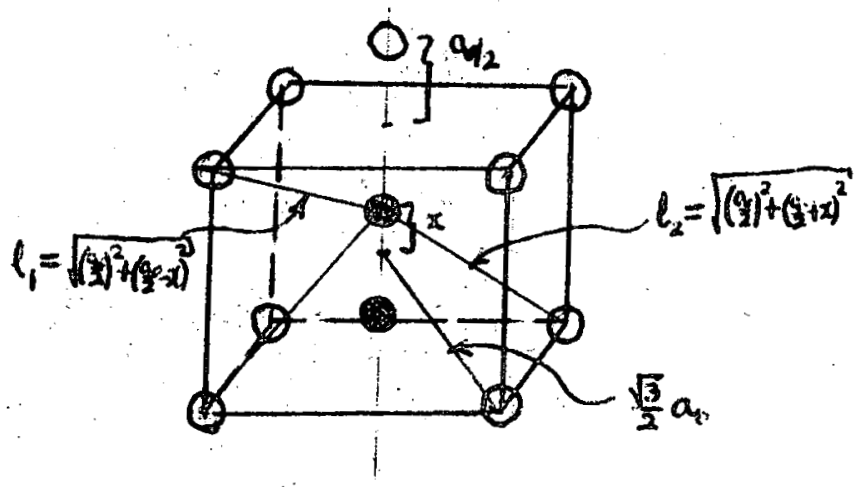

o

$E_{T}=$ Increase in energy of crystal when one interstitial atom is added. It includes only those atom-atom interactions which are different from those in perfect crystal

(a) Octahedral Interstitial

$$
E_{I}=2 \phi\left(\frac{a_{0}}{2}\right)+4 \phi\left(\frac{a_{0}}{\sqrt{2}}\right)+4 \phi\left(\sqrt{\frac{3}{2}} a_{0}\right)+\phi\left(\frac{3}{2} a_{0}\right)
$$

(b) Split Interstitial

Compute the energy of the split configuration and subtract the energy of one normal atom before the interstitial was added:

$$
\begin{aligned}
E_{I}=2\left[4 \phi\left(l_{1}\right)+\phi\left(a_{0}-x\right)\right. & \left.+\frac{1}{2} \phi(2 x)+4 \phi\left(l_{2}\right)+\phi\left(a_{0}+x\right)\right] \\
& -\left[8 \phi\left(\frac{\sqrt{3}}{2} a_{0}\right)+3 \phi\left(a_{0}\right)\right]
\end{aligned}
$$

(The factor $\frac{1}{2}$ in front of $\phi(2 x)$ is necessary to avid counting twice) 
112

From $\frac{d E_{I}}{d x}=0$ at equilibrium, solve for $x=x_{e q}$.

Then $E_{I}\left(x_{\text {eq }}\right)$ should be compared with" $E_{I}$ for octahedral interstitial, The configuration which has smaller value of $E_{I}$ is energetically favored. 
$1 / 3$

Problem 6.4

(a)

BCC - Octahedral sites

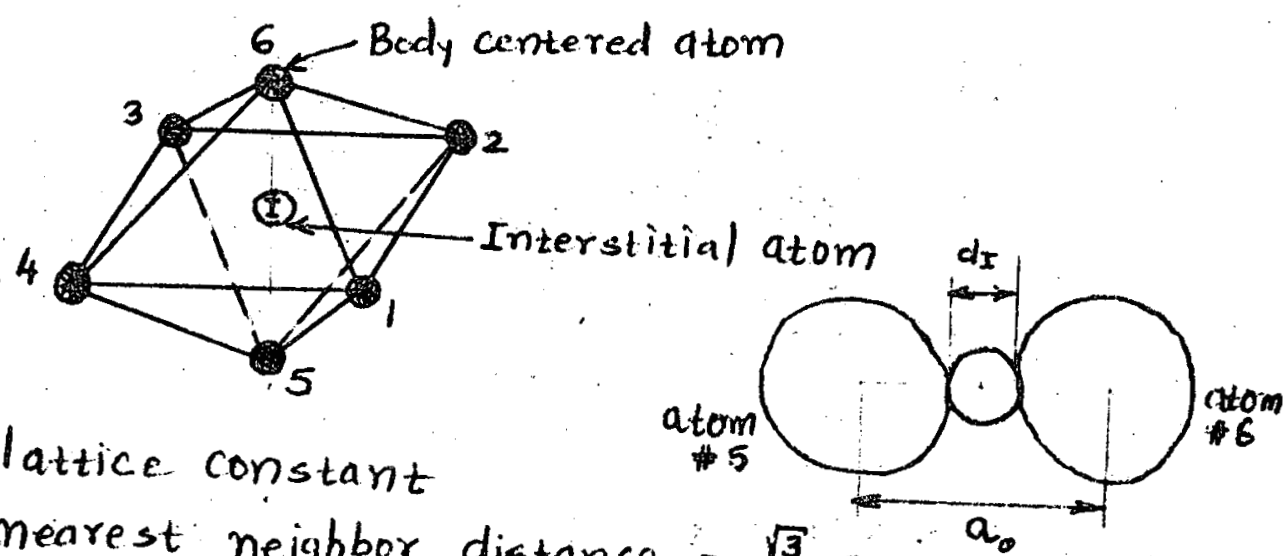

$a_{0}=$ lattice constant
$d_{m}=$ nearest neighbor distance $=\frac{\sqrt{3}}{2} a_{0}$ (see problem 3.2)

If the atoms of the lattice are regarded as hard spheres, the diameter of a lattice atom is equal to the nearest neighbor distance ie. $\frac{\sqrt{3}}{2} a_{0}$

For an impurity of diameter $d_{I}$ to fit in the center of the octahedral site, it must fit between atoms " 5 " and " 6 ", since the distances between other atoms are larger than the distance between " 5 " and " 6 ", which is the shortest and equal to $a$.

Hence $\frac{\sqrt{3}}{2} a_{0}+\left(d_{I}\right)_{\max }=a_{0}$

$$
\begin{gathered}
\therefore\left(d_{I}\right)_{\text {max }}=\left(1-\frac{\sqrt{3}}{2}\right) a_{0}=\left(\frac{2}{\sqrt{3}}-1\right) \frac{\sqrt{3}}{2} a_{0} \\
\therefore\left(d_{I}\right)_{\max }=\left(\frac{2}{\sqrt{3}}-1\right) d_{m} \\
\text { or } \quad\left(d_{I}\right)_{\max }=0.155 d_{m}
\end{gathered}
$$


114

$B C C$ - Tetrahedral site

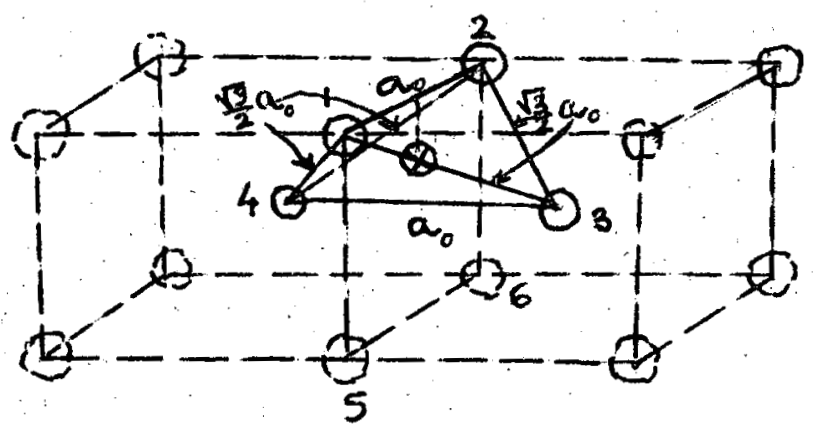

The interstitial site is $\frac{1}{4} a_{0}$ down from the middle of the unit cell edge between atoms 1 and 2. Taking a plane through atoms 1,5,6 and 2

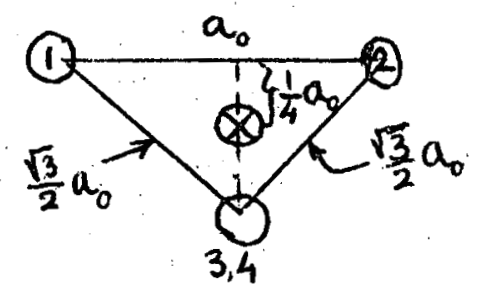

If $d_{I-1}$ or $d_{I-2}$ are the distances of the interstitial atom from the atoms 1 or 2 respectively, then

$$
d_{x-1} \text { or } d_{x-2}=\sqrt{\left(\frac{1}{4} a_{0}\right)^{2}+\left(\frac{1}{2} a_{0}\right)^{2}}=\frac{\sqrt{5}}{4} a_{0}
$$

Next, taking a plane through atoms 3 and 4 which is perpendicular to the unit cell edge $1-2$ and includes the interstitial site, again we can 
115

calculate $d_{I-3}$ or $d_{I-4}$ in precisely the same manner. "Since in this plane the relative positions of the atoms are same as in the previous plane we get,

$$
d_{1-3} \text { or } d_{1-4}=\frac{\sqrt{5}}{4} a_{0}
$$

Since $d_{m}=\frac{\sqrt{3}}{2} a_{0}$ (nearest neighbor distance)

$$
\begin{gathered}
\frac{1}{2}\left(d_{1}\right)_{\text {max }}+\frac{1}{2} d_{m}=\frac{\sqrt{5}}{4} a_{0} \\
\therefore \frac{1}{2}\left(d_{1}\right)_{\text {max }}=\frac{\sqrt{5}}{4} a_{c}-\frac{\sqrt{3}}{4} a_{0} \\
\therefore\left(d_{2}\right)_{\max }=\frac{1}{2}(\sqrt{5}-\sqrt{3}) a_{0} \\
\therefore\left(d_{1}\right)_{\max }=\left(\sqrt{\frac{5}{3}}-1\right) d_{m} \\
\therefore\left(d_{1}\right)_{\text {max }}=0.290 d_{m}
\end{gathered}
$$

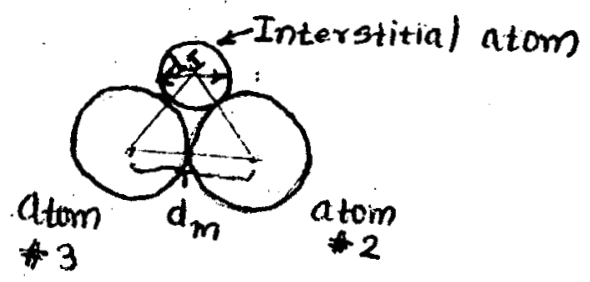

FCC -Lattice (see fig. 6.2 in chapter 6)

$$
d_{m}=\frac{a_{0}}{\sqrt{2}}
$$

FCC - Octahedral site

$$
\begin{gathered}
\left(d_{I}\right)_{\text {max }}+2 \cdot \frac{a_{0}}{2 \sqrt{2}}=a_{0} \\
\therefore\left(d_{1}\right)_{\max }=a_{0}\left(1-\frac{1}{\sqrt{2}}\right)=\left(\frac{2}{\sqrt{2}}-1\right) d_{m} \\
\therefore\left(d_{x}\right)_{\text {max }}=0.414 d_{m}
\end{gathered}
$$

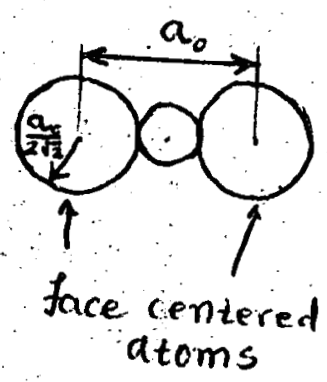


$1 / 6$

FCC - Tetrahedral site

Interstitial site is at

$$
\begin{aligned}
& \left(\frac{1}{4}, \frac{1}{4}, \frac{1}{4}\right) \\
& d_{p-3}=\sqrt{\left(\frac{1}{\sqrt{2}}\right)^{2}-\left(\frac{1}{2 \sqrt{2}}\right)^{2}} \quad a_{0} \\
& =\frac{\sqrt{3}}{2 \sqrt{2}} a_{0}=d_{p_{-2}} \\
& d_{H-p}=\frac{1}{3} \frac{\sqrt{3}}{2 \sqrt{2}} a_{0}
\end{aligned}
$$

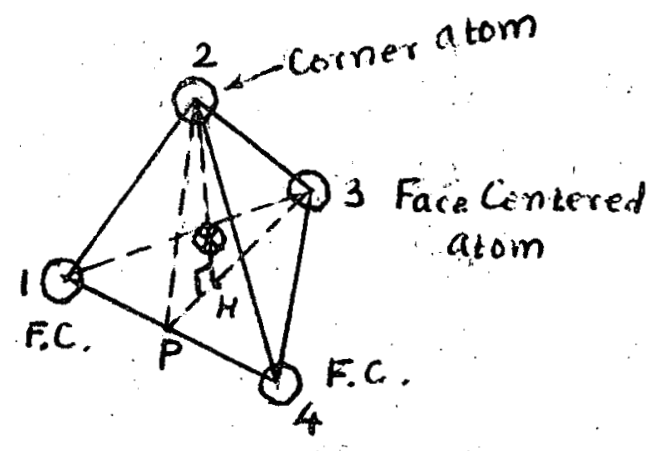

$$
\begin{aligned}
& \therefore d_{H-2}=\sqrt{d_{P-2}^{2}-d_{H-2}^{2}} \\
&=\sqrt{\left(\frac{\sqrt{3}}{2 \sqrt{2}}\right)^{2}-\left(\frac{1}{3} \frac{\sqrt{3}}{2 \sqrt{2}}\right)^{2}} a_{0} \\
&=\frac{a_{0}}{\sqrt{3}} \\
& d_{I-2}=\frac{3}{4} d_{H-2}=\frac{\sqrt{3}}{4} a_{0} \\
& \therefore \frac{1}{2}\left(d_{I}\right)_{\max }+\frac{1}{2} \frac{a_{0}}{\sqrt{2}}=\frac{\sqrt{3}}{4} a_{0} \\
& \therefore\left(d_{I}\right)_{\max }=a_{0}\left(\frac{\sqrt{3}}{2}-\frac{\sqrt{2}}{2}\right) \\
& \therefore\left(d_{1}\right)_{\text {max }}=d_{m}\left(\sqrt{\frac{3}{2}}-1\right) \\
& \therefore\left(d_{I}\right)_{\max }=0.223 d_{m}
\end{aligned}
$$


$1 / 7$

(b) FCC -Tetrahedral

FCC has $6 \times \frac{1}{2}+8 \times \frac{1}{8}=4$ atoms per unit cell and there are 8 tetrahedral sites.

Then the chemical formula with saturated tetrahedral sites becomes

$M I_{2}$

Interstitial atoms form SC lattice interceding FCC of $M$ This is like sc lattice of 0 atoms interceding $\mathrm{FCC}$ lattice of $U$ atoms in $\mathrm{UO}_{2}$ Therefore we have Fluorite structure.

FCC - octahedral

Interstitial site/ unit cell $=1+12 \times \frac{1}{4}=4$ (center (edge-center
of
the cell) sites)

$\therefore$ Chemical formula is MI

$B C C$ - Tetrahedral

Interstitial sites/unit cell $=4 \times 6 \times \frac{1}{2}=12$ (since there are 4 sites on each of the 6 faces and each site is shared by two unit cells)

Host atoms/urit cell $=2$

$\because$ Chemical formula is $M I_{6}$ 
118

BC.C-Octahedral

Interstitial sites/ unit cell $=6 \times \frac{1}{2}+12 \times \frac{1}{4}=6$

Con the (on the faces) edges)

$\therefore$ Chemical formula is $\mathrm{MI}_{3}$ 
119

Problem 6.5

Schottky Defects in $M x_{2}$

$$
\begin{aligned}
& G-G(0)=N_{V_{M}} \in_{V M}+N_{V_{x}} \in_{v_{x}}-k T \ln W \\
& N_{s M}=\text { Cation sites, }, N_{m}=\text { Cations, } \\
& N_{v_{M}}=\text { Cation vacancies, } N_{D M}=\text { Cation impurity, } \\
& N_{s x}=\text { Anion sites, } N_{x}=\text { Anions, } \\
& N_{v_{x}}=\text { Anion vacancies } \\
& N_{S x}=2 N_{S M}
\end{aligned}
$$

Electrical neutrality gives,

$$
\begin{aligned}
& q_{M} N_{M}+q_{D} N_{D_{M}}=q_{x} N_{x} \\
& N_{S M}=N_{M}+N_{U M}+N_{D M} \\
& N_{S x}=N_{x}+N_{V x} \\
\therefore & q_{M}\left(N_{S M}-N_{V M}-N_{D M}\right)+q_{D} N_{D M}=q_{x}\left(N_{S x}-N_{U x}\right)
\end{aligned}
$$

Dividing by $q_{m}$ gives,

$$
\begin{aligned}
& N_{s M}-N_{v M}+\left(\frac{q_{D}}{q_{m}}-1\right) N_{D M}=\frac{q_{x}}{q_{m}} N_{s x}-\frac{q_{x}}{q_{m}} N_{v x} \\
& \text { but } \quad \frac{q_{x}}{q_{m}}=\frac{1}{2}
\end{aligned}
$$$$
\therefore \frac{q_{X}}{q_{M}} N_{S K}=\frac{1}{2} \times 2 N_{S M}=N_{S M}
$$ 
120

$$
\begin{aligned}
& \therefore\left(\frac{q_{D}}{q_{m}}-1\right) N_{D m}+\frac{1}{2} N_{v x}=N_{v M} \\
& \therefore \frac{d N_{v M}}{d N_{v x}}=\frac{1}{2} \\
& \therefore \frac{\partial G}{\partial N_{v x}}=\frac{\partial N_{v M}}{\partial N_{v x}} \epsilon_{v v_{1}}+\epsilon_{v x}-k T\left[\frac{1}{2}\left(\frac{\partial \ln W_{v M}}{\partial N_{v M}}\right)+\frac{\partial \ln W_{x}}{\partial N_{v x}}\right] \\
& \text { [using (i)] } \\
& =\frac{1}{2} \epsilon_{v m}+\epsilon_{v x}-k T\left[\left(\frac{\partial \ln w_{m}}{\partial N_{v_{m}}}\right) \frac{1}{2}+\frac{\partial \ln w_{x}}{\partial N_{i x}}\right] \\
& \text { [using }(1) \text { ] } \\
& \therefore \text { In equilibrium } \\
& 0=\frac{\partial G}{\partial N_{v x}}=\frac{1}{2} \epsilon_{s}-k T\left[-\frac{1}{2} \ln x_{r_{p 1}}-\ln x_{v_{x}}\right] \\
& \text { (where } \epsilon_{s}=\epsilon_{i m}+2 \epsilon_{i x}
\end{aligned}
$$

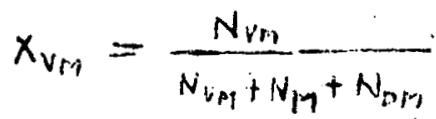

$$
\begin{aligned}
& \left.x_{v x}=\frac{N_{v x}}{N_{v_{x}}+N_{x}}\right) \\
& \therefore \quad-\frac{\epsilon_{s}}{k T}=\ln x_{v m}+2 \ln x_{v x}=\ln \left(x_{v m} x_{v K}^{2}\right) \\
& \text { or } \quad x_{V M} x_{v_{i}}^{2}=e^{-\epsilon_{s} / k T}
\end{aligned}
$$


121

Problem 6.6

Let. $N_{s M}=N_{s x}=N$ be the number of sites (cation or anion)

$$
\begin{aligned}
N_{m} & =\text { number of cations on cation sublattice. } \\
N_{U_{n}} & =\text { number of vacancies on cation sublattice } \\
N_{x} & =\text { number of anions on anion sublattice. } \\
N_{V_{x}} & =\text { number of vacancies on anion sublattice } \\
N_{S I} & =\text { number of interstitial sites } \\
N_{I M} & =\text { number of cation interstitial }
\end{aligned}
$$

Then

$$
\begin{aligned}
& N_{S M}=N_{M}+N_{V_{M}} \\
& N_{S x}=N_{x}+N_{V_{x}}
\end{aligned}
$$

Also $\quad K_{s}=\frac{N_{v m} N_{v x}}{N_{s m} N_{s x}}=x_{v_{m}} \cdot x_{v_{x}}[$ [eq. (6.32)]

For Frankel defect

$$
K_{F M}=\frac{N_{V M} N_{2 M}}{N_{S M} N_{S I}}=X_{V M} \cdot X_{I M}[e q(6.34)]
$$

For electrical neutrality,

$$
N_{m}+N_{t m}=N_{x}
$$

Substituting (1) and (2) into (5) and using

$$
\begin{aligned}
& N_{s M}=N_{s x} \text { we get } \\
& \left(N_{s M}-N_{V M}\right)+N_{I M}=N_{s x}-N_{V x}
\end{aligned}
$$


122

or

$$
N_{v i}=N_{v x}+N_{1 m}
$$

Dividing by $N_{s m}=N_{s x}$ and using the definition

$$
X_{v m}=\frac{N_{v m}}{N_{s m}}, \quad X_{v x}=\frac{N_{v x}}{N_{s x}}, X_{I m}=\frac{N_{\text {rm }}}{N_{s I}}
$$

and also using $\frac{N_{s I}}{N_{s M}}=\beta$, we get

$$
x_{v_{M}}=x_{v_{x}}+\beta x_{I M}
$$

Solving eqs. (3), (4) and (6) for $x_{v m}, x_{v x}$ and $x_{I m}$ we have, multiplying (6) by $x_{v M}$ and substituting (3) and (4)

$$
\begin{aligned}
x_{V_{M}}^{2} & =x_{v_{M}} \cdot x_{v x}+\beta x_{V M} \cdot x_{I M} \\
& =k_{s}+\beta K_{F M} \\
\therefore \quad x_{V M} & =\sqrt{K_{S}+\beta K_{F M}}
\end{aligned}
$$

then from (3) and (4)

$$
\cdot x_{v_{x}}=\frac{K_{s}}{\sqrt{K_{s}+\beta \cdot K_{r m}}}
$$

and

$$
x_{I M}=\frac{K_{F M}}{\sqrt{K_{S}+\beta K_{F M}}}
$$


1223

Problem 6.7

$$
\begin{gathered}
\text { Atom (A site) } \rightleftharpoons \text { Atom (B site) } \\
\mu_{A}=\mu_{B} \\
\mu_{i}=-k T\left(\frac{\partial \ln Z}{\partial N_{i}}\right)_{T, V, N_{j}}
\end{gathered}
$$

If the lattice modes are unaffected $z$ is given by Eq. (5.41):

$$
\begin{aligned}
& Z=\frac{N_{S A} !}{N_{A} !\left(N_{S A}-N_{A}\right) !} z_{A}^{N_{A}} \cdot \frac{N_{S B} !}{N_{B} !\left(N_{S B}-N_{B}\right) !} z_{B}^{N_{B}} \\
& N_{E}=\text { number of host atoms on normal lattice sites } \\
& N_{A}=\text { number of impurity atoms on } A \text { sites } \\
& N_{B}=\text { number of impurity atoms on } B \text { sites }
\end{aligned}
$$

When the host atoms are present, their vibrational lattice modes will be affected by the presence of impurity atoms on $A$ and $B$ sites. Then the partition function $Z$ of eq .(1) must be multiplied by

$$
\prod_{i=1}^{3 N_{l}}\left(1-e^{-h \gamma_{i} / k T}\right)^{-1}
$$

let $\alpha_{A}=$ number of lattice modes which are affected by atoms in $A$ site. The frequency of these modes is changed from $x_{i}$ to $x_{l A}$ 
124

$\alpha_{B}=$ number of lattice modes affected by an atom on $B$ site, with mode frequency change from $y_{l}$ to $y_{t B}$

$$
\begin{aligned}
& \therefore \prod_{i=1}^{3 N_{k}}\left[1-e^{-h \nu_{i} / k T}\right]^{-1}=\left[1-e^{-h \nu_{L n} / k T}\right]^{-\alpha_{A} N_{A}}\left[1-e^{-h \gamma_{k B} k_{k}}\right]^{-\alpha_{B} N_{B}} \\
& {\left[1-e^{-h \nu_{t} / R_{T} T}\right]^{-\left(3 N_{t}-\lambda_{B} N_{A}-\alpha_{B} N_{t}\right)}} \\
& \cong\left(\frac{k T}{h \gamma_{l A}}\right)^{\alpha A N_{R}}\left(\frac{k T}{h \gamma_{1 B}}\right)^{\alpha_{B} N_{B}}\left(\frac{k T}{h \gamma_{l}}\right)^{3 N_{l}-\alpha_{A} N_{A}-\alpha_{B} N_{B}} \\
& =\left(\frac{k T}{h y_{c}}\right)^{3 N_{l}}\left(\frac{\nu_{L}}{\nu_{L A}}\right)^{\alpha_{A} N_{A}}\left(\frac{\nu_{l}}{\nu_{l_{B}}}\right)^{\alpha_{B} N_{B}} \\
& =Z_{l}^{*}\left(\frac{\nu_{l}}{\nu_{i A}}\right)^{R N_{A}}\left(\frac{\nu_{C}}{\nu_{l, B}}\right)^{\alpha_{B} N_{B}}
\end{aligned}
$$

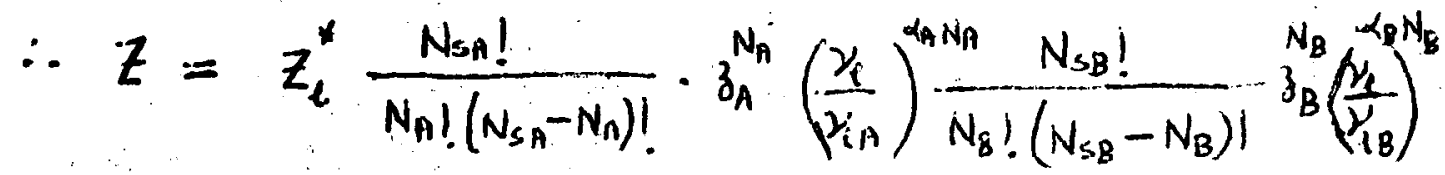

$$
\begin{aligned}
& \therefore \mu_{A}=-k T\left(\frac{\partial \ln Z}{\partial N_{A}}\right)_{T, V, N_{B}}=-k T \ln \left[\frac{\partial_{A}}{x_{A}}\right]-k T \alpha_{A} \ln \left(\frac{\gamma_{l}}{\nu_{l A}}\right) \\
& =-k T \ln \left[\frac{z_{A}}{x_{A}}\left(\frac{\partial_{A}}{\partial_{i H}}\right)^{\alpha_{A}}\right]
\end{aligned}
$$

[The first term on the RHS in eq.(2) follows from Eq .(5.42)]

Similarly $\mu_{B}=-k T \ln \left[\frac{3_{B}}{x_{B}}\left(\frac{\nu_{P}}{\nu_{B}}\right)^{\alpha_{B}}\right]$

Equating $\mu_{A}$ and $\mu_{B}$ in equilibrium and 
125

factoring out ground state energy from $z_{A}$ and $z_{B}$ we get in the equilibrium,

$$
\frac{x_{A}}{x_{B}}=\left(\frac{\partial_{A}^{0}}{z_{B}^{0}}\right)\left[\left(\frac{\nu_{\ell}}{\nu_{\ell_{B}}}\right)^{\alpha_{A}} /\left(\frac{\nu_{Q}}{\nu_{l_{B}}}\right)^{\alpha_{B}}\right] e^{-\Delta \epsilon_{0} / k T}
$$

The additional factor due to the alteration of lattice modes is

$$
\frac{\left(\frac{\nu_{l}}{\nu_{C A}}\right)^{\alpha_{A}}}{\left(\frac{\nu_{A}}{\nu_{C_{B}}}\right)^{\gamma_{B}}}
$$


126

Problem 6.8

(a) Since the formula is $M X_{2}$

$$
N_{S x}=2 N_{S M}
$$

The number of anion interstitial sites in the fluorite structure is equal to the number of cation lattice sites

$$
N_{S I}=N_{S M}
$$

Electrical neutrality gives,

$$
4 N_{m}^{+4}+q N_{m}^{+2}=2 N_{X}+2 N_{x I}
$$

Filling of sublattice sites gives.

Cation: $\quad N_{\text {sm }}=N_{M}^{+4}+N_{M}^{+2}$

(assume. perfect)

Anion: $\quad N_{s x}=N_{x}+N_{v x}$

(b)

$$
\frac{\text { Oxygen }}{\text { Uranium }}=\frac{N_{x}+N_{x s}}{N_{M}^{+4}+N_{m}^{+q}}=2+x
$$

ort from eq. (4),

$$
\frac{N_{x}+N_{x I}}{N_{S M}}=2+x
$$

Using eq:(5),

$$
\frac{N_{S x}-N_{V_{x}}+N_{X I}}{N_{S M}}=2+x
$$


$|2\rangle$

using (1) we get,

$$
x=2\left(\frac{N_{X I}-N_{v X}}{N_{5 x}}\right)
$$

When hyperstoichiometric $(x>0) \quad N_{x I}>N_{V x}$, when hypostoichiometric $(x<0) \quad N_{x_{I}}<N_{v_{x}}$

(c)

Combining eqs (3) and (E)

$$
4 N_{m}^{+4}+2 N_{p q}^{+q}=2(2+x)\left(N_{m}^{+4}+N_{m}^{+q}\right)
$$

let $\quad \gamma=\frac{N_{m}^{+q}}{N_{m}^{+4}}$

then dividing $\theta(\varepsilon)$ by $N_{m}^{+4}$ gives,

$$
\begin{aligned}
4+q \gamma & =2(2+x)(1+\gamma) \\
& =2(2+x)+2 \gamma(2+x) \\
\therefore 4-2(2+x)=y[2(2+x)-q] & \\
\therefore \quad & =\frac{2 x}{q-4-2 x} \\
\therefore \quad \frac{N_{m}^{+q}}{N_{S M}} & =\frac{N_{m}^{+q}}{N_{m i}^{+4}+N_{m}^{+2}}=\frac{\gamma}{1+x}=\frac{2 x}{q-4}
\end{aligned}
$$


128

(d)

$$
K_{F X}=\frac{N_{V x} N_{x I}}{N_{S x} N_{s I}}=\frac{2 N_{v x} N_{x I}}{N_{S_{X}}^{2}}
$$

Solving eq (7) and (10) simultaneously for $N_{i x}$ and $N_{x I}$ gives,

$$
\begin{aligned}
& \text { from (7), } N_{x I}=\frac{1}{2} x N_{s x}+N_{v x} \\
& \text { into (10) }, \frac{1}{2} N_{s x}^{2} K_{F x}=N_{v_{x}}\left(N_{v x}+\frac{1}{2} \times N_{S x}\right)
\end{aligned}
$$

let. $\quad y_{v x}=\frac{N_{v x}}{N_{s x}}$ (site fraction of uncancies on anion sublattice)

$$
\begin{aligned}
& \frac{1}{2} k_{F x}=y_{v_{x}}\left(y_{v_{x}}+\frac{1}{2} x\right) \\
& \therefore y_{v x}=\frac{1}{4} x\left\{ \pm \sqrt{1+\frac{8 k_{F x}}{x^{2}}}-1\right\}
\end{aligned}
$$

If $x \geqslant 0$, choose + sign in the root,

$$
y_{v_{x}}=\frac{1}{4} x\left\{\sqrt{1+\frac{9 k_{F x}}{x^{2}}}-1\right\}
$$

If $x<0$, choose - sign in the root,

$$
\begin{aligned}
& y_{v x}=\frac{1}{4}(-x)\left\{\sqrt{1+\frac{g K_{F x}}{x^{2}}}+1\right\} \\
& \text { At } x=0, \quad y_{v x}=\frac{1}{\sqrt{2}} \sqrt{k_{F x}}
\end{aligned}
$$


129

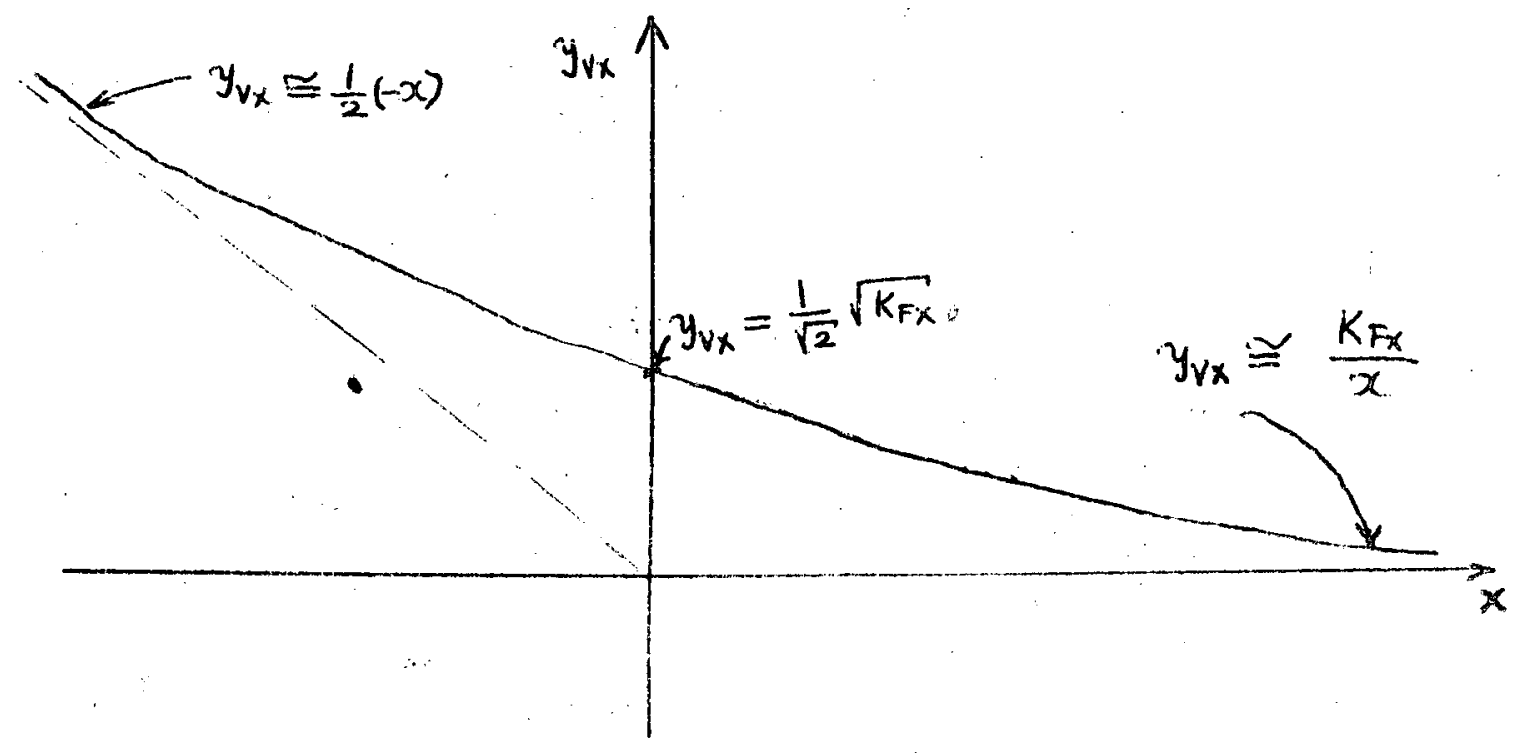


130

Problem 6.9

$B C C$ lattice - Octahedral interstitial sites

(a) $\alpha=$ number of interstitial sites per lattice site $\alpha=3$ for BCC - octahedral interstitial sites (from problem 6.3)

$\beta=$ number of interstitial sites adjacent to a lattice site

$\beta=6$ for $B C C$-octahedral interstitial sites

$\beta^{\prime}=$ number of lattice sites adjacent to an interstitial site

$$
\beta^{\prime}=\frac{\beta}{\alpha}=\frac{6}{3}=2
$$

(b) $G$, the total free energy is a function of (1) the number of free vacancies $\left(N_{v}^{f}\right)$

(2) the number of free interstitial $\left(N_{b}-N_{p}\right)$

(3) the number of bound pairs $\left(N_{p}\right)$

$$
G=G(0,0,0)+N_{v}^{f} g_{v}+\left(N_{b}-N_{p}\right) g_{b}+N_{p} g_{p}-k T \ln W
$$

(c) $g_{p}$ can be written as

$$
g_{p}=g_{v}+g_{b}-B_{p}
$$

where $B_{p}$ is the binding energy of the pair. It is the energy released when a free vacancy and a free interstitial come together.

$$
+\otimes=\square \otimes
$$


131

(d) Combinatorial factor $W$

$$
W=W_{p} W_{b} w_{v}
$$

$W_{p}=$ number of ways of arranging $N_{p}$ pairs among available sites.

$W_{b}=$ number of ways of arranging $N_{b}-N_{p}$ free. interstitial atoms on sites available to them

$N_{v}=$ number of ways of arranging $N_{v}^{*}$ vacancies on sites available to them.

Modification of divacancy method to calculate $W_{p}$

(1) Start with perfect lattice.

(2) Withdraw an atom (create a vacancy) - add an interstitial

(3) withdraw another atom - add another interstitial

until $N_{p}$ pairs are created

lIst pair

vacancy - withdraw atom from any of $N_{s}$ sites. interstitial - add to any of $\beta$ adjacent sites

$\therefore \beta N_{s}$ ways of creating the first pair.

[Do not divide by 2 as in the divacancy case because the two partners are different entities on different sites] 
132

$2^{\text {nd }}$ pair

$N_{s}-1$ lattice atoms available to be withdrawn.

(neglect possible exclusion of atoms surrounding.

the existing pair)

$\beta\left(N_{s}-1\right)$ ways of making second pair

$3^{\text {rd }}$ pair

$\beta\left(N_{s}-2\right)$ ways of making third pair

$\beta\left[N_{s}-\left(N_{p}-1\right)\right]$ ways of making $N_{p}^{t h}$ pair

$\therefore$ Total number of ways

$$
\begin{aligned}
& =\left[\beta N_{s}\right]\left[\beta\left(N_{s}-1\right)\right]\left[\beta\left(N_{s}-2\right)\right] \ldots\left[\beta\left(N_{s}-\left[N_{p}-1\right)\right]\right. \\
& =\beta^{N_{p}} N_{s}\left(N_{s}-1\right) \ldots \ldots\left[N_{s}-\left(N_{p}-1\right)\right] \\
& =\beta^{N_{p}} \frac{N_{s} !}{\left(N_{s}-N_{p}\right) !}
\end{aligned}
$$

But remove permutations among the $N_{p}$ pairs by dividing by $N_{p}$ !

$$
\therefore W_{p}=\frac{\beta^{N_{p}} N_{s} !}{\left(N_{s}-N_{p}\right) ! N_{p} !}
$$


Calculation of $W_{b}$

If there are $N_{f}$ pairs, there are $N_{b}-N_{p}$ free impurity atoms to be distributed among $N_{s I}-N_{p}$ sites.

$$
\text { But } N_{S I}=\alpha N_{S}
$$

The number of vacant interstitial sites is

$$
\begin{aligned}
& \left(\alpha N_{s}-N_{p}\right)-\left(N_{b}-N_{p}\right)=\alpha N_{s}-N_{b} \\
& \therefore W_{b}=\frac{\left(\alpha N_{s}-N_{p}\right) !}{\left(N_{b}-N_{p}\right) !\left(\alpha N_{s}-N_{b}\right) !}
\end{aligned}
$$

Calculation of $W_{V}$

If there are. $N_{p}$ pairs and $N_{b}-N_{p}$ free interstitials in the lattice, the $N_{v}^{f}$ free vacancies can be distributed among $N_{s}$ lattice sites except those. that are

(1) already accounted for in the vacancy-interstitial pairs

(2) nearest neighbois to one of the $N_{b}-N_{p}$ interstitial atoms

$\therefore$ available lattice sites on which to distribute

$N_{v}^{f}$ free vacancies is

$$
=N_{s}-N_{p}-\beta^{\prime}\left(N_{b}-N_{p}\right)
$$


134

number of filled lattice sites

$$
\begin{array}{r}
=N_{s}-N_{p}-\beta^{\prime}\left(N_{b}-N_{p}\right)-N_{v}^{f} \\
\therefore W_{v}=\frac{\left[N_{s}-N_{p}-\beta^{\prime}\left(N_{b}-N_{p}\right)\right] !}{\left[N_{s}-N_{p}-\beta^{\prime}\left(N_{b}-N_{p}\right)-N_{v}^{f}\right] ! N_{v}^{f} !}
\end{array}
$$

(e) Equilibrium occurs when

$$
\left(\frac{\partial G}{\partial N_{v}}\right)_{N_{p}}=0 \text { and }\left(\frac{\partial G}{\partial N_{p}}\right)_{N_{v}}=0
$$

[assuming that the total number of solute atoms and the total number of solvent atoms remain constant]

$$
\begin{aligned}
G=G(0,0,0)+N_{v}^{f} g_{v} & +\left(N_{b}-N_{p}\right) g_{b}+N_{p} g_{p} \\
& -k T \ln W_{p} W_{b} W_{v}
\end{aligned}
$$

where $W_{p}, W_{b}$ and $W_{v}$ are given by eqs.(1) $-(3)$

$$
N_{v}^{f}=N_{v}-N_{p}(4), \quad N_{s}=N_{a}+N_{v}
$$

where $N_{v}=$ total number of vacancies and $\mathrm{Na}_{\mathrm{a}}=$ total number of solvent atoms Writing, $\left(\frac{\partial G}{\partial N_{p}}\right)_{N_{a}, N_{b}, N_{v}}=0$ we get 
135

$$
\begin{aligned}
& -g_{v}-g_{b}+g_{p}-k T \ln \beta-k T \ln \left(N_{s}-N_{p}\right)+k T \ln N_{p} \\
& +k T \ln \left(\alpha N_{s}-N_{p}\right)-k T \ln \left(N_{b}-N_{p}\right)-k T\left(\beta^{\prime}-1\right) \ln \left[N_{s}-\beta^{\prime}\left(N_{b}-N_{p}\right)-N_{p}\right] \\
& +\beta^{\prime} k T \ln \left[N_{s}-\beta^{\prime}\left(N_{b}-N_{p}^{\prime}\right)-N_{v}\right]-k T \ln \left(N_{v}-N_{p}\right)=0 \\
& \therefore-B_{p}-k T \ln \beta=k T \ln \left\{\frac{N_{s}-N_{p}}{N_{p}} \cdot \frac{N_{b}-N_{p}}{\left\langle N_{s}-N_{p}\right.} \cdot \frac{N_{s}-\beta^{\prime}\left(N_{b}-N_{p}\right)-N_{p}}{\left[N_{s}-\beta^{\prime}\left(N_{b}-N_{p}\right)-N_{v}\right]^{2}}\right. \\
& \left.\quad\left(N_{v}-N_{p}\right)\right\}
\end{aligned}
$$

(since $\beta^{\prime}=3, \therefore \beta^{\prime}-1=2$ )

$$
\therefore e^{\left(B_{p}+k T \ln \beta\right) / h T}=\frac{N_{p}}{N_{b}-N_{p}} \cdot \frac{\alpha N_{s}-N_{p}}{N_{V}-N_{p}} \cdot \frac{\left[N_{s}-\beta^{\prime}\left(N_{b}-N_{p}\right)-N_{p}\right]^{2}}{N_{s}-\beta^{\prime}\left(N_{b}-N_{p}\right)-N_{p}}
$$

Now since $N_{p} \ll N_{s}$

$$
N_{b}:<N_{s}
$$

and $N_{v} \ll N_{s}$ we can neglect $N_{p}, N_{v}$ or $\beta^{\prime}\left(N_{b}-N_{p}\right)$ in comparison to $N_{s}$ or $\alpha N_{s}$

$$
\therefore \exp \left[\left(B_{p}+k T \ln \beta\right) / k T\right] \cong \frac{N_{p}}{N_{b}-N_{p}} \cdot \frac{\alpha N_{s}}{N_{y}-N_{p}} \cdot \frac{N_{s}^{2}}{N_{s}} \cdot \frac{1}{N_{s}}
$$


136

$\alpha N_{S}=N_{S I}$, number of octahedral interstitial sites

$N_{b}-N_{p}=N_{b}^{f}$, number of free solute atoms

$N_{v}-N_{p}=N_{v}^{*}$, number of free vacancies

$$
\therefore \frac{N_{p} N_{s I}}{N_{b}^{f} N_{v}^{f}}=\exp \left[\left(B_{p}+k T \ln \beta\right) / k T\right]
$$

where $B_{p}$ is the binding energy of the pair, ie.

$$
B_{p}=g_{y}+g_{b}-g_{p}
$$

Now taking, $\left(\frac{\partial G}{\partial N_{v}}\right)_{N_{a}, N_{b}, N_{p}}=0$ we get, since $\because \ldots$

$$
\begin{aligned}
& N_{v}^{f}=N_{v}-N_{p} \text { and } N_{s}=N_{a}+N_{v} \\
& g_{v}-k T \ln N_{s}+k T \ln \left(N_{s}-N_{p}\right)-\alpha k T \ln \left(\alpha N_{s}-N_{p}\right)+\alpha k T \ln \left[\alpha N_{s}-N_{b}\right) \\
& -k T \ln \left[N_{s}-N_{p}-\beta^{\prime}\left(N_{b}-N_{p}\right)\right]+k T \ln N_{v}^{f}=0 \\
& \therefore \exp \left[-g_{v} / k T\right]=\frac{N_{s}-N_{p}}{N_{s}} \cdot \frac{\left[\alpha N_{s}-N_{b}\right]^{\alpha}}{\left[N_{s}-N_{p}\right]^{\alpha}} \cdot \frac{N_{v}^{f}}{\left[N_{s}-N_{p}-\beta^{\prime}\left(N_{b}-N_{p}\right)\right]}
\end{aligned}
$$

Again neglecting $N_{p}, N_{b}$ in comparison to $\alpha N_{s}$ we get,

$$
\frac{N_{v}^{f}}{N_{s}}=\left[1-\beta^{\prime} \frac{N_{b}-N_{p}}{N_{s}-N_{p}}\right] \exp [-g v / k T]
$$


137

Again neglecting $N_{p}$ in comparison to $N_{s}$, we get,

$$
\frac{N_{v}^{f}}{N_{s}}=\left[1-\beta^{\prime} \frac{N_{b}-N_{p}}{N_{s}}\right] \exp [-q v / k T]
$$

Now let us define $x_{p}=\frac{N_{p}}{N_{s}}$

$$
\begin{gathered}
x_{v}^{f}=\frac{N_{v}^{f}}{N_{s}}-(9) ;\left(x_{b}^{f}\right)^{\prime}=\frac{N_{b}^{f}}{N_{s I}} \\
x_{b}^{f}=\frac{N_{b}^{f}}{N_{s}}=\alpha\left(x_{b}^{f}\right)^{\prime} \quad \ldots \text { (II) }
\end{gathered}
$$

Therefore from eq (6),

$$
\begin{aligned}
& \frac{x_{p}}{x_{v}^{f}\left(x_{b}^{f}\right)}=\exp \left[\left(B_{p}+k T \ln \beta\right) / k T\right] \\
& \text { or } \quad x_{p}=x_{v}^{f}\left(x_{b}^{f}\right)^{\prime} \cdot \beta \exp \left(B_{p} / k T\right) \\
& =x_{v}^{f} x_{b}^{f} \frac{\beta}{\alpha} \exp \left(B_{p} / k T\right) \text { from eq.(ii) }
\end{aligned}
$$

- If we define

$$
\begin{aligned}
x_{v}=x_{v}^{f}+x_{p}=\frac{N_{v}}{N_{s}} & =x_{v}^{f}\left[1+\frac{x_{p}}{x_{v}^{f}}\right] \\
& =x_{v}^{f}\left[1+\frac{\beta}{\alpha} x_{b}^{f} \exp \left[B_{p} / k T\right]\right\}
\end{aligned}
$$

From eq. (f) we get,

$$
x_{v}^{f}=\left(1-\beta^{\prime} x_{b}^{f}\right) \exp \left[-g_{v} / k T\right]
$$


138

Defining $x_{b}=\frac{N_{b}}{N_{s}}$, we have $x_{b}^{f}=x_{b}-x_{p}$

Substituting this in eq.(15) and neglecting second order terms in concentration, $x_{v}$ becomes,

$$
\begin{aligned}
x_{y}= & x_{v}^{f}\left\{1+\frac{\beta}{\alpha} x_{b}^{f} \exp \left[B_{p} / k T\right] \quad[\text { from eq. (14)] }\right. \\
= & \left(1-\beta^{\prime} x_{b}^{f}\right)\left\{1+\beta^{\prime} x_{b}^{f} \exp \left[B_{p} / k T\right]\right\} \exp \left[-g_{v} / k T\right] \\
& {[\text { from eq. (15)] }}
\end{aligned}
$$

(since $\left.\beta^{\prime}=\frac{\beta}{\alpha}\right)$

$$
\therefore x_{v} \cong\left(1-\beta^{\prime} x_{b}^{f}\right) \exp \left[-g_{v} / k T\right]+\beta^{\prime} x_{b}^{f} \exp \left[-\left(g_{v}-B_{p}\right) / k T\right]
$$

Since $x_{b}=x_{b}^{f}+x_{p}=x_{b}^{f}\left[1+\beta^{\prime} x_{v}^{f} \exp \left(B_{p} / k T\right)\right]$ [from eq .(12)]

$$
\left.=x_{b}^{f}\left[1+\beta^{\prime}\left(1-\beta^{\prime} x_{b}^{f}\right) \exp \left(-g_{v} / k T\right)\right] \text { fromeq.(15) }\right]
$$

then $x_{b}^{f}$ can be solved for in terms of $x_{b}$ and substituted in eq. (16). Thus ie ob tain $x_{v}$ in terms of $x_{b}$.

(f) For the "reaction" the law of mass action is

$$
\begin{aligned}
& \frac{x_{p}}{\left(\bar{x}_{b}^{\prime}-x_{p}\right) x_{v}^{t}}=\exp \left(B_{p} / k T\right) \\
& \text { where } x_{b}^{\prime}=\frac{N_{b}}{N_{s I}}=\frac{x_{b}}{\alpha}
\end{aligned}
$$


139

$$
x_{v}^{f}=\exp \left(-\epsilon_{v} / k T\right)=\frac{N_{v}^{*}}{N_{s}}=\text { site fraction of free }
$$

vacancies

Eq.(17) must be compared with eq. (16) for the results of the two approaches. 
140

Problem 6.10

Starting with Eq. (6.i1)

$$
\begin{aligned}
& \text { Starting with } E q \cdot(6.1)) \\
& G\left(N_{v}\right)-G(0)=N_{v} g_{v}-k T \ln \left[\frac{\left(N+N_{v}\right) !}{N ! N_{v} !}\right]
\end{aligned}
$$

Neglecting excess entropy

$$
\begin{gathered}
g_{v} \cong \epsilon_{v} \\
\mu_{v}=\left(\frac{\partial G}{\partial N_{v}}\right)_{N, T, p} \\
G-G(0)=N_{v} \epsilon_{v}-k T\left[\left(N+N_{v}\right) \ln \left(N+N_{v}\right)-(N+N)-N \ln N\right. \\
\left.\quad+N-N v \ln N_{v}+N v\right] \\
\therefore \frac{\partial G}{\partial N_{v}}=\epsilon_{v}-k T\left[\ln \left(N+N_{v}\right)-\ln N_{v}\right] \\
\therefore \mu_{v}=\epsilon_{v}+k T \ln \left[\frac{N_{v}}{N+N_{v}}\right]=\epsilon_{v}+k T \ln x_{v}
\end{gathered}
$$

From Eq. (6.12), the equilibrium concentration of vacancies is

$$
x_{v}^{e q}=e^{-\epsilon_{v} / k T}
$$

$\therefore$ at equilibrium $\mu_{v}=0$ 
141

Problem 6.11

Let $n_{v}=$ atom fraction vacancies at time $t$ then $\frac{d n_{V}}{d t}=k^{+}-\bar{k} n_{V}$

As $t \rightarrow \infty$, equilibrium is approached

$$
\begin{aligned}
& \therefore \quad\left(n_{v}\right)_{e q .}=e^{-\epsilon_{v} / k T}=\frac{k^{+}}{k^{-}} \\
& \therefore \frac{d n_{v}}{d t}=-k^{+}\left[\frac{n_{v}}{\left(n_{v}\right)_{e q}}-1\right]
\end{aligned}
$$

Integrating eq. (3) and using the initial condition

$$
n_{v}(0)=n_{v}^{0}
$$

yeilds

$$
\frac{n_{v}-\left(n_{v}\right)_{e q}}{n_{v}^{0}-\left(n_{v}\right)_{e q}}=e^{-k^{+} t}
$$

When the correct value of $n_{v}^{0}$ is used, the left hand side of eq. (5) will plot as a straight line on a semilog plot vs. $t$. The value of $n_{v}^{0}$ which yeilds a straight line on such a plot is the correct value and the slope of the line is $k^{+}$. The rate constant $k^{-}$ can then be determined from eq. (2). 
142

Problem. 6.12.

(a) Anneal of the Irradiated Sample (First Anneal)

$$
M C_{p} \frac{d T}{d t}=K[200-T]+M q[T(t)]
$$

(Second Anneal)

(Stored energy has been dissipated by first. anneal)

$$
M c_{p} \frac{d T^{\prime}}{d t}=K\left[200-T^{\prime}\right]
$$

Subtract the two equations,

$$
M C_{p} \frac{d}{d t}\left(T-T^{\prime}\right)=-K\left(T-T^{\prime}\right)+M q(T)
$$

Integrating,

$$
Q \equiv \int_{0}^{\infty} q[T(t)] d t=C_{p} \int_{0}^{\infty} d\left(T-T^{\prime}\right)+\frac{K}{M} \int_{0}^{\infty}\left(T-T^{\prime}\right) d t
$$

The first integral on the right hand side is zero; since in the curve of two anneals, $\left(T-T^{\prime}\right)$ at both the end points are zero.

$$
\therefore Q=\frac{K}{M} \int_{0}^{\infty}\left(T-T^{\prime}\right) d t
$$

This is the area enclosed by the two curves of two anneals. It can be evaluated graphically. 
143

$$
Q=\frac{0.44}{10}\left(4.2 \times 10^{3}\right)=184.8 \text { Joules } / \mathrm{gm}
$$

Alternate method

Assume temperature spike - heat released instantaneously at $T_{c}=128^{\circ} \mathrm{C}$. Temperature rises to $T_{\max }=279^{\circ} \mathrm{C}$. in very short time. Neglect the heat exchange term $K(200-T)$ during the interval compared to $M C_{p} \frac{d r}{d t}$

$$
\begin{aligned}
Q=\int_{0}^{\infty} q[T(t)] d t & =C_{p} \int_{0}^{\infty} \frac{d T}{d t} d t=C_{p} \int_{T_{0}}^{T_{\text {max }}} d T \\
& =C_{p}\left(T_{\text {max }}-T_{c}\right) \\
\therefore Q & =1.26(151)=190.3 \text { Joules } / g m
\end{aligned}
$$

(b) Energy released per vacancy -interstitial recombination:

Step:1 Move an atom from surface to vacant lattice site inside the solid - releases $\epsilon_{y}$ of energy and removes vacancy.

Step:2 Move an atom from interstitial site inside solid to surface - releases $\epsilon_{i}$ of energy and removes an interstitial. 
144

$$
\begin{aligned}
\therefore \text { Total energy released per } & =\epsilon_{v}+\epsilon_{i} \\
v-i \text { recombination } & =5.2+13.9 \\
& =19.1 \mathrm{eV}
\end{aligned}
$$

Let $x_{i}=x_{v}=$ Frankel pair concentrations prior to anneal (atom fractions)

$N_{c}=$ number of atoms per $\mathrm{gm}$ of graphite, then

$$
\begin{aligned}
& N_{c}=\frac{1 \mathrm{gmatom}}{12 \mathrm{gm}} \times \frac{6 \times 10^{23} \text { atoms }}{\mathrm{gm} \mathrm{atom}}=5 \times 10^{22} \frac{\text { atoms }}{\mathrm{gm}} \\
& x_{i}=x_{v}=\frac{Q \mathrm{ev} / g_{m}}{\left(\epsilon_{i}+\epsilon_{v}\right)^{e v / F_{\text {renkel }}} * N_{c} \frac{a+c m s}{g m}}=\frac{Q}{N_{c}\left(\epsilon_{j}+\epsilon_{v}\right)} \\
& =\frac{190.26}{19.1 \times 1.6 \times 10^{-19} \times 5 \times 10^{22}} \\
& =\frac{190.26}{152.3} \times 10^{-3} \\
& =1.25 \times 10^{-3}
\end{aligned}
$$


145

CHAPTER 7

Problem 7.1

(a) Since Frankel disorder on the oxygen sublattice predominates, the concentration of oxygen vacancies can be obtained from the results of the previous problem 6.2, ie. from eq. (1), (2), (6) and (8) in the problem 6.2

$$
\frac{N_{v x}}{N_{5 x}}=x_{v x}=\frac{1}{2} \sqrt{2 K_{F x}}=\sqrt{\frac{K_{F x}}{2}}
$$

The vacancy concentration on the cation sublattice is obtained for Schotiky. disorder by the law of mass action

$$
x_{v x}^{2} x_{v m}=k_{s}
$$

Combining (1) and (2)

$$
x_{V M}=\frac{2 K_{s}}{K_{F x}}
$$

and

$$
\begin{aligned}
K_{F x} & =e^{\Delta S_{F \times} / k} e^{-E_{F x / k T}} \\
& =e^{\frac{63}{8.314}} e^{-\frac{297 \times 10^{8}}{8.314 \times 1673}}=10^{-5} \\
K_{S} & =e^{\Delta S_{s} / k} e^{-E_{s} / k T} \\
& =1 e^{-\frac{544 \times 10^{3}}{8.314 \times 1673}}=1^{-16.93}
\end{aligned}
$$


146

Substituting eqs.(4) and (5) into (1) and (3) we get,

$$
\left.\begin{array}{l}
x_{v x}=7.24 \times 10^{-4} \\
x_{v m}=2.24 \times 10^{-11}
\end{array}\right\} \begin{aligned}
& \text { for pure } \\
& \mathrm{UO}_{2}
\end{aligned}
$$

(b) Charge balance in $\mathrm{NO}_{2}$ doped with $\mathrm{NDm}$ cation impurity atoms of charge +5 is:

$$
4 N_{M}+5 N_{D M}=2 N_{X}+2 N_{X I}
$$

but: $N_{S M}=N_{M}+N_{D M}+N_{V M}$

and

$$
\begin{aligned}
& N_{s x}=N_{x}+N_{v x} \\
& N_{S M}=\frac{1}{2} N_{s x}, N_{s I}=\frac{1}{2} N_{s x} \\
& \therefore 4\left(N_{S M}-N_{D M}-N_{V M}\right)+5 N_{D M}=2\left(N_{S X}-N_{V X}\right) \\
& +2 N_{x I}
\end{aligned}
$$

Neglecting $N_{U m}$ by assumption,

$$
N_{D M}=2\left(N_{X I}-N_{v X}\right)
$$

Dividing by $N_{S M}=\frac{1}{2} N_{s x}$

$$
\frac{N_{D M}}{N_{S M}}=4\left(\frac{N_{x I}}{N_{S x}}-\frac{N_{v x}}{N_{S x}}\right)
$$

let $\frac{N_{D M}}{N_{S M}}=X_{D M}, \frac{N_{v X}}{N_{S_{X}}}=X_{v X}$ 
147

and by $N_{S I}=\frac{1}{2} N_{S x}$

$$
\frac{N_{X I}}{N_{s X}}=\frac{N_{X I}}{2 N_{S I}}=\frac{1}{2} x_{x I}
$$

Therefore electrical neutrality gives,

$$
x_{D M}=2 x_{x I}-4 x_{v x}
$$

Law of mass action for Frenkel defects of anion sublattice gives,

$$
K_{F x}=x_{v_{x}} x_{x I}
$$

Solving es. (11) and (12) for $x_{v_{x}}$ gives,

$$
X_{v_{x}}=\frac{1}{8} X_{D M}\left(\sqrt{1+32 \frac{K_{F x}}{x_{D M}^{2}}}-1\right)
$$

From eq (2),

Limiting case

$$
x_{v_{m}}=\frac{K_{s}}{x_{v_{x}}^{2}}
$$
Expanding the square root term in Taylor Series and taking the first 2 -terms

$$
\begin{array}{r}
x_{V X}=\frac{1}{8} x_{D M}\left(1+16 \frac{K_{F X}}{x_{D V}^{2}}-1\right)=2 \frac{K_{F X}}{x_{D M}} \\
\therefore x_{V X}=\frac{2 K_{F X}}{x_{D M}}
\end{array}
$$


148

(2) Small impurity concentration $\frac{32 K_{F x}}{x_{D P}^{2}}>1$

$$
\begin{aligned}
& \therefore X_{V X} \cong \frac{1}{8} X_{D M} \sqrt{\frac{32 K_{F x}}{x_{D M}^{2}}} \\
& \text { or } X_{V x}=\sqrt{\frac{K_{F x}}{2}}
\end{aligned}
$$

(c)

Using the calculated value of $K_{F x}$ and $x_{D M}=0.1$ in eq.(13), we get,

$$
\begin{aligned}
x_{v x} & =\frac{1}{8}(0.1)\left(\sqrt{1+32 \frac{10^{-5.98}}{10^{-2}}}-1\right) \\
& =2.1 \times 10^{-5}
\end{aligned}
$$

Now using this in eq.(2) to get $x_{u n}$

$$
\left.\begin{array}{rl}
x_{v m}=\frac{K_{s}}{x_{v i}^{2}} & =\frac{10^{-16.93}}{\left(2.1 \times 10^{-5}\right)^{2}}=\frac{10^{-6.93}}{(2.1)^{2}} \\
& \cong 2.66 \times 10^{-8} \\
\therefore \quad x_{v x} & =2.1 \times 10^{-5} \\
x_{v m} & =2.66 \times 10^{-8}
\end{array}\right\} \text { in doped } 40_{2}
$$

For both $U$ and $O$,

$$
\frac{D_{\text {(loped) }}}{D_{\text {(pure) }}}=\frac{\left.X_{v} \text { (doper) }\right)}{X_{v} \text { (pure) }}
$$

for $U$

$$
\frac{D_{u \text { (copal) }}}{D_{u \text { (pure) }}}=\frac{\left(x_{v_{m}}\right)_{\text {(doped })}}{\left(x_{v_{m}}\right)_{\text {(pure) }}}=\frac{2.7 \times 10^{-8}}{2.24 \times 10^{-11}} \cong 1.2 \times 10^{3}
$$

for $0 \quad \frac{D_{0} \text { (doped) }}{D_{0} \text { (pure) }}=\frac{\left(x_{v_{x}}\right)_{\text {(doped })}}{\left(x_{v_{x}}\right)_{\text {(pure) }}}=\frac{2.1 \times 10^{-5}}{7.24 \times 10^{-4}}=2.9 \times 10^{-2}$ 
149

Problem 7.2

$$
\begin{aligned}
K & =\left(\frac{d^{2} \varphi}{d x^{2}}\right)_{x=0} \\
\varphi(x) & =\frac{1}{2} \epsilon^{*}\left[1-\cos \left(\frac{2 \pi x}{a_{0}}\right)\right] \\
\frac{d \phi}{d x} & =\frac{1}{2} \epsilon^{*}\left(\frac{2 \pi}{a_{0}}\right) \sin \left(\frac{2 \pi x}{a_{0}}\right) \\
\frac{d^{2} \varphi}{d x^{2}} & =\frac{1}{2} \epsilon^{*}\left(\frac{2 \pi}{a_{c}}\right)^{2} \cos \left(\frac{2 \pi x}{a_{c}}\right) \\
\therefore K & =\frac{2 \pi^{2}}{a_{c}^{2}} \epsilon^{*}
\end{aligned}
$$

Frequency of vibration is:

$$
\begin{aligned}
& \left.\nu=\frac{1}{2 \pi} \sqrt{\frac{k}{m}}=\frac{1}{2 \pi} \sqrt{\frac{(2 \pi)^{2} \epsilon^{*}}{2 m a_{0}^{2}}} \quad \text { [from }(1)\right] \\
& \because \nu=\sqrt{\frac{\epsilon^{*}}{2 m a_{0}^{2}}} \\
& \text { Jump frequency } w=\nu e^{-\epsilon^{*} / k T} \\
& \therefore W=\sqrt{\frac{\epsilon^{*}}{2 m a_{c}^{2}}} e^{-\epsilon^{*} / k T}
\end{aligned}
$$


150

Problem 7.3

(a) For a single degree of vibrational freedom, the energy of the oscillator above the minimum in the potential curve is :

$$
\epsilon_{j}=\left(j+\frac{1}{2}\right) h \nu
$$

The partition function of a single oscillator is:

$$
\begin{aligned}
z= & \sum_{j=0}^{\infty} e^{-\epsilon_{j} / k T}=\sum_{j=0}^{\infty} e^{-\left(j+\frac{1}{2}\right) \frac{h \nu}{k T}} \\
= & e^{-\frac{1}{2} \frac{h \nu}{k T}} \sum_{j=0}^{\infty} e^{-j\left(\frac{h \nu}{k T}\right)}
\end{aligned}
$$

or $z=e^{-\frac{1}{2}\left(\frac{h \nu}{k T}\right)}\left(1-e^{-\frac{h \nu}{k T}}\right)^{-1}$

$$
\begin{aligned}
& \text { let } \quad x=\frac{h \nu}{k T} \\
& \therefore \quad z=e^{-\frac{x}{2}}\left(1-e^{-x}\right)^{-1}=\frac{1}{x}\left[\frac{x}{e^{x / 2}-e^{-x / 2}}\right]
\end{aligned}
$$

The bracketed term is the correction factor $f(x)=$

$$
\begin{array}{r}
\therefore \quad f(x)=\frac{e^{x / 2}-e^{-x / 2}}{x}=\frac{2 \sinh \left(\frac{x}{2}\right)}{x} \\
\text { or } f(x)=\frac{\sinh \left(\frac{x}{2}\right)}{(x)}
\end{array}
$$


151

(b) From Eq. (7.34), neglecting lattice effect

$$
W=\frac{z^{*}}{z_{\text {eq. }}} \cdot \frac{\bar{v}_{x}}{\delta} e^{-\epsilon^{*} / k r}
$$

where $x^{*}=\frac{h \nu^{*}}{k T}$

$$
\begin{aligned}
z_{e q} & =\frac{1}{[x f(x)]^{3}} \\
\therefore \quad W & =\delta\left(\frac{2 \pi m k T}{h^{2}}\right)^{1 / 2} \frac{[x f(x)]^{3}}{\left[x^{*} f\left(x^{*}\right)\right]^{2}}\left(\frac{k T}{2 \pi m}\right)^{1 / 2} \frac{1}{\delta} e^{-\epsilon^{*} / k T} \\
\therefore W & =\frac{k T}{h}\left(\frac{h \nu}{k T}\right)^{3}\left(\frac{k T}{h \nu^{*}}\right)^{2} \frac{[f(x)]^{3}}{\left[f\left(x^{*}\right)\right]^{2}} e^{-\epsilon^{*} / k T} \\
\therefore & =\frac{\nu^{3}}{\left(2^{*}\right)^{2}} \frac{[f(x)]^{3}}{\left[f\left(x^{*}\right)\right]^{2}} e^{-\epsilon^{*} / k T}
\end{aligned}
$$

(c) From Random Walk Theory

$$
\begin{aligned}
& D=\frac{1}{6} \lambda^{2} \Gamma=\frac{1}{6} \lambda^{2} \beta W \\
& E=-k \frac{d \ln D}{\ln D}=-k \frac{d \ln W}{\ln }
\end{aligned}
$$


152

(because $\lambda$ and $\beta$ are temperature independent)

From part (b):

$$
\begin{gathered}
\ln W=\ln \left[\frac{\nu^{3}}{\left(\nu^{*}\right)^{2}}\right]+3 \ln [f(x)]-2 \ln \left[f\left(x^{*}\right)\right] \\
-\frac{\epsilon^{*}}{k T} \\
\frac{d \ln W}{d(1 / r)}=3\left(\frac{h \nu}{k}\right)\left[\frac{d \ln f(x)}{d x}\right]_{x=\frac{h \nu}{k T}}-2\left(\frac{h \nu^{*}}{k}\right)\left[\frac{d \ln f(x)}{d x}\right]_{x=\frac{h \nu^{*}}{k T}} \\
-\frac{\epsilon^{*}}{k}
\end{gathered}
$$

From part (a):

$$
\frac{d \ln f(x)}{d x}=\frac{1}{2} \frac{1}{f} \frac{d f}{d\left(\frac{x}{2}\right)}=\frac{1}{2} \frac{\left[\frac{\cosh \left(\frac{x}{2}\right)}{\left(\frac{x}{2}\right)}-\frac{\sinh \left(\frac{x}{2}\right)}{\left(\frac{x}{2}\right)^{2}}\right]}{\frac{\sinh \left(\frac{x}{2}\right)}{\left(\frac{x}{2}\right)}}
$$

or $\frac{d \ln f(x)}{d x}=\frac{1}{2}\left[\operatorname{coth}\left(\frac{x}{2}\right)-\frac{1}{\left(\frac{x}{2}\right)}\right]$

$$
\begin{array}{r}
\therefore E=-k \frac{d \ln W}{d(1 / r)}=\epsilon^{*}+k\left\{2\left(\frac{h \nu^{*}}{k}\right) \frac{1}{2}\left[\operatorname{coth}\left(\frac{h \nu^{*}}{2 k^{*}}\right)-\frac{2 k T}{h \nu^{*}}\right]\right. \\
\left.-3\left(\frac{h \nu}{k}\right) \frac{1}{2}\left[\operatorname{coth}\left(\frac{h \nu}{2 k T}\right)-\frac{2 k T}{h \nu}\right]\right\}
\end{array}
$$


153

$$
\therefore E=\epsilon^{*}+k T\left\{1+\left(\frac{h \nu^{*}}{k T}\right) \operatorname{coth}\left(\frac{h \nu^{*}}{2 k T}\right)-\frac{3}{2}\left(\frac{h \nu}{k T}\right) \operatorname{coth}\left(\frac{h \nu}{2 k T}\right)\right\}
$$

(d) Isotope effect

$$
\begin{aligned}
& D_{1}=\text { diffusivity of hydrogen } \\
& D_{2}=\text { diffusivity of deuterium }
\end{aligned}
$$

$D=\frac{1}{6} \lambda^{2} \beta w$, but $\lambda$ and $\beta$ are the properties of the host lattice, not the diffusing atom, therefore

$$
\frac{D_{1}}{D_{2}}=\frac{W_{1}}{W_{2}}
$$

Using the result of the part (b), ie. eq .(2) for $w_{1}$ and $W_{2}$ :

$$
\frac{D_{1}}{D_{2}}=\left(\frac{y_{1}}{y_{2}}\right)^{3}\left(\frac{y_{2}^{*}}{\nu_{1}^{*}}\right)^{2}\left[\frac{f\left(x_{1}\right)}{f\left(x_{2}\right)}\right]^{3}\left[\frac{f\left(x_{2}^{*}\right)}{f\left(x_{1}^{*}\right)}\right]^{2}
$$

For simple harmonic oscillator,

$$
\nu=\frac{1}{2 \pi} \sqrt{\frac{k}{m}}
$$

where $k$ is the force constant for the second derivative of the potential energy curve) and $m$ is the mass of the diffusing atom: 
154

Since $K$ is the same for both the isotopes

$$
\begin{aligned}
& \left(\frac{\nu_{1}}{\nu_{2}}\right)^{3}=\left(\frac{m_{2}}{m_{1}}\right)^{3 / 2}=2^{3 / 2} ; \\
& \left(\frac{\nu_{2}^{*}}{\nu_{1}^{*}}\right)^{2}=\left(\frac{m_{1}}{m_{2}}\right)=\frac{1}{2} \\
& x_{2}=\frac{h \nu_{2}}{k T}=\left(\frac{h \nu_{1}}{k T}\right)\left(\frac{\nu_{2}}{\nu_{1}}\right)=x_{1}\left(\frac{m_{1}}{m_{2}}\right)^{1 / 2}=\frac{x_{1}}{\sqrt{2}} \\
& \text { similarly, } \quad x_{2}^{*}=\frac{x_{1}^{*}}{\sqrt{2}}
\end{aligned}
$$

Dropping the subscript 1 on $x_{1}$, the diffusivity ratio is:

$$
\frac{D_{1}}{D_{2}}=\sqrt{2}\left[\frac{f(x)}{f\left(\frac{x}{\sqrt{2}}\right)}\right]^{3}\left[\frac{f\left(\frac{x^{*}}{\sqrt{2}}\right)}{f\left(x^{*}\right)}\right]^{2}
$$

(e) At $300^{\circ} \mathrm{K}$,

$$
x=\frac{h \nu}{k T}=\frac{1350}{300}=4.5 ; \frac{x}{\sqrt{2}}=3.182
$$

and $x^{*}=\frac{h \nu^{*}}{k T}=\frac{2300}{300}=7.66 ; \frac{x^{*}}{\sqrt{2}}=5.42$

$$
\therefore \quad \frac{D_{\text {Hydrogen }}}{D_{\text {Deuterium }}}=\frac{D_{1}}{D_{2}}=\sqrt{2}\left[\frac{f(x)}{f\left(\frac{x}{\sqrt{2}}\right)}\right]^{3}\left[\frac{f\left(\frac{x^{*}}{\sqrt{2}}\right)}{f\left(x^{*}\right)}\right]^{2}
$$


155

$$
\begin{aligned}
& \therefore \frac{D_{\text {Hod. }}}{D_{\text {Deut }}}=\sqrt{2}\left[\frac{\left(\frac{e^{\frac{4.5}{2}}-e^{-\frac{4.5}{2}}}{4.5}\right)}{\left(\frac{e^{\frac{3.18 .3}{2}}-e^{-\frac{3.132}{2}}}{3.182}\right)}\right]^{3}\left[\frac{\left(\frac{e^{\frac{5.42}{2}}-e^{-\frac{5.42}{2}}}{5.42}\right)}{\left(\frac{e^{\frac{7.66}{2}}-e^{-\frac{7.66}{2}}}{7.66}\right)}\right]^{2} \\
& =\sqrt{2} \times 2.8 \times 0.211 \\
& =0.836 \\
& \text { At } 1000^{\circ} \mathrm{K} \text {, } \\
& x=\frac{h \nu}{k T}=1.35, \quad \frac{x}{\sqrt{2}}=0.95 \\
& \text { and } x^{*}=\frac{h \nu^{*}}{k T}=\frac{2.30}{\vdots}, \frac{x^{*}}{\sqrt{2}}=1.63
\end{aligned}
$$

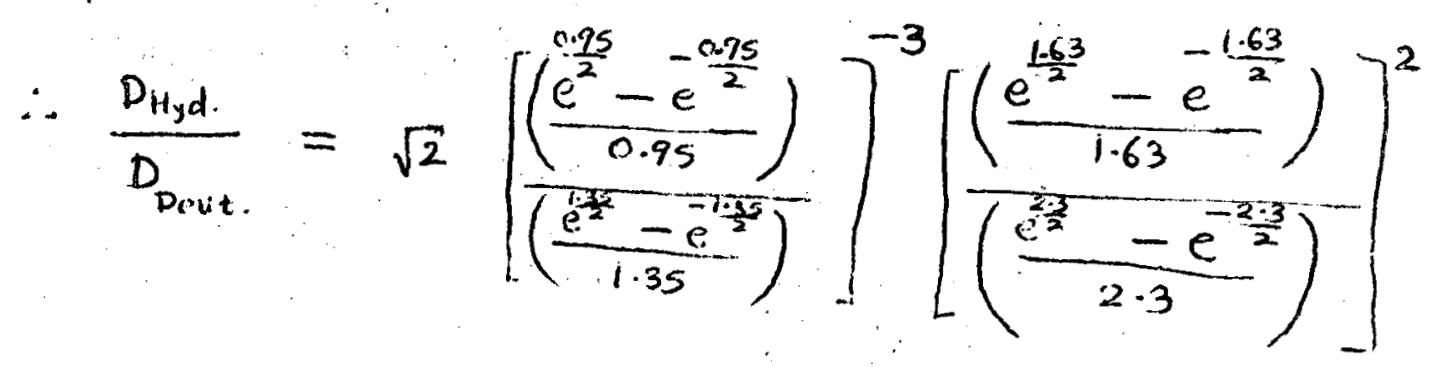

$$
\begin{aligned}
& =\sqrt{2} \times 1.1 \times 0.81 \\
& =1.26
\end{aligned}
$$


156

Problem 7.4

Substitutional Migration with FCC lattice octahedral equilibrium sites

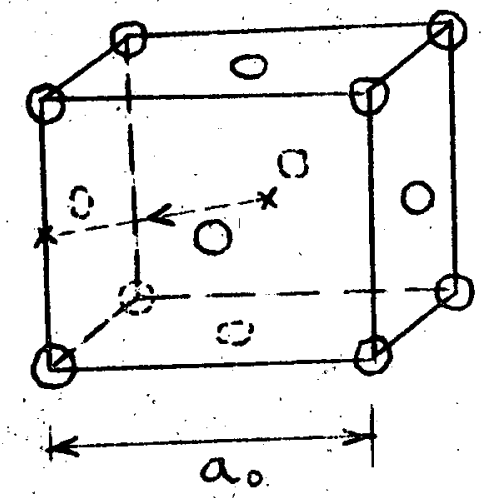

$$
\begin{aligned}
\lambda=\frac{a_{0}}{\sqrt{2}} \quad, \quad \beta=12 ; \quad \text { (all edge center } \\
\text { positions) }
\end{aligned}
$$


Problem 7.5

(a) Either by (i) the Einstein relation

$$
\overline{x^{2}} \cong 6 D t
$$

or by (ii) recognising that solutions of the unsteady state diffusion problems of this sort depend upon the variable $\left(x^{2} / 4 D t\right)$, and a value of the variable of approximately unity is needed to produce a significant change in the concentration,

$$
\begin{aligned}
\therefore \quad D_{\min } \cong \frac{\left(\bar{x}^{2}\right)_{\min }}{6 t_{\max }} & =\frac{\left(2 \times 10^{-4}\right)^{2} \mathrm{~cm}^{2}}{6 \times 3.14 \times 10^{7} \mathrm{sec}} \\
& =2 \times 10^{-16} \mathrm{~cm}^{2} / \mathrm{sec}
\end{aligned}
$$

(b) For $B C C$ interstitial diffusion,

$$
\begin{aligned}
D & =\frac{1}{6} a_{0}^{2} \nu e^{s^{*} / k} e^{-\epsilon^{*} / k T} \\
\epsilon^{*} & =60 \mathrm{kcal} / \mathrm{mole}
\end{aligned}
$$

Estimates

$$
\begin{aligned}
& a_{0} \cong 3 A^{0}=3 \times 10^{-8} \mathrm{~cm} \\
& \nu \cong 10^{13} \sec ^{-1} \\
& S^{*} \cong 0 \\
& \therefore \quad \frac{\epsilon^{*}}{k T_{\min }}=\ln \left(\frac{a_{0 \nu}^{2}}{6 D}\right) \cong \ln \left(10^{3^{*} / k}\right)=30
\end{aligned}
$$

$\therefore k T_{\min }=\frac{60}{30}=2 \quad, \quad T_{\min }=1000^{\circ} \mathrm{K}$. 
158

CHAPTER 8

Problem 8.1

(a) $\frac{a_{0}}{2}[110]$ edge dislocation in the [III] plane in the FCC lattice.

The extra half sheet of atoms which forms the edge dislocation must be perpendicular to the slip plane [ie., the (III) plane] and also must be perpendicular to the Burgers vector (ie. [ilo])

extra hals sheet

of atoms: (110) planes

[10]

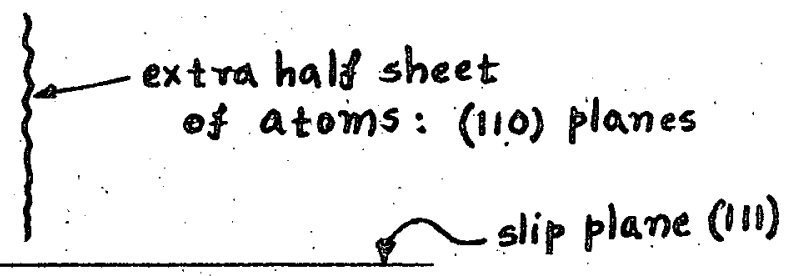

The extra half sheet in the fec lattice is very wrinkled. We need to pick out a row of atoms in the (III) planes which is perpendicular to the [110] direction. A cut 1 to the extra half sheet would reveal that it consists of the atoms shown with an " $x$ " in the sketch below.

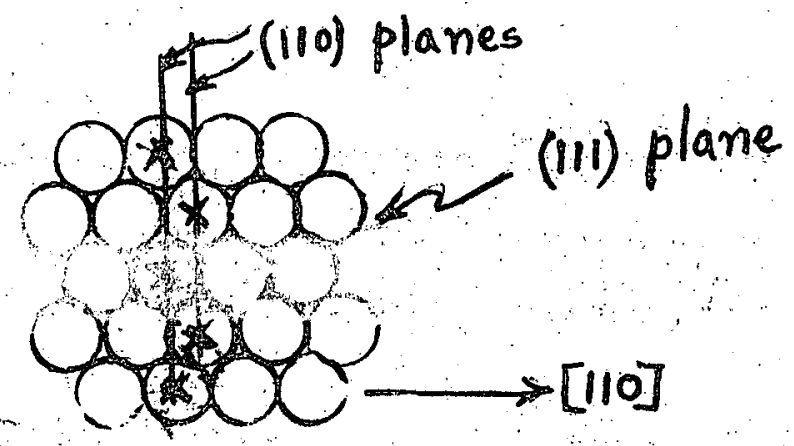


159

Continuation of the " $x$ " atoms in the (111) planes above and below the one in the sketch shows that the extra half sheet consists of two adjacent: (110) planes in the fac structure.

b)

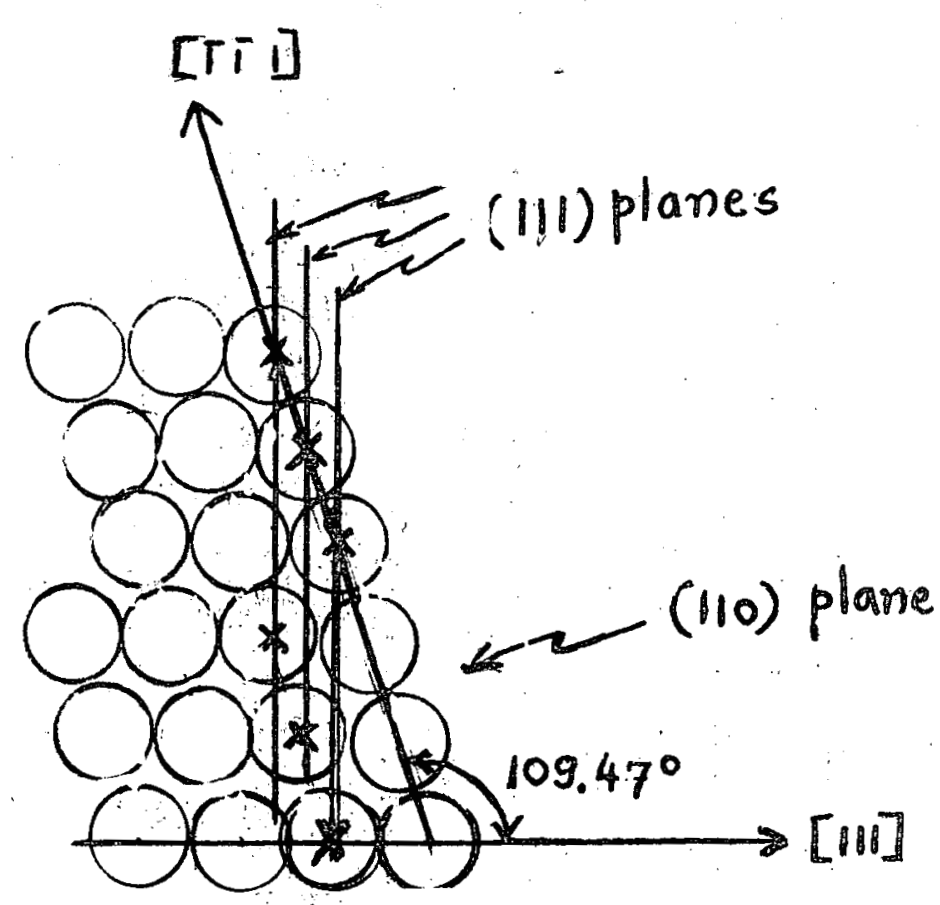

$\frac{a_{0}}{2}$ [111] edge dislocation in the (110) plane of be lattice. Extra half sheet must simultaneously be $\perp$ to [iII] direction and to the (110) plane. Half sheet consists of three adjacent (III) planes. ( $x^{\prime}$ s in sketch) 
160

Problem 8.2

$$
\begin{aligned}
E_{e l}=\frac{1}{2 E}\left(\sigma_{r r}^{2}+\sigma_{\theta \theta}^{2}\right. & \left.+\sigma_{z z}^{2}\right)-\frac{\nu}{E}\left(\sigma_{r r} \sigma_{z z}+\sigma_{r \gamma} \sigma_{\theta \theta}+\sigma_{\theta \theta} \sigma_{z z}\right) \\
& +\frac{1}{2 G}\left(\sigma_{r \theta}^{2}+\sigma_{\theta z}^{2}+\sigma_{r z}^{2}\right)-\left[E_{q \cdot}(A-26)\right]
\end{aligned}
$$

From Fig. 8.8

$$
\begin{aligned}
& \sigma_{r r}=\sigma_{\theta \theta}=\frac{G b}{2 \pi(1-\nu)} \frac{\sin \theta}{r}=\frac{K \sin \theta}{r} \\
& \text { [where } \left.K=\frac{G b}{2 \pi(1-\nu)}\right] \\
& \sigma_{r \theta}=-\frac{G b}{2 \pi(1-\nu)} \frac{\cos \theta}{r} \\
& =-\frac{K \cos \theta}{r} \\
& \sigma_{z z}=\frac{a b \nu}{2 \pi(1-\nu)} \frac{\sin \theta}{r}=2 \nu \sigma_{r r}=\frac{2 k \nu \sin \theta}{r}
\end{aligned}
$$

Substituting:

$$
\begin{aligned}
E_{e l}= & \frac{1}{2 E} \frac{K^{2}}{r^{2}}\left(\sin ^{2} \theta+\sin ^{2} \theta+4 \nu^{2} \sin ^{2} \theta\right) \\
& -\frac{\nu K^{2}}{E r^{2}}\left(2 \nu \sin ^{2} \theta+\sin ^{2} \theta+2 \nu \sin ^{2} \theta\right) \\
& +\frac{1}{2 G} \frac{K^{2}}{\gamma^{2}} \cos ^{2} \theta \\
\therefore E_{e l}= & \frac{K^{2}}{r^{2}}\left\{\frac{2}{2 E} \sin ^{2} \theta\left(1+2 \nu^{2}\right)-\frac{\nu}{E} \sin ^{2} \theta(1+4 \nu)\right. \\
\therefore E_{e l}= & \frac{K^{2}}{r^{2}} \frac{1}{E} \sin ^{2} \theta\left[1+2 \nu^{2}-\nu-4 \nu^{2}\right]+\frac{K^{2}}{r^{2}}\left(\frac{1+\nu}{E}\right) \cos ^{2} \theta \\
2 G= & \frac{E}{1+\lambda} \quad-\quad-[E q \cdot(A-20)]
\end{aligned}
$$


161

$$
\therefore \quad E_{e l}=\frac{K^{2}}{r^{2}} \frac{1}{E}\left\{(1-2 \nu)(1+\nu) \sin ^{2} \theta+(1+\nu) \cos ^{2} \theta\right\}
$$

Replace $\frac{1+y}{E}$ by $\frac{1}{2 G}$

$$
\begin{aligned}
& \frac{K^{2}}{2 G}=\frac{G^{2} b^{2}}{4 \pi^{2}(1-\nu)^{2}} \frac{1}{2 G}=\frac{G b^{2}}{8 \pi^{2}(1-\nu)^{2}} \\
& \therefore E_{e l}=\frac{G b^{2}}{8 \pi^{2}(1-\nu)^{2}} \cdot \frac{1}{r^{2}}\left\{(1-2 \nu) \sin ^{2} \theta+\cos ^{2} \theta\right\}
\end{aligned}
$$

Line Tension:

$$
\begin{aligned}
& \tau=\int_{0}^{2 \pi} d \theta \int_{r_{d}}^{R} r d r E_{e l} \\
& \therefore \quad \tau=\frac{G b^{2}}{8 \pi^{2}(1-\nu)^{2}} \int_{r_{d}}^{R} \frac{d r}{r}\left\{(1-2 \nu) \int_{0}^{2 \pi} \sin ^{2} \theta d \theta+\int_{0}^{2 \pi} \cos ^{2} \theta d \theta\right\} \\
&=\frac{G b^{2}}{8 \pi^{2}(1-\nu)^{2}} \ln \left(\frac{R}{r_{d}}\right) \pi\{(1-2 \nu)+1\} \\
& \therefore \quad \tau=\frac{G b^{2}}{4 \pi(1-\nu)} \ln \left(R / r_{d}\right)
\end{aligned}
$$


162

Problem 8.3

a)

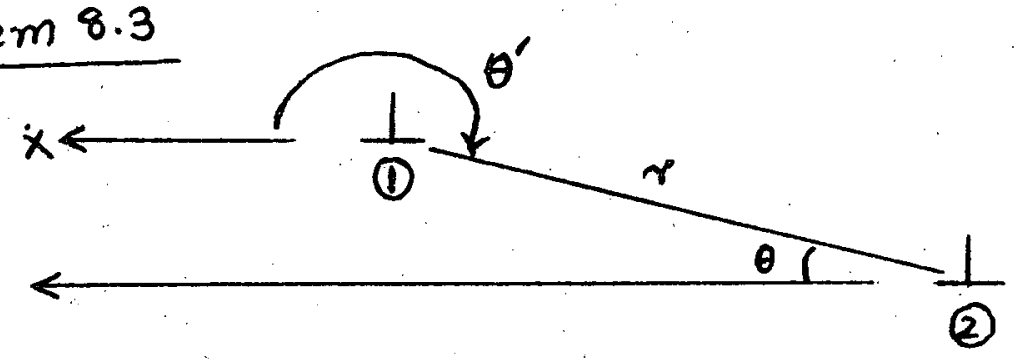

$$
F_{12}=\text { Force exerted by (1) on (2) }=-F_{21}
$$

From Eq. (8.13)

$$
F_{12}=-\left(\sigma_{x y}\right)_{1} b \underline{q}+\left(\sigma_{x x}\right)_{1} b \underline{\jmath} \quad\left[E_{q} \cdot(8.13)\right]
$$

where $\left(\sigma_{x y}\right)$, and $\left(\sigma_{x x}\right)$, are the stress fields of (1) at the position of (2).

Eq. (8.22):

$$
\begin{aligned}
& \left(\sigma_{x y}\right)_{1}=\left(\cos ^{2} \theta-\sin ^{2} \theta\right)\left(\sigma_{r \theta}\right)_{1} \\
& \left(\sigma_{x x}\right)_{1}=\left(\sigma_{r \gamma}\right)_{1}-2 \sin \theta \cos \theta\left(\sigma_{r \theta}\right)_{1}
\end{aligned}
$$

Apply equations in Fig. 8.8 to (1)

(note: use $\theta^{\prime}$, not $\theta$, here)

$$
\begin{aligned}
& \left(\sigma_{r \gamma}\right)_{1}=\frac{G b}{2 \pi(1-\nu)} \frac{\sin \theta^{\prime}}{r}=\frac{K \sin \theta^{\prime}}{r} \\
& \left(\sigma_{r \theta}\right)_{1}=-\frac{G b}{2 \pi(1-\nu)} \frac{\cos \theta^{\prime}}{r}=-\frac{K \cos \theta^{\prime}}{r}
\end{aligned}
$$

where $K=\frac{G b}{2 \pi(1-\nu)}$

$$
\theta^{\prime}=\pi+\theta
$$


163

$$
\begin{aligned}
\cos \theta^{\prime} & =-\cos \theta, \quad \sin \theta^{\prime}=-\sin \theta \\
\left(\dot{\sigma}_{r r}\right)_{1} & =-\frac{k}{r} \sin \theta, \quad\left(\sigma_{r \theta}\right)_{1}=\frac{k}{r} \cos \theta \\
\therefore \quad\left(\sigma_{x y}\right)_{1} & =\frac{k}{r}\left(\cos ^{2} \theta-\sin ^{2} \theta\right) \cos \theta \\
\left(\sigma_{x x}\right)_{1} & =\frac{k}{r}\left(-\sin \theta-2 \sin \theta \cos ^{2} \theta\right)=-\frac{k}{r} \sin \theta\left(1+2 \cos ^{2} \theta\right)
\end{aligned}
$$

Substituting in Eq. (8.13) we get,

$$
\begin{aligned}
& F_{12}=\frac{K b}{r}\left[-\cos \theta\left(\cos ^{2} \theta-\sin ^{2} \theta\right) \underline{i}-\sin \theta\left(1+2 \cos ^{2} \theta\right) \perp\right] \\
& \therefore F_{12}=-\frac{G b^{2}}{2 \pi(1-2) r}\left[\cos \theta\left(\cos ^{2} \theta-\sin ^{2} \theta\right) \underline{i}+\sin \theta\left(1+2 \cos ^{2} \theta\right) \underline{]}\right]
\end{aligned}
$$

This is Eq. (8.23) For $F_{21}$, remove minus sign.

b)

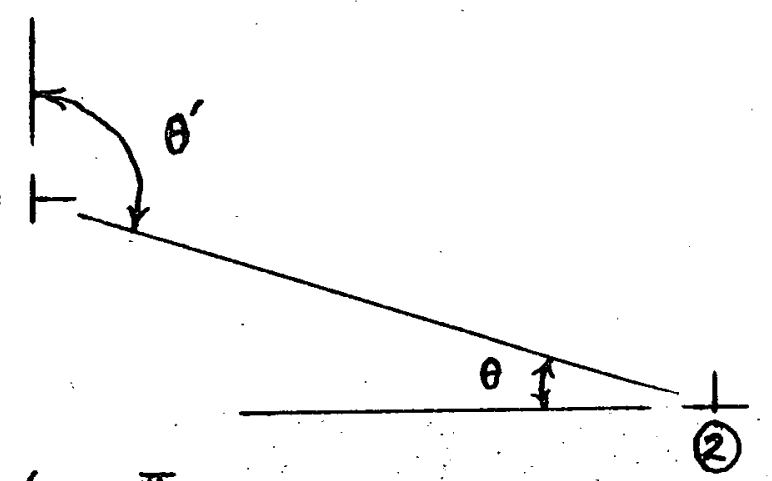

$$
\begin{aligned}
& \theta^{\prime}=\frac{\pi}{2}+\theta \\
& \sin \theta^{\prime}=\cos \theta, \quad \cos \theta^{\prime}=-\sin \theta \\
& \left(\sigma_{r r}\right)_{1}=\frac{K}{r} \cos \theta, \quad\left(\sigma_{r \theta}\right)_{1}=\frac{k}{r} \sin \theta \\
& \left(\sigma_{x y}\right)_{1}=\frac{k}{r}\left(\cos ^{2} \theta-\sin ^{2} \theta\right) \sin \theta \\
& \left(\sigma_{x x}\right)_{1}=\frac{k}{r}\left(\cos \theta-2 \sin ^{2} \theta \cos \theta\right) \\
& \therefore F_{12}=\frac{K b}{r}\left[-\sin \theta\left(\cos ^{2} \theta-\sin ^{2} \theta\right) \underline{i}+\cos \theta\left(1-2 \sin ^{2} \theta\right) \underline{\jmath}\right]
\end{aligned}
$$


164

but $\quad 1-2 \sin ^{2} \theta=\cos ^{2} \theta-\sin ^{2} \theta$

$$
F_{12}=-\frac{G b^{2}}{2 \pi(1-2)^{r}}\left(\cos ^{2} \theta-\sin ^{2} \theta\right)(\sin \theta \underline{i}-\cos \theta \underline{j})
$$

For $F_{21}$, remove minus sign. 


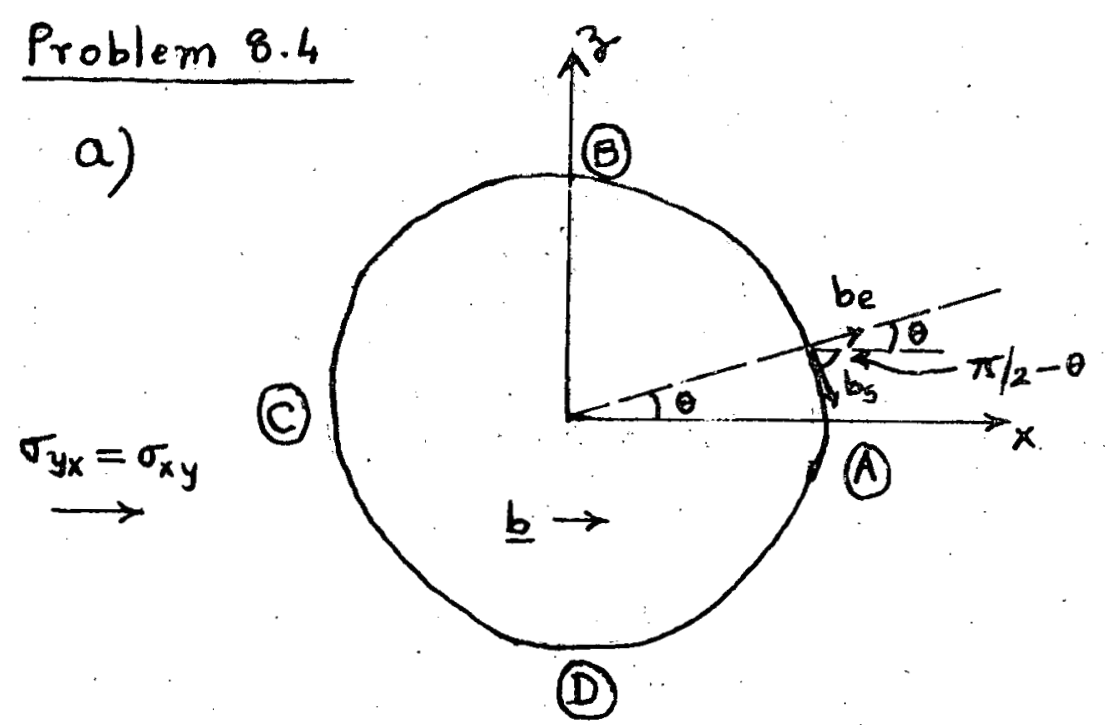

For $\underline{b}$ to be independent of $\theta$ :

$$
\begin{aligned}
& b_{e} \cos \theta+b_{s} \cos (\pi / 2-\theta)=b \\
& b_{e} \sin \theta=b_{s} \sin (\pi / 2-\theta)
\end{aligned}
$$

Solving:

$$
\begin{aligned}
& b_{s}=b \sin \theta \\
& b_{e}=b \cos \theta
\end{aligned}
$$

At (A)

$$
\left.\begin{array}{l}
b_{e}=b \\
b_{s}=0
\end{array}\right\} \text { pure edge }
$$

At (C)

$$
\left.\begin{array}{l}
b_{e}=-b \\
b_{s}=0
\end{array}\right\} \begin{aligned}
& \text { pure edge, of opposite } \\
& \text { sign as at (A) }
\end{aligned}
$$

At (B)

$$
\left.\begin{array}{l}
b_{e}=0 \\
b_{s}=b
\end{array}\right\} \text { pure screw }
$$

At (D)

$$
\left.\begin{array}{l}
b_{e}=0 \\
b_{s}=-b
\end{array}\right\} \begin{aligned}
& \text { pure screw of opposite } \\
& \text { sign as at. (B) }
\end{aligned}
$$


166

b)

$$
\begin{gathered}
F_{e}=\text { Force on edge component (in } x \text {-direction) } \\
F_{e}=\sigma_{y x} b_{e} \quad-\left[E_{q \cdot}(8.13)\right]
\end{gathered}
$$

[Choose sign to give + force at (A)]

- $F_{s}=$ Force on screw component

$$
\text { (in z-direction) }
$$

Fig. (8.11): Shear stress in the direction of screw dislocation produces force at right angles to it. For geometry of circular loop, screw component lies along

$$
x \text {-axis, not } z \text {-axis. }
$$

$F=$ resultant force

$\theta^{\prime}=$ angle of resultant

(c)

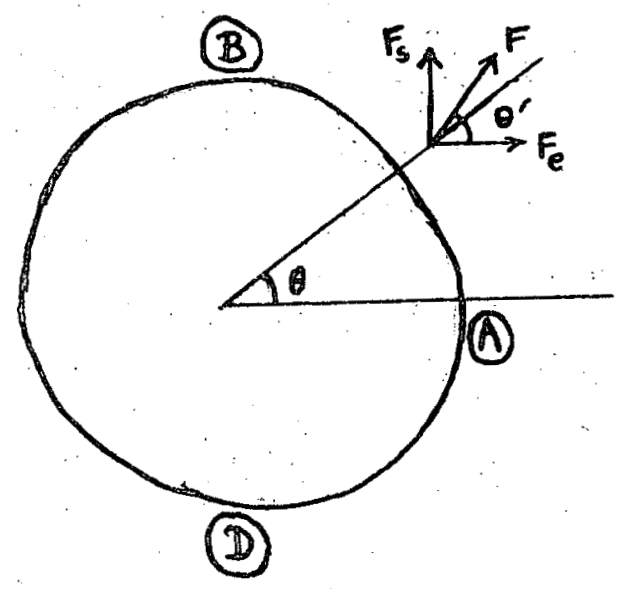
force from $x$-axis

$$
\tan \theta^{\prime}=\frac{F_{s}}{F_{e}}=\frac{b_{s}}{b_{e}}=\frac{\sin \theta}{\cos \theta}=\tan \theta
$$

$\therefore \theta^{\prime}=\theta$, or the resultant force is along radially outward.

$$
\begin{aligned}
F=\sqrt{F_{s}^{2}+F_{e}^{2}} & =\sigma_{y x} \sqrt{b_{e}^{2}+b_{s}^{2}} \\
& =\sigma_{y x b} \sqrt{\cos ^{2} \theta+\sin ^{2} \theta} \\
\therefore F & =\sigma_{y x} b
\end{aligned}
$$


167

Problem 8.5

a)

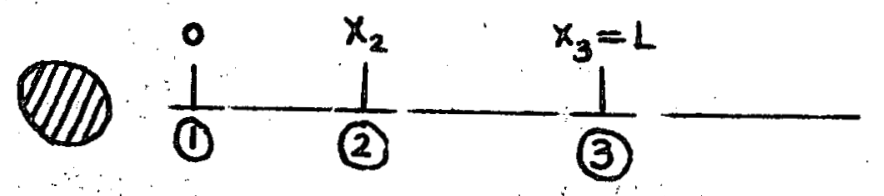

$n=3$

Let $\quad K^{\prime}=\frac{G b}{2 \pi(1-y) \sigma_{x y}}$

$$
x_{2}=d, x_{3}=L
$$

Eq. (8.38) for $i=2,3$ :

$$
\begin{aligned}
& \frac{d}{d}-\frac{1}{L-d}=\frac{1}{k^{\prime}} \quad \ldots(1) \\
& \frac{1}{L}+\frac{1}{L-d}=\frac{1}{K^{\prime}} \quad \ldots(2) \\
& \text { From (1) Let } D=d / k^{\prime} \\
& \mathscr{L}=L / K^{\prime} \\
& \therefore \frac{1}{D}-\frac{1}{L-D}=1---(3) \\
& \frac{1}{2}+\frac{1}{1-D}=1
\end{aligned}
$$

From (3)

$$
\mathcal{L}=\frac{2 D-D^{2}}{1-D}--(5)
$$

Substitute (s) into (4):

$$
\frac{1-D}{D(2-D)}+\frac{1-D}{D}=1
$$

or:

$$
2 D^{2}-6 D+3=0
$$


168

Solutions: $\quad \mathcal{D}=2.37, L=0.64$ [from (s)] and $D=0.64, L=2.37$

Reject the first set because $\mathscr{L}$ must be greater than $D$.

$$
\therefore D=0.64 \text { and } L=2.37 K^{\prime}
$$

From Eq.(8.39), for $n=3, L=6 K^{\prime}$

b)

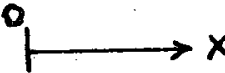

$$
n
$$

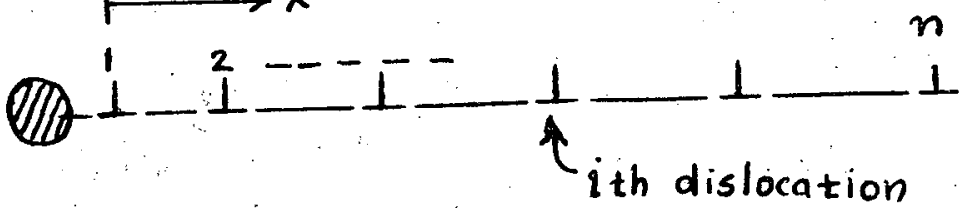

$$
\begin{aligned}
K^{\prime}= & \frac{G b}{2 \pi(1-\nu) \sigma_{x y}}, \text { units of } \mathrm{cm} \\
& K^{\prime} \sum_{\substack{j=1 \\
j \neq i}}^{n} \frac{1}{x_{i}-x_{j}}=1
\end{aligned}
$$

Continuous distribution:

$$
\begin{aligned}
f\left(x^{\prime}\right) d x^{\prime}=\text { number of dislocations in } x^{\prime} \text { to } x^{\prime}+d x^{\prime} \\
k^{\prime} \lim _{\epsilon \rightarrow 0}\left\{\int_{0}^{x-\epsilon} \frac{f\left(x^{\prime}\right) d x^{\prime}}{x-x^{\prime}}+\int_{x+\epsilon}^{L} \frac{f\left(x^{\prime}\right) d x^{\prime}}{x-x^{\prime}}\right\}=1-(2) \\
\int_{0}^{L} f(x) d x=n-(3)(f=0 \text { for } x>L)
\end{aligned}
$$

Solution is: $\quad f(x)=\frac{2 n}{\pi L}\left(\frac{L-x}{x}\right)^{1 / 2}$

and $L=2 n K^{\prime}$ 
169

Check integrals:

Let $x=L \sin ^{2} \theta$

$$
\frac{L-X}{X}=\frac{L-L \sin ^{2} \theta}{L \sin ^{2} \theta}=\frac{\cos ^{2} \theta}{\sin ^{2} \theta}
$$

Eq.(3) becomes,

with $d x=2 L \sin \theta \cos \theta d \theta$

and when $x=0, \theta=0$

$$
\begin{gathered}
\text { and } \quad x=L, \theta=\pi / 2 \\
\therefore \frac{2 n}{\pi L} \int_{0}^{\pi / 2} L \frac{\cos \theta}{\sin \theta}(2 \sin \theta \cos \theta) d \theta=\frac{4 n}{\pi} \int_{0}^{\pi / 2} \cos ^{2} \theta d \theta \\
=\frac{4 n}{\pi} \frac{\pi}{4}=n \quad(0 k)
\end{gathered}
$$

Eq. (2) gives with $x^{\prime}=L \sin ^{2} \theta$ and since $k^{\prime}=\frac{L}{2 n}$

$$
\begin{aligned}
& \frac{L}{2 n} \lim _{\epsilon \rightarrow 0}\left\{\int_{0}^{x-\epsilon} \frac{f\left(x^{\prime}\right) d x^{\prime}}{x-x^{\prime}}+\int_{x+\epsilon}^{L} \frac{f\left(x^{\prime}\right) d x^{\prime}}{x-x^{\prime}}\right\} \\
& =\frac{2}{\pi} \lim _{\delta \rightarrow 0}\left\{\int_{0}^{\sin \frac{\sqrt{x}-\delta}{L}} \frac{\cos ^{2} \theta d \theta}{\frac{x}{L}-\sin ^{2} \theta}+\int_{\sin ^{-1} \frac{\pi}{L}+\sigma^{2}}^{\frac{\cos ^{2} \theta d \theta}{L}-\sin ^{2} \theta}\right\}
\end{aligned}
$$

The term in \{\} above can be shown to be equal to $\pi / 2$ Thus eq (2) is satisfied also.

Evaluation of the integral in \{\} (Courtesy of HAmster)

Let

$$
I \equiv \lim _{\delta \rightarrow 0}\left[\int_{0}^{\eta-\delta} \frac{\cos ^{2} \theta d \theta}{\sin ^{2} \eta-\sin ^{2} \theta}+\int_{\eta+\delta}^{\pi / 2} \frac{\cos ^{2} \theta d \theta}{\sin ^{2} \eta-\sin ^{2} \theta}\right]
$$


170

where $\frac{x}{L}=\sin ^{2} \eta$ or $\eta=\sin ^{-1} \sqrt{\frac{x}{L}}$

This is known as the "Cauchy Principal Value" of the integral (sometimes called the "Principal Part" of the integral) and written.

$$
I=p \int_{0}^{\pi / 2} \frac{\cos ^{2} \theta d \theta}{\sin ^{2} \eta-\sin ^{2} \theta}
$$

Without the $P$ (ie. with $\delta=0$ ) the integral does not exist (even though its "Principal Part" does.)

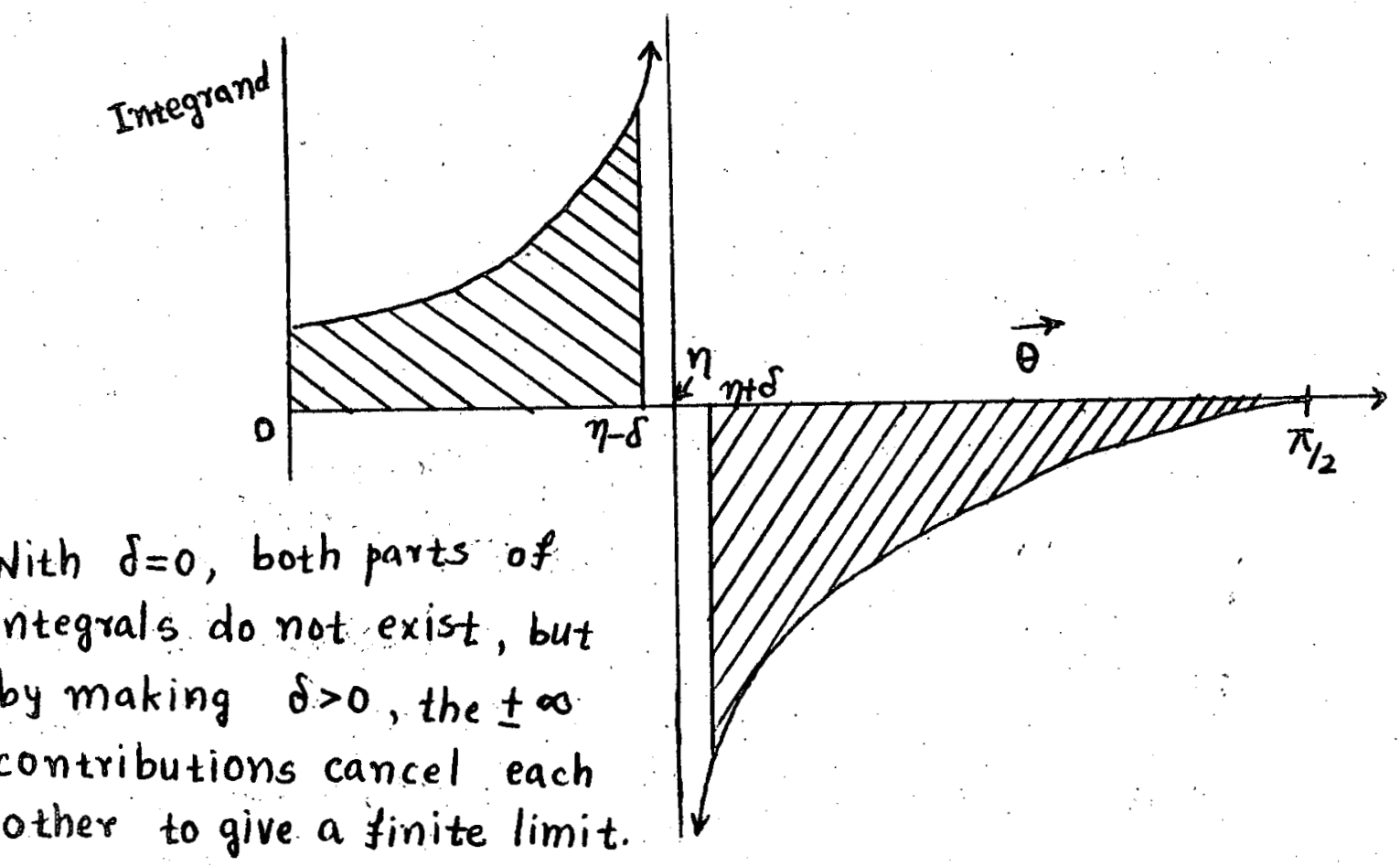


171

Since there is no closed contour, the contour integration cant help. The indefinite integral must be sought.

$$
\begin{aligned}
\int \frac{\cos ^{2} \theta d \theta}{\sin ^{2} \eta-\sin ^{2} \theta} & \equiv \int \frac{\left(\sin ^{2} \eta-\sin ^{2} \theta\right)+1-\sin ^{2} \eta}{\sin ^{2} \eta-\sin ^{2} \theta} d \theta \\
& =\theta+\cos ^{2} \eta \int \frac{d \theta}{\sin ^{2} \eta-\sin ^{2} \theta}
\end{aligned}
$$

Consider

$$
\begin{aligned}
& \left.\theta]_{\theta=0}^{\eta-\delta}+\theta\right]_{\eta+\delta}^{\pi / 2}=\eta-\delta+\pi / 2-(\eta+\delta) \\
& =\pi / 2-2 \delta \underset{\delta \rightarrow 0}{\underset{\longrightarrow}{\longrightarrow}} \pi / 2
\end{aligned}
$$

Thus, $\quad I=\pi / 2+J \cos ^{2} \eta$

where $J=P \int_{0}^{\pi / 2} \frac{d \theta}{\sin ^{2} \eta-\sin ^{2} \theta}$.

See Dwight P.94: $b=1 ; a=\sin \eta$

$$
\begin{aligned}
& \therefore b^{2}>a^{2} \& \sqrt{b^{2}-a^{2}}=\cos \eta \\
& \left.\therefore \int \frac{d \theta}{\sin ^{2} \eta-\sin ^{2} \theta}=\frac{1}{2} \frac{1}{\cos \eta \sin \eta}|| \frac{\cot \eta \tan \theta+\sin \eta]}{\cos \eta \tan \theta-\sin \eta}\right] \mid \\
& J=\lim _{\delta \rightarrow 0} \frac{1}{2} \frac{1}{\cos \eta \sin \eta} \ln \left|\frac{[\theta=\eta-\delta][\theta=\pi / 2]}{[\theta=0][\theta=\eta+\delta]}\right|
\end{aligned}
$$


172

$$
\begin{aligned}
\therefore \cos ^{2} \eta J & =\lim _{\delta \rightarrow 0} \frac{1}{2} \cot \eta \ln \left|\frac{\left[\frac{\cos \eta \tan (\eta-\delta)+\sin \eta}{\cos \eta \tan (\eta-\delta)-\sin \eta}\right][1]}{[-1]\left[\frac{\cos \eta \tan (\eta+\delta)+\sin \eta}{\cos \eta \tan (\eta+\delta)-\sin \eta}\right]}\right| \\
& =\frac{1}{2} \cot \eta \ln 1=0 \\
\therefore \quad I & =\pi / 2+J \cos ^{2} \eta \\
& =\pi / 2
\end{aligned}
$$


173

Problem 8.6

a)

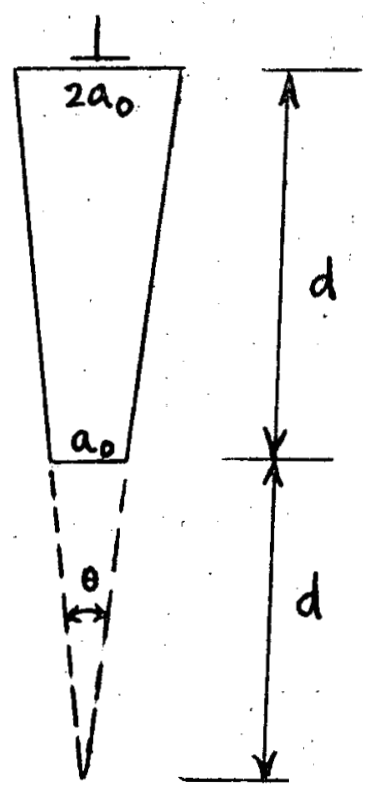

$$
\begin{gathered}
\sin \frac{\theta}{2}=\frac{a_{0}}{2 d} \cong \frac{\theta}{2} \\
\therefore \theta=\frac{a_{0}}{d}
\end{gathered}
$$

b) Energy/unit length of edge dislocation $\perp$ to the diagram $=\tau_{d}$

Each edge dislocation is associated with a distance $d$ in the direction along the grain boundary in the diagram.

$$
\begin{aligned}
& \gamma_{g b}=\frac{\tau_{d}}{d}=\frac{G b^{2}}{4 \pi(1-\nu)} \ln \left(\frac{d}{b}\right) / d \\
& d=\frac{a_{0}}{\theta} \cong \frac{b}{\theta} \quad \text { or } \frac{b}{d}=\theta \\
\therefore & \gamma_{g b}=-\frac{G b}{4 \pi(1-\nu)} \theta \ln \theta
\end{aligned}
$$




\section{CHAPTER 9}

Problem 9.1

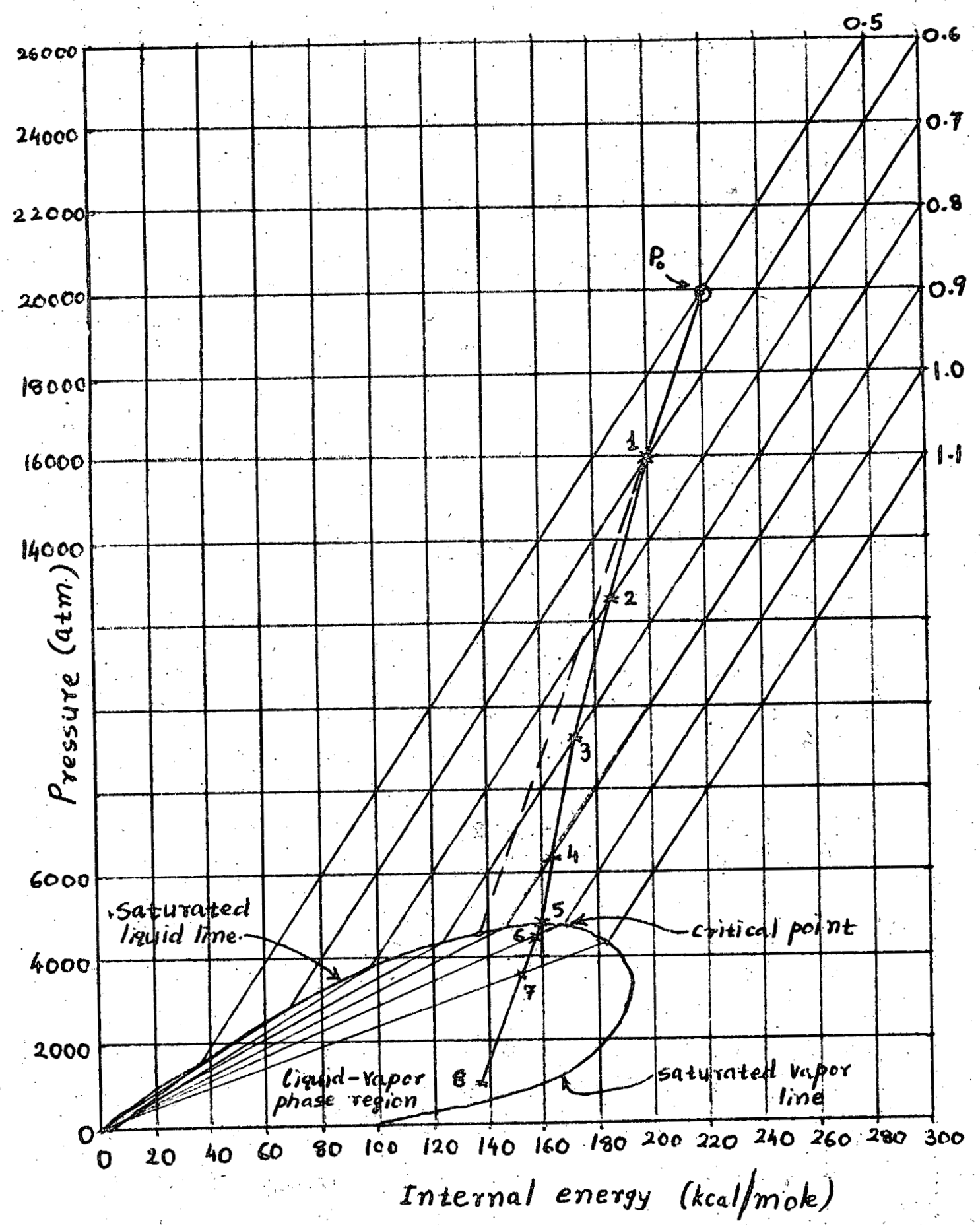


175

From fundamental thermodynamic relation,

$$
d u=T d s-p d v
$$

For an isentropic process, $d s=0$

$$
\begin{gathered}
\therefore d u=-p d v \quad d v=v_{c} d v_{r} \\
\therefore \Delta u=-p \Delta v=-p v_{c} \Delta v_{r} .
\end{gathered}
$$

(a)

$$
\begin{aligned}
\Delta u_{0} & =-\frac{20000 \times 1.01 \times 10^{6} \times 414 \times 0.1}{4.186 \times 10^{10}} \cong-2000 \times 10^{-3} \\
& \cong-20 \mathrm{kcal} / \mathrm{mole}=-83.72 \mathrm{~kJ} / \mathrm{mole}
\end{aligned}
$$

The curve $P_{0}-1$ plotted on the diagram shows this process.

(6) The path of expansion can be obtained by the following iterative scheme

$$
\begin{aligned}
& u_{1}=u_{0}+\Delta u_{0}=220-20=200 \mathrm{kcal} / \mathrm{mole} . \\
& \text { From } v_{r}=0.6, u_{1}=200 \mathrm{kca} / \mathrm{mol},
\end{aligned}
$$

From $v_{r}=0.6, u_{1}=200 \mathrm{kcal} / \mathrm{mole}$, find

$$
\begin{aligned}
& P_{1}=16000 \text { atm } \\
& \Delta u_{1} \cong-16000 \times 10^{-3} \cong-16 \mathrm{kcal} / \mathrm{mole} \\
& u_{2}=u_{1}+\Delta u_{1}=200-16=184 \mathrm{kcal} / \mathrm{mole} \text {. } \\
& v_{r}=0.7, u_{2}=184 \mathrm{kcal} / \mathrm{mole} \text {, find } P_{2}=12500 \mathrm{~atm} \\
& \Delta u_{2} \cong-12500 \times 10^{-3}=-12.5 \mathrm{kcall} / \mathrm{mole}
\end{aligned}
$$




$$
\begin{aligned}
& u_{3}=u_{2}+\Delta u_{2}=184-12.5=171.5 \mathrm{kcal} / \mathrm{mole} \\
& P_{3}=9200 \mathrm{~atm}, \Delta u_{3}=-9.2 \mathrm{kcal} / \mathrm{mole} \\
& u_{4}=u_{3}+\Delta u_{3}=171.5-9.2=162.3 \mathrm{kcal} / \mathrm{mole} \\
& P_{4}=6200 \mathrm{~atm}, \Delta u_{4}=-6.2 \mathrm{kcal} / \mathrm{mole} \\
& u_{5}=158 \mathrm{kcal} / \mathrm{mole}, v_{r} \cong 0.97, P_{5}=4600 \mathrm{~atm} \\
& u_{6}=157.3 \mathrm{kcal} / \mathrm{mole}, \quad v_{r}=1.0, P_{6}=4400 \mathrm{~atm} \\
& u_{7}=154.9 \mathrm{kcal} / \mathrm{mole}, \quad v_{r}=1.1, P_{7}=3400 \mathrm{~atm}
\end{aligned}
$$

(1) Because along the isentropic path, $d u=-p d y$ and $p$ decreases as the fuel expands. Thus although $\Delta v$ is uniformly changed, $\Delta u$ keeps decreasing as the state of the material moves down the path. The dotted line is the straight tine extension of the line segment $P_{0}-1$.

(ii) The discontinuity occurs at the saturated liquid line. After this the fluid is in the two phase region In this region $d v$ is much larger, due to vaporization, so that the slope is different from outside.

(c) At $p=1000 \mathrm{~atm}$, the fluid is in a liquid-gas. phase. 
177

Problem 9.2

Neglecting electronic excitation, the specific heat at constant volume of gaseous $\mathrm{PuO}_{2}$ at high temperature will have contributions from translation, rotation and vibration of the molecule.

Linear Molecule.

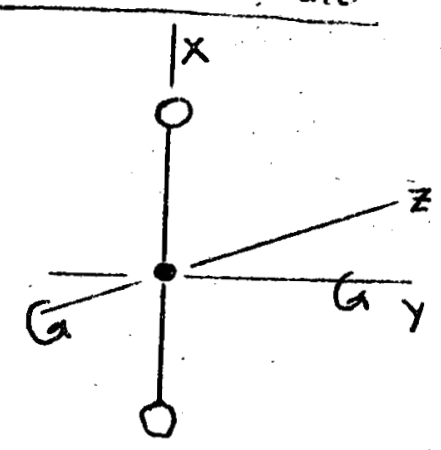

$$
\begin{aligned}
& \text { = Plutonium } \\
& 0=\text { Oxygen }
\end{aligned}
$$

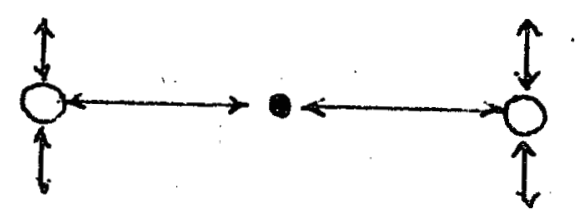

(a) Rotation

(b) Vibration

As in the case of $\mathrm{UO}_{2}$ there will be three translational modes giving $\frac{3}{2} R$, two rotational modes giving $2 \times \frac{1}{2} R$ and four vibrational modes giving

$$
\begin{aligned}
4 \times\left(2 \times \frac{1}{2} R\right) \quad \therefore \text { Molar heat capacity } \begin{aligned}
C_{y} & =\left(\frac{3}{2}+1+4\right) R \\
& =\frac{13}{2} R
\end{aligned} \\
\text { Nonlinear Molecule }
\end{aligned}
$$

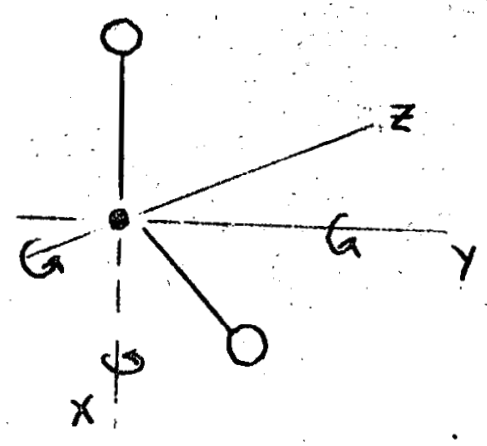

Rotation 
178

For a nonlinear molecule, there will be an additional rotational mode about $x$-axis as shown. However for the nonlinear molecule there will be only $3 N-6=3 \times 3-6=3$ vibrational modes as against $3 N-5=4$ for the linear molecule. (see Thermodynamics by

Lewis, Randall, Pitzer and

Brewer P. 59-60)

Therefore we have $\frac{3}{2} \&$ from translation, $\frac{3}{2} R$. from rotation and $3 \times\left(2 \times \frac{1}{2} R\right)$ from Vibration.

$$
\begin{aligned}
\text { Molar heat capacity }=C_{V} & =\left(\frac{3}{2}+\frac{3}{2}+3\right) R \\
& =6 R
\end{aligned}
$$


179

Problem 9.3

The vapor pressure of the solid is

$$
-R T \ln P_{S}^{\circ}=\Delta H_{s}-T \Delta S_{s}
$$

The vapor pressure of the liquid is

$$
-R T \ln P_{l}^{0}=\Delta H_{l}-T \Delta S_{l}
$$

At the melting point $T=T_{f}$, and the solid and liquid phases are in equilibrium,

$$
\begin{gathered}
G_{l}=G_{s} \\
H_{l}-T_{f} S_{l}=H_{s}-T_{f} S_{s} \\
\therefore H_{l}-H_{s}=T_{f}\left(S_{l}-S_{s}\right) \\
\therefore \Delta S_{f}=S_{l}-S_{s}=\frac{\Delta H_{f}}{T_{f}} \\
\therefore \Delta S_{s}=S_{g}-S_{s}=S_{g}-S_{l}+S_{l}-S_{s} \\
\therefore \Delta S_{l}=\Delta S_{s}-\frac{\Delta H_{f}}{T_{f}}
\end{gathered}
$$

At the triple point the heat of sublimation equals the sum of the heats of fusion and the heat of vaporization:$$
\ell_{13}=l_{12}+l_{23}
$$$$
\text { or } \Delta H_{s}=\Delta H_{t}+\Delta H_{f} \quad \therefore \Delta H_{l}=\Delta H_{s}-\Delta H_{f}-(4)
$$ 
180

If the enthalpy differences are independent of $T$, then eq. (4) holds at all temperatures

Substituting eqs. (3) and (4) into (2) and using (1) for $\Delta H_{s}$ we get,

$$
\begin{aligned}
-R T \ln P_{l}^{0} & =\Delta H_{s}-\Delta H_{f}-T \Delta s_{s}+\frac{T}{T_{f}} \Delta H_{f} \\
& =-R T \ln P_{s}^{0}-\Delta H_{f}\left(1-\frac{T}{T_{f}}\right) \\
\therefore R T \ln \frac{P_{l}^{0}}{P_{s}^{3}}=\Delta H_{f}\left(1-\frac{T}{T_{f}}\right) & \\
\therefore P_{l}^{0} & =P_{s}^{0} \exp \left[\frac{\Delta H_{f}}{R}\left(\frac{1}{T}-\frac{1}{T_{f}}\right)\right]
\end{aligned}
$$

(for $T>T_{f}, P_{s}^{0}(T)$ is the extrapolated vapor pressure curve over the solid)

Start from the ciausius-Clapeyron equation for the solid-liquid equilibrium:

$$
\begin{aligned}
& \frac{d p}{d T_{f}}=\frac{\Delta H_{f}}{T_{f} \Delta V_{f}} \\
& \therefore \frac{d T_{f}}{T_{f}}=\frac{\Delta v_{f}}{\Delta H_{f}} d_{p}
\end{aligned}
$$

Integrating:

$$
\ln \frac{T_{f 2}}{T_{f 1}} \cong \frac{\Delta v_{f}}{\Delta H_{f}}\left(p_{2}-p_{1}\right)
$$


where $h_{1}$ is 1 atmosphere and $T_{f 1}$ is the melting point at $1 \mathrm{~atm}$

$$
\text { or } T_{f_{2}}=T_{f_{1}} \exp \left[\frac{\Delta v_{f}}{\Delta H_{f}}\left(p_{2}-p_{1}\right)\right]
$$

Therefore for an increasc in the applied pressure

$$
\left(p_{2}-p_{1}\right)>0 \text {, there are two cases for the }
$$
melting point temperature:

i) $T_{f 2}>T_{f 1}$, if $\Delta V_{f}>0$ (i.e. if the material expands on melting)

ii) $T_{f 2}<T_{f 1}$, if $\Delta v_{f}<0$ (i.e. if the material expands with solidification) 
182

Problem 9.4

(a) From Fig. $9.4, p \cong 0.04 \mathrm{~atm}$ at $T=3100^{\circ} \mathrm{K}$.

$$
\begin{aligned}
& v_{g}=\frac{R T}{P_{g}}=82 \frac{\mathrm{cm}^{3}-\mathrm{atm}}{\text { mole } \mathrm{oK}} \times \frac{3100^{\circ} \mathrm{K}}{04 \mathrm{~atm}} \\
& =6 \times 10^{6} \mathrm{~cm}^{3} / \mathrm{mole} \\
& v_{t}=\frac{(238+32) \mathrm{gm} / \mathrm{mole}}{s_{\mathrm{l}} \mathrm{gm} / \mathrm{cm}^{3}}
\end{aligned}
$$

From Fig. $9.3 \quad \rho_{i}=8.8 \mathrm{gm} / \mathrm{cm}^{3}$

$$
\begin{aligned}
& \therefore v_{l}=\frac{270}{8.8} \cong 30 \mathrm{~cm}^{3} / \mathrm{mole} \\
& v=v_{l} x_{l}+\left(1-x_{l}\right) v_{g}=60 \mathrm{~cm}^{3} / \text { mole }
\end{aligned}
$$

solving for $x_{t}$ gives,

$$
\begin{aligned}
\underset{\text { fraction }}{\operatorname{mole}} x_{t}=\frac{1-v / v_{g}}{1-v_{i} / v_{g}}=1-\frac{v-v_{l}}{v_{g}} & =1-\frac{30}{6 \times 10^{6}} \\
= & 1-5 \times 10^{-6}
\end{aligned}
$$

(b)

volume

fraction of liquid $=\frac{x_{e} v_{l}}{v} \cong \frac{v_{l}}{v}=\frac{1}{2}$

$$
\begin{aligned}
U & =x_{l} U_{l}+\left(1-x_{l}\right) U_{g} \\
\Delta H_{v a p}= & \text { latent heat of vaporization }=119.1 \mathrm{kcal} / \text { male } \\
& =498.6 \mathrm{~kJ} / \mathrm{male}
\end{aligned}
$$


183

$$
\Delta H_{\text {rap }}=H_{g}-H_{l}=U_{g}-U_{l}+p\left(v_{g}-v_{l}\right)
$$

$v_{\ell}$ is negligible compared to $v_{j}$ :

$$
\begin{aligned}
\therefore \Delta H_{v a p} & =U_{g}-U_{l}+p v_{g}=U_{g}-U_{l}+R T \\
\text { or } \quad U_{g}-U_{l}=\Delta H_{v a p}-R T & =119.1-1.98 \times 10^{-3} \times 3100 \\
\therefore \Delta U_{v a p}=U_{g}-U_{l} & =119.1-6.1=113 \mathrm{kcal} / \mathrm{mole} \\
& =472.3 \mathrm{~kJ} / \mathrm{mole}
\end{aligned}
$$

With reference state as ideal gas at $0^{\circ} \mathrm{K}$.

$$
\begin{aligned}
& U_{g}=\frac{13}{2} R T=40 \mathrm{kcal} / \text { mole } \\
= & 167.2 \mathrm{~kJ} / \text { mole } \\
U= & x_{l} U_{l}+\left(1-x_{l}\right) U_{g} \\
\cong & U_{l}+5 \times 10^{-6} U_{g} \cong U_{l} \quad\left(\begin{array}{l}
\text { (as the second term } \\
\text { is negligible) }
\end{array}\right.
\end{aligned}
$$

(c) From Eq. (9.35)

$$
\begin{aligned}
& \left(\frac{d U}{d T}\right)_{s} \cong\left(\frac{d V_{l}}{d T}\right)_{s}=C_{p l}-T\left(\frac{d V_{l}}{d T}\right)_{p}\left(\frac{d p}{d T}\right)-p\left(\frac{d V_{l}}{d T}\right)_{s} \\
& \alpha_{l}=\text { liquid thermal coefficient of expansion } \\
& \alpha_{l}=\frac{1}{v_{l}}\left(\frac{d V_{l}}{d T}\right)_{p}=-\frac{1}{S_{l}}\left(\frac{d S_{l}}{d T}\right)_{p} \quad\left(\rho_{l}=\text { liquid density }\right)
\end{aligned}
$$


184

From Fig 9.3 ,

$$
\alpha_{l}=7.5 \times 10^{-5} \mathrm{ok}^{-1}
$$

Assume

$$
\begin{aligned}
\left(\frac{d V_{l}}{d T}\right)_{p} & \cong\left(\frac{d V_{l}}{d T}\right)_{\text {saturation }} \quad(\text { sec Fig. } 0 \cdot 1) \\
& =\alpha_{l} V_{l}
\end{aligned}
$$

$$
\begin{aligned}
& \left(\frac{d U_{l}}{d T}\right)_{s}=C_{p l}-\left[T \frac{d p}{d T}+p\right]\left(\frac{d V_{l}}{d T}\right)_{S} \\
& =c_{p l}-\left[v_{g} T \frac{d p}{d T}+p v_{g}\right] \alpha_{l}\left(\frac{v_{l}}{v_{i j}}\right) \\
& P V_{g}=R T \quad \therefore \quad V_{g}=\frac{R T}{P} \\
& \therefore\left[V_{g} T \frac{d p}{d T}+p V_{g}\right]=\frac{R T^{2}}{p} \frac{d p}{d T}+R T \\
& =-R \frac{d \ln _{\mathrm{P}} P}{d(1 / T)}+R T=-\Delta H_{v_{\mathrm{ap}}}+R T \\
& =-\Delta U_{v_{n p}} \\
& \therefore\left(\frac{d U_{l}}{d T}\right)_{s}=c_{p l}+\Delta U_{\text {vp }} \alpha_{l} \frac{U_{l}}{V_{j}} \\
& =C_{p l}\left[1+\frac{\Delta U_{v C_{p} p}}{C_{p l}} \alpha_{l} \frac{V_{l}}{V_{q}}\right] \\
& \frac{\Delta U_{\text {hap }}}{C_{p t}} \alpha_{l} \frac{V_{p}}{v_{g}}=\frac{113 \times 10^{3} \mathrm{cal} / \mathrm{mcle}}{18 \mathrm{cal} / \mathrm{mdle} \cdot 0 \mathrm{~K}} \times 7.5 \times 10^{-5} \times \frac{30}{6 \times 10^{6}} \cong 2.35 \times 10^{-6} \\
& \therefore\left(\frac{d U_{t}}{d T}\right)_{s} \cong C_{p t}
\end{aligned}
$$


185

Problem 9.5

From $E_{q} \cdot(9.37)$ :

$$
\begin{aligned}
& \frac{1}{\gamma}=\frac{1}{v}\left(\frac{\partial U}{\partial p}\right)_{V} \\
& \text { But }\left(\frac{\partial U}{\partial p}\right)_{V}=\left(\frac{\partial U}{\partial T}\right)_{V}\left(\frac{\partial T}{\partial p}\right)_{V} \\
& \text { and }\left(\frac{\partial U}{\partial T}\right)_{V}=C_{V},\left(\frac{\partial T}{\partial p}\right)_{V}=\frac{\beta}{\alpha} \\
& \text { (from problem } 1.5 \mathrm{~d} \text { ) } \\
& \therefore \quad \frac{1}{\gamma}=\frac{1}{v} c_{v} \frac{\beta}{\alpha} \\
& \text { or } \gamma=\frac{\alpha V}{\beta C_{V}}
\end{aligned}
$$


186

CHAPTER 10

Problem 10.1

$$
\begin{aligned}
& P_{u}^{239}+n \stackrel{\sigma_{P_{u}}}{\longrightarrow} 2 n+\text { F.P. (neglect capture } \\
& \text { by } P_{u}^{2.33} \text { to for } m \\
& P_{u}^{240} ; P_{u}^{241} \text { etc.) } \\
& U^{239}+n \stackrel{\sigma_{u}^{f}}{\longrightarrow} 2 n+\text { FP. } \\
& U^{238}+n \stackrel{\alpha_{v} \sigma_{v}^{f}}{\longrightarrow} U^{239} \stackrel{\bar{\beta}}{\longrightarrow} N_{p}^{233} \stackrel{\bar{\beta}}{\longrightarrow} P_{u}^{239}
\end{aligned}
$$

where $\alpha_{v} \sigma_{u}^{f}$ is the capture cross-section of $U^{238}$

Nuclide Balances

for Pu:

$$
\frac{d N_{p_{u}}}{d t}=-\sigma_{P_{u}} N_{P_{u}} \phi+\alpha_{v} \sigma_{u}^{f} N_{u} \phi
$$

for $u^{238}$ :

$$
\frac{d N_{u}}{d t}=-\left(1+\alpha_{u}\right) \sigma_{u}^{j} N_{u} \phi
$$

for F.P. pairs: $\frac{d N_{F . P .}}{d t}=\sigma_{P_{u}} N_{P_{u}} \phi+\sigma_{u}^{*} N_{U} \phi$

Definition of Burnup : $\quad \beta \equiv \frac{N_{F P}}{N_{4}^{0}+N_{P_{u}^{0}}^{0}}$

Combine eq. (3) and (4)

$$
\frac{d \beta}{d t}=\frac{\sigma_{p_{u}} N_{R_{u}}+\sigma_{u}^{f} N_{u}}{N_{u}^{0}+N_{P_{u}}^{0}} \phi
$$

Transform the time variable to the burnup variable. in eq (1) and (2) by use of eq. (5),

3 
187

ie. $\frac{d}{d \beta}=\frac{d}{d t} \frac{d t}{d \beta}$ this gives,

$$
\frac{d N_{P_{u}}}{d \beta}=\frac{d N_{P_{u}} / d t_{i}}{d \beta / d t}=\frac{-\sigma_{P_{u}} N_{P_{u}}+\alpha_{u} \sigma_{u}^{f} N_{u}}{\sigma_{u} N_{P_{u}}+\sigma_{v}^{f} N_{U}}\left(N_{u}^{0}+N_{P_{u}}^{0}\right)
$$

and $\frac{d N_{v}}{d \beta}=-\frac{\left(1+\alpha_{u}\right) \sigma_{u}^{f} N_{u}}{\sigma_{R_{u}} N_{P_{u}}+\sigma_{u}^{f} N_{u}}\left(N_{u}^{0}+N_{P_{u}}^{0}\right)$

Solve eq. (6) and (7) subject to $N_{p_{v}}(0)=N_{P_{u}}^{0}$ and

$$
N_{u}(0)=N_{u}^{0}
$$

Approximate Solution

Rewrite (6) and (7) as,

$$
\begin{aligned}
& \frac{d N_{p_{u}}}{d \beta}=\frac{-1+\alpha_{u}\left(\frac{\sigma_{u}^{t} N_{u}}{\sigma_{r_{u}} N_{u}}\right)}{1+\left(\frac{\sigma_{v}^{t} N_{u}}{\sigma_{R_{i}} N_{p_{u}}}\right)}\left(N_{v}^{0}+N_{p_{u}}^{c}\right) \\
& \frac{d N_{u}}{d \beta}=-\frac{(1+\alpha u)\left(\frac{\sigma_{u}^{f} N_{u}}{\sigma_{p_{u}} N_{R_{u}}}\right)}{1+\left(\frac{\sigma_{v}^{f} N_{u}}{\sigma_{u} N_{p_{v}}}\right)}\left(N_{u}^{0}+P_{u}^{0}\right)
\end{aligned}
$$

The conversion ratio is:

$$
c=\frac{\alpha_{u} \sigma_{u}^{f} N_{u} \phi}{\sigma_{P_{u}} N_{P_{u}} \phi}=\frac{\alpha_{u} \sigma_{u}^{f} N_{u}}{\sigma_{P_{u}} N_{P_{u}}}
$$

Then eq (8) and (9) become,

$$
\frac{d N_{P_{u}}}{d \beta}=\frac{-(1-c)\left(N_{u}^{c}+N_{P_{u}}^{0}\right)}{1+\left(c \beta_{u}\right)}
$$


188

$$
\frac{d N_{u}}{d \beta}=-\left(\frac{1+\alpha_{u}}{x_{u}}\right) c \frac{\left(N_{u}^{0}+N_{p_{u}}^{c}\right)}{1+\left(c \alpha_{u}\right)}
$$

The approximation consists of setting $C \cong$ constant, independent of burnup for the purpose of solving ens. (11) and (12).

This gives:

and

$$
\begin{aligned}
& N_{P_{u}}=N_{P_{u}}^{c}-\left(\frac{1-c}{1+c \alpha_{u}}\right)\left(N_{u}^{c}+N_{P_{u}}^{c}\right) \beta \\
& N_{u}=N_{u}^{c}-\left[\frac{\left(\frac{1+\alpha_{u}}{x_{u}}\right) c}{1+c / \alpha_{u}}\right]\left(N_{u}^{c}+N_{P_{u}}^{c}\right) \beta
\end{aligned}
$$

The plutonium fraction is:

$$
q=\frac{N_{P_{u}}}{N_{P_{u}}+N_{u}}
$$

Substituting ens (13) and (14) into eq. (15) gives,

$$
q=\frac{q_{0}-\left(\frac{1-c}{1+c \alpha_{i}}\right) \beta}{1-\beta}
$$


189

Problem 10.2

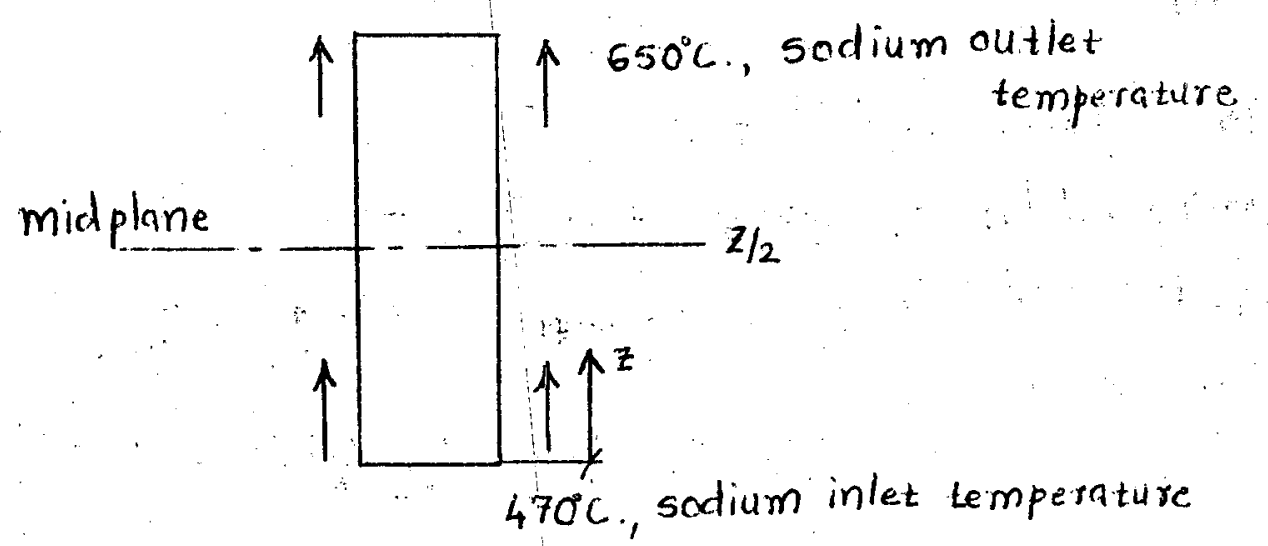

$$
\begin{aligned}
T_{\text {Na }}=\begin{array}{c}
\text { sodium temperature at } \\
\text { mid plane }
\end{array} & =470+\frac{1}{2}(650-470) \\
& =560^{\circ} \mathrm{C}
\end{aligned}
$$

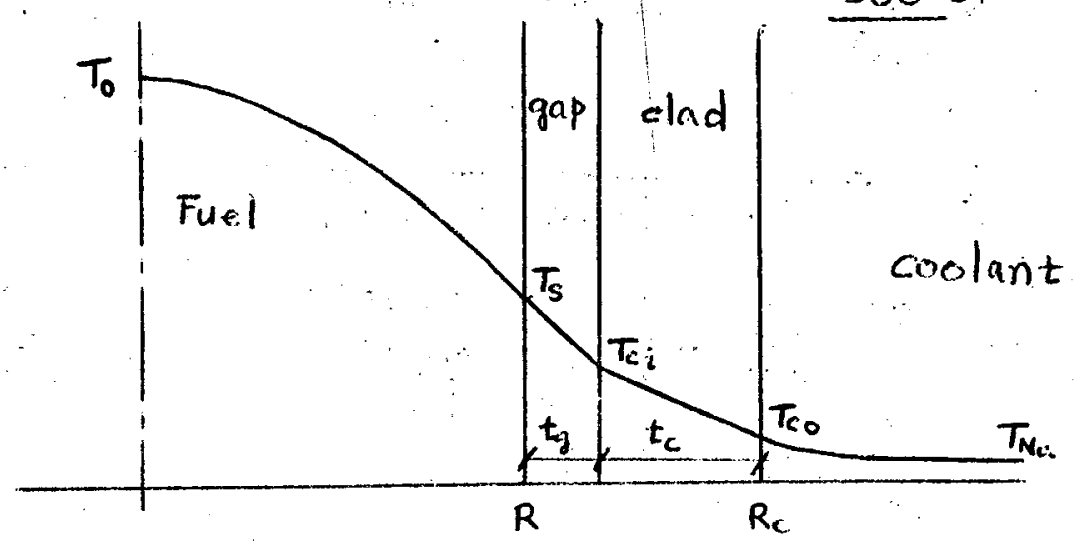

Sodium Film Temperature Drop:-

$$
\begin{aligned}
& q_{N_{a}}=\text { heat flux from clad surface to coolant } \\
& q_{N_{a}}=\frac{P}{2 \pi R_{c}}=h_{N_{a}}\left(T_{c_{a}}-T_{N_{a}}\right) \\
\therefore \quad & T_{c_{c}}-T_{N_{a}}=\frac{P}{2 \pi R_{c} h_{N_{a}}}=\frac{550}{2 \pi \times 0.315 \times 12}=23^{\circ} \mathrm{C}
\end{aligned}
$$

(since $\theta=550$ watts $/ \mathrm{cm}, 2 R_{c}=0.63 \mathrm{~cm}$.

and $h_{N_{a}}=12$ watts $/ \mathrm{cm}^{2}-{ }^{\circ} \mathrm{C}$.) 
190

$$
\begin{aligned}
\therefore T_{c o} & =T_{\mathrm{Na}}+23^{\circ} \mathrm{C} \\
& =560+23=583^{\circ} \mathrm{C}
\end{aligned}
$$

Temperature Drop through cladding :-

Average heat flux through cladding $=q_{c}$

$$
q_{c}=\frac{P}{2 \pi\left(R_{c}-\frac{1}{2} t_{c}\right)}=\frac{k_{c}}{t_{c}}\left(T_{c l}-T_{c c}\right)
$$

For stainless steel, $k_{c}=0.22$ watts $/ \mathrm{cm}^{\circ}{ }^{\circ} \mathrm{C}$.

$$
\begin{aligned}
& \frac{k_{c}}{t_{c}}=\frac{22 \times 10^{-2}}{4 \times 10^{-2}}=5.5 \text { watts } / \mathrm{cm}^{2}-{ }^{\circ} \mathrm{C} . \\
& \therefore T_{c_{i}}-T_{c_{c}}=\frac{550}{2 \pi(0.315-0.02) \times 5.5}=54^{\circ} \mathrm{C} \\
& \therefore T_{c i}=637^{\circ} \mathrm{C} \\
& q_{f}=\text { surface heat flux from fuel } \\
& =\frac{P}{2 \pi R}
\end{aligned}
$$

From Eq. (10.63) $\quad \int_{T_{s}}^{T_{0}} k d T=\frac{P}{4 \pi}$

or. $\int_{0}^{T_{6}} k d T-\int_{0}^{T_{5}} k d T=\frac{P}{4 \pi}=\frac{550}{4 \pi}=43.8$ Watts $k \mathrm{~cm}$.

For the gas conductivity use Eq .(10.102),

$$
k_{g}=15.8 \times 10^{-6}(900)^{0.79}=3.4 \times 10^{-3} \frac{\text { watts }}{c_{m-x}}
$$

(Average temperature in the gas $=900^{\circ} \mathrm{K}$ ) 
191

Then $q_{f}=\frac{k_{g}}{t_{g}}\left(T_{s}-T_{c i}\right)=\frac{P}{2 \pi R}$

Gap Closure

Fuel thermal expansion $=\frac{\Delta R}{R}=\alpha_{F}\left(\bar{T}_{f}-T_{a}\right)$

$$
\left(T_{a}=\text { fabriantion temp }=25^{\circ} \mathrm{C}\right)
$$

clad thermal expansion $=\frac{\Delta R_{c}}{R_{c}}=\alpha_{c}\left(\bar{T}_{c}-T_{a}\right)$

Hot fuel radius $=R_{f}^{\text {hot }}=R_{f}^{\text {cold }}\left[1+\alpha_{F}\left(\bar{T}_{f}-T_{a}\right)\right]$

Hot clad inner radius $=R_{c}^{\text {hot }}-t_{c}^{\text {hot }}$

$$
R_{c i}^{\text {hot }}=R_{c}^{c c i d}\left[1+\alpha_{c}\left(\bar{T}_{c}-T_{a}\right)\right]-t_{c}^{\text {hot }}
$$

$\left.\begin{array}{rlrl}\text { gap width } & t_{g}^{\text {hot }}=R_{c_{i}}^{\text {hot }}-R_{f}^{\text {hot }} \\ \text { and. } & t_{g}^{\text {coil d }}=R_{c i}^{\text {cold }}-R_{f}^{\text {cold }}\end{array}\right\} \Rightarrow$

$$
t_{y}^{\text {hot }}-t_{g}^{\text {cold }}=R_{c i}^{\text {call }} \alpha_{c}\left(\overline{T_{c}}-T_{a}\right)-R_{f}^{\text {cold } \alpha_{f}}\left(\bar{T}_{f}-T_{a}\right)
$$

Inserting numbers,

$$
\begin{aligned}
& T_{c}=\frac{1}{2}(637+58)=610^{\circ} \mathrm{C},, T_{a}=25^{\circ} \mathrm{C} \\
& \alpha_{f} \cong 1.2 \times 10^{-5}{ }^{-1} \quad\left(F_{i g} 10.8\right) \\
& \alpha_{c} \cong 1.8 \times 10^{-5} C^{-1} \quad\left(F_{i g} \cdot 10.9\right) \\
& R_{f}^{\text {cold }}=0.315-0.04-0.001=0.268 \mathrm{~cm} \\
& R_{c i}^{\text {cold }}=0.315-0.04=0.275 \mathrm{~cm} \\
& \therefore t_{g}^{\text {hot }}-t_{g}^{\text {cold }}=0.275 \times 1.8 \times 10^{-5}(610-25) \\
& \quad-0.268 \times 1.2 \times 10^{-5}\left(T_{f}-T_{a}\right)
\end{aligned}
$$


192

$$
\therefore t_{g}^{\text {hot }}-t_{g}^{\text {cold }}=2.9 \times 10^{-3}-3.22 \times 10^{-6}\left(T_{f}-T_{h}\right)
$$

Iterative Procedure

$$
\text { Assume } t_{y}=t_{y}^{\text {cold }}=7 \times 10^{-3} \mathrm{~cm} \text {. }
$$

Then from eq. (3).

$$
\begin{aligned}
T_{s}-T_{c i} & =\frac{P}{2 \pi R\left(\frac{k_{g}}{y}\right)} \\
& =\frac{550}{2 \pi \times 0.265 \times\left(\frac{3.4 \times 10^{-3}}{7 \times 10^{3}}\right)} \\
& =695^{\circ} \mathrm{C} \\
\therefore T_{s}=637+ & 695=1332 \mathrm{C}
\end{aligned}
$$

From Fig.(10.20): $\int_{0}^{1332} k d T=51 \mathrm{Watts} / \mathrm{cm}$

Therefore from eq. (2) $\int_{0}^{T_{c}} k d T=43.8+51$

$$
=94.8 \text { wattsicm }
$$

From Fig $(10.20) \therefore T_{0} \cong 2700^{\circ} \mathrm{C}$

Estimating $\bar{T}_{f}$

Assuming a parabolic profile $\frac{T-T_{S}}{T_{0}-T_{s}}=1-\left(\frac{r}{R}\right)^{2}$

$$
T=T_{s}+\left(T_{0}-T_{s}\right)\left[1-\left(\frac{\gamma}{R}\right)^{2}\right]=T_{0}-\left(T_{0}-T_{s}\right)\left(\frac{r}{R}\right)^{2}
$$

Then

$$
\begin{aligned}
\bar{T}_{f} & \cong \frac{1}{\pi R^{2}} \int_{0}^{R}\left[T_{0}-\left(T_{0}-T_{5}\right)\left(\frac{r}{R}\right)^{2}\right] 2 \pi r d r \\
& =\frac{2}{R^{2}} T_{0} \frac{R^{2}}{2}-\frac{2}{R^{4}}\left(T_{0}-T_{5}\right) \frac{R^{4}}{t_{7}}
\end{aligned}
$$


19.3

$$
\therefore \bar{T}_{f}=T_{0}-\frac{1}{2}\left(T_{0}-T_{s}\right)=\frac{1}{2}\left(T_{0}+T_{s}\right)
$$

Then $\bar{T}_{f}=\frac{1}{2}(1332+2700)=2016^{\circ} \mathrm{C}$

From eq. (4)

$$
\begin{aligned}
t_{g}^{\text {hot }} & =t_{g}^{\text {cold }}+2.9 \times 10^{-3}-3.22 \times 10^{-6}(2016-25) \\
& =7 \times 10^{-3}+2.9 \times 10^{-3}-6.45 \times 10^{-3} \\
& =3.45 \times 10^{-3} \mathrm{~cm}
\end{aligned}
$$

Sst Iteration

$$
\begin{aligned}
& \frac{k_{g}}{t_{g}}=\frac{3.4}{3.45}=0.985 \text { watts } / \mathrm{cm}^{2}-x \\
\therefore T_{s}-T_{c i} & =\frac{550}{2 \pi \times 0.268\left[1+1.2 \times 10^{-5}(2016-25)\right] \times 0.985} \\
& =\frac{550}{2 \pi \times 0.275 \times 0.785}=323^{\circ} \mathrm{C} . \\
\therefore T_{s}^{(2)} & =637+323=960^{\circ} \mathrm{C}
\end{aligned}
$$

From Fig. (10.20)

$$
\begin{aligned}
& \int_{0}^{960} k d T=41 \mathrm{watl} / \mathrm{cm} \\
\therefore & \int_{0}^{T_{c}} k d T=43.8+41=84.8 \mathrm{~cm} \\
\therefore & T_{0}^{(2)}=2520^{\circ} \mathrm{C} \quad[\text { from Fig. (10.20)] } \\
\therefore \quad & T_{f}=\frac{1}{2}(2520+960)=1740^{\circ} \mathrm{C}
\end{aligned}
$$

$$
\text { and } \begin{aligned}
t^{\text {hot }} & =7 \times 10^{-3}+2.9 \times 10^{-3}-3.22(1740-25) \times 10^{-6} \\
& =4.525 \times 10^{-3} \mathrm{~cm}
\end{aligned}
$$

Repeat the iterations until convergence

Final values $T_{0} \cong 2530^{\circ} \mathrm{C}, T_{s} \cong 1050^{\circ} \mathrm{C}$ 
194

Problem 10.3

a) For no loss of Plutonium:

$$
\begin{aligned}
& \frac{1}{\pi R^{2}} \int_{0}^{R} \frac{q(r)}{q_{0}} 2 \pi r d r=1 \quad \ldots(1) \\
& \therefore \quad 1=1+\frac{D}{\pi R^{2}} \int_{0}^{R}\left\{\exp \left[-2 \alpha\left(\frac{r-r^{*}}{R}\right)\right]-2 \exp \left[-\alpha\left(\frac{r-r^{*}}{R}\right)\right]\right\} 2 \pi r d r \\
& \text { or } \int_{0}^{R} \exp \left[-2 \alpha\left(\frac{\gamma-r^{*}}{R}\right)\right] r d \gamma=2 \int_{0}^{R} \exp \left[-\alpha\left(\frac{r-r^{*}}{R}\right)\right] r d \gamma
\end{aligned}
$$

Let $\eta=\frac{r}{R}, \quad \eta^{*}=\frac{r^{*}}{R}$

Then $\int_{0}^{1} \exp \left[-2 \alpha\left(\eta-\eta^{*}\right)\right] \eta d \eta=2 \int_{0}^{1} \exp \left[-x\left(\eta-\eta^{*}\right)\right] \eta d \eta$

or $e^{2 x \eta^{*}} \int_{0}^{1} \eta e^{-2 x \eta} d \eta=2 e^{\alpha \eta^{+}} \int_{0}^{1} \eta e^{-\alpha \eta} d \eta$

Integrating,

$$
\frac{1}{8} e^{\alpha \eta^{*}}\left[1-(2 \alpha+1) e^{-2 \alpha}\right]=1-(\alpha+1) e^{-\alpha}
$$

For $\alpha=10 \quad e^{-2 \alpha} \cong 0$ and $e^{-\alpha} \cong 0$

Therefore $\quad e^{\alpha \eta^{*}}=8$

- or $\eta^{+}=\frac{1}{\alpha} \ln 8=\frac{\ln 8}{10}=0.207$

b) $\frac{q(0)}{q_{0}}=1+D\left\{e^{2 \alpha \eta^{*}}-2 e^{\alpha \eta^{*}}\right\}$ 
195

$$
\begin{aligned}
\therefore \frac{q(0)}{q_{c}}=1+10^{-2}\{64-2 \times 8\} & =1+10^{-2} \times 48 \\
& =1.48
\end{aligned}
$$

Then $q(0)=1.48 \times 20 \% \cong 30 \%$

For the minimum Plutonium concentration:,

Making $\frac{d q(r)}{d r}=0$ and with a little of algebra yeilds,

$q(\gamma)$ is minimum at $r=\gamma^{*}$

$$
\frac{q\left(r^{*}\right)}{q_{0}}=1-D=0.99
$$

or $q\left(r^{*}\right)=0.99 \times 20 \%=19.8 \%$

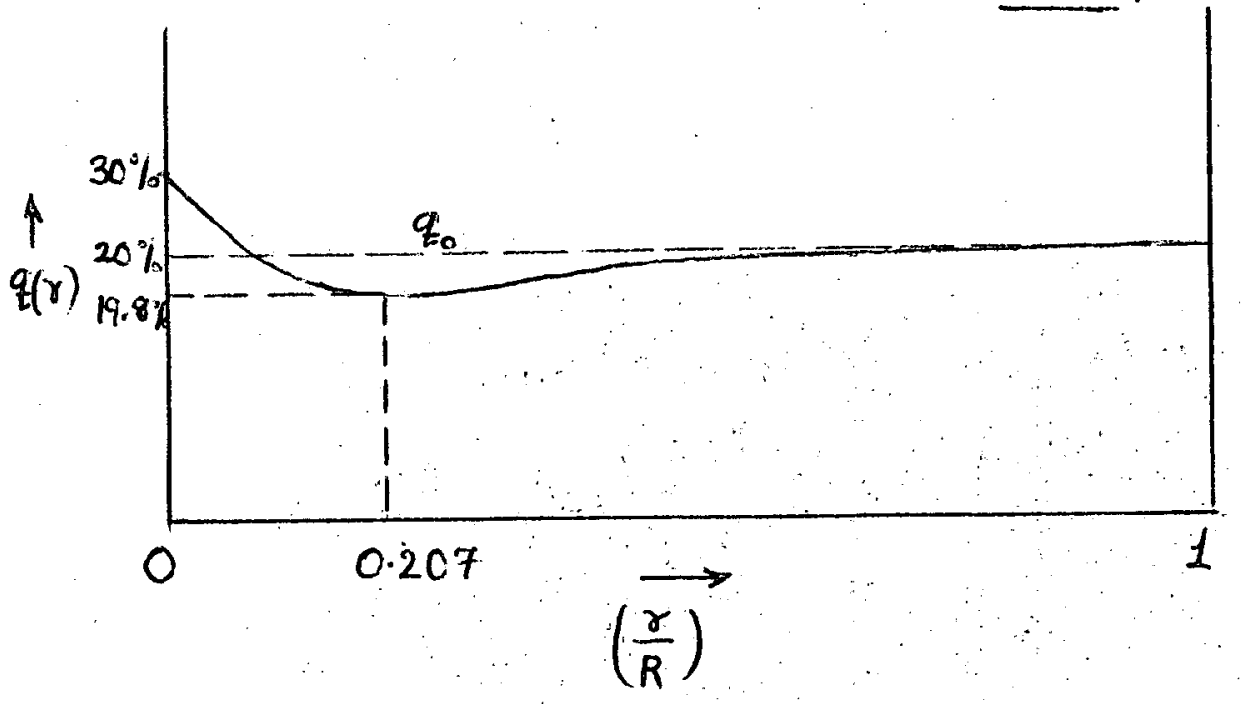


196

c) $\quad H(\gamma)=\bar{H} \cdot \frac{q(\gamma)}{q_{0}}$

with $\bar{H}=$ Average power density of fuel

With constant thermal conductivity Eq. (10.49) reduces to:

$$
\frac{1}{r} \frac{d}{d r}\left(\gamma \frac{d T}{d r}\right)=-\frac{\bar{H}}{k} \frac{q(\gamma)}{q_{0}} \ldots \ldots(2)
$$

Boundary Conditions:

i) $T(R)=T_{5}$

(i) $T(0)=T_{0}$

For the initial plutonium distribution, $\frac{q(\gamma)}{q_{0}}=1$, $\bar{H}=H_{0}$, initial power density.

Temperature distribution is given by $E_{q}$ (10.52):

$$
T-T_{s}=\frac{1}{4} \cdot \frac{H_{0} R^{2}}{k}\left[1-\left(\frac{r}{R}\right)^{2}\right]
$$

For non-uniform plutonium distribution:

Integrating en (2),

$$
r \frac{d T}{d r}=-\frac{\bar{H}}{k} \int r \frac{q(r)}{q_{0}} d r+A
$$

Changing variable to $\eta=\frac{\gamma}{R}$;

$$
\eta \frac{d T}{d \eta}=-\frac{\bar{H} R^{2}}{k} \int \eta \frac{\eta(\eta)}{q_{0}} d \eta+A
$$


197

$$
\begin{aligned}
& \therefore \eta \frac{d T}{d \eta}=-\frac{\bar{H} R^{2}}{2 k} \eta^{2}-\frac{\bar{H} R^{2} D}{k}\left\{e^{2 \alpha \eta^{*}} \int \eta e^{-2 \alpha \eta} d \eta\right. \\
&\left.-2 e^{\alpha \eta^{*}} \int \eta e^{-\alpha \eta} d \eta\right\}+A \\
&=-\frac{\bar{H} R^{2}}{2 k} \eta^{2}+\frac{\bar{H} R^{2} D}{\alpha^{2} k}\left\{\frac{e^{2 \alpha \eta^{*}} e^{-2 \alpha \eta}}{4}(1+2 \alpha \eta)\right. \\
&\left.-2 e^{\alpha \eta^{*}} e^{-\alpha \eta}(1+\alpha \eta)\right\}+A
\end{aligned}
$$

BiC.

$$
\begin{aligned}
& \text { At } \eta=0, \frac{d T}{d \eta}=0 \\
& \therefore \quad A=-\frac{\bar{H} R^{2} D}{\alpha^{2} k}\left\{\frac{1}{4} e^{2 \alpha \eta^{*}}-2 e^{\alpha \eta^{*}}\right\} \\
& \text { with } \quad e^{\alpha \eta^{*}}=8, e^{2 \alpha \eta^{*}}=64 \\
& A=-\frac{\bar{H} R^{2} D}{\alpha^{2} k}\left\{\frac{64}{4}-2 \times 8\right\}=0
\end{aligned}
$$

Then

$$
\begin{array}{r}
\frac{d T}{d \eta}=-\frac{F R^{2}}{k}\left\{\frac{\eta}{2}-\frac{D}{4 \alpha^{2}} e^{2 \alpha \eta^{*}}\left(\frac{1+2 \alpha \eta}{\eta}\right)\right. \\
\left.+\frac{2 D}{\alpha^{2}} e^{\alpha \eta^{*}}\left(\frac{1+\alpha \eta}{\eta}\right) e^{-\alpha \eta}\right\}
\end{array}
$$

Integrating from $\eta=1$ to $\eta=\eta ; T=T_{s}$ to $T=T$ Yeilds:

$$
\begin{array}{r}
T-T_{s}=\frac{\bar{H} R^{2}}{k}\left\{\frac{1}{4}\left(1-\eta^{2}\right)-\frac{D}{4 \alpha^{2}} e^{\alpha \alpha \eta}\left[F(2 \alpha, \eta)+\left(e^{-2 \alpha \eta}-e^{-2 \alpha}\right)\right]\right. \\
\left.+\frac{2 D}{\alpha^{2}} e^{\alpha \eta^{*}}\left[F(\alpha, \eta)+\left(e^{-\alpha \eta}-e^{-\alpha}\right)\right]\right\} \therefore
\end{array}
$$


198

with $\quad F(\alpha, \eta)=\int_{\eta}^{1} \frac{e^{-\alpha \eta}}{\eta} d \eta$;

d)

$H \sim P$ (linear power of rod)

For $T_{0}$ to be the same before and after redistribution:

$$
\begin{aligned}
\frac{P_{0}}{P}=\frac{H_{0}}{\bar{H}}=1+\frac{D}{\alpha^{2}}\left\{\delta e^{\alpha \eta^{*}}\right. & {\left[F(\alpha, 0)+\left(1-e^{-\alpha}\right)\right] } \\
& \left.-e^{2 \alpha \eta^{*}}\left[F(2 \alpha, 0)+\left(1-e^{-2 \alpha}\right)\right]\right\}
\end{aligned}
$$

(using ens. (3) and (4) and putting $\eta=0$ in these)

since $8 e^{\alpha \eta^{*}}=e^{2 \alpha \eta^{*}}$ and neglecting $e^{-\alpha}$ and $e^{-2 \alpha}$. we get,

$$
\begin{aligned}
\frac{P_{0}}{P} & =1+\frac{8 D}{\alpha^{2}} e^{\alpha \eta^{*}}[F(\alpha, 0)-F(2 \alpha, 0)] \\
& =1+\frac{8 D}{\alpha^{2}} e^{\alpha \eta^{*}} \int_{0}^{1} \frac{e^{-\alpha \eta}-e^{-2 \alpha \eta}}{\eta} d \eta
\end{aligned}
$$

Examine the behavior of the integral near $\eta=0$

$$
\begin{aligned}
e^{-\alpha \eta} & \rightarrow 1-\alpha \eta+\frac{1}{2} \alpha^{2} \eta^{2}-\ldots+\ldots \\
e^{-2 \alpha \eta} \rightarrow 1-2 \alpha \eta+2 \alpha^{2} \eta^{2} & \rightarrow+\ldots \\
\therefore & \frac{e^{-\alpha \eta}-e^{-2 \alpha \eta}}{\eta}=\alpha-\frac{3}{2} \alpha^{2} \eta+\ldots(\text { negligible near } \eta=0)
\end{aligned}
$$




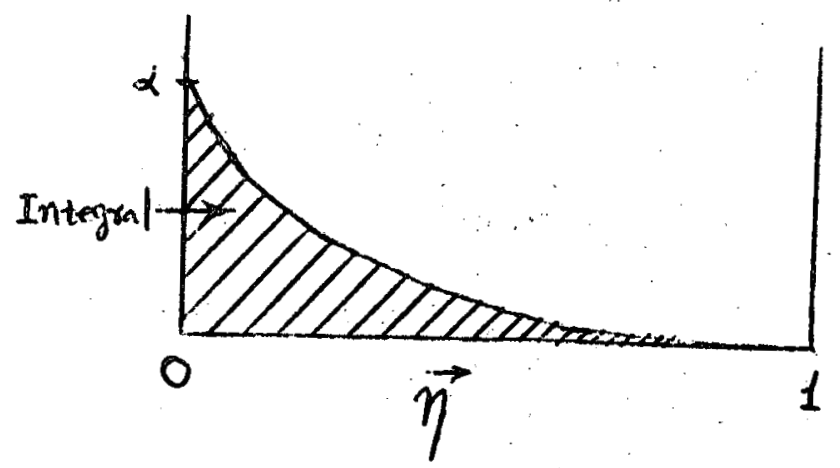

$\therefore$ Integral $\cong \frac{1}{5} \alpha$ (estimate of area on plot).

$$
\begin{aligned}
\frac{P_{0}}{P}=1+\frac{8 D e^{\alpha \eta^{*}}}{\alpha^{2}}\left(\frac{1}{5} \alpha\right) & =1+\frac{64}{5} \times 10^{-3} \\
& =1.013
\end{aligned}
$$

Approximately $1.3 \%$ reduction in power is needed in order to keep To constant. The effect is small because volume where $q$ changes (near $r=0$ ) contains little mass. 
Problem 10.4

(?)

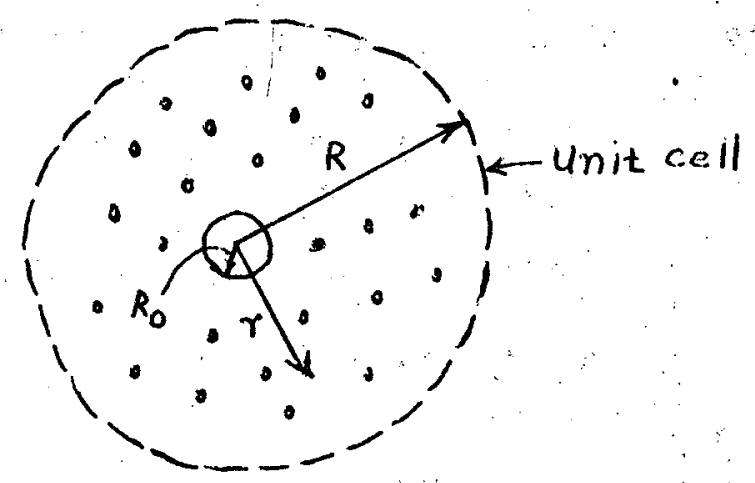

$\circ$

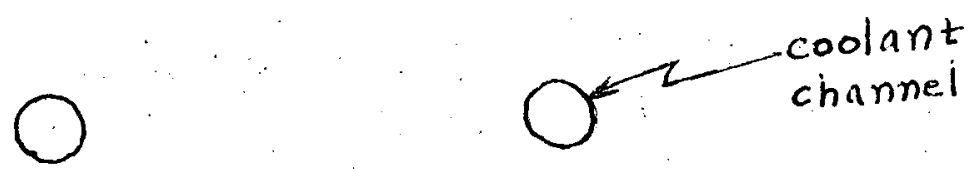

a)

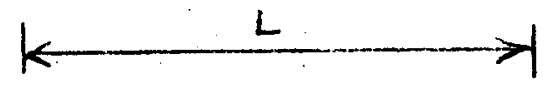

Area per channel $=L^{2}=\pi R^{2}=$ Area of unit cell

$$
\therefore R=\frac{L}{\sqrt{\pi}}
$$

There are $N_{p}$ fuel pellets $/ \mathrm{cm}^{3}$, ench of radius $R_{p}$ and contains fully enriched $U^{235} \mathrm{O}_{2}$.

Heat produced $/$ pellet $=\sigma_{\text {fisr }} N_{f} \phi\left(\frac{4}{3} \pi R_{p}^{3}\right) \times 3.07 \times 10^{11}$

Hent generation rate $/ \mathrm{cm}^{3}=H=3.07 \times 10^{\prime \prime} \times \sigma_{\text {fiss }} \times N_{f} \phi\left(\frac{4}{3} \pi R_{p}^{3}\right) \times N_{p}$

$$
\begin{aligned}
& P=\text { Linear power per channel } \\
& P=\pi\left(R^{2}-R_{0}^{2}\right) H
\end{aligned}
$$


b) Temperature distribution in graphite:

$$
\frac{1}{r} \frac{d}{d r}\left(r k_{g} \frac{d T}{d r}\right)+H=0
$$

where $H$ is given by (1).

$$
k_{g}=\text { thermal conductivity of graphite }
$$

B.C

$$
\begin{aligned}
& T\left(R_{0}\right)=T_{s}=\begin{array}{l}
\text { temperature of the surface of } \\
\text { coolant channel }
\end{array} \\
& \left(\frac{d T}{d r}\right)_{r=R}=0 \quad[\text { by definition of unit cell] }
\end{aligned}
$$

Integrating we get

$$
r \frac{d T}{d r}=-\frac{1}{2} \frac{H}{k_{g}} r^{2}+c_{1}
$$

using (s) we get,

$$
c_{1}=\frac{1}{2} \frac{H}{k_{g}} R^{2}
$$

Integrating once more

Using (4) we get,

$$
T=-\frac{1}{4} \frac{H}{k_{g}}+\frac{1}{2} \frac{H}{k_{g}} R^{2} \ln r+C_{2}
$$

$$
T_{s}=-\frac{1}{4} \frac{H}{k_{g}} R_{0}^{2}+\frac{1}{2} \frac{H}{k_{g}} R^{2} \ln R_{0}+C_{2}
$$

$\therefore$ Temperature distribution in graphite is

$$
T-T_{s}=\frac{1}{4} \frac{H}{k_{g}}\left\{R_{0}^{2}-r^{2}+2 R^{2} \ln \left(\frac{r}{R_{c}}\right)\right\}
$$


c) Temperature distribution in and around pellet:

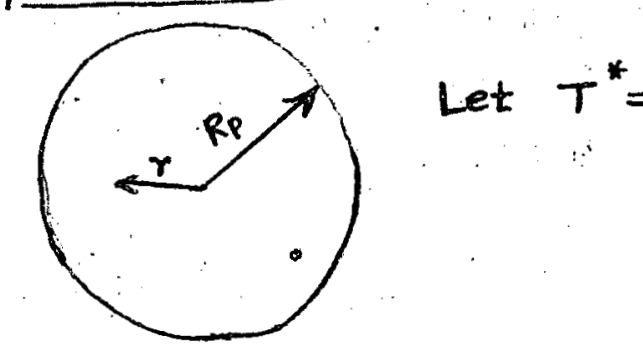

Temperature far away from pellet equal to the value given by (7) for radial location $r$ of pellet in unit cell.

$$
\begin{aligned}
& H_{p}=\text { power density in pellet } \\
& H_{p}=\sigma_{\text {fiss. }} N_{f} \phi \times 3.07 \times 10^{11} \ldots(8) \\
& \frac{1}{\gamma^{2}} \frac{d}{d r}\left(r^{2} k_{p} \frac{d T}{d r}\right)+H_{p}=0 \quad \ldots(9) \\
& k_{p}=\text { thermal conductivity of fuel material in pellet. }
\end{aligned}
$$

Integrate once:

$$
\begin{array}{r}
r^{2} \frac{d T}{d r}=-\frac{1}{3} \frac{H_{p}}{k_{p}} r^{3}+c_{1} \\
\frac{d T}{d r}=0 \text { at } r=0 . \quad \therefore c_{1}=0
\end{array}
$$

Integrate once more:

$$
T=-\frac{1}{6} \frac{H_{p}}{k_{p}} r^{2}+C_{2} \quad \ldots(10)
$$

In graphize near pellet

$$
\frac{1}{r^{2}} \frac{d}{d r}\left(r^{2} \operatorname{kg} \frac{d T}{d r}\right)=0
$$

Integrate once:

$$
\dot{r}^{2} \frac{d T}{d r}=c_{3}
$$


Determine $C_{3}$ by relating heat flows at interface: $\left(\gamma=R_{P}\right)$

From (10) and (11)

$$
-\left.k_{p} \frac{d T}{d r}\right|_{\substack{R_{p} \\ \text { (peillt) }}}=-\left.k_{g} \frac{d T}{d r}\right|_{\substack{R_{p} \\ \text { (qraphite) }}}
$$

$$
\begin{gathered}
-k_{p}\left(-\frac{1}{3} \frac{H_{p}}{k_{p}} R_{p}\right)=-k_{g}\left(\frac{C_{3}}{R_{p}^{2}}\right) \\
\therefore C_{3}=-\frac{1}{3} \frac{H_{p}}{k_{g}} R_{p}^{3}
\end{gathered}
$$

Integrating (11) once more

$$
T=-\frac{C_{3}}{r}+C_{4}=\frac{1}{3} \frac{H_{p}}{k_{g}} \frac{R_{R}^{3}}{r}+C_{4} \ldots(13)
$$

Now the temperature in graphite far from pellet is equal to $T^{*}$ given by (7).

$$
\therefore c_{4}=T^{*}
$$

Equality of temperatures at the interface, $r=R_{p}$ gives, from (ic) and (13)

$$
-\frac{1}{6} \frac{H_{p}}{k_{p}} R_{p}^{2}+C_{2}=\frac{1}{3} \frac{H_{p}}{k_{g}} \frac{R_{p}^{3}}{R_{p}}+T^{*}
$$

This determines $C_{2}$.

Therefore the distribution in the pellet is from (10)

$$
T-T^{*}=\frac{1}{6} \frac{H_{p}}{k_{p}}\left(R_{p}^{2}-r^{2}\right)+\frac{1}{3} \frac{H_{p}}{k_{g}} R_{p}^{2} \quad\left(0 \leq r \leq R_{p}\right)
$$

and in the graphite. near the pellet is from (13)

$$
T-T^{*}=\frac{1}{3} \frac{R_{p}}{\mathrm{~kg}_{\mathrm{g}}}
$$


204

Problem 10.5

Random Spherical Pores of Same Diameter Obstacks in Glide Plane Analysis:

$C=$ Number of spherical pores / cc (total Volume)

$r_{p}=$ Radius of each pore.

(a)

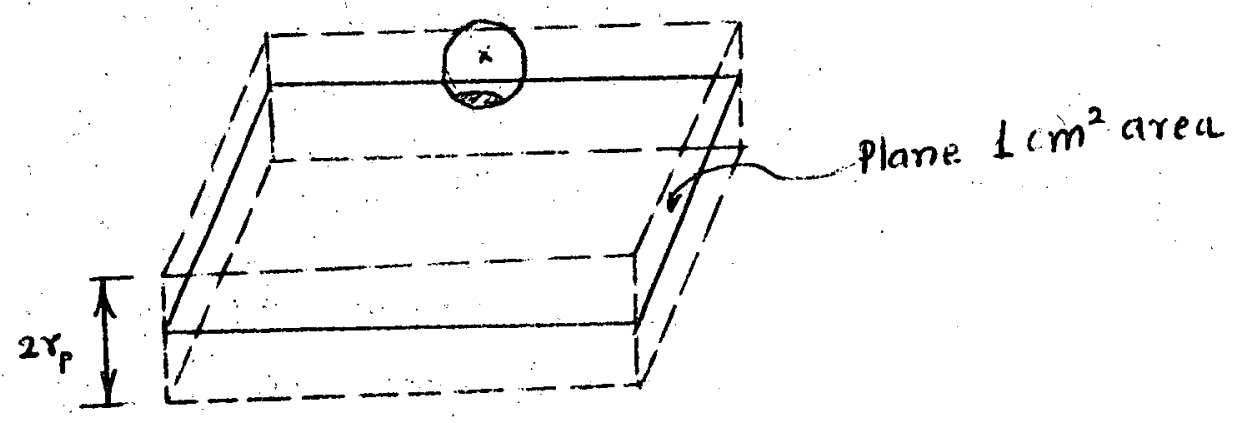

The volume $\left(2 \gamma_{p}\right)\left(1\left(\mathrm{~m}^{2}\right)\right.$ contains the centers of $c\left(2 r_{p}\right)$ pores, each of which intersects the plane $\therefore$ There are $2 \gamma_{p} c$ intersections $/ \mathrm{cm}^{2}$.

$\therefore$ Area / Intersection $=\frac{1}{2 \gamma_{p} C}=\ell^{2}$

$\therefore$ Average distance betucen intersections $=\ell=1 / \sqrt{2 r_{p} c}$

(b) $\rho=$ radius of circle formed by intersection of pore with plane

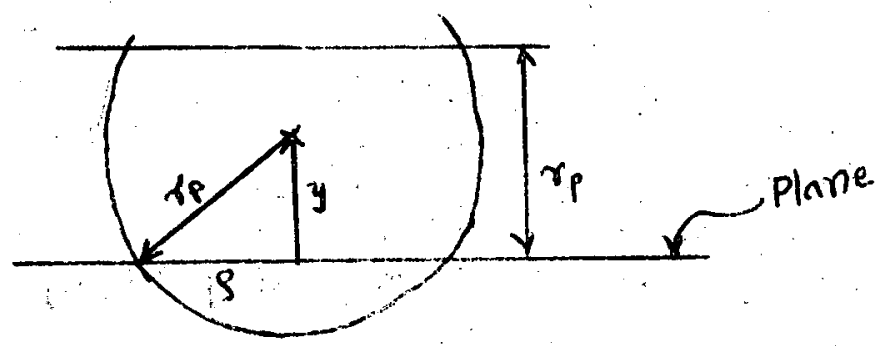


205

$$
\rho=r_{p} \sqrt{1-\left(y / r_{p}\right)^{2}} \quad(\text { length of chord) }
$$

Assume - of the pores which intersect the plane, the distances of their centers from the plane is randomly distributed

or

$p(y) d y=$ probability of a given pore being within a distance $y$ and $y+d y$ from the plane:

$$
p(y)=\frac{1}{\gamma_{p}} \quad\left(\text { since } \int_{0}^{\gamma_{p}} p(y) d y=\int_{0}^{\gamma_{p}} \frac{d y}{\gamma_{p}}=1\right) .
$$

$A(y)=$ Area of circle formed in the plane with center of the pore a distance $y$ from the plane.

$$
\begin{aligned}
& A(y)=\pi \rho^{2}=\pi r_{p}^{2}\left[1-\left(y / r_{p}\right)^{2}\right] \\
& \bar{A}=\text { Average area of circle } \\
& \bar{A}=\int_{0}^{\gamma_{p}} A(y) p(y) d y=\frac{\pi \gamma_{p}^{2}}{\gamma_{p}} \int_{c}^{\gamma_{p}}\left[1-\left(y / r_{p}\right)^{2}\right] d y \\
& \eta=y
\end{aligned}
$$

Let $\eta=\frac{y}{\gamma_{p}}$.

$$
\begin{aligned}
\therefore \bar{A} & =\pi \gamma_{p}^{2} \int_{0}^{1}\left(1-\eta^{2}\right) d \eta \\
& =\frac{2}{3} \pi r_{p}^{2}
\end{aligned}
$$

(c)

$P_{c}^{\prime \prime}=$ Fraction of area of plane occupied by intersecting circles 
206

$$
\begin{aligned}
P_{c} & =\left(\frac{\text { intersections }}{c m^{2}}\right)\left(\frac{\text { area }}{\text { intersection }}\right) \\
& =\left(2 \gamma_{p} c\right) \bar{A} \\
& =2 \gamma_{p} C\left(\frac{2}{3} \pi \gamma_{p}^{2}\right) \\
& =\frac{4}{3} \pi \gamma_{p}^{3} C \\
& =p \quad(\text { porosity) }
\end{aligned}
$$


Problem 10.6

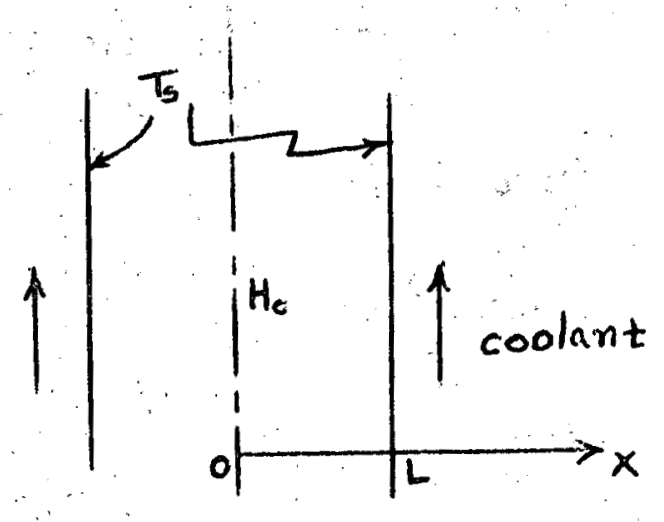

Initial porosity $=P_{0}$

Conductivity is given by Eq. (10.40);

$$
k_{0}=k_{s}\left(1-p^{2 / 3}\right) \text { independent of temperature }
$$

a) Energy Equation

$$
k_{0} \frac{d^{2} T}{d x^{2}}+H_{0}=0
$$

Integrating: $\quad \frac{d T}{d x}=-\frac{H_{c}}{k} x+A ;$

Boundary Condition: i) At $x=0, \frac{d T}{d x}=0$

This gives $A=0$

Integrating again: $T=-\frac{1}{2} \frac{H_{0}}{k_{c}} x^{2}+B$

This gives $B=T_{s}+\frac{1}{2} \cdot \frac{H_{c}}{k_{c}} L^{2}$ 
208

Therefore: $\quad T-T_{s}=\frac{1}{2} \frac{H_{0}}{k_{0}} L^{2}\left(1-\frac{x^{2}}{L^{2}}\right)$

Also: $\quad T(0)=T_{m}$.

$$
\therefore H_{0}=\frac{2 k_{0}\left(T_{m}-T_{s}\right)}{L^{2}}=\frac{2 k_{s}\left(1-P_{0}^{2 / 3}\right)\left(T_{m}-T_{s}\right)}{L^{2}}
$$

b) After restructuring

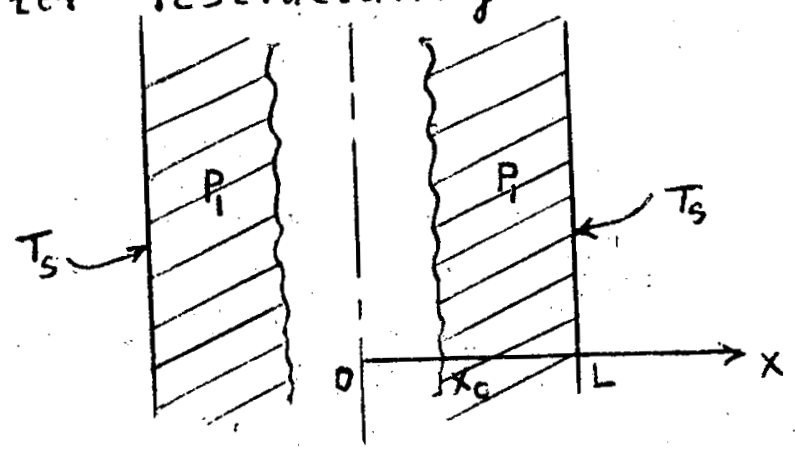

From Eq. $(10.40) \quad k_{1}=k_{s}\left(1-p_{1}^{2 / 3}\right)$

$$
T=-\frac{1}{2} \cdot \frac{H}{k_{1}} x^{2}+A x+B
$$

and $\frac{d T}{d x}=-\frac{H}{k_{1}} x+A$

B.C. i) $\frac{d T}{d x}=0$ at $x=x_{0}$

This gives, $A=\frac{H}{k_{1}} x_{0}$

B.C. ii)

$$
T=T_{s} \text { at } x=L
$$


209

This gives, $B=T_{s}+\frac{1}{2} \frac{H}{k_{1}} L^{2}-\frac{H x_{0} L}{k_{1}}$

Mass balance gives, $\rho_{0} L=\rho_{1}\left(L-x_{0}\right)$

$$
\therefore \frac{X_{0}}{L}=1-\frac{\rho_{0}}{\rho_{1}}=1-\frac{1-P_{0}}{1-P_{1}}
$$

Now we have $T\left(x_{0}\right)=T_{m}$, then

$$
\begin{aligned}
& T_{m}=-\frac{1}{2} \frac{H}{k_{1}} x_{0}^{2}+\frac{H x_{0}^{2}}{k_{1}}+\left(T_{s}+\frac{1}{2} \frac{H L^{2}}{k_{1}}-\frac{H x_{0} L}{k_{1}}\right) \\
& \therefore T_{m}-T_{s}=\frac{H x_{0}^{2}}{2 k_{1}}+\frac{H L^{2}}{2 k_{1}}-\frac{H x_{0} L}{k_{1}}
\end{aligned}
$$

or

$$
\begin{aligned}
H & =\frac{T_{m}-T_{s}}{\frac{1}{2}\left(\frac{L^{2}}{k_{1}}\right)\left[1-2 \frac{x_{0}}{L}+\left(\frac{x_{0}}{L}\right)^{2}\right]} \\
& =\frac{2 k_{1}\left(T_{m}-T_{s}\right)}{L^{2}\left(1-\frac{x_{0}}{L}\right)^{2}}=\frac{2 k_{1}\left(T_{m}-T_{s}\right)}{L^{2}\left(S_{2} / s_{1}\right)^{2}}
\end{aligned}
$$

or rearranging:

$$
\begin{aligned}
H & =\frac{2 k_{0}\left(T_{m}-T_{s}\right)}{L^{2}} \cdot \frac{k_{1}}{k_{0}} \cdot \frac{L^{2}}{L^{2}\left(1-\frac{k_{0}}{L}\right)^{2}} \\
& =H_{0}\left(\frac{1-P_{1}^{2 / 3}}{1-P_{0}^{2 / 3}}\right)\left(\frac{1-P_{1}}{1-P_{0}}\right)^{2} \cdot
\end{aligned}
$$

Therefore $\quad \frac{H}{H_{0}}=\left(\frac{1-P_{1}^{2 / 3}}{1-P_{0}^{2 / 3}}\right)\left(\frac{1-p_{1}}{1-P_{0}}\right)^{2}$ 
Problem 10.7

a)

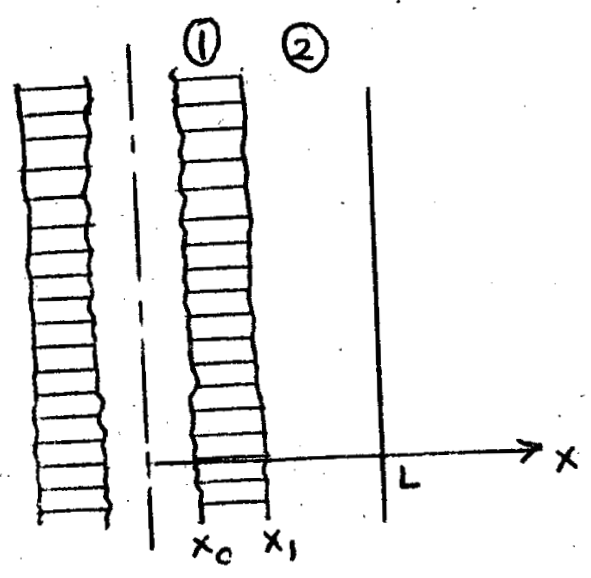

After half restructuring we have two regions and void.

Region (1) = Columnar grain region with density $=\rho_{1}$

Region (2) = Unrestructured region with density $=\rho_{0}$

(initial value)

Since reactor power is constant the power density in the unrestructured region is $\mathrm{H}_{2}=\mathrm{H}_{0}$

$$
\begin{aligned}
H_{2} & =\text { power density in region (2) } \\
H_{1} & =\text { power density in region (1) } \\
\frac{H_{1}}{H_{2}} & =\frac{\rho_{1}}{\rho_{0}} \quad \ldots . .(1)
\end{aligned}
$$

Mass balance (per unit area)

$$
\begin{aligned}
\text { Initial } & =\rho_{0} L \\
\text { Final } & =\rho_{0}\left(L-x_{1}\right)+\rho_{1}\left(x_{1}-x_{0}\right)
\end{aligned}
$$

Equating and dividing by $S_{c} L$

$$
1=\left(1-\frac{x_{1}}{L}\right)+\frac{\rho_{1}}{\rho_{0}}\left(\frac{x_{1}}{L}-\frac{x_{0}}{L}\right)
$$


211

$$
\therefore\left(\frac{\rho_{1}}{\rho_{0}}-1\right)\left(\frac{X_{1}}{L}\right)=\left(\frac{\rho_{1}}{\rho_{0}}\right)\left(\frac{X_{0}}{L}\right)
$$

Final value of the central void occurs when $X_{1}=L$ or

$$
\frac{x_{c f}}{L}=\frac{\rho_{1} / s_{c}-1}{\rho_{1} / s_{0}}=1-\frac{\rho_{0}}{\rho_{1}}
$$

For this problem $\frac{x_{0}}{x_{0 f}}=\frac{1}{2}$

or

$$
\frac{X_{0}}{L}=\frac{1}{2}\left(1-\frac{\rho_{0}}{\rho_{1}}\right)
$$

using this in eq .(2) we get,

$$
\begin{aligned}
\left(\frac{\rho_{1}}{\rho_{0}}-1\right)\left(\frac{x_{1}}{L}\right) & =\left(\frac{\rho_{1}}{\rho_{c}}\right) \frac{1}{2}\left(1-\frac{\rho_{c}}{\rho_{1}}\right) \\
\therefore \frac{x_{1}}{L} & =\frac{1}{2}
\end{aligned}
$$

b) Integrating the heat conduction equation, we get

Region (1): $\quad T_{1}(x)=-\frac{1}{2} \frac{H_{1}}{k_{1}} x^{2}+A_{1} x+B_{1} ; \frac{d T_{1}}{d x}=-\frac{H_{1}}{k_{1}} x+A_{1}$

Region (2): $T_{2}(x)=-\frac{1}{2} \frac{H_{2}}{k_{2}} x^{2}+A_{2} x+B_{2} ; \frac{d T_{2}}{d x}=-\frac{H_{2}}{k_{2}} x+A_{2}$

B.C.

i) $\left(\frac{d T_{1}}{d x}\right)_{x_{0}}=0$

ii)

$$
k_{1}\left(\frac{d T_{1}}{d x}\right)_{x_{1}}=k_{2}\left(\frac{d T_{2}}{d x}\right)_{x_{1}}
$$


212

iii) $T_{1}\left(x_{1}\right)=T_{2}\left(x_{1}\right)$

iv) $\quad T_{2}(L)=T_{5}$
- $-(10)$

(11)

From (8):

$$
\begin{aligned}
A_{1}= & \frac{H_{1}}{k_{1}} x_{0}=\frac{H_{2}}{k_{2}} L\left(\frac{H_{1}}{H_{2}}\right)\left(\frac{k_{2}}{k_{1}}\right)\left(\frac{X_{0}}{L}\right) \\
= & \frac{H_{2}}{k_{2}} L\left(\frac{\rho_{1}}{S_{c}}\right)\left(\frac{k_{2}}{k_{1}}\right) \frac{1}{2}\left(1-\frac{S_{0}}{S_{1}}\right) \\
& {[\text { using (1) and (4)].. }}
\end{aligned}
$$

From (9) :

$$
\begin{aligned}
-H_{1} x_{1}+k_{1} A_{1} & =-H_{2} x_{1}+k_{2} A_{2} \\
\therefore-H_{1} x_{1}+H_{1} x_{0} & =-H_{2} x_{1}+k_{2} A_{2} \text { [using (12)] } \\
\therefore \quad A_{2} & =\frac{1}{k_{2}}\left\{H_{1} x_{0}-\left(H_{1}-H_{2}\right) x_{1}\right\} \\
& =\frac{H_{1} x_{0}}{k_{2}}\left\{1-\left(1-\frac{\rho_{0}}{S_{1}}\right)\left(\frac{x_{1}}{x_{0}}\right)\right\} \quad[4 \operatorname{sing}(1)] \\
& =\frac{H_{1} x_{0}}{k_{2}}\left\{1-\frac{\left(1-\frac{\rho_{0}}{\rho_{1}}\right) \frac{\rho_{1}}{s_{0}}}{\left(\frac{\rho_{1}}{\rho_{r}}-1\right)}\right\} \quad[u \operatorname{sing}(2)] \\
& =\frac{H_{1} x_{0}}{k_{2}}\{1-1\}=0 .(13)
\end{aligned}
$$

From (11):

$$
\begin{aligned}
& T_{5}=-\frac{1}{2} \frac{H_{2}}{k_{2}} L^{2}+B_{2} \\
& \therefore B_{2}=T_{5}+\frac{1}{2} \frac{H_{2}}{k_{2}} L^{2}
\end{aligned}
$$


213

From (10):

$$
\begin{gathered}
-\frac{1}{2} \frac{H_{1}}{k_{1}} x_{1}^{2}+\frac{H_{1}}{k_{1}} x_{0} x_{1}+B_{1}=-\frac{1}{2} \frac{H_{2}}{k_{2}} x_{i}^{2}+T_{5}+\frac{1}{2} \frac{H_{2}}{k_{2}} L^{2} \\
\quad[\text { using (i2);(13) and (14)] } \\
\therefore B_{1}=T_{5}+\frac{1}{2} \frac{H_{2}}{k_{2}} L^{2}\left[1-\left(\frac{x_{1}}{L}\right)^{2}\right]+\frac{1}{2} \frac{H_{1}}{k_{1}} L^{2}\left\{\left(\frac{x_{1}}{L}\right)^{2}-2\left(\frac{x_{0}}{L}\right)\left(\frac{x_{1}}{L}\right)\right\} \\
\text { or } B_{1}=T_{5}+\frac{1}{2} \frac{H_{2}}{k_{2}} L^{2}\left[1-\left(\frac{x_{1}}{L}\right)^{2}\right]+\frac{1}{2} \frac{H_{2}}{k_{2}} L^{2}\left(\frac{H_{1}}{H_{2}}\right)\left(\frac{k_{2}}{k_{1}}\right)\left\{\left(\frac{x_{1}}{L}\right)\left[\left(\frac{x_{L}}{L}\right)-2\left(\frac{x_{0}}{L}\right)\right]\right\} \\
\text { or } \left.B_{1}=T_{5}+\left(\frac{1}{2} \frac{H_{2}}{k_{2}} L^{2}\right)\left\{1-\left(\frac{x_{1}}{L}\right)^{2}+\left(\frac{s_{1}}{S_{2}}\right)\left(\frac{k_{2}}{k_{1}}\right)\left(\frac{x_{1}}{L}\right)\left(\frac{x_{1}}{L}-2 \frac{x_{0}}{L}\right)\right\}\right] \text { (15) }
\end{gathered}
$$

This determines the temperature profiles in the two regions.

c) At the edge of the central void

$$
\begin{aligned}
T_{1}\left(x_{0}\right)=-\frac{1}{2} \frac{H_{1}}{k_{1}} x_{0}^{2}+ & A_{1} x_{0}+B_{1} \ldots(16) \\
-\frac{1}{2} \frac{H_{1}}{k_{1}} x_{0}^{2}+A_{1} x_{c}= & -\left(\frac{1}{2} \frac{H_{2}}{k_{2}} L^{2}\right)\left(\frac{S_{1}}{S_{c}}\right)\left(\frac{k_{2}}{k_{1}}\right)\left(\frac{x_{0}}{L}\right)^{2}+ \\
& 2\left(\frac{1}{2} \frac{H_{2}}{k_{2}} L^{2}\right)\left(\frac{S_{1}}{S_{0}}\right)\left(\frac{k_{2}}{k_{1}}\right)\left(\frac{x_{0}}{L}\right)^{2} \\
& {[\text { using (1) and (12)] }} \\
= & \frac{1}{2} \frac{H_{2}}{k_{2}} L^{2}\left(\frac{g_{1}}{S_{0}}\right)\left(\frac{k_{2}}{k_{1}}\right)\left(\frac{x_{0}}{L}\right)^{2}
\end{aligned}
$$

combining with (i5) and (16): 
$T_{1}\left(x_{c}\right)-T_{s}=\left(\frac{1}{2} \frac{H_{2}}{k_{2}} L^{2}\right)\left\{\frac{g_{1}}{s_{1}} \frac{k_{2}}{k_{1}}\left(\frac{x_{c}}{L}\right)^{2}+1-\left(\frac{x_{1}}{L}\right)^{2}+\frac{\rho_{1}}{\rho_{0}} \frac{k_{2}}{k_{1}}\left(\frac{x_{1}}{L}\right)\left(\frac{x_{1}}{L}-2 \frac{x_{c}}{L}\right)\right\}$

But from Problem 10.6, with $H_{0}=H_{2}$,

$$
\frac{1}{2} \frac{H_{2}}{k_{2}} L^{2}=T_{m}-T_{s} ;\left(T_{m}=\begin{array}{l}
\text { initial centerline } \\
\text { temperature before } \\
\text { restructuring })
\end{array}\right.
$$

$$
\begin{aligned}
\therefore \frac{T_{1}\left(x_{c}\right)-T_{s}}{T_{m}-T_{s}} & =\frac{\rho_{1}}{\rho_{c}} \frac{k_{2}}{k_{1}}\left[\left(\frac{x_{2}}{L}\right)^{2}+\left(\frac{x_{1}}{L}\right)^{2}-2\left(\frac{x_{1}}{L}\right)\left(\frac{x_{0}}{L}\right)\right]+1-\left(\frac{x_{1}}{L}\right)^{2} \\
& =\frac{\rho_{1}}{\rho_{c}} \frac{k_{2}}{k_{1}}\left(\frac{x_{1}}{L}-\frac{x_{c}}{L}\right)^{2}+1-\left(\frac{x_{1}}{L}\right)^{2} \ldots(1)
\end{aligned}
$$

with $k_{2}=k_{c}$ and from Prablem 10:6 $\frac{\rho_{1}}{s_{c}}=\frac{1-P_{1}}{1-P_{0}}$

$$
\text { and } \frac{k_{2}}{k_{1}}=\frac{1-P_{c}^{2 / 3}}{1-p_{1}^{2 / 3}}
$$

using (4) and (5)

$$
\begin{aligned}
& \frac{x_{1}}{L}-\frac{x_{0}}{L}=\frac{1}{2}-\frac{1}{2}+\frac{1}{2} \frac{S_{0}}{S_{1}}=\frac{1}{2} \frac{1-P_{0}}{1-P_{1}} \\
& 1-\left(\frac{x_{1}}{L}\right)^{2}=1-\left(\frac{1}{2}\right)^{2}=\frac{3}{4} \\
\therefore & \frac{T_{1}\left(x_{0}\right)-T_{s}}{T_{m}-T_{s}}=\frac{1}{4}\left(\frac{1-P_{0}}{1-P_{1}}\right)\left(\frac{1-P_{0}^{2 / 3}}{1-P_{0}^{2 / 3}}\right)+\frac{3}{4}
\end{aligned}
$$




\section{Problem 10.8}

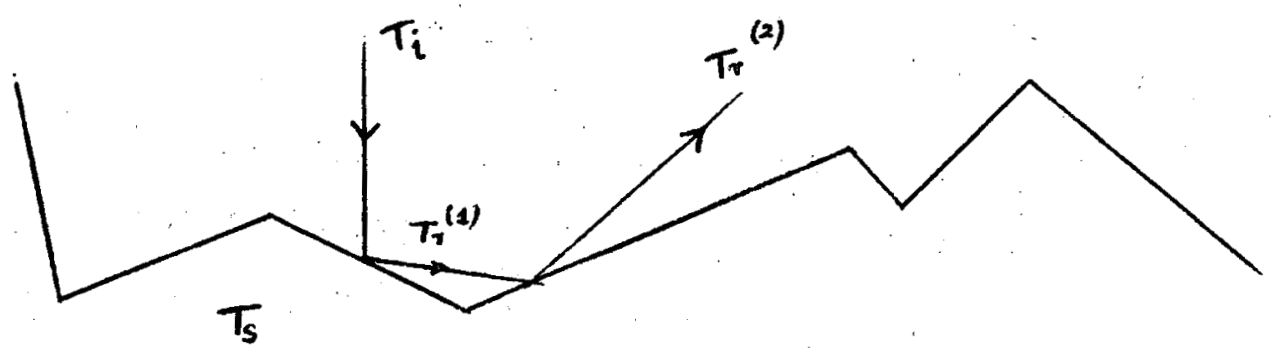

Multicallision Effect upon $\alpha$

$$
\begin{aligned}
T_{r}^{(1)} & =T_{i}+\alpha\left(T_{s}-T_{i}\right)=\alpha T_{s}+(1-\gamma) T_{i} \\
\alpha & =\frac{T_{r}^{(2)}-T_{\gamma}^{(1)}}{T_{s}-T_{r}^{(1)}} \\
\therefore T_{r}^{(2)} & =T_{r}^{(1)}+\alpha\left[T_{s}-T_{r}^{(1)}\right]=\alpha T_{s}+(1-\alpha) T_{r}^{(1)} \\
& =\alpha T_{s}+(1-\alpha)\left\{\alpha T_{s}+(1-\alpha) T_{i}\right\} \\
& =[\alpha+\alpha(1-\alpha)] T_{s}+(1-\alpha)^{2} T_{i}
\end{aligned}
$$

If there are 3 collisions;

$$
\begin{aligned}
T_{\gamma}^{(3)} & =T^{(2)}+\alpha\left(T_{s}-T_{\gamma}^{(2)}\right)=\alpha T_{s}+(1-\alpha) T_{r}^{(2)} \\
& =\alpha T_{s}+(1-\alpha) \alpha(2-\alpha) T_{s}+(1-\alpha)^{3} T_{i} \\
& =\alpha T_{s}[1+(1-\alpha)(2-\alpha)]+(1-\alpha)^{3} T_{i} \\
\alpha_{\text {eff }} & =\frac{T_{\gamma}^{(3)}-T_{i}}{T_{s}-T_{i}}
\end{aligned}
$$




$$
\begin{aligned}
& \alpha_{\text {eff. }}=\frac{\alpha[1+(1-\alpha)(2-\alpha)] T_{s}+\left[(1-\alpha)^{3}-1\right] T_{i}}{T_{s}-T_{i}} \\
& (1-\alpha)^{3}-1=-3 \alpha+3 \alpha^{2}-\alpha^{3}=\alpha\left(-3+3 \alpha-\alpha^{2}\right) \\
& \therefore \quad \frac{\alpha e f f}{\alpha}=\frac{[1+(1-\alpha)(2-\alpha)] T_{s}-\left(\alpha^{2}-3 \alpha+3\right) T_{i}}{T_{s}-T_{i}} \\
& \quad=\frac{\left[1+2-3 \alpha+\alpha^{2}\right] T_{3}-\left(\alpha^{2}-3 \alpha+3\right) T_{i}}{T_{s}-T_{i}} \\
& \therefore \alpha_{\text {eff }}=\alpha\left(\alpha^{2}-3 \alpha+3\right)=\alpha[1+(1-\alpha)(2-\alpha)]
\end{aligned}
$$

General expression for $m$ collisions is:

$$
\alpha_{\text {eff. }}=\alpha[1+(1-\alpha)(2-\alpha) \ldots([n-1]-\alpha)]
$$


217

Problem 10.9

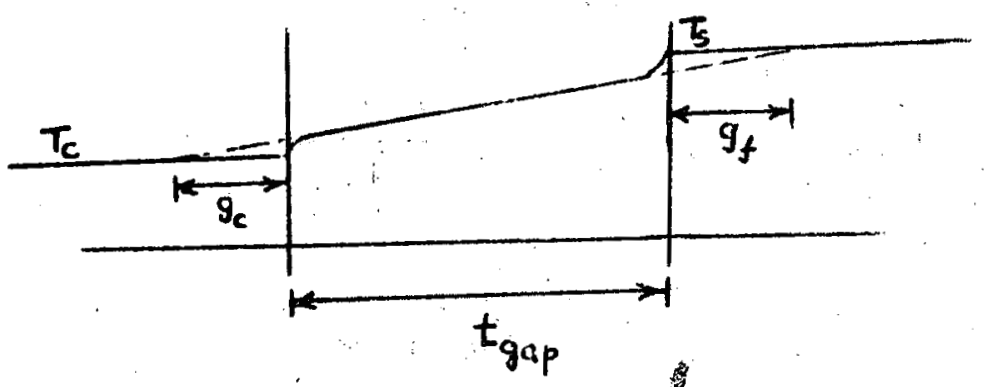

a)

The slope of temperature in the gap is

$$
\frac{d T}{d x}=-\frac{T_{s}-T_{c}}{g_{f}+t_{g_{a p}}+g_{c}}
$$

Heat flux in the gap $=q=-k_{\text {ip }} \frac{d T}{d x}=h_{g a p}\left(T_{s}-T_{c}\right) \ldots$ (2) From (1) and (2), we get,

$$
\begin{gathered}
k_{\text {gap }} \frac{\left(T_{s}-T_{c}\right)}{g_{f}+t_{\text {gap }}+g_{c}}=h_{g a p}\left(T_{s}-T_{c}\right) \\
\therefore \quad h_{\text {gap }}=\frac{k_{\text {gap }}}{g_{f}+t_{g a p}+g_{c}}
\end{gathered}
$$

b)

$$
g_{f}=2\left(\frac{2-\alpha}{\alpha}\right)\left(\frac{\gamma}{1+\gamma}\right)\left(\frac{k}{\mu c_{p}}\right) \lambda
$$

For He, $Y=\frac{5}{3}$ For $\mathrm{VO}_{2} \alpha=0.1$

$$
\frac{k}{\mu C_{p}} \cong 0.7, \lambda=\frac{\lambda_{c}}{p}\left(\frac{T}{273}\right)
$$


218

Mean free path in Helium at $1 \mathrm{~atm}$ pressure and room temperature is $0.17 \mu \mathrm{m}$ (see p.70, chapter 10)

$$
\begin{aligned}
& \therefore \text { At } 1000^{\circ} \mathrm{K} . \quad \lambda=\frac{0.17 \times 10^{-6} \times 1000}{300 \ldots} \\
& =0.056 \times 10^{-5} \mathrm{~m} \text {. } \\
& =5.6 \times 10^{-7} \mathrm{~m} \text {. } \\
& \therefore y_{f}=2\left(\frac{\frac{5}{3}}{1+\frac{5}{3}}\right) \times 0.7 \times 5.6 \times 10^{-7} \times\left(\frac{2-\alpha}{\alpha}\right)_{\text {fuel }} \\
& =\frac{5}{4} \times 0.7 \times 5.6 \times 10^{-7} \times\left(\frac{2-\alpha}{\alpha}\right)_{f u e l} \\
& =4.9 \times 10^{-7} \times\left(\frac{2-0.1}{0.1}\right) \\
& =19 \times 4.9 \times 10^{-7}=93.1 \times 10^{-7} \mathrm{~m} \text {. } \\
& =\underline{9.31} \mu \mathrm{m} \\
& g_{c}=4.9 \times 10^{-7}\left(\frac{2-\alpha}{\alpha}\right)_{\text {cladding }} \\
& =4.9 \times 10^{-7}\left(\frac{2-0.2}{0.2}\right)=9 \times 4.9 \times 10^{-7} \mathrm{~m} \\
& =\underline{4.41} \mu \mathrm{m} \\
& k_{\text {gap }}=15.8 \times 10^{-6} \times(1000)^{0.79}=15.8 \times 10^{-6} \times 235 \\
& =3.72 \times 10^{-3} \text { watts } / \mathrm{cm}-\mathrm{ec} \\
& \therefore\left|\frac{d T}{d x}\right|=\text { slope }=\frac{q}{k_{\text {gap }}}=\frac{1200}{3.72 \times 10^{-3}} \\
& =3.22 \times 10^{5} \mathrm{o} / \mathrm{cm}
\end{aligned}
$$


$\therefore$ Temperature jump at

$$
\begin{aligned}
\text { fuel end } & =g_{f} \times \text { slope } \\
& =9.31 \times 10^{-4} \mathrm{~cm} \times 3.22 \times 10^{5} \mathrm{o} / \mathrm{cm} \\
& =300^{\circ} \mathrm{C}
\end{aligned}
$$

Temperature jump at

$$
\begin{aligned}
\text { cladding end } & =g_{c} \times \text { slope } \\
& =4.41 \times 10^{-4} \times 3.22 \times 10^{5}{ }^{\circ} \mathrm{C} \\
& =142^{\circ} \mathrm{C}
\end{aligned}
$$


220

Problem 10.10

We have from Eq. (10.94) for overall heat transfer coefficient $U$,

$$
\begin{aligned}
\frac{1}{U} & =\frac{1}{h_{\text {gap }}}+\frac{t_{c}}{k_{c}}+\frac{1}{h_{\text {cocinnt }}} \\
& =1+\frac{1}{9}+\frac{1}{12}=\frac{36+4+3}{36}=\frac{43}{36} \\
\therefore U & =0.84 \text { Watts } / \mathrm{cm}^{2}-{ }^{\circ} \mathrm{C} .
\end{aligned}
$$

$H_{\text {eat }}$ flux $=U\left(T_{s}-T_{\text {coolant }}\right)=\frac{P}{2 \pi R_{C}}$

( $R_{c}$ - outside radius of cladding)

$$
\begin{aligned}
& \therefore q= \frac{800}{\pi(0.7)}=0.84\left(T_{s}-500\right) \\
& \therefore T_{s}=500+433=933^{\circ} \mathrm{C} . \\
& \int_{5}^{T_{m p}}=\int_{0}^{T_{m p}} k d T-\int_{0}^{T_{s}} k d T=93-40 \\
&=53 \text { watts } / \mathrm{km}
\end{aligned}
$$

[using Eq. (10.66) and Fig. (10.40)]

From Eq. (10.65) with $T^{*}=T_{\text {mp }}$

$$
\int_{T_{s}}^{T_{m p}} k d T=\frac{P}{4 \pi}\left[1-\left(\frac{r_{m}}{R}\right)^{2}\right]
$$


221

$$
\begin{array}{r}
\therefore\left(\frac{Y_{m}}{R}\right)^{2}=1-\frac{4 \pi}{P} \int_{T_{S}}^{T_{M P}} k \\
=1-0.83=0.17 \\
\therefore\left(\frac{r_{m}}{R}\right)=\sqrt{0.17}=0.412
\end{array}
$$


222

CHAPTER 11

Problem 11.1

For the gaseous state $\mathrm{H}_{2} \mathrm{O} / \mathrm{H}_{2}$ equilibrium:

$$
\begin{aligned}
& \mathrm{H}_{2}(\mathrm{~g})+\frac{1}{2} \mathrm{O}_{2}(\mathrm{~g}) \rightleftharpoons \mathrm{H}_{2} \mathrm{O}(\mathrm{g}) \\
& \text { At } 1500^{\circ} \mathrm{K}, \\
& \Delta G_{\mathrm{H}_{2} \mathrm{O}}^{0^{*}}=-58.9+13.1 \times 1.5=-39.2 \mathrm{kcal} / \text { mole }
\end{aligned}
$$

* This value is given in metric system of units in the problem statement.

$$
\Delta G_{\mathrm{H}_{2} \mathrm{O}}^{\circ}=-R T \ln K_{\mathrm{H}_{2} \mathrm{O}}=-R T \ln \left[\frac{P_{\mathrm{H}_{2} \mathrm{O}}}{P_{\mathrm{H}_{2}} \sqrt{\mathrm{P}_{2}}}\right]
$$

For $\frac{P_{\mathrm{H}_{2} \mathrm{O}}}{\mathrm{P}_{\mathrm{H}_{2}}}=1$,

$$
R T \ln P_{\mathrm{O}_{2}}=2 \Delta G_{\mathrm{H}_{2} \mathrm{O}}^{\circ}=-78.4
$$

$\mathrm{O}_{2}$ equilibrium above $\mathrm{UO}_{2+x}$ at $1500^{\circ} \mathrm{K}$.

$$
\overline{\Delta G}_{\mathrm{O}_{2}}=R T \ln P_{\mathrm{O}_{2}}=\overline{\Delta H}_{\mathrm{O}_{2}}^{*}-\overline{\Delta S}_{\mathrm{O}_{2}}^{*}\left(\frac{T}{10^{3}}\right) \mathrm{kcal} / \mathrm{mole}
$$

* These values are given in metric units in the problem statement.

Using $\mathrm{RTPl}_{\mathrm{P} \mathrm{O}_{2}}$ value from eq. (1), we get,

$$
\begin{aligned}
-78.4 & =-125+1.6 \times 10^{4} x-1.5\left(-29+6.6 \times 10^{3} x\right) \\
& =-125+43.5+1.6 \times 10^{4} x-0.99 \times 10^{4} x .
\end{aligned}
$$


223

$$
\begin{aligned}
\therefore \quad-78.4 & =-81.5+0.61 \times 10^{4} x \\
\therefore \quad x & =\frac{3.1}{0.61 \times 10^{4}} \\
& =5.1 \times 10^{-4}
\end{aligned}
$$

The stoichiometry is:

$$
\mathrm{NO}_{2.0005}
$$

$\Theta$ 
224

Problem 11.2

$$
\begin{aligned}
& P_{\mathrm{O}_{2}}=\text { constant (with radius) } \\
& \overline{\Delta G}_{\mathrm{O}_{2}}=R T \ln P_{\mathrm{O}_{2}}=\overline{\Delta H}_{\mathrm{O}_{2}}-T \overline{\Delta S}_{\mathrm{O}_{2}}^{*}
\end{aligned}
$$

* These values are given $=-65+120 R T x$ $\left.\begin{array}{c}\text { in metric units in the } \\ \text { problem statement. }\end{array}\right]$ ( $T$ in unite of $10^{3} \mathrm{~K}$ )

$$
\therefore \quad x=\frac{1}{120}\left\{\ln P_{\mathrm{O}_{2}}+\frac{65}{R T}\right\}
$$

Parabolic Profile:

$$
\frac{T-T_{s}}{T_{0}-T_{s}}=1-\left(\frac{\gamma}{R}\right)^{2}
$$

a) Average value of $x=\bar{x}$

$$
\overline{\bar{x}}=\frac{1}{\pi R^{2}} \int_{0}^{R} 2 \pi r x(r) d r
$$

let $\eta=\left(\frac{r}{R}\right)^{2}$; then $T=T_{s}+\left(T_{r}-T_{s}\right)(1-\eta)$

and

$$
\begin{aligned}
\bar{x}=\int_{0}^{1} x(\eta) d \eta=\frac{1}{120} \int_{0}^{1} & {\left[\ln P_{c_{2}}+\frac{65}{R T_{5}\left[1+\frac{T_{0}-T_{5}}{T_{5}}(1-\eta)\right]}\right\} d \eta } \\
& {[u \operatorname{sing}(1) \text { and }(2)] }
\end{aligned}
$$

Integrating and solving for $l_{n} P_{c_{2}}$, we obtain:

$$
\ln P_{O_{2}}=120 \bar{x}-\frac{65}{R\left(T_{0}-T_{5}\right)} \ln \left(\frac{T_{5}}{T_{5}}\right)
$$


225

$$
\begin{aligned}
\bar{x} & =.05 \\
\therefore \quad \ln P_{O_{2}} & =120 \times .05-\frac{65}{1.98 \times 1.5} \ln (2.5) \\
& =-14.1 \\
\therefore P_{O_{2}} & =7.5 \times 10^{-7} \mathrm{~atm}
\end{aligned}
$$

b)

$$
\begin{aligned}
& \text { From eq. (1) } \\
& x=\frac{1}{120}\left\{\ln P_{C_{2}}+\frac{65}{R} \frac{1}{T}\right\} \\
& =-\frac{14.1}{120}+\frac{65}{120 \times 1.98} \frac{1}{T} \quad(T \text { in units of } \\
& =-0.1175+\frac{0.274}{T}
\end{aligned}
$$

$$
\left.10^{3} \circ k\right)
$$

\begin{tabular}{|c|c|c|c|}
\hline$\gamma / R$ & $\eta$ & $T / 10^{3}(0 \mathrm{~K})$ & $x$ \\
\hline 0 & 0 & 2.50 & $(-0.0079)$ \\
0.2 & 0.04 & 2.44 & $(-0.0052)$ \\
0.4 & 0.16 & 2.26 & $(+0.0037)$ \\
0.6 & 0.36 & 1.96 & +0.022 \\
0.8 & 0.64 & 1.54 & +0.060 \\
1.0 & 1.00 & 1.00 & +0.156 \\
\hline
\end{tabular}

(Note: The negative values of $x$ are not correct. The $\overline{\Delta G}_{\mathrm{O}_{2}}$ approximation used here is vair only for

$$
x>\sim+.005 \text { ) }
$$




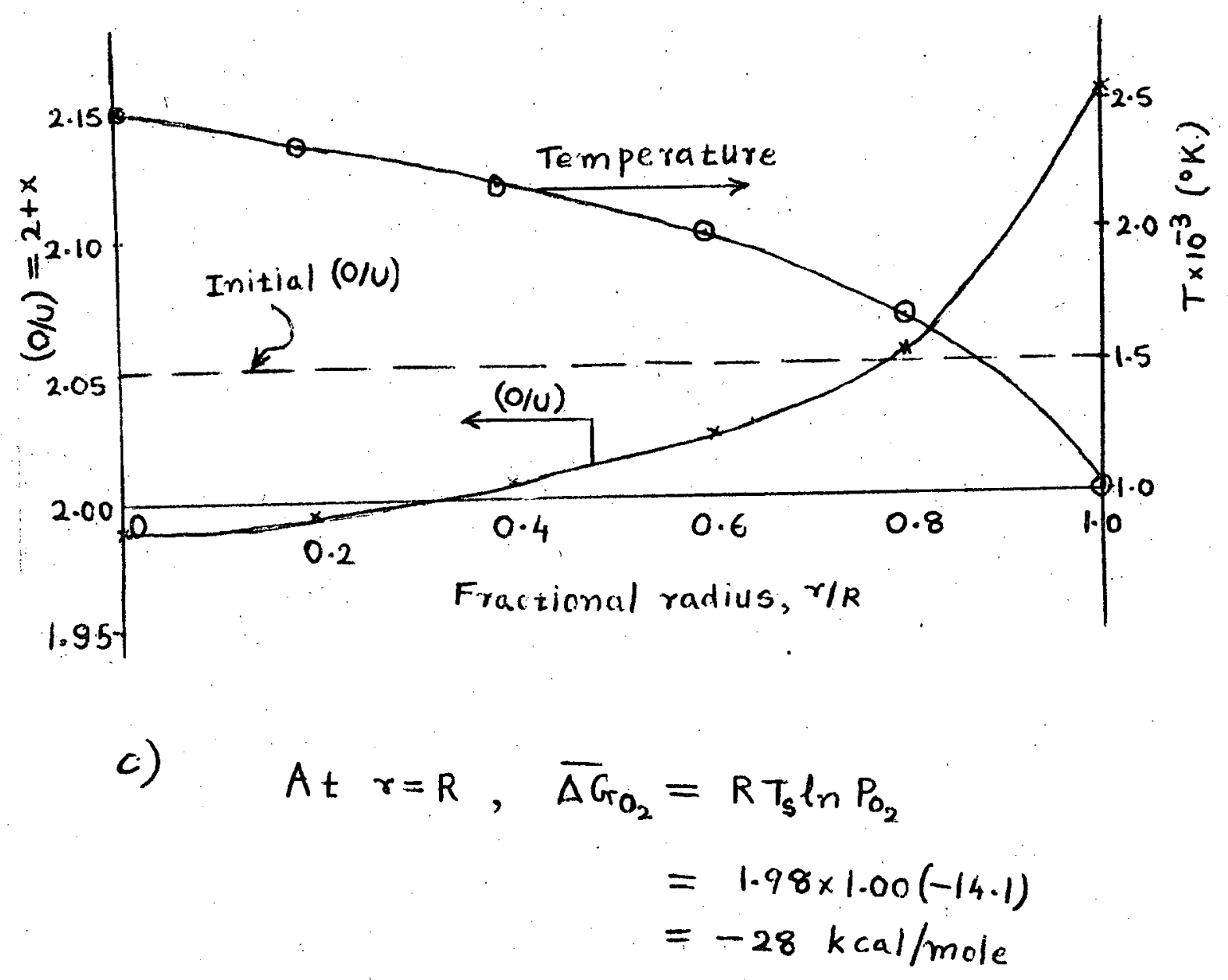

From Fig. 12.7, $\Delta G_{z_{r}}^{0}$ for the reaction,

$$
Z_{r}+O_{2}(g)=Z_{r} O_{2}
$$

at $1000^{\circ} \mathrm{K}$ is $\cong-220 \mathrm{kcal} /$ male

Since $\overline{\Delta G}_{\mathrm{O}_{2}}>\Delta G_{Z_{r}}^{0}$, the cladding should be oxidized thermodynamically. However, as soon as the axide layer is formed the rate of further oxidation reduces drastically as the oxygen has to diffuse ihrough the oxide layer. This prevents catastrophic corrosion of the cladding. 
227

Problem 11.3

(a)

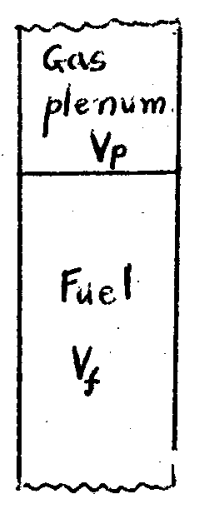

From Fig. 10.4, the volume of Gas plenum

$$
\begin{aligned}
V_{p} & =\frac{\pi}{4}(0.6)^{2} \times 66 \\
& =18.66 \mathrm{~cm}^{3}
\end{aligned}
$$

Volume of fuel

$$
\begin{aligned}
V_{f} & =\frac{\pi}{4}(0.6)^{2} \times[112+30+46] \\
& =\frac{\pi}{4} \times 0.36 \times 188 \\
& =53.16 \mathrm{~cm}^{3}
\end{aligned}
$$

Let us assume that the density of fuel is $90 \%$ of theoretical density.

ie. $\rho_{f}=0.9 \times 10.43 \mathrm{gm} / \mathrm{cm}^{3}$

Then the number of fut atoms in a rad are

$$
\begin{aligned}
N_{f} & =\frac{\rho_{f} V_{f}}{M_{f}} N_{\text {AV }} \\
& =\frac{0.9 \times 10.43 \times 6.02 \times 10^{23} \times 53.16}{270}=1.17 \times 10^{24}
\end{aligned}
$$

If $N_{c}$ are number of carbon atoms, then

$$
\frac{N_{c} M_{c}}{N_{f} M_{f}}=\frac{5}{10^{6}}
$$

(ice: 5 ppm by weight) 
228

$$
\begin{aligned}
\therefore N_{C} & =\frac{5}{10^{6}} \times \frac{270}{12} \times 1.17 \times 10^{24} \\
& =1.32 \times 10^{20}
\end{aligned}
$$

Then the number of carbon atoms in the gas

$$
\text { plenum }=N_{C}^{\prime}=\frac{N_{c}}{2}=0.66 \times 10^{20} \text { atoms }
$$

Let us assume that $\mathrm{CO}$ and $\mathrm{CO}_{2}$ are ideal gases.

Then their partial pressures $p_{c o}$ and $p_{\mathrm{CO}_{2}}$ will be proportional to their numbers of molecules. ire.

$$
\begin{aligned}
\frac{P_{\mathrm{CO}_{2}}}{P_{\mathrm{CO}}}=\frac{N_{\mathrm{CO}_{2}}}{N_{\mathrm{CO}}} \quad \text { or } \frac{P_{\mathrm{CO}_{2}}}{P} & =\frac{N_{\mathrm{CO}_{2}}}{N_{\mathrm{CO}_{2}}+N_{\mathrm{CO}_{\mathrm{O}}}} \\
& =\frac{N_{\mathrm{CO}_{2}}}{N_{C}^{\prime}}
\end{aligned}
$$

where $p$ is the total pressure

$$
\begin{aligned}
& \therefore \quad p V_{p}=N_{c}^{\prime} k T \\
& k=1.38 \times 10^{-16} \mathrm{erg} / \text { molecule }-{ }^{\circ} \mathrm{K} \\
& T \cong 900^{4} \mathrm{~K}, 1 \mathrm{~atm}=1.0133 \times 10^{6} \text { dynes } / \mathrm{cm}^{2} \\
& \therefore p=\frac{N_{c}^{\prime} k T}{V_{p}}=\frac{0.66 \times 10^{20} \times 1.38 \times 10^{-16} \times 900}{18.66}=0.44 \times 10^{6} \mathrm{dyod}_{\mathrm{cm}}^{2} \\
&=
\end{aligned}
$$


$228 a$

(b)

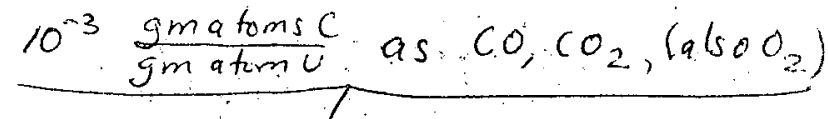

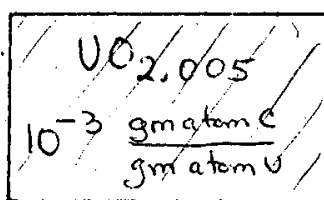

$$
\begin{aligned}
& \longrightarrow \frac{15 \text { gas }}{y / 0 k+\alpha / 7}
\end{aligned}
$$

Oxygen balance:

(neglect oxygen in $\mathrm{O}_{2}$ )

Basis I gm mole $\mathrm{UO}_{2,005}$

initial $0=2.005 \mathrm{gm}$ atoms 0 in fuel

finial $0 \Rightarrow$ infuel $=2+x \mathrm{gm}$ atoms 0

in gas, let $\gamma \equiv\left(\mathrm{Pco}_{2} / \mathrm{PCO}_{\mathrm{O}}\right)$

$$
\begin{gathered}
N_{c}=\text { gm atoms carbon per mole } \mathrm{VO}_{2} \\
N_{c}=44 \times 10^{-6} \times \frac{270}{12}=10^{-3}
\end{gathered}
$$

Let

$f \equiv$ fraction of gas as $\mathrm{CO}_{2}$

$$
f=\frac{P_{\mathrm{CO}_{2}}}{P_{\mathrm{CO}}+P_{\mathrm{CO}_{2}}}=\frac{\gamma}{\gamma+1}
$$

Now,

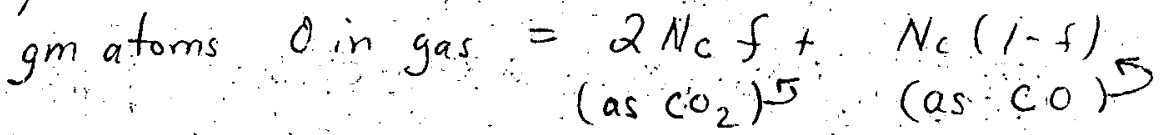

$$
\begin{aligned}
& =N_{c}\left(\frac{2 \gamma}{1+\gamma}+\frac{1}{i+\gamma}\right) \\
& =\left(\frac{2 \gamma+1}{\gamma+1}\right) N_{c} \\
& \therefore 2,005=(2+x)+\left(\frac{2 \gamma+1}{\gamma+1}\right) \times \\
& \text { til } 0 \text { final } 0 \\
& \text { infuel in fuel o incas as } \mathrm{CO}, \mathrm{CO}_{2}
\end{aligned}
$$


$228 \mathrm{~b}$

or,

$$
0.005=x+\left(\frac{2 \gamma+1}{\gamma+1}\right) 10^{-3}
$$

Equlibrium

gos phase:

$$
\begin{array}{cl}
C O+\frac{1}{2} O_{2}=C_{2} \quad \Delta G_{C}^{\circ} & =-282+86.8 \mathrm{~T} / 10^{3} \\
& \text { in }[\mathrm{kJ} / \mathrm{mole}] \\
= & -151.8 @ 1500^{\circ} \mathrm{k}
\end{array}
$$

$\frac{P_{\mathrm{CO}_{2}} / P_{C \mathrm{C}}}{\sqrt{P_{C}}}=e^{-\Delta G_{C}^{\circ} / R T}$

or, $R T \ln \gamma=\frac{1}{2}{\overline{\Delta G_{0}}}-\Delta G_{C}^{\circ}$

$$
\overline{\Delta G}_{\mathrm{O}_{2}}=R T \sin \mathrm{PO}_{2}
$$

fuel oxygen potential:

$$
\begin{gathered}
\overline{\Delta G}_{O_{2}}=\overline{\Delta H}_{O_{2}}-T{\overline{\Delta S O_{2}}} \\
\left.\overline{\Delta G}_{2}=-523+121\left(\frac{T}{10^{3}}\right)+\left(6.7 \times 10^{4}-2.8 \times 10 \frac{4 T}{10^{3}}\right)\right) \times \\
\text { at } T=1500 \mathrm{~K} \\
\overline{\Delta G}_{2}=-341.5+2.5 \times 10^{4} \times\left[\frac{\mathrm{kJ}}{\text { mole }}\right]
\end{gathered}
$$

Now, using $\Delta G_{C}^{c} \& \overline{\Delta G}_{O_{2}}$ in (2), obtain

$$
\ln \gamma=-1.52+10^{3} x
$$

Combine (1) \& (3)

$$
5=\ln \gamma+1.52+\left(\frac{2 \gamma+1}{\gamma+1}\right)
$$

Solving (4) $y=5$ Substititing (3) yields $x=0,003$ 
229

Problem 11.4

$$
\left(U_{0.8} P_{u_{0.2}}\right) O_{1.98}
$$

Since it is a hypo stoichiometric mixed oxide,

$$
\begin{aligned}
& V_{u}=4 ; V_{P_{u}}=4-\frac{2 x}{q} \quad \text { [from Eq .(11.15)] } \\
& q=0.2, \quad x=0.02 \quad \text { (since } 0 / m=1.78 \text { ) } \\
& \therefore V_{P_{u}}=4-\frac{0.04}{0.2}=3.80
\end{aligned}
$$

From Fig.(11.3) using Saved and Roberts plot (as it corresponds to a temperature range $1400-1700^{\circ} \mathrm{C}$. Which is closer to $2241^{\circ} \mathrm{C}$ than Martin and MaIner plot temperature range)

$$
\overline{\Delta S}_{\mathrm{O}_{2}}^{*}=-55 \text { eu (for } V_{\mathrm{P}_{4}}=3.8 \text { ) }
$$

From Fig. (11.4) $\overline{\Delta H}_{\mathrm{O}_{2}}^{*}=-220 \mathrm{kcal} /$ mole

$$
\begin{aligned}
& \therefore \overline{\Delta G} * \text { [saved \& Robertsplot] } \\
& \therefore \overline{\Delta G}_{O_{2}}=\Delta \bar{H}_{\mathrm{O}_{2}}-\overline{\Delta S}_{\mathrm{O}_{2}}\left(T / 10^{3}\right) \quad\left[\text { from } \mathrm{Eq}_{\mathrm{q}(11.14)]}\right. \\
& =-220+55 \times 2.241 \\
& \cong-220+123=-97 \mathrm{keal} / \mathrm{mole}
\end{aligned}
$$

Then from Eq: (11.4)

$$
R T \ln P_{O_{2}}=\overline{\Delta G} O_{2}=-97 \mathrm{kcal} / \mathrm{mole}_{\mathrm{l}}
$$

( These values are given in metric units in the text.) 
230

For atomic oxygen

$$
\frac{1}{2} O_{2}(g)=O(g)
$$

Equilibrium requires

$$
\begin{aligned}
K_{p} & =\frac{P_{0}}{\sqrt{P_{O_{2}}}} \\
\therefore \quad \ln K_{p} & =\ln P_{0}-\frac{1}{2} \ln P_{O_{2}} \\
\therefore \quad \ln P_{0} & =\ln K_{P}+\frac{1}{2} \ln P_{O_{2}}
\end{aligned}
$$

From Table 11.2 , for the reaction

$$
O_{2}(g)=20(9)
$$

$\Delta H^{*}=122.5 \mathrm{keal} /$ mole, $\Delta S^{*}=32.2 \mathrm{H}$.

$$
\begin{aligned}
& \therefore \quad K=\frac{P_{0}^{2}}{P_{O_{2}}}=K_{P}^{2}=e^{-\Delta G^{\circ} / R T} \\
& \Delta G^{0}=\Delta H^{*}-\Delta s^{*}\left(T / 10^{3}\right) \quad\left[K_{p} \text {-defined in eq. (2) }\right] \\
& =12.2 .5-2.241 \times 32.2 \\
& =122.5-72.1=50.4 \mathrm{kcal} / \mathrm{mcle} \\
& \therefore \quad K_{p}=e^{-\frac{\Delta G_{c}}{2 R T}}=e^{-\frac{50.4}{2 \times 1.78 \times 2.241}} \\
& \therefore \ln K_{p}=-\frac{50.4}{2 \times 1.98 \times 2.241}
\end{aligned}
$$

[ These values are given in metric units in the text.] 
231

From eq. (3),

$$
\begin{aligned}
\ln P_{0} & =\ln K_{P}+\frac{1}{2} \ln P_{O_{2}} \\
& =-\frac{50.4}{2 \times 1.98 \times 2.241}-\frac{97}{2 R T} \\
& =-\frac{50.4+97}{2 \times 1.98 \times 2.21} \\
& =-\frac{147.4}{8.86}=-16.6 \\
\therefore \log P_{0} & =-\frac{16.6}{2.303} \\
& =-\frac{7.21}{10^{-7.21}} \mathrm{~atm} \\
\therefore \quad P_{0} & =\frac{11)]}{}
\end{aligned}
$$


232

Problem 11.5

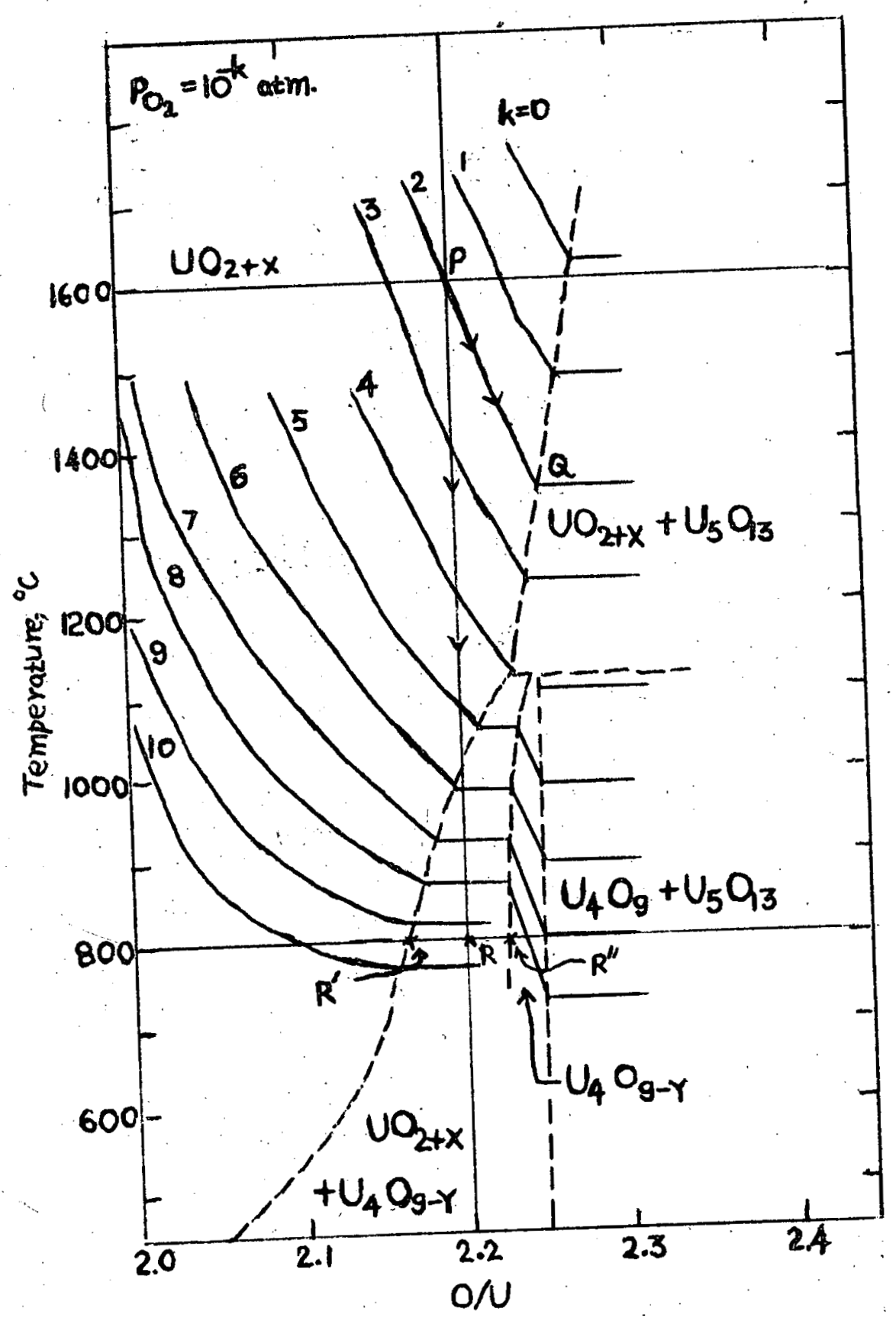


233

$\mathrm{VO}_{2.2}$ at $1600^{\circ} \mathrm{C}$.

(a)

$$
T=1600+273=1873^{\circ} \mathrm{K} .
$$

$$
\overline{\Delta G}_{\mathrm{O}_{2}}={\overline{\Delta \mathrm{H}_{2}}}_{2}(\mathrm{x})-\left(\frac{T}{10^{3}}\right){\overline{\Delta S_{\mathrm{O}_{2}}}}_{(x)}
$$

From Fig. 11.10, for $x=0.2$

$$
\begin{aligned}
& \overline{\Delta H}_{\mathrm{O}_{2}}=-315 \mathrm{~kJ} / \mathrm{mole} \\
\overline{\Delta S_{O_{2}}} & =-126 \mathrm{~J} / \mathrm{mole}^{0} \mathrm{~K} . \\
\therefore \overline{\Delta G O_{2}} & =-315+1.873 \times 126 \\
= & -79 \mathrm{~kJ} / \mathrm{mole} \\
\therefore P_{\mathrm{O}_{2}}=\exp \left(\frac{\overline{\Delta G_{O_{2}}}}{\mathrm{RT}}\right) & =\exp \left(-\frac{79}{8.31 \times 1.873}\right) \\
& =6.2 \times 10^{-3} \mathrm{~atm} .
\end{aligned}
$$

From Fig.11.6, $P_{\mathrm{O}_{2}}=10^{-2} \mathrm{~atm}$.

(b) Following $P_{O_{2}}=10^{-2} \mathrm{~atm}$ isobar, $\mathrm{U}_{5} \mathrm{O}_{13}$ precipitates out at point $Q$. (se effing.) $T=1350^{\circ} \mathrm{C}$. The other equilibrium phase is $\mathrm{UO}_{2.256}$. (last digit approximate)

(c) Cooling at constant $0 / U$ to $800^{\circ} \mathrm{C}$, (point R) the two phases present are $\mathrm{UO}_{2 \cdot 17}$ at $R^{\prime}$ and $\mathrm{UO}_{2.23}$ at $\mathrm{R}^{\prime \prime}$.

Relative amounts:-

Basic -1 mole of $\mathrm{UO}_{2-2}$ initially. 
234

Let $f=$ fraction of $\mathrm{UO}_{2.17}$ in the mixture oxygen balance: $\quad 2.17 f+2.23(1-f)=2.2$

$$
\begin{gathered}
\therefore f=0.5 \\
P_{O_{2}} \cong 5 \times 10^{-10} \mathrm{~atm} \text { at this temp. }
\end{gathered}
$$




\section{CHAPTER 12}

Problem 12.1

Elemental yield $=\frac{\text { Number of Zr atoms at time } T}{\text { Number of henvy metal atomsfissioned }}$

$$
=\frac{1}{\dot{F} T} \sum_{j=90}^{99} N_{z_{v}, j}
$$

$A=90$

Neglecting species with half lives shorter than $\sim$ day, the decay chain reduces to fission $\rightarrow 28 y-5 x^{90} \longrightarrow$ stable $z_{r}^{90}$

\subsection{7}

The growth of $\mathrm{Sr}^{90}$ is governed by ;

$$
\begin{aligned}
& \frac{d N_{s_{r}}}{d t}=y_{g_{0}} \ddot{F}-N_{s_{r}} \lambda_{s_{r}} ; \text { and } N_{s_{r}}(0)=0 \\
& \therefore N_{S_{r}}(T)=\frac{Y_{g_{0}} \dot{F}}{\lambda_{S_{r}}}\left(1-e^{-\lambda_{s r} T}\right) \\
& \lambda_{\text {Sr70 }}=\frac{0.693}{28 \times 365}=0.678 \times 10^{-4} \text { days }^{-1} ; T=100 \text { days; } \\
& Y_{90}=5.77 \\
& \therefore N_{S T} 90(T)=\frac{5.77 F}{0.678 \times 10^{-4}}\left(1-e^{-0.678 \times 10^{-4} \times 10^{2}}\right) \\
& =851 \times 10^{2} \dot{F} \times 0.00675=574.42 \dot{F} \\
& \therefore N_{z_{r}}{ }^{90}(T)=Y_{90} \dot{F} T-N_{s_{r}}=(577-574.42) \dot{F}=2.57 \dot{F}
\end{aligned}
$$


236

$A=91$

The decay chain reduces to:

fission $\rightarrow 58 d-y^{91} \rightarrow$ stable $z_{r}^{91}$

$$
x_{91}=5.4
$$

and fission $\rightarrow$ stable $z_{r}^{91} \quad\left(y_{91}^{\prime}=5.84-5.4=0.44\right)$

For the first reaction:

$$
\begin{aligned}
& \frac{d N_{y}}{d t}=\gamma_{q 1} \dot{F}-\lambda_{y} N_{y} \text {, and } N_{y}(0)=0 \text { gives } \\
& N_{y q 1}(T)=\frac{X_{91} \dot{F}\left(1-e^{-\lambda_{y}{ }^{91} T}\right)}{\lambda_{y 91}} ; \lambda_{y^{91}}=\frac{0.693}{58 d}=0.0119 \mathrm{day}^{-1} \\
& =\frac{5.4 \dot{F}}{0.0119}\left(1-e^{-1.19}\right)=F \times 453.78 \times 0.6958=315.7 \dot{F} \\
& \therefore N_{z_{\gamma} g^{91}}(T)=Y_{q 1} \dot{F} T-N_{y^{g i}}(T)=(540-315.7) \dot{F}=224.3 \dot{F}
\end{aligned}
$$

From the second reaction:

$$
N_{z_{r}}{ }^{11}(T)=\gamma_{a 1}^{\prime} \cdot \dot{F} T=44 \dot{F}
$$

So:

$$
N_{Z_{\gamma}}{ }^{91}(T)=(224.3+44) \dot{F}=268.3 \dot{F}
$$

$A=92$ The decay chain reduces to:

fission $\longrightarrow$ stable $z_{r}^{92}$

$$
y=6.03
$$

$$
\therefore N_{z_{\gamma}^{92}}(T)=Y_{z_{r}^{92}} F T=603 \dot{F}
$$

$A=93$ The decay chain reduces to:

$$
\begin{aligned}
& \text { fission } \rightarrow \text { (almost) stable } Z_{\gamma}^{93} \quad(\gamma=6.45) \\
& \therefore N_{Z_{\gamma}{ }^{93}(T)}=Y_{Z_{r}{ }^{93}} \dot{F} \cdot T=645 F
\end{aligned}
$$


237

$A=94$

The decay chain reduces to:

$$
\begin{aligned}
& \text { fission } \longrightarrow \text { stable } Z_{r}{ }^{94} \quad(Y=6.4) \\
& \therefore N_{z r}{ }^{94}(T)=Y_{Z_{r}}{ }^{94} F T=640 \dot{F}
\end{aligned}
$$

$A=95$

The decay chain reduces to:

fission $\longrightarrow 65 \mathrm{~d}-\mathrm{Zr}_{r}^{95} \longrightarrow \mathrm{Nb}^{95}$$$
\lambda_{z_{r}{ }^{95}=\frac{0.693}{65 d}=0.01066 \mathrm{day}^{-1}}
$$$$
\frac{d N_{Z_{\tau}}^{95}}{d t}=Y_{95} \dot{F}-N_{Z_{7} 95} \lambda_{Z_{\gamma}{ }^{95}} ; N_{z_{7} 95}(0)=0 \text { gives }
$$$$
N_{z r 95}(T)=\frac{6.2 \dot{F}}{0.01066}\left(1-e^{-0.01066 \times 100}\right)=381.3 \dot{F}
$$

$A=96$

The decay chain reduces to:

$$
\begin{aligned}
& \text { fission } \longrightarrow z_{r}{ }^{96} \quad(Y=6.33) \\
& \therefore N_{z_{r} 96}(T)=Y_{96} \dot{F} T=633 \dot{F}
\end{aligned}
$$

$A=97$

The decay chain reduces to:

$$
\begin{aligned}
& \text { fission } \longrightarrow 17 \mathrm{~h}-\mathrm{Z}_{r}{ }^{97} \longrightarrow \mathrm{N}_{b} 97 \\
& (y=5.9) \\
& \lambda_{z_{r} 97}=\frac{0.693}{17 h_{r}}=\frac{0.693}{\frac{17}{24} \text { day }}=0.978 d a y^{-1}
\end{aligned}
$$

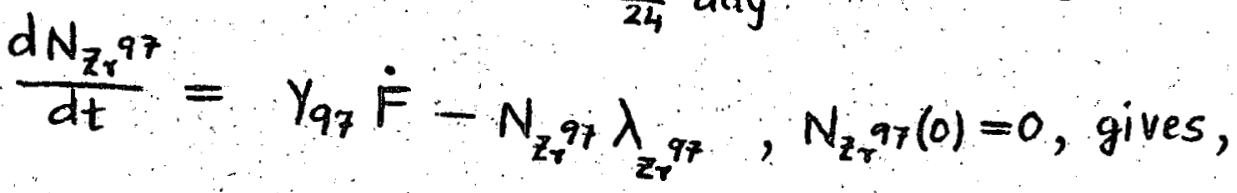

$$
\begin{aligned}
& N_{Z_{r}}(T)=\frac{5.9 F}{0.978}\left(1-e^{-97.8}\right)=6.03 F
\end{aligned}
$$




$$
\begin{array}{ll}
A=98 & \begin{array}{l}
\text { Neglect } N_{z_{r}^{98}} \text { as } N_{z_{r}^{98}}(T) \text { will be } \\
\text { very small }
\end{array} \\
A=99 . & \text { Neglect } N_{z_{r}^{99}(T)}
\end{array}
$$

Therefore:

$$
\begin{aligned}
& \text { Elemental yield }=\frac{1}{\dot{F} T} \sum_{A=90}^{99} N_{Z_{r}^{A}}(T) \\
& Y_{z_{r}}=\frac{1}{100 F}(2.57+268.3+603+645+640+ \\
&=\frac{3179.2 \dot{F}}{100 \dot{F}}=31.79 \% \\
& \therefore \quad Y_{Z_{r}}=31.79 \% \quad \text { (after } 100 \text { days of } \\
& \text { irradiation) }
\end{aligned}
$$


239

Problem 12.2

a) $10^{12}$ oxide phase particle $/ \mathrm{cm}^{3}$ of fuel.

$\therefore$ The volume occupied by one particle is

$$
\begin{aligned}
& \frac{4}{3} \pi R^{3}=10^{-12} \mathrm{~cm}^{3} . \\
& \therefore \quad R=0.62 \times 10^{-4} \mathrm{~cm} .
\end{aligned}
$$

b) Since $Y_{Z r}>Y_{\mathrm{Ba}-s_{r}}$, growth of oxide particles is controlled by rate of diffusion of $\mathrm{Ba}-\mathrm{Sr}$ to oxide particle surface.

Diffusion of $\mathrm{Ba}-\mathrm{Sr}$ in fuel annulus

$$
\dot{F}=\text { fissions } / \mathrm{cm}^{3}-\mathrm{sec} ; \quad Y_{B a-5 r}=\begin{aligned}
& \text { elemental yield } \\
& \text { of } B a-s r
\end{aligned}
$$

Quasi -stationary approximation is used since concentration of $\mathrm{Ba}-\mathrm{Sr}$ in fuel is a slowly increasing function of time.

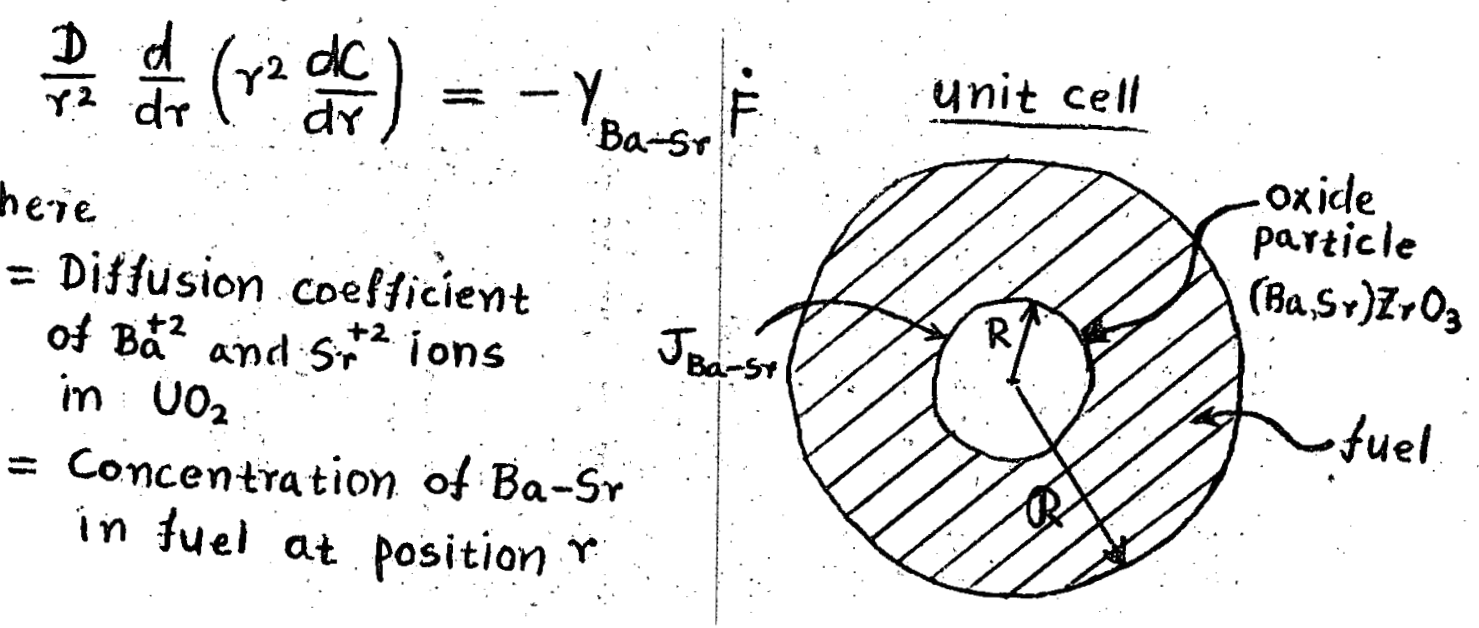


240

Boundary Conditions:-

i) $C(R)=0$ (oxide particle a perfect sink)

ii) $\left(\frac{d C}{d r}\right)_{R}=0$ (by definition of "unit cell")

Solution Let $u=r c$, then:

$$
\begin{array}{r}
\frac{d^{2} u}{d r^{2}}=-\left(\frac{Y_{B a-s r} \dot{F}}{D}\right) r=-k r \\
\text { where } k=\frac{Y_{B a-s r}}{D} \dot{F}
\end{array}
$$

Integrating twice we get

$$
u(r)=-\frac{1}{6} k r^{3}+A r+B ; \quad \frac{d u}{d r}=-\frac{1}{2} k r^{2}+A
$$

B.C. i) $\quad U(R)=0$

ii) $\frac{1}{R}\left(\frac{d u}{d r}\right)_{R}-\frac{u(R)}{R^{2}}=0$

From i) $\quad 0=-\frac{1}{6} k R^{3}+A R+B$

From ii) $\quad 0=-\frac{1}{2} K R+\frac{A}{R}+\frac{1}{6} k Q-\frac{A}{R}-\frac{B}{R^{2}}$

$$
\begin{gathered}
\therefore \quad B=-\frac{1}{3} K R^{3}, A=\frac{\frac{1}{3} K R^{3}+\frac{1}{6} K R^{3}}{R} \\
\therefore \quad C(r)=\frac{U(r)}{r}=-\frac{1}{6} k r^{2}+\frac{1}{6} k R^{2}+\frac{1}{3} k \frac{R^{3}}{R} \\
-\frac{1}{3} k \frac{R^{3}}{r}
\end{gathered}
$$


241

c) Growth of oxide particle

$J_{B a-s r}=F l u x$ of $B a-S r$ ions to outer surface of oxide particle, atoms $/ \mathrm{cm}^{2}-\mathrm{sec}$ :

$$
V_{\text {ox }} \cong 7 \times 10^{-23} \mathrm{~cm}^{3} / \mathrm{atom}
$$

$=$ Volume of oxide phase per atom of $\mathrm{Ba}$ or $\mathrm{Sr}$.

Number of atoms of $\mathrm{Ba}-\mathrm{Sr}$ in an oxide

$$
\text { particle }=\frac{\frac{4}{3} \pi R^{3}}{V_{0 x}}
$$

The rate of influx of $\mathrm{Ba}-\mathrm{Sr}$ atoms in the oxide particle $=4 \pi R^{2} J_{B a-S r}$

$$
\begin{aligned}
\therefore \quad \frac{d}{d t}\left[\frac{\frac{4}{3} \pi R^{3}}{V_{0 x}}\right] & =4 \pi R^{2} J_{B a-S r} \\
\therefore \quad \frac{d R}{d t} & =V_{\text {Ox }} J_{B a-S r}
\end{aligned}
$$

$$
\begin{aligned}
J_{B_{a-S r}=D\left(\frac{d C}{d r}\right)_{R}} & =\frac{D}{R}\left[\left(\frac{d u}{d r}\right)_{R}-\frac{u}{R}\right] \\
& =D\left[-\frac{1}{3} K R+\frac{1}{3} K \frac{R^{3}}{R^{2}}\right] \\
& =\frac{K D}{3 R^{2}}\left[Q^{3}-R^{3}\right] \cong \frac{K D R^{3}}{3 R^{2}}
\end{aligned}
$$$$
K D=\gamma_{B a-S \gamma} \dot{F}
$$ 
242

$$
\therefore \frac{d R}{d t}=\frac{Y_{B a-S} \cdot \dot{F} R^{3} V_{O x}}{3 R^{2}}
$$

Integrating with $R(0)=0$, we get,

$$
R^{3}=V_{0 x} Y_{B a-5 x} \dot{F} t R^{3}
$$

This result could be arrived at by assuming that all Ba-sr created in volume $\frac{4}{3} \pi R^{3}$ collect in sphere of radius $R$.

As a function of burnup:

$$
\beta=\frac{\dot{F} t}{N_{H M}^{0}}, \text { where } N_{H M}^{0}=\begin{aligned}
& \text { Heavy meta atom } \\
& \text { density of fuel } \\
& \text { at time } t=0 .
\end{aligned}
$$

Then

$$
\frac{R}{R}=\left(V_{O x} Y_{B M-S Y} N_{H M}^{0} P\right)^{1 / 3}
$$

d) Inserting numbers:

$$
\begin{aligned}
\therefore \frac{R}{R} & =\left[7 \times 10^{-23} \times 0.1 \times 2.5 \times 10^{22} \times 0.05\right]^{1 / 3} \\
& =0.26 \\
\therefore R & =0.26 R=0.26 \times 62 \times 10^{-6}=16 \times 10^{-6} \mathrm{~cm} . \\
& =0.16 \mu \mathrm{m}
\end{aligned}
$$

Concentration profile:-

$$
C=-\frac{1}{6} K r^{2}+\frac{1}{3} K \frac{Q^{3}}{R}+\frac{1}{6} K R^{2}-\frac{1}{3} K \frac{R^{3}}{r} .
$$




$$
\text { or } \begin{array}{r}
C(\gamma)=\frac{Y_{B a-5 \gamma} \dot{F}}{6 D}\left[2 R^{3}\left(\frac{r-R}{r R}\right)-\left(r^{2}-R^{2}\right)\right] \\
(r \geqslant R)
\end{array}
$$

or

[Eq. (13.60)]

$\begin{aligned} & \text { atom fraction } \\ & \text { Ba-Sr in fuel }\end{aligned}=\frac{C(r)}{N_{H M}^{0}}=\left(\frac{Y_{B_{a}-S_{r}} \dot{F} R^{2}}{6 N_{H M}^{\circ} D}\right)\left\{2\left[\frac{(\gamma / R)-(R / R)}{(\gamma / R)(R / R)}\right.\right.$

$$
\left.-\left[\left(\frac{r}{R}\right)^{2}-\left(\frac{R}{R}\right)^{2}\right]\right\}
$$

\begin{tabular}{|c|c|}
\hline$\frac{r}{R}$ & $\begin{array}{c}\text { Atom fraction } \\
\text { Ba-sr in fue } \\
\left(\times 10^{9}\right)\end{array}$ \\
\hline 0.26 & 0 \\
0.30 & 2.0 \\
0.40 & 5.2 \\
0.50 & 7.0 \\
0.60 & 8.1 \\
0.70 & 8.8 \\
0.80 & 9.2 \\
0.90 & 9.4 \\
1.00 & 9.5 \\
\hline
\end{tabular}

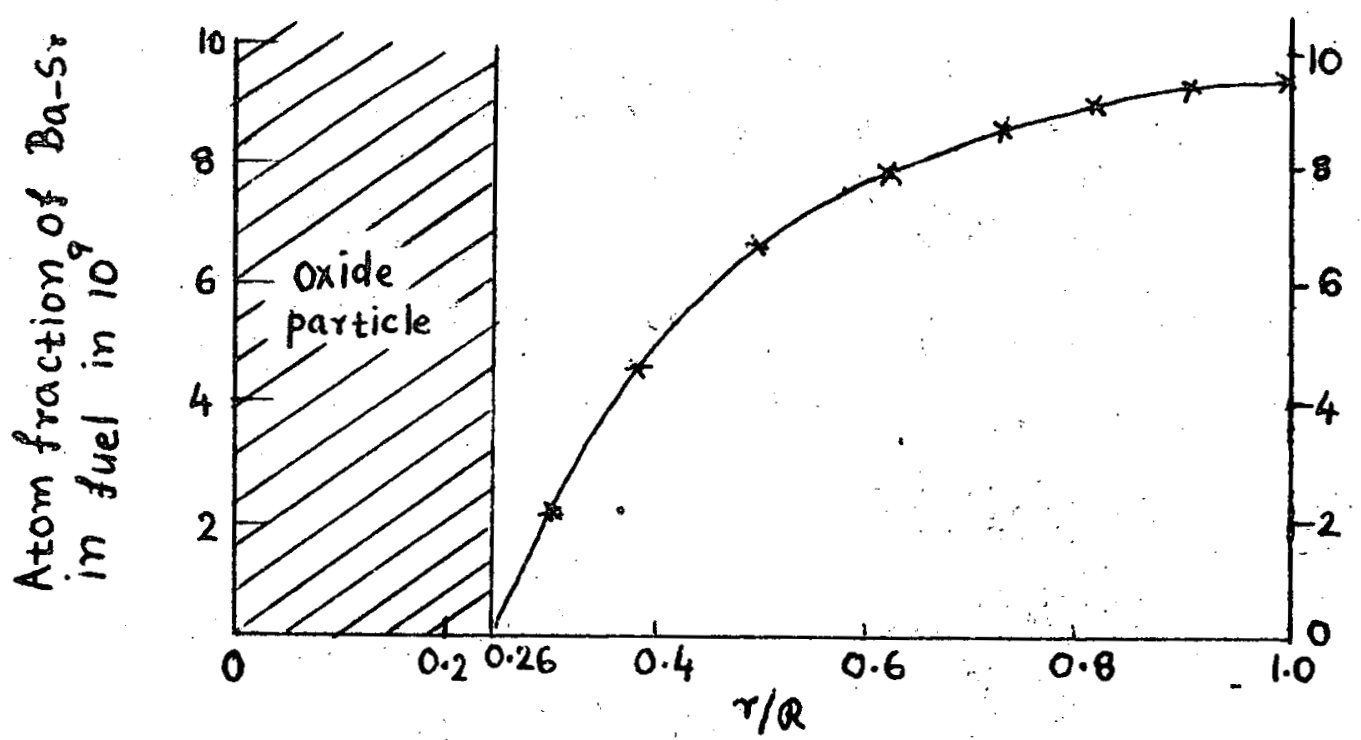


244

Problem 12.3

a)

$$
\begin{aligned}
\overline{\Delta H}_{\mathrm{O}_{2}}^{*} & =-65 \mathrm{kcal} / \mathrm{mole} \\
V_{U} & =2(2+x)=4+2 x \\
\therefore x & =\frac{1}{2}\left(V_{v}-4\right) \\
\overline{\Delta S}_{\mathrm{O}_{2}}^{*} & =-120 \mathrm{Rx} \text { call mole }-{ }^{\circ} \mathrm{K}
\end{aligned}
$$

[* These values are given in metric.

[Sec problem 11.2]

units in the problem statement.]

$$
\begin{aligned}
& =-120 \times 1.98 \times \frac{1}{2}\left(v_{v}-4\right) \\
& =-118.8\left(v_{v}-4\right) \mathrm{cal} / \mathrm{mole}-{ }^{\circ} \mathrm{K} .
\end{aligned}
$$

b) Initial number of atoms:

$$
\begin{aligned}
& \text { uranium }=N_{u}^{0} \\
& \text { oxygen }=\left(\frac{O}{M}\right) N_{U}^{0}=2 N_{U}^{0}=N_{0}^{0}
\end{aligned}
$$

Final number of atoms:

$$
N_{u}=(1-\beta) N_{u}^{0}
$$

Let $N_{1}$ be the number of atoms of +3 valence and $N_{2}$ be the number of atoms of zero valence (element)

Then since $Y=1$ for both fop. 1 and 2

$$
\begin{aligned}
& N_{1}=\beta N_{u}^{0} \\
& N_{2}=\beta N_{v}^{0}
\end{aligned}
$$

charge balance gives,

$$
2 N_{0}^{0}=V_{U} N_{U}+3 N_{1}+O N_{2}
$$


245

$$
\begin{aligned}
& \therefore \quad 2 N_{0}^{0}=V_{u}(1-\beta) N_{u}^{0}+3 \beta N_{u}^{0} \\
& \therefore \quad 2\left(\frac{N_{0}^{0}}{N_{u}^{0}}\right)=V_{u}(1-\beta)+3 \beta \\
& \text { But } \quad \frac{N_{0}^{0}}{N_{u}^{0}}=2 \\
& \therefore \quad V_{u}=\frac{4-3 \beta}{1-\beta} ; \\
& \text { or } \quad V_{u}-4=\frac{4-3 \beta}{1-\beta}-4=\frac{\beta}{1-\beta} \\
& \text { At } \quad 5 \% \text { burnup }, \beta=0.05 \\
& \therefore \quad V_{u}-4=\frac{0.05}{0.95}=0.0526 \\
& \therefore \quad \frac{\Delta S_{0}}{O_{2}}=-118.8 \times 0.0526 \quad[\text { using eq. (2)] } \\
&=-6.25 \text { cal/ mole- } \mathrm{K}
\end{aligned}
$$

$\therefore$ Oxygen potential at $1000^{\circ} \mathrm{K}$ is

$$
\begin{aligned}
\overline{\Delta G}_{\mathrm{O}_{2}} & =\overline{\Delta H}_{\mathrm{O}_{2}}-T \overline{\Delta S}_{\mathrm{O}_{2}} \\
& =-65+\frac{1000 \times 6.25}{10^{3}} \mathrm{kcal} / \mathrm{mole} \\
& =-65+6.25 \mathrm{kal} / \mathrm{mole} \\
& =-58.75 \mathrm{~kJ} / \mathrm{mole} \\
& =-246 \mathrm{k}
\end{aligned}
$$


246

Problem 12.4

a) For ideal solution of Mo in metallic inclusions and of $\mathrm{MoO}_{2}$ in fuel, the distribution of molybdenum is governed by:

$$
K=e^{-\Delta G_{m_{c}}^{0} / R T}=\frac{y_{m_{0}}^{f}}{y_{M_{0}}^{m} P_{O_{2}}}\left[E_{q}(12 \cdot 17)\right]
$$

At $2000^{\circ} \mathrm{K}$

$$
\begin{aligned}
\Delta G_{M_{0}}^{0} & =-574+164 \times 2 \\
= & -246 \mathrm{~kJ} / \text { mole } \\
\therefore \text { Oxygen potential } & =\overline{\Delta G}_{O_{2}}=R T \ln P_{O_{2}} \\
& =\Delta G_{m_{0}}^{0}+R \cdot \ln \left(\frac{y_{m_{0}}^{f}}{y_{m_{0}}^{m}}\right) \\
\operatorname{RT} \ln \left(\frac{y_{m_{0}}^{f}}{y_{M_{0}}^{m}}\right)= & 8.28 \times 2 \times \ln \left(\frac{0.08}{10}\right) \\
= & -80 \mathrm{~kJ} / \text { mole } \\
\therefore \frac{\Delta G_{O_{2}}}{A} & =-246-80=-326 \mathrm{~kJ} / \text { mole }
\end{aligned}
$$

b)

There are $10^{-2} \mathrm{gms}$ of metallic inclusions per $\mathrm{gm}$. of fuel.

or $10^{-2} / 100 \mathrm{gm}$ atoms of metallic inclusions per $1 / 10 \mathrm{~cm}^{3}$ of fuel But $10 \%$ of the inclusions consists of Mo.

$\therefore$ the Noble Metal concentration (gmatom $/ \mathrm{cm}^{3} \mathrm{fuel}$ ) is:

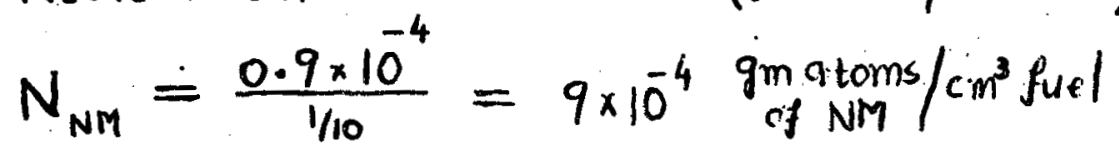


247

$$
\begin{aligned}
N_{u}^{0}+N_{p_{u}}^{0}=\frac{\text { the initial heavy }}{\text { metal concentration }} & =10 \mathrm{gms} / \mathrm{cm}^{3} \times \frac{1}{270 \mathrm{gm} / \mathrm{gmatom}} \\
& =3.7 \times 10^{-2} \frac{\mathrm{gmatom} \text { of } \mathrm{HM}}{\mathrm{cm}^{3} \text { of fuel }}
\end{aligned}
$$

Using Eq. (12.16)

$$
\begin{aligned}
\beta & =\frac{1}{Y_{N M}} \frac{N_{N M}}{\left(N_{U}^{0}+N_{P_{U}}^{0}\right)} \\
& =\frac{1}{0.456} \times \frac{9 \times 10^{-4}}{3.7 \times 10^{-2}}=0.053
\end{aligned}
$$

( $Y_{N M}$ obtained from Table 12.1 , using the value for mixed oxide)

Therefore the burnup $\beta=5.3 \%$

c) If no $M_{0}$ is lost or gained in unit volume, $\left(y_{M_{0}}^{*}\right)^{*}$ and $\left(y_{M_{0}}^{m}\right)^{*}$ would have been the compositions of the fuel and metallic inclusions respectively.

Expected total Mo concentration after $5.3 \%$ burnup from Eq $(12.16)$ is:

$$
\begin{aligned}
N_{M_{0}}^{*} & =(0.206) \times \beta \times\left(N_{u}^{0}+N_{p_{v}^{0}}^{0}\right) \quad(u \text { sing Table 12.1) } \\
& =(0.206) \times(0.053) \times\left(3.7 \times 10^{-2}\right) \quad[\text { using part (b)] } \\
& =4 \times 10^{-4}
\end{aligned}
$$


248

$\left(N_{M_{0}}^{\text {ind }}\right)^{*}=M_{0}$ in metallic inclusions, gmatom $/ \mathrm{cm}^{3}$ fuel

$$
\cong 10^{-3} \frac{\text { gmatoms inclusions }}{\mathrm{cm}^{3} \text { of fuel }} \times\left(y_{m_{0}}^{m}\right)^{*}
$$

[where it is assumed that the metallic inclusions are principally Noble Metals and then the result of part (b) is used.]

$$
\begin{aligned}
\left(N_{m_{0}}^{f}\right)^{*} & =M_{0} \text { in fuel matrix, gmatom } / \mathrm{cm}^{3} \text { fuel } \\
& =3.7 \times 10^{-2} \cdot \frac{\text { gmatom of fuel }}{c m^{3} \text { of fuel }} \times\left(y_{M_{0}}^{f}\right)^{*} \\
\therefore N_{m_{0}}^{*} & =\left(N_{m_{0}}^{\text {incl }}\right)^{*}+\left(N_{m_{0}}^{f}\right)^{*}=10^{-3}\left(y_{M_{0}}^{m}\right)^{*}+3.7 \times 10^{-2}\left(y_{m_{0}}^{*}\right)
\end{aligned}
$$

However, the ratio $\left(y_{m_{0}}^{f}\right)^{*} /\left(y_{m_{0}}^{m}\right)^{*}$ is fixed by $\overline{\Delta G}_{\mathrm{O}_{2}}$ and is the same as the measured value (despite loss or gain of total $M_{0}$ )

$$
\therefore\left(y_{m_{0}}^{*}\right)^{*}=\left(\frac{.08}{10}\right)\left(y_{m_{0}}^{m}\right)^{*}
$$

and

$$
\begin{aligned}
N_{m_{0}}^{*} & =\left(y_{m_{0}}^{m}\right)^{*}\left[10^{-3}+3.7 \times 10^{-2} \times \frac{.08}{10}\right] \\
& =1.3 \times 10^{-3}\left(y_{m_{0}}^{m}\right)^{*} \\
\therefore\left(y_{m_{0}}^{m}\right)^{*}=\frac{N_{m_{0}}^{*}}{1.3 \times 10^{-3}} & =\frac{4 \times 10^{-4}}{1.3 \times 10^{-3}} \\
& =0.31
\end{aligned}
$$

$\therefore$ The metallic inclusions should have contained 
249

about $31 \% M_{0}$ if none of this element had been lost from this spot in the fuel. The fact that only $10 \%$ of the inclusions was Mo means that $\sim 2 / 3$ of the Mo produced by fission at the radial location where the measurements were made was removed by some migration process to other radial positions in the fuel pin. 
250

Problem 12.5

(a) $\quad R T \ln P_{\mathrm{O}_{2}}=\overline{\Delta G}_{\mathrm{O}_{2}}=\Delta \bar{H}_{\mathrm{O}_{2}}-\overline{\Delta S}_{\mathrm{O}_{2}}\left(T / 10^{3}\right)$

$\therefore$ At $T=1000^{\circ} \mathrm{K}$, using the results of problem 12.3 , expressed in metric units

$$
\overline{\Delta G}_{\mathrm{O}_{2}}{ }^{1}=-272+500\left(\mathrm{~V}_{\mathrm{v}}-4\right) \mathrm{kJ} / \mathrm{mole}
$$

Oxygen Balance:-

Let $N_{0}^{0}=$ Initial number of oxygen atoms per unit volume of fuel

$N_{u}=$ Number of uranium atoms/unit volume.

$N_{1}=$ Number of +3 valence fission proiluet atoms/unit volume

$N_{0}^{c}=$ Number of oxygen atoms absorbed per unit area of cladding

charge balance in fuel gives,

$$
\begin{aligned}
& 2\left(N_{0}^{0}-\frac{2 \pi R}{\pi R^{2}} N_{0}^{c}\right)=V_{U} N_{U}+3 N_{1} \ldots(3) \\
& N_{0}^{0}=\left(\frac{0}{M}\right) N_{U}^{0}=2 N_{i}^{0} \quad \text { (for initially } \\
& N_{U}=(1-\beta) N_{u}^{0} \\
& N_{1}=\beta N_{u}^{0}
\end{aligned}
$$

Then eq.(3) gives,

$$
2\left(2-\frac{2}{R} \frac{N_{0}^{c}}{N_{v}^{c}}\right)=(1-\beta) V_{u}+3 \beta \ldots(4)
$$


251

Solving, we get,

$$
V_{u}-4=\frac{\beta}{1-\beta}-\frac{4 N_{0}^{c}}{(1-\beta) R N_{u}^{0}}
$$

Substituting eq .(5) into eq .(2) we get,

$$
\begin{aligned}
& \overline{\Delta G}_{O_{2}}=-272+500\left[\frac{\beta}{1-\beta}-\frac{4 N_{0}^{e}}{(1-\beta) R N_{0}^{c}}\right] \\
& \text { At } T=1000^{\circ} \mathrm{K} \text {, } \\
& \overline{\Delta G}_{\mathrm{O}_{2}}=R T \ln \mathrm{P}_{\mathrm{O}_{2}}=8.314 \ln \mathrm{P}_{\mathrm{O}_{2}} \mathrm{~kJ} / \mathrm{mole} \\
& {[R=8.314 \mathrm{~J} / \mathrm{mole}-0 \mathrm{~K} .]} \\
& \therefore P_{\mathrm{O}_{2}}=\exp \left\{-32.7+60\left(\frac{\beta}{1-\beta}-\frac{4 N_{0}^{e}}{(1-\beta) R N_{v}^{0}}\right)\right\}
\end{aligned}
$$

Cladding corrosion kinetics:-

$$
\begin{aligned}
\quad \frac{d N_{0}^{c}}{d t} & =C=k_{\text {corr }} P_{O_{2}} \\
\beta & =\frac{\dot{F} t}{N_{u}^{0}}
\end{aligned}
$$

Then using eq. (6) in (7) and converting from time. variable to $\beta$ variable, we get,

$$
\dot{F} \frac{d}{d \beta}\left(N_{0}^{c} / N_{u}^{c}\right)=k_{\text {corr }} \exp \left\{-32 \cdot 7+60\left(\frac{\beta}{1-\beta}-\frac{4 N_{0}^{c}}{(1-\beta) R N_{v}^{0}}\right)\right\}
$$

Fraction of oxygen absorbed by cladding 
252

$$
\begin{aligned}
\therefore \quad x & =\frac{2 \pi R N_{0}^{c}}{\pi R^{2} N_{0}^{0}} \\
& =\frac{N_{0}^{c}}{R\left(\frac{1}{2} N_{0}^{0}\right)}=\frac{N_{0}^{c}}{R N_{0}^{0}} \quad\left[\left(\frac{0}{m}\right)_{0}=2\right]
\end{aligned}
$$

Let $A=\frac{k_{\text {corr }}}{R \dot{F}}=\underset{\text { rate }}{\text { dimensionless corrosion }}$

Then eq. ( 8 ) becomes,

$$
\frac{d x}{d \beta}=A \exp \left\{-32 \cdot 7+60\left(\frac{\beta}{1-\beta}-\frac{4 x}{1-\beta}\right)\right\}
$$

with initial condition $x(0)=0$.

$--(9)$

(b) En (9) is a nonlinear equation, which is to be integrated numerically with $A=10^{12}$ to obtain $X(\beta)$ as a function of burnup, $\beta$

Combining eqs (2) and (5) we get,

$$
\overline{\Delta G}_{\mathrm{O}_{2}}(\beta)=-272+500\left[\frac{\beta}{1-\beta}-\frac{4 \times(\beta)}{1-\beta}\right]
$$

(10) 
This is to be compared with the result from problem 12.3, namely,

$$
\Delta G_{O_{2}}(\beta)=-272+500 \frac{\beta}{1-\beta} \ldots-c
$$

\begin{tabular}{|c|c|c|c|}
\hline $\begin{array}{l}\text { Burnup } \\
\beta \quad(\%)\end{array}$ & \begin{tabular}{|} 
Fraction * \\
of oxygen \\
absorbed by clad \\
X( $\beta$ ( $)$
\end{tabular} & \begin{tabular}{|c|}
$\overline{\Delta G}_{\mathrm{O}_{2}}(\beta)$ \\
for problem 12.5 \\
eq. (10) (kJtroke
\end{tabular} & 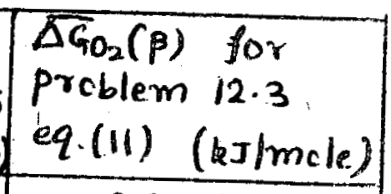 \\
\hline 0.0 & 0.0 & -272.00 & -272.00 \\
\hline 0.5 & $0.037 \times 10^{-3}$ & -269.56 & -269.49 \\
\hline 1.0 & $0.086 \times 10^{-3}$ & -267.12 & -266.95 \\
\hline 1.5 & $0.152 \times 10^{-3}$ & -264.69 & -264.39 \\
\hline 2.0 & $0.240 \times 10^{-3}$ & -262.29 & -261.80 \\
\hline 2.5 & $0.358 \times 10^{-3}$ & -259.91 & -259.18 \\
\hline 3.0 & $0.513 \times 10^{-3}$ & -257.59 & -256.54 \\
\hline 3.5 & $0.718 \times 10^{-3}$ & -255.35 & -253.87 \\
\hline 4.0 & $0.983 \times 10^{-3}$ & $-253 \cdot 22$ & -251.17 \\
\hline 4.5 & $1.324 \times 10^{-3}$ & -251.21 & -248.44 \\
\hline 5.0 & $1.752 \times 10^{-3}$ & -249.37 & -245.68 \\
\hline 5.5 & $2.279 \times 10^{-3}$ & -247.72 & -242.90 \\
\hline 6.0 & $2.913 \times 10^{3}$ & $-246 \cdot 28$ & -240.09 \\
\hline 6.5 & $3.655 \times 10^{-3}$ & -245.06 & -237.24 \\
\hline 7.0 & $4.504 \times 10^{-3}$ & -244.05 & -234.37 \\
\hline 7.5 & $5.449 \times 10^{-3}$ & -243.24 & -231.46 \\
\hline 8.0 & $6.480 \times 10^{-3}$ & -242.61 & -228.52 \\
\hline 8.5 & $7.582 \times 10^{-3}$ & $-242 \cdot 12$ & -225.55 \\
\hline 9.0 & $8.741 \times 10^{-3}$ & -241.76 & -222.55 \\
\hline 9.5 & $9.945 \times 10^{-3}$ & -241.49 & -219.51 \\
\hline 10.0 & $11.180 \times 10^{-3}$ & -241.29 & -216.44 \\
\hline
\end{tabular}

Eq. (9) was solved numerically for burnup ranging from 0 to $10 \%$, using a Runge-Kutta and a predictor - corrector method. The resilts of the two methods agree well with each other and are shown in the table above. 

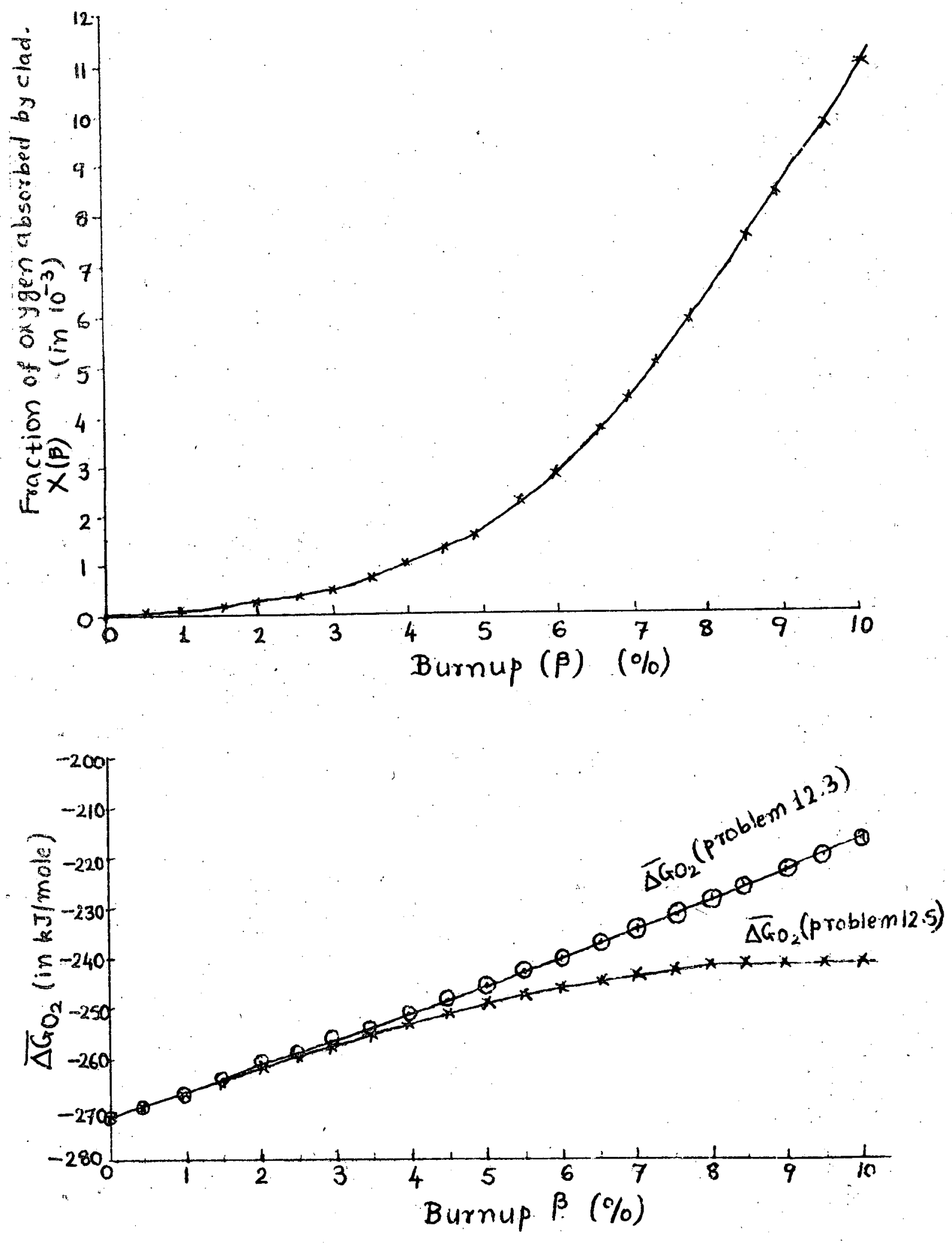
255

PROBLEM 12.6

(a) Substitute $(12.13)-(12.16)$ into $(12.22)$ and $(12.23)$

$$
\begin{aligned}
& Y_{M_{0}}^{f}=\frac{f_{M_{0}} Y_{M_{c}} \beta}{(1-q)(1-\beta)+q(1-\beta)+\left(Y_{Y-R E}+f_{M_{1}} Y_{M_{0}}+Y_{Z_{V}-N_{n}}-Y_{e_{n}-B_{r}}\right) \xi_{i}} \\
& =\frac{f_{M c} Y_{M c}}{\frac{1-\beta}{\beta}+Y_{Y-R E}+f_{M c} Y_{M c}+Y_{Z_{r}-N_{b}}-Y_{B_{A}-S_{r}}}-\cdots(1)
\end{aligned}
$$

and

$$
Y_{M_{0}}^{m}=\frac{\left(1-f_{M_{0}}\right) Y_{M_{0}}}{Y_{N M}+\left(1-f_{M_{0}}\right) Y_{M_{0}}}-\ldots . . .(2)
$$

given $\beta=0.08$ and $f_{H 0}=0.5$, ane wig the yield e in the last column of table 12.1

$$
\begin{aligned}
& Y_{M_{0}}^{f}=\frac{(0.5)(0.206)}{\frac{1-0.08}{0.08}+0.493+(0.5)(0.206)+0.219+0.109}=0.0084 \\
& Y_{M_{0}^{m}}^{m}=\frac{(0.5)(0.206)}{(0.456)+(0.5)(0.206)}=0.184
\end{aligned}
$$

(b) For an ideal solution of $\mathrm{MOO}_{0}$ an the fuel. Racictio fac is obeyed

and $P_{\mathrm{MCO}_{2}}=\mathrm{P}_{\mathrm{B}: 0 \mathrm{O}}^{\mathrm{O}}$

where $\mathrm{PAoc}_{\mathrm{M}_{0} \mathrm{O}}$ is the vapor presume of pare

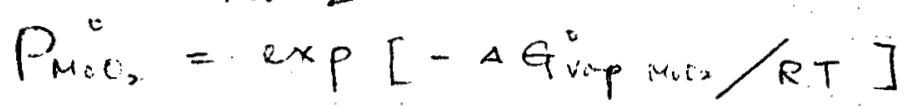

at $2000^{\circ} \mathrm{K} P_{M C_{2}}^{0}=\exp \left[-\frac{561-303(3.0)}{(8.3 .1)(2.0)}\right]=8.9 \times 10^{-5} \mathrm{~atm}$ 
256

$$
\therefore \mathrm{PMOO}_{\mathrm{O}}=(0.0084)\left(8.9 \times 10^{-.5}\right)=7.48 \times 10^{-7} \mathrm{~atm}
$$

This presume is for to low for vapor transept of $\mathrm{MoO}_{2}$

(c) Consider the reactions

$$
\mathrm{Mo}(s)+\mathrm{O}_{2}(g)=\mathrm{M}_{0} \mathrm{O}_{2} \text { (solution in fuel) }
$$

$\Delta G_{1}{ }^{c}$ is queen by equation $(12.18)=-137.1+39 \cdot 3\left(\mathrm{Tal} / \mathrm{i}^{3}\right)$

$$
\begin{aligned}
& \frac{3}{2} M_{0} O_{2}(s)=\frac{1}{2} M_{0}(s)+M_{0} O_{3}(g) \\
& \Delta G_{2}{ }^{\circ}=510-194(T / 1000), \mathrm{kJ} / \mathrm{malc}
\end{aligned}
$$

Multiply reaction " 1 ) by $1 / 2$, and add to reation(2)

$$
\begin{aligned}
M_{0} O_{2}(s)+\frac{1}{2} O_{2}=M_{0} O_{3}(g) & (3) \\
\Delta G_{3}^{c} & =\frac{1}{2} \Delta G_{1}^{0}+\Delta G_{2}^{0}=\frac{1}{2}(-574)+510+\left[\frac{1}{2}(164)-194\right]\left(T / 10^{3}\right) \\
& =223-112\left(T / 10^{3}\right)
\end{aligned}
$$

at $T=\operatorname{soc}^{\circ} \mathrm{K} \quad \Delta G_{3}^{\circ}=-1.3^{\prime} \mathrm{KJ} /$ male The equilithim of equation ( 3 ) is

$$
\exp \left(-\frac{\Delta G_{3}^{\circ}}{R T}\right)=\frac{P_{\mathrm{HCO}_{3}}}{\sqrt{P_{O_{2}}} \cdot Y_{M_{0}}}
$$

or $R T \ln P_{M_{1} O_{3}}=\frac{1}{2} R T \ln P_{O_{2}}+R T \ln Y_{M i}{ }^{f}-\Delta \theta_{3}{ }^{0}$ But $R T \ln P_{\mathrm{O}_{2}}=\Delta \bar{G}_{\mathrm{O}_{2}}=$ oxygen potential of fuel

$$
\therefore P_{M_{0} O_{3}}=Y_{M_{0}}^{f} \exp \left[\frac{\frac{1}{2} \Delta \bar{G}_{c_{2}}-\Delta G_{3}^{c}}{R T}\right]
$$


257

$$
\begin{aligned}
& \begin{array}{l}
\text { at }>0000^{\circ} K \\
P_{M_{0} O_{3}}=c_{0084} \exp \left[\frac{\frac{1}{2}(-3 \pi)-(-1.3)}{(8.31)(2.0)}\right]
\end{array} \\
& =1.1 \times 10^{-6} \cdot a t_{\text {in }}
\end{aligned}
$$

This presume w al io too low for vapor 
2570

Problem 12.7

Eg. $(12.44)$

$$
\left(\frac{\Delta V}{V}\right)_{\text {solid fp }}=\left(\sum y_{i} \cdot \frac{v_{i}}{v_{v}}-1\right) \beta
$$

If the fission gases are treated as a solid:

$$
\begin{aligned}
& y_{x_{e}-k_{r}}=0.25 \\
& v_{x_{e}-k_{r}}=B=85 \AA^{3} \quad\left(v_{v}=41 A^{3}\right)
\end{aligned}
$$

$0.25\left(\frac{85}{41}\right)=0.518$ be added to table 12.6

$\therefore$ The sum tabulated in Tab. 12.6 becomes: $1.324+0.518$

$$
=1.842
$$

and $\left(\frac{\Delta V}{V}\right)_{\text {solid }}=0.842 \%$

or

$0.84 \%$ atom \% bursup 
2574

Problem 12.8

at equilibrium. the oxygen partial pressure established by. the reactor: $\frac{2}{3} C_{r}(s)+O_{2}(g)=\frac{2}{3} c_{r_{2}} O_{3}$ (s) must be the same as that over the final orly of the furl. the fuel $0 / \pi$ is reduced from 2.01 to a value $(0 / m)_{f}$ at which

$$
\left(\overline{\Delta C_{\mathrm{O}_{2}}}\right)_{f}=\Delta G_{\mathrm{ucc}_{3} / \mathrm{c}_{2}}^{0}=-560 \mathrm{~kJ} / \mathrm{mo} / \mathrm{e}
$$

From Fig. 11.12 at $800^{\circ} \mathrm{C}$, we find $(\mathrm{o} / \mathrm{n} / \mathrm{f}=1.994$. The oxygen removed from the fuel sea cts with chavaraien to form a layer if $\mathrm{Cr}_{2} \mathrm{O}_{3}$ the thicleness of tab layer is fund bs an oxygen balance:

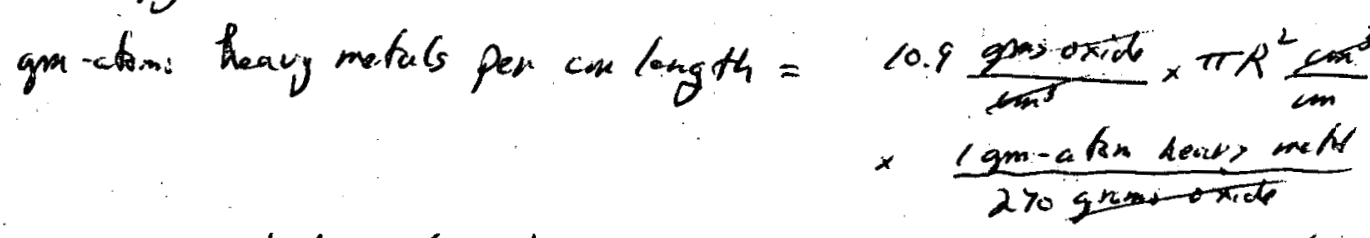

oxygen reacted with chromium $=(2.01-1.994) \times 10.9 \times \pi R^{\prime} / 2>0$ gin-atims chromium oridryed $=\frac{2}{3}(2.01-1.994) \times 10.7 \times \pi R^{2} / \alpha>0$

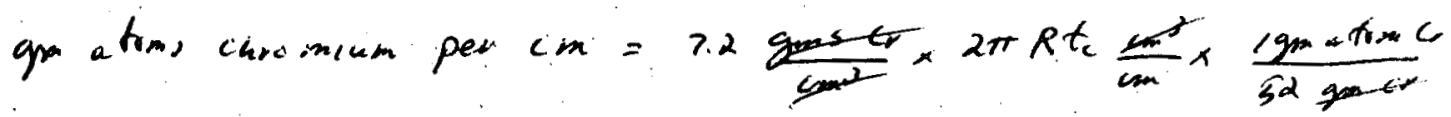

fraction od cr oxidized $=\frac{\text { gmatoms oxidized }}{\text { gmatoms cor }}$

$$
=\frac{\frac{2}{3}(201-1.994) \times 10.9 \times \pi R^{2} / 270}{22 \times 2 \pi R t_{c} / 52}
$$

using $R=3 \mathrm{~mm}, t_{c}=0.4 \mathrm{~mm}$,

fraction or oxidized $=0.012(1.2 \%)$ 
258

CHAPTER 13

Problem 13.1

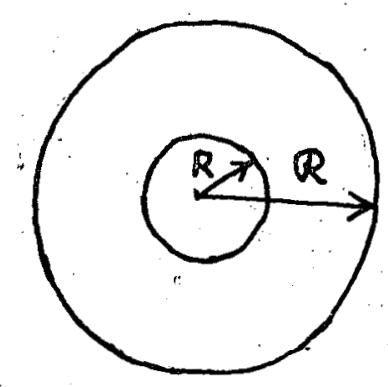

a) Determine $R:-$

$$
\begin{aligned}
& \left(\frac{4}{3} \pi R^{3}\right) N=1 \\
& \therefore R=\left(\frac{3}{4 \pi N}\right)^{1 / 3}
\end{aligned}
$$

Small bubble (dens egos limit)

$$
m=\frac{4}{3} \frac{\pi R^{3}}{B}
$$

Initial State:-

$N$ bubbles/cc of radius $R_{0}$, matrix gas concentration $=C_{0}$;

Balance of gas in bubble:

$$
\frac{d m}{d t}=4 \pi R D_{x e} c-b m
$$

(2) $\left[E_{2} \cdot(13.157)\right]$

Balance of gas in fuel + bubble

$$
\begin{aligned}
& C=\text { average concentration at time } t \\
& \text { (assuming } Q \gg R_{0} \text { ) }
\end{aligned}
$$

Total gas (bubble +fuel) at time $t$

$$
=\left(\frac{4}{3} \pi R^{3}\right) c_{0}+\frac{\frac{4}{3} \pi R_{0}^{3}}{B}+\left(Y_{x e} \dot{F} t\right)\left(\frac{4}{3} \pi R^{3}\right)
$$


259 ie. Total gas $=\underset{\text { in bubble }+ \text { fuel }}{\text { in present }}+\begin{gathered}\text { that produced } \\ \text { by fission }\end{gathered}$

This is also equal to:

$$
\underset{\text { Total gas at }}{\text { time } t}=\left(\frac{4}{3} \pi R^{3}\right) c+\left(\frac{\frac{4}{3} \pi R^{3}}{B}\right)
$$

Equating the two we get,

$$
Q^{3}\left(C-c_{0}\right)+\frac{1}{B}\left(R^{3}-R_{0}^{3}\right)=R^{3} Y_{X_{e}} \dot{F} t
$$

From eq .(1) we get,

$$
\frac{d m}{d t}=\frac{4 \pi R^{2}}{B} \frac{d R}{d t}
$$

Equating with (2), we get,

$$
\begin{aligned}
& \frac{4 \pi R^{2}}{B} \frac{d R}{d t}=4 \pi R D_{x_{e}} C-\frac{\frac{4}{3} \pi R^{3}}{B} b \\
& \text { or } \frac{d R}{d t}=\frac{B D_{x_{e}} C}{R}-\frac{R}{3} b
\end{aligned}
$$

Equations (3) and (4) must be solved simultaneously (numerically);

b) Define:

$$
\begin{aligned}
& \tau=b t=\text { dimensionless time } \\
& \eta=\frac{R}{R_{0}}=\text { dimensionless bubble } \\
& \theta=\frac{c}{C_{0}}=\text { dimensionless } \\
& \text { concentration }
\end{aligned}
$$

With these definitions eq. (3) and (4) reduce to: 
260

$$
\theta=1+A_{2} \tau+A_{3}\left(1-\eta^{3}\right)
$$

and $\frac{d \eta}{d \tau}=A_{1} \frac{\theta}{\eta}-\frac{1}{3} \eta$

where:

$$
\text { re: } \begin{aligned}
A_{1} & =\frac{B D_{x_{e}} C_{0}}{b R_{0}^{2}} \\
A_{2} & =\frac{\ddot{Y}_{x_{e}} \dot{F}}{C_{0} b} \\
\text { and } \quad A_{3} & =\frac{R_{0}^{3}}{R^{3} C_{0} B}
\end{aligned}
$$

Combining (5) and (6) we get,

$$
\frac{d \eta}{d \tau}=\frac{A_{1}\left(1+A_{3}\right)}{\eta}+\frac{A_{1} A_{2} \tau}{\eta}-A_{1} A_{3} \eta^{2}-\frac{1}{3} \eta
$$

with initial condition $\eta(0)=1$

c) For initial shrinkage by resolution, we must have, $\frac{d \eta}{d \tau}<0$ for $\tau=0$ and $\eta(0)=1$. ie we must have $A_{1}<\frac{1}{3}$ from eq. (10)

Substituting numerical values

$$
\begin{aligned}
A_{1} & =\frac{85 \times 10^{-24} \mathrm{~cm}^{3} \times 10^{-14} \mathrm{~cm}^{2} / \mathrm{sec} \times 5.2 \times 10^{17} \text { gas atoms } / \mathrm{cm}^{3}}{10^{-5} \mathrm{sec} !} \times 400 \times 10^{-16} \mathrm{~cm}^{2} \\
& =\frac{85 \times 5.2}{400}=1.105
\end{aligned}
$$

Since $A_{1}>\frac{1}{3}$, there is no initial shrinkage. 
261

$$
\begin{aligned}
A_{2}= & \frac{0.26 \times 10^{13} \text { fissions } / \mathrm{cm}^{3} \mathrm{sec}}{5.2 \times 10^{17} \text { gas atoms } / \mathrm{cm}^{3} \times 10^{-5} \mathrm{sec}^{-1}} \\
= & \frac{2.6}{5.2}=\frac{0.50}{A_{3}=\frac{R_{0}^{3}}{a^{3} C_{0} B}}=\frac{\frac{R_{0}^{3}}{4 \pi N} C_{0} B}{=}=\frac{4 \pi N R_{0}^{3}}{3 C_{0} B} \\
& =\frac{8 \times 10^{3} \times 10^{-24} \mathrm{~cm}^{3} \times 4 \times \pi \times 1.3 \times 10^{13} \mathrm{bubbles} / \mathrm{cm}^{3}}{3 \times 5.2 \times 10^{17} \mathrm{gas} \mathrm{atoms} / \mathrm{cm}^{3} \times 85 \times 10^{-24} \mathrm{~cm}^{3}} \\
& =\frac{32 \pi \times 1.3 \times 10^{-1}}{3 \times 5.2 \times 85}=0.98 \times 10^{-2}
\end{aligned}
$$

Introducing these values in eq. (10) we get,

$$
\begin{gathered}
\frac{d \eta}{d \tau}=\frac{1.115}{\eta}+0.552 \frac{\tau}{\eta}-0.010 \eta^{2}-\frac{1}{3} \eta \\
\text { with } \eta(0)=1
\end{gathered}
$$

Finite Difference scheme for solving eq. (II):

$$
\begin{aligned}
& \frac{d \eta}{d \tau} \cong \frac{\eta_{i+1}-\eta_{i}}{h} \quad \text { (Forward difference scheme) } \\
& \text { or } \eta_{i+1}=\eta_{i}+h\left[\frac{1115}{\eta_{i}}+0.552 \frac{i h}{\eta_{i}}-0.01 \eta_{i}^{2}-\frac{1}{3} \eta_{i}\right]
\end{aligned}
$$

(where $\tau=i h$ has been used)

and $\eta_{0}=1$

This equation was solved with $h=0.2$ and the following results were obtained. 
262

For $h=0.2$

\begin{tabular}{|c|c|c||c|c|c|}
\hline$i$ & $\tau$ & $\eta$ & $i$ & $\tau$ & $\eta$ \\
\hline 0 & 0 & 1.00 & 11 & 2.2 & 2.17 \\
\hline 1 & 0.2 & 1.17 & 12 & 2.4 & 2.24 \\
\hline 2 & 0.4 & 1.32 & 13 & 2.6 & 2.30 \\
\hline 3 & 0.6 & 1.44 & 14 & 2.8 & 2.37 \\
\hline 4 & 0.8 & 1.56 & 15 & 3.0 & 2.43 \\
\hline 5 & 1.0 & 1.66 & 16 & 3.2 & 2.50 \\
\hline 6 & 1.2 & 1.76 & 17 & 3.4 & 2.56 \\
\hline 7 & 1.4 & 1.85 & 18 & 3.6 & 2.61 \\
\hline 8 & 1.6 & 1.93 & 19 & 3.8 & 2.67 \\
\hline 9 & 1.8 & 2.01 & 20 & 4.0 & 2.73 \\
\hline 10 & 2.0 & 2.09 & & & \\
\hline
\end{tabular}

The shape of this function is:

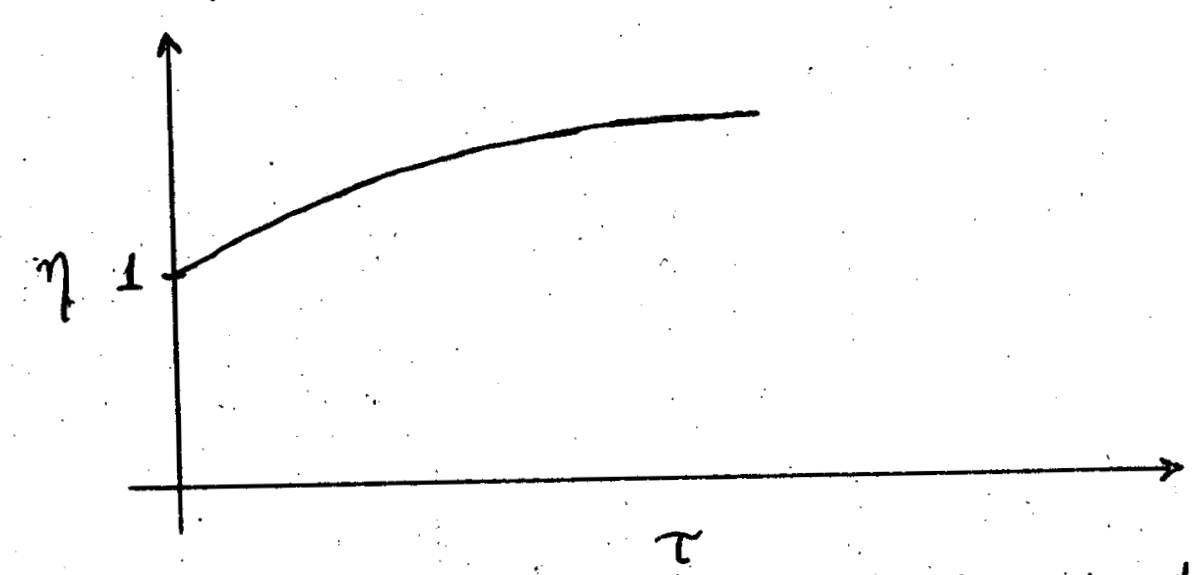

A more interesting case would be when $A_{1}<\frac{1}{3}$. For this case, there is a minimum bubble radius. 
The differential equation (10), with values:

$A_{1}=0.1, \quad A_{2}=0.5 ; A_{3}=1$ was solved numerically $\therefore \frac{d \eta}{d \tau}=\frac{0.2}{\eta}+0.05 \frac{\tau}{\eta}-0.1 \eta^{2}-\frac{1}{3} \eta ; \quad \eta(0)=1$

The result is the following: $h=0.2$

\begin{tabular}{|c|c|c||c|c|c|}
\hline$i$ & $\tau$ & $\eta$ & $i$ & $\tau$ & $\eta$ \\
\hline 0 & 0.0 & 1.000 & 11 & 2.2 & 0.840 \\
\hline 1 & 0.2 & 0.955 & 12 & 2.4 & 0.846 \\
\hline 2 & 0.4 & 0.919 & 13 & 2.6 & 0.853 \\
\hline 3 & 0.6 & 0.891 & 14 & 2.8 & 0.862 \\
\hline 4 & 0.8 & 0.869 & 15 & 3.0 & 0.870 \\
\hline 5 & 1.0 & 0.854 & 16 & 3.2 & 0.880 \\
\hline 6 & 1.2 & 0.843 & 17 & 3.4 & 0.890 \\
\hline 7 & 1.4 & 0.837 & 18 & 3.6 & 0.900 \\
\hline 8 & 1.6 & 0.834 & 19 & 3.8 & 0.910 \\
\hline 9 & 1.8 & 0.834 & 20 & 4.0 & 0.921 \\
\hline 10 & 2.0 & 0.836 & & & \\
\hline
\end{tabular}

The shape of this function is:

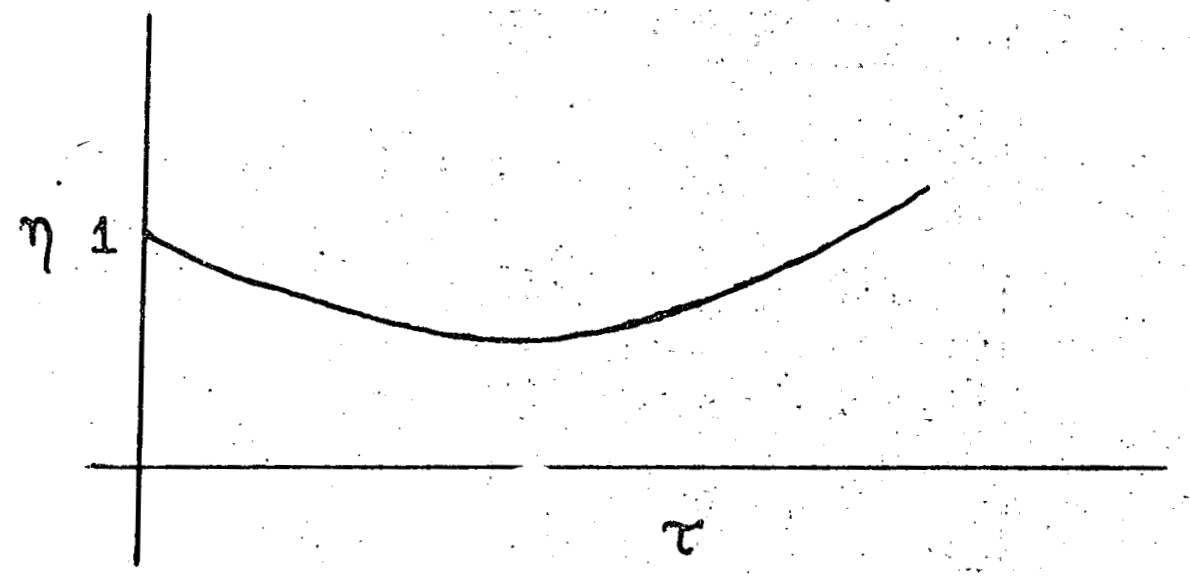


264

Problem 13.2

a) From Eq. (7.24),

$$
\begin{array}{r}
\text { Eq. (7.24), } \\
\sqrt{r^{2}}=\sqrt{6 D_{b} t}=\frac{\sqrt{\frac{9 a^{4} D_{s} t}{\pi R^{4}}}}{\text { [using Eq. (13.214)] }}
\end{array}
$$

Numerically,

$$
\begin{aligned}
a & =3 A^{\circ} \\
t & =40 \times 24 \times 3600=3.456 \times 10^{6} \mathrm{sec} . \\
R & =10 \mathrm{~A}^{\circ} \\
T & =1400^{\circ} \mathrm{C}=1673^{\circ} \mathrm{K} \\
D_{S} & =4 \times 10^{5} \mathrm{e}^{-108 / \mathrm{RT}} \quad \frac{\mathrm{cm}^{2}}{\mathrm{sec}} \quad[\mathrm{Eq} \cdot(13.216)] \\
& =2.77 \times 10^{-9} \frac{\mathrm{cm}^{2}}{\mathrm{sec}}
\end{aligned}
$$

Inserting in (1),

$$
\begin{aligned}
\sqrt{\bar{r}^{2}} & =\left(\frac{9 \times 3^{4} \times A^{04} \times 2.77 \times 10^{-9} \frac{\mathrm{cm}^{2}}{\mathrm{sec}} \times 3.456 \times 10^{6} \mathrm{sec}}{\pi 10^{4} A^{-4}}\right)^{1 / 2} \\
& =0.015 \mathrm{~cm} .
\end{aligned}
$$

b) Thermal Gradient Migration:-

$$
\begin{aligned}
r & =\frac{3 D_{s} Q_{s}^{*} a}{R k T^{2}}\left(\frac{d T}{d x}\right) t \quad[E q .(13.219)] \\
& =\frac{3 \times 2.77 \times 10^{-9} \frac{\mathrm{cm}^{2}}{5 e c} \times 100 \times 10^{3} \frac{\mathrm{cal}}{\mathrm{mcle}} \times 4.18 \frac{\text { joule }}{\mathrm{cal}} \times 3 A^{\circ} \times 2000^{0 \times} \mathrm{K} / \mathrm{cm}}{10 A^{0} \times 1.386 \times 10^{5} \mathrm{sec}} \\
& =0.310 \mathrm{~cm} .
\end{aligned}
$$


265

Problem 13.3

Overall gas balance (neglecting $c_{4}, c_{5}, \ldots$ etc.)

$$
c_{0}=C_{1}+2 C_{2}+3 C_{3}
$$

a) Kinetic Equations:

$$
\begin{aligned}
& \frac{d c_{1}}{d t}=-2 k_{11} c_{1}^{2}-k_{12} c_{1} c_{2} \\
& \frac{d c_{2}}{d t}=k_{11} c_{1}^{2}-k_{12} c_{1} c_{2} \\
& \frac{d c_{3}}{d t}=k_{12} c_{1} c_{2}
\end{aligned}
$$

(neglect $k_{13} c_{1} c_{3}$ term)

This is a self consistent set. Note that $\frac{d}{d t}$ of eq. (1) is satisfied identically by eggs. (2) - (4).

Initial conditions for these eq. are

$$
\begin{aligned}
& C_{1}(0)=C_{0} \\
& C_{2}(0)=C_{3}(0)=0
\end{aligned}
$$

$k_{11}$ and $k_{12}$ are given by,

$$
k_{11}=\frac{z_{11} \Omega D_{x e}}{a^{2}} ; z_{11} \cong 12 ; k_{12}=\frac{z_{12 \Omega} D_{x e}}{a^{2}} ; z_{12} \cong 24
$$

b)

Let

$$
\begin{aligned}
& \theta_{1}=\frac{c_{1}}{c_{0}} ; \theta_{2}=\frac{c_{2}}{c_{0}} ; \theta_{3}=\frac{c_{3}}{c_{0}} \\
& \tau=c_{0} k_{11} t
\end{aligned}
$$

Then ens (2) - (4) become:

$$
\frac{d \theta_{1}}{d \tau}=-2 \theta_{1}^{2}-\frac{i}{2} \theta_{1} \theta_{2}
$$


2,66

$$
\begin{aligned}
\frac{d \theta_{2}}{d \tau} & =\theta_{1}^{2}-\frac{1}{2} \theta_{1} \theta_{2} \\
\frac{d \theta_{3}}{d \tau} & =\frac{1}{2} \theta_{1} \theta_{2}
\end{aligned}
$$

Initial Conditions:-

$$
\theta_{1}(0)=1 ; \quad \theta_{2}(0)=\theta_{3}(0)=0
$$

Numerical solutions of eos. (7), (8) and (9) are as follows:

$$
\begin{aligned}
& \theta_{1}^{n+1}=\theta_{1}^{n}+h\left(-2 \theta_{1}^{n}-\frac{1}{2} \theta_{1}^{n} \theta_{2}^{n}\right) \\
& \theta_{2}^{n+1}=\theta_{2}^{n}+h\left(\theta_{1}^{n}-\frac{1}{2} \theta_{1}^{n} \theta_{2}^{n}\right) \\
& \theta_{3}^{n+1}=\theta_{3}^{n}+h\left(\frac{1}{2} \theta_{1}^{n} \theta_{2}^{n}\right) \\
& \text { with } \theta_{1}^{0}=1, \quad \theta_{2}^{0}=\theta_{3}^{0}=0
\end{aligned}
$$

(superscript $n$ stands for the value at $n$th time step.)

Solved numerically for $h=0.2$ gives:

$h=0.2$\begin{tabular}{|c|c|c|c|c|c|}
\hline$i$ & $\tau$ & $\theta_{1}$ & $\theta_{2}$ & $\theta_{3}$ & $\theta_{1}+2 \theta_{2}+3 \theta_{3}$ \\
\hline 0 & 0.0 & 1.000 & 0.000 & 0.000 & 1.000 \\
\hline 1 & 0.2 & 0.600 & 0.200 & 0.000 & 1.000 \\
\hline 2 & 0.4 & 0.444 & 0.260 & 0.012 & 1.000 \\
\hline 3 & 0.6 & 0.353 & 0.287 & 0.023 & 1.000 \\
\hline 4 & 0.8 & 0.293 & 0.302 & 0.033 & 0.999 \\
\hline 5 & 1.0 & 0.250 & 0.311 & 0.042 & 0.999 \\
\hline 6 & 1.2 & 0.217 & 0.315 & 0.050 & 0.999 \\
\hline 7 & 1.4 & 0.191 & 0.318 & 0.057 & 0.999 \\
\hline
\end{tabular}


267

\begin{tabular}{|c|c|c|c|c|c|}
\hline$i$ & $\tau$ & $\theta_{1}$ & $\theta_{2}$ & $\theta_{3}$ & $\theta_{1}+2 \theta_{2}+3 \theta_{3}$ \\
\hline 8 & 1.6 & 0.170 & 0.319 & 0.063 & 0.999 \\
\hline 9 & 1.8 & 0.153 & 0.319 & 0.068 & 0.999 \\
\hline 10 & 2.0 & 0.139 & 0.319 & 0.073 & 0.999 \\
\hline 11 & 2.2 & 0.127 & 0.319 & 0.078 & 1.000 \\
\hline 12 & 2.4 & 0.116 & 0.318 & 0.082 & 1.000 \\
\hline 13 & 2.6 & 0.107 & 0.317 & 0.085 & 1.000 \\
\hline 14 & 2.8 & 0.099 & 0.316 & 0.089 & 1.000 \\
\hline 15 & 3.0 & 0.092 & 0.315 & 0.092 & 1.000 \\
\hline 16 & 3.2 & 0.085 & 0.313 & 0.095 & 1.000 \\
\hline 17 & 3.4 & 0.080 & 0.312 & 0.098 & 1.000 \\
\hline 18 & 3.6 & 0.075 & 0.311 & 0.100 & 1.000 \\
\hline 19 & 3.8 & 0.070 & 0.310 & 0.102 & 1.000 \\
\hline 20 & 4.0 & 0.066 & 0.309 & 0.105 & 1.000 \\
\hline
\end{tabular}

The shapes of these curves are:

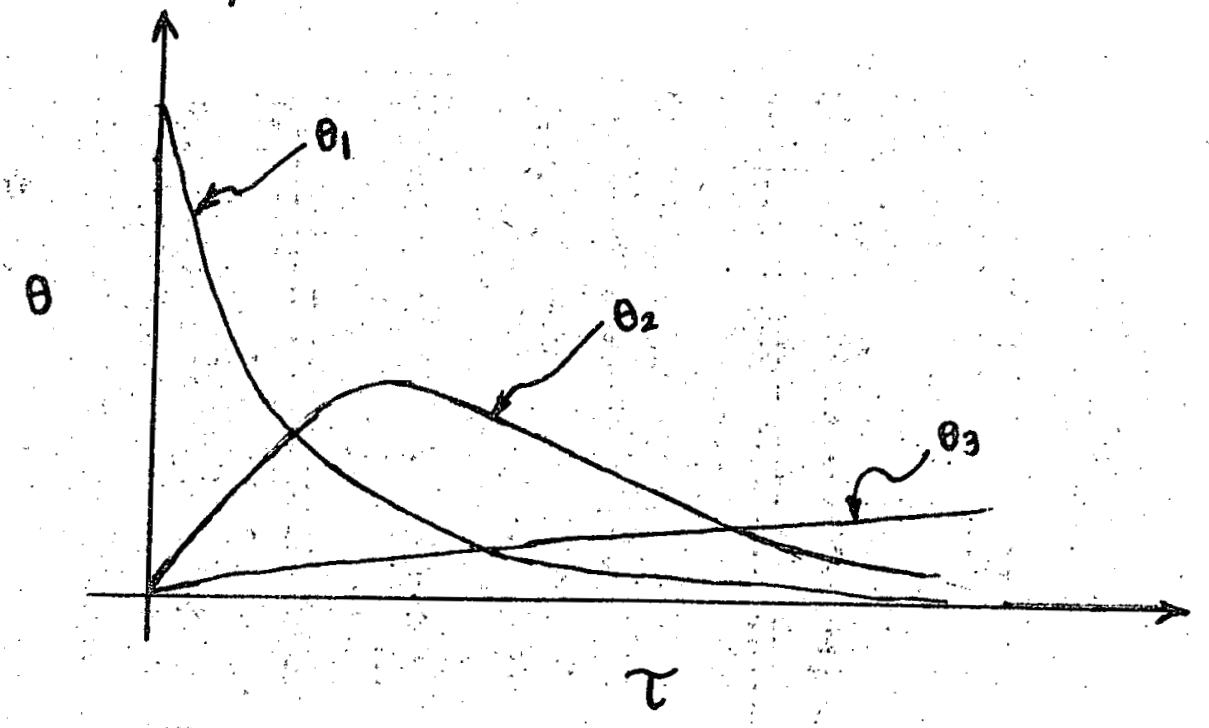


268

C) The nucleation time $t_{c}$ is given by:

$$
\left(\frac{d C_{2}}{d t}\right)_{t_{c}}=0 \quad\left[E_{q} .(13.123)\right]
$$

From the numerical solution $\theta_{2}$ is maximum for $\tau=1.9$

$$
\therefore t_{c}=\frac{\tau}{c_{0} k_{11}}
$$

with $k_{11}=\frac{12 \times \Omega \times D_{x e}}{a^{2}}=12 \times a \times D_{x_{e}}$

$$
\begin{aligned}
& =12 \times 3 \times 10^{-8} \mathrm{~cm} \times 10^{-1 / 4} \mathrm{~cm}^{2} / \mathrm{sec} \\
& =36 \times 10^{-22} \mathrm{~cm}^{3} / \mathrm{sec} .
\end{aligned}
$$

and,

$$
\begin{aligned}
t_{c} & =\frac{1.9}{10^{17} \mathrm{kcc} \times 36 \times 10^{-22} \mathrm{cc} / \mathrm{sec}} \\
& =5.27 \times 10^{3} \mathrm{sec}=1.46 \mathrm{hr}
\end{aligned}
$$

$$
\text { At } \begin{aligned}
& t=t_{c} \\
& \frac{c_{1}}{c_{0}}=\theta_{1} \cong 0.146=14.6 \% \text { remains atomically } \\
& 2 \theta_{2} \cong 0.638=63.8 \% \text { in diatomic dusters } \\
& 3 \theta_{3} \cong 0.216=21.6 \% \text { in triatomic clusters }
\end{aligned}
$$


269

Problem 13.4

$$
\begin{aligned}
& \text { Initially : type(I) :- } \quad p_{1}=\frac{2 \gamma}{r_{1}} \\
& \text { type(II) :- } \quad p_{2}=\frac{2 \gamma}{\gamma_{2}} \\
& \gamma_{2}=0.5 r_{1}
\end{aligned}
$$

Initial volume

occupied by bubbles $=\frac{4}{3} \pi n\left(r_{1}^{3}+r_{2}^{3}\right)$,

where $n=$ number of bubbles of each type (total $2 n$ bubbles)

By ideal gas law, let $N_{i}$ be the number of gas atoms in bubble type (i):

$$
\begin{aligned}
N_{i} & =p_{i}\left(\frac{4}{3} \pi r_{i}^{3}\right) / k T \\
& =\frac{4}{3} \pi\left(\frac{2 \gamma^{2}}{k T}\right) r_{i}^{2} \quad(i=1,2)
\end{aligned}
$$

We have 3 types of collisions:

$$
\left.\begin{array}{l}
\text { (I) }+ \text { (I) } \\
\text { (I) }+ \text { (II) } \\
\text { or } \\
\text { (II) }+ \text { (I) }
\end{array}\right\} \text { same type }
$$

Since in actual process the rate of coalescence of smaller bubbles may be faster than that for larger ones, it is not possible to get explicit expression due to complex process of collision, simply an approach is indicated by random motion of bubbles.

Assume that the probability of each

type of collision is the same $=\frac{1}{4}$ 
$2>0$

Then we finish with $n$ total bubbles: $\frac{n}{4}$ bubbles of type $[(I)+(I)] ; \frac{n}{4}$ bubbles of type $[(I I)+(I I)]$; $\frac{n}{2}$ bubbles of type $[(I)+(I)]$

Moles of gas in bubbles (or number of gas atoms)

$$
N=N_{i}+N_{j}=\frac{4}{3} \pi\left(\frac{2 \gamma}{k T}\right)\left(\gamma_{i}^{2}+\gamma_{j}^{2}\right)
$$

For ideal gas $p=\frac{2 \gamma}{\gamma}$ and $p\left(\frac{4}{3} \pi r^{3}\right)=N k T$

$$
\therefore r=\frac{2 \gamma}{p}=2 \gamma\left(\frac{N k T}{\frac{4}{3} \pi r^{3}}\right)^{-1} \text {. }
$$

This gives $r=\sqrt{\gamma_{i}^{2}+r_{j}^{2}}$.

$\therefore$ Final volume of gas is:

$$
\begin{gathered}
V=\frac{4}{3} \pi n\left\{\frac{1}{4}\left(r_{1}^{2}+r_{1}^{2}\right)^{3 / 2}+\frac{1}{4}\left(r_{2}^{2}+r_{2}^{2}\right)^{3 / 2}+\frac{1}{2}\left(r_{1}^{2}+r_{2}^{2}\right)^{3 / 2}\right\} \\
\therefore \quad \text { Swelling }=\frac{V-V_{\text {initial }}}{V_{\text {initial }}}=\frac{V}{V_{\text {initial }}}-1 \\
\text { initial }=\frac{4}{3} \pi n\left(r_{1}^{3}+r_{2}^{3}\right) \\
\therefore \text { Swelling. } \frac{\Delta V}{V}=\frac{\frac{1}{\sqrt{2}}\left(r_{1}^{3}+r_{2}^{3}\right)+\frac{1}{2}\left(r_{1}^{2}+r_{2}^{2}\right)^{3 / 2}}{r_{1}^{3}+r_{2}^{3}}-1 \\
r_{2}=0.5 r_{1} \quad \therefore \frac{\Delta V}{V}=\frac{1}{\sqrt{2}}+\frac{\frac{1}{2} r_{2}^{3}(1+4)^{3 / 2}}{r_{2}^{3}(1+8)}-1 \\
=0.707+\frac{5 \sqrt{5}}{18}-1 \\
=0.328
\end{gathered}
$$


271

Problem 13.5

a) For no radiation: $x_{v}=x_{v}^{e q}=e^{-\varepsilon_{v} / k T}$ with $E_{F}=$ energy of formation of

$$
\therefore D_{U}^{t h}=a_{0}^{2} \nu e^{-\left(\epsilon_{v}^{*}+\varepsilon_{y}\right) / k T}
$$

b) When recombination is the only means of destruction, then equilibrium of destruction and production rates gives [see Eqs.(13.186) and

$$
Y_{v i} \dot{F}=k_{i v} C_{v} C_{i}
$$$$
(13.187)]
$$

[Where it is assumed that

$$
c_{v}^{\text {eq. }} \text { and } c_{i}^{\text {eq. }} \cong 0 \text { ] }
$$

$$
\begin{gathered}
\text { But } c_{v}=c_{i} \text {, and } c_{v}=\frac{x_{v}}{\Omega} \cong \frac{x_{v}}{a_{0}^{3}} \\
\therefore \quad x_{v}=a_{0}^{3} c_{v}=a_{0}^{3} \sqrt{\frac{y_{v i} F}{k_{i v}}} \quad[u \operatorname{sing}(1)]
\end{gathered}
$$

From Eq. (13.42), $\quad k_{i v}=\frac{z_{i v} \Omega D_{i}}{a_{0}^{2}}=z_{i v} a_{0} D_{i}$

Where. $D_{i}=$ interstitial diffusion coefficient

Then:

$$
x_{v}=a_{0}^{3} \sqrt{\frac{y_{v i F}}{z_{i v} a D_{i}}} x_{v} \text {, replaces } x_{v}
$$

and

$$
D_{v}^{\mathrm{rad}}=a_{0}^{2} \nu\left\{a_{0}^{3} \sqrt{\frac{y_{v i} F}{z_{i v} a_{0} D_{i}}}\right\} e^{-\varepsilon_{v}^{*} / k T}
$$

but since, $D_{i}=a_{0}^{2} \nu e^{-\varepsilon_{i}^{*} / k T}$

We get:

$$
D_{u}=\left(\frac{a_{0}^{7} Y_{V i} \dot{F} \nu}{Z_{i v}}\right)^{1 / 2} e^{-\left(\varepsilon_{v}^{*}-\frac{1}{2} \varepsilon_{i}^{*}\right) / k T}
$$


c)

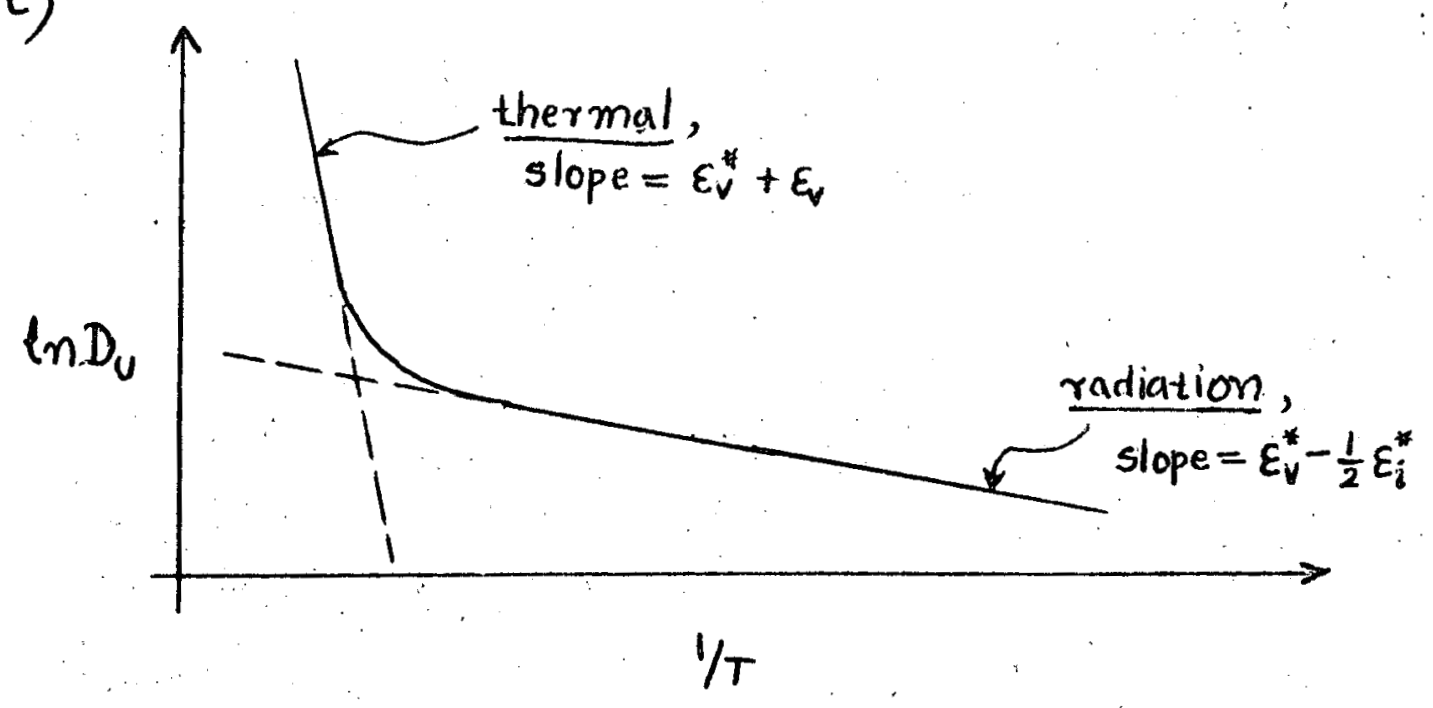


273

Problem 13.6

Condition of mechanical equilibrium for bubble of radius $r$ is:

$$
\begin{gathered}
p=\frac{2 r}{r} \text { and } p\left(\frac{4}{3} \pi r^{3}\right)=m k T \\
\therefore m=\left[\frac{8 \pi}{3} \frac{\gamma}{k T}\right] r^{2}=c r^{2}-\ldots(1) \\
\text { Eq. (1) holds for all bubbles. }\left(r=R \text { or } r=R^{*}\right)
\end{gathered}
$$

If the large bubble expands by $d R^{*}$, it sweeps out a volume of $4 \pi R^{* 2} d R^{*}$. This volume contains $N$ bubbles of radius $R$ per $C C$, and each bubble contains

$$
m=C R^{2}
$$

gas atoms by eq. (1). Therefore the number of additional gas atoms acquired by the large bubble as a result of expansion by $d R^{*}$ is

$$
d m^{*}=4 \pi R^{* 2} d R^{*} N C R^{2}
$$

However to maintain mechanical equilibrium according to eq.(1), the number of additional gas atoms required is given by

$$
\begin{aligned}
d m^{* \prime} & =\frac{d\left(C R^{* 2}\right)}{d R^{*}} d R^{*} \\
& =2 C R^{*} d R^{*} \ldots \ldots \text { (3) }
\end{aligned}
$$

The bubble will tend to grow spontaneously if

$$
d m^{*}>d m^{*}
$$


274

or if

$$
4 \pi R^{2} N C R^{2}>2 C R^{*}
$$

$\therefore$ The critical radius is given by

$$
R^{*} \geqslant \frac{1}{2 \pi N R^{2}}
$$

If $\frac{R^{*}}{R}=10$, then from eq. (4),

$$
N=\frac{1}{2 \pi \times 10 \times R^{3}}
$$

But $\quad V_{b}=\frac{4}{3} \pi R^{3} \quad$ (Volume of a bubble)

$$
\therefore N v_{b}=\Delta V=\frac{\frac{4}{3} \pi R^{3}}{2 \pi \times 10 \times R^{3}}=\frac{2}{30}=0.0667
$$

$\therefore$ Swelling is

$$
\begin{aligned}
\frac{\Delta v}{v} & =\frac{0.0667}{1-0.0667} \\
& =\frac{0.0667}{0.9333}=7.2 \%
\end{aligned}
$$


275

Problem 13.7

a)

$$
v=M F \quad[\text { from Eq. (13.220)] }
$$

where $F=$ force on interstitial $=-\nabla V$

$$
\begin{aligned}
M & =\text { mobility of interstitial } \\
& =\frac{D_{i}}{k T} \quad \text { [from Eq. (13.221)] } \\
\therefore & =-\frac{D_{i}}{k T} \nabla v
\end{aligned}
$$

[from Eq .(13.223)]

b)

$$
\begin{aligned}
& J_{i}=\text { interstitial flux } \\
& J_{i}=\underbrace{-D_{i} \nabla C_{i}}_{\text {concentration drift }}+\underbrace{C_{i} v}_{i} \\
& \text { gradient } \\
& \therefore J_{i}=-D_{i}\left(\nabla C_{i}+\frac{C_{i}}{k T} \nabla V\right)
\end{aligned}
$$

c) Continuity equation is:

$$
\begin{aligned}
& \frac{\partial C_{i}}{\partial t}=-\nabla \cdot J_{i} \\
& \therefore \quad \frac{\partial C_{i}}{\partial t}=D_{i} \nabla \cdot\left(\nabla C_{i}+\frac{C_{i}}{k T} \nabla V\right) \text { [fromeg.(2)] }
\end{aligned}
$$

or

$$
\frac{\partial C_{i}}{\partial t}=D_{i}\left(\nabla^{2} C_{i}+\frac{C_{i}}{k T} \nabla^{2} V+\frac{1}{k T} \nabla C_{i} \cdot \nabla V\right)
$$


276

d) Steady state: $\frac{\partial C_{i}}{\partial t}=0$

$$
v=-B / \gamma
$$

In cylindrical coordinates,

$$
\begin{gathered}
\nabla^{2} C_{i}=\frac{1}{r} \frac{d}{d r}\left(r \frac{d C_{i}}{d r}\right) \\
\nabla^{2} V=\frac{1}{r} \frac{d}{d r}\left[r \frac{d}{d r}\left(-\frac{B}{r}\right)\right]=-\frac{B}{r^{3}} \\
\nabla C_{i} \cdot \nabla V=\frac{d C_{i}}{d r} \cdot \frac{d V}{d r}=\frac{B}{r^{2}} \frac{d C_{i}}{d r}
\end{gathered}
$$

Continuity equation becomes,

$$
\frac{1}{r} \frac{d}{d r}\left(r \frac{d C_{i}}{d r}\right)-\frac{A C_{i}}{r^{3}}+\frac{A}{r^{2}} \frac{d C_{i}}{d r}=0
$$

[Using (4), (5), (6) and (7) in (3)]

where $A=B / k T=$ characteristic range of

Let $\quad C_{i}(\gamma)=e^{A / r} u(r)$ interaction

then substituting this in (8) we get,

$$
\frac{d^{2} u}{d r^{2}}+\left(\frac{1}{r}-\frac{A}{r^{2}}\right) \frac{d u}{d r}=0
$$

e)

$$
\text { With } A=0, \quad c_{i}(r)=U(r)
$$

and the differential equation (9) becomes,

$$
\frac{d^{2} C_{i}}{d r^{2}}+\frac{1}{r} \frac{d C_{i}}{d r}=0
$$

By inspection the solution to this equation is seen to be 
277

$$
c_{i}(r)=k \ln r+k^{\prime} \ldots(11)
$$

where $K$ and $K^{\prime}$ are constants of integration. Using the boundary conditions specified in the problem.

$$
c_{i}\left(R_{d}\right)=c_{i}^{e q}=0
$$

and $\quad C_{i}(R)=C_{i}$

(specified)

$---(13)$

$$
\left.\therefore 0=k \ln R_{d}+k^{\prime} \quad \text { [using (12) in (11) }\right]
$$

and $C_{i}=k \ln Q+k^{\prime} \quad$ [using (13) in (ii)]

Subtracting we get,

$$
c_{i}=K \ln \left(\frac{R}{R_{d}}\right)
$$

or $K=\frac{C_{i}}{\ln \left(\frac{R}{R_{i}}\right)}$

The flux per unit length of dislocation line (with flux positive, when directed towards the dislocation line) is:

$$
\begin{aligned}
J_{i}^{d} & =2 \pi R_{d}\left[D_{i}\left(\frac{d C_{i}}{d r}\right)_{R_{d}}\right] \\
& \left.=2 \pi R_{d}\left[\frac{D_{i} K}{R_{d}}\right]=\frac{2 \pi D_{i} C_{i}}{\ln \left(R / R_{d}\right)} \text { [using }(14)\right] \\
\therefore J_{i}^{d} & =\frac{2 \pi D_{i} C_{i}}{\ln \left(R / R_{d}\right)} \text {-(15) [Compare this with Eq .(13.89)] }
\end{aligned}
$$


$2>8$

f) Let $w=\frac{d u}{d r}$

Substituting in eq .(q) we get,

$$
\begin{aligned}
& \frac{d W}{d r}+\left(\frac{1}{r}-\frac{A}{r^{2}}\right) W=0 \\
& \text { or } \quad \frac{d W}{W}=-\left(\frac{1}{r}-\frac{A}{r^{2}}\right) d r
\end{aligned}
$$

Integrating:

$$
\begin{aligned}
& \ln w=-\ln r-\frac{A}{r}+\ln K_{1} \quad\left[\begin{array}{l}
\ln K_{1} \text { is a } \\
\text { constant of }
\end{array}\right. \\
& \text { or } \left.w=\frac{d u}{d r}=k_{1} \frac{e^{-A / r}}{r}--(16)^{\text {integration }}\right]
\end{aligned}
$$

Boundary conditions:

$$
\begin{gathered}
\text { At } r=R_{d}, \quad C_{i}=C_{i}^{e q} \cong 0 \\
\therefore u\left(R_{d}\right)=C_{i}^{e q} e^{-A / R_{d}} \cong 0 \\
\text { At } r=R, \quad C_{i}=C_{i} \text { (specified) } \\
\therefore U(R)=C_{i} e^{-A / R} \ldots(18)
\end{gathered}
$$

Now integrate (16) from $R_{d}$ to $Q$ :

$$
+\int_{u\left(R_{d}\right)}^{u(R)} d u=u(R)-u\left(R_{d}\right)=k_{1} \int_{R_{d}}^{R} \frac{e^{-A / r}}{r} d r
$$

or the constant $K_{1}$ is:

$$
K_{1}=\frac{C_{i} e^{-A / R}}{\int_{R_{d}}^{R} \frac{e^{-A / r}}{r} d r} \quad[u \operatorname{sing}(17) \text { and }(18)
$$


$2>9$

Flux at the core of dislocation from eq. (2) is

$$
\begin{aligned}
& J=-D_{i}\left[\left(\frac{d C_{i}}{d r}\right)_{R_{d}}+\frac{C_{i}^{e q}}{k T} \cdot \frac{B}{R_{d}^{2}}\right] \\
& \text { since } C_{i}^{e q} \cong 0 \\
& \text { but } \left.\frac{d C_{i}}{d r}=-\frac{A}{r^{2}} e^{A / r} u+e^{A / r} \frac{d u}{d r}\right)_{R_{d}} \\
& \therefore\left(\frac{d C_{i}}{d r}\right)_{R_{d}}=-\frac{A}{R_{d}^{2}} e^{A / R_{d}} C_{i}^{e q} e^{-A / R_{d}}+e^{A / R_{d}}\left(\frac{d u}{d r}\right)_{R_{d d}} \\
& \text { Again since } c_{i}^{e q} \simeq 0
\end{aligned}
$$

Again since $c_{i}^{e q} \cong 0$,

$$
\begin{aligned}
\therefore\left(\frac{d C_{i}}{d r}\right)_{R_{d}} & =e^{A / R_{d}}\left(\frac{d u}{d r}\right)_{R_{d}} \ldots-(21) \\
\therefore J \cong-D_{i} e^{A / R_{d}}\left(\frac{d u}{d r}\right)_{R_{d}} \quad[\text { from (20) and (21)] } & --(22) \\
\text { But }\left(\frac{d u}{d r}\right) & =K_{1} \frac{e^{-A / R_{d}}}{R_{d}} \quad[\text { from eq (16)] } \\
& =\frac{C_{i} e^{\left(-n / R_{d}-A / R\right)}}{R_{d} \int_{R_{d}}^{R} \frac{e^{-A / r}}{r} d r}
\end{aligned}
$$

Now since $A / Q \cong 0$, then

$$
\left(\frac{d u}{d r}\right)_{R_{d}}=\frac{C_{i} e^{-A / R_{d}}}{R_{d} \int_{R_{d}}^{Q} \frac{e^{-A / r}}{r} d r}
$$


280

From (22) and (23),

$$
\left.J=-\frac{D_{i} C_{i}}{R_{d} \int_{R_{d}}^{R} \frac{e^{-A / r}}{r} d r} \quad \begin{array}{l}
\text { (This current is positive, when } \\
\text { directed outward from } \\
\text { dislocation line }
\end{array}\right)
$$

The area per Unit length of dislocation line is:

$$
2 \pi R_{d}
$$

$\therefore$ Flux of interstitial into a unit length of dislocation line is:

$$
\begin{aligned}
& J_{i}^{d}=-2 \pi R_{d} J \\
& \therefore J_{i}^{d}=\frac{2 \pi D_{i} C_{i}}{\int_{R_{d}}^{R} \frac{e^{-\pi / r}}{r} d r}[u \operatorname{sing}(24)] \ldots \ldots \text { (25) } \\
& \text { [This equation replaces }
\end{aligned}
$$$$
\text { Eq. (13.89) for }
$$$$
\text { interstitial] }
$$

Now in order that the solution to part e) ie. eq. (15) equals that of part f) ie eq.(25), replace $R_{d}$ in eq.(15) by $R_{d i}$ and equate the resulting $J_{i}^{d}$ expression to that in (25). We obtain

$$
\ln \left(R / R_{d i}\right)=\int_{R_{d}}^{R} \frac{e^{-A / r}}{r} d r \quad-(26)
$$

Let

$$
\begin{aligned}
& \eta=r / R_{d}, \quad \eta_{f}=R / R_{d} \\
& \frac{A}{R_{d}}=0.1
\end{aligned}
$$


281

$$
\ln \left[\frac{\eta_{f}}{\left[\left(R_{d i} / R_{d}\right)\right.}\right]=\int_{1}^{\eta} \frac{e^{-0.1 / \eta}}{\eta} d \eta
$$

Now write: $e^{-0.1 / \eta}=1-\left(1-e^{-0.1 / \eta}\right)$

$$
\begin{gathered}
\therefore \ln \eta_{f}-\ln \left(R_{d i} / R_{d}\right)=\int_{1}^{\eta_{f}} \frac{d \eta}{\eta}-\int_{1}^{\eta_{f}} \frac{1-e^{-0.1 / \eta}}{\eta} d \eta \\
\text { But } \int_{1}^{\eta_{f}} \frac{d \eta}{\eta}=\ln \eta_{f} \\
\therefore \ln \left(R_{d i} / R_{d}\right)=\int_{1}^{\eta_{f}} \frac{1-e^{-0.1 / \eta}}{\eta} d \eta \\
\therefore \frac{R_{d i}}{R_{d}}=\exp \left\{\int_{1}^{\eta_{f}}\left(\frac{1-e^{-0.1 / \eta}}{\eta}\right) d \eta\right\}
\end{gathered}
$$

Expand $e^{-0.1 / \eta}$ in Taylor Series:

$$
e^{-0.1 / \eta}=1-\frac{0.1}{\eta}+\frac{1}{2} \frac{0.01}{\eta^{2}}-\frac{1}{6} \frac{0.001}{\eta^{3}}+\ldots
$$
we can replace the limit of integration from $\eta_{f}$ to $\infty$.

$$
\begin{aligned}
& \therefore \quad \int_{1}^{\infty}\left(\frac{1-e^{-0.1 / \eta}}{\eta}\right) d \eta \\
& =0.1 \int_{1}^{\infty} \frac{d \eta}{\eta^{2}}-\frac{0.01}{2} \int_{1}^{\infty} \frac{d \eta}{\eta^{3}}+\frac{0.001}{6} \int_{1}^{\infty} \frac{d \eta}{\eta^{4}}+\cdots \\
& =0.1-\frac{0.01}{2 \times 2}+\frac{0.001}{6 \times 3}+
\end{aligned}
$$


282

$$
\begin{aligned}
\therefore \int_{1}^{\infty}\left(\frac{1-e^{-0.1 \eta}}{\eta}\right) d \eta & =0.1-2.5 \times 10^{-3}+5.5 \times 10^{-5}+\cdots \\
& =0.0976 \\
\therefore \frac{R_{d i}}{R_{d}} & =\exp [0.0976] \\
& =1.10
\end{aligned}
$$

g) For $\rho_{d}=10^{10} \mathrm{~cm}^{-2}, \quad Q$ is determined by

$$
\begin{aligned}
& \pi R^{2}=1 / \rho_{d} \\
& \therefore R=\frac{1}{\sqrt{\pi \rho_{d}}}=5.6 \times 10^{-6} \mathrm{~cm} .
\end{aligned}
$$

For vacancies:

$$
\begin{aligned}
& \frac{2 \pi}{\ln \left(R / R_{d}\right)}=\frac{2 \pi}{\ln \left(\frac{5.6 \times 10^{-6}}{6 \times 10^{-8}}\right)}=1.38 \\
& \therefore \quad Z_{V}=\frac{1}{\frac{1}{24}+\frac{1}{1.38}}=1.31
\end{aligned}
$$

For interstitial:

$$
\begin{gathered}
\frac{2 \pi}{\ln \left(R / R_{d i}\right)}=\frac{2 \pi}{\ln \left(\frac{5.6 \times 10^{-6}}{1.1 \times 6 \times 10^{-3}}\right)}=1.41 \\
Z_{i}=\frac{1}{\frac{1}{24}+\frac{1}{1.41}}=1.335 \\
\therefore \frac{z_{i}-z_{V}}{z_{V}} \cong 2 \%
\end{gathered}
$$


283

Problem 13.8

Mean ff path length through bubble of radius $R$ Assume parallel beam of $f^{\prime} s$.

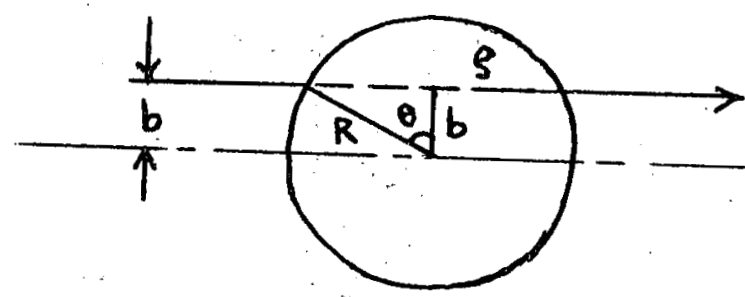

$\rho=$ track length for impact parameter $b$

$=$ chord length through circle

$$
=2 R \sin \theta=2 R \sqrt{1-\left(\frac{b}{R}\right)^{2}}
$$

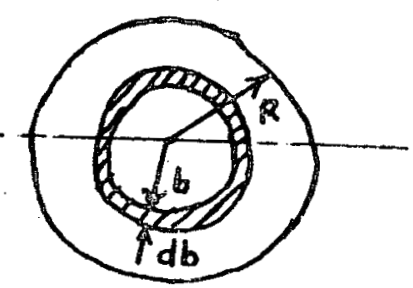

Head on view

Let $\quad P(b) d b=$ probability of ff striking with impact parameter in $(b, d b)$ (assuming it in fact does strike. the bubble!)

$$
P(b) d b=K 2 \pi b d b=\frac{2}{R^{2}} b d b
$$

since $\int_{0}^{R} P(b) d b=1=2 \pi K \int_{0}^{R} b d b=\pi R^{2} K$

$$
\begin{gathered}
\therefore \quad \bar{\rho}=\int_{0}^{R} P(b) \rho(b) d b \\
=4 R \int_{0}^{R} \sqrt{1-\left(\frac{b}{R}\right)^{2}} \frac{b d b}{R^{2}}=4 R \int_{0}^{1} \sqrt{1-\eta^{2}} \eta d \eta \\
\text { Let } \omega=\eta^{2} \quad \therefore \quad d \omega=2 \eta d \eta \\
\therefore \frac{\bar{\rho}}{R}=2 \int_{0}^{1} \sqrt{1-\omega} d \omega=2\left[-\frac{2}{3}(1-\omega)^{3 / 2}\right]_{0}^{1}=\frac{4}{3} \\
\therefore \bar{\rho}=\frac{4}{3} R
\end{gathered}
$$

$\widehat{n}$ 
284

Problem 13.9

a) After low temperature irradiation, $t_{1}$

$$
\begin{aligned}
c_{01}=y_{x_{e}} \dot{F} t_{1} & =0.25 \times 5 \times 10^{13} \times 10^{6} \\
& =1.25 \times 10^{19} \frac{a \text { toms }}{c c}
\end{aligned}
$$

b)

Let $m_{1}=$ gas atoms/bubble

$$
c_{01}=m_{1} N
$$

From Eq .(13.16),

$$
m_{1}=\frac{4}{3} \pi R_{1}^{2}\left(\frac{2 \gamma}{k T}\right)
$$

(assuming $R_{i}$ large enough for ideal gas law

Using (1) and (2) and solving for $R_{1}$. to apply)

$$
R_{1} \cong \frac{1}{2} \sqrt{\frac{k T}{2 x} \frac{Y_{x_{e}} \dot{F} t_{i}}{N}}
$$

[where $\frac{4}{3} \pi \cong 4$ is used]

Putting numbers,

$$
R_{1}=175 A^{\circ}
$$

c) Swelling

$$
\begin{aligned}
\left(\frac{\Delta V}{V}\right)_{1} & =\left(\frac{4}{3} \pi R_{1}^{3}\right) N \\
& =2.1 \times 10^{-3}=0.2 \%
\end{aligned}
$$

d) After second low -temperature irradiation, $\therefore$ consider one bubble. From Eq. (13.117)

$\frac{\text { gas atoms redissolved }}{\mathrm{sec}-\text { bubble }}=b m--(6)$ 
285

$$
\therefore \quad \frac{d m}{d t}=-b m
$$

Integrate from $m=m_{1}$ at $t=0$ to $m=m_{2}$ at $t=t_{2}$

$$
\frac{m_{2}}{m_{1}}=e^{-b t_{2}}
$$

and:

$$
\begin{aligned}
& \quad b t_{2}=\left(2 \times 10^{-19}\right)\left(5 \times 10^{13}\right) \times 10^{5}=1 \\
& \therefore \quad \frac{m_{2}}{m_{1}}=0.37
\end{aligned}
$$

using (3)

$$
\begin{aligned}
\frac{R_{2}}{R_{1}} & =\sqrt{\frac{m_{2}}{m_{1}}}=0.61 \\
\therefore R_{2} & =106 \mathrm{~A}
\end{aligned}
$$

e) Swelling at $t_{2}$;

$$
\begin{aligned}
\left(\frac{\Delta V}{V}\right)_{2} & =\left(\frac{4}{3} \pi R_{2}^{3}\right) N=\left(\frac{\Delta V}{V}\right)_{1} \cdot\left(\frac{R_{2}}{R_{1}}\right)^{3} \\
& =2.1 \times 10^{-3} \times(0.61)^{3}=4.7 \times 10^{-4} \cong 0.05 \%
\end{aligned}
$$

f) Gas in solution at $t_{2}$;

$$
\begin{aligned}
& \text { Total gas present }=Y_{x_{e}} \dot{F}\left(t_{1}+t_{2}\right) \\
&=\gamma_{\text {ie }} \dot{F} t_{1}\left(1+\frac{t_{2}}{t_{1}}\right)=c_{01}\left(1+\frac{t_{2}}{t_{1}}\right)
\end{aligned}
$$

Gas in bubbles at $t_{2}=m_{2} N=\left(\frac{m_{2}}{m_{1}}\right) m_{1} N=\left(\frac{m_{2}}{m_{1}}\right) c_{01}$

$$
\begin{gathered}
C_{02}=\text { gas in solution at } t_{2}=C_{01}\left(1+\frac{t_{2}}{t_{1}}-\frac{m_{2}}{m_{1}}\right) \\
=C_{01}(1+0.1-0.37)=0.73 C_{01} \\
\therefore \quad C_{02}=0.91 \times 10^{19} \frac{\text { gas atoms }}{c^{3}}
\end{gathered}
$$


286

Problem 13.10

a)

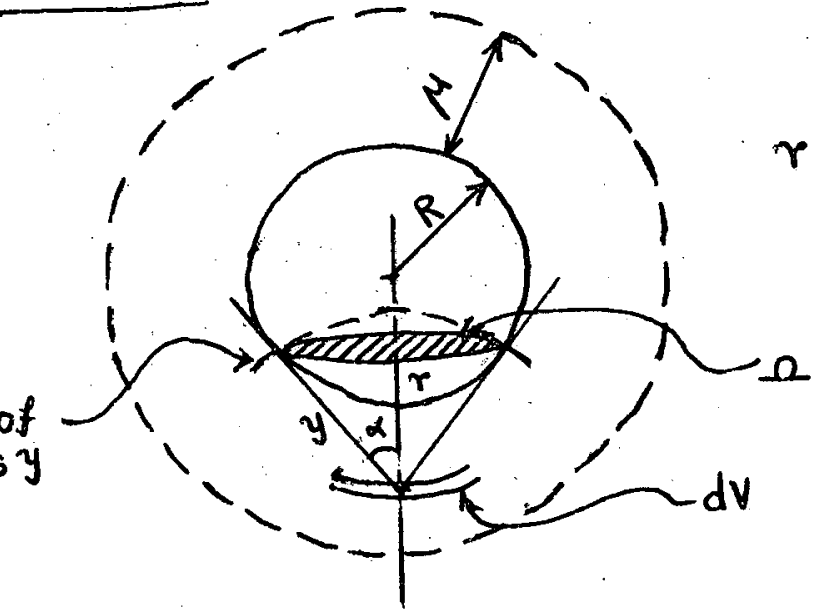

$r=$ distance of spherical shell $d V$ from the center of the bubble

$d V=$ spherical shell of thickness $d r$; where $R \leq r \leq R+\mu$

$\Omega=$ probability of striking the bubble $=$ solid angle subtended by bubble from $d V=\frac{A}{4 \pi y^{2}}$

where $A=$ area of spherical cap of sphere of radius $=y$ and angle $=\alpha$

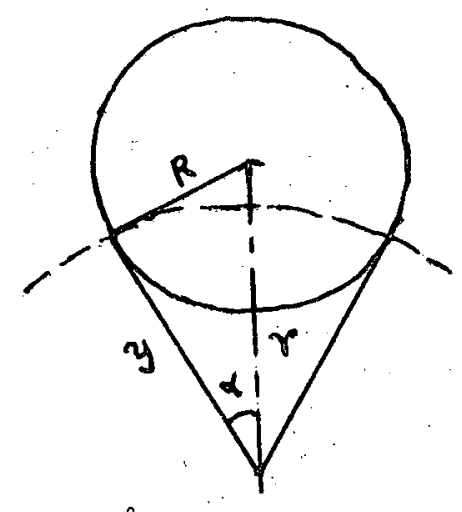

$$
\begin{aligned}
d A & =2 \pi y^{2} d(\cos \theta) \\
A & =2 \pi y^{2}(1-\cos \alpha) \\
& =2 \pi y^{2}\left[1-\sqrt{1-(R / \gamma)^{2}}\right]
\end{aligned}
$$

$$
\begin{aligned}
& y=\sqrt{R^{2}-r^{2}} \\
& \sin \alpha=\frac{R}{r} \\
& \cos \alpha=\sqrt{1-\left(\frac{R}{r}\right)^{2}}
\end{aligned}
$$

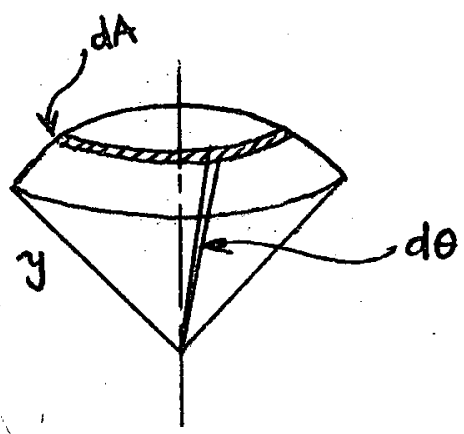


287

$$
\therefore \Omega=\frac{A}{4 \pi y^{2}}=\frac{1}{2}\left[1-\sqrt{1-(R / r)^{2}}\right]
$$

. Check on limits:

$$
\begin{aligned}
& \left(\frac{R}{r}\right)^{2} \longrightarrow 0 ; \Omega \longrightarrow \frac{R^{2}}{4 r^{2}}(O K) \\
& \left(\frac{R}{r}\right)^{2} \longrightarrow 1 ; \Omega \longrightarrow \frac{1}{2} \quad(O K)
\end{aligned}
$$

let. $Q=f f^{\prime}$ cutting bubble $/ \mathrm{sec}=2 \dot{F} \int_{R}^{R+\mu} \Omega 4 \pi r^{2} d r$

$$
Q=4 \pi \dot{F} \int_{R}^{R+\mu}\left[1-\sqrt{1-(R / r)^{2}}\right] r^{2} d r
$$

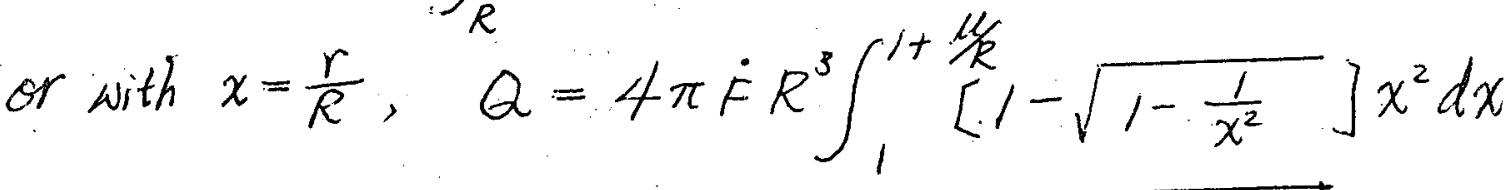

Let $\quad G(\mu / R)=\int_{1}^{1+\mu / / R}\left[1-\sqrt{1-\frac{1}{x^{2}}}\right] x^{2} d x$

$$
\begin{gathered}
G=\int_{1}^{1+\mu / R}\left[x^{2}-x \sqrt{x^{2}-1}\right] d x=\left[\frac{x^{3}}{3}-\frac{\left(x^{2}-1\right)^{\frac{3}{2}}}{3}\right]_{1}^{1+M / R} \\
G=\frac{1}{3}\left\{\left(1+\frac{M}{R}\right)^{3}-1-\left[\left(1+\frac{M}{R}\right)^{2}-1\right]^{\frac{3}{2}}\right\}
\end{gathered}
$$


288

Limit of $G$ as $\mu / R>>1$ :

let $1+\frac{M}{R}=A$

$$
\begin{aligned}
G & =\frac{1}{3}\left\{A^{3}-1-\left[A^{2}-1\right]^{\frac{3}{2}}\right\}=\frac{1}{3}\left\{A^{3}-1-A^{3}\left(1-\frac{1}{A^{2}}\right)^{\frac{3}{2}}\right\} \\
& \cong \frac{1}{3}\left\{A^{3}-1-A^{3}\left(1-\frac{3}{2 A^{2}}\right)\right\}=\frac{1}{3}\left(\frac{3 A}{2}-1\right) \\
& \cong \frac{1}{2} A=\frac{1}{2}\left(1+\frac{M}{R}\right) \simeq \frac{1}{2} \frac{M}{R} \\
\therefore Q & =4 \pi \dot{F} R^{3}\left(\frac{1}{2} \frac{M}{R}\right)=2 \pi \dot{F} R^{2} \mu
\end{aligned}
$$

Limit of $G$ as $\mu / R \longrightarrow 0$ :

$$
G=\frac{1}{3}\left\{\left(1+\frac{3 M}{R}\right)-1-\left[\left(1+\frac{2 M}{R}\right)-1\right]^{\frac{3}{2}}\right\}=\frac{1}{3}\left\{\frac{3 M}{R}-\left(\frac{2 M}{R}\right)^{\frac{3}{2}}\right\} \cong \frac{M}{R}
$$

and $Q=4 \pi \dot{F} R^{3}\left(\frac{\mu}{R}\right)=4 \pi \dot{F} R^{2} \mu$

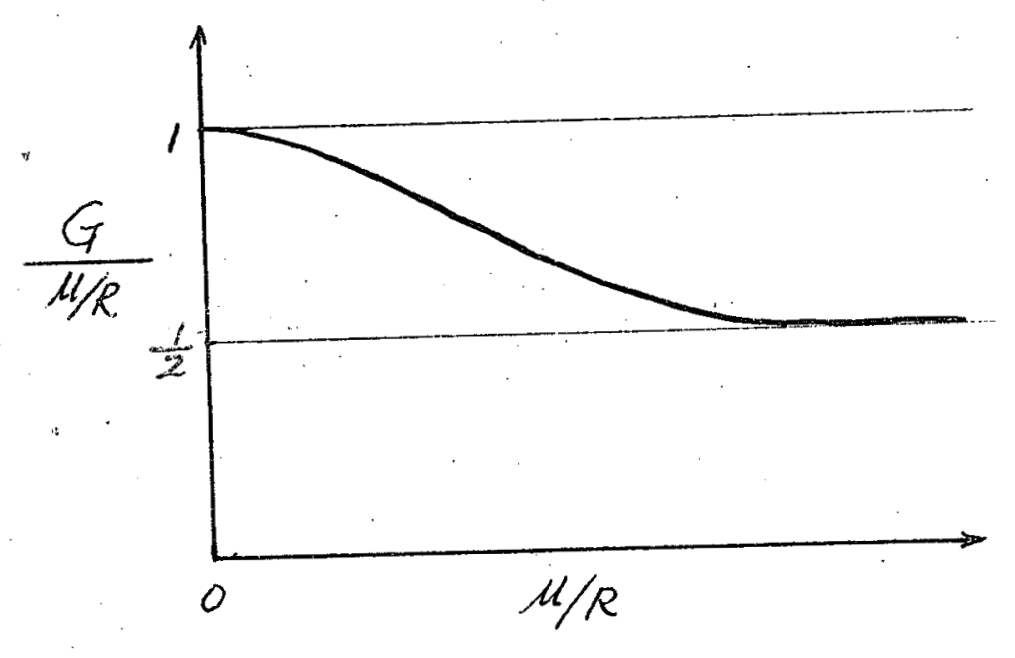


289

b) Each ff which intersects bubble redissolves $\Delta m$ gas atoms

$$
\begin{aligned}
& \therefore \frac{\text { gas atoms redissolved }}{\text { sec. - bubble }}=Q \Delta m=4 \pi \dot{F} R^{3} G(\mu / R) \Delta m \\
& \text { or } \quad b=4 \pi \dot{F} R^{3} G(\mu / R) \frac{\Delta m}{m}
\end{aligned}
$$

where $m=$ Number of gas atoms per bubble (equilibrium bubble)

$$
\begin{gathered}
P\left(\frac{4}{3} \pi R^{3}\right)=m k T \text { and } P=\frac{2 \gamma}{R} \\
\therefore m=\frac{8 \pi r R^{2}}{3 k T}\left(\frac{\text { gas atoms }}{\text { bubble }}\right) \\
\therefore \quad b=\frac{3 k T \Delta m R G(M / R)}{2 \gamma}
\end{gathered}
$$

for $\mu / R>>1$

$$
b=\frac{3 k T \Delta m M}{4 \gamma}
$$

for $\mu / R<1$

$$
b=\frac{3 k T \Delta m \mu}{2 \gamma}
$$




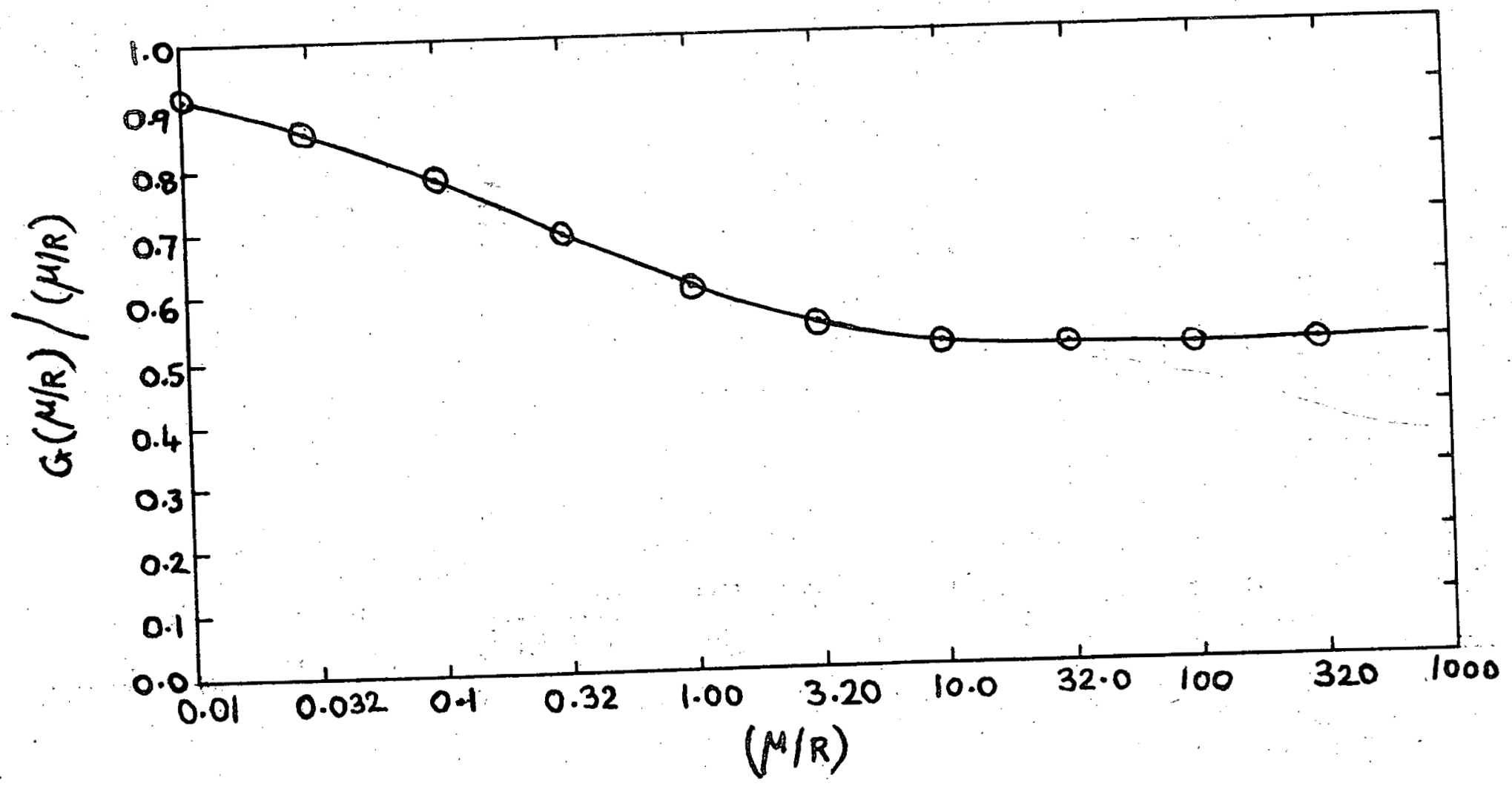

$\frac{1}{0}$ 
291

CHAPTER 14

Problem 14.1

a)

Radius of central void after restructuring is complete: All the pores in the region $0 \leq r \leq 0.8 R$ contribute to the final central void.

$$
P_{0} \pi(0.8 R)^{2}=\pi r_{\text {of }}^{2}
$$

or $\frac{\gamma_{\text {of }}}{R}=0.8 \sqrt{P_{0}}$

b) For constant thermal conductivity, the heat conduction equation in the fuel is:

$$
\frac{k}{r} \frac{d}{d r}\left(r \frac{d T}{d r}\right)=-H
$$

BiC.

$$
\begin{aligned}
& T(R)=T_{s} \\
& \left(\frac{d T}{d r}\right)_{r_{0}}=0
\end{aligned}
$$

If the rod is operated at constant linear power, the average heat generation rate in the columnar grain region is [.From Eq. (10.54)]

$$
\bar{H}=\frac{P}{\pi R^{2}\left(1-\frac{r_{0}^{2}}{R^{2}}\right)}=\frac{H_{0}}{1-\left(\frac{r_{0}}{R}\right)^{2}} 1028-(5)
$$

Where $H_{0}$ is the initial power density of the fuel since $x_{0}$ starts at $O$ and increases 
292

to the value given by eq. (1) when restructuring is complete, $r_{0} / R$ increases with time and therefore $\bar{H}$ increases with time

Assume that the local power density in the columnar region is equal to the average power density throughout the region, or that $H$ in eq. (2) is equal to $\bar{H}$ given by eq. (5) for all $r_{0} \leqslant \gamma \leqslant 0.8 R$.

Then the solution to eq. (2) $-(4)$ in the zone $\tau_{0} \leq r \leq 0.8 R$ is

$$
T_{1}(r)=-\frac{1}{4} \frac{\bar{H}}{k} r^{2}+A \ln r+B
$$

where $T_{1}(r)$ is the temperature distribution in this region. $A$ and $B$ are constants of integration.

From B.C. (4),

$$
A=\frac{1}{2} \frac{\bar{H}}{k} r_{0}^{2}=\frac{1}{2} \frac{H_{0} R^{2}}{k} \cdot\left(\frac{\bar{H}}{H_{0}}\right)\left(\frac{Y_{0}}{R}\right)^{2}
$$

In the unrestructured region $0.8 R \leq r \leq R, H$ in eq.(2) is equal to $H_{0}$, and the temperature distribution $T_{2}(r)$ is given by:

$$
T_{2}(r)=-\frac{1}{4} \frac{H_{0}}{k} r^{2}+c \ln r+D
$$

Using B.C. (3),

$$
T_{s}=-\frac{1}{4} \frac{H_{0}}{k} R^{2}+c \ln R+D
$$


293

Matching the two solutions at $r=0.8 R$ :

and

$$
T_{1}(0.8 R)=T_{2}(0.8 R)
$$

$$
\left(\frac{d T_{1}}{d r}\right)_{0.8 R}=\left(\frac{d T_{2}}{d r}\right)_{0.8 R}
$$

[Note: $k$ is constant throughout the

$$
\text { region } \left.r_{0} \leq r \leq R\right] \text {. }
$$

Using (6) and (8) in (11) yields:

$$
\begin{aligned}
-\frac{1}{4} \frac{H}{k}(0.8 R)^{2}+A \ln (0.8 R)+B= & -\frac{1}{4} \frac{H_{0}}{k}(0.8 R)^{2} \\
& +C \ln (0.8 R)+D
\end{aligned}
$$

Using (6) and (8) in (12) yields:

Solving (14) for $C$ gives:

$$
C=A-\frac{1}{2}\left(\frac{\bar{H}-H_{0}}{k}\right)(0.8 R)^{2}=A-\frac{1}{2} \frac{H_{0} R^{2}}{k}(0.64)\left(\frac{H}{H_{0}}-1\right)
$$

Using eq. (f) for $A$, we get,$$
C=\frac{1}{2} \frac{H_{0} R^{2}}{k}\left[\left(\frac{r_{0}}{R}\right)^{2}\left(\frac{\bar{H}}{H_{0}}\right)-0.64\left(\frac{\bar{H}}{H_{0}}-1\right)\right]
$$

Solving eq. (q) for $D$

$$
D=T_{S}+\frac{H_{0} R^{2}}{k}-C \ln R--(17)
$$

where $C$ is given by eq. (16) 
2.94

Now using (13) and (17) to determine $B$ :

$$
\begin{gathered}
B=\frac{1}{4}\left(\frac{\bar{H}-H_{0}}{k}\right)(0.8 R)^{2}+(C-A) \ln (0.8 R)+T_{S}+\frac{1}{4} \frac{H_{0}}{k} R^{2} \\
-C \ln R
\end{gathered}
$$

Substituting into (6):

$$
\begin{aligned}
& T_{1}(r)-T_{5}=-\frac{1}{4} \frac{\bar{H}^{\prime}}{k} r^{2}+A \ln r+\frac{1}{4}\left(\frac{\bar{H}-H_{0}}{k}\right)(0.8 R)^{2} \\
&+(C-A) \ln (0.8 R)+\frac{1}{4} \frac{H_{0}}{k} R^{2}-C \ln R \\
& \text { but } C \ln R= {[A+(C-A)] \ln R=A \ln R+(C-A) \ln R } \\
& \therefore T_{1}(r)-T_{5}=-\frac{1}{4} \frac{\bar{H}}{k} r^{2}+\frac{1}{4} \frac{H_{0} R^{2}}{k}+\frac{1}{4}\left(\frac{\bar{H}-H_{0}}{k}\right)(0.8 R)^{2} \\
&+A \ln \left(\frac{\gamma}{R}\right)+(C-A) \ln (0.8)
\end{aligned}
$$

Using (7) for $A$ and (15) for $(C-A)$ we get:

$$
\begin{aligned}
T_{1}(r)-T_{S}= & \frac{1}{4}\left(\frac{H_{0} R^{2}}{k}\right)\left\{0.36+\frac{\bar{H}}{H_{0}}\left(0.64-\frac{r^{2}}{R^{2}}\right)\right\} \\
& +\frac{H_{0} R^{2}}{2 k}\left(\frac{\bar{H}}{H_{0}}\right)\left(\frac{Y_{0}}{R}\right)^{2} \ln \left(\frac{r}{R}\right)-\frac{H_{0} R^{2}}{2 k}[0.64 \ln (0.8)]\left(\frac{\bar{H}}{H_{0}}-1\right)
\end{aligned}
$$

But $\quad \frac{\bar{H}}{H_{0}}=\frac{1}{1-\left(\frac{x_{0}}{R}\right)^{2}} \quad[$ from eq. (s) $]$

Also $H_{0}=\frac{P}{\pi R^{2}}$, then

$$
\left.+2\left[\frac{\left(\gamma_{0} / R\right)^{2}}{1-\left(\gamma_{0} / R\right)^{2}}\right]\left[\ln \left(\gamma_{R}\right)-0.64 \ln (0.8)\right]\right\}
$$


Eq. (18) gives the temperature distribution in the columnar grain region as a function of $\left(r_{0} / R\right)$. The central void radius starts at $r_{0}=0$ when $t=0$ and grows to $r_{0}$ given by eq. (1) at the end of restructuring. We have assumed that the variation of $\left(r_{0} / R\right)$ is known as a function of time.

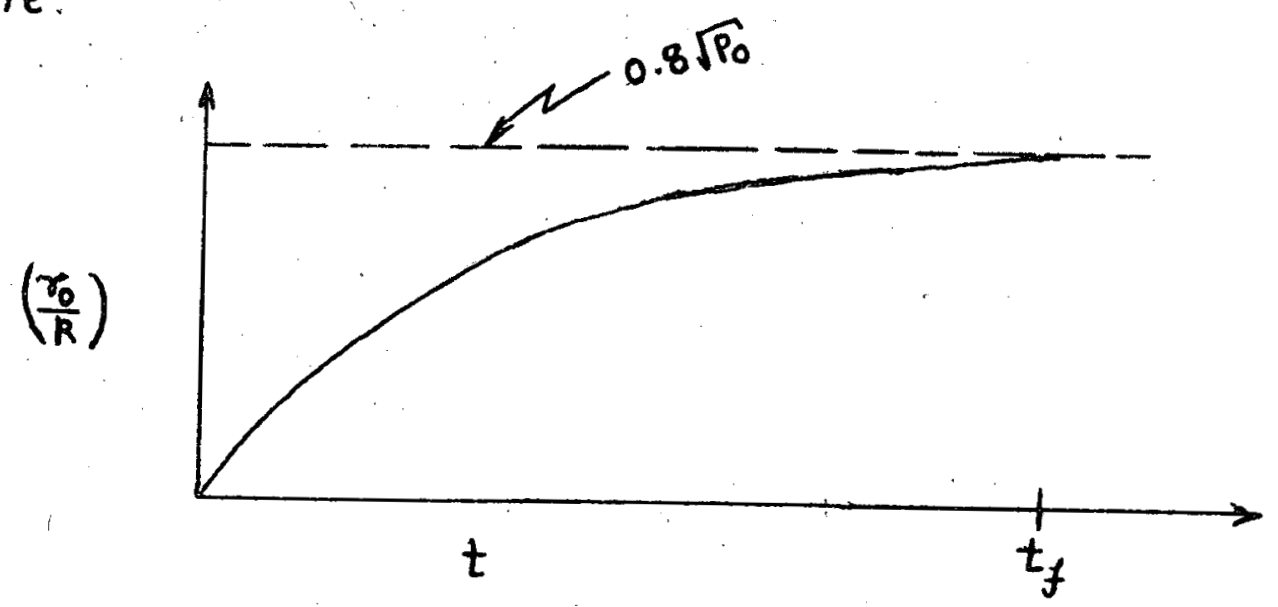

Therefore the temperature distribution in the columnar grain region is a function of both $r$ and $t$, or $T(r, t)$. c) The time to complete restructuring

$$
\begin{aligned}
& \text { Time for a pore at the } \\
& \text { outer edge of columnar } \\
& \text { grain region to migrate } \\
& \text { to the final central void } \\
& \text { position } r_{0}=r_{\text {of }}
\end{aligned}
$$

The pore velocity is a known function of temperature, but as a result of the solution in b), the temperature is a function of $r$ and $t$. Therefore the velocity of $a$ pore is a function of $r$ and $t, v_{p}(r, t)$, which is presumed known. 
296

Let $r=$ radial position of a pore which started at $r=0.8 R$ at $t=0$.

$$
\begin{aligned}
& v_{p}(r, t)=\frac{d \gamma}{d t} \\
& \gamma(0)=0.8 R
\end{aligned}
$$

Eq. (19) must be integrated numerically, obtaining $\nu_{p}$ as a function of $T$. from pore migration theory and $T_{1}(r, t)$ from eq. (18). Eq. (1.9) must be integrated from $r=0.8 R$ up to $r=r_{0 f}=0.8 \sqrt{R} R$. The time at which this is reached is $t_{f}$.

d) The pore velocity is given by combination of Ens. (14.6) and (14.9):

$$
v_{p}(T, p)=\frac{C}{p T^{3 / 2}} \exp \left(-\Delta H_{\text {rap }} / R T\right) \ldots(21)
$$

where $c$ is a constant

In the as -fabricated (cold) fuel, the number of gas atoms in each pore is given by:

$$
p_{0}\left(\frac{4}{3} \pi r_{p_{0}}^{3}\right)=m k T_{a} \quad \ldots(22)
$$

When heated to a temperature $T$ and subject to the condition of mechanical equilibrium:

$$
p\left(\frac{4}{3} \pi r_{p}^{3}\right)=m k T
$$

and

$$
p=\frac{2 r}{r_{p}}
$$


Dividing (22) by (23) we get:

$$
\frac{p_{a}}{p}\left(\frac{r_{p_{a}}}{r_{p}}\right)^{3}=\frac{T_{a}}{T} \ldots(25)
$$

Eliminating $r_{p}$ between (24) and (25) yields:

$$
p=\left(\frac{1}{p_{0}} \frac{T_{a}}{T}\right)^{1 / 2}\left(\frac{2 \gamma}{\gamma_{p_{0}}}\right)^{3 / 2} \ldots-(26)
$$

Substituting (26) into (21) gives:

$$
V_{p}(T)=C\left(\frac{P_{0}}{T_{a}}\right)^{1 / 2}\left(\frac{\gamma_{p o}}{2 \gamma^{2}}\right)^{3 / 2} \frac{\exp \left(-\Delta H_{\text {vap }} / R T\right)}{T}
$$


298

Problem 14.2

a) Pore Migration in Mixed Oxide Fuels (Raqudt's Law binary)

See figure on the next page.

$\Delta q=$ mole fraction of $\mathrm{PuO}_{2}$ on hot side - that on -

$q_{0}=$ mole fraction of $\mathrm{PuO}_{2}$ on cold side

cold side

At steady state:

$$
\begin{aligned}
& \frac{J_{P_{u}}}{J_{u}+J_{P_{u}}}=q_{0} \\
& J_{u}=\frac{D_{Q}}{k T_{\delta}}\left[p_{u}^{H}-p_{u}^{c}\right] \\
& J_{P_{u}}=\frac{D_{Q}}{k T^{\prime} \delta}\left[p_{P_{u}}^{H}-p_{P_{u}}^{c}\right]
\end{aligned}
$$

where $p_{i}^{H}=$ partial pressure of $i$ on hot side of pore

$p_{i}^{c}=$ partial pressure of $i$ on cold side of pore

$$
(i=U, P u)
$$

Assuming Raoul's Law for both $\mathrm{UO}_{2}$ and $\mathrm{PuO}_{2}$ in mixture:

$$
\begin{array}{ll}
p_{U}^{H}=\left(1-q_{H}\right) f_{U}\left(T_{H}\right)_{\ldots} & \left(q_{H}=q_{0}+\Delta q\right) \\
p_{P_{U}}^{H}=q_{H} p_{P_{U}}^{0}\left(T_{H}\right) \ldots \ldots(5) & {\left[\begin{array}{l}
T_{H}=\text { hot side temp. } \\
T_{C}=\text { cold side temp. }
\end{array}\right]}
\end{array}
$$




$$
\text { (11) }-\left(\frac{14}{? H \nabla}\right) d x z \frac{{ }^{\perp y}}{? H \nabla !}=\frac{1 p}{\frac{?}{d d p}}
$$

$74 a ! p b \alpha b$ asnjosaduaz ayz s! $\frac{x p}{I p}$ aiaym

$$
\text { (8) moey }
$$

$$
\begin{aligned}
& \text { (oi) }-\left(\frac{x p}{I p}\right)\left(\frac{L p}{0 d p}\right)=\frac{x p}{\delta_{0}^{d} d p} \\
& \text { (6) }-\rho\left(\frac{x p}{g_{0}^{d} p}\right)+\left(\nu_{L}\right)_{0}^{d}=\left(H_{L}\right)_{0}^{b} d
\end{aligned}
$$

(acod $u ! 47)$ llbus

5! alod ay7 550xob doep aenzbeadulaz ay7 azU!s

$$
\begin{aligned}
& \text { (8) } \cdots\left(\frac{1 y}{! H \nabla}-\right) d \times \partial^{2} \forall=(1)_{0}^{?} d \text { do }(1)^{2} f \\
& (t)-\left(\nu_{1}\right)_{0}^{n_{d}} o_{b}={ }_{\partial_{d}}^{n_{d}} \\
& \text { (9) - (1) } n_{f}\left(o_{b}-1\right)={ }_{d}^{n}
\end{aligned}
$$

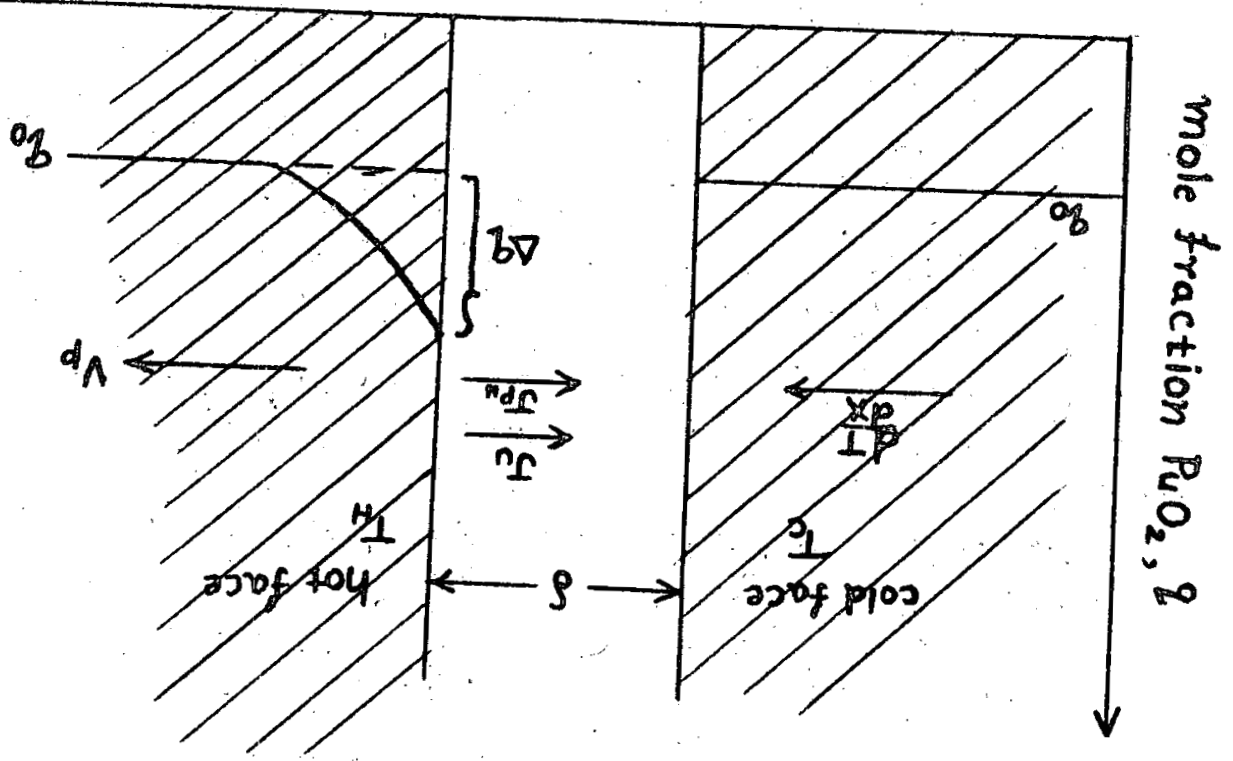

$662^{\circ}$ 


$$
\therefore \quad P_{i}^{0}\left(T_{H}\right) \cong P_{i}^{0}\left(T_{C}\right)+\frac{A_{i} \Delta H_{i}}{k T_{c}^{2}} \exp \left(-\frac{\Delta H_{i}}{k T_{c}}\right) \frac{d T}{d x} \delta
$$

Using. (12) with $i=U$ in (4):

$$
p_{u}^{H}=\left(1-q_{H}\right)\left[f_{u}+\frac{A_{u} \Delta H_{u}}{k T^{2}} \exp \left(-\frac{\Delta H_{u}}{k T}\right)\left(\frac{d T}{d x}\right) \delta\right]
$$

where the subscript " $C$ " used on temperature was dropped.

Using (13) and (6) into (2) we get,

$$
\begin{gathered}
J_{U}=\left(\frac{D_{g}}{k T \delta}\right)\left\{\left(1-q_{H}\right)\left[f_{U}+\frac{A_{U} \Delta H_{U}}{k T^{2}} \exp \left(-\frac{\Delta H_{U}}{k T}\right)\left(\frac{d T}{d x}\right) \delta\right]\right. \\
\left.-\left(1-q_{0}\right) f_{U}\right\}
\end{gathered}
$$

[we approximate $f_{U}\left(T_{C}\right) \cong f_{U}\left(T_{H}\right)=f_{U}(T)$ ]

Using (8) for $f_{U}$ :

$$
\begin{aligned}
J_{u}=\left(\frac{D_{q}}{k T \delta}\right)\left\{\frac{\left(1-q_{H}\right)\left(\frac{d T}{d x}\right) \delta A_{u} \Delta H_{u} \exp \left(-\frac{\Delta H_{u}}{k T}\right)}{k T^{2}}\right. & \left.-A_{U} \exp \left(-\frac{\Delta H_{u}}{k T}\right)\left(q_{H}-q_{0}\right)\right\}
\end{aligned}
$$

Similarly for $\mathrm{PuO}_{2}$ :

$$
--(14)
$$

Replace $\left(1-q_{H}\right)$ by $q_{H}$ and $\left(1-q_{0}\right)$ by $q_{0}$

$$
\begin{array}{r}
J_{P_{u}}=\left(\frac{D_{g}}{k T \delta}\right)\left\{\frac{q_{H}\left(\frac{d T}{d x}\right) \delta A_{P_{u}} \Delta H_{P_{u}} \exp \left(-\frac{\Delta H_{P_{u}}}{k T}\right)}{k T^{2}}\right. \\
\left.+A_{P_{u}} \exp \left(-\frac{\Delta H_{P_{u}}}{k T}\right)\left(q_{H}-q_{0}\right)\right\}
\end{array}
$$


In order to determine $q_{H}$, substitute (14) and (15) into (1):

- Let

$$
B_{i}=\frac{A_{i} \Delta H_{i} \exp \left(-\frac{\Delta H_{i}}{k T}\right)\left(\frac{d T}{d x}\right) \delta}{k T^{2}}\left(i=U, P_{U}\right)
$$

Also note that:

$$
f_{u} \text { or } p_{P_{u}}^{0}=A_{i} \exp \left(-\frac{\Delta H_{i}}{k T}\right)
$$

Combining the above two equations:

$$
B_{u}=\frac{\Delta H_{u} f_{u}}{k T^{2}} \frac{d T}{d x} \delta \text { and } B_{P_{u}}=\frac{\Delta H_{P_{u}} P_{P_{u}}^{0}}{k T^{2}} \frac{d T}{d x} \delta
$$

Eqs. (14) and (15) become

$$
\begin{aligned}
& J_{U}=\frac{D_{g}}{k T \delta}\left[B_{U}\left(1-q_{H}\right)-f_{U}\left(q_{H}-q_{0}\right)\right] \\
& J_{P_{U}}=\frac{D_{g}}{k T \delta}\left[B_{P_{U}} q_{H}+p_{P_{U}}^{0}\left(q_{H}-q_{0}\right)\right]
\end{aligned}
$$

Substituting into eq. (1):

$$
q_{0}=\frac{B_{p_{u}} q_{H}+p_{p_{u}}^{0}\left(q_{H}-q_{0}\right)}{B_{U}+\left(B_{p_{u}}-B_{U}\right) q_{H}+\left(p_{p_{u}}^{0}-f_{u}\right)\left(q_{H}-q_{0}\right)}
$$

Assume $q_{H} \cong q_{0}$, except in $q_{H}-q_{0}=\Delta q$

$$
\begin{array}{r}
\therefore B_{u} q_{0}+\left(B_{p_{u}}-B_{u}\right) q_{0}^{2}+\left(p_{p_{u}}^{0}-f_{u}\right) q_{0} \Delta q \\
=B_{p_{u}} q_{0}+p_{p_{u}}^{0} \Delta q
\end{array}
$$


or $\Delta q=q_{H}-q_{0}$

$$
=\frac{B_{u}-B_{p_{u}}}{\frac{f_{u}}{1-q_{0}}+\frac{p_{p_{u}}^{0}}{q_{0}}}
$$

using (16):

$$
\Delta q=\left[\frac{\Delta H_{u} f_{u}-\Delta H_{p_{u}} p_{p_{u}}^{0}}{\frac{f_{u}}{1-q_{0}}+\frac{p_{p_{u}}^{0}}{q_{0}}}\right] \frac{\delta}{k T^{2}}\left(\frac{d T}{d x}\right)
$$

b) Pore Velocity:

$$
v_{p}=\frac{J_{P u}+J_{u}}{N}
$$

where $N=$ total density of heavy metal

$$
\text { atoms }(u+P u) / c c
$$

Using (14a) and (15a):

$$
J_{p_{u}}+J_{u}=\frac{D_{q}}{k T \delta}\left[B_{u}+\left(B_{p_{u}}-B_{u}\right) q_{H}+\left(p_{p_{u}}^{0}-f_{u}\right) \Delta q\right]
$$

Approximate $q_{H}$ by $q_{0}$ in the above:

$$
\begin{aligned}
\therefore v_{p}=\frac{D_{g}}{k T \delta N}[ & {\left[\Delta H_{u} f_{u}\left(1-q_{0}\right)+\Delta H_{p_{u}} p_{p_{u}}^{0} q_{0}\right] } \\
& \left.-\frac{\left(f_{u}-p_{P_{u}}^{0}\right)\left(\Delta H_{u} f_{u}-\Delta H_{p_{u}} p_{p_{u}}^{0}\right)}{\frac{f_{u}}{1-q_{0}}+\frac{p_{p_{u}}^{0}}{q_{0}}},\right\}\left(\frac{\delta}{k T^{2}}\right)\left(\frac{d T}{d x}\right)
\end{aligned}
$$

In pure $\mathrm{VO}_{2}$, the pore velocity is:

$$
\left(U_{p}\right)_{\mathrm{VO}_{2}}=\frac{D_{q} \Delta H_{u} P_{u}^{0}}{k^{2} T^{3} N}\left(\frac{d T}{d x}\right) \quad \begin{aligned}
& \text { [Note: Here } \Delta H_{u} \text { and } R^{\circ} \\
& \text { refer to pure } \left.\mathrm{UO}_{2}\right]
\end{aligned}
$$


303

$$
\therefore \frac{v_{p}}{\left(v_{p}\right)_{v_{2}}}=\left(1-q_{0}\right) \frac{f_{u}}{p_{u}^{0}}+r q_{0}-\frac{\left(f_{u}-p_{p_{u}}^{0}\right)\left(\frac{f_{u}}{p_{u}^{0}}-r\right)}{\frac{f_{u}}{1-q_{0}}+\frac{p_{p u}^{0}}{q_{0}}}
$$

where $\gamma^{2}=\frac{\Delta H_{P u} P_{P u}^{0}}{\Delta H_{u} P_{u}^{0}}$

is a known function.

of temperature

c)

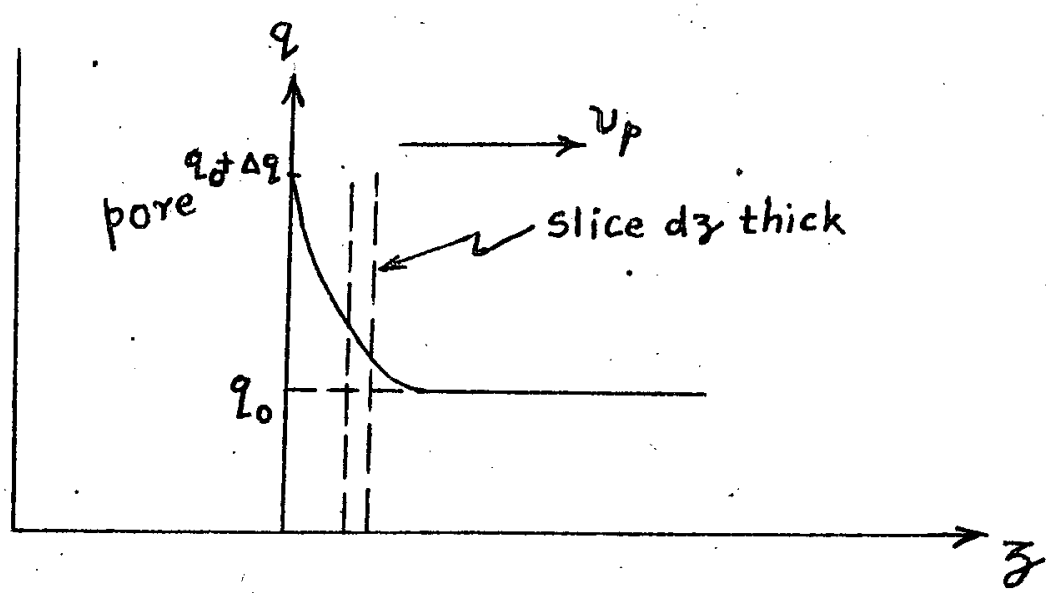

Pu Diffusion Equation:

Coordinate system moves with velocity $v_{p}$ of pore. In the coordinate system the solid "flows" in $-z$ direction with velocity $u_{p}$. Balance over slice $d z$ :

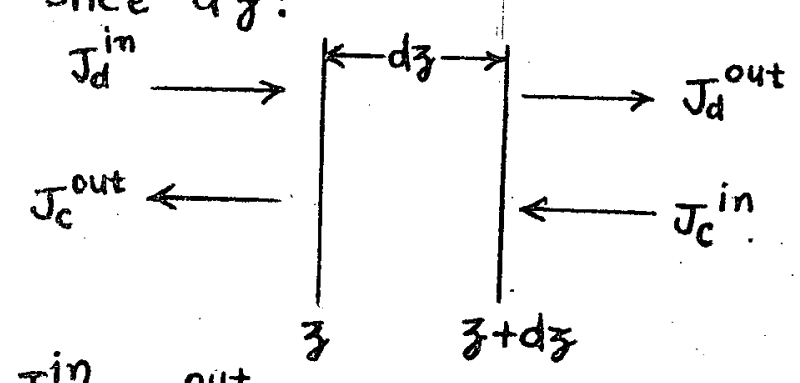

$J_{d}^{\text {in }}-J_{d}^{\text {out }}=$ net diffusion current of $P_{u}$ into slice 
304

$J_{c}^{\text {in }}-J_{c}^{\text {out }}=$ net convection of Pu into slice

$C=\begin{gathered}\text { concentration of } P u \\ \text { in } \mathrm{gms} / \mathrm{cc}\end{gathered} ; D_{S}=\begin{aligned} & \text { diffusion coefficient } \\ & \text { of } P_{u} \text { in solid }\end{aligned}$

$$
\begin{aligned}
& J_{d}^{\text {in }}=-D_{s} \frac{d C}{d z} \\
& J_{d}^{\text {out }}=J_{d}^{\text {in }}+\left(\frac{d J_{d}^{i n}}{d z}\right) d z=J_{d}^{\text {in }}-\left(D_{s} \frac{d^{2} c}{d z^{2}}\right) d z
\end{aligned}
$$

$\therefore$ net input by diffusion $=D_{s} \frac{d^{2} c}{d z^{2}} d z$

Also:

$$
\begin{aligned}
& J_{c}^{\text {in }}=v_{p} c(z+d z)=v_{p}\left[c+\frac{d c}{d z} d z\right] \\
& J_{c}^{\text {out }}=v_{p} c
\end{aligned}
$$

$\therefore$ net input by convection $=v_{p} \frac{d C}{d z} d z$

At steady state, with no sources or sinks: net input by diffusion

$$
+ \text { net input by convection }=0
$$

or $D_{s} \frac{d^{2} C}{d z^{2}}+v_{p} \frac{d C}{d z}=0$

Converting to $q$

$$
\begin{aligned}
& q=\frac{c \frac{g m s p_{u}}{c c}}{\rho_{s} \frac{g m s\left(u+p_{u}\right)}{c c}} \quad\left(\rho_{s}=\right.\text { density of } \\
& \therefore \quad D_{S} \frac{d^{2} q}{d z^{2}}+v_{p} \frac{d q}{d z}=0
\end{aligned}
$$

B.C. i) $q(0)=q_{0}+\Delta q$; ii) $q(\infty)=q_{0}$ 
305

Solution:

$$
\begin{aligned}
& \quad \text { let } u=\frac{d q}{d z} \\
& \therefore \quad \frac{d u}{d z}+\frac{v_{p}}{D_{s}} u=0
\end{aligned}
$$

Integrating we get,

$$
u=\frac{d q}{d z}=A e^{-\frac{v_{p}}{D_{s}} z} \text {. (where } A \text { is a constant) }
$$

Integrating again:

$$
\begin{aligned}
q & =\frac{A D_{s}}{v_{p}} e^{-\frac{v_{p}}{D_{s}} \xi}+B \\
& =A^{\prime} e^{-\frac{v_{p}}{D_{s}} z}+B
\end{aligned}
$$

From B.C. ii), , $B=q_{0}$

From B.C. i) , $q_{0}+\Delta q=A^{\prime}+q_{0}$

$$
\therefore \quad A^{\prime}=\Delta q
$$

Therefore the complete solution is:

$$
q(z)-q_{0}=\Delta q \exp \left(-\frac{v_{p}}{D_{s}} z\right)
$$


306

Problem 14.3

a) The excess plutonium contained in the distribution in front of each moving pore is given by multiplying the answer to problem 14.2 c) by the solid density $s_{s}$ and integrating over all distances from the front face:

$$
\begin{array}{r}
A=\rho_{s}\left(\pi R_{p}^{2}\right) \int_{0}^{\infty}\left(q-q_{0}\right) d z \text { atoms excess } P_{u} / \text { pore } \\
{\left[R_{p}=\text { radius of a pore }\right]}
\end{array}
$$

$z$ is the distance from the front face. Using the expression for $q-q_{0}$ :

$$
A=\rho_{s}\left(\pi R_{p}^{2}\right) \Delta q \int_{0}^{\infty} e^{-\frac{v_{p}}{D_{s}} z} d z=\frac{\rho_{s} \pi R_{p}^{2} \Delta q D_{s}}{v_{p}} \ldots(1)
$$

where the radius of the pore $r_{p}$ is given by:

$$
V_{p}=\pi R_{p}^{2} \delta
$$

b) Since the excess $P_{4}$ in front of each pore is equal to the deficiency of $\mathrm{Pu}$ left behind at the initial location of the pore, the quantity of $P_{u}$ lost from the volume element as a result of departure of, the initial number $N_{p o}$ of pores is No. The lost pores are replaced by $N_{p}$ pores which have migrated to radial position $r$ from positions further out. Each of the newly arrived pores supplies an excess of $P u$ of amount $A$ per pore. Therefore the net gain of $P u$ in the volume element due to pore motion is:

$\left(N_{p}-N_{p o}\right)$ A per unit volume. 
307

In the solid at time $t$, the porosity $P$ is related to the pore concentration by:

$$
P=N_{p} V_{p}
$$

The total mass of heavy metals in the volume element is $\rho_{S}(1-P)$, and in the absence of the Pu carried by the pores (ie. if $A$ were zero), the amount of $P_{4}$ in the unit volume would be $q_{0} g_{s}(1-p)$.

When $P_{u}$ is carried by the pores, the amount of $\mathrm{Pu}_{4}$ in the volume element is:

$$
q_{0} \rho_{s}(1-p)+\left(N_{p}-N_{p o}\right) A \text {. }
$$

or the average. Pu fraction in the volume element is:

$$
\bar{q}=\frac{q_{0} \rho_{s}(1-p)+\left(N_{p}-N_{p o}\right) A}{\rho_{s}(1-p)}
$$

Using (1) and (2) in (3) we get:

$$
\bar{q}=q_{0}+\left(\frac{p-p_{c}}{1-p}\right) \frac{\Delta q D_{s}}{\delta v_{p}} \ldots-(4)
$$

Now $\Delta q$ is given by the final eq. (iv) in problem 14.2 a) solution. Similarly, $v_{p}$ is given by:

$$
v_{p}=\left\{D_{g} \frac{\Delta H_{u} P_{u}^{0} \Omega}{k^{2} T^{3}}\left(\frac{d T}{d r}\right)\right\} x
$$

where $x$ is given by eqs (18) and (19) of problem 14.2 .

Also $\Delta q$ from eq (17) of problem 14.2 is:

$$
\Delta q=Y \frac{\delta}{k T^{2}}\left(\frac{d T}{d r}\right)--(6)
$$

Where $y$ is the bracketed coefficient in the formula in the problem 14.2 a) solution. 
Inserting (5) and (6) into (4) yields:

$$
\bar{q}=q_{0}+\left(\frac{p-p_{0}}{1-p}\right) \frac{y}{x \Delta H_{u}} \frac{D_{s}}{D_{g}} \frac{k T}{p_{u}^{0} \Omega} \ldots(7)
$$

The variation of $p_{u}^{0}$ and $D_{s}$ with temperature is given by:

$$
\begin{aligned}
& P_{u}^{0}=10^{6} \exp \left(\Delta S_{U} / R\right) \exp \left(-\Delta H_{U} / R T\right) \ldots\left[E_{q} \cdot(14.4)\right] \\
& D_{s}=D_{s}^{0} \exp \left(-E_{d} / R T\right) \quad \ldots(9)
\end{aligned}
$$

where $E_{d}$ is the activation energy for solid state diffusion of $\mathrm{Pu}$ in the mixed oxide and $D_{s}^{\circ}$ is the pre-exponential factor of the diffusivity.

$$
\text { Substituting (8) and (9) into (7) yields: }
$$

$$
\bar{q}=q_{0}+C\left(\frac{p-p_{0}}{1-p}\right) \exp \left(\frac{\Delta H_{u}-E_{d}}{R T}\right)
$$

where $C$ is a dimensionless number which is approximately temperature independent.

$$
C=\frac{Y}{X \Delta H_{u}} \frac{D_{s}^{0}}{D_{g}} \frac{k T}{10^{6} \exp \left(\Delta S_{U} / R\right) \Omega}
$$

Thus, plutonium redistribution by pore migration depends directly upon the porosity redistribution through the term $\left(p-p_{0}\right) /(1-p)$ and upon the temperature distribution which appears in the exponential term. The temperature distribution is also affected by restructuring, so that porosity redistribution affects plutonium redistribution indirectly through the temperature profile $T(r)$. 
309

Problem 14.4

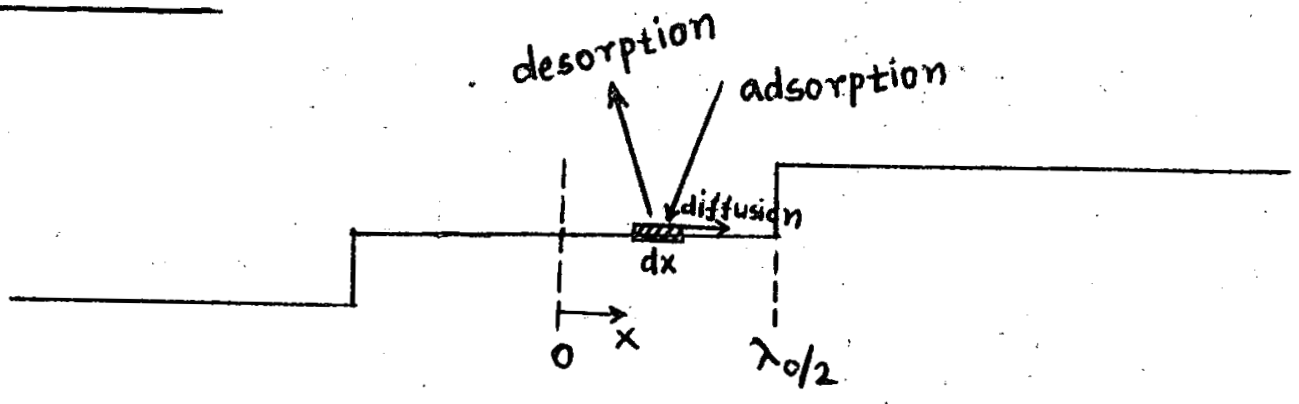

Balance on slice $d x$ wide and unit length 1 to diagram on terrace (steady state)

$$
\begin{gathered}
n=\text { surface concentration of adsorbed atoms } \\
\text { (atoms } / \mathrm{cm}^{2} \text { ) }
\end{gathered}
$$

Net input by surface diffusion $=D_{s} \frac{d^{2} n}{d x^{2}} d x$

Let $\quad z=\frac{p}{\sqrt{2 \pi m k T}}=$ impingement rate from gas, atoms $/ \mathrm{cm}^{2}-\mathrm{sec}$.

Input from gas phase $=\xi d x$

Let $k=$ desorption rate constant

= reciprocal of mean life time on surface

$K=\nu_{1} \exp \left(-E_{b} / k T\right), \sec ^{-1}$

Output by desorption $=k n d x$

Balance:

$$
D_{s} \frac{d^{2} n}{d x^{2}}=-z+k n
$$

Let $\quad \xi=\frac{x}{\left(\lambda_{0} / 2\right)}=$ dimensionless distance 
310

Balance becomes:

$$
\frac{d^{2} n}{d \xi^{2}}=\beta^{2} n-c \text { or } \frac{d^{2} n}{d \xi^{2}}-\beta^{2} n=c
$$

where $\beta^{2}=\frac{\xi \lambda_{0}^{2}}{4 D_{s}}-(2 a), \quad c=\frac{3 \lambda_{0}^{2}}{4 D_{s}}--(2 b)$

Homogeneous Solution:

$$
n_{H}(\xi)=A \sinh \beta \xi+B \cosh \beta \xi
$$

Particular solution:

$$
\begin{gathered}
n_{p}(\xi)=C / \beta^{2} \\
\therefore n(\xi)=A \sinh \beta \xi+B \cosh \beta \xi+C / \beta^{2}
\end{gathered}
$$

Boundary conditions:

$$
\begin{aligned}
& \left(\frac{d n}{d g}\right)_{0}=0 \quad \text { (by symmetry) } \\
& n(1)=0 \quad \text { (ledges are perfect sinks) }
\end{aligned}
$$

From first B.C. $A=0$.

From second B.C.

$$
\begin{aligned}
& \text { second B.C. } \quad B=-\frac{C}{\beta^{2} \cosh \beta} \\
& \therefore n(\xi)=\frac{C}{\beta^{2}}\left[1-\frac{\cosh \beta \xi}{\cosh \beta}\right]
\end{aligned}
$$

$$
J=\text { flux to ledge }=-D_{s}\left(\frac{d n}{d x}\right)_{x=\lambda_{0 / 2}}=-\frac{2}{\lambda_{0}} D_{s}\left(\frac{d n}{d s}\right)_{1}
$$

From eq. (6),

$$
\frac{d n}{d \xi}=-\frac{C}{\beta} \frac{\sinh \beta \xi}{\cosh \beta}
$$


311

$$
\therefore J=\frac{2 C D_{s}}{\lambda_{0} \beta} \tanh \beta
$$

Total rate at which atoms strike one-half of terrace $=z \frac{\lambda_{0}}{2}$

$$
\begin{aligned}
\therefore \alpha & =\text { condensation coefficient } \\
& =\frac{J}{3\left(\lambda_{0} / 2\right)} \\
\therefore \alpha & =\frac{2 C D_{s}}{3 \lambda_{0}\left(\lambda_{0} / 2\right)} \frac{\tanh \beta}{\beta}
\end{aligned}
$$

Using definition of $C$ from eq. (rb)

$$
\begin{aligned}
\frac{4 C D_{S}}{3 \lambda_{0}^{2}} & =1 \\
\therefore \alpha & =\frac{\tanh \beta}{\beta}
\end{aligned}
$$

where $\beta$ is given by eq. (2a). 
$3 / 2$

Problem 14.5

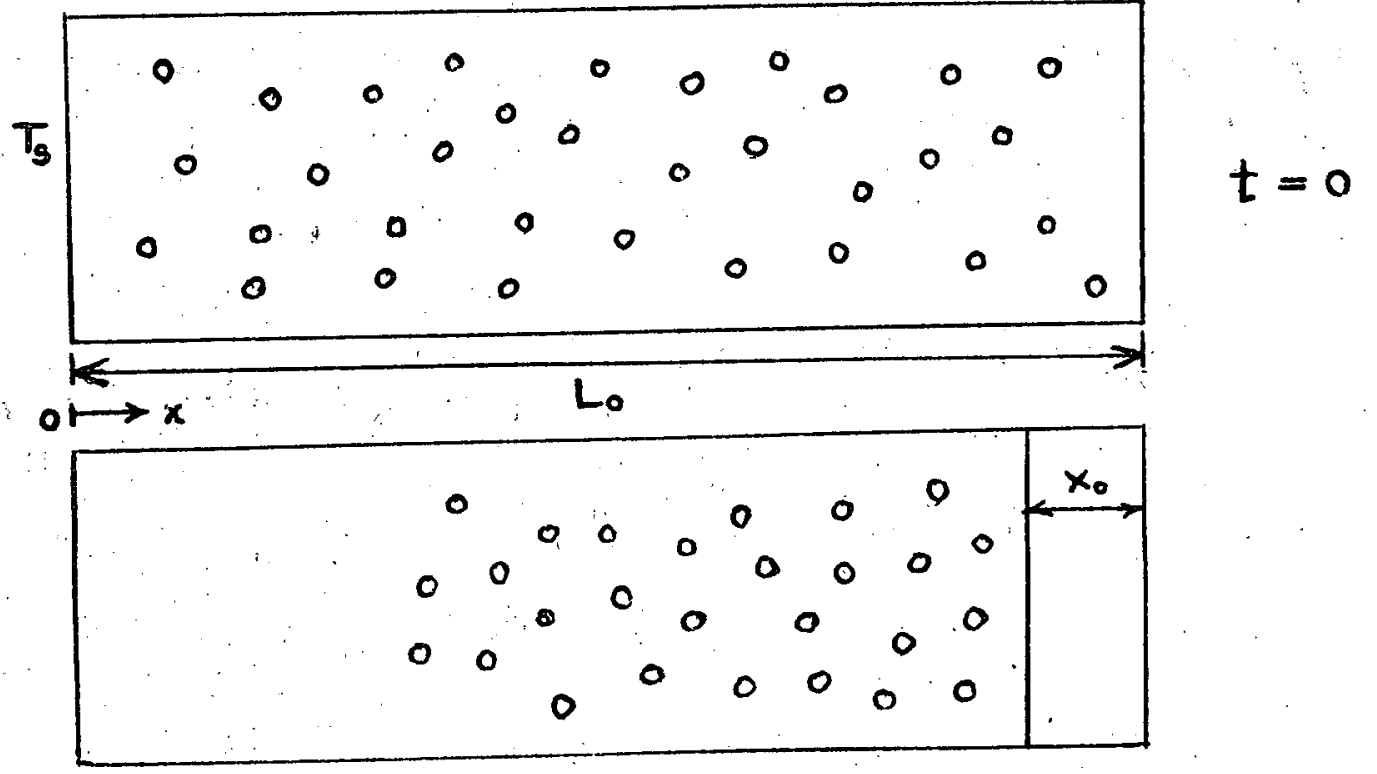

a) Fixed temperature distribution :

$$
\begin{gathered}
T=T_{s}+\left(\frac{d T}{d x}\right) x \\
t\left(T, T^{\prime}\right)=\text { Time for pore to move from } T \text { to } T^{\prime} \\
d t=\frac{d x}{v_{p}(x)}=\frac{d T}{\left(\frac{d T}{d x}\right) v_{p}(T)} \\
t\left(T, T^{\prime}\right)=\frac{1}{\left(\frac{d T}{d x}\right)} \int_{T}^{T^{\prime}} \frac{d T}{v_{p}(T)} \\
v_{p}=v_{p}^{+} \exp \left(-\Delta H_{\text {rap }} / k T\right)\left(\frac{d T}{d x}\right)
\end{gathered}
$$




$$
\therefore t\left(T, T^{\prime}\right)=\frac{1}{\left(\frac{d T}{d x}\right)^{2}} \cdot \frac{1}{V_{p}^{+}} \int_{T}^{T^{\prime}} e^{\Delta H_{v a p} / k T} d T
$$

Text, following Eq. (14.38):

$$
\int_{T}^{T^{\prime}} e^{\Delta H_{\text {vap }} / k T} d T=-\frac{\Delta H_{\text {vap }}}{k} \int_{\frac{\Delta H_{\text {vap }}}{k T}}^{\frac{\Delta H_{\text {vap }}}{k T^{\prime}}}\left(\frac{e^{u}}{u^{2}}\right) d u
$$

Since $T<T^{\prime}$, neglect the second term $\exp \left(\frac{\Delta H_{k p p}}{k T^{\prime}}\right)$

$$
\therefore t(T) \cong \frac{1}{\left(\frac{d T}{d x}\right)^{2} v_{p}^{+}} \cdot \frac{\Delta H_{\text {vap }}}{k}\left(\frac{k T}{\Delta H_{\text {vap }}}\right)^{2} \exp \left(\frac{\Delta H_{\text {vap }}}{k T}\right){ }_{-(1)}^{\exp \left(\frac{\Delta H_{\text {kq }}}{k T^{\prime}}\right)}
$$

Note: $\quad t$ is independent of $T^{\prime}$ because most of the time is spent in moving in the low temperature region.
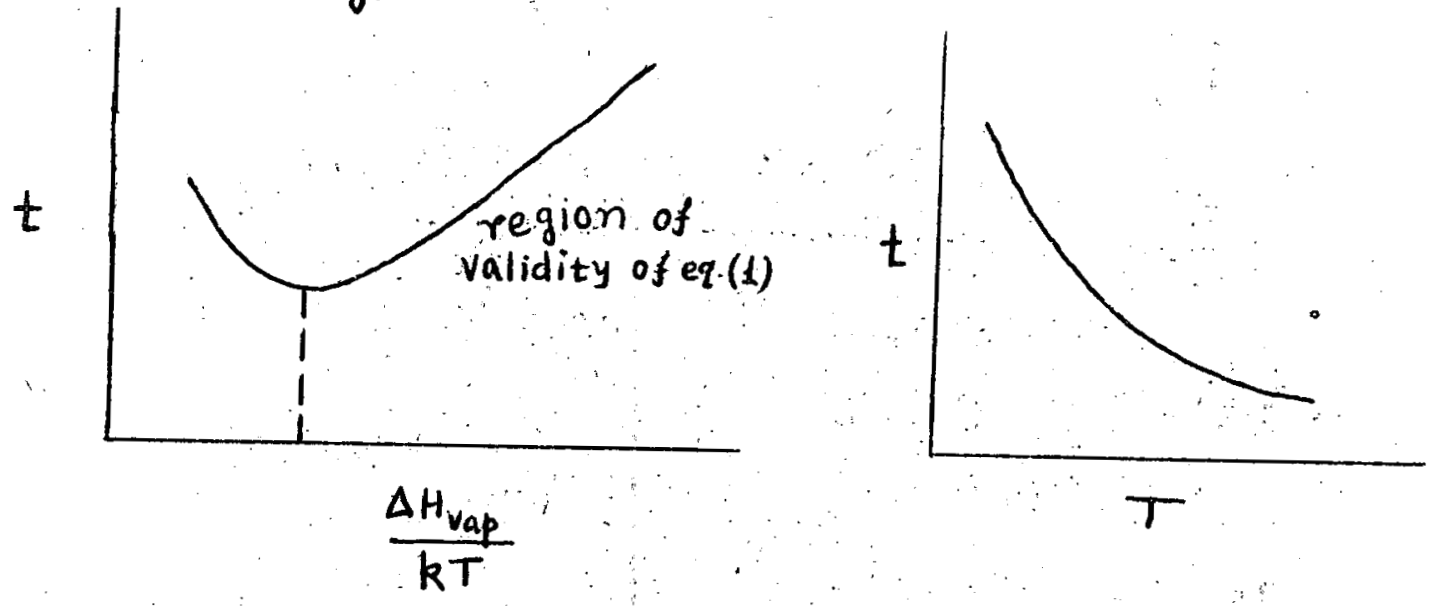
314

b) At time $t$ all pores, which were originally at positions, where temperature $\geq T$, reach the hot face.

Let $T(t)$ be the inverse of eq. (1). The position from which pores just reach the hot face in time $t$ is:

$$
X=\frac{T(t)-T_{s}}{(d T / d x)}
$$

All porosity from $x$ to $L_{0}$ is removed from the slab in time $t$.

Initial void volume in zone from $x$ to $L_{0}$ (per unit area $\perp$ to $\left.\frac{d T}{d x}\right)=P_{0}\left(L_{0}-\dot{x}\right)$

All of this volume is removed, or the thickness of the densified slab is:

or

$$
\begin{aligned}
& L=L_{0}-P_{0}\left(L_{0}-x\right), \\
& L(t)=L_{0}\left(1-P_{0}\right)+P_{0} \frac{T(t)-T_{s}}{(d T / d x)}
\end{aligned}
$$

where $T(t)$ is obtained from solving eq. (1) for $T$ in terms of $t$. 
315

Problem 14.6

Grain size obeys the following equation with time:

$$
\frac{d(d)}{d t}=k\left(\frac{1}{d}-\frac{1+2000 \Omega \dot{F} t}{d_{m}^{0}}\right)
$$

[Eq. $(14.63)]$

where $k=5.2 \times 10^{7} \exp \left[-\frac{270}{R\left(T / 10^{3}\right)}\right] \quad$ [Eq. (14.61)]

and $\quad d_{m}^{0}=2200 \exp \left[-\frac{63}{R\left(T / 10^{3}\right)}\right] \quad\left[E_{q .}(14.62)\right]$

At $T=1600^{\circ} \mathrm{K}$

$$
\begin{aligned}
k & =5.2 \times 10^{7} \exp \left[-\frac{270}{8.314 \times 1.6}\right] \\
& =\frac{0.0803}{\mu m^{2}}=\frac{1.925}{\mathrm{hr}} \frac{\mu \mathrm{m}^{2}}{\text { days }} \\
d_{m}^{0} & =2200 \exp \left[-\frac{63}{8.314 \times 1.6}\right] \\
& =\frac{19.35 \mu \mathrm{m}}{\Omega=} \quad \frac{41 \times 10^{-24} \mathrm{~cm}^{3}, \dot{F}=6.1 \times 10^{12} \times 24 \times 3600 \frac{\text { fissions }^{3}}{\mathrm{~cm}^{3} \text { days }}}{} \\
\Omega & =5.28 \times 10^{7} \frac{\text { fissions }}{\mathrm{cm}^{3} \text {-days }}
\end{aligned}
$$

Then eq. (1) becomes,

$$
\frac{d(d)}{d t}=1.925\left(\frac{1}{d}-\frac{1+2 \times 10^{3} \times 41 \times 10^{-23} \times 5.28 \times 10^{7}+}{d_{m}^{0}}\right)
$$




$$
\therefore \quad \frac{d(d)}{d t}=1.925\left(\frac{1}{d}-\frac{1+0.043 t}{19.35}\right) \ldots(2)
$$

In absence of irradiation, $\dot{F}=0$,

$$
\therefore \frac{d(d)}{d t}=1.925\left(\frac{1}{d}-\frac{1}{19.35}\right)-\ldots \text { (3) }
$$

In both eqs (2) and (3) time $t$ is in days and $d$ is in $\mu m$. They are solved numerically using a Runge-Kutta method. The initial condition

\begin{tabular}{|c|c|c|c|}
\hline \multicolumn{2}{|c|}{$\dot{F}=6.1 \times 10^{12} \frac{\text { fissions }}{\mathrm{cm}^{3}-\sec }$} & \multicolumn{2}{|l|}{$\dot{F}=0$} \\
\hline Time $t$ (Days) & Grainsize d (um) & \pm (days) & $d(\mu m)$ \\
\hline 0 & 9.00 & 0 & 9.00 \\
\hline 3 & 9.31 & 40 & $|2 \cdot 2|$ \\
\hline 6 & 9.57 & 80. & 14.07 \\
\hline 9 & 9.77 & 120 & 15.32 \\
\hline 12 & 9.92 & 160 & $=16.22$ \\
\hline 15 & 10.03 & 200 & 16.89 \\
\hline 18 & 10.09 & gur & 10.07 \\
\hline 21 & $10 \cdot 12$ & 240 & 17.40 \\
\hline 24 & 10.10 & $\begin{array}{l}280 \\
320\end{array}$ & 17.79 \\
\hline 27 & 10.05 & 360 & 18.10 \\
\hline 30 & 9.96 & & 18.37 \\
\hline 33 & 9.84 & 400 & 18.54 \\
\hline $\begin{array}{l}36 \\
39\end{array}$ & $\begin{array}{l}9.69 \\
9.45\end{array}$ & & \\
\hline
\end{tabular}
in both the equations is $d_{0}=9 \mu \mathrm{m}$. 

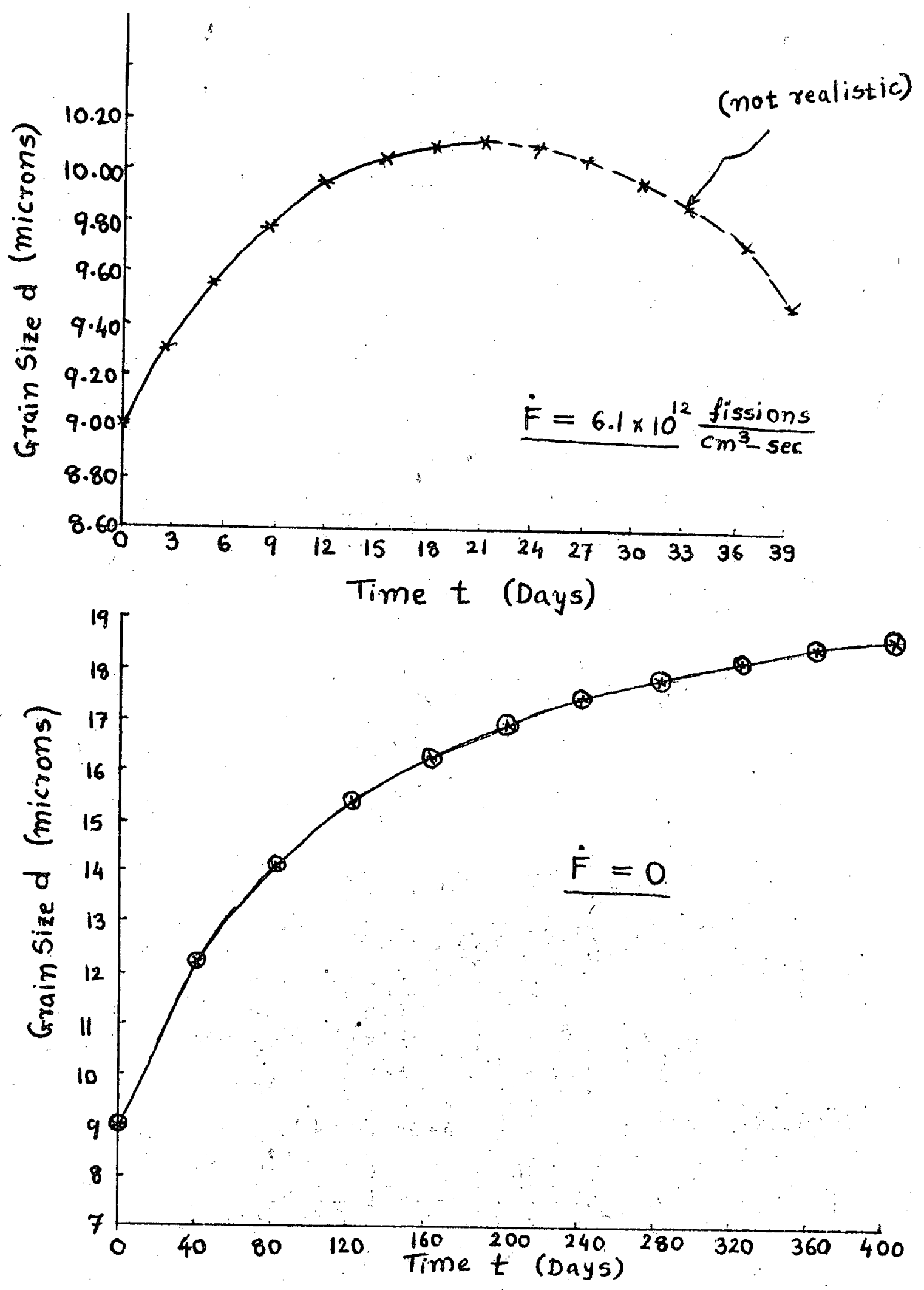
318

Problem 14.7

a) The velocity of the particle according to the surface diffusion mechanism is given by Eq. (13.219):

$$
v=\frac{3 D_{s} Q_{s}^{*} a_{0}}{k T^{2} R}\left(\frac{d T}{d x}\right)
$$

The distance covered in time $d t$ is:

$$
d x=v d t
$$

or $t=\int_{0}^{x} \frac{d x}{v}=\int_{T_{0}}^{T(x)} \frac{d T}{v\left(\frac{d T}{d x}\right)}$

But $\frac{d T}{d x}$ is a constant

$$
\begin{aligned}
& \therefore \quad T(x)=T_{0}+\left(\frac{d T}{d x}\right) \times \\
& \therefore t=\frac{1}{(d T / d x)} \int_{T_{0}}^{T_{0}+(d T / d x) x} \frac{d T}{v}--(2)
\end{aligned}
$$

The principal temperature variation of $V$ is due to the surface diffusivity $D_{S}=D_{o s} e^{-E_{s} / k}$, so that (1) becomes:

$$
v=\frac{3 D_{e s} Q_{s}^{*} a_{0}\left(\frac{d T}{d x}\right)}{k T_{0}^{2}} \frac{e^{-E_{s} / k t}}{R}
$$

Substituting (Ia) into (2) yields:

$$
t=\frac{k T_{0}^{2} R}{3 D_{0 S} Q_{s}^{*} a_{0}\left(\frac{d T}{d x}\right)^{2}} \int_{T_{0}}^{T_{0}+(d T / d x) x} \exp \left(E_{s} / k T\right) d T
$$


319

Let $\theta=\frac{T}{T_{0}}$ and $C=\frac{E_{s}}{k T_{0}}$

$$
\therefore \int_{T_{0}}^{T_{0}+(d T / d x) x} \exp \left(E_{s} / k T\right) i T=T_{0} \int_{1}^{1+\frac{1}{T_{c}}\left(\frac{d T}{d x}\right) x} \exp (c / \theta) d \theta
$$

Using approximate method following Eq. (14.38):

$$
\begin{aligned}
& \int_{1}^{1+\frac{1}{T_{0}}\left(\frac{d T}{d x}\right) x} \exp (c / \theta) d \theta=\frac{1}{c}\left[\exp (c)-\exp \left(\frac{c}{1+\frac{1}{T_{0}}\left(\frac{d T}{d x}\right) x}\right)\right] \\
& \therefore \quad t=\frac{\left(k T_{0}\right)^{2} T_{0}^{2} R}{3 D_{0 s} Q_{s}^{*} a_{0}\left(\frac{d T}{d x}\right)^{2} E_{s}}\left\{\exp (c)-\exp \left(\frac{c}{1+\frac{1}{T_{0}}\left(\frac{d T}{d x}\right) x}\right)\right\}
\end{aligned}
$$

For small $x$ :

$$
\begin{aligned}
\exp \left(\frac{c}{1+\frac{1}{T_{0}}\left(\frac{d T}{d x}\right) x}\right) & \cong \exp \left[c\left(1-\frac{1}{T_{0}}\left(\frac{d T}{d x}\right) x\right)\right] \\
& =e^{c} \exp \left(-\frac{c}{T_{0}}\left[\frac{d T}{d x}\right] x\right)
\end{aligned}
$$

or $t=A R\left\{1-\exp \left[-\frac{c}{T_{0}}\left(\frac{d T}{d x}\right) x\right]\right\}$

where $\quad A=\frac{\left(k T_{0}\right)^{2} T_{0}^{2} e^{c}}{3 D_{0 S} Q_{s}^{*} a_{0}(d T / d x)^{2} E_{S}}$

or

$$
x=-\frac{T_{0}}{c\left(\frac{d T}{d x}\right)} \ln \left[1-\frac{t}{A R}\right]
$$


320

b) The particles found in the range $x$ to $x+d x$ at time $t$ are those which have a radius between $R$ and $R+d R$, where $x$, $R$ and $t$ are related by eq. (4) of part $a$ )

$$
\begin{gathered}
g d x=f d R \\
\text { or } \quad g(x, t)=\frac{f(R)}{\left|\left(\frac{\partial x}{\partial R}\right)_{t}\right|}
\end{gathered}
$$

where $\left(\frac{\partial X}{\partial R}\right)_{t}$ is obtained from $x(R, t)$ of part $A$, ie. eq. (4). 
$320 a$

14.8

(a) velocity of migration in temperature gradient.

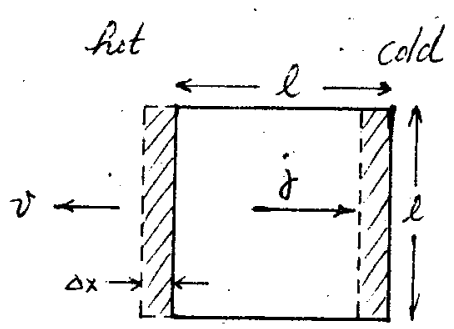

let $j=$ flux of salt from hot $\rightarrow$ cold; $\frac{\text { gmo }}{\cos ^{2}-\sec }$ In time $\Delta t, j \ell^{2} \Delta t$ gems of salt are transferred from the hot to the cold end. This corresponds to $j l^{2} \Delta t / \rho_{s} \mathrm{~cm}^{3}$ of salt transferred. But the volume. transported is also given by $l^{2} \Delta x$, so that

$$
\begin{aligned}
& \frac{j l^{2} \Delta t}{l_{s}}=l^{2} \Delta x \\
& v=\frac{\Delta x}{\Delta t}=j / P_{s}
\end{aligned}
$$

Pick's Law:

$$
\begin{aligned}
& j=\frac{D}{l} \Delta C \simeq \frac{D}{l}\left(\frac{d C}{d T}\right) \Delta T=D\left(\frac{d C}{d T}\right) \nabla T \\
& \frac{d c}{d T}=A \frac{\Delta H}{R T^{2}} e^{-\Delta H / R T}
\end{aligned}
$$

Combining (1)-(3)

$$
v=\frac{D \Delta H A e^{-\Delta H / R T}}{P_{S} R T^{2}} \nabla T
$$

* note this problem does not involve thermal diffusion (ice. the Sort effect) 
3206

(b) Rate of brine flow to a pot

Let $J_{B C}=r$-flux of brine cubes at $r$ and $t, \mathrm{~cm}^{-2}-\sec ^{-1}$

$$
J_{B C}=N v
$$

General Conservation Equation: $\frac{\partial N}{\partial t}+\nabla \cdot J_{B C}=0$

Most frequently, the flux in continuity equations is given by Fuck's first law (ie. diffusion). In the present case, no diffusion (or random walk) occurs. the flux is given by (1). In spherical coordinates (radial component only), the continuity equation is:

$$
\frac{\partial N}{\partial t}+\frac{1}{r^{2}} \frac{\partial}{\partial r}\left(r^{2} J_{B C}\right)=0
$$

Substituting (1) into (2) yields:

$$
\frac{\partial N}{\partial t}=\left(2 \frac{v}{r}+\frac{d v}{d r}\right) N+v \frac{\partial N}{\partial r}
$$

the sign of $v$ has been changed and positive $v$ means movement inwards. the coefficients of $N$ and $\partial N / \partial r$ in $\varepsilon_{q} .(3)$ are known functions of $r . . \Sigma q .(3)$ is to be solved subject to the conditions (only two needed):

$$
N(r, 0)=N_{0} \text { and } N(\infty, t)=N_{0}
$$

Finally, the volume of brine reaching the pot surface per unit time

$$
=4 \pi R^{2} \cdot J_{B C}(R) l^{3}=4 \pi R^{2} \cdot l^{3} v(R) N(R, t)
$$


325

Problem 14.9

Eq 14.6:

$$
v_{p}=\frac{(\text { cons }) \times D_{g} e^{-\Delta H / K T}}{(K T)^{2}} \cdot \frac{1}{T}\left(\frac{d T}{d x}\right)
$$

Eq 14.9:

$$
D_{g}=(\text { cont }) \times\left(\frac{T^{3 / 2}}{P}\right)
$$

therefore,

$$
v_{p}=\left(\text { constr) } \times \frac{e^{-\Delta H / k T}}{p T^{3 / 2}} \cdot \frac{d T}{d x}\right.
$$

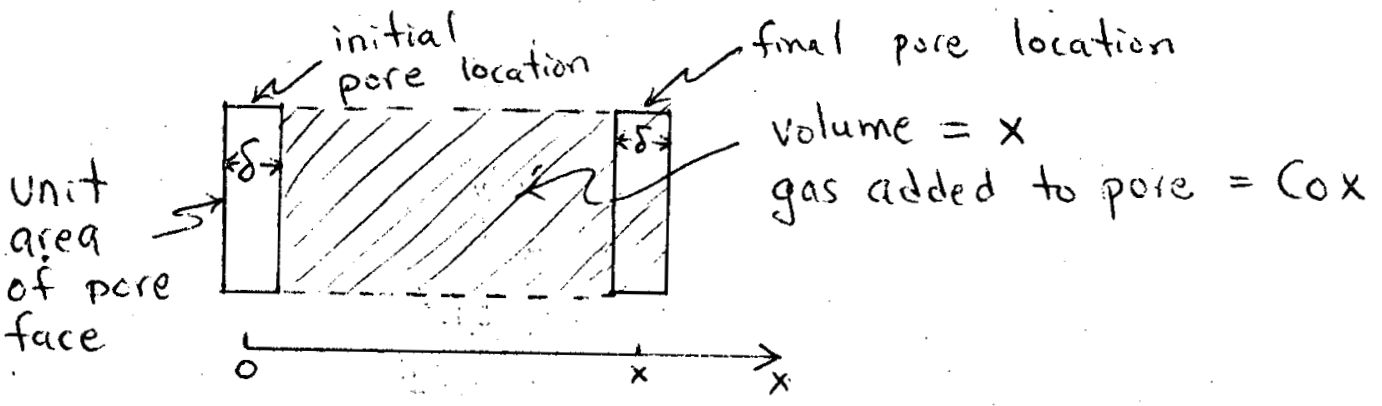

let $n_{0}=$ initial number of gas atoms in pore per $\mathrm{cm}^{2}$ pore area

Ideal gas' law then 'becomes,

$$
\begin{aligned}
\delta p_{0} & =n_{0} k T_{0} \\
\therefore \quad n_{0} & =\frac{\delta p_{0}}{k T_{0}}
\end{aligned}
$$

Gas atoms added to pore per $\mathrm{cm}^{2}$ area in traveling distance $x=C_{0} x$ 
$320 d$

Thus,

$$
\begin{aligned}
& \delta p=\left(n_{0}+c_{0} x\right) \hat{k} T \\
& \frac{p}{p_{0}}=\left(1+\frac{c_{0} x}{n_{0}}\right) \frac{T_{0}}{T_{0}}
\end{aligned}
$$

or

But, $T(x)=T_{0}+\left(\frac{d T}{d x}\right) \times$ for linear temp distrib.

$$
\therefore \quad x=\frac{T-T 0}{(d T / d x)}
$$

and therefore

$$
\text { and therefore } \frac{P}{P_{0}}=\left[1+\frac{C_{0}}{n_{0}}\left(\frac{T-T_{0}}{d T / d x}\right)\right] \frac{T}{T_{0}}
$$

Substituting into (1) and using. (2) for $n_{0}$, obtain:

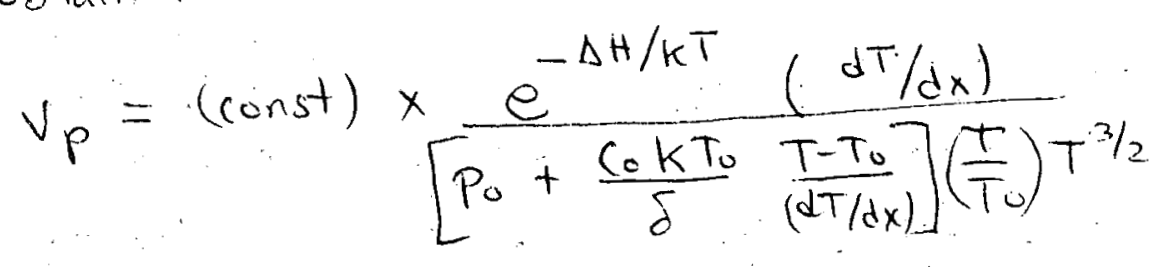


3200

Problem 14.10

(a) Molecular diffusion a of $00_{2}$ in the inert gas in the control void causes $\mathrm{UO}_{2}$ to be vapiright from. kit portions of the inner surface if the central rid and be com den see on colder portions. In diagram (a), the plugs are hotter than the casals af the segments of central void, as cen be shown by an approximate temperature calculation (the method of ob tain ing the exact temperature distribution is outlined in part (b) of this solution):

The temperature on the fuel axis in the plug is

$$
T_{0}^{0}=T_{5}+1+R^{2} / 4 k
$$

If the initial radius at the centrallvid is to i. rae temperature at the edge of the cental wo il in aicyram(al is:

$$
T\left(r_{i i}\right)=T_{s}+\left(\frac{H R}{4 R}\right)\left\{1-\left(\frac{r_{i}}{R}\right)^{2}+\left(\frac{x_{i}}{R}\right)^{2} \ln \left(\frac{r_{i}}{R}\right)^{2}\right\}(2)
$$

Since the bracketeil term in $\xi_{g}$ (2) is a (wars <1, then $T_{0}^{0}>T_{0}\left(r_{b i}\right)$. Hence the ripon pressure of the fuel is greater at the surface of the plugs exposed to the centre "vide then it is on the cylincleicat in lis of the cantor void segments, 5 VO $\mathrm{O}_{2}$ vaporize from the plugs moves. along the central width by aliffinsion in the gros and anchusin on the wells of the central wit segment As the play shrinks in size (and centrally open up). the

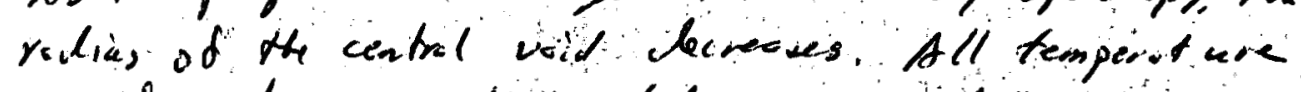

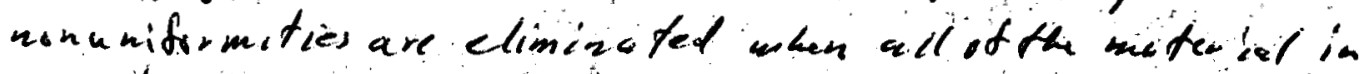
the plugs is redeposited uniformly on the eccles of the central void, which then dore decrees in sochivi.

* $\xi(2)$ is the solution of $\Sigma_{9}(1044)$ in the text with bender conditions given by \&o (10.50) ane (10.51) 
3206

(b) at any time teduring restructuring, let, $r_{0}(z)=$ vadius of Central videt at oxiel location 3 , inclading the shape of the plugat the end meary $=0$ (see drawing on right of sicyrom do cosidinute systema).

rhe temperature sisitribution in the solid potim of the fual is seterminel by solution of the condention 7 cation:

$$
\nabla^{2} \tau_{z}-H_{k}
$$

(3)

chere:

$$
\nabla^{2} T=\frac{1}{\partial} \frac{\partial}{\partial r}\left(r \frac{\partial T}{\partial r}\right)+\frac{\partial^{2} T}{\partial z^{2}}
$$

Bounitary canditions for eq (3) ase:

$$
\begin{aligned}
& \left(07 / \partial_{3}\right)_{3>0}=0 \\
& \text { (b) syimmetri) }
\end{aligned}
$$

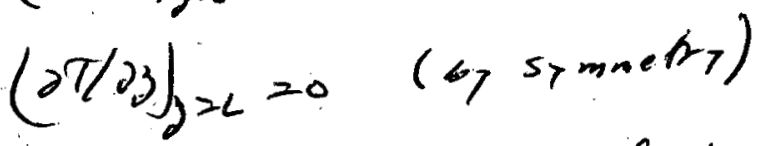

$$
\begin{aligned}
& T(R, z)=T_{3} \text { (at outer fuel surfice) ( } 2 \text { ) } \\
& (\nabla T)_{r \times V_{0}(z)}=0 \quad \text { (neglectiong hat trousport }
\end{aligned}
$$

The diffusion guation is solved for th testarbating of the $v_{2}$ fortial pross une in the gose $\left.p(r, z)\right)$ :

$$
\frac{1}{r} \frac{\partial}{\partial r}\left(r \frac{\partial r}{\partial r}\right)+\frac{\partial^{2} r}{\partial y}>0 \text {. }
$$

Biendeary conditions:

$$
\begin{aligned}
& (\partial P / \partial y)_{z=4}=0 \quad \text { (symmetry) }(10) \\
& (\partial P / \partial 3)_{r=0}=0 \quad \text { (Syminetry) }(11)
\end{aligned}
$$
$\left.P\left(x_{1, j}\right), 3\right)=P\left[T\left(x_{2}(3), 3\right] \quad(\right.$ dris demperature solution) 
$320 \mathrm{~g}$

The time dependence enters win the rate of change of the

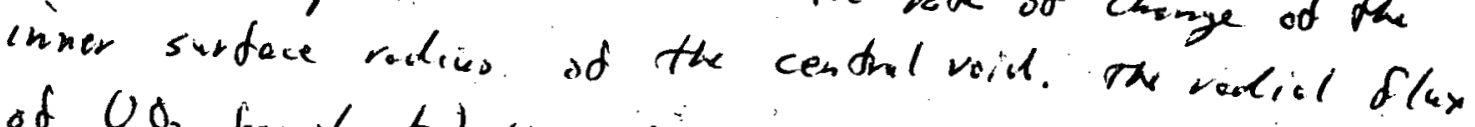
of $U O_{2}$ from (ont or the vapor and the surface es

$$
J_{r}=-\frac{D_{g}}{R T_{0}}\left(\frac{\partial \rho}{\partial r}\right)_{r=r_{0}}
$$

The velour $\sqrt{7}$ of growth Lon shrinkage of the solid surtuie is:

$$
\frac{d r_{0}}{d t}=\int J_{r}=-\int_{R T_{0}}\left(\frac{\partial \rho}{\partial r}\right)_{r=r_{s}}
$$

where $\beta=$ solid fuel density.

Solution Procedure

1. Shooting at timon $t$ when $r_{0}(y)$ is known, solves iss (3)-(8) for $T(x, z)$ in the soling end hance for $T\left(r_{0}(z), z\right)$, at the central void suntuce. This

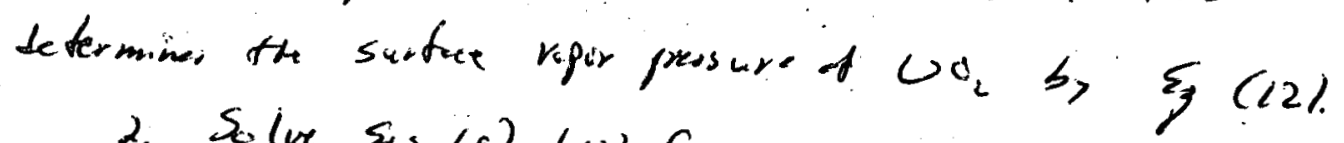

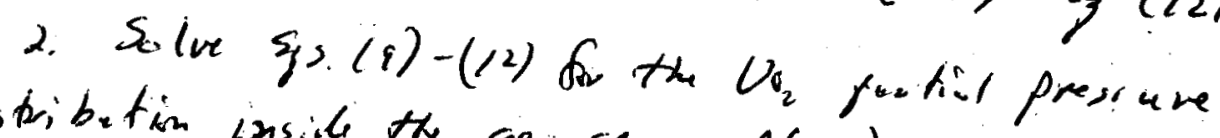
distribution inside the gas spout $\rho(v, z)$.

3. Calculate the time rate of change of the cental wild radii, as, atunctin of 3 by is (ii).

4. Taking a small time step $\Delta t$, calculate to new shape of the central ride at $t+\Delta t$ st:

$$
r_{0}(3) /\left.{ }_{\text {test }} 2 r_{0}(3)\right|_{t}+\left(\frac{d K_{1}}{d t}\right)_{\Delta t_{3}} \Delta t
$$

(I)

5. Return to step 1 a repeat until thy dime state is achieved (ie until $x$ no linger achenes upon a). 


\section{CHAPTER 15}

Problem 15.1

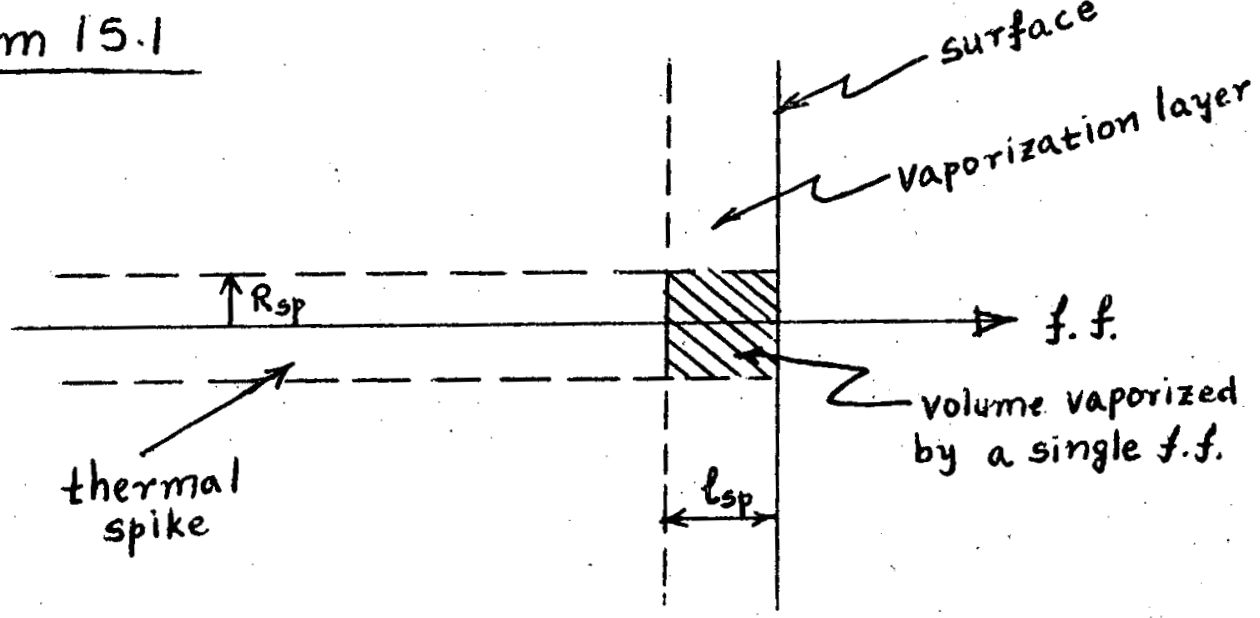

Assume all f.f. tracks are 1 to surface

F.P. balance in vaporization zone $\left(0 \leq x \leq l_{\mathrm{sp}}\right) \& 1 \mathrm{~cm}^{2}$ area

$$
\left(C_{i}=\text { conc. of f.p. } i\right. \text { in vaporization zone) }
$$

1) Input by stopping of recoils $=\left(\frac{1}{2} y_{i} \dot{F}\right) l_{s p} \frac{\text { atoms } i}{\sec -\mathrm{cm}^{2}}$

(only $\frac{1}{2}$ of recoils which stop

in bulk, stop in surfacelayer)

2) Output by vaporization:

$$
I_{f f}^{r e c}=\frac{1}{4}(2 \dot{F}) \mu_{f f}=f f^{\prime} \text { crossing unit } / \text { area of surface } / \text { sec }
$$

Each f.f. removes $\left(\pi R_{s p}^{2} l_{s p}\right) C_{i}$ atoms

$$
\therefore \text { output }=I_{f f}^{\text {rec }}\left(\pi R_{s p}^{2} l_{s p}\right) C_{i} \text { atoms } / \mathrm{sec}-\mathrm{cm}^{2}
$$

3) At steady state:

$$
\frac{1}{2} y_{i} \dot{F}=I_{f f}^{r e c} \pi R_{s p}^{2} C_{i}
$$


322

$I_{i}^{T s}=$ release rate of fop. $i$ by thermal spike mechanism

then $\quad I_{i}^{T s}=I_{f f}^{r e c} \pi R_{s p}^{2} l_{s p} C_{i}=\frac{1}{2} Y_{i} \dot{F} l_{s p}$

$$
\begin{aligned}
& \frac{I_{i}^{T s}}{I_{i}^{r e c}}=\frac{\frac{1}{2} Y_{i} \dot{F} l_{s p}}{\frac{1}{4} Y_{i} \dot{F} \mu_{f f_{i}}}=\frac{1}{2} \frac{l_{s p}}{\mu_{f f i}}=\frac{1}{2} \frac{70}{7 \times 10^{4}} \\
& \therefore \quad I_{i}^{T s}=\left(\mu_{f f i} \cong 7 \mu\right)
\end{aligned}
$$

$\therefore$ mechanism is negligible

$$
\begin{aligned}
& \alpha_{u}=\frac{U O_{2} \text { molecules vaporized }}{f f \text { recoil }}=\frac{\pi R_{s p}^{2} l_{s p}}{\Omega} \\
& \left(\Omega=\text { volume } / \text { vo }_{2} \text { molecule } \cong 41 \AA^{3}\right) \\
& \therefore \alpha_{u}=\frac{\pi(20)^{2} 70}{41} \cong 2000
\end{aligned}
$$


323

Problem 15.2

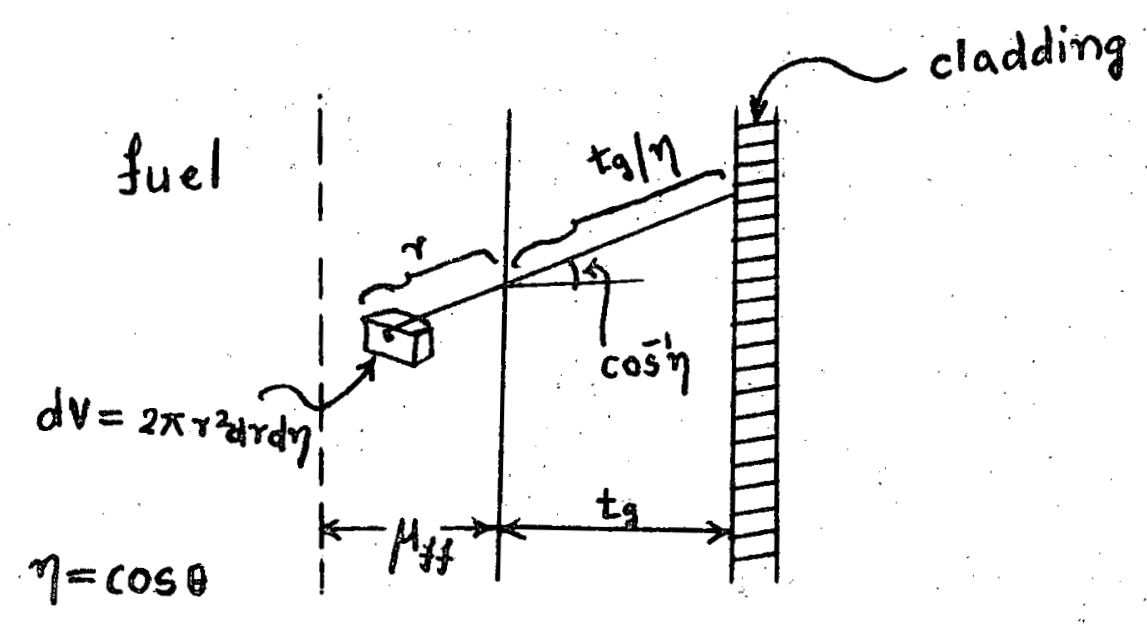

Consider a fission fragment emerging from surface at angle $\cos ^{-1} \eta$ after traversing a distance $r$ of fuel:

a) distance through gas $=t_{g} / \eta$

b) energy after passage through gas in gap $=E$

$$
E=E_{s}\left(1-\frac{t_{g}}{\eta \mu_{g}}\right)=E_{s}\left(1-\frac{t_{g} E_{o}}{\eta \mu_{g}^{\circ} E_{s}}\right)
$$

If $E=0$, when ff. particle reaches clad, then

$$
\begin{gathered}
1-\frac{t_{g} E_{0}}{\eta \mu_{g}^{0} E_{s}}=0 \\
\therefore \quad E_{s}=\frac{t_{g} E_{0}}{\eta \mu_{g}^{0}}=E_{0}\left(1-\frac{r}{\mu_{f f}}\right)
\end{gathered}
$$

or

$$
1-\frac{r}{\mu_{f f}}=\frac{t_{g}}{\eta \mu_{g}^{\circ}}
$$


324

c) At a given polar angle $\cos ^{-1} \eta$, all the fils created at $r$, where $r_{m}<r<\mu_{f f}$ will be stopped in the gas, where $r_{m}$ is defined by eq. (1).

or

$$
\begin{aligned}
& \text { or } \quad \frac{\gamma_{m}}{\mu_{f f}}=1-\frac{t_{g}}{\eta \mu_{g}^{0}} \text { and } \\
& \quad 0 \leq \frac{r_{m}}{\mu_{f f}} \leq 1 \text { or } \eta>\frac{t_{g}}{\mu_{g}^{\circ}} \\
& y \dot{F}=\text { source strength of fff.s in ff.s/ } / \mathrm{cm}^{3}-\mathrm{sec}
\end{aligned}
$$
ff. current at surface of fuel due to generation in $d v$ :

$$
d j=\frac{y \dot{F} d V}{4 \pi}\left(\frac{\eta}{r^{2}}\right)=\frac{y \dot{F}}{2} d r d \eta \eta
$$

Let $j^{\prime}=$ current at surface which will stop in the gas

$$
\text { then } \begin{aligned}
j^{\prime} & =\frac{y \dot{F}}{2} \int_{0}^{1} d \eta\left[\int_{r_{m}}^{\mu_{f f}} d r\right] \eta \\
& =\frac{y \dot{F}}{2} \int_{0}^{1}\left[\mu_{f f}-r_{m}(\eta)\right] \eta d \eta \\
& =\frac{1}{4} \mu_{f f} Y \dot{F}\left\{1-2 \int_{0}^{1} \frac{r_{m}(\eta)}{\mu_{f f}} \eta d \eta\right\}
\end{aligned}
$$

for $\eta$ integration: $\eta>\frac{t_{g}}{\mu_{g}}$, use above formula and for $\eta<\frac{t_{g}}{\mu_{g}^{0}}, r_{m}=0$ 
325

$$
\begin{aligned}
\therefore 2 \int_{0}^{1} \frac{r_{m}(\eta)}{\mu_{f f}} \eta d \eta & =2 \int^{1}\left(1-\frac{t_{g}}{\eta \mu_{g}^{0}}\right) \eta d \eta \\
& =\left(1-\frac{t_{g}}{\mu_{g}^{0}}\right)^{2}
\end{aligned}
$$

Therefore:

$$
j^{\prime}=\frac{1}{4} \mu_{f f} \gamma \dot{F}\left[1-\left(1-\frac{t_{g}}{\mu_{g}^{g}}\right)^{2}\right]
$$

By comparison with the recoil formula:

$$
I^{\text {rec }}=\frac{1}{4} \mu_{f f} \gamma \dot{F}
$$

and

$$
\eta_{\text {rec }}=\frac{j^{\prime}}{I^{\text {rec }}}=1-\left(1-\frac{t_{g}}{\mu_{y}^{\prime}}\right)^{2}
$$

for $\frac{t_{g}}{\mu_{g}^{\circ}} \leqslant 1$

$$
\eta_{\text {rec }} \cong 2 \frac{t_{g}}{\mu_{g}^{\circ}}
$$

Typical fuel-clad gap $=8 \times 10^{-3} \mathrm{~cm}$;

$$
\begin{aligned}
\mu_{g}^{0} & =2 \mathrm{~cm}(\mathrm{at} 1 \mathrm{~atm}) \\
\therefore \eta_{\mathrm{rec}} & =\frac{2 \times 8 \times 10^{-3}}{2} \\
& =8 \times 10^{-3}
\end{aligned}
$$


326

Problem 15.3

a)

$P=$ power/ unit length;

$H=$ volumetric heat source in fuel rod, watts/ce

$\dot{F}=$ fission density, fissions/cc-sec

$H_{0}=3.07 \times 10^{-11} \frac{\text { watt }- \text { sec }}{\text { fission }} \dot{F}$

$P=\pi \cdot R^{2} H_{0}$

$Y=$ fission product yield (elemental)

combining :

$$
y \dot{F}=\frac{Y P}{\pi\left(3.07 \times 10^{-11}\right) R^{2}}
$$

b)

$Y \dot{F}=$ rate of production of fop./ unit volume

$\therefore$ Diffusion equation is:

$$
\frac{\partial C}{\partial t}=D \frac{1}{r} \frac{\partial}{\partial r}\left(r \frac{\partial C}{\partial r}\right)+Y \dot{F}
$$

IFC.

$$
c(r, 0)=0
$$

B.C.

i) $\left(\frac{d c}{d r}\right)_{r=0}=0$

ii) $C(R)=0$

Define Dimensionless variables:

$$
\xi=\frac{r}{R} ; \quad T=\left(\frac{D}{R^{2} Y F}\right) C ; \theta=\frac{D t}{R^{2}}
$$


327

Eq (1)-(4) become:

$$
\frac{\partial T}{\partial \theta}=\frac{1}{\xi} \frac{\partial}{\partial \xi}\left(\xi \frac{\partial T}{\partial \xi}\right)+1
$$

I.C.

$$
T(\xi, 0)=0
$$

BC.

i) $T(0, \theta)=$ finite.

$-1-(7)$

ii) $T(1, \theta)=0$

C) Solution by Laplace Transform Method

$$
\begin{aligned}
& p=\text { transform variable: } \\
& q=\sqrt{p} \\
& \bar{C}(\xi)=\text { Laplace transform of } T(\xi, \theta)
\end{aligned}
$$

Transform of $\frac{\partial T}{\partial \theta}=p \bar{C}(\xi)$ since $T(\xi, 0)=0$

$$
\begin{aligned}
\therefore \quad q^{2} \bar{c} & =\frac{1}{\xi} \frac{d}{d \xi}\left(\xi \frac{d \bar{c}}{d \xi}\right)+\frac{1}{p} \\
\bar{c}(0) & =\text { finite } \quad--(10) \\
\bar{c}(1) & =0
\end{aligned}
$$

Let $\quad \eta=q \xi$

then: $\quad \frac{d^{2} \bar{c}}{d \eta^{2}}+\frac{1}{\eta} \frac{d \bar{c}}{d \eta}-\bar{c}=-\frac{1}{p^{2}}$

Homogeneous Solution:

$$
\bar{C}_{H}=A I_{0}(\eta)+B K_{0}(\eta)
$$

$I_{0}, K_{0}=$ modified Bessel functions

By B.C., eq (10): B must be zero, as $\bar{C}(0)$ is finite. 
328

Particular Solution:

$$
\bar{c}_{p}=\frac{1}{p^{2}}
$$

$\therefore$ General solution is:

$$
\begin{aligned}
\bar{C}(\eta) & =A I_{0}(\eta)+\frac{1}{p^{2}} \\
\text { or } \bar{C}(\xi) & =A I_{0}(q \xi)+\frac{1}{p^{2}}
\end{aligned}
$$

using (11):

$$
\begin{aligned}
& 0=A I_{0}(q)+\frac{1}{p^{2}} \\
\therefore & A=-\frac{1}{p^{2} I_{0}(q)} \\
\therefore \quad & \bar{C}(\xi)=\frac{1}{p^{2}}\left[1-\frac{I_{0}(q \xi)}{I_{0}(q)}\right]
\end{aligned}
$$

Surface flux of f.p.s

$$
\begin{aligned}
J & =-D\left(\frac{\partial C}{\partial \gamma}\right)_{R}=-\frac{D}{R}\left(\frac{\partial C}{\partial \xi}\right)_{1} \\
\left(\frac{J}{R Y \dot{F}}\right) & =-\frac{D}{R^{2} Y \dot{F}}\left(\frac{\partial C}{\partial \xi}\right)_{1}=-\left(\frac{\partial T}{\partial \xi}\right)_{1}
\end{aligned}
$$

Taking transform:

$$
\frac{\bar{J}}{R V \bar{F}}=-\left(\frac{d \bar{C}}{d \xi}\right)_{i}
$$

From (13):

$$
\begin{aligned}
\frac{d \bar{C}}{d \xi}=-\frac{1}{p^{2} I_{0}(q)} \frac{d}{d \xi} I_{0}(q \xi)=-\frac{q}{p^{2} I_{0}(q)} \frac{d}{d(q \xi)}\left[I_{0}(q \xi)\right] \\
=-\frac{q}{p^{2}} \frac{I_{1}(q \xi)}{I_{0}(q \xi)}
\end{aligned}
$$


therefore:

$$
\frac{\bar{J}}{R Y \dot{F}}=\frac{1}{q^{3}} \frac{I_{1}(q)}{I_{0}(q)}
$$

Long time approximation:

$$
\begin{aligned}
& \text { As } t \rightarrow \infty, \quad q \rightarrow 0 \\
& I_{1}(q) \rightarrow \frac{1}{2} q, I_{0}(q) \rightarrow 1 \\
& \therefore \quad \frac{\bar{J}}{R Y \dot{F}}=\frac{1}{2 p}
\end{aligned}
$$

Inverting:

$$
\frac{J}{R Y \dot{F}}=\frac{1}{2}
$$

Short time approximation:

$$
\begin{aligned}
& \text { As } t \rightarrow 0, q \rightarrow \infty \\
& I_{1}(q) \rightarrow \frac{e^{q}}{\sqrt{2 \pi q}}\left[1-\frac{3}{8 q}+\cdots\right] \\
& I_{0}(q) \rightarrow \frac{e^{q}}{\sqrt{2 \pi q}}\left[1+\frac{1}{8 q}+\cdots\right] \\
& \therefore \frac{\bar{J}}{R Y \dot{F}}=\frac{1}{q^{3}}\left[\frac{1-\frac{3}{8 q}}{1+\frac{1}{8 q}}\right]-\frac{1}{q^{3}}\left(1-\frac{1}{2 q}+\cdots\right)
\end{aligned}
$$

Inverting: (Carslaw \& Jaeger, app I, nos 8 \&9)

$$
\frac{J}{R Y \dot{F}}=2 \sqrt{\frac{\theta}{\pi}} \div \theta
$$


330

Problem 15.4

Diffusion equation in the cladding is:

$$
\frac{\partial C_{c l}}{\partial t}=D_{c l} \frac{\partial^{2} C_{c l}}{\partial x^{2}}
$$

Initial Condition:

$$
C_{c 1}(0, x)=0
$$

Boundary Conditions:

i) $C_{c 1}(t, \infty)=0$

ii) Let $C_{N_{a}}=$ concentration of

distribution coefficient

$$
m=\frac{C_{c 1}(t, 0)}{C_{N_{a}}(t)}=\text { constant }
$$

Balance on F.P. in the sodium

$$
\operatorname{tg} \frac{d C_{N a}}{d t}=J_{-}-\left[-D_{c l}\left(\frac{\partial C_{c l}}{\partial x}\right)_{0}\right]
$$

where $J$ is a constant given by eq.(15) of problem 15.3;

$$
\therefore \frac{t_{g}}{m}\left(\frac{\partial C_{c 1}}{\partial t}\right)_{x=0}=J+D_{c 1}\left(\frac{\partial C_{c 1}}{\partial x}\right)_{0}
$$

Dimensionless variables:-

$$
\theta=\frac{D_{c 1} t}{t_{g}^{2}} \text {, dimensionless time }
$$


331

$\zeta=\frac{x}{t_{g}}$, dimensionless distance

$C=\left(\frac{D_{c l}}{m t_{g} J}\right) C_{c l}$, dimensionless concentration

Diffusion Equation becomes

$$
\frac{\partial C}{\partial \theta}=\frac{\partial^{2} C}{\partial \rho^{2}}
$$

with

$$
\begin{aligned}
& C(0, y)=0 \\
& C(\theta, \infty)=0
\end{aligned}
$$

and

$$
\left(\frac{\partial C}{\partial \theta}\right)_{g=0}=1+m\left(\frac{\partial C}{\partial s}\right)_{0}
$$

Solution by Laplace Transform

Let $p=$ transform variable and $q=\sqrt{p}$

Transform eq.(1) with initial condition (2):

$$
\frac{d^{2} \bar{c}}{d s^{2}}=q^{2} \bar{c} \quad \text { or } \quad \frac{d^{2} \bar{c}}{d s^{2}}-q^{2} \bar{c}=0
$$

Transform of (3): $\quad \bar{C}(\infty)=0$

Transform of (4):

$$
q^{2} \bar{C}(0)=\frac{1}{q^{2}}+m\left(\frac{d \bar{c}}{d s}\right)
$$

Solution of (5) is

$$
\bar{C}=A e^{-q s}+B e^{q s}
$$

$B=0$ to satisfy

(6) 
To satisfy (7):

$$
q^{2} A=\frac{1}{q^{2}}-m A q
$$

or $A=\frac{1}{q^{3}(m+q)}$

Therefore:

$$
\bar{c}(s, q)=\frac{e^{-q s}}{q^{3}(m+q)}
$$

To invert, see $p .495$, Carslaw \& Jaeger, no 16.

Inverting:

$$
\begin{array}{r}
C(\theta, j)=\frac{1}{m^{2}}\left\{e^{m s} e^{m^{2} \theta} \operatorname{erfc}\left(m \sqrt{\theta}+\frac{s}{2 \sqrt{\theta}}\right)\right. \\
+\frac{1}{m}\left\{2 \sqrt{\frac{\theta}{\pi}} e^{-s^{2} / 4 \theta}-\operatorname{erfc}\left(\frac{s}{2 \sqrt{\theta}}\right)\right\} \\
\end{array}
$$


33.3

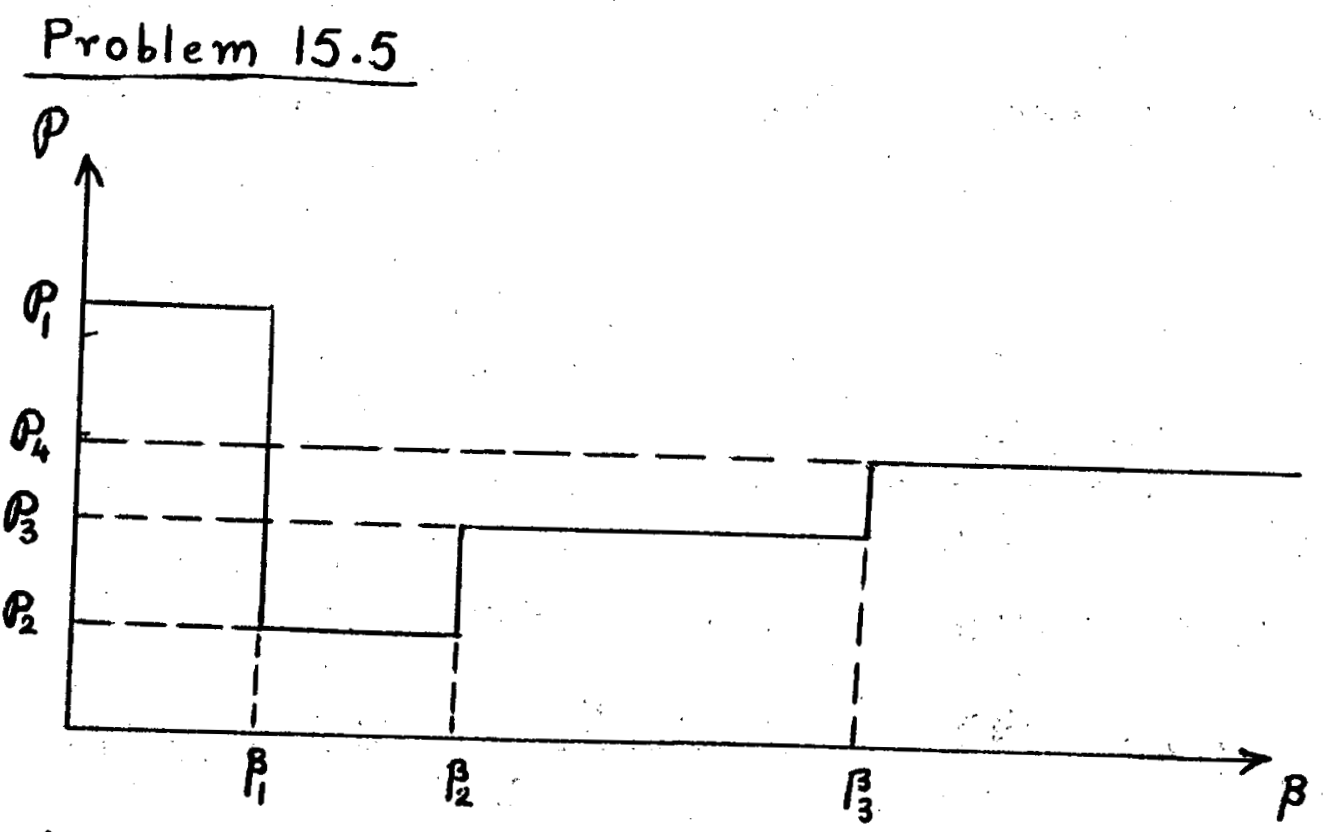

a) $n_{g}\left(\beta_{1}\right)=n_{0}+\left(\frac{Y_{x e} V_{f}}{\Omega}\right) \beta_{1} \bar{f}^{*}\left(R_{1}\right) \ldots(1)$

Since initial high power operation does not enhance release in subsequent lower power cycle:

$$
n_{g}\left(\beta_{2}\right)=n_{g}\left(\beta_{1}\right)+\left(\frac{Y_{e} V_{f}}{\Omega}\right)\left(\beta_{2}-\beta_{1}\right) \overline{f^{*}}\left(P_{2}\right)
$$

Adding we get;

$$
n_{g}\left(\beta_{2}\right)=n_{0}+\left(\frac{Y_{x_{e}} V_{f}}{\Omega}\right)\left[\beta_{1} \bar{f}\left(\beta_{1}\right)+\left(\beta_{2}-\beta_{1}\right) \bar{f}\left(\beta_{2}\right)\right]
$$

For the third power cycle at $P_{3}$, the fractional $-(2)$ release is the same as if the fractional had been at $p_{3}$

Using (1):

$$
n_{g}\left(\beta_{3}\right)=n_{g}\left(\beta_{1}\right)+\left(\frac{\psi_{e} V_{f}}{\Omega}\right)\left(\beta_{3}-\beta_{1}\right) \overline{f^{*}}\left(\beta_{3}\right)
$$

$$
n_{g}\left(\beta_{3}\right)=n_{0}+\left(\frac{Y_{x_{e}} Y_{f}}{\Omega}\right)\left\{\beta_{1} \bar{f}^{*}\left(P_{1}\right)+\left(\beta_{3}-\beta_{1}\right) \bar{f}^{*}\left(P_{3}\right)\right\}
$$


334

b)

$$
\begin{aligned}
\text { Coolant outlet temperature } & =\begin{array}{c}
\text { Temperature of gas } \\
\text { in plenum }
\end{array} \\
& =T_{p}
\end{aligned}
$$

Also: Coolant temperature rise, $\left(T_{p}-T_{i n}\right) \propto$ linear

0

$$
\therefore \frac{T_{p}-T_{\text {in }}}{T_{1}-T_{\text {in }}}=\frac{P}{\rho_{1}}
$$

Only open voidage $=$ that of plenum, volume $v_{p}$

$$
\begin{gathered}
P_{g}=\frac{n_{g} R T_{p}}{V_{p}}=\frac{n_{g} R T_{i n}}{V_{p}}\left[1+\frac{p}{Q_{1}}\left(\frac{T_{1}}{T_{\text {in }}}-1\right)\right] \\
{[u \operatorname{sing}(4)]}
\end{gathered}
$$

$$
0 \leq \beta \leq \beta_{1}, \quad \ddot{n}_{g}=n_{0}, P=p_{1}
$$

$\therefore$ pressure between $0 \leq \beta \leq \beta_{1}$ is:

$\left(P_{g}\right)_{0-1}=\frac{n_{0} R T_{\text {in }}}{V_{p}}\left(\frac{T_{1}}{T_{\text {in }}}\right)$

$$
\begin{aligned}
& \beta_{1} \leq \beta \leq \beta_{2}, \quad n_{g}=n_{g}(\beta) \quad\left[E_{q}(1)\right], \quad P=P_{2} \\
& \left(p_{g}\right)_{1-2}=\left(\frac{R T_{i n}}{V_{p}}\right)\left[1+\frac{P_{2}}{P_{1}}\left(\frac{T_{1}}{T_{i n}}-1\right)\right]\left[n_{0}+\left(\frac{x_{e} V_{f}}{\Omega}\right) p_{1} F_{p}(p)\right] \\
& \therefore \quad\left(p_{g}\right)_{1-2}=\left[1+\frac{P_{2}}{P_{1}}\left(\frac{T_{1}}{T_{i n}}-1\right)\right]\left[\frac{\left(p_{g}\right)_{o_{-1}}}{\left(T_{1} / T_{i n}\right)}+\left(\frac{Y_{x e} R T_{i n}}{\Omega} \frac{V_{f}}{V_{p}}\right) \beta_{1} f\left(p_{g}\right)\right] \\
& \beta_{2} \leq \beta \leq \beta_{3}, \quad \beta=p_{3}, \quad n_{g}=n_{g}\left(\beta_{2}\right),[E q \cdot(2)]
\end{aligned}
$$




$$
\begin{aligned}
& \left(p_{g}\right)_{2-3}=\left(\frac{R T_{\text {in }}}{V_{p}}\right)\left[1+\frac{P_{3}}{P_{1}}\left(\frac{T_{1}}{T_{i n}}-1\right)\right]\left\{n_{0}+\left(\frac{Y_{\text {Xe }} V_{p}}{\Omega}\right)\left[\beta_{1} f^{\pi}\left(P_{1}\right)+\left(\beta_{2}-\beta_{1}\right) f^{*}\left(P_{2}\right)\right]\right\} \\
& \text { or }\left(P_{g}\right)_{2-3}=\left[1+\frac{P_{3}}{P_{1}}\left(\frac{T_{1}}{T_{\text {in }}}-1\right)\right]\left\{\frac{\left(P_{g}\right)_{0-1}}{\left(T_{1} / T_{i n}\right)}+\left[\frac{V_{x e R} T_{\text {in }}}{\Omega} \frac{V_{f}}{V_{p}}\right]\left[\beta_{1} \bar{f}^{*}\left(P_{1}\right)+\left(\beta_{2}-\beta\right) \overline{f^{*}\left(p_{2}\right)}\right]\right\} \\
& \beta>\beta_{3} \\
& \text {-.-(8) }
\end{aligned}
$$

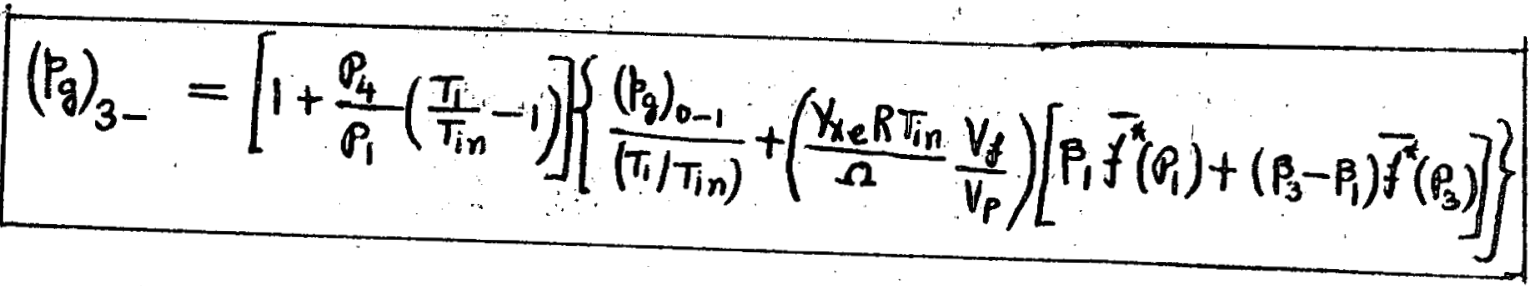$$
---(9)
$$

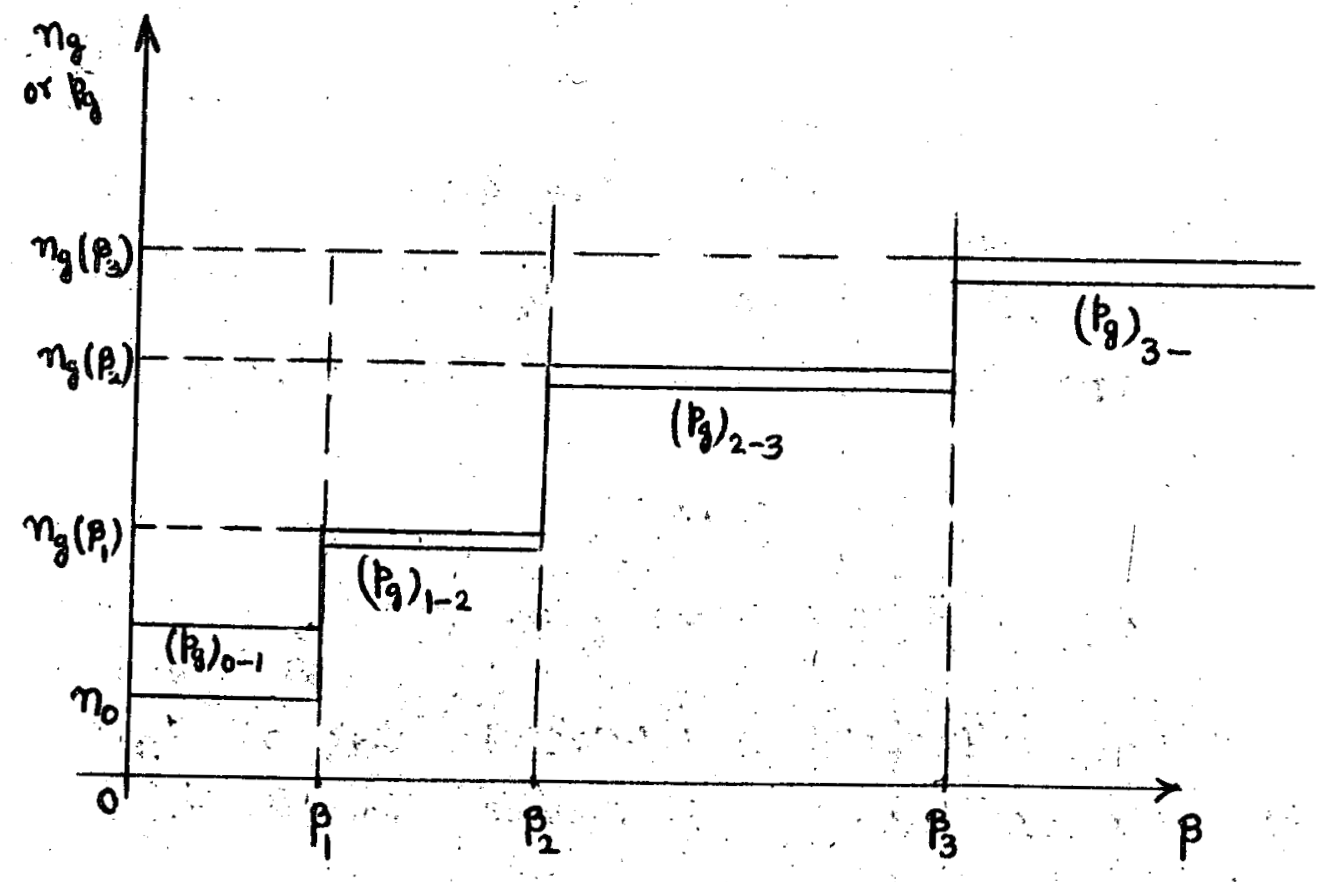


336

c) According to the "life-fraction rule", failure occurs when:

$$
\frac{t_{1}}{t_{R}\left(p_{g_{0-1}}\right)}+\frac{t_{2}-t_{1}}{t_{R}\left(p_{g_{1-2}}\right)}+\frac{t_{3}-t_{2}}{t_{R}\left(p_{g_{2-3}}\right)}+\frac{t_{f}-t_{3}}{t_{R}\left(p_{g_{3-f}}\right)}=1
$$

where $t_{1}, t_{2}$ and $t_{3}$ are the times corresponding to burnups $\beta_{1}, \beta_{2}$ and $\beta_{3}$ and $t_{f}$ is the time at which cladding failure occurs.

Using $t_{R}=\frac{k}{k_{g}^{n}}$, eq. (10) becomes:

$$
t_{1} p_{g_{0-1}}^{n}+\left(t_{2}-t_{1}\right) p_{g_{1-2}}^{n}+\left(t_{3}-t_{2}\right) p_{g_{2-3}}^{n}+\left(t_{f}-t_{3}\right) p_{g_{3}-}^{n}=k
$$

$P_{g_{0}-1}, P_{g_{-2}}, P_{g_{2-3}}$ and $P_{g_{3}}$ are given by eq (6) through (9) in problem 15.5 b). There remains to calculate $t_{1}, t_{2}$ and $t_{3}$ : The burnup is given by:

$$
\beta=\dot{F} \Omega t
$$

where $F=$ fissions $/ \mathrm{cm}^{3}-\sec$ (average over fuel cross section)

$\Omega=$ volume per uranium atom, $\mathrm{cm}^{3}$ and the fission density is related to the linear power by:

$$
\frac{P}{\pi R^{2}}=3.07 \times 10^{-11} \dot{F}
$$


337

$$
\therefore \quad \beta=k P t
$$

where $K=\frac{\Omega}{3.07 \times 10^{-11} \pi R^{2}}$.

$$
\begin{aligned}
\therefore t_{1} & =\frac{1}{k} \frac{\beta_{1}}{P_{1}} \cdots(13 a) \\
t_{2}-t_{1} & =\frac{1}{k} \frac{\beta_{2}-\beta_{1}}{P_{2}} \ldots(13 b) \\
t_{3}-t_{2} & =\frac{1}{K} \frac{\beta_{3}-\beta_{2}}{P_{3}} \ldots(13 c) \\
t_{f}-t_{3} & =\frac{1}{k} \frac{\beta_{f}-\beta_{3}}{P_{4}} \cdots(13 d)
\end{aligned}
$$

Substituting eqs(13) into eq.(11) yields:

$$
\begin{gathered}
\frac{\beta_{1} p_{g_{0-1}}^{n}}{p_{1}}+\frac{\left(\beta_{2}-\beta_{1}\right) p_{p_{1-2}}^{n}}{p_{2}}+\frac{\left(\beta_{3}-\beta_{2}\right) p_{g_{2-3}}^{n}}{p_{3}}+\frac{\left(\beta_{3}-\beta_{3}\right) p_{g_{3-}-}^{n}}{p_{4}} \\
=K k
\end{gathered}
$$

This can be solved for $\beta_{f}$

5 
338

Problem 15.6

$$
\begin{aligned}
& \frac{\partial C}{\partial t}=D \nabla^{2} C+S=0 \text { in steady state } \\
& \therefore D \frac{1}{r^{2}} \frac{d}{d r}\left(r^{2} \frac{d C}{d r}\right)-\lambda C=-y \dot{F}
\end{aligned}
$$

Let $\frac{1}{L^{2}}=\frac{\lambda}{D}$, then a particular solution is: $\quad C_{p}=\frac{Y \dot{F}}{\lambda}$ for homogeneous equation: $\quad C=\frac{A}{r} \sinh \frac{x}{L}+\frac{B}{r} \cosh \frac{R}{L}$

Boundary Conditions: (1) $C(0)$ is finite.

This gives $B=0$

(2) $\quad c(a)=0$

This gives $\frac{y \dot{F}}{\lambda}+\frac{A}{a} \sinh \frac{a}{L}=0$

$$
\begin{aligned}
& \therefore \quad A=-\frac{Y \dot{F}}{\lambda} \frac{a}{\sinh \frac{a}{L}} \\
& \therefore \quad C(r)=\frac{Y \dot{F}}{\lambda}\left(1-\frac{a}{r} \frac{\sinh \frac{r}{L}}{\sinh \frac{a}{L}}\right) \\
& f=\frac{R}{P}=\frac{\text { total rate of release }}{\text { total rate of production }}=\frac{4 \pi a^{2} j(a)}{\frac{4}{3} \pi a^{3} y \dot{F}} \\
&=-\left.\frac{3 D}{a Y \dot{F}} \frac{d C}{d r}\right|_{r=a}
\end{aligned}
$$


339

$$
\begin{aligned}
\therefore f & =-\frac{3 D}{a y \dot{F}} \cdot \frac{y \dot{F}}{\lambda}\left(\frac{a}{r^{2}} \frac{\sinh \frac{r}{L}}{\sinh \frac{a}{L}}-\frac{a}{r L} \frac{\cosh \frac{r}{L}}{\sinh \frac{a}{L}}\right)_{r=a} \\
& =-\frac{3 D}{\lambda}\left(\frac{1}{a^{2}}-\frac{1}{a L} \operatorname{coth} \frac{a}{L}\right) \\
\therefore f & =\frac{3 D}{\lambda a^{2}}\left(-1+\sqrt{\frac{a^{2} \lambda}{D}} \operatorname{coth} \sqrt{\frac{a^{2} \lambda}{D}}\right)
\end{aligned}
$$

Using the radioactive half life $z_{1 / 2}=\frac{\ln 2}{\lambda}$, or for simplicity, the radioactive mean life $z=\frac{1}{\lambda}$ and the diffusion mean life $z_{D}=\frac{a^{2}}{D}$, we get:

$$
f=3 \frac{z}{z_{D}}\left(\sqrt{\frac{z_{D}}{z}} \operatorname{coth} \sqrt{\frac{z_{D}}{z}}-1\right)=\frac{3}{x^{2}}(x \operatorname{coth} x-1)
$$

where $x^{2}=\frac{z_{D}}{z}$

For $z_{D} \gg z$, ie. $x \gg 1$

$$
\begin{aligned}
& \operatorname{coth} x=\frac{e^{x}+e^{-x}}{e^{x}-e^{-x}} \cong 1 \quad \text { as } x \rightarrow \infty \\
& \therefore \quad f=\frac{3}{x^{2}}(x-1) \cong \frac{3}{x^{2}} \cdot x=\frac{3}{x}=3 \sqrt{\frac{z}{z_{D}}}=\frac{3}{a} \sqrt{\frac{D}{\lambda}}
\end{aligned}
$$

For $Z_{D} \ll z$, ie. $x \ll 1$

This means that the decay rate is much smaller than the rate of release by diffusion; in this case we can approximate the diffusion equation by:

$$
\begin{aligned}
& D \frac{1}{r^{2}} \frac{d}{d r}\left(r^{2} \frac{d C}{d r}\right)=-y \dot{F} \\
& \therefore r^{2} \frac{d C}{d r}=-\frac{y \dot{F}}{D}\left[\frac{r^{3}}{3}+c_{1}\right] \\
& \therefore \frac{d C}{d r}=-\frac{y \dot{F}}{3 D}\left[r+\frac{3 C_{1}}{r^{2}}\right]
\end{aligned}
$$


340

$$
\begin{aligned}
& C_{1}=0, \text { since }\left.\frac{d C}{d r}\right|_{r=0}=0 \\
& \therefore \quad c=-\frac{y \dot{F}}{6 D} r^{2}+C_{2}
\end{aligned}
$$

Since $\quad C(a)=-\frac{y \dot{F} a^{2}}{6 D}+c_{2}=0$,

we get $\quad C_{2}=\frac{Y \dot{F} a^{2}}{6 D}$

$$
\therefore \quad C=\frac{y \dot{F} a^{2}}{6 D}\left(1-\frac{r^{2}}{a^{2}}\right)
$$

and $f=-\left.\frac{3 D}{y \dot{F} a} \frac{d C}{d r}\right|_{r=a}=\frac{3 D}{a y \dot{F}} \frac{y \dot{F} a}{3 D}=1$

$$
\therefore f=1
$$


341

Problem 15.7

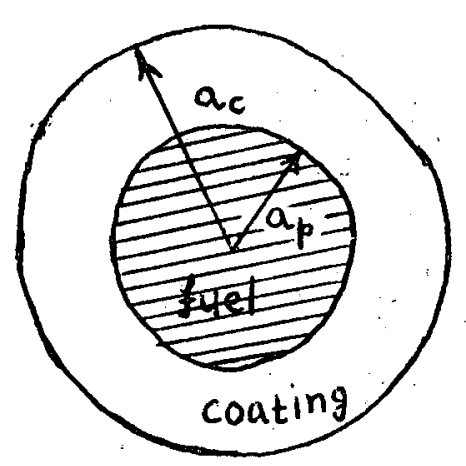

a) Diffusion in coating.

$$
-D \frac{1}{r^{2}} \frac{d}{d r}\left(r^{2} \frac{d c}{d r}\right)+\lambda C=S(r)
$$

where $S(r)=$ fission fragments from fuel pellet deposited in coating at $r$ per ce per sec

B.C. (1) $\left(\frac{d C}{d r}\right)_{a_{p}}=0$ (No transfer back to pellet)

(2) $\quad c\left(a_{c}\right)=0$

To solve the eq. (1) for arbitrary $s(r)$,

let $u=r c$, then (1) becomes

$$
D \frac{d^{2} u}{d r^{2}}-\lambda u=-r s(r)
$$

B.C. (1)

$$
\begin{gathered}
a_{p}\left(\frac{d u}{d r}\right)_{a_{p}}=u\left(a_{p}\right) \\
u\left(a_{c}\right)=0
\end{gathered}
$$

Then, the general solution to $\left(1^{\prime}\right)$ is given by

$$
u(r)=A e^{-\sqrt{\lambda / p} r}+B e^{\sqrt{\lambda / D} r}+u_{p}(r)
$$

where $u_{p}(r)$ is a particular solution to $\left(1^{\prime}\right)$ 
$34-2$

But, by method of variation of constants, we can assume the trial function is as

$$
u_{p}(r)=v(r) e^{-\sqrt{\lambda / D} r}+\omega(r) e^{\sqrt{\lambda / D} r}
$$

For simplicity let $\quad y_{1}(r)=e^{-\sqrt{\lambda_{D D}} \gamma}$ and $y_{2}(\gamma)=e^{\sqrt{\lambda_{D}} r}$ and since $y_{1}(r)$ and $y_{2}(r)$ satisfy the homogeneous equation.

$$
D \frac{d^{2} u}{d r^{2}}-\lambda u=0
$$

First taking a derivative of $u_{p}(r)$ w.r.t. $r$

$$
\begin{aligned}
\quad u_{p}(r) & =v(r) y_{1}(r)+\omega(r) y_{2}(r) \\
\therefore \frac{d u_{p}}{d r} & =\left(y_{1} \frac{d v(r)}{d r}+y_{2} \frac{d \omega(\gamma)}{d r}\right)+\left(v(r) \frac{d y_{1}(r)}{d r}+\omega(\gamma) \frac{d y_{2}(r)}{d r}\right)
\end{aligned}
$$

So, assume $y_{1} v^{\prime}+y_{2} \omega^{\prime}=0$

Then

$$
\begin{aligned}
\frac{d^{2} u_{k}}{d r^{2}}=\left(v(r) \frac{d^{2} y_{1}(r)}{d r^{2}}+\omega(r) \frac{d^{2} y_{2}(r)}{d r^{2}}\right) & \\
& +\left(\frac{d v(r)}{d r} \cdot \frac{d y_{1}(r)}{d r}+\frac{d \omega(r)}{d r} \cdot \frac{d y_{2}(r)}{d r}\right)
\end{aligned}
$$

Substituting (2) and (5) into (1') and using the fact that $y_{1}(r), y_{2}(r)$ satisfy

$D u^{\prime \prime}-\lambda u=0$, then we have

$$
v^{\prime} \cdot y_{1}^{\prime}+\omega^{\prime} y_{2}^{\prime}=-r s(r)
$$

Substituting for $y_{1}(r)$ and $y_{2}(r)$ into $(4)$ and (6)

$$
e^{-\sqrt{\lambda / D} r} v^{\prime}+e^{\sqrt{\lambda / D} r} \omega^{\prime}=0
$$


343

and $\quad-e^{-\sqrt{\lambda / D} r} v^{\prime}+e^{\sqrt{\lambda / D} r} \omega^{\prime}=-\sqrt{D / \lambda} r s(r)$

Solving $\left(4^{\prime}\right)$ and $\left(6^{\prime}\right)$ for $v^{\prime}$ and $\omega^{\prime}$

$$
v^{\prime}=\frac{d v}{d r}=\frac{1}{2} \sqrt{\frac{D}{\lambda}} r s(r) e^{\sqrt{\lambda / D} r}
$$

and $\omega^{\prime}=\frac{d \omega}{d r}=-\frac{1}{2} \sqrt{\frac{D}{\lambda}} r s(r) e^{-\sqrt{\lambda l D} r}$

$$
\begin{gathered}
\therefore \quad v(r)=\frac{1}{2} \sqrt{\frac{D}{\lambda}} \int_{a_{p}}^{r} \rho s(\rho) e^{\sqrt{\lambda / D} \rho} d \rho \\
\omega(\gamma)=-\frac{1}{2} \sqrt{\frac{D}{\lambda}} \int_{a_{p}}^{r} \rho s(\rho) e^{-\sqrt{\lambda / D} \rho} d \rho \\
\therefore \quad u(r)=[A+v(r)] e^{-\sqrt{\lambda / D} r}+[B+\omega(\gamma)] e^{+\sqrt{\lambda / D} \gamma}
\end{gathered}
$$

$A$ and $B$ can be determined from B.C. (1) and (2)

Then the general solution to eq. (1) is given by,

$$
C(r)=\frac{1}{r}[A+v(r)] e^{-\sqrt{\lambda_{1 D}} r}+\frac{1}{r}[B+\omega(r)] e^{\sqrt{\lambda / D} r}
$$

Rate of release from the surface of the coating $=-4 \pi a_{c}^{2} D\left(\frac{d C}{d r}\right)_{a_{c}}$

Rate of production in the pellet $=\frac{4}{3} \pi a_{p}^{3} Y_{i} \dot{F}$

$\therefore \quad$ fractional release $f=-\left(\frac{3 a_{c}^{2} D}{a_{p}^{3} y_{i} F}\right)\left(\frac{d c}{d r}\right)$

... (8) 
b)

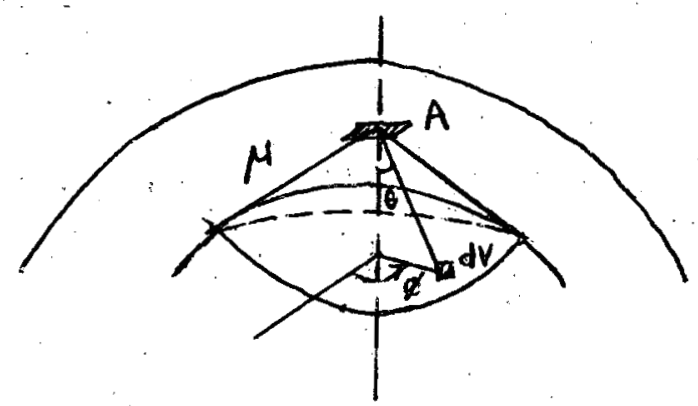

Current through unit area at $r$ (point A) comes from material in pellet which is contained within a spherical cap of radius $\mu$ with its center at point $A$, at which the current is normal.

Consider a volume element $d v$ in pellet at polar angle $\theta$ from the vertical axis and a distance $\rho$ from point $A . \quad(\rho<\mu)$

Rate of "emission" of fission product " $i$ " from $d v$ is:

\section{$y_{i} \dot{F} d v$}

Solid angle subtented by unit area perpendicular to the vertical axis at $d v$ is:

$$
\frac{\cos \theta}{4 \pi \rho^{2}}
$$

Then $d J=$ rate at which ff's from $d v$ cross the unit area at point $A$

$$
\begin{aligned}
& =y_{i} \dot{F} d V \frac{\cos \theta}{4 \pi \rho^{2}}=-y_{i} \dot{F} \rho^{2} d \phi d \rho d(\cos \theta) \cdot \frac{\cos \theta}{4 \pi \rho^{2}} \\
d J & =-\frac{1}{4 \pi} y_{i} \dot{F} \cos \theta d \phi d \rho d(\cos \theta)
\end{aligned}
$$

To get $J(r)$, integrate $\rho$ from $\rho_{0}$ to $\rho_{1} ; \theta$ from 0 to $\theta_{f}$, $\phi$ from 0 to $2 \pi$ These limits differ depending upon $r$. 
Now define $r_{c}$ and $\theta_{c}$ such that

$$
\begin{aligned}
r_{c} & =\sqrt{a_{p}^{2}+\mu^{2}} \ldots(10) \\
\cos \theta_{c} & =\mu / r_{c} \ldots \ldots \text { (11) }
\end{aligned}
$$

Then we have 3 distinct regians

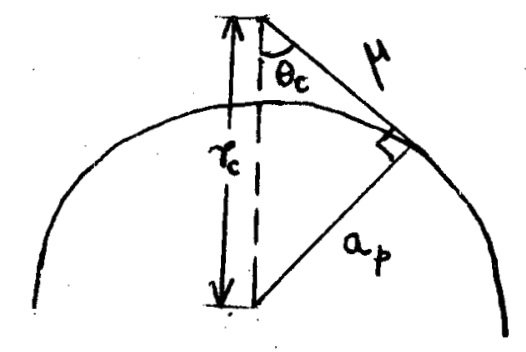
of $J(r)$

I) $\quad a_{p}<r \leq r_{c}$

Define $\theta_{f}$ and $\theta_{\mathfrak{i}}$ such that

$$
\begin{aligned}
\sin \theta_{f} & =\frac{a_{p}}{r} \\
\text { or } \cos \theta_{f} & =\sqrt{1-\left(\frac{a_{p}}{\gamma}\right)^{2}}
\end{aligned}
$$

and $a_{p}^{2}=\mu^{2}+r^{2}-2 \mu r \cos \theta_{i}$

$$
\text { or } \cos \theta_{i}=\frac{\mu^{2}+r^{2}-a_{p}^{2}}{2 \mu r}--(13)
$$

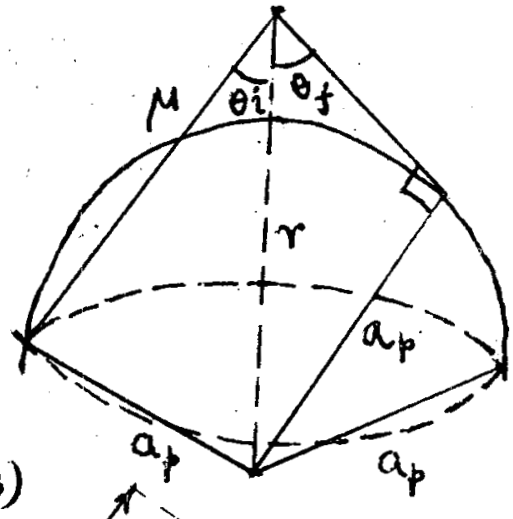

Then, for $\theta_{i} \leq \theta \leq \theta_{f}$ Limits of $\rho$ integration: $a_{p}^{2}=r^{2}+\rho^{2}-2 \rho r \cos \theta$

or $\rho_{0}=r \cos \theta-\sqrt{(r \cos \theta)^{2}-\left(r^{2}-a_{p}^{2}\right)}$

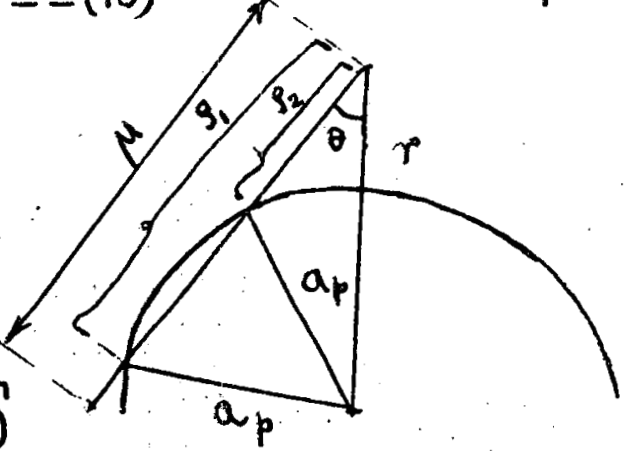

and $\rho_{1}=r \cos \theta+\sqrt{(r \cos \theta)^{2}-\left(r^{2}-a_{p}^{2}\right)}$

for $\quad 0 \leqslant \theta \leqslant \theta_{i}$

$$
\rho_{0}=r \cos \theta-\sqrt{(r \cos \theta)^{2}-\left(r^{2}-a_{p}^{2}\right)}
$$

and $\rho_{1}=\mu$ 
346

Now let $\eta=\cos \theta$

Then for $a_{p} \leq r \leq r_{c}$

$$
\begin{aligned}
J(r) & =\int_{V} d J=\frac{1}{4 \pi} \int_{0}^{2 \pi} d \phi \int_{\rho_{0}}^{\rho_{1}} d \rho \int_{1}^{\cos \theta_{f}} y_{i} \dot{F} \cos \theta d(\cos \theta) \\
& =-\frac{1}{2} y_{i} \dot{F}\left[\int_{1}^{\cos \theta_{i}} d \eta \int_{\rho_{0}}^{\mu} d \rho+\int_{\cos \theta_{i}}^{\cos \theta_{\rho}} \int_{\rho_{0}}^{\rho_{1}} d \rho\right] \\
& =-\frac{1}{2} y_{i} \dot{F}\left[\int_{1}^{\cos \theta_{i}}\left(\mu-\rho_{0}\right) \eta d \eta+\int_{\cos \theta_{i}}^{\cos \theta_{\rho}}\left(\rho_{1}-\rho_{0}\right) \eta d \eta\right]
\end{aligned}
$$

Substituting (12) through (16) we get,

$$
\begin{aligned}
J_{I}(r)=y_{i} \dot{F}\left[\frac{\int_{r^{2}+\mu^{2}-a_{p}^{2}}^{1} \eta\left[\mu-r \eta+\sqrt{(r \eta)^{2}-\left(r^{2}-a_{p}^{2}\right)}\right] d \eta}{2 \mu r}\right. & \frac{r^{2}+\mu^{2}-a_{p}^{2}}{2 r \mu} \\
& \left.+2 \int^{\frac{r^{2}}{\eta\left(a_{p} / r\right)^{2}}} \frac{\sqrt{(r \eta)^{2}-\left(r^{2}-a_{p}^{2}\right)}}{2 \eta}\right]
\end{aligned}
$$

III) $r_{c}<r \leq a_{p}+\mu$

In this region only the first term in (17) remains.

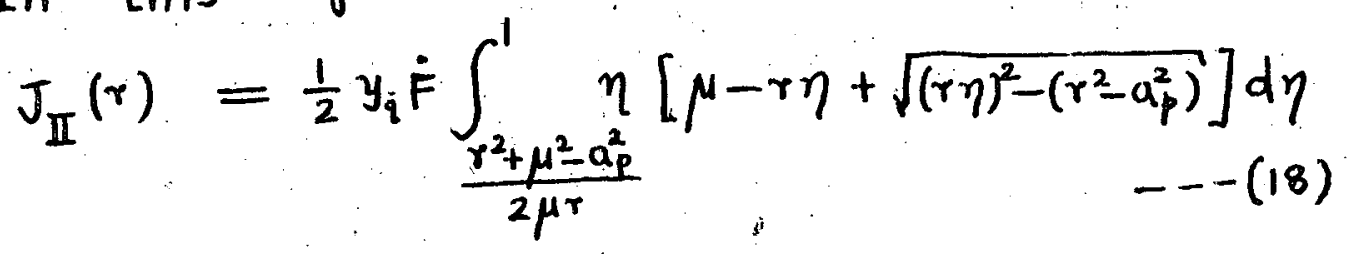

III) $r>a_{p}+\mu$

Since this is beyond the range of the f $f^{\prime}$ ',

$$
J_{\text {III }}(r) \equiv 0
$$


347

(ii) Evaluation of $S(v)$ due to Recoil from pellet

Since $\mu<\left(a_{c}-a_{p}\right)$, fragments can not recoil completely out of particle and since $\mu$ is of the order of $a_{p}$, spherical coordinates must be used.

Let $J(\gamma)=f_{\text {fission fragment current at }}$ location $r, a_{p}<r<a_{p}+\mu$

ie. $J(r)=$ number of fission fragments crossing unit area 1 to radius at $r$ per sec.

Then $N(r)=$ number of f's crossing spherical surface at $r=4 \pi r^{2} J(r)$

and $4 \pi r^{2} d r=$ volume of spherical element between $r$ and $r+d r$

$\therefore S(r)=\frac{N(r)-N(r+d r)}{4 \pi r^{2} d r}=-\frac{d N(r)}{4 \pi r^{2}}$

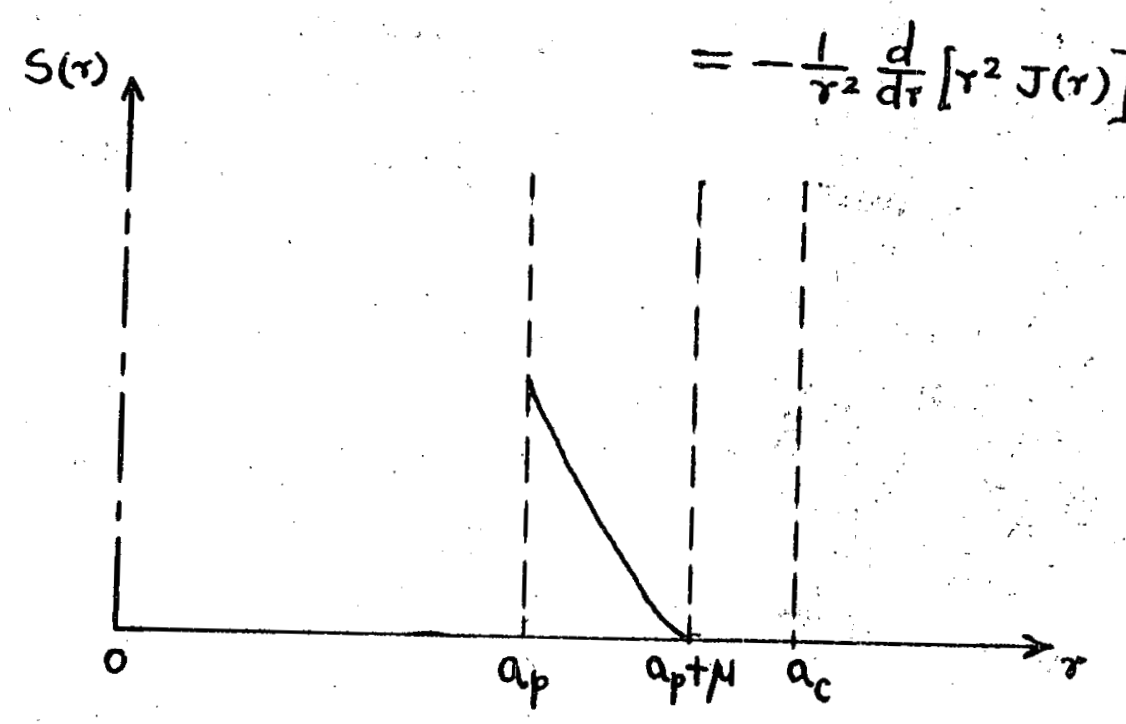


348

Problem 15.8

Stable isotope: atoms $/ \mathrm{cm}^{3}$ of fuel produced $=y \dot{F} t$ Radioactive isotope: atoms $/ \mathrm{cm}^{3}$-sec produced $=\dot{y}^{*} \dot{F}$

fractional release of stable isotope

$$
f=\frac{4}{\sqrt{\pi}} \sqrt{D^{\prime} t} \ldots[\text { Eq. }(15.89)]
$$

fractional release rate of radioactive

$$
\text { isotope }=3 \sqrt{\frac{D^{\prime}}{\lambda}}--\left[E_{q} \cdot(15.92)\right]
$$

Stable isotope at time $t$,

$$
\begin{gathered}
N=\text { number of atoms of stable fission } \\
\text { gas released }
\end{gathered}
$$$$
N=\left[\frac{4}{\sqrt{\pi}} \sqrt{D^{\prime} t}\right] y \dot{F} t
$$

Radioactive isotope:

$$
\begin{aligned}
R & =\text { rate of release of isotope } \\
& =\left[3 \sqrt{\frac{D^{\prime}}{\lambda}}\right] y^{*} \dot{F}
\end{aligned}
$$

Nuclear Balance on radioactive species:

$$
N^{*}=\text { atoms of radioactive gas in plenum }
$$

$$
\begin{aligned}
& \frac{d N^{*}}{d t}=R-\lambda N^{*} \\
\therefore \quad & N^{*}(t)=\frac{R}{\lambda}\left(1-e^{-\lambda t}\right) \cong \frac{R}{\lambda} \quad \text { (for large) } \\
& N^{*}=3 \sqrt{\frac{D}{\lambda}} \frac{y^{*} \dot{F}}{\lambda}
\end{aligned}
$$


Isotopic ratio

$$
\begin{aligned}
& \frac{N^{*}}{N}=\frac{3 \sqrt{D^{\prime}} y^{*} \dot{F}}{\lambda^{3 / 2}} \frac{\sqrt{\pi}}{4} \frac{1}{\sqrt{D^{\prime}} y \dot{F} t^{3 / 2}} \\
& \therefore \frac{N^{*}}{N}=\frac{3 \sqrt{\pi}}{4} \frac{y^{*}}{y} \frac{1}{(\lambda t)^{3 / 2}}
\end{aligned}
$$


$349 a$

Problem 15.9

let $L_{f}=$ length of fuel $(\mathrm{cm})$ Total power of fuel element $=P L_{f}$ watts

(neglecting axial power variations)
Total fissions in fuel rod in time $t=\frac{P L_{f} \text { watts } \times t \mathrm{sec}}{3.2 \times 10^{-11} \text { watt } / \mathrm{fissions} / \mathrm{sec}}$

$\left(\begin{array}{c}\text { Total number of stable } \\ \text { fission gas atoms produced } \\ \text { in fuel nod }\end{array}\right)=\frac{Y_{X_{e}} P L_{f} t}{3.2 \times 10^{-11}} \quad\left(\begin{array}{c}Y_{x_{e}}=0.25=\text { yield of } \\ \text { stable } X_{e}-K_{r}\end{array}\right)$

$\bar{f}=$ radially -averaged fractional release from fuel

$n_{g}=$ gin-atoms fission gases released

$$
=\frac{\bar{f} Y_{x_{e}} P L_{f} t}{\left(3.2 \times 10^{-1 \prime}\right)\left(6.023 \times 10^{23}\right)}
$$

Cross sectional area of fuel

plenum volume $=V_{p}=L_{p} A^{\sim} \quad$ (table 10.2)

$$
\begin{aligned}
& P_{g}=\frac{n_{g} R T_{p}}{V_{p}}=\frac{\bar{f} Y_{x_{e}} P\left(L_{f} / L_{A}\right) R T_{p} t}{A\left(3.2 \times 10^{-11}\right)\left(6.023 \times 10^{23}\right)} \\
& A=\frac{\pi}{4}(1.07-2 \times .061)^{2}=0.71 \mathrm{~cm}^{2}
\end{aligned}
$$

(a) Empirical diffusion coefficient method: For $\theta=500$ watt $/ \mathrm{cm}$

Fig. 15.16 gives $\overline{\bar{D}}^{\prime}=\bar{D}^{\prime} \cong 5 \times 10^{-10} \sec ^{-1}$ 
3496

$$
\begin{aligned}
\text { for } t & =3 \text { yrs }=9.4 \times 10^{7} \mathrm{sec} \\
\bar{f} & =\frac{4}{\sqrt{\pi}} \sqrt{\left(5 \times 10^{-10}\right)\left(9.4 \times 10^{7}\right)}=0.49 \\
\therefore P_{g} & =\frac{(0.49)(0.25)(500)(4)(82)(320+273)\left(9.4 \times 10^{7}\right)}{(0.71)\left(3.2 \times 10^{-11)}\left(6.023 \times 10^{23}\right)\right.} \\
& =82 \mathrm{~atm}
\end{aligned}
$$

(b) Engineering fission gas release method:

$$
\begin{aligned}
T_{0}-T_{s} & =\frac{500}{4 \pi(0.03)}=1326{ }^{\circ} \mathrm{C} \\
T_{s} & =400^{\circ} \mathrm{C} \\
T_{0} & =1726{ }^{\circ} \mathrm{C} \\
\therefore \quad & \bar{f}=\frac{0.3(1400-400)+0.5(1726-1460)}{1326}=0.35 \\
P_{g} & =\frac{0.35}{0.49} \times 82=58 \mathrm{~atm}
\end{aligned}
$$


350

CHAPTER 16

Problem 16.1

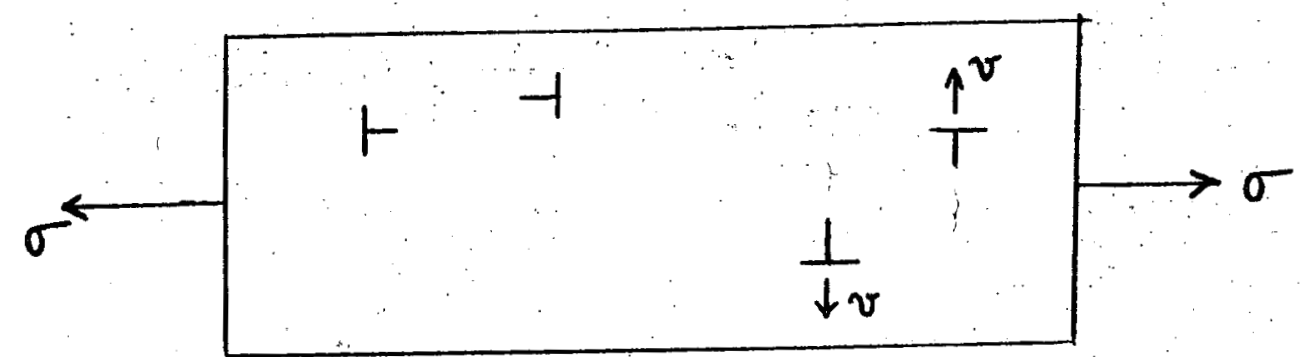

a) The notation for an edge dislocation means:

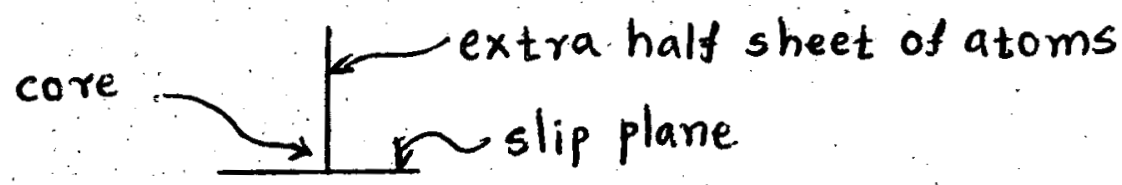

- When the tensile stress is normal to the extra sheet of atoms, the vacancy concentration at the core increases to the value given in b) below. This concentration is greater than that in the bulk, so that the dislocation emits vacancies and therefore moves perpendicular to the slip plane (climb) in a manner that will increase the size of the extra sheet of atoms.

b) See the derivation leading to Eq. (13.175). Replace $p$ in the formula by $-\sigma$ and set $r=0$. Divide by $\Omega$ to give proper units,

$$
C_{v}\left(R_{d}\right)=C_{v}^{\text {eq }} \exp \left(\frac{\sigma \Omega}{k T}\right)
$$


351

c) For $1 \mathrm{~cm}$. length along dislocations, the volume of solid associated with $1 \mathrm{~cm}$. of dislocation line $=\pi R^{2}$

or $\rho_{d}=\frac{1}{\pi R^{2}}$

or $R=\frac{1}{\sqrt{\pi s_{A}}}-\ldots(2) \quad\left[E_{q} \cdot(13.79)\right]$

d) Diffusion Equation in Cylindrical Coordinates:

$$
\frac{1}{r} \frac{d}{d r}\left(r \frac{d C_{v}}{d r}\right)=0 \quad\left[E_{q}(13.85)\right]
$$

Solution is given by Eq. (13.87), flux by Eq. (13.88).

Use $C_{V}(R)=C_{y}^{e q}$ and $C_{R_{d}}$ given by eq. (1), then

$$
2 \pi R_{d} J=\frac{2 \pi D_{v}\left[C_{v}^{e q} \exp \left(\frac{\sigma \Omega}{k T}\right)-C_{v}^{e q}\right]}{\ln \left(R / R_{d}\right)}
$$

Since $\frac{\sigma \Omega}{k T} \ll 1$, the above equation reduces to

$$
2 \pi R_{d} J=\frac{2 \pi D_{v} C_{v}^{e q} \sigma}{k T \ln \left(R / R_{d}\right)} \frac{\text { vacancies }}{\sec -c_{m} \text { of line }}
$$

e)

volume / vacancy $=\Omega$

Flow of volume away from $1 \mathrm{~cm}$.

of line $=\left(2 \pi R_{d} J\right) \Omega$

But the sheet of atoms has thickness $=b$,

(the Burgers vector)

$\therefore$ Flow of volume away from line $=(b)(1)(v)$

$$
\begin{array}{r}
\therefore v=\frac{2 \pi R_{d} J \Omega}{b}=2 \pi R_{d} J b^{2}-(4) \\
\text { (where } \Omega=b^{3} \text { ) }
\end{array}
$$


352

Use (3) in (4) to get,

$$
v=\frac{2 \pi D_{V} C_{v}^{e q} \sigma \Omega b^{2}}{k T \ln \left(R / R_{d}\right)}
$$

Also note that $D_{\text {vol }}=D_{y} C_{V}^{e q} \Omega$

f) Consider a block of solid of volume $V=x y z$, which consists $\rho_{d} x y$ dislocations, half of which climb when the tensile stress is applied in the $x$-direction.

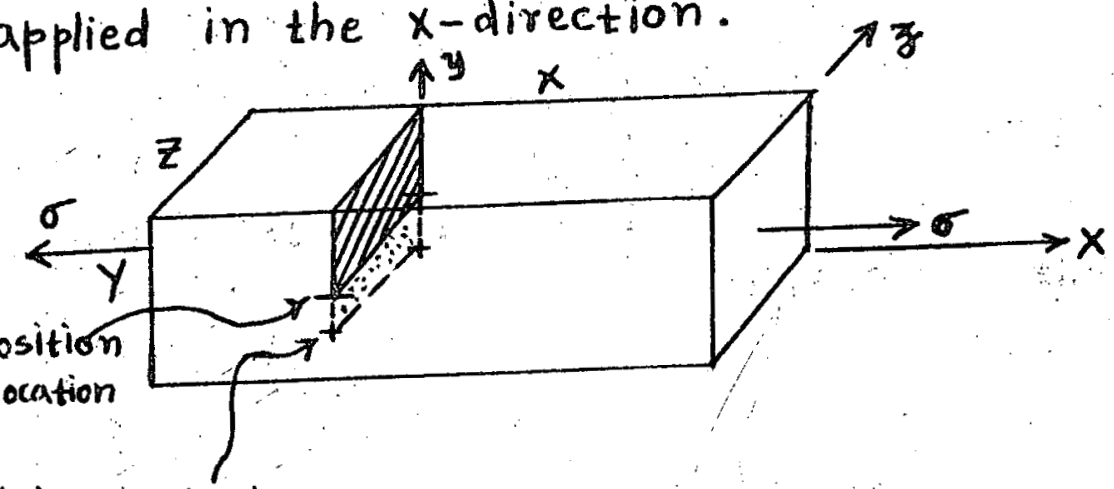

Position of the line after time $\Delta t$ under tensile stress

In time $\Delta t$, each properly oriented edge dislocation limbs a distance $v_{c} \Delta t$ in the $y$-direction. For each dislocation, the area swept out is $\left(v_{c} \Delta t\right) z$. Since $\frac{1}{2} \rho_{d}(x y)$ dislocations climb like this, the total. area added to planes perpendicular to the $x$-axis is:

$$
\left(v_{c} \Delta t\right) z \frac{1}{2} \rho_{d}(x y) \mathrm{cm}^{2}
$$

or the equivalent of $\frac{\left(\nu_{E} \Delta t\right) z\left(\frac{1}{2} \rho_{d} x y\right)}{y z}$ complete. planes of atoms have been added to the surface perpendicular to the $x$-direction. 
Since each plane has a thickness $b$, the strain in the $x$-direction is:

$$
\Delta x=\frac{\left(v_{c} \Delta t\right) z \frac{1}{2} \rho_{\text {et }} x y_{b}}{y z}
$$

The creep (or strain) rate in the direction of the applied stress is:

$$
\dot{\epsilon}=\frac{1}{x} \frac{\Delta x}{\Delta t}=\frac{1}{2} \rho_{d} v_{c} b
$$

The above equation is the same as Eq. (8.21) except for a factor of $\frac{1}{2}$, which corrects from total dislocation density to the density of mobile dislocations. Use (5), (6) and $b^{3}=\Omega$ in (7) to get

$$
\dot{\epsilon}=\frac{\pi \rho_{d} \mathbb{D}_{\text {Vol }} \Omega \sigma}{k T \ln \left(R / R_{d}\right)}
$$

This is a form of Nabarro-Herring creep. 
354

Problem 16.2

Fission Gas Swelling Law using continuum creep

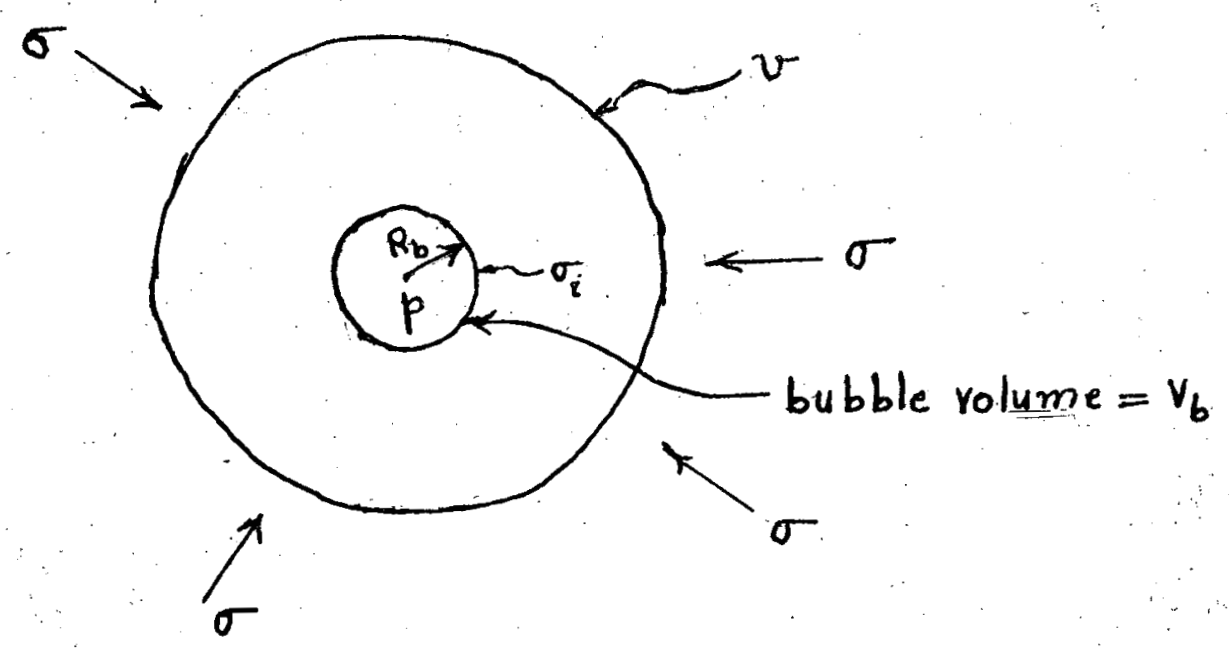

$N_{b}=$ bubble density, $\quad v=\frac{1}{N_{b}}$

Mechanical balance at bubble surface: [Eq. (13.6)]

$p=\frac{2 \gamma}{R_{b}}+\sigma_{i} \quad$ ( $\sigma_{i}$ positive if directed radially inward)

Ideal gas law:

$$
p V_{b}=m k T
$$

Gas atoms/bubble

Assume all gas in $v$ goes to bubble.

$$
m=Y_{x_{e}} \dot{F} t\left(v-V_{b}\right)=\begin{aligned}
& \text { gas atoms generated } \\
& \text { in time } t \text { in volume } V_{b}
\end{aligned}
$$

Combining (1) to (3)

$$
\sigma_{i}=p-\frac{2 \gamma}{R_{b}}=\frac{m k T}{V_{b}}-\frac{2 \gamma}{R_{b}}
$$


355

$$
\therefore \sigma_{i}=Y_{x_{e}} \dot{F} t\left(\frac{v}{V_{b}}-1\right) k T-\frac{2 x}{R_{b}}
$$

where $V_{b}=\frac{4}{3} \pi R_{b}^{3}=$ volume of bubble External work: (by both inner and outer surfaces) replace Eq. (16.183) - by

$$
\left(p-\frac{2 x}{R_{b}}\right) \dot{V}_{b}=\text { rate of work done in solid by internal }
$$

gas pressure (less surface tension)

$-\sigma \dot{v}=$ work done on solid by applied

Add and use (1) and note that $\dot{v}_{b}=\dot{v}$

$$
\therefore \dot{W}=\left(\sigma_{i}-\sigma\right) \dot{V}
$$

$\sigma_{i}$ is a function of $v_{b}$ and $t$ by eq. (4)

Rest of derivation is same as in text except Eq. (16.183) replaced by eq.(5) above.

Eq.(16.185) becomes:

$$
\left(\sigma_{i}-\sigma\right) \dot{v}=\frac{2}{3}|\dot{v}| \int_{v_{b}}^{v} \sigma^{*} \frac{d v}{v}
$$

Eq. (16.190) becomes:

(replacing $\dot{p}$ by $\dot{s}=$ gas swelling rate

$$
\dot{s}=\frac{q}{4} \alpha\left(\sigma_{i}-\sigma\right) s
$$

Eq. (4) becomes,

$$
\sigma_{i}=y_{x_{e}} \dot{F} k T\left(\frac{1}{s}-1\right) t-2 x\left(\frac{4 \pi N_{b}}{3}\right)^{1 / 3} / s^{1 / 3}
$$

since $R_{b}=\left(\frac{3 V_{b}}{4 \pi}\right)^{1 / 3}=\left(\frac{3 \cdot v}{4 \pi}\right)^{1 / 3} s^{1 / 3}=\left(\frac{3}{4 \pi N_{b}}\right)^{1 / 3} s^{1 / 3}$ 
356

Eq. (7) becomes:

$$
\frac{\left.5 \dot{s}=\frac{9}{4} \alpha \sigma\left\{\frac{Y_{x e} \dot{F} k T}{\sigma}\left(\frac{1-s}{s}\right) t-\frac{2 \gamma\left(\frac{4 \pi N_{b}}{3}\right)^{1 / 3}}{\sigma} s^{-1 / 3}-1\right\}\right]}{\text { (swelling law) }}
$$

When the fuel is weak, $\alpha \rightarrow \infty$, that is the creep rate becomes very large for any non-zero deviatoric stress. For s to be finite, \{\} in eq.(8) must go to zero. (Neglecting $s$ compared to unity in (8))

or : $\quad \frac{Y_{x e} \dot{F} k T_{t}}{s}-\frac{2 \gamma\left(\frac{4 \pi N_{b}}{3}\right)^{1 / 3}}{3^{1 / 3}}-\sigma=0$

or, with $\sigma=0$,

$$
s=\left(\frac{y_{x e} \dot{F}_{t k} T}{2 \gamma}\right)^{3 / 2}\left(\frac{3}{4 \pi N_{b}}\right)^{1 / 2}
$$

This is Eq. (13.146). 
357

Problem 16.3

Power Law Creep

$$
\begin{aligned}
& \dot{e}_{g}=A S_{g}^{n} \\
& \sigma=\frac{2}{3} \int_{v_{p}}^{v}\left[-\frac{2}{3 s} \frac{v}{v} \frac{\dot{p}}{1-p}\right]^{\frac{1}{n}} \frac{d v}{v} \\
& \frac{3}{2} \sigma=\left(\frac{2}{\partial s}\right)^{1 / n}\left(\frac{-\dot{p}}{1-p}\right)^{1 / n} \int_{p}^{1} \frac{d x}{x^{\frac{1}{n}+1}} \\
& \int_{p}^{1} \frac{d x}{x^{\frac{1}{n}+1}}=-\left[\frac{n}{x^{1 / n}}\right]_{p}^{1}=\left[\frac{n}{x^{1 / n}}\right]_{1}^{p}=\frac{n}{p^{1 / n}}-n \\
& \therefore\left(\frac{3}{2} \sigma\right)=\left(\frac{2}{3 s}\right)^{1 / n}\left(\frac{-\dot{p}}{1-p}\right)^{1 / n} n\left(\frac{1}{p^{1 / n}}-1\right) \\
& \text { or } \frac{3 \sigma}{2 n}=\left(\frac{2}{3 s}\right)^{1 / n}\left(\frac{-\dot{p}}{1-p}\right)^{1 / n}\left(\frac{1}{p^{1 / n}}-1\right) \\
& =\left(\frac{2}{3 s}\right)^{4 / n}\left(\frac{-\dot{p}}{1-p}\right)^{1 / n} \frac{1}{p^{1 / n}}\left(1-p^{1 / n}\right) \\
& \therefore\left(\frac{3 \sigma}{2 n}\right)^{n}=\left(\frac{2}{35}\right)\left(\frac{-p}{1-p}\right) \frac{1}{p}\left(1-p^{1 / n}\right)^{n} \\
& \text { or } \quad \dot{p}=-\left(\frac{3 \sigma}{2 n}\right)^{n}\left(\frac{3 s}{2}\right) \frac{(1-p) p}{\left(1-p^{1 / n}\right)^{n}}
\end{aligned}
$$


358

Problem 16.4

a) From problem 13.10, the number of fission fragments intersecting a spherical pore of radius $R_{p}$ per sec. is:

$4 \pi R_{p}^{3} G\left(\mu / R_{p}\right) \dot{F}$, where $\dot{F}$ is the fission density and $G$ is the function plotted in the solution to problem 13.10. For $\mu / R_{p} \ll 1$, the limiting form of $G$ is $\mu / R_{p}$ and the rate. at which. a pore is pierced by fission fragments is:

$4 \pi R_{p}^{2} \mu \dot{F} \quad \sec ^{-1}$

$\therefore \quad \frac{\text { vacancies redissolved }}{\text { sec }- \text { pore }}=4 \pi R_{p}^{2} \mu \Delta m_{v} \dot{F}$

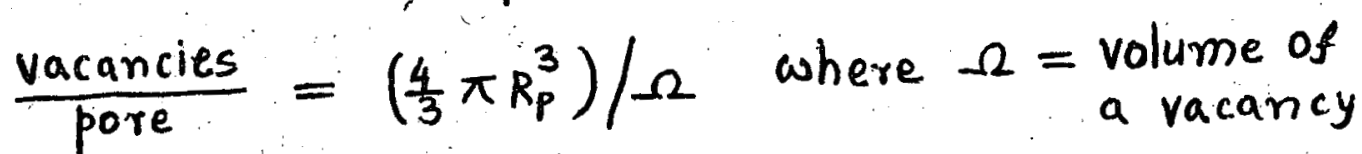

dividing:

$$
b=\frac{4 \pi R_{p}^{2} \mu \Delta m_{v} \dot{F}}{\left(4 / 3 \pi R_{p}^{3}\right) / \Omega}=\frac{3 \Omega \Delta m_{v} \mu \dot{F}}{R_{p}} \sec ^{-1}
$$

b) Let $b=b_{0}\left(\frac{R_{p o}}{R_{p}}\right)$

where $b_{0}=\frac{3 \Omega \Delta m_{y} \mu \dot{F}}{R_{p o}}$ and $R_{p o}$ is the initial pore radius.

Eq. (16.143), neglecting vacancy emission from surface, is: 
359

$$
4 \pi R_{p}^{2} \frac{d R_{p}}{d t}=-\frac{Q_{v}^{\text {Tesol }} \Omega}{N_{p}} \quad\left[E_{q}(16,143)\right]
$$

Eq. (16.146) is:

$$
\begin{gathered}
\frac{a_{y}^{r e s o l} \Omega}{N_{p}}=\left(\frac{4}{3} \pi R_{p}^{3}\right) b \quad\left[E_{q}(16.146)\right] \\
\therefore 4 \pi R_{p}^{2} \frac{d R_{p}}{d t}=-\frac{4}{3} \pi R_{p}^{3} b .
\end{gathered}
$$

or using the expression for $b$ :

$$
\begin{aligned}
& \frac{d R_{p}}{d t}=-\frac{1}{3} R_{p o} b_{0} \\
& \text { using } \quad y=\frac{R_{p}}{R_{p 0}} \\
& \frac{d y}{d t}=-\frac{1}{3} b_{0}
\end{aligned}
$$

Integrating: $\quad y=1-\frac{1}{3} b_{0} t$

Using this expression for $y$ in Eq. (16.166):

$$
\left.\dot{p}=\frac{d p}{d t}=-b P_{0} y^{3}=-b_{0} P_{0} y^{2} \text { [since } b=\frac{b_{0}}{y}\right]
$$

But $\frac{d}{d t}=\frac{d y}{d t} \frac{d}{d y}=-\frac{1}{3} b_{0} \frac{d}{d y}$

or $\frac{d P}{d y}=3 p_{0} y^{2}$

Integrating with $P=P_{0}$ at $y=1$, we get,

$$
P_{0}-P=P_{0}\left(1-y^{3}\right)
$$

or

$$
P_{0}-P=P_{0}\left[1-\left(1-\frac{1}{3} b_{0} t\right)^{3}\right]
$$


360

Problem 16.5

For parabolic temperature profile in fuel:

$$
T_{0}-T_{s}=P / 4 \pi \bar{k}
$$

Therefore thermal stress may be expressed in terms of linear power. In addition, cracking occurs when $\sigma_{\theta}=-\sigma_{f}$. Therefore the crack root $r_{c}$ is given by

$$
-\sigma_{f}=\frac{\alpha E Q}{16 \pi \bar{k}(1-\nu)}\left[1-3\left(\frac{r_{c}}{R}\right)^{2}\right]
$$

$\sigma_{f}=$ fracture strength (in tension)

Cracking first begins at the surface, or $\frac{r_{C}}{R}=1$

Eq. (2) becomes

$$
\frac{\alpha E(100)}{16 \pi \bar{k}(1-\nu)}[-2]=-\sigma_{f}
$$

At $500 \mathrm{w} / \mathrm{cm}$, eq. (2) is:

$$
\frac{\alpha E(500)}{16 \pi \bar{k}(1-\nu)}\left[1-3\left(\frac{r_{c}}{R}\right)^{2}\right]=-\sigma_{f}
$$

Equating left hand sides of (3) and (4) (neglect change in $\sigma_{f}$ with $T$ ):

$$
\begin{aligned}
-2(100) & =500\left[1-3\left(\frac{r_{C}}{R}\right)^{2}\right] \\
-0.4 & =1-3\left(\frac{\gamma_{C}}{R}\right)^{2}
\end{aligned}
$$


361

$$
\begin{aligned}
\therefore \quad \frac{x_{C}}{R} & =\sqrt{\frac{1}{3}(1+0.4)} \\
& =0.68
\end{aligned}
$$

$T_{c}=$ Temperature at the root of crack. For parabolic temperature distribution:

$$
\begin{aligned}
\frac{T_{c}-T_{s}}{T_{0}-T_{s}} & =1-\left(\frac{r_{C}}{R}\right)^{2} \\
\therefore T_{c} & =T_{s}+\left(T_{0}-T_{s}\right)\left[1-\left(\frac{r_{C}}{R}\right)^{2}\right] \\
& =T_{s}+\frac{\rho}{4 \pi \bar{k}}\left[1-\left(\frac{r_{C}}{R}\right)^{2}\right]
\end{aligned}
$$

where $\bar{k}=0.03$ watts $/ \mathrm{cm}-{ }^{\circ} \mathrm{C}$ = thermal conductivity

$$
\begin{aligned}
\therefore \quad T_{c} & =700+\frac{500}{4 \pi(0.03)}(0.53) \\
& =1403{ }^{\circ} \mathrm{C}
\end{aligned}
$$


362

CHAPTER 17

PROBLEM 17

a) $\left(Z_{1}\right)$ eff where

$$
\begin{aligned}
& \hbar=1.05 \times 10^{-27} \text { erg -sec, } e=4.8 \times 10^{-10} \mathrm{esu} \\
& \Rightarrow\left(Z_{1}\right)_{\text {eff }}=22.6
\end{aligned}
$$

b)

Considering the quantum mechanical correction factor of 2 . Bethe formula he comes,

$$
\frac{d E}{d x}=\frac{2 \pi N\left(Z_{1}\right)_{\text {eff }}^{2} Z_{2} e^{4}\left(M_{1} / m_{e}\right)}{E} \ln \left[\frac{4 E}{\left(M_{1} / m_{e}\right) \bar{I}}\right]
$$

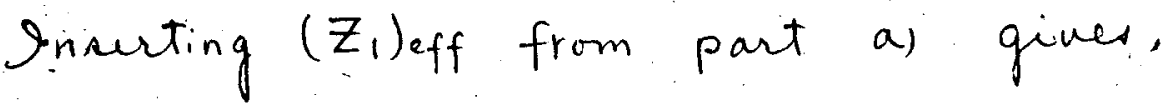

$$
\left(\frac{d E}{d x}\right)_{0}=4 \pi Z_{1}^{2 / 3} \frac{\hbar^{2}}{m_{e}} N_{0} Z Z_{0} \ln \left[\frac{\Delta m_{e} E}{M_{1} I_{b}}\right]
$$

Let $k=4 \pi Z_{1}^{2 / 3} \frac{\hbar^{2}}{m_{e}} N_{u} Z_{u}$

$$
\gamma=\frac{2 Z_{0}}{Z_{u}} \text { and } E_{j}^{*}=\frac{M_{1}}{4 m_{e}} \bar{I}_{0}
$$

then

$$
\left(\frac{d E}{d x}\right)_{\text {tot }}=2 K\left(Z_{0} / Z_{U}\right) \ln \left[\frac{4 m_{e} E}{M_{1} \bar{I}_{0}}\right]+K \ln \left[\frac{4 m_{e} E}{M_{1} \bar{I}_{0}}\right]
$$

or

$$
\left(\frac{d E}{d x}\right)_{\text {tot }}=K\left[\gamma \ln \left(\frac{E^{*}}{E_{0}^{*}}\right)+\ln \left(\frac{E^{\prime}}{E_{u}^{*}}\right)\right]=K \ln \left[\frac{E^{\gamma+1}}{\left(E_{0}^{*}\right)^{\gamma} E_{u}^{*}}\right]
$$

Let $A=\left[\left(E_{0}^{*}\right)^{\gamma}\left(E_{\nu}^{*}\right)\right]^{-1}$

So that $\left(\frac{d E}{d x}\right)_{\text {tot }}=K \ln \left[A E^{\gamma+1}\right]$

Where $A=\left(\frac{4 m_{e}}{M_{1}}\right)^{r+1}\left(\frac{1}{\bar{I}_{0}}\right)^{\gamma}\left(\frac{1}{\bar{I}_{u}}\right)=1.32 \times 10^{5}$ 
363

and $\begin{aligned} N_{u} & =\frac{10.98 \mathrm{~g} / \mathrm{cm}^{3}}{23.8 \mathrm{~g} / \mathrm{mole}^{3}} \times 6.02 \times 10^{23} \mathrm{atoms} / \mathrm{mole}=2.8 \times 10^{22} \mathrm{Lat \text {ond }} \\ Y & =\frac{2 \times 8}{9}=\frac{1 \mathrm{~cm}^{3}}{}\end{aligned}$ $\bar{I} \frac{92}{92}=0.174$

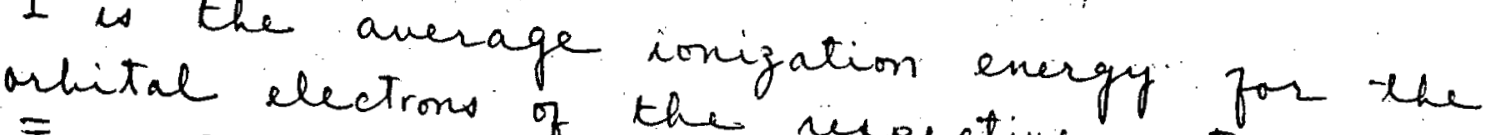
$\bar{I}=8.8 \mathrm{z}$
$\bar{I}=8 \mathrm{tal}$

$$
\begin{aligned}
& \bar{I}=8.8 \mathrm{Z} \\
& \Rightarrow \bar{I}_{0}=8.8 \times 8=70.4 \mathrm{gV} \quad I_{U}=8.8 \times 92 \cong 810 \mathrm{eV} \\
& K=4 \pi(42)^{2 / 3} \frac{\left(1.05 \times 10^{-27}\right)^{2}}{0.911 \times 10^{-27}} \times 2.8 \times 10^{22} \times 92 \\
& =0.428 \\
& \left(\frac{d E}{d x}\right)_{\text {tat }}=K \ln A+K(r+1) \ln E=2.19+0.503 \ln E
\end{aligned}
$$

i) Numerical evaluation

the total distance traveled heforeaRutherford callinion is $x_{c}=2 \times 10^{-4} \mathrm{~cm}$

Let's divide $x_{C}$ into four equal interval. of each $0.5^{\circ} \times 10^{-4} \mathrm{~cm}=\Delta x$

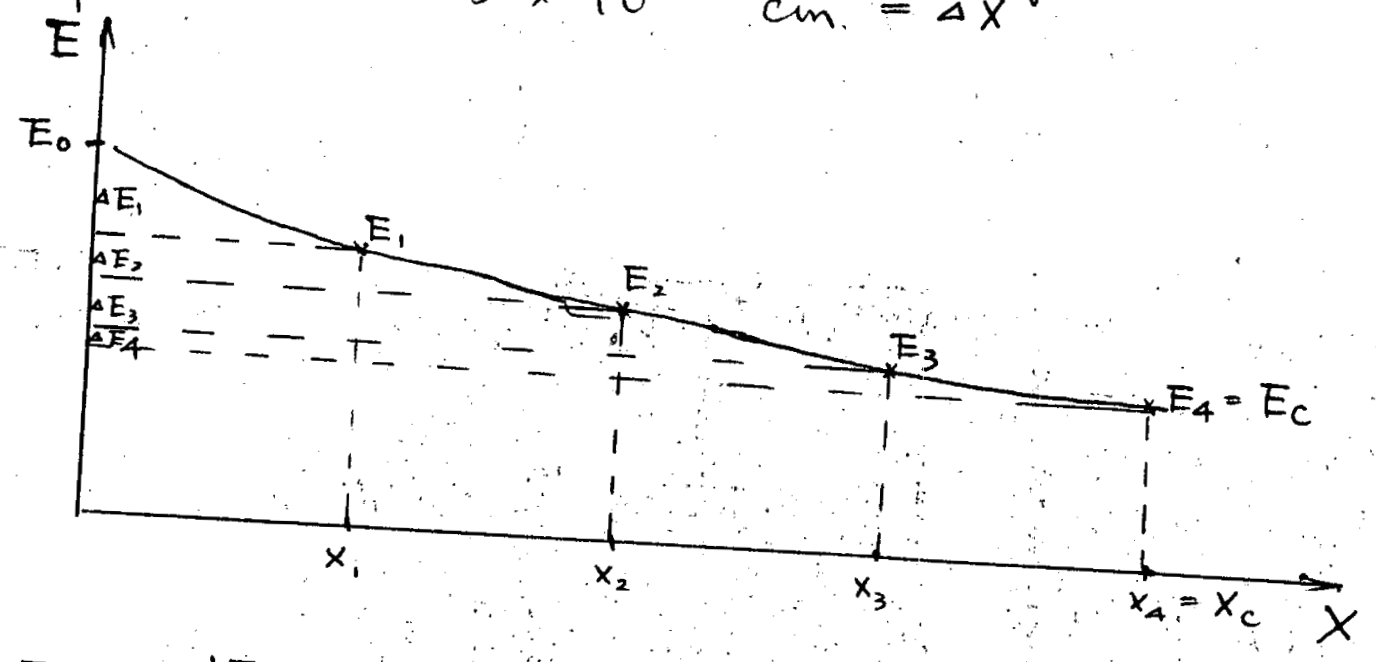

$$
\begin{aligned}
& \Delta E=\left(\frac{d E}{d x}\right)_{\text {tat }} \Delta x=1095 \times 10^{-4}+0260 \times 10^{-4} \ln E \\
& E_{0}=10^{8} \mathrm{ev}=1.6 \times 10^{-4} \mathrm{erg}
\end{aligned}
$$


364

$$
\begin{aligned}
& \Delta E_{1}=1.095 \times 10^{-4}+0.260 \times 10^{-4} \ln \left(1.6 \times 10^{-4}\right)=0.11 \times 10^{-4} \mathrm{lzg} \\
& \dot{E}_{1}=(1.60-0.11) \times 10^{-4} \mathrm{erg}=1.49 \times 10^{-4} \mathrm{ag} \\
& \Delta E_{2}=1.095 \times 10^{-4}+0.0 t \times 10^{-4} \ln \left(1.49 \times 10^{-\Delta}\right)=0.101 \times 10^{-4} \\
& E_{2}=(1.49-0.101) \times 10^{-4}=1.389 \times 10^{-4} \text { eng } \\
& \Delta E_{3}=0.095 \times 10^{-4} \mathrm{erg} \\
& F_{3}=1.94 \times 10^{-4} \mathrm{eqg} \\
& \Delta E_{4}=0 \therefore 085 \times 10^{-4} \mathrm{erg} \\
& E_{4}=E_{C}=1: 209 \times 10^{-4} \mathrm{erg}=75.6 \mathrm{MeV} \\
& \therefore E \subset \cong 75.6 . M E V
\end{aligned}
$$

ii) Analytic solution

$$
\left(\frac{d E}{d x}\right) \operatorname{tat}=K \ln \left[A E^{r+1}\right]
$$

Integrate $\Rightarrow \dot{k} x_{c}=\int_{E_{0}}^{E_{c}} \frac{d E^{\prime}}{\ln \left[A E^{\cdot r+1}\right]}$

Let $u=A E^{r+1}$ or $E=\frac{1}{A^{(r+1)^{-1}} u^{\left(\frac{1}{r+1}\right)}}$

then $d u=A(r+1) E^{\gamma} d E=(r+1) A^{\left(\frac{1}{r+1}\right)} u^{\left(\frac{r}{r+1}\right)} d E$

$$
\begin{aligned}
& u_{i}=A E_{v}^{r+1} \\
& s_{0} \\
& K x_{c}^{c}=\int \frac{a u}{(r+1) A^{\frac{1}{r+1}} u^{\frac{r}{r+1}} \ln u}
\end{aligned}
$$

Let $\alpha=\frac{\gamma}{r+1}$$$
K(r+1) A^{\left(\frac{1}{r+1}\right)} \quad X_{c}=\int_{u_{0}}^{u_{c}} \frac{d u}{u^{x} c_{u} u}=\operatorname{Li}\left(\frac{1}{u_{c}^{x-1}}\right)-\operatorname{Li}\left(\frac{1}{u_{e}^{x-1}}\right)
$$$$
K(r+1) A^{\left(\frac{1}{r}+1\right)} x_{c}=2=4=L_{i}\left(u_{i}^{0.32}\right) \cdots L_{i}\left(u_{0}^{0.352}\right)
$$$$
u_{0}=37.73 \quad u_{0}^{0 . \% 2}=20
$$

Thus $\operatorname{Li}\left(U_{c}^{8.85}\right)=2.24+L^{3}(2.2)$ lie and Ec can be haled for fum graph of Li function. 
365

c)

$$
\begin{aligned}
& T=\frac{1}{2} \Lambda E(1-\cos \theta), \Lambda=\frac{4 M_{1} M_{2}}{\left(M_{1}+M_{2}\right)^{2}} \\
& \tan \phi_{1}=\frac{\left(M_{2} / M_{1}\right) \mu \operatorname{cin} \theta}{1+\left(M_{2} / M_{1}\right) \cos \theta}
\end{aligned}
$$

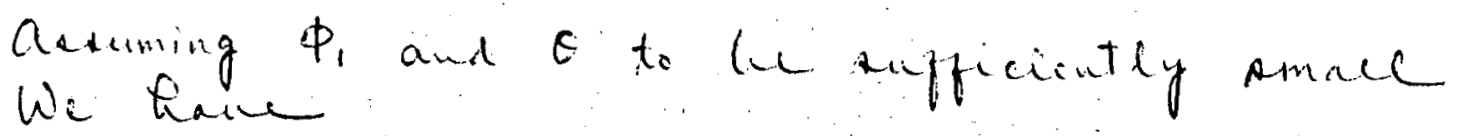

$$
\phi_{1}=\frac{\left(M_{2} / M_{1}\right)}{1+\left(M_{2} / M_{1}\right)} \theta \Rightarrow \theta=\left(1+\frac{M_{1}}{M_{2}}\right) \phi_{1}
$$

also $\cos \theta \cong 1-\frac{\theta^{2}}{2}$

$$
T \simeq \frac{M_{1}}{M_{2}} E \phi_{1}^{2}
$$

i)

$$
\begin{aligned}
T_{0} & \simeq E_{c}\left(\frac{M_{1}}{M_{0}}\right) \phi_{1}^{2}=\frac{100}{1 t}\left(\frac{5}{360} \times 2 \pi\right)^{2} \times 7.5 .6 \\
& =3.57 \mathrm{MeV}
\end{aligned}
$$

ii)

$$
\begin{aligned}
T_{u} & =E_{c}\left(\frac{H_{1}}{M_{u}}\right) \phi_{1}{ }^{2} \\
& =0.156 M_{e} \mathrm{~V}
\end{aligned}
$$


366.

PROBLEM 17.2

Froni $E_{q}:(T \cdot z T) \cdot \tau(E, T)=\pi z_{1}^{2} z_{2}^{2} e^{4}\left(\frac{M_{1}}{\mu_{2}}\right) \frac{1}{E T}=-.(1)$

$$
\begin{aligned}
& \text { From } E_{q}(17.22) \quad T(E, \theta)=\frac{1}{2 \pi} \sigma(E, T)\left|\frac{d T}{d \cos \theta}\right|-(2) \\
& \text { From } E_{q}(17.9) \quad T=\frac{1}{2} \cap E(1-\cos \theta)
\end{aligned}
$$

$$
\left|\frac{d T}{d \cos \theta}\right|=\frac{1}{2} \wedge E=\frac{1}{2} \frac{4 M_{1} M_{2}}{\left(M_{1}+M_{2}\right)^{2}} E \ldots-(4)
$$

Siluticute (1) and (4) inte (>)

$$
\begin{aligned}
& \sigma(E \cdot C)=z_{1}^{2} z_{2}^{2} \in^{4}\left(\frac{H_{1}}{M_{1}+M_{2}}\right)^{2}-\frac{1}{T^{2}} \ldots(15) \\
& \text { Firm (3) } T=\frac{>M_{1} M_{2}}{\left(M_{1}+M_{2}\right)^{2}} \times 2 M^{2} \frac{\theta}{2} \ldots-(16)
\end{aligned}
$$

Silutitite (6) into $(5)$

$$
\begin{aligned}
& \text { Sulutitite } \\
& \sigma(E, \theta)
\end{aligned}=\frac{z_{1} z_{2} e^{4}}{16}\left(\frac{M_{1}+M_{2}}{M_{0}}\right)^{2} \frac{1}{E^{2} M^{4}\left(\frac{\theta}{2}\right)}
$$


367

PROBLEM 17.3

Screed coulomb potential $V_{s c}(r)=\frac{z_{1} z_{2} e^{2}}{r} e^{-r / a}$ Inverse power potential $V_{p}(r)=A / r^{3}$

Ct the distance of claret approach

$$
\begin{aligned}
V_{p}\left(r_{m}\right)= & \frac{A}{r_{m}{ }^{3}}=\frac{E^{*}}{2} \Longrightarrow r_{m}=\left(\frac{2 A}{E^{*}}\right)^{1 / s} \\
\text { and } V_{s c}\left(r_{m}\right) & =\frac{z_{1} z_{2} e^{2}}{r_{m}} e^{-r_{m} / a} \\
& =\frac{z_{1} z_{2} e^{2}}{\left({ }^{2 A} / E\right)^{1 / s}} \exp \left[-\left(\frac{2 A}{E^{*}}\right)^{1 / s} / a\right]=\frac{E^{*}}{2}
\end{aligned}
$$

So,

$$
\begin{aligned}
& \exp \left[-\left(\frac{2 A}{E^{*}}\right)^{1 / 3} / a\right]=\frac{E^{*}}{2 z_{1} z_{2} e^{2}}\left(\frac{2 A}{E^{*}}\right)^{1 / 3} \\
& \text { or }-\left(\frac{2 A}{E^{*}}\right)^{1 / 3}=a \ln \left[\left(\frac{2 A^{*}}{E^{*}}\right)^{1 / 3} \frac{E^{*}}{2 z_{1 \cdot z_{2} e^{2}}}\right]
\end{aligned}
$$
The intersection of the plats of hath rides

$$
a=\frac{\sqrt{2} \lambda a_{B}}{\left(z_{1}^{2 / 3}+z_{2}^{2 / 3}\right)^{1 / 2}}
$$

where $a_{B}$ is the Bohr radius 
368

PROBLEM 12.4

a) Each atom has 8 nearest neighbors in the b.c.C. structure

$\Delta E_{\text {pah }}\left(\right.$ at $\left.O^{\circ} \mathrm{K}\right)=\frac{1}{2} \times 8 \phi_{B}=4 \phi_{B}$

$\phi_{B}=\frac{8.1}{4}=2.02 \mathrm{eV}$

b) It was shown in Chapter 4 , that the compresichility is related to the second derivative of the crete energy by.

$$
\frac{1}{\beta}=\dot{V}_{\text {eq }}\left(\frac{d^{2} U}{d V^{2}}\right)_{e q}
$$

where veg is the specific volume $-\frac{a_{0}^{3}}{2}$ for $b c c$ $a_{0}$ is the lattice constant

Let $r_{e q}$ be the distance between nearest neighbor $=\frac{\sqrt{3}}{2} a_{0}$. For $b c c$ stricture.

$$
\begin{aligned}
& \Rightarrow \quad a_{0}=\frac{2}{\sqrt{3}} r_{e q} \\
& \therefore V_{e q}=\frac{a_{0}^{3}}{>}=\frac{4}{3 \sqrt{3}} r_{e q}^{3}-0--(2) \\
& \frac{d U}{d v}=\frac{d U}{d r} \frac{d r}{d V} \\
& \frac{d^{2} U}{d v}=\frac{d}{d V}\left(\frac{d U}{d V}\right)=\frac{d^{2} r}{d V^{2}} \cdot \frac{d U}{d r}+\frac{d^{2} U}{d r^{2}}\left(\frac{d r}{d V}\right)^{2}-(3)
\end{aligned}
$$

The first term on the right hand side of

(3) is zero, $\left.|\sin c| \frac{d u}{d r}\right|_{r=r_{\text {eg }}}=0 \ldots-(4)$

Sulititute (3), (4), (2) into (1)

$$
\frac{1}{\beta}=\frac{1}{4 \sqrt{3} r_{e q}}\left(\frac{d^{2} U}{d r}\right)_{e q}-\cdots--(5)
$$


369

$$
\begin{aligned}
& U(r)=\frac{1}{2} \times 8 \times V(r)=4 V(r) \\
& \therefore \frac{1}{\beta}=\frac{1}{4 \sqrt{3} r_{\text {q }}} \times 4 \frac{d^{2} v}{d r^{2}}=\frac{k}{\sqrt{3} r_{\text {eq }}} \\
& =\frac{k}{\sqrt{3} \cdot \frac{\sqrt{3}}{2} a_{0}}=\frac{2 k}{3 a_{0}}=\frac{k a_{0}^{2}}{\frac{3}{2} a_{0}^{3}} \\
& =\frac{k a_{0}^{2}}{3 \cdot V_{e q}} \\
& \therefore k \cdot a_{0}^{2}=\frac{3 V_{2}}{\beta} \\
& =3 \times \frac{1}{16.6 \mathrm{~g} \cdot \mathrm{cm}^{-3}} \times \frac{181 \mathrm{~g} / \mathrm{mole}}{6.02 \times 10^{23} \mathrm{atom} / \mathrm{male}} \\
& x \frac{1}{0.52 \times 10^{-12} \mathrm{an}^{2} \text { dyne }} \\
& \cong 65 \mathrm{eV}
\end{aligned}
$$

c) The Miller indices of direction of displacement
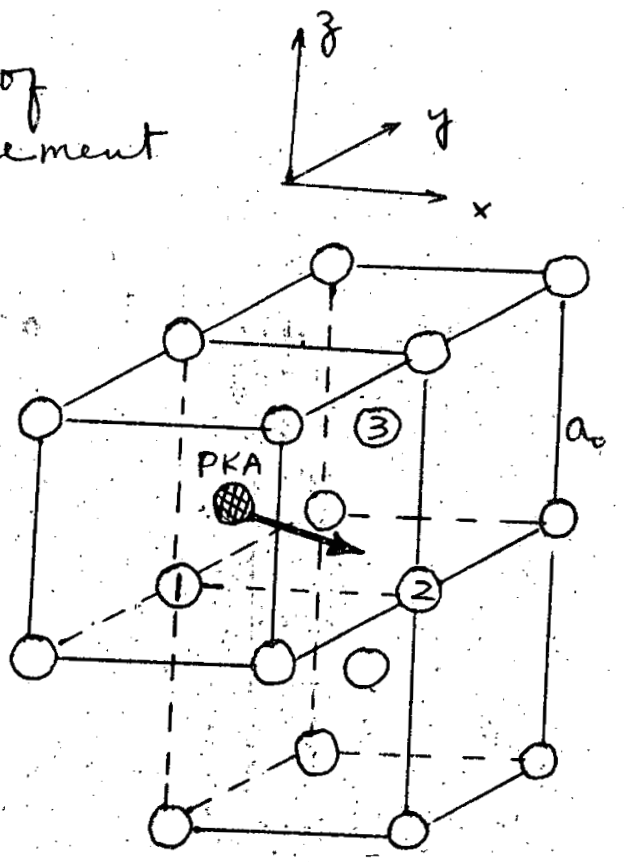
370

d) The cressy is maximum whin the PKA passes through the triangle formed by atoms 1,2 and 3 . (Ar ec the drawing in part $c$ ) $V(r)=-D+\frac{1}{2} k\left(r_{\text {eq }}-r\right)^{2}$

The planar configuration for the raddle point is:

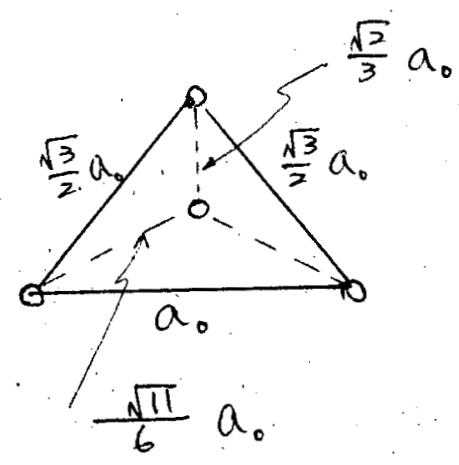

$\varepsilon^{*}$ - potential energy at the saddle point

$$
\begin{aligned}
& =2\left[-D+\frac{1}{2} k\left(r_{\text {eq }}-\frac{\sqrt{11}}{6} a_{0}\right)^{2}\right]+\left[-D+\frac{1}{2} k\left(r_{\text {eq }}-\frac{\sqrt{2}}{3} a_{0}\right)^{2}\right] \\
& =-3 D+0.178 k a_{0}^{2} \\
& =-3 \Phi_{B}+0.178 k a_{0}^{2} .
\end{aligned}
$$

Eq. potential unify in the equilibimin $=-8 \phi_{B}$

$\therefore \varepsilon_{d}=$ displacement energy

$$
\begin{aligned}
& =\varepsilon^{*}-\varepsilon=5 \phi_{B}+0.178 \mathrm{ka}^{2} \\
& =21.5 \mathrm{eV}
\end{aligned}
$$


371

e)

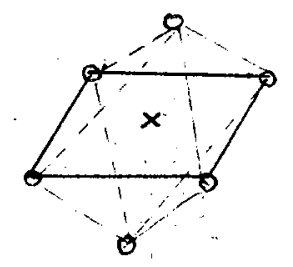

$$
\begin{aligned}
& \varepsilon_{\text {act }}= 4\left[-\phi_{B}+\frac{1}{2} k\left(\frac{\sqrt{3}}{2}-\frac{\sqrt{2}^{2}}{2}\right)^{2} a_{0}^{2}\right] \\
&+2\left[-\phi_{B}+\frac{1}{2} k\left(\frac{1}{2}-\frac{\sqrt{3}}{2}\right) a_{0}^{2}\right] \\
&=-6 \phi_{B}+0.150 k a_{0}^{2} \\
& \mathcal{E}_{\text {oct }}-\mathcal{E}_{\text {eq }}=2 \phi_{B}+0.150 \mathrm{k} a_{0}^{2}=13.69 \mathrm{eV} .
\end{aligned}
$$

f)

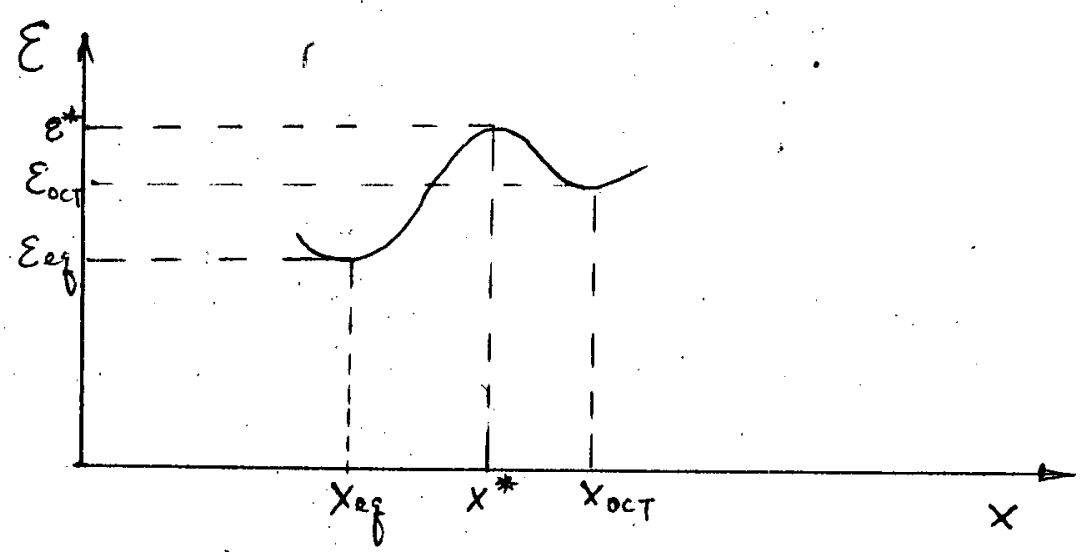

distance along $\langle 0,2, T\rangle$ 
372

PROBLEM 17.5

a) Since $A_{1}, A_{2}$ and $a$ atom lie on a $(111)$ plane, they form an equilateral triangle with the rides equal to $D=\frac{a_{0}}{\sqrt{2}}$

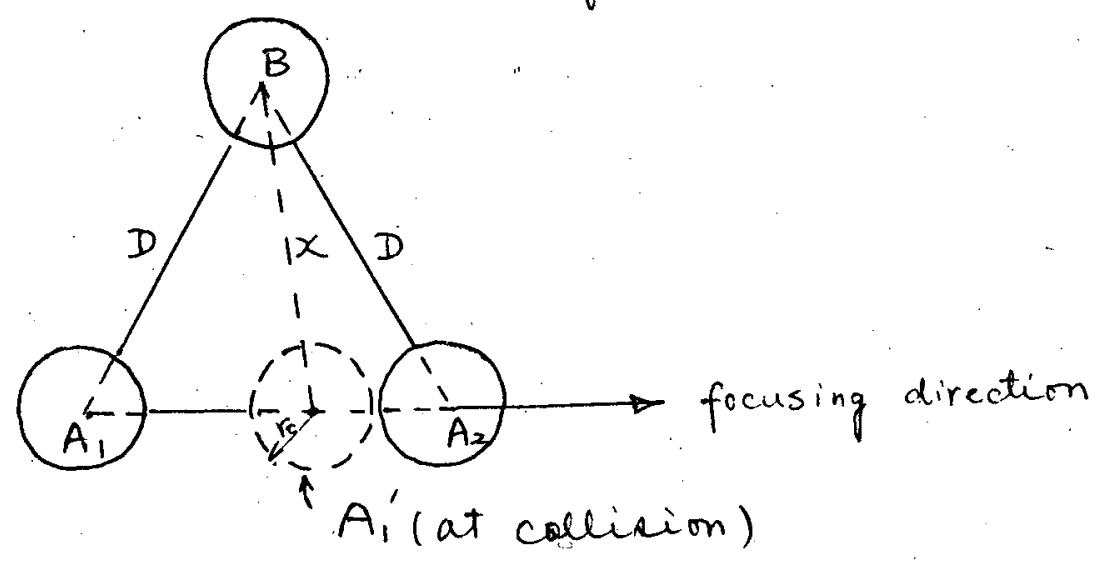

$x=A_{1}-B$ distance at collision. Apply the Law of confines to triangle $\left(A_{1}^{\prime} A_{2} B\right)$ :

$$
\begin{aligned}
\dot{x}^{2} & =D^{2}+\left(2 r_{0}\right)^{2}-2\left(2 r_{0}\right) D \cos 60^{\circ}=D^{2}+4 r^{2}-2 r_{0} D \\
& =D^{2}\left[1+4\left(\frac{r_{0}}{D}\right)^{2}-2\left(\frac{r_{0}}{D}\right)\right]=D^{2}\left[\frac{3}{4}+\frac{1}{4}\left(4 \frac{r_{0}}{D}-1\right)^{2}\right]-(1)
\end{aligned}
$$

But, from equation $(17.41)$. $r_{0}$ ss defined by,

$$
r_{0}=\frac{1}{2} \rho \ln (2 A / E) \ldots \ldots
$$

where and $A$ are the constants of the Born - Mayer potential function

$D$ may he expressed in terms of Ex by eq. (17.95)

$$
D=>\rho \ln \left(\frac{\geq A}{E_{f}}\right)
$$

the (2) and (3) to eliminate the ratio $\frac{r_{0}}{D}$ in (1)

$$
\Rightarrow \frac{r_{0}}{D}=\frac{1}{4} \frac{\ln \left(\frac{2 A}{E}\right)}{\ln \left(\frac{2 A}{E_{f}}\right)}=\frac{1}{4} \frac{\ln \left(2 A_{E_{f}}\right)+\ln \left(E_{1 / E}\right)}{\ln \left(2 A / E_{f}\right)}
$$


373

$$
=\frac{1}{4}\left[1+\frac{\ln \left(E_{f} / E\right)}{(D / 2 \rho)}\right]
$$

$O R$ (1) hecomes

$$
x=D\left\{\frac{3}{4}+\left[\left(\frac{\rho}{D}\right) \ln \left(\frac{E_{f}}{E}\right)\right]^{2}\right\}^{1 / 2} \ldots
$$

b)

$$
\begin{aligned}
\Delta E & =A_{1}-4 B \text { interaition energy change. } \\
& =4[V(x)-V(D)]
\end{aligned}
$$

where $V(D)$ is the $A_{1}-B$ interaition energy at equilibrimm

ling Barn - Mayir Potential

$$
\Delta E=4 A[\exp (-x / \rho)-\exp (-D / \rho)]
$$

$\Delta E$ is a function of energy, Rince $x$ is

c) at the $n^{\text {th }}$ callixion, the energy locs is $\triangle E(E)$. If $\Delta E$ is smale companed to $E$. khe encigy loss process may be considued contimuous, and:

$$
\frac{d E}{d n}=-\Delta E(E)-\ldots-\ldots-\ldots \text { - (6) }
$$

When $E$ dicrianes to $\frac{1}{4} E_{f}$, the replacement procen ceaces. Call the number of collicion at this pint $n_{f}$. From (6)

$$
\int_{E_{1}}^{\frac{1}{4} E_{f}} \frac{d E}{\Delta E(E)}=-\int_{1}^{n_{f}} d n=-\left(n_{f}-1\right)
$$

or $n_{f}=1+\int_{\frac{1}{4} E_{f}}^{E_{1}} \frac{d E}{\Delta E(E)}$

fet $y=\frac{E}{E_{f}}$. 
374

$$
n_{f}=1+E_{f} \int_{1 / 4}^{E_{1} / E_{f}} \frac{d y}{\Delta E(y)^{x}} \cdots \cdots(7)
$$

Using (4) and (5) in 171

$$
n_{f}=1+\left(\frac{E_{f}}{4 A}\right) \int_{1 / 4}^{E_{1} / E_{f}} \frac{d y}{\exp \left\{-\left(\frac{D}{g}\right)\left[\frac{3}{4}+\left(\frac{p}{D} \ln \frac{1}{y}\right)^{2}\right]^{1 / 2}\right\}-\exp \left(-\frac{D}{\rho}\right)}
$$

Note = Denomination of ( 8$) \rightarrow 0$ as the term in the squame bracket $\rightarrow 1$, this determines a minimum value of $y\left(y_{\min }\right)$ which mut he $<1 / 4$ for the integral to be valid. $y_{\text {min }}$ may. le found from.

$$
\frac{\rho}{D} \ln \left(\frac{1}{y_{\text {min }}}\right)=\frac{1}{2} \text {. }
$$

or $\ln \left(\frac{1}{y_{\min }}\right)=\frac{D}{\partial \rho}=\ln \left(\frac{2 A}{E_{f}}\right) \quad($ using (3)) or $y_{\min }=\frac{E_{f}}{2 A}-\ldots-\ldots$ (9)

From Fig $17.5 \quad A \simeq 2 \times 10^{4} \mathrm{ev}$ and $E_{f} \simeq 10^{2} \mathrm{eV}$ $y_{\min }=2.5 \times 10^{-3}$, which is $<1 / 4$. 
375

PROBLEM 17.6

Lind hand stopping formula =

$$
\begin{array}{ll}
\left(\frac{d E}{d x}\right)_{e}=k \sqrt{E} & E_{q}(17.52) \\
k=0.3 \mathrm{NZ} z^{2 / 3}, e v^{1 / 2} / \AA & E q(17.53 a)
\end{array}
$$

Let $x$ he the distance before decharaching

$$
\begin{aligned}
& \int_{E_{0}}^{E_{c h}} \frac{d E^{\prime}}{\sqrt{E^{\prime}}}=k x=2\left[\sqrt{E^{\prime}}\right]_{E_{c h}}^{E_{0}}=2\left(\sqrt{E_{0}}-\sqrt{\left.E_{c h}\right)}\right. \\
& x=2 \frac{1-\sqrt{E_{c h / E_{0}}}}{k / \sqrt{E_{0}}} \\
& E_{c h}=300 \mathrm{eV}, Z=29 \cdot N=0.085 \AA^{-3} \\
& E_{0}=30 \mathrm{keV} \\
& \sqrt{\frac{E_{c h}}{E_{0}}}=0.1 \\
& \therefore k=0.24 \\
& x=\frac{2(1-0.1)}{0.24 / \sqrt{3 \times 10^{4}}}=1.3 \times 10^{3} \AA
\end{aligned}
$$


376

PROBLEM 17.7

a) Accime nentron as at reet initially. Momentim conservation requires that ofter the reation $\because \frac{E_{r}}{C}=P_{\gamma}=P_{57}$

$$
\begin{aligned}
& \Rightarrow E_{57}=\frac{\left(P_{57}\right)^{2}}{2 M}=\frac{E_{r}^{2}}{2 M c^{2}} \\
& \therefore E_{57}=\frac{7^{2}}{2 \times 56.9354 \times 9.3148 \times 10^{2}}=4.62 \mathrm{eV}
\end{aligned}
$$

b) $P_{K P}$ - number of displacement calculated hy Kinchin - Prace formula

$$
D_{k p}=\frac{E}{2 E_{d}}-\bmod (1) \frac{E}{2 E_{d}}=9.42-0.42=9 \text {. }
$$

D.. numbir of dipplacement calculated ly Findhords

$$
=\zeta(E) \gamma_{K p}=\frac{\gamma_{K p}}{1+0.13\left(3.4 \varepsilon^{1 / 6}+0.4 \varepsilon^{3 / 4}+\varepsilon\right)}
$$

when $\varepsilon=\frac{E}{\left(\frac{z e^{2}}{a}\right)}, a=\frac{0.88 a_{B}}{z^{1 / 3}}$

$$
\begin{gathered}
a_{B}=\frac{h^{20}}{\mu e^{2}}=0.53 \times 10^{-8} \mathrm{~cm}, z=26 \\
\therefore a=\frac{0.88 \times 0.53^{2} \times 10^{-8}}{26 / 3}=1.97 \times 10^{-9} \mathrm{~cm} \\
\varepsilon=4.62 \times 1.6 \times 10^{-12} \times \frac{1.97 \times 10^{-9}}{2 \times 26 \times\left(4.8 \times 10^{-10}\right)^{2}}=0.122 \\
\Rightarrow D_{L}=\frac{9.42}{1+0.13\left[3.4 \times(0.122)^{1 / 6}+0.4(0.122)^{3 / 4}+0.122\right]}=7
\end{gathered}
$$


377

$$
\left(R_{d}\right)_{\gamma}=\frac{99.6}{100} N_{56} \phi_{\text {th }} \bar{\sigma}_{56} \gamma_{k p}
$$

where $\bar{\sigma}_{56}$ is the abruption choir section for $F_{0}-56$ in thermal flux

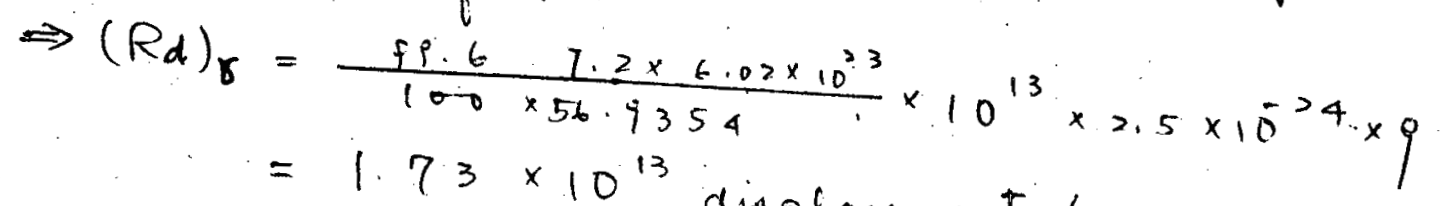

$$
\begin{aligned}
& =1.73 \times 10^{13} \text { displacements } / \text { sec. } \\
& \begin{array}{l}
\phi_{f}\left(E_{n}\right)=10^{5} \delta\left(E_{n}-0.5\right) \\
\left(R_{d}\right)_{f}=N_{F e} \int_{E_{d} / \Lambda}^{\infty} \sigma_{d}\left(E_{n}\right) \phi_{f}\left(E_{n}\right) \propto E_{n}
\end{array} \\
& =N_{F e} \sigma_{d}(0.5 \mathrm{MeV}) \times 10^{15}
\end{aligned}
$$

For elastic and inotropic Accttung

$$
\begin{aligned}
& \sigma\left(E_{n}, E\right)=\frac{\sigma_{e l}}{\Lambda E_{n}}, \Lambda=\frac{4 M_{n} \bar{M}_{F e}}{\left(M_{F e}+1\right)^{2}} \approx 0.069 \\
& \text { and } \sigma_{d}\left(E_{n}\right)=\int_{E_{d}}^{\Lambda E_{n}} \frac{\sigma_{e l}}{\Lambda E_{n}} \cdot \frac{E}{2 E_{d}} d E=\frac{\Lambda E_{n}}{4 E_{s}} \sigma_{e l}
\end{aligned}
$$

From $B N L-325$, the cross section for iron at $0.5 \mathrm{MeV}$ is 3 baud

$$
\begin{aligned}
\text { Re } & \sigma_{d}(0.5 \mathrm{MeV})=\frac{0.069 \times 0.5 \times 10^{6} \times 3 \times 10^{-24}}{4 \times 25}=1.15 \times 10^{-21} \mathrm{~cm}^{2} \\
& =1150 \text { barns }
\end{aligned}
$$$$
\left(R_{d}\right)_{f}=10^{15} \mathrm{~N}_{\mathrm{Fe}} \cdot \sigma_{d}(0.5 \mathrm{MeV})
$$$$
=10^{15} \times\left(\frac{7.2}{5.7} \times 6.02 \times 10^{23}\right) \times 1: 15 \times 10^{-21}
$$$$
=8 \times 10^{16.7} \text { displacements } / \mathrm{sec}
$$ 
378

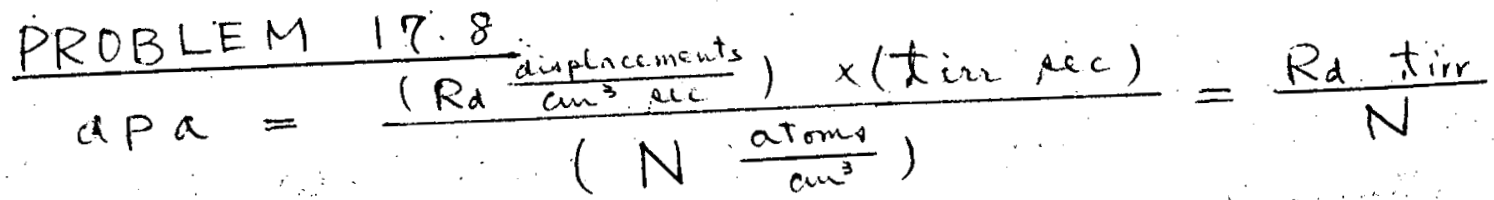

$$
\begin{aligned}
& \text { From eff }(17.115) \quad R_{d}=N \int_{E_{d} / \Lambda}^{\infty} \sigma_{d}\left(E_{n}\right) \phi\left(E_{n}\right) d E_{n} \\
& d p_{a}=\operatorname{tirr} \int \sigma_{d}\left(E_{n}\right) \phi\left(E_{n}\right) d E_{n}=\Phi \operatorname{tirr} \int \sigma_{\lambda}\left(E_{n}\right) \cdot \delta\left(E_{n}-0.5\right) d E_{n} \\
& \simeq[\Phi \operatorname{tirr}] \sigma_{d}(0.5 \mathrm{MeV})
\end{aligned}
$$

From problem $17.7 \quad \sigma_{d}(0.5 \mathrm{MeV})=1150$ bant

$$
\Rightarrow d P_{a}=10^{22} \times 1.15 \times 10^{-21} \approx 12
$$


$3>9$

PROBLEM 1?:9

For isotropic (in the C.M. ryotemin), Llactic Reattering, the: energy tropfer croct rection is

$$
\begin{aligned}
& \sigma\left(E_{n}, E\right)=\frac{\sigma\left(E_{n}\right)}{\Lambda E_{n}} \ldots \ldots \text { (1) } \\
& \text { where } \quad \Lambda=\frac{4 A}{(A+1)^{2}}-\ldots \text { (2) }
\end{aligned}
$$
energy is

$$
\bar{E}\left(E_{n}\right)=\frac{\int_{0}^{\Lambda E_{n}} E \sigma\left(E_{n}, E\right) d E}{\int_{0}^{\Lambda E_{n}} \sigma\left(E_{n}, E\right) d E} \cdots(3)
$$

Uning (1) in (3)

$$
\bar{E}\left(E_{n}\right)=\frac{1}{2} \wedge E_{n}-\cdots-\cdots \text { (4) }
$$

In a nentron jeux rpectrum $P\left(E_{n}\right)$, the auerage PKA energy is oltained ly weighting $\bar{E}\left(E_{n}\right)$ given by (4), with the Acabting rote $\sigma\left(E_{n}\right) \phi_{\left(E_{n}\right)}$

$$
\overline{\bar{E}}=\frac{\int_{0}^{\infty} \sigma\left(E_{n}\right) \phi\left(E_{n}\right) \bar{E}\left(E_{n}\right) d E_{n}}{\int_{0}^{\infty} \sigma\left(E_{n}\right) \phi\left(E_{n}\right) d E_{n}}
$$$$
----(5)
$$

Wing Eq (1), (4) and the fincion nutron spectrum $\phi\left(E_{n}\right)$. in eq. (5)

$$
\overline{\bar{E}}=\frac{1}{2} n \frac{\int_{0}^{\infty} e^{-E_{n}} \mu h \sqrt{2 E_{n}} d E_{n}}{\int_{0}^{\infty} \frac{1}{E_{n}} e^{-E_{n}} \operatorname{rinh} \sqrt{2 E_{n}} d E_{n}}
$$


380 Evaluating che aboue integrali numerically
$\bar{E}$ for iron $(n=0.065)$ is found to be

$$
\overline{\bar{E}}=0.33 \mathrm{MeV} \ldots \ldots(7)
$$

The avernge nutron energy in the firsion rpection is

$$
\begin{aligned}
\bar{E}_{n} & =\frac{\int_{0}^{\infty} \phi\left(E_{n}\right) E_{n} d E_{n}}{\int_{0}^{\infty} \phi\left(E_{n}\right) d E_{n}}=\frac{\int_{0}^{\infty} E_{n} e^{-E_{n}} \sinh \sqrt{2 E_{n}} d E_{n}}{\int_{0}^{\infty} e^{-E_{n}} \sinh \sqrt{2 E_{n}} d E_{n}} \\
& =\text { 2.0.MeV }-\ldots-\ldots \text { (8) }
\end{aligned}
$$

The anenge PKA energy of the aneige nentron is oltained by neing (8) in (A).

$$
\overline{\bar{E}}=\frac{1}{2} \cap \bar{E}_{n}=0.065 \mathrm{MeV}-\ldots-191
$$

Note: She carrect remelt is $E_{q}(7)$, NOT Eq (9). 
381

PROBLEM 17.10

ATom displacements per neutron

$=\frac{\text { displacement } / \mathrm{an}^{3}-\mu e c}{\text { neutron collision / } \mathrm{an}^{2}-\mu \mathrm{cc}}$

From Eq $(17.115)$

$$
R_{d}=\frac{\text { displacements }}{\operatorname{con}^{3} \mu c}=N \int_{E d / \Lambda}^{\infty} \sigma_{d}\left(E_{n}\right) \phi\left(E_{n}\right) d E_{n}
$$

For monoenergetic neutron flux

$$
R_{a}=N \sigma_{d}\left(E_{n}\right) \Phi, \Phi \text { is the total neutron flux }
$$

Neutron collision rate $=N r_{\text {scat }}\left(E_{n}\right) \Phi$

where Frat includes both elastic and inelastic scattering croce section

$$
\therefore \frac{\text { displacements }}{\text { neutron cilliuon }}=\frac{\sigma_{d}\left(E_{n}\right)}{\sigma_{\text {seat }}\left(E_{n}\right)}=\frac{3200}{2 \cdot 3} \simeq 1400
$$

$$
\begin{aligned}
& \text { o. } 5 \\
& 14 .
\end{aligned}
$$




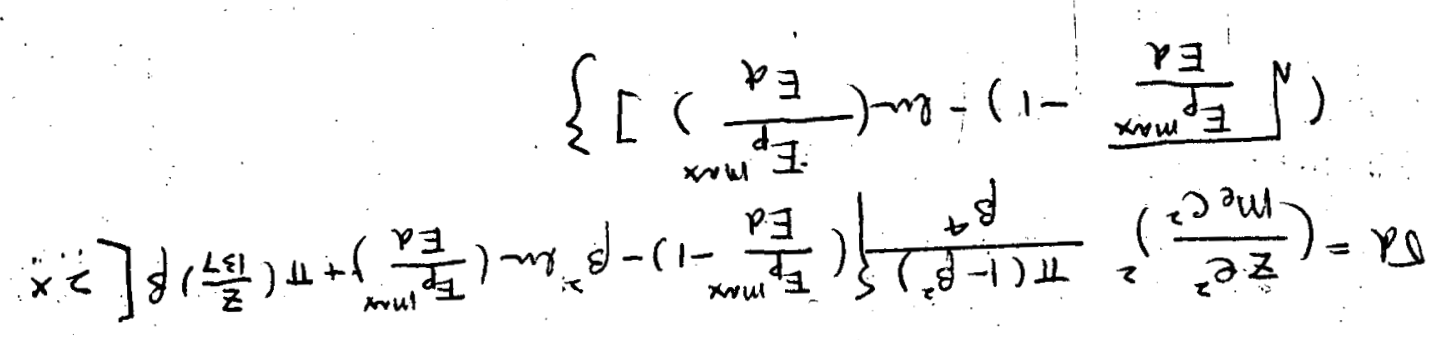

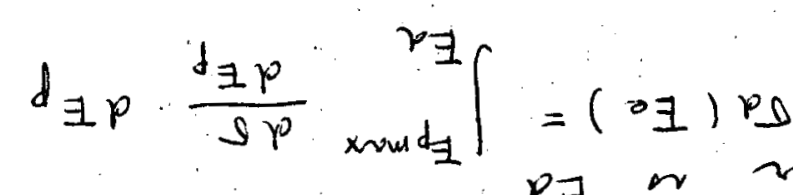

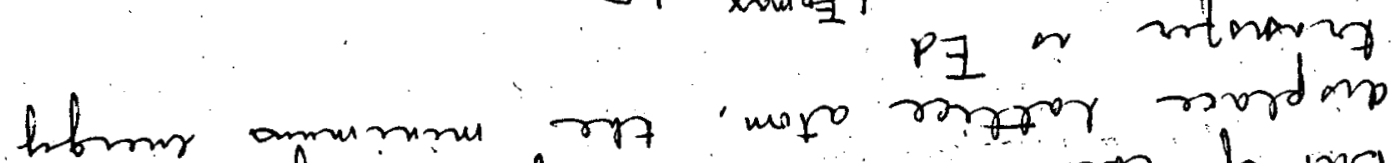

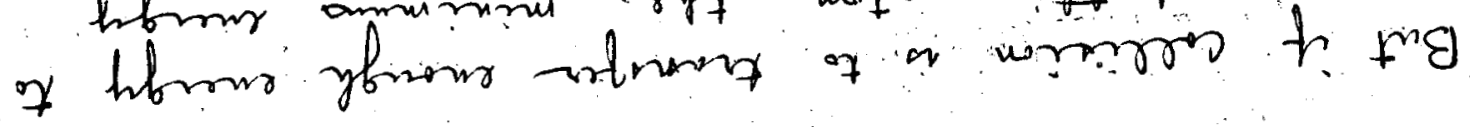

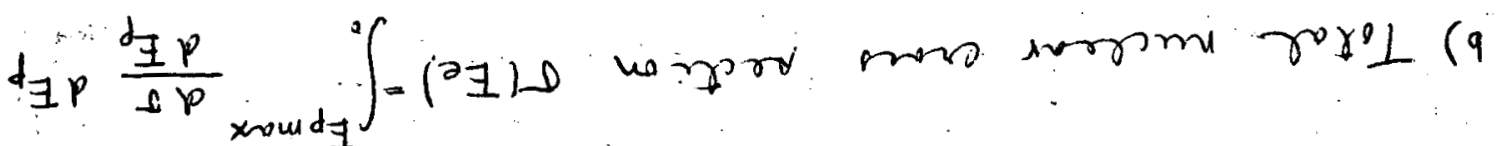
(z) $\ldots-\left(g_{-} 01 \times \gamma \exists \frac{\partial \mu b}{W}\right)=\left(\ln ^{2} \exists+1\right)_{\text {nim }}^{2} \exists$

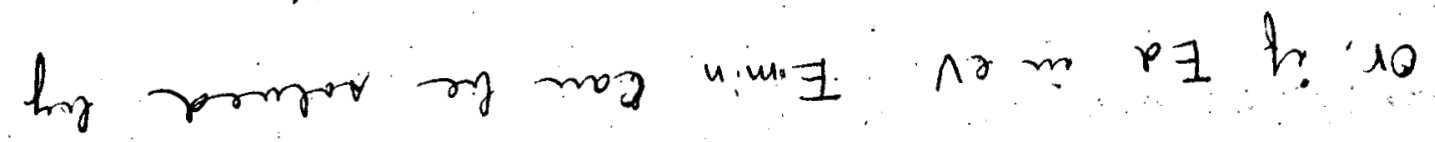

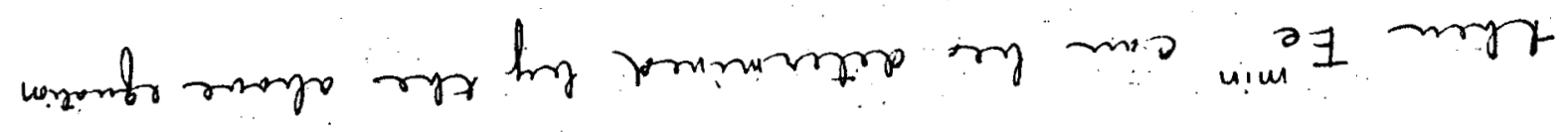

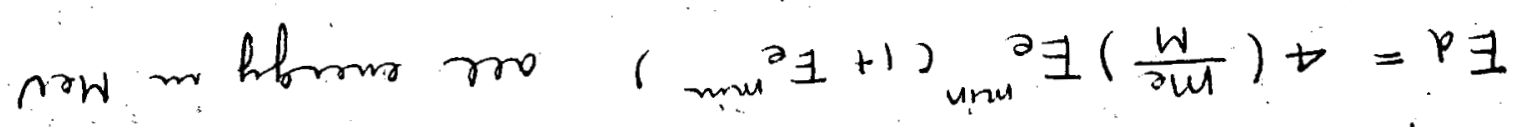

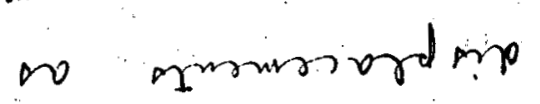

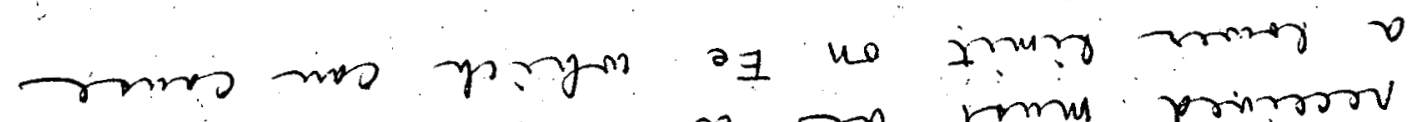

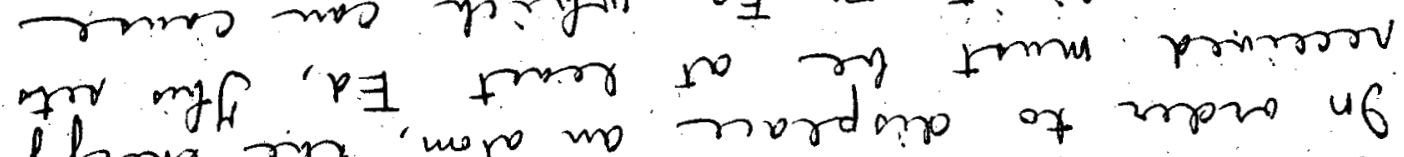
librow rist "me no nordino of mper 46

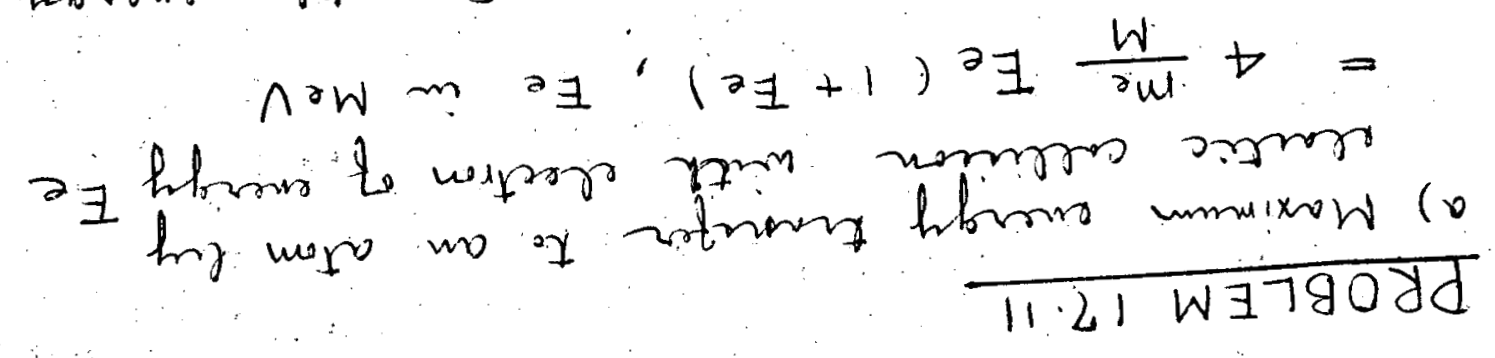


383

Number of primary knock on d.

$P_{d}(x) d x=$ probability of displacement collision in $(x, d x)$

$x$ : path length

$P_{d}(x) d x=\sigma_{d} N d x$

$$
d x=\frac{d E_{e}}{\left(\frac{d E_{e}}{d x}\right)_{e}}
$$

$P_{d}\left(E_{e}\right) d E_{e}$ probability of displacement collision during plowing down from $E_{e}$ to $E_{e}-d E_{e}$

$$
P_{d}\left(E_{e}\right) d E_{e}=\frac{N \sigma_{a}}{\left(d E_{e} / d x\right)_{e}} d E_{e}
$$

also $P a\left(E_{e}\right)=$ average number of displacement callioin per unit every lows

total number of displacement collision

$$
=\int_{E_{e}^{\min }}^{E_{e}} P_{\alpha}\left(E_{e}^{\prime}\right) d E_{e}^{\prime}
$$

$\frac{\text { total number of PKA }}{\text { incident electron }}=N \int_{E_{e}^{\text {min }}}^{E_{e}} \frac{\sigma d\left(E_{e}\right)}{\left(-d E_{e} / d x\right)} d E_{e}^{\prime}$

Total number of displace st atoms.

But, the formula gives the total nimble of atoms displaced by the electron under comiduation, NOT the total number if displaced atoms - ie. it gives the number of PKA's 
384

Each PKA of energy EP gives $D\left(E_{P}\right)$ displaced atoms. Redo calculations for probabilities of PKA in ( $E_{p}$. $d E_{p}$ ) yields.

$P_{d}\left(E_{e}, E_{p}\right) d E_{p}$ : aneinge number of displacement collision per cunt energy lows with the verge of $P K A$ (E $E_{p} d E_{p}$ )

$$
P_{d}\left(E_{e}, E_{p}\right)=\frac{N\left(\frac{d \sigma}{d E_{p}}\right)}{\left(d E_{e} / d x\right)}
$$

average number of displaced atoms per unit energy lows produced by $P_{K} A$ in $\left(E_{p}\right.$ \& $\left.E_{p}\right)=P_{a}\left(E_{e}, E_{p}\right) P\left(E_{p}\right)$ d $E_{p}$

$$
\begin{aligned}
& \bar{n}\left(E_{e}\right)=\frac{\text { total number of displaced atoms }}{\text { incident electron }} \\
& =N \int_{E_{e}^{\prime m i n}}^{E_{e}} \frac{1}{-\left(d E_{e} / d x\right)_{E_{e}^{\prime}}}\left[\int_{E_{a}}^{E_{p}^{\max }\left(E_{e}^{\prime}\right)}\left(\frac{d \sigma}{d E_{p}}\right) \nu\left(E_{p}\right) d E_{p}\right] d E_{e}^{\prime} \\
& e^{-}-\bar{e} \text { collision }
\end{aligned}
$$

c) Special cure

$\left(\frac{E_{p}^{\max }}{E_{d}}\right)$ jut rightly greater than unity or $\left(\frac{E e}{E_{e} e^{\min }}\right)$ joint rightly lagger than 1 
385

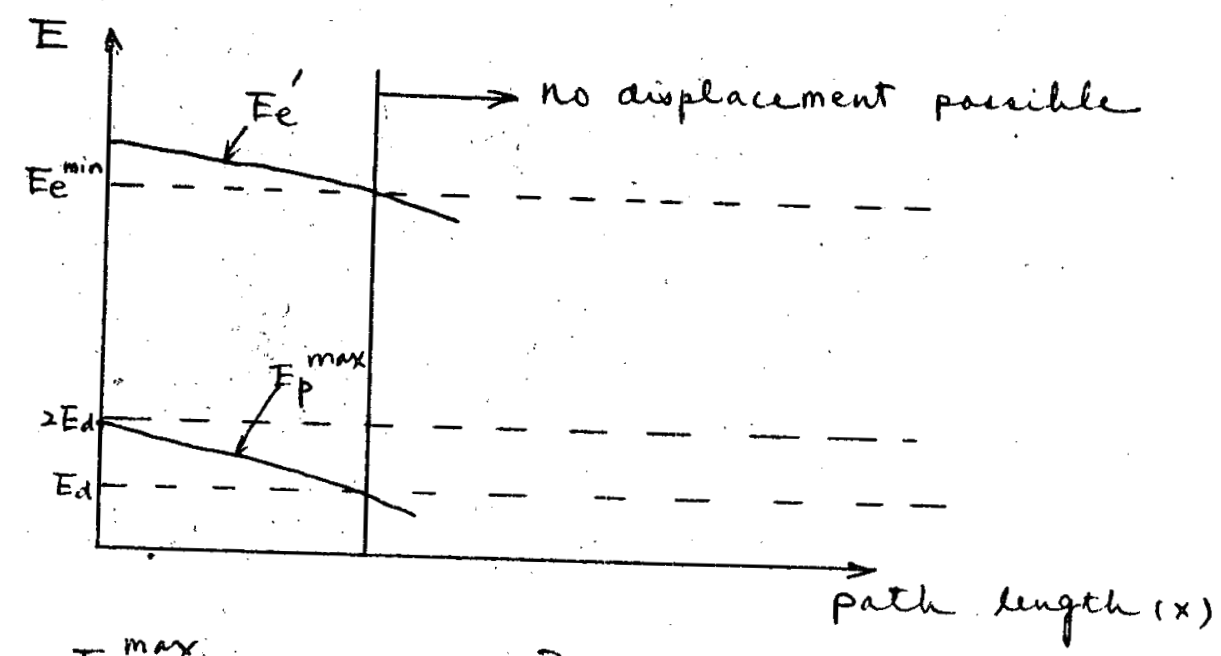

Since $E_{d}<E_{p}^{\max }<>E_{d}, \quad P\left(E_{p}\right)=1$

$$
\begin{aligned}
& \because \int_{E_{d}}^{E_{p}^{\max }}\left(\frac{d \sigma}{d E_{p}}\right) \gamma\left(E_{p}\right) d E_{p}=\sigma_{d}\left(E_{e}\right) \\
& \simeq\left(\frac{z e^{2}}{m_{e} c^{2}}\right)^{2} \frac{\pi\left(1-\beta^{2}\right)}{\beta^{4}}\left[\frac{E_{p}^{\max }}{E_{d}}-1\right] \\
& \text { Since } E_{e} \simeq E_{e}^{\text {min }} \frac{d E_{e}}{d x}=\text { constant at all } E_{e} \text { value } \\
& \Rightarrow \bar{n}\left(E_{e}\right)=\frac{N\left(E_{e}-E_{e}^{\min }\right)}{(-d E / d x)_{E_{e}}}\left(\frac{z e^{2}}{m_{e} c^{2}}\right)^{2} \frac{\pi}{\beta^{4}\left(1-\beta^{2}\right)}\left[\frac{E_{p}^{\max }\left(E_{e}\right)}{E_{d}}-1\right]
\end{aligned}
$$


386

$\therefore \quad$ PROBLEM 17.12

a) nimbler of Compton electrons produced/cis - pec in energy range (Fe. $d E_{e}$ ) lo photons in energy range (Er, $\left.d E_{r}\right)$

$$
=(Z N) \phi\left(E_{\gamma}\right) d E_{r} \sigma_{c}\left(E_{\gamma}, E_{e}\right) d E_{e}
$$

multiply by $\bar{n}\left(E_{e}\right)=$ number of displaced atoms per electron of energy $E_{e}$ and integrate over all energies (For $r$-Ray and electron)

$$
R_{d}=Z N \int_{E_{r}^{\min }}^{E_{r}^{c}} \phi\left(E_{r}\right)\left\{\int_{E_{e} \min }^{E_{e}^{\max }} \sigma_{c}\left(E_{Y}, E_{e}\right) \bar{n}\left(E_{e}\right) d E_{e}\right\} d E_{r}-(1)
$$

Since the compton electrons are of energy $\sim 1 \mathrm{MeV}$, We.mint cue relativistic kinematics. $E^{m i n}$ calculated in problem 17.11 as a Solution $E_{e}^{\min }\left(1+E_{e}^{\min }\right)=\left(\frac{M}{4 m_{e}} E_{d} \times 10^{-t}\right)-\cdots(2)$

Whir e $E e^{\min }$ in MeV and $E d$ in e $\dot{V}$ $E_{e}^{\max }$ : the maximum energy of a compton electron

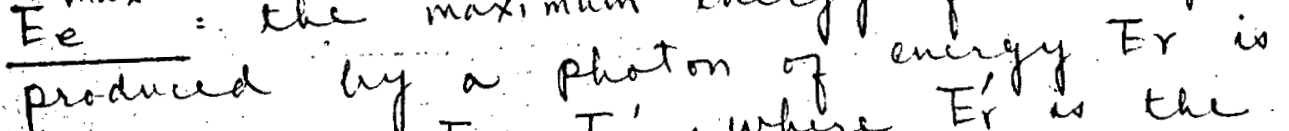
$E_{e}^{\max }=E_{r}-E_{r}^{\prime}$, whine $E_{r}^{\prime}$ is the energy of the backacattered $\left(\theta=180^{\circ}\right)$ phot ins.

$$
E_{r}^{\prime}=\frac{E_{r}}{1+2 \frac{E_{r}}{m_{e} c^{2}}}=\frac{E_{r}}{1+4 E_{r}}, \operatorname{Er} \text { in } M e V
$$

$$
\because E^{\max }=\frac{4 E_{r}^{2}}{1+4 E_{r}}-\cdots(3)
$$


387

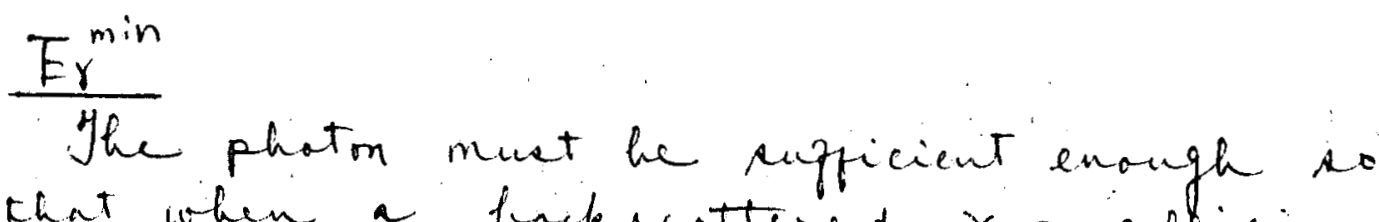
that when a tackacattered $\gamma$-e collision occurs the secondary electron energy is $E_{e}^{\text {min }}$ or $E_{r}^{\text {min }}$ is determined by.

$$
\frac{4\left(E_{\gamma}^{\min }\right)^{2}}{1+4\left(E_{\gamma}^{\min }\right)}=E^{\text {min }}=\frac{1}{2}\left[\sqrt{1+4\left(\frac{M}{m_{e}} E_{d} \times 10^{-6}\right)}-1\right]--(4)
$$

b) No damage wile occur if $E_{\dot{P}}^{0}$ is less than or equal to $E_{r}^{\text {min }}$, as determined by (4)

$$
\text { Let } E^{*}=\frac{M}{4 m_{e}} E_{d} \times 10^{-6} \cdot M e V
$$$$
\frac{x=E_{\gamma}^{\min }}{1+4 x}=\frac{1}{2}\left(\sqrt{1+4 E^{*}}-1\right)=A
$$

$$
\begin{aligned}
& 4 x^{2}-4 A x-1=0 \\
x= & E_{r}^{\min }=\frac{A+\sqrt{A^{2}+1 / 4}}{2} \\
= & \frac{1}{2}\left[\frac{1}{2}\left(\sqrt{1+4 E^{*}}-1\right)+\sqrt{\frac{1}{4}\left(\sqrt{1+4 E^{*}}-1\right)^{2}+\frac{1}{4}}\right]
\end{aligned}
$$

$$
\therefore E_{r}^{\min }=\frac{1}{4}\left[\left(\sqrt{1+4 E^{*}}-1\right)+2 \sqrt{\left(\sqrt{1+4 E^{*}}-1\right)^{2}+1}\right]
$$


388

$$
\begin{aligned}
& \frac{\text { PROBLEM } 17.13}{\text { From } E_{q}(17.15 .1)} \\
& b=\int_{T_{\text {min }} / \Lambda}^{n_{n}} d E_{n} \phi\left(E_{n}\right) \int_{T_{\text {min }}}^{\wedge E_{n}} \sigma\left(E_{n}, T\right) d T
\end{aligned}
$$

Where $\Lambda E_{n}$ is the maximum energy trans fer to a lat ice atom by a neutron of energy $E_{n}$ and $\Lambda=\frac{4 A}{(A+1)^{2}}$

$\sigma\left(E_{n}, T\right)$ is the energy trapper cross section. for neutron acatising

Since this is a monoenergetic neutron flux

$$
\phi\left(E_{n}\right)=\Phi \delta\left(E_{n}-0.5\right), E_{n} \text { in MeV. }
$$

Acme the scattering process is clastic and inotropic in C.M. Ayctim and $\sigma_{e l}=10 \times 10^{-24} \mathrm{~cm}^{2}$

$$
\begin{aligned}
& \Rightarrow \sigma\left(E_{n}, T\right)=\frac{\sigma_{e 1}}{\Lambda E_{n}} \\
& \therefore b=\Phi \int_{T_{\min }}^{\wedge E_{n}} \frac{\sigma_{e 1}}{\Lambda E_{n}} d T=\Phi \sigma_{e 1}\left(1-\frac{T_{\min }}{\Lambda E_{n}}\right) \\
& \Rightarrow b=\Phi \sigma_{e 1}\left(1-\frac{T_{\min }}{\Lambda E_{n}}\right) \\
& \quad T_{\min }=25 \mathrm{eV} . \\
& \therefore b=10^{-8} \cdot \mathrm{Rec}^{-1} .
\end{aligned}
$$


389

- Ruclution by firsion fragment recrils

$$
b=1.7 \times 10^{-17} \dot{\mathrm{F}} \mathrm{rec}^{-1}
$$

From Eq $(10.1)$

$$
\dot{F}=q \cdot \sigma_{f} N_{f} \cdot \phi \text {. }
$$

Where $f$ is the enrichment, $=0.15$

$\sigma_{f}$ : the effective fision crous rection for the fircile species in the appropriate nuitron energi Apectrum. for a fact reactor

$$
\sigma_{f}=1.8 \text { barno }=1.8 \times 10^{-24} \mathrm{~cm}^{2}
$$

Nf: loxal monlee density of the heony metal atron, In TBR this numlier is abut the Aame as the chanium atm density in peise

$$
\begin{aligned}
& \phi=10^{15} \delta\left(E_{n}-0.5\right) \\
& \therefore \dot{F}=0.15 \times 1.8 \times 10^{-24} \times 2.4 \times 10^{22} \times 10^{15}=0.65 \times 10^{13} \mathrm{~cm}^{-3} \mathrm{\mu t}^{-1} \\
& b=1.7 \times 10^{-17} \times \dot{F}=1.10 \times 10^{-4} \mathrm{rec}^{-1} \\
& \text { bfret reutron }=10^{-8} \mathrm{pec}^{-1} \\
& \text { brecril }=1.1 \times 10^{-4} \mathrm{rec}
\end{aligned}
$$

The magnitide of brecal a four oider o the recilution offect as moce important for the fision fiagment chac that for fast nentron 
390

PROBLEM 17.14

a) Resolition due to direct neutron collicion: Sinilar to $\varepsilon q(13.116)$ for He bibleles an a runtro flux is

$$
\text { bdirect }=\int_{T_{\text {min }}}^{\text {a noutron }} d E_{n} \phi\left(E_{n}\right) \int_{T_{\text {min }}}^{\Lambda_{r E} E_{n}} \sigma_{H_{e}-n}\left(E_{n} . T\right) d T
$$

For elactic, isctropic scattering of neutron on He atorms,

$$
\begin{aligned}
& \sigma_{H e-n}\left(E_{n}, T\right)=\frac{\sigma_{H e}-n}{\Lambda_{H e} E_{n}} \\
& \Lambda_{H e}=\frac{4(4),}{(1+4)^{2}}=0.64 \\
& \text { bdirect }^{\infty}=\sigma_{H e-n} \int_{\text {Tmin }}^{\infty} \phi\left(E_{n}\right)\left(1-\frac{T_{\text {min }}}{\Lambda_{H e} E_{n}}\right) d E_{n}
\end{aligned}
$$

For monoenergetic flux

$$
\begin{aligned}
& \text { bolirect }=\sigma_{H e-n} \Phi\left(1-\frac{T_{\min }}{\Lambda_{H E} E_{n}}\right) \\
& \text { where } \frac{T_{\min }}{\Lambda_{H e} E_{n}} \simeq 0 \\
& \sigma_{H E-n} \cong 1 \times 10^{-24} \mathrm{~cm}^{2} \\
& \Phi=10^{15} \mathrm{~cm}^{-2}-\mathrm{rec}^{-1} \\
& \therefore \text { bdirect }=\left(10^{-24}\right)\left(10^{15}\right)=10^{-9} \mathrm{rec}^{-1}
\end{aligned}
$$

b) Ruclution of He in Atamles ituel due ti recil $\phi\left(E_{r}\right)$ flux ppetum of iros reciels

Er reciel atom crierger $\sigma_{F e-H e}(E . T)$ energy tranifer cros pection fre iron-Helium interation 
391.

Following Eq (17.151)

$$
\begin{gathered}
\text { brecie }=\int_{T_{\text {min }} / \Lambda} d E_{\gamma} \phi\left(E_{r}\right) \int_{T_{\text {min }}}^{\Lambda E_{r}} \sigma_{F_{e}-H_{e}}\left(E_{Y}, T\right) d T \ldots(1) \\
\Lambda=\frac{4 M_{F e} M_{H e}}{\left(M_{F e}+M_{H e}\right)^{2}}=0.25 \ldots \ldots \text { (2) }
\end{gathered}
$$

$T_{\text {min }}$ minimum energy of He atom for ricolution Following $E_{q}(17.156)-(17.157)$

$$
\sigma_{F_{e}-H_{e}}\left(E_{r}, T\right)=\frac{K}{E_{r}^{2}} \ldots \ldots \ldots \text { (3) }
$$

where

$$
\begin{aligned}
& K=\frac{\sqrt{2} \pi Z_{F e} Z_{H e} a_{B} e^{2} \exp (-1)}{n\left(Z_{F e}^{3 / 3}+Z_{H E}^{3 / 3}\right)^{1 / 2}}\left(\frac{M_{F e}+M_{H E}}{M_{H E}}\right)--(4) \\
& z_{\mathrm{Te}}=26 \\
& \left.\begin{array}{l}
z_{H e}=26 \\
Z_{B} e^{2}=7.5 \times 10^{-16} \mathrm{eV}-\mathrm{am}^{2}
\end{array}\right\} \Rightarrow K=1.2 \times 10^{-12} \mathrm{eV}-\mathrm{cm}^{2}
\end{aligned}
$$

The recoil glux mpectrum is given by $E_{i}(17.166)$ with apprepriate changen from $f f$ to $n$ and $W t_{2} F_{e}$

$$
\phi\left(E_{r}\right)=\frac{4}{E_{r}^{2} \sigma_{F e}-F_{e}\left(E_{r}\right)} \int_{E_{r} / \Lambda_{n}}^{\infty} d E_{n} \phi\left(E_{n}\right) \int_{E_{r}}^{\Lambda_{n} E_{n}} E \sigma_{F_{E}}\left(E_{n}, E\right) d E-(5)
$$

Where: $\Lambda_{n}=\frac{4 \times 56}{(1+56)^{2}}=0.069 \cdots \cdots$ (6) $T_{F e}-F_{e}=$ iron - irm atomic Acatting crons pection $\sigma_{F e}\left(E_{n}, E\right)$, enirgy trinffer cros section foi nentrons on 
392

$\phi\left(E_{n}\right)=$ nutron flux Apectrum

For isotropic, elastic neutron, acattiring

$$
\sigma_{F \in}\left(E_{n}, E\right)=\frac{\sigma_{F E}-n\left(E_{n}\right)}{\lambda_{n} E_{n}} \cdots \cdots
$$

$\sigma_{F e-n}\left(E_{n}\right)$ : elactic peattering corrs section for iron Unig (7), in (5):

$$
\left.\phi\left(E_{Y}\right)=\frac{2}{\Lambda_{n} E_{r}^{2} \sigma_{F_{\epsilon}-F e}} \int_{E_{r} / \Lambda_{n}}^{\infty} \frac{\sigma_{E \in-n}\left(E_{n}\right) \phi\left(E_{n}\right)\left(n_{n}^{2} E_{n}^{2}-E_{r}^{2}\right)}{E_{n}} d E_{n}-18\right)
$$

Cheiming an eneigy dependent seattering cross section and a monoenergete feux at $E_{n}, \phi\left(E_{n}^{\prime}\right)=\Phi \delta\left(E_{n}^{\prime}-E_{n}\right)$

$$
\left.\Rightarrow \phi\left(E_{r}\right)=2\left(\frac{\Lambda_{n}^{2} E_{n}^{2}-E_{r}{ }^{2}}{\Lambda_{n} E_{n} E_{r}{ }^{2}}\right)\left(\frac{\vdots \sigma_{F e-n}}{\sigma_{F e-F e}}\right) \Phi-\cdots-19\right)
$$

where $E_{n}$ is given

Unig (2), (3), (4), (6), and (9) in (1)

$$
b_{\text {recoil }}=2 K \Phi\left(\frac{\sigma_{E e-n}}{\sigma_{F e}-F_{e}}\right) \int_{T_{m: n} / n}^{\Lambda_{n} E_{n}}\left(\frac{\Lambda_{n}^{2} E_{n}^{2}-E_{r}^{2}}{\Lambda_{n} E_{n} E_{r}^{2}}\right)\left(\frac{1}{T_{\text {min }}}-\frac{1}{\Lambda E_{r}}\right) d E_{r}
$$

Where $\Lambda_{n} E_{n}$ is the maximum necail enirgy an moncenegitio neutron flux

$$
\begin{aligned}
& \therefore \text { brecail }=2 K\left(\frac{\sigma_{F e}-n}{\sigma_{F e}-F_{e}}\right) \Phi\left[\left(\frac{\Lambda_{n} E_{n}}{T_{\min }}\right)\left(\frac{\Lambda}{T_{\text {min }}}-\frac{1}{\Lambda_{n} E_{n}}\right)\right. \\
& -\left(\frac{1}{\Lambda_{n} E_{n} T_{\text {min }}}\right)\left(\Lambda_{n} E_{n}-\frac{T_{\min }}{\Lambda}\right)-\left(\frac{\Lambda_{n} E_{n}}{2 \Lambda}\right)\left(\frac{\Lambda^{2}}{T_{n \text { min }}^{2}}-\frac{1}{\Lambda_{n}^{2} E_{n}^{2}}\right) \\
& \left.+\frac{1}{\Lambda \Lambda_{n} E_{n}} \ln \left(\frac{\Lambda_{n} \Lambda E_{n}}{T_{\min }}\right)\right]
\end{aligned}
$$


393

when $\sigma_{F e-n} \simeq 3 \times 10^{-24} \mathrm{~cm}^{2}$

$$
\begin{aligned}
& \sigma_{F e}-F_{e} \simeq 5 A^{2}=5 \times 10^{-16} \mathrm{~cm}^{2} \\
& K=1.2 \times 10^{-12} \mathrm{eV}-\mathrm{cm}^{2} \\
& \Phi=10^{15} \text { neutrois } / \mathrm{cm}^{2} / \mathrm{sec} \\
& \begin{array}{l}
\cap_{n} E_{n}=(0.069) \times\left(0.5 \times 10^{6}\right)=3 \times 10^{4} \mathrm{eV} \\
T_{\text {min }}=200 \mathrm{eV} .
\end{array} \\
& T_{\min } / \Lambda=800 \mathrm{eV} \text {. }
\end{aligned}
$$

$$
\begin{aligned}
& \therefore \text { brecril }=2 \times\left(13 \times 10^{-12}\right)\left(\frac{3 \times 10^{-24}}{5 \times 10^{-6}}\right)\left(10^{15}\right)\left[\frac{3 \times 10^{4}}{2 \times 10^{2}} \frac{1}{8 \times 10^{2}}\right. \\
& \left.-\frac{1}{2 \times 10^{2}}-\frac{3 \times 10^{4}}{0.5} \times \frac{1}{\left(8 \times 10^{2}\right)^{2}}+\frac{1}{125 \times 3 \times 10^{4}} \ln \left(\frac{3 \times 10^{4}}{8 \times 10^{2}}\right)\right] \\
& =1.3 \times 10^{-6} \sec ^{-1} \\
& \Rightarrow \text { brecal }=1.3 \times 10^{-6} \mathrm{sec}^{-1}
\end{aligned}
$$


394

CHAPTER 18

Problem 18.1

(A)

boundary conditions:

1) at void $C_{v}(R)=C_{v}^{e q} \exp \left[\frac{2 \gamma}{R} \frac{\Omega}{k T}\right]$

2) in bulk $C_{v}(\infty)=C_{v}^{e q}$

Diffusion equation for vacancies, source free, $r$ dependence only, becomes:

$$
\begin{aligned}
& \quad \frac{d}{d r}\left(r^{2} \frac{d C_{v}}{d r}\right)=0 \Rightarrow \frac{d C_{v}}{d r}=\frac{C_{1}}{r^{2}} \\
& \therefore C_{v}(r)=-\frac{C_{1}}{r}+C_{2}
\end{aligned}
$$

Apply foundry conditions:

$$
\begin{aligned}
& r \rightarrow \infty \\
& C_{v}(\infty)=C_{2}=C_{v}^{e q} \\
& r=R \\
& C_{v}(R)=-\frac{C_{1}}{R}+C_{v}^{e q}=C_{v}^{e q} \exp \left[\frac{2 \gamma}{R} \frac{\Omega}{k T}\right] \\
& \therefore C_{1}=R C_{v}^{e q}\left[1-\exp \left(\frac{2 \gamma^{\mu}}{R} \cdot \frac{\Omega}{k T}\right)\right]
\end{aligned}
$$

Thus,

$$
\left.\frac{d C_{v}(r)}{d r}\right)_{R}=\frac{C_{1}}{R^{2}}=\frac{C_{j}^{e q}}{R}\left[1-\exp \left(\frac{2 \gamma}{R} \frac{\Omega}{K T}\right)\right]
$$

For $\frac{2 \gamma}{R} \frac{\Omega}{K T} \ll 1$, we may expand exponent

$\left.\therefore \frac{d c v}{d r}\right)_{R} \cong-\frac{C_{v}^{\varepsilon q}}{R^{2}} \frac{2 \gamma \Omega}{k T}$

Equating the volumetric loss rate of the void. with the outward current of volume carried by diffusing vacancies, obtain $\therefore$ 
395

$$
\begin{aligned}
-\frac{d}{d t}\left(\frac{4}{3} \pi R^{3}\right) & =-D_{v}\left(\frac{d C_{v}}{d r}\right)_{R} 4 \pi R^{2} \Omega \\
& =4 \pi D_{v} c_{v}^{e q} \frac{2 \gamma \Omega^{2}}{k T}
\end{aligned}
$$

$\therefore$ integrating, obtain

$$
R^{3}=-3 D_{v} C_{v}^{e q} \frac{2 \gamma \Omega^{2}}{k T} t+\text { (constant) }
$$

but, constant $=R_{0}^{3}$, since $R(t=0)=R_{0}$

$$
\therefore \quad R_{0}^{3}-R^{3}=3 D_{v} \Omega^{2} C_{v}^{e}\left(\frac{2 \gamma}{k T}\right) t
$$

or

$$
R\left(R_{0}, t\right)=\left\{R_{0}^{3}-Q t\right\}^{1 / 3} \text { where } Q=3 D_{v} C_{v}^{e q}\left(\frac{2 \gamma}{k T}\right) \Omega^{2}
$$

(B)

All voids in the range $R$ to $(R+d R)$ at time $t$ are due to those present at $t=0$ in the range $R_{0}$ to $\left(R_{0}+d R_{0}\right)$, this:

$$
\begin{aligned}
& N^{\prime}(R, t) d R=N_{0}\left(R_{0}\right) d R_{0}
\end{aligned}
$$

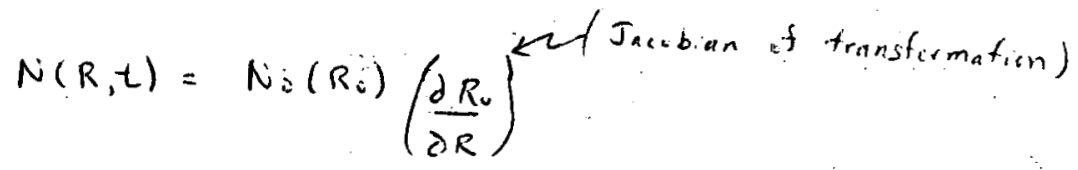

From part (A) solve for $R_{0}(R, t)$ :

$$
\begin{aligned}
& R_{0}(R, t)=\left(R^{3}+Q t\right)^{1 / 3} \\
\therefore \quad \frac{\partial R_{0}}{\partial R} & =R^{2}\left(R^{3}+Q t\right)^{-2 / 3} \\
\therefore \quad N(R, t) & =N_{0}\left(R_{0}(R, t)\right) \cdot R^{2}\left(R^{3}+Q t\right)^{2 / 3}
\end{aligned}
$$


396

(C)

Gaussian distribution is given by

$$
N_{0}\left(R_{0}\right)=k \exp \left(-\frac{\left(R_{0}-\bar{R}_{0}\right)^{2}}{2 \sigma^{2}}\right)
$$

To find $k$, use normalizing condition:

$$
\begin{aligned}
N_{T}^{0} & =\int_{0}^{\infty} N_{i}\left(R_{0}\right) d R_{0} \\
& =k \int_{0}^{\infty} \exp \left(-\frac{\left(R_{0}-\bar{R}_{0}\right)^{2}}{2 \sigma^{2}}\right) d R_{0}
\end{aligned}
$$

let $x=\frac{R_{0}-\bar{R}_{0}}{\sqrt{2 \sigma}} \rightarrow d x=\frac{d R_{0}}{\sqrt{2} \sigma}$

Thus,

$$
\begin{aligned}
& N_{T}^{0}=\sqrt{2} k \sigma \int_{-\bar{R}_{0} / \sqrt{2} \sigma}^{\infty} e^{-x^{2}} d x \\
& =\sqrt{2} k \sigma\left\{\int_{0}^{\infty} e^{-x^{2}} d x+\int_{0}^{\overline{R_{0}} / \sqrt{2} \sigma} e^{-x^{2}} d x\right\} \\
& =\sqrt{2} k \sigma\left\{\frac{\sqrt{\pi}}{2}+\frac{\sqrt{\pi}}{2} \operatorname{erf}\left(\frac{\bar{R}_{0}}{\sqrt{2} \sigma}\right)\right\} \\
& \therefore k=\frac{\sqrt{\frac{2}{\pi}} N^{0} T}{\sigma\left[1+\operatorname{erf}\left(\bar{R}_{0} / \sqrt{2} \sigma\right)\right]} \cong \frac{\sqrt{\frac{2}{\pi}} \times 10^{15}}{50[1+\operatorname{erf}(5.61)]} \\
& \cong \sqrt{\frac{2}{\pi}} \times \frac{10^{15}}{50(1+1)} \\
& \cong 7.95 \times 10^{12} \text { voids } / \mathrm{cm}^{3}-\AA
\end{aligned}
$$

When average void disappears,

$$
\begin{aligned}
& R\left(\bar{R}_{0}, t\right)=0 \Rightarrow \bar{R}_{0}^{3}=Q t_{d \text { (Ave) }} \quad \text { (fro mpart A) } \\
& \text { or } Q=\frac{(400)^{3}}{3 \times(60)^{2}} \cong 5.9 \times 10^{3} \mathrm{sec}^{-1}
\end{aligned}
$$


397

Thus,

$$
\begin{aligned}
& N(R, t)=K \exp \left\{-\frac{\left[\left(R^{3}+Q t\right)^{1 / 3}-\bar{R}_{0}\right]^{2}}{2 \sigma^{2}}\right\} \cdot R^{2}\left(R^{3}+Q t\right)^{-2 / 3} \\
& \text { for } t=2 \mathrm{hrs}=2 \times 60^{2} \mathrm{sec} \\
& Q t=2 \times 60^{2} \times 5.9 \times 10^{3} \cong 4.25 \times 10^{7} \\
& \therefore N(R, 2 \text { hrs })=7.95 \times 10^{12} \exp \left\{\frac{\left[\left(R^{3}+4.25 \times 10^{7}\right)^{1 / 3}-400\right]^{2}}{2 \cdot(50)^{2}}\right\} \cdot R^{2}\left(R^{2}+4.25 \times 10^{7}\right)^{-2 / 3}
\end{aligned}
$$

and

$$
N_{0}\left(R_{0}\right)=7.95 \times 10^{12} \exp \left\{-\frac{\left(R_{0}-400\right)^{2}}{2 \cdot(50)^{2}}\right\}
$$

in units of $\left[\frac{\text { voids }}{\mathrm{cm}^{3}-A}\right]$

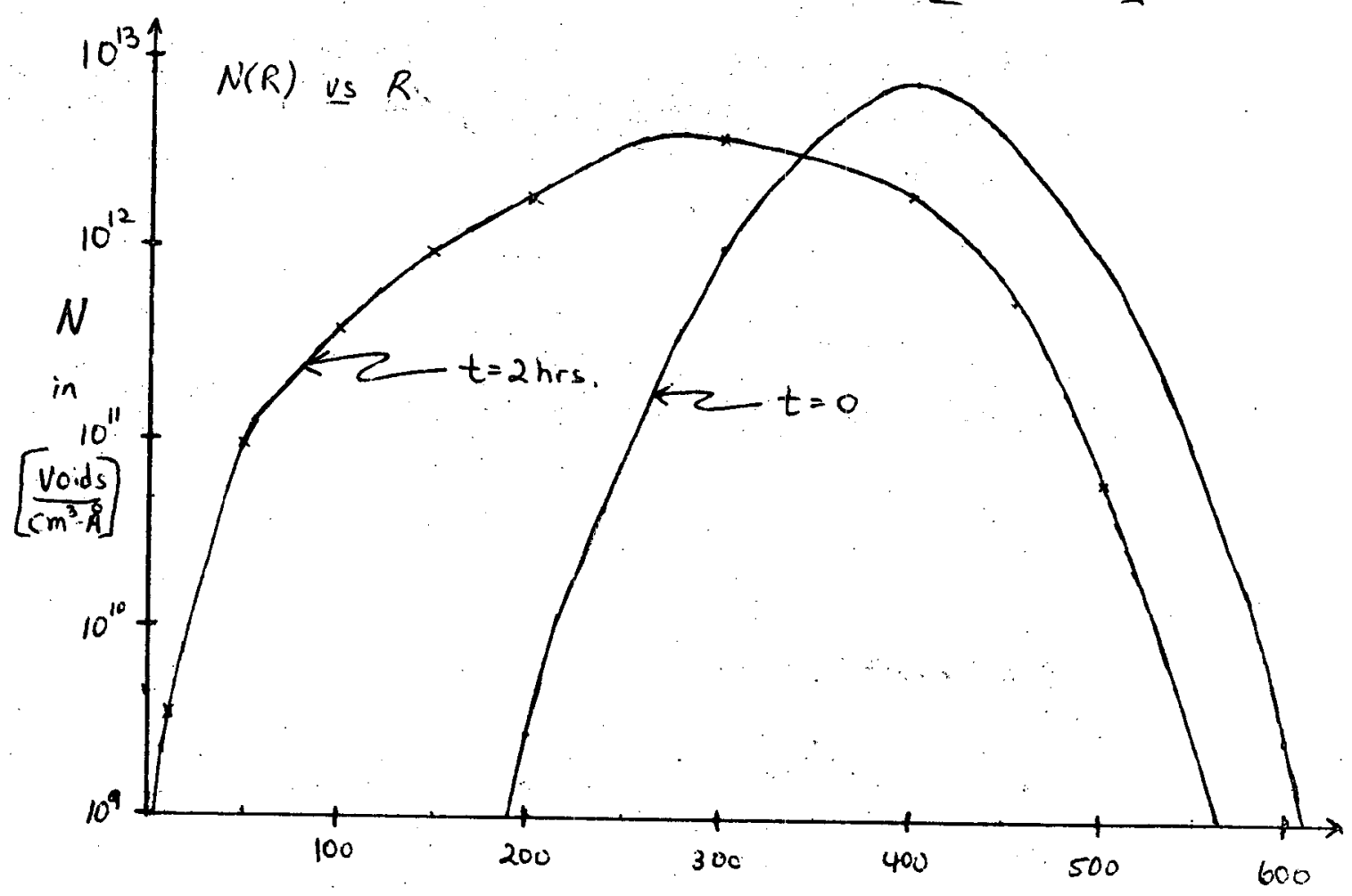

$R$ in $[\AA]$ 
398

Problem 18.2

$$
\begin{aligned}
& \frac{d \sigma}{d \epsilon}=\sigma \\
& \text { at onset of } \\
& \text { plastic instability } \\
& \left(E_{q n} \cdot 18.7\right) \\
& \text { With } \\
& \sigma=k \epsilon^{n} \text {, obtain } \\
& k n \epsilon^{n-1}=k \epsilon^{n} \\
& \text { or: } \\
& n=\epsilon \text { uTs } \\
& \therefore \Delta \epsilon_{u T S}=\left(\epsilon_{\text {uts }}\right)_{\text {unirr }}-\left(\epsilon_{\text {ers }}\right)_{\text {rad }}=\Delta n
\end{aligned}
$$

ie, reduction in uniform elongation due to irradiation $=\Delta n$. 
399

Problem 18.3

(A)

$$
\begin{aligned}
& \leftarrow a \longrightarrow 0<\text { pinning } \\
& \text { pent }
\end{aligned}
$$

$\therefore$ area $a^{2}$ has $\left(4 \times \frac{1}{4}+1\right)=2$ pinning points

$\therefore \frac{\text { obstacles }}{\text { area }}=\frac{2}{a^{2}}=\frac{1}{a^{2}}$

$$
\therefore \quad l=\frac{a}{\sqrt{2}}
$$

case : :

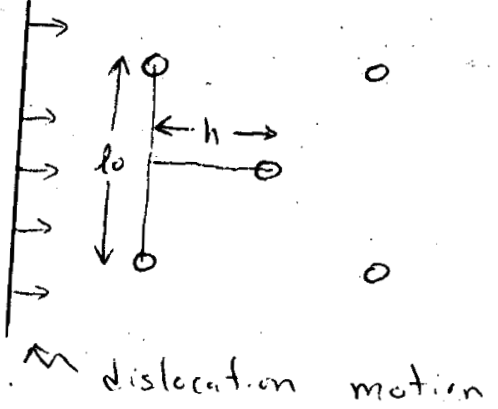

for cast 1

$$
\begin{aligned}
l_{0} & =a \\
h & =a / 2 \\
& : \\
\therefore l_{0} & =\left(\frac{a}{2}\right)(a)=\frac{a^{2}}{2}=l^{2}
\end{aligned}
$$

case 2:

0

o.

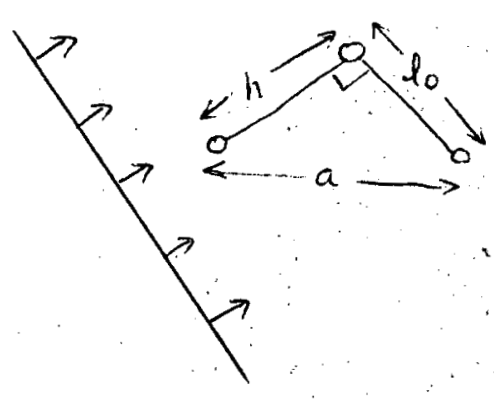

$$
\begin{aligned}
& \text { for case } 2 \\
& \qquad 0=a / \sqrt{2} \\
& h=a / \sqrt{2} \\
& \therefore h l o=(a / b)\left(\frac{a}{\sqrt{2}}\right)=a^{2} / 2=l^{2} \mathrm{~V}
\end{aligned}
$$


400

(B)

$$
Y=1-\frac{1}{1+e^{m}}-A_{m}+\text { constant }
$$

where $\eta=\frac{x}{r^{\prime}} \quad \& \quad A=\frac{\sigma b l_{0} r^{\prime}}{v_{0}}$

(i)

$Y$ reaches maximum (barrier exists) only if

$$
\begin{aligned}
& \frac{d Y}{d \eta}=0 \\
& -\frac{d}{d \eta}\left(\frac{1}{1+e^{n}}\right)-A=0 \\
& \left(\frac{1}{1+e^{m}}\right)^{2} e^{\eta}-A=0 \\
& \text { let } z=e^{n} \quad ; z \in\{\text { Reals }>0\}
\end{aligned}
$$

$$
\frac{z}{(1+z)^{2}}-A=0
$$

Solve for $z$

$$
\begin{aligned}
& z-A-2 A z-A z^{2}=0 \\
& z^{2}+\left(2-\frac{1}{A}\right) z+1=0 \\
& \therefore z=\frac{\left(\frac{1}{A}-2\right) \pm \sqrt{\left(\frac{1}{A}-2\right)^{2}-4}}{2} \\
&=\left(\frac{1}{2 A}-1\right) \pm \sqrt{\left(\frac{1}{2 A}-1\right)^{2}-1} \\
& \text { Since }\left|\frac{1}{2 A}-1\right|>\left|\sqrt{\left(\frac{1}{2 A}-1\right)^{2}-1}\right|,
\end{aligned}
$$

$$
\left(\frac{1}{2 A}-1\right)>0 \text { for } z>0
$$

For $z \in \mathbb{R}_{\text {R ea }}$,

$$
\left(\frac{1}{2 A}-1\right)^{2}-1>0 \text {. }
$$


401

$$
\left(\frac{1}{2 A}-1\right)^{2}>1
$$$$
\frac{1}{2 A}-1>1
$$

(positive root taken since $\left(\frac{1}{\partial A}-1\right)>0$ )

$\therefore \frac{1}{4}>A$, if barrier exists.

$\therefore \quad$ if $A \geq 1 / 4$

barrier height disappears (barrier

(ii) does not exist)

$$
\begin{gathered}
\epsilon=1-4 A \\
\epsilon-1=-4 A \\
\therefore \frac{1}{2 A}=\frac{2}{1-\epsilon} \\
\therefore\left(\frac{1}{2 A}-1\right)=\frac{2}{1-\epsilon}-1=\frac{1+\epsilon}{1-\epsilon}
\end{gathered}
$$

Thus,

$$
\begin{aligned}
z & =\left(\frac{1+\epsilon}{1-\epsilon}\right) \pm \sqrt{\left(\frac{1+\epsilon}{1-\epsilon}\right)^{2}-1} \\
& =\frac{1+\epsilon}{1-\epsilon}\left[1 \pm \sqrt{1-\left(\frac{1-\epsilon}{1+\epsilon}\right)^{2}}\right]
\end{aligned}
$$

expand for small $E$

$$
\begin{aligned}
& z \cong(1+\epsilon)(1+\epsilon)\left[1 \pm \sqrt{1-[(1-\epsilon)(1-\epsilon)]^{2}}\right] \\
& \cong(1+2 \epsilon)\left[1 \pm \sqrt{1-(1-\epsilon)^{4}}\right] \\
& \cong(1+2 \epsilon)[1 \pm \sqrt{1-(1-4 \epsilon)}] \\
& \cong(1+2 \epsilon)[1 \pm 2 \sqrt{\epsilon}] \\
& \cong 1 \pm 2 \sqrt{\epsilon}+2 \epsilon \text { to first order in } \epsilon
\end{aligned}
$$


402

$$
\begin{aligned}
& \therefore e^{n \pm \cong 1 \pm 2 \sqrt{\epsilon}+2 \epsilon} \\
& \therefore n_{ \pm} \cong \ln (1 \pm 2 \sqrt{\epsilon}+2 \epsilon) \\
& \text { using } \ln (1+x) \cong x-\frac{1}{2} x^{2}+\cdots \text { for }|x|<1 \\
& \text { obtain, } \\
& m_{ \pm} \cong \pm 2 \sqrt{\epsilon} \text {. to lowest order in } \epsilon
\end{aligned}
$$

(iii)

$$
\begin{aligned}
& Y\left(\eta_{+}\right)=1-\left(\frac{1}{1+e^{n_{+}}}\right)-A n_{+}+\text {cons } \\
& Y\left(m_{-}\right)=1-\left(\frac{1}{1+e^{n_{-}}}\right)-A \eta_{-}+\text {cons }
\end{aligned}
$$

where

$$
\begin{aligned}
A & =\frac{1-\epsilon}{4} \\
\Delta Y & =Y\left(\eta_{+}\right)-Y\left(\eta_{-}\right) \\
& =-\left(\frac{1}{1+e^{n}}\right)+\left(\frac{1}{1+e^{n_{-}}}\right)-A n_{+}+A \eta_{-} \\
& \cong-\left(\frac{1}{1+(1+2 \sqrt{\epsilon})}\right)+\left(\frac{1}{1+(1-2 \sqrt{\epsilon})}\right)+\frac{1-\epsilon}{4}(-2 \sqrt{\epsilon}-2 \sqrt{\epsilon}) \\
& \cong \frac{1}{2}\left\{\frac{1}{1-\sqrt{\epsilon}}-\frac{1}{1+\sqrt{\epsilon}}\right\}+\frac{(\epsilon-1)}{\not \epsilon} \cdot \not 2 \\
& \cong \frac{\epsilon^{1 / 2}}{1-\epsilon}+\epsilon^{3 / 2}-\epsilon^{1 / 2} \\
& \cong \epsilon^{1 / 2}(1+\epsilon)+\epsilon^{3 / 2}-\epsilon^{1 / 2} \\
& \cong 2 \epsilon^{3 / 2}
\end{aligned}
$$


403

$\frac{\text { Problem 18.4 }}{\text { Eqn 18.39 may be written as }}$

$$
\sigma_{s}^{2 / 3}=\left(\sigma_{s}^{0}\right)^{2 / 3}-\left(\frac{\sigma_{i}^{0}}{T_{c}}\right)^{2 / 3} T^{2 / 3}
$$

For the Ni data of Fig 18.20:

$$
\begin{aligned}
& \left(\sigma_{s}^{0}\right)_{A} \cong 8200^{3 / 2} \text { \& }\left(\sigma_{S}^{\circ}\right)_{B} \cong(6750)^{3 / 2} \\
& \therefore \frac{\left(\sigma_{S}^{0}\right)_{4}}{\left(\sigma_{s}\right)_{B}} \cong\left(\frac{8200}{6750}\right)^{3 / 2} \cong 1.34 \\
& \left(\frac{\sigma_{s}^{0}}{T_{c}}\right)_{A} \cong 66^{3 / 2} \&\left(\frac{\sigma_{S}^{0}}{T_{C}}\right)_{B} \cong 28^{3 / 2} \\
& \therefore \quad \frac{\left(T_{C}\right)_{A}}{\left(T_{C}\right)_{B}}=\frac{\left(\sigma_{S} / T_{C}\right)_{B}}{\left(\sigma_{S} / T_{C}\right)_{A}} \frac{\left.\left(\sigma_{S}\right)^{\circ}\right)_{A}}{\left(\sigma_{S}\right)_{B}} \\
& \cong(1.34)\left(\frac{28}{66}\right)^{3 / 2} \\
& \cong 0.37 \text {. } \\
& \text { From En } 18.39, \quad T_{c} \propto U_{0} \text { or }
\end{aligned}
$$$$
\frac{\left(V_{0}\right)_{A}}{\left(V_{c}\right)_{B}}=\frac{\left(T_{C}\right)_{A}}{\left(T_{C}\right)_{B}} \cong 0.37
$$$$
\text { but. } U_{0} \propto r^{2} \text { (se tent) }
$$$$
\therefore \quad \frac{r_{A}}{r_{B}}=\sqrt{\frac{\left.U_{0}\right)_{A}}{\left(U_{0}\right)_{B}}}
$$$$
\cong \sqrt{0.37}
$$$$
\cong 0.61
$$

From Eq 18.37,

$$
N \propto\left[\frac{r \sigma_{s}^{0}}{\left(v_{0}\right)^{3 / 2}}\right]^{2}
$$




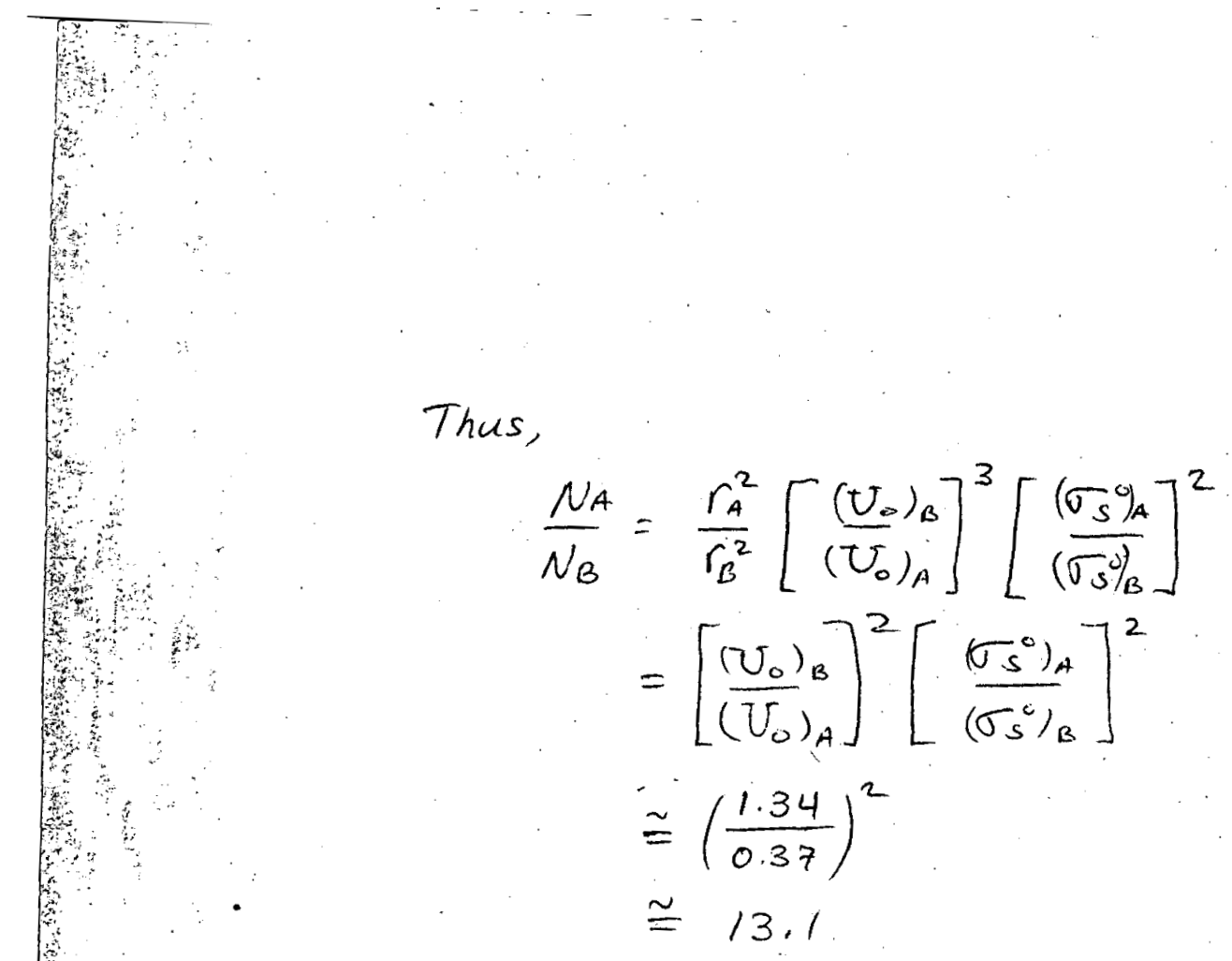

404 
405

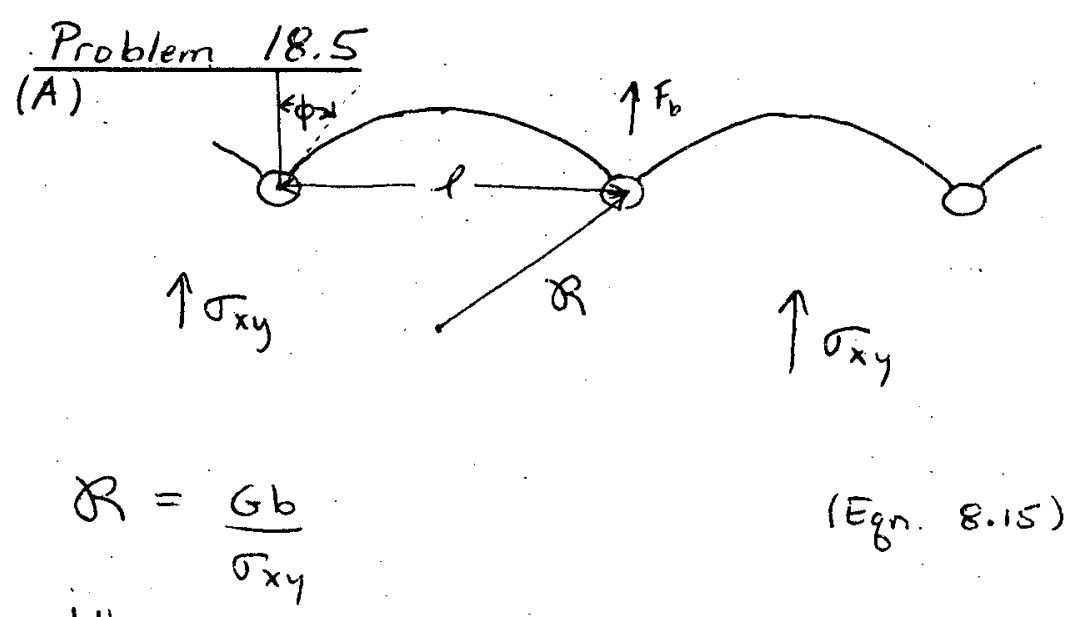

When $x=1 / 2$, dislocation line pinches off and leaves bubble.

$\therefore\left(\sigma_{x y}\right)_{\text {crit i..1 }}=\frac{2 G b}{l}$ ( $E_{q n} 816$ or $E_{q n 18.53)}$

For sweeping,

$$
\sigma_{x y}<\left(\sigma_{x y}\right)_{\text {critical }} \text { : }
$$

Since bubble spacing along line is given by:

$$
l=\frac{1}{\sqrt{2 R N}} \quad\left(E_{q n} 18.25\right)
$$

we have:

$$
\sigma_{x y}<2^{3 / 2} G b \sqrt{R N}
$$

(B)

for bubble sweeping by dislocation line.

Let $V_{b}=$ velocity of dislocation line with bubbles

then,

$v_{b}=$ velocity of bubbles due to force $F_{b}$

$$
\begin{array}{cl}
V_{b}=\Gamma_{b} F_{b} \quad & F_{b}=2 \tau_{d} \cos \phi \quad(E q n \text { 13.276) } \\
\Gamma_{b}=\frac{D_{b}}{K T} \quad \text { (Nernst - Einstein relation) }
\end{array}
$$


406

also,

$$
\begin{aligned}
D_{b} & =\frac{3 a_{0}^{4} D_{s}}{2 \pi R^{4}} \quad\left(E_{(n, 13.214)}\right. \\
\& & \cos \phi=\frac{(l / 2)}{R}=\frac{l}{2 R}=\frac{l \sigma_{x i}}{2 G_{b}}
\end{aligned}
$$

thus,

$$
V_{b}=\frac{3 a_{0}^{4} D_{s}}{2 \pi R^{4} k T} 2 \tau_{d} \frac{l \sigma_{x y}}{2 G b}
$$

Initially,

$$
\begin{array}{ll}
l=\frac{1}{\sqrt{2 R N}} & \left(E_{8 n} 18.25\right) \\
\left(\operatorname{lo}^{2}\right. & \left(E_{\text {n }} 8.10\right)
\end{array}
$$$$
\text { and } \quad \tau_{d}=b^{2}
$$

$$
\therefore \quad\left(v_{b}\right)_{0}=\frac{3 a_{c}^{4} D_{s} b \sigma_{x y}}{2 \pi R^{9 / 2} \sqrt{N} k T}
$$

$\cdot(c)$

The density of bubbles on the slip plane is 2RN. After the dislocation: line has moved a distance $x$, each unit length of line has suet up all bubbles in the area $x, i$ behind it:

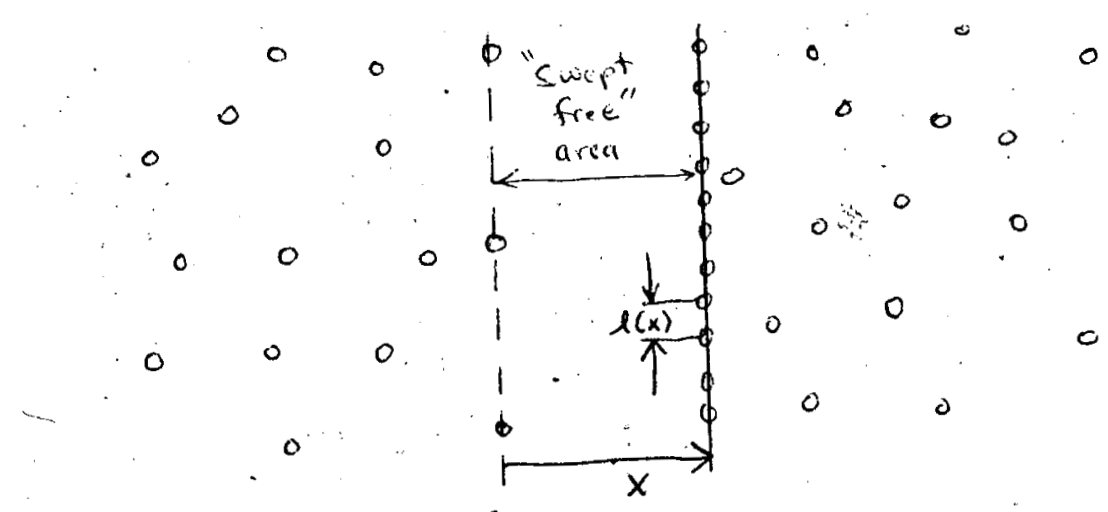

initial position 
407

Number of bubbles per unit length of dislocation line at $x$ is the original number, $\sqrt{2 R N}$, plus the number swept up, $(2 R N)_{x}$

$\therefore$ average bubble spacing $f(x)$ is

$$
l(x)=\frac{1}{\sqrt{2 R N}+2 R N_{x}}=\frac{1}{\sqrt{2 R N}}\left\{\frac{1}{1+x \sqrt{2 R N}}\right\}
$$

The velocity at $x$ is therefore. (since $\left.v_{b} \propto l\right)$ :

$$
V_{b}(x)=\frac{\left(V_{b}\right)_{0}}{1+x \sqrt{2 R N}}
$$

(ie. $\left.\frac{v_{b}(x)}{\left(v_{b_{0}}\right)}=\frac{l(x)}{l_{0}}\right)$ 
408

Problem 18.6

(A)

Force balances $\therefore$ (independent of assumptions of elasticity theory)

Axial:

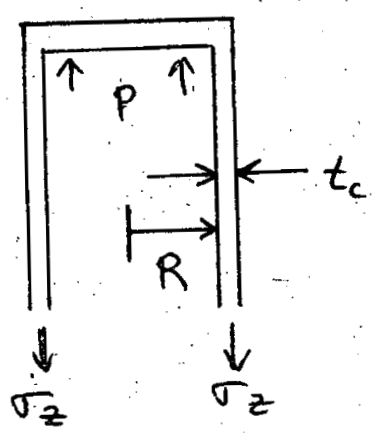

$$
\begin{aligned}
& t_{c} \ll R \\
& 2 \pi R t_{c} \sigma_{z}=\pi R^{2} p \\
& \therefore \sigma_{z}=\frac{R p}{2 t_{c}}
\end{aligned}
$$

Azimuthal:
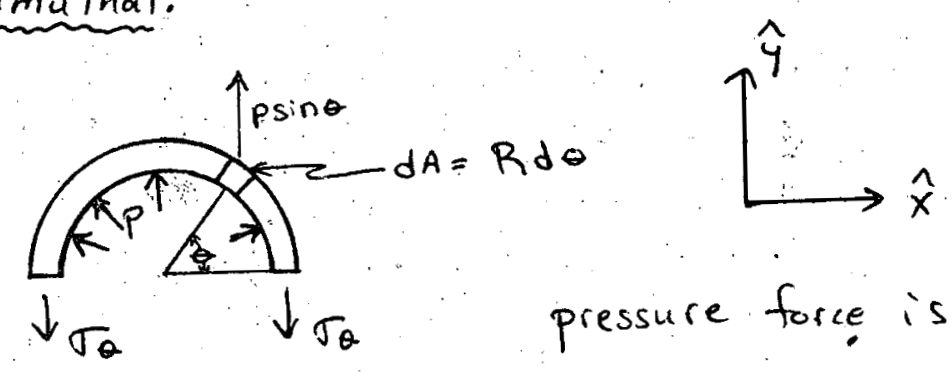

pressure force is in $\hat{r}$ direction $\hat{y}$ component of pressure force, on $d A=p \sin \theta d A$

$\therefore$ total $\hat{y}$ pressure force over 1/2 of tube is given by:

$$
P R \int_{0}^{\pi} \sin \theta d \theta=2 P R
$$

$$
\begin{aligned}
& \therefore 2 P R=2 \sigma_{\theta} t_{c} \\
& \therefore \quad \sigma_{\theta}=\frac{R P}{t_{c}}
\end{aligned}
$$

(force balance) 
409

(B)

From appendix to Ch 8 .

$$
\left.\begin{array}{l}
\epsilon_{r}=\frac{1}{E}\left[\sigma_{r}-\nu\left(\sigma_{\theta}+\sigma_{z}\right)\right] \\
\epsilon_{\theta}=\frac{1}{E}\left[\sigma_{\theta}-\nu\left(\sigma_{r}+\sigma_{z}\right)\right] \\
\epsilon_{z}=\frac{1}{E}\left[\sigma_{z}-\nu\left(\sigma_{r}+\sigma_{\theta}\right)\right]
\end{array}\right\} \begin{aligned}
& \text { from } \\
& (\text { A.21) }
\end{aligned}
$$

Equilibrium conditions:

$$
\begin{aligned}
& \frac{d \sigma_{r}}{d r}+\frac{\sigma_{r}-\sigma_{0}}{r}=0 \\
& \frac{d \sigma_{z}}{d z}=0
\end{aligned} \quad \begin{aligned}
& \text { from } \\
& (A, 29)
\end{aligned}
$$

(7)

Stran-displacement:

$$
\begin{aligned}
& \epsilon_{r}=\frac{d u}{d r} \\
& \epsilon_{0}=\frac{u}{r}
\end{aligned} \quad\{(A .32)
$$

(9)

Plane strain:

$$
\epsilon_{z}=\text { constant }
$$

from (8) \& (9) obtain,

$$
\frac{d \epsilon_{\theta}}{d r}+\frac{E_{\theta}-\epsilon_{r}}{r}=0
$$

Solve (s) for $\sigma_{z}$

(compatiblity condition)

$$
\sigma_{z}=\nu\left(\sigma_{r}+\sigma_{\theta}\right)+E \epsilon z
$$


410

Substitute into (3) \&(4)

(13)

$$
\begin{aligned}
& E \epsilon_{r}=\left(1-\nu^{2}\right) \sigma_{r}-\nu(1+\nu) \sigma_{e}-\nu E \epsilon_{z} \\
& E \epsilon_{\theta}=\left(1-\nu^{2}\right) \sigma_{\theta}-\nu(1+\nu) \sigma_{r}-\nu E \epsilon_{z}
\end{aligned}
$$

(14)

substitute into. (11), recalling plane strain $\rightarrow \frac{\partial \epsilon z}{\partial r}=0$

$$
(1-\nu) \frac{d \sigma_{\theta}}{d r}-\nu \frac{d \sigma_{r}}{d r}+\frac{\sigma_{r}-\sigma_{\theta}}{r}=0
$$

eliminate $\sigma_{\theta}$ by ( 6 ):

$$
\frac{d^{2} \sigma_{r}}{d r^{2}}+\frac{3}{r} \frac{d \sigma_{r}}{d r}=\frac{1}{r^{3}} \frac{d}{d r}\left(r^{3} \frac{d \sigma_{r}}{d r}\right)=0
$$

integrate twice

$$
\sigma_{r}=-\frac{c_{1}}{r^{2}}+c_{2}
$$

Boundary conditions:

$$
\begin{aligned}
& \sigma_{r}\left(R_{i}\right)=-p \\
& \sigma_{r}\left(R_{0}\right)=0
\end{aligned}
$$

$R_{0}=$ outer radius

$$
\therefore \sigma_{r}(r)=-\frac{P}{\left(\frac{R_{0}^{2}}{R^{2}}-1\right)}\left(\frac{\left.R_{0}^{2}-1\right)}{r^{2}}\right)
$$

Use, (17) inc 6) to obtain:

$$
\begin{aligned}
& \sigma_{\theta}=\sigma_{r}+r \frac{d \sigma r}{d r} \\
& \sigma_{\theta}=\left[\frac{P}{\left(\frac{R_{0}^{2}}{R_{i}^{2}}-1\right)} \cdot\left(\frac{R_{0}^{2}}{r^{2}}+1\right)\right]
\end{aligned}
$$

$(18)$

Thin wall approx:

$$
\frac{R_{0}^{2}}{r^{2}} \cong 1
$$

(in $\sigma \operatorname{orn}(y)$ 
411

$$
\begin{aligned}
\frac{R_{0}^{2}}{R_{i}^{2}}-1 \cong \frac{1}{R_{i}^{2}}\left(R_{0}^{2}-R_{i}^{2}\right) & \cong \frac{R_{0}+R_{i}}{R_{i}^{2}}\left(R_{0}-R_{i}\right) \\
& \cong \frac{2}{R} t_{c} \\
\sigma_{\theta}=\frac{R_{p}}{t_{c}} \quad \& \quad \sigma_{r} & =-P\left(\frac{R_{0}-r}{t_{c}}\right) \\
& \approx V \text { with }(i)
\end{aligned}
$$

(c)

Strain components:

substitute (17) \& (12)

$$
\begin{aligned}
& \epsilon_{r}=-\frac{P(1+\nu)}{E\left(\left(R_{0}^{2} / R_{i}^{2}\right)-1\right)}\left[\frac{R_{0}^{2}}{r^{2}}-(1-2 \nu)\right]-\nu \epsilon_{z} \\
& \epsilon_{\theta}=\frac{P(1+\nu)}{\epsilon\left(\left(R_{0}^{2} / R_{i}^{2}\right)-1\right)}\left[\frac{R_{0}^{2}}{r^{2}}+(1-2 \nu)\right]-\nu \epsilon_{z}
\end{aligned}
$$

thin wall:

$$
\epsilon_{0}=\frac{\left(1-\nu^{2}\right) R P}{\epsilon t_{c}}-\nu \epsilon_{z}
$$

axial strain: use (s) with $\sigma_{z}+\sigma_{0}$ from force balance. $\left(\mathrm{eq}_{\mathrm{q}}(1)\right.$-(2)) noting that $\sigma_{r} \ll \sigma_{\theta}$

$$
\epsilon_{z}=\frac{1}{E}\left(\sigma_{z}-\nu \sigma_{c}\right)=\frac{R p}{2 E t_{c}}(1-2 \nu)
$$

(D)

Eliminate strains by combining (3)-(5) with (8) \& (9) a Solve for stress components:

$$
\begin{aligned}
& \sigma_{r}=\frac{E}{1+\nu}\left\{\frac{d u}{d r}+\frac{\nu}{1-2 v}\left(\frac{d u}{d v}+\frac{u}{r}+\epsilon_{z}\right)\right\} \\
& \sigma_{0}=\frac{E}{1+v}\left\{\frac{u}{r}+\frac{\nu}{1-2 v}\left(\frac{d u}{d r}+\frac{u}{r}+\epsilon_{z}\right)\right\}
\end{aligned}
$$


412

$$
\sigma_{z}=\frac{E}{1+\nu}\left\{\epsilon_{z}+\frac{\nu}{1-2 \nu}\left(\frac{d u}{d r}+\frac{u}{r}+\epsilon_{z}\right)\right\} \quad:(2 t)
$$

substitute (24) \&(25) into (6):

$$
\frac{d}{d r}\left(\frac{1}{r} \frac{d(r u)}{d r}\right)=0
$$

(27)

Solution:

$$
u(r)=\frac{c_{1}}{r}+c_{2}
$$

(28)

Cunstants $C_{1} \& C_{2}$ may be determined by substituting (28) into (24) \& applying $B C$ 's (16). u(r), however, is most easily determined bu

(q) \& (21)

$$
\begin{aligned}
& u(r)=r \epsilon_{\theta}=\frac{p(1+\nu) r}{E\left(\left(R_{0}^{2} / R_{i}^{2}\right)-1\right)}\left[\frac{R_{0}^{2}}{r^{2}}+(1-2 \nu)\right]-\nu \epsilon_{z} r
\end{aligned}
$$

(E)

$$
\Delta t_{c}=u\left(R_{0}\right)-u\left(R_{i}\right)
$$

but in thin-wall approx:

$$
\Delta t_{c}=u\left(R_{0}\right)-u\left(R_{i}\right) \cong\left(\frac{d u}{d r}\right) t_{c}
$$

Using (8) obtain,

$$
\therefore \quad \epsilon_{r}=\frac{\Delta t_{c}}{t_{c}}
$$


Problem 18.7

The yield stress is the sum of the long-range
stresses due to the dislocation netwo stresses due to voids \& network and the

$$
\sigma_{y}=\sigma_{L_{R}}+\sigma_{\text {loop }}+\sigma_{\text {void }} \text {. }
$$

From $\left(E_{q i 1} 18.23\right) \&\left(E_{q n} 18.24\right)$

$$
\sigma_{L_{R}}=\frac{G b}{2 \pi l}=\frac{G b \sqrt{P_{d}}}{2 \sqrt{3} \pi}
$$

From $\left(E_{q n}\right.$ 18.62); with $\beta \sim 3$

$$
\sigma_{\text {loop }}=\frac{\sqrt{2} G b}{3} \sqrt{R_{l} N_{l}}
$$

Before unfaulting

$$
\sigma_{y}=G b\left[\frac{\sqrt{P_{d}}}{2 \sqrt{3} \pi}+\frac{\sqrt{2}}{3} \sqrt{R_{l} N_{l}}\right]+\sigma_{\text {oid }}
$$

After unfaulting, loops join dislocation network

$$
\rho_{d}^{\prime}=\rho_{d}+2 \pi R_{l} N_{l} \text { join dislocation network }
$$

$\therefore \sigma_{y}^{\prime}=G b\left[\frac{\sqrt{\rho_{d}+2 \pi R_{l} N_{l}}}{2 \sqrt{3} \pi}\right]+\sigma_{v o d}$.

$\begin{aligned} \therefore\left(\Delta \sigma_{y}\right)_{\text {unfauting }} & =\sigma_{y}^{\prime}-\sigma_{y} \\ & =G\left\{\frac{\sqrt{\rho_{d}}}{2 \sqrt{3} \pi}\left[\sqrt{1+\frac{2 \pi R_{l} N_{l}}{\rho_{d}}-1}\right]-\frac{\sqrt{2}}{3} \sqrt{R_{l} N_{l}}\right\}\end{aligned}$ 
s.

414

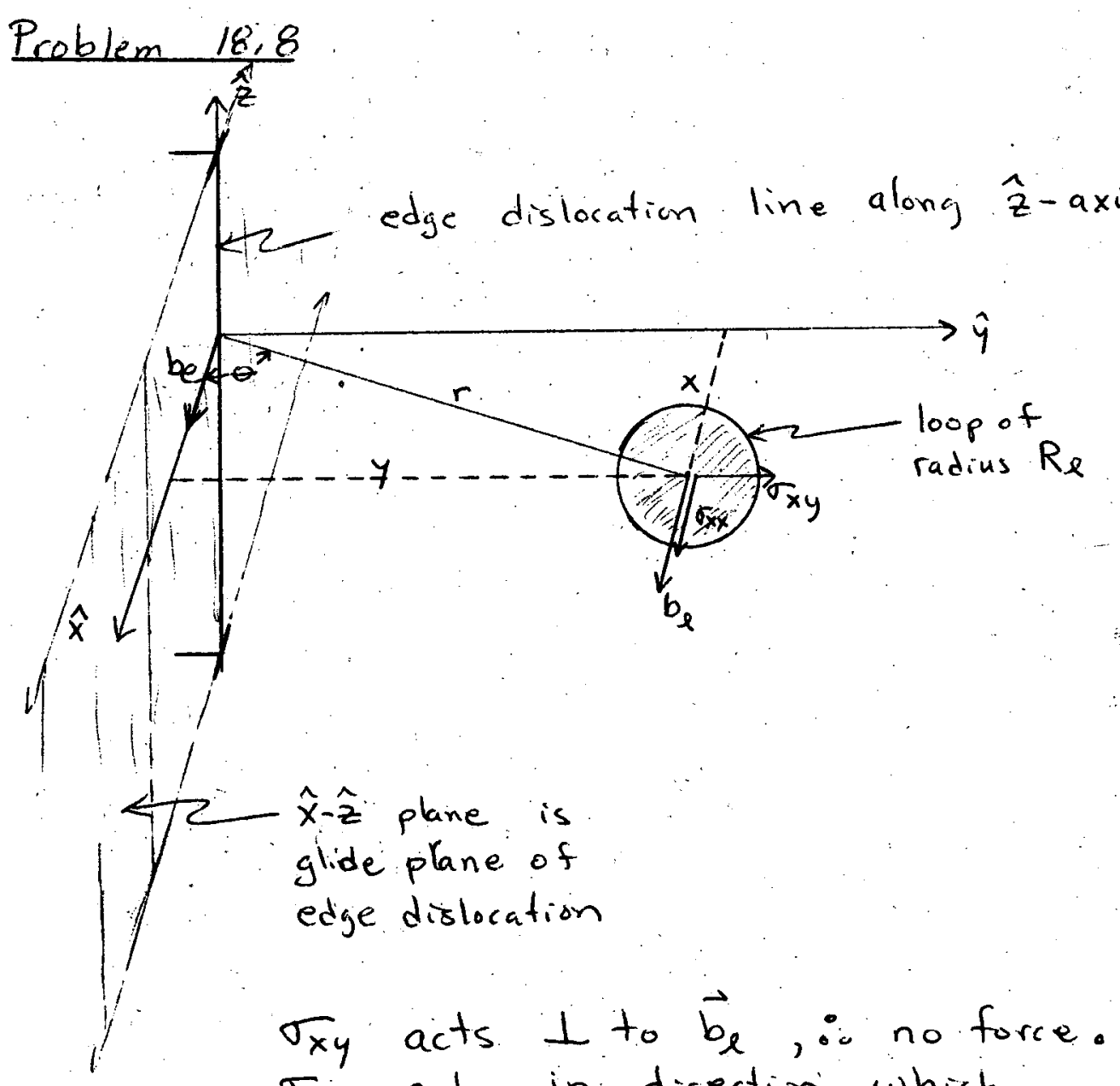
$\sigma_{x x}$ acts in direction which gives force $=b_{l} \sigma_{x x}$ (see Fig 8.10b)

total force on loop by edge dislocation $=\sigma_{x x} b_{l}\left(2 \pi R_{l}\right)$ let $d w_{l}=$ work dine by dislocation as loop expands by $d R_{e}$

$$
d W=\sigma_{x \times} b_{l}\left(2 \pi R_{l}\right) d R_{l}
$$

Integrate to obtain,

$$
W=\sigma_{x x} b_{l} \cdot \pi R_{l}^{2}
$$


415

Stress field of edge dislocation at center of loop (position $(x, y)$ ) given by.

$$
\sigma_{x x}=\sigma_{r r}-2 \sin \theta \cos \theta \sigma_{r}
$$

where

$$
\left(\epsilon_{g \times r} 8.229\right)
$$

$$
\left.\begin{array}{l}
\sigma_{r r}=y \frac{\sin \theta}{r} \\
\sigma_{\theta r}=-\frac{\cos \theta}{r}
\end{array}\right\}(\text { see Fig 8.8) }
$$

\& where $H^{\prime}=\frac{G b_{e}}{2 \pi(1-\nu)}$

Converting to cartesian coordinates

$$
\begin{aligned}
& y=r \sin \theta ; x=r \text { wow } \theta \\
& \therefore \sigma_{r r}=\mathscr{y} \frac{y}{x^{2}+y^{2}} \\
& \text { To }=-y \frac{x}{x^{2}+y^{2}} \\
& \therefore \sigma_{x x}=y\left[\frac{y}{x^{2}+y^{2}}+\frac{2 x y}{x^{2}+y^{2}} \cdot \frac{x}{x^{2}+y^{2}}\right] \\
& =\frac{x y}{\left(x^{2}+y^{2}\right)^{2}}\left[y\left(x^{2}+y^{2}\right)+2 x^{2} y\right] \\
& =\frac{\mathscr{y} y\left(3 x^{2}+y^{2}\right)}{\left(x^{2}+y^{2}\right)^{2}} \\
& \dot{\alpha}=\frac{G b_{e}}{2 \pi(1-\nu)}
\end{aligned}
$$

$$
\omega=\pi R_{l}^{2} b_{e} \mathscr{y} \frac{\left(3 x^{2}+y^{2}\right)}{\left(x^{2}+y^{2}\right)^{2}}
$$


416

Now,

$$
\begin{aligned}
F_{x} & =\frac{d w}{d x} \\
& =\pi R_{l}^{2} b_{l} \& y\left[\frac{6 x}{\left(x^{2}+y^{2}\right)^{2}}-\frac{4 x\left(3 x^{2}+y^{2}\right)}{\left(x^{2}+y^{2}\right)^{3}}\right] \\
& =\pi R_{l}^{2} b_{l} H \frac{2 x y\left(-3 x^{2}+y^{2}\right)}{\left(x^{2}+y^{2}\right)^{3}}
\end{aligned}
$$

or

$$
\begin{array}{r}
\frac{F_{x}}{\pi b_{l} y}=2 \eta \xi \frac{\left(-3 \eta^{2}+\xi^{2}\right)}{\eta^{2}+\xi^{2}} \equiv f(\eta) \\
\quad \eta=\frac{x}{R_{l}}, \quad \xi=\frac{y}{R_{l}}
\end{array}
$$

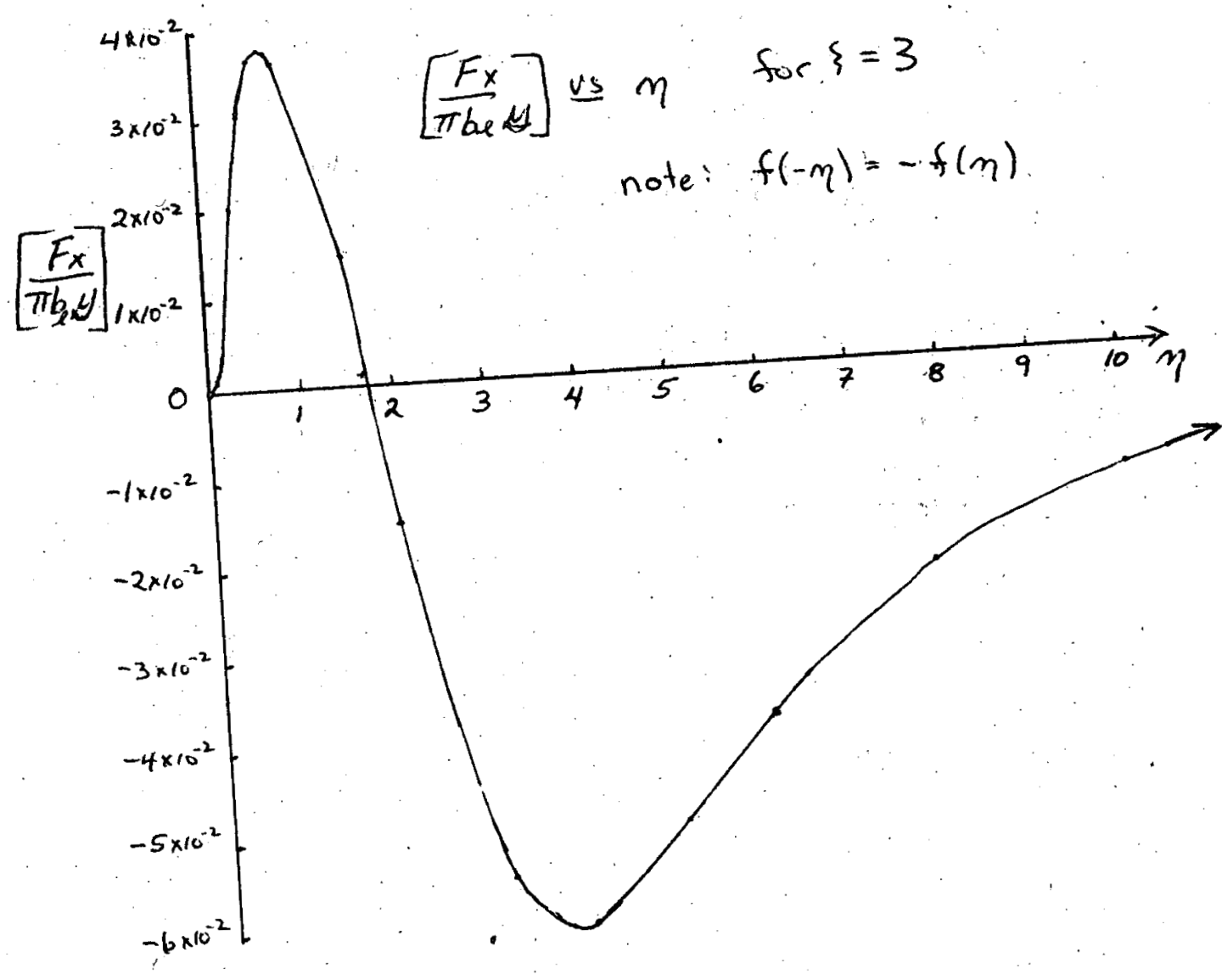


417

Problem 18.9

(A)

There are 36 edges where helium collects.

Each edge is shared between 3 grains

$\therefore$ There are 12 edges per tetrakaidecahedron to accept the $m\left(8 \sqrt{2} l^{3}\right)$ atoms of helium

$\therefore$ Each edge contains:

$$
m=\frac{m 8 \sqrt{2} l^{3}}{12}=\frac{2 \sqrt{2}}{3} m l^{3} \text { atoms He }
$$

(B)

Work to form crack given by "Eq 18,68 with $\sigma$ replaced by $(\sigma+p)$, plus the $p-v$ work of expanding He in the crack:

$$
\begin{aligned}
& W=\frac{-\pi(1-\nu) c^{2}(\sigma+p)^{2} l}{8 G}+2 c \gamma l+ \\
& +\frac{G(n b)^{2} l}{4 \pi(1-\nu)} \ln \left(\frac{4 x}{c}\right)-(\sigma+p) V_{c}-\int_{V_{0}}^{V_{c}} p d V_{c} \\
& \quad \text { where } \quad V_{c}=\frac{1}{2} n b c l . \\
& \text { extra term due } \\
& \text { to gas (p.vwik) }
\end{aligned}
$$

Use ideal gas law

$$
\begin{aligned}
& \therefore P V_{c}=m R T \\
& \therefore P=\frac{2 m R T}{n b \cdot C \cdot l}
\end{aligned}
$$

since

$$
\begin{aligned}
& d V_{c}=\frac{1}{2} n b l d c \\
& p d V_{c}=\frac{2 m R T}{n b c l} \cdot \frac{1}{2} n b l d c=m R T \frac{d c}{c}
\end{aligned}
$$


418

$$
\int_{V_{0}}^{V_{c}} p d V_{c}=m R T \ln \left(\frac{c}{c_{0}}\right)
$$

Thus,

where $C_{0}=$ initial crack length.

$$
\begin{aligned}
W & =\left\{-\frac{\pi(1-\nu) \sigma^{2} \lambda}{8 G}\left[1+\left(\frac{2 m R T}{n b l \sigma}\right) \frac{1}{c}\right]^{2} c^{2}+\right. \\
2 \gamma l c & +\frac{\ell G(n b)^{2}}{4 \pi(1-\nu)} \ln \left(\frac{4 \pi}{c}\right)-\frac{1}{2} n b l \sigma\left[1+\left(\frac{2 m R T}{n b l \sigma}\right) \frac{1}{c}\right] c+ \\
& \left.+\left(-m R T \ln \frac{c}{c_{0}}\right)\right\}
\end{aligned}
$$

(c)

$$
\begin{aligned}
& \text { Set } \frac{d W}{d c}=0 \\
& \left.0=-\frac{\pi(1-\nu) \sigma^{2} l}{8 G}\left\{2\left[1+\left(\frac{2 m R T}{n b l \sigma}\right) \frac{1}{c}\right]^{2} c-\left(\frac{2 m R T}{n b l \sigma}\right) \frac{1}{c^{2}}\left[1+\left(\frac{2 m R T}{n b l \sigma}\right) \frac{I}{c}\right]\right]^{2}\right\} \\
& +2 \gamma l-\frac{G(n b)^{2} l}{4 \pi(1-\nu)} \frac{1}{c}-\frac{1}{2} n b l \sigma-m R T \frac{1}{c} \\
& 0=-\frac{2 \pi(1-\nu) \sigma^{2} l}{8 G} c^{2}-\frac{3 \pi(1-\nu) \sigma^{2} l}{8 G} a c+\frac{\pi(1-\nu) \sigma^{2} l}{8 G} a^{2}+ \\
& +2 \gamma l c-\frac{G(n b)^{2} l}{4 \pi(1-\nu)}-\frac{1}{2} n b l \sigma c-\frac{1}{2} n b l \sigma a
\end{aligned}
$$

where $a \equiv\left(\frac{2 m R T}{n b l \sigma}\right)$

Divide by $\ell$ \& collect coefficients of powers of $c$ :

$$
\begin{aligned}
0= & {\left[\frac{2 \pi(1-\nu) \sigma^{2}}{8 G}\right] c^{2}-\left[-\frac{1}{2} n b \sigma+2 \gamma-\frac{3 \pi(1-\nu) \sigma^{2}}{8 G} a\right] c+} \\
& +\left[\frac{G(n b)^{2}}{4 \pi(1-\nu)}-\frac{\pi(1-\nu) \sigma^{2}}{8 G} a^{2}+\frac{1}{2} n b \sigma a\right]
\end{aligned}
$$


419

Multiply by $\left(\frac{8 G}{2 \pi(1-v) \sigma^{2}}\right)$ to cobain:

$$
\begin{aligned}
0= & C^{2}-\frac{8 \gamma^{1} G}{\pi(1-\nu) \sigma^{2}}\left[1-\frac{n b \sigma}{4 \gamma}-\frac{3 \pi(1-\nu) \sigma^{2}}{16 \gamma^{2} G} a\right]+ \\
& +\frac{8 \gamma G}{\pi(1-\nu) \sigma^{2}} \cdot \frac{G(n b)^{2}}{8 \pi \gamma^{\prime}(1-\nu)}\left[1+\frac{\sigma 4 \pi(1-\nu)}{2 G(n b)} a+\right. \\
& \left.+\left(-\frac{\pi(1-\nu) \sigma^{2}}{8 G \gamma}\right)\left(\frac{8 \pi(1-\nu) \gamma}{G(n b)^{2}}\right) \frac{a^{2}}{2}\right] \\
B_{y} \text { defining } & \quad ; B \equiv \frac{8 \gamma G}{\pi(1-\nu) \sigma^{2}}
\end{aligned}
$$

we may rewrite the above equation as

$$
O=c^{2}-B\left[1-2 \sqrt{\frac{A}{B}}-\frac{3 a}{2 B}\right] C^{2}+A B\left[1+\frac{2 a}{\sqrt{A B}}-\frac{a^{2}}{2 A B}\right]
$$

Condition for neutral stability is obtained by setting discriminant of quadratic formula for $c$ equal to 0 . (Sectext) ie:

$$
\begin{gathered}
B^{2}\left(1-2\left(\sqrt{\frac{A}{B}}-\frac{3 a}{2 B}\right)^{2}=4 A B\left(1+\frac{2 a}{\sqrt{A B}}-\frac{a^{2}}{2 A B}\right)\right. \\
\left(1-4 \sqrt{\frac{A}{B}}+4 \frac{A}{B}\right)-\left(\frac{3}{B}-\frac{6 \sqrt{A}}{B^{3 / 2}}\right) a+\frac{9}{4 B^{2}} a^{2}=\frac{4 A}{B}+ \\
\quad+\frac{8 \sqrt{A}}{B^{3 / 2}} a-\frac{2}{B^{2}} a^{2}
\end{gathered}
$$

Thus,

$$
0=1-4 \sqrt{\frac{A}{B}}-\left(3+2 \sqrt{\frac{A}{B}}\right)\left(\frac{a}{B}\right)+\frac{17}{4}\left(\frac{a}{B}\right)^{2}
$$


420

Upon substitution for $a, A, \& B$ in the above equation, one obtains a quadratic in $\sigma$ which may be solved algebraically for $\sigma$ since the result yields little physical insight, we will make a low pressure approximation, ie $\left(\frac{a}{B}\right) \ll 1$

first order approx:

(1) neglect $(a / B)^{2}$

(2) when $(a / B)=0$ result is

$$
\left(\frac{A}{B}\right)_{0}=\frac{1}{16}
$$

(3) Use 2 in coefficient of $a / B$

$$
\begin{aligned}
& 3+2 \sqrt{\frac{A}{B}} \cong 3+2 \cdot \frac{1}{4} \cong \frac{7}{2} \\
\therefore \quad 1 \cong 4 \sqrt{\frac{A}{B}}+\frac{7}{2}\left(\frac{a}{B}\right) & \left.11 \frac{7(a / B)}{8(\sqrt{a / B})}\right) \\
1 & \cong 4 \sqrt{\frac{A}{B}} \cong \frac{1}{4} \\
1 & \cong 16 \frac{A}{B}\left(1+\frac{7}{2}\left(\frac{a}{B}\right)\right)^{2} \\
\therefore \quad B & \cong 16 A(1+7(9 / B))
\end{aligned}
$$

Using definitions of $A \& B$ in above,

$$
\begin{aligned}
& \frac{8 \gamma^{\prime} G}{\pi(1-v) \sigma^{2}}=\frac{G(n b)^{2} / 6}{8 \pi \gamma^{\prime}(1-\alpha)}\left(1+7 \frac{a}{B}\right) \\
& \frac{8^{2} \gamma^{2}}{4^{2}}=\sigma^{2}(n b)^{2}\left(1+7\left(\frac{a}{B}\right)\right)
\end{aligned}
$$

Taking $\sqrt{ } \&$ using $n b$ from Eq 18.74 obtain:

$$
\sigma_{\text {rr t }}\left(\sigma_{x_{y}}-\sigma_{i}\right) \cong \frac{2 G \gamma}{d}\left(1+7 \frac{a}{B}\right)^{-1 / 2} \cong \frac{2 G \gamma}{d}\left(1-\frac{z}{2} \frac{a}{B}\right)
$$


421

Now,

$$
\left(\frac{a}{B}\right)=\frac{2 m R T_{\pi}(1-\nu) \sigma_{c r}^{2} t}{n b l \sigma_{c r}+8 \gamma G}=\frac{m R T_{\pi}(1-\nu) \sigma_{c r} t}{4\left(\sigma_{x y}-\sigma_{i}\right) d / G l \gamma^{\prime} G}
$$

\& assuming

$$
\begin{aligned}
& \sigma_{\text {crit }} \cong 2\left(\sigma_{x y}-\sigma_{i}\right) \text {; ie } \sigma_{i} \cong 0,2 \sigma_{x y} \cong \sigma \\
& \left(\frac{a}{B}\right) \cong \frac{\pi(1-y) m R T}{2 l \mu^{n} d}
\end{aligned}
$$

Substituting for $m$ from part (a)

$$
\left(\frac{a}{B}\right) \cong \frac{\pi(1-\nu) 2 \sqrt{2} m l^{3} R T}{6 l d \gamma}
$$

\& taking $l \cong d$, obtain

- $\left(\frac{a}{B}\right) \cong \frac{\pi \sqrt{2}(i-\nu) m \mu R T}{3 \mu}$

Thus with $\left(\sigma_{x y}-\sigma_{i}\right) \cong \frac{1}{2} \sigma_{\text {crit }}$

$$
\begin{aligned}
\therefore \sigma_{\text {crit }}= & \left(\frac{4 G H}{D}\right)^{1 / 2}\left(1-\frac{7}{2}\left(\frac{a}{B}\right)\right) \\
= & \left(\sigma_{\text {crit }}\right)_{n_{0}}\left(1-\frac{7 \sqrt{2} \pi(1-\nu) m l R T}{12 \gamma}\right) \\
& \eta_{h}
\end{aligned}
$$

pressure of gas reduces initial stress 
422

Problein 18.10

(A)

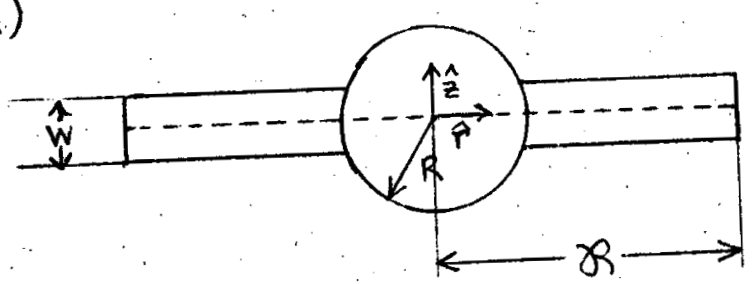

cylindrical coordinates

Vacancy diffusion equation in cylindrical coordinates is.

$$
\begin{aligned}
\frac{1}{r} \frac{\partial}{\partial r}\left(r \frac{\partial C_{v}}{\partial r}\right)+\frac{\partial^{2} C_{v}}{\partial z^{2}} & =0 \\
& <\left\{\begin{array}{l}
\text { source free }\left(G_{v}=0\right) \\
\text { no } \phi \text { dependence }
\end{array}\right\}
\end{aligned}
$$

2 et

$$
\theta=\frac{C_{v}-C_{v}^{e q} \exp \left(\frac{2 \gamma}{R} \frac{\Omega}{k T}\right)}{C_{v}^{e q} \exp \left(\frac{\sigma \Omega}{K T}\right)-C_{v}^{\text {eq }} \exp \left(\frac{2 \gamma}{R} \frac{\Omega}{K T}\right)}
$$

then,

$$
\frac{1}{r} \frac{\partial}{\partial r}\left(r \frac{\partial \theta}{\partial r}\right)+\frac{\partial^{2} \theta}{\partial z^{2}}=0
$$

with foundry conditions:

(1) $C_{V}(R, z)=C_{v}^{e g} \exp \left(\frac{2 \gamma}{R} \cdot \frac{\Omega}{k T}\right)$

$(E \overline{8}+18.80)$

(2) $\left.\frac{\partial c_{v}(r ; z)}{\partial r}\right|_{r=q}=0$

$$
\left(E_{8 n} 18.81\right)
$$

(3) $\left.\frac{\partial C_{v}(r, z)}{\partial z}\right)_{z=0}=0$

(4) $C_{v}\left(r, \pm \frac{w}{2}\right)=C_{v}^{e q g} \exp \left(\frac{\sigma \Omega}{k T}\right)$.

Transforming to $\Theta$, these B.C.'s become.

(i) $\theta(R, z)=0$

$\left.(2)^{\prime} \cdot \frac{\partial \theta}{\partial r}\right)_{r=\gamma}=0$ 


$$
\begin{aligned}
& \text { (3) } \left.\frac{\partial \theta}{\partial z}\right)_{z=0}=0 \\
& \text { (4) } \theta\left(r, \pm \frac{w}{2}\right)=1
\end{aligned}
$$

(B)

Let $\theta(r, z)=P(r) Y(z)$

Insert into diffusion equation \& divide by $G$ :

Thus,

$$
\frac{1}{\operatorname{Pr}} \frac{d}{d r}\left(r \frac{d P}{d r}\right)=-\frac{1}{Y} \frac{d^{2} \tau}{d z^{2}}=-\alpha^{2}
$$

$$
\frac{d^{2} Y}{d z^{2}}-\alpha^{2} Y=0 \Rightarrow \sin ^{-} Y(z)=A \sinh \alpha z+B \cosh \alpha z
$$

Apply $B C$ * (3)' to obtain

$$
A=0 \Rightarrow Y(z)=B \cosh \alpha z
$$

Also,

$$
\frac{1}{r} \cdot \frac{d}{d r}\left(r \frac{d P}{d r}\right)+\alpha^{2} P=0 \text { (Bessel's equation of order } 0 \text { ) }
$$

which has solution

$$
P(r)=E J_{0}(\alpha r)+G N_{0}(\alpha r)
$$

where. Jo \& $N_{0}$ are Bessel functions of first and seicond kind, respictively.

Apgly $B C * 25$ i to pbtain:

$$
E J_{0}^{\prime}(\alpha x)+G N_{0}^{\prime}(\alpha \gamma)=0
$$

Since

$$
\& \begin{aligned}
& J_{0}^{\prime}(z)=J_{1}(z) \\
& N_{0}^{\prime}(z)=N_{1}(z)
\end{aligned}
$$

we cobtain

$\in J_{1}(\alpha X)+G N_{1}(\alpha R)=0$ 
424

Thus,

$$
P(r)=E\left[J_{0}(\alpha r)-\frac{J_{1}(\alpha \alpha)}{N_{1}(\alpha(R)} N_{0}(\alpha r)\right]
$$

Applying. $B C$ \# (I) we find.

$$
P(R)=0 \Rightarrow J_{0}(\alpha R) N_{1}(\alpha R)=J_{1}(\alpha R) N_{c}(\times R)
$$

The roots satisfying the above equation prow de: $\alpha_{n}$ such that:

$$
O(r ; z)=\sum_{n=0}^{\infty} A_{n} \cosh \left(\alpha_{n} z\right)\left\{J_{0}\left(\alpha_{n} r\right)-\frac{J_{1}\left(\alpha_{n} \alpha\right)}{N_{1}\left(\alpha_{n}\right)} N_{0}\left(\alpha_{n} r\right)\right\}(z)
$$

Finely, applying $B C \neq$ (u) ,

$$
\begin{aligned}
& \theta\left(r_{1} \pm \frac{\omega}{2}\right)=1=\sum_{n=0}^{\infty} A_{n} \cosh \left(\alpha_{n} \frac{\omega}{2}\right)\left\{J_{0}\left(\alpha_{n} r\right)-\frac{J_{1}\left(\alpha_{n} X\right)}{N_{1}\left(\alpha_{n} R\right)} n_{0}\left(\alpha_{n} r\right)\right\} \\
& \text { define }\left\{M_{0}\left(\alpha_{n} r\right)\right\}
\end{aligned}
$$

$$
1=\sum_{n=0}^{\infty} A_{n} \cosh \left(\alpha_{n} \frac{\omega}{2}\right)\left\{M_{0}\left(\alpha_{n} r\right)\right\} \text {. }
$$

To find coefficients $A_{n}$, multiply both sides of above equation by: $r M_{0}(\alpha m r)$ \& integrate from $R$ to $R$ By orthogonality property obtain

$$
\begin{aligned}
\frac{m \neq n]}{I} \equiv & \int_{R}^{\alpha} r m_{0}\left(\alpha_{n} r\right) m_{0}\left(\alpha_{m} r\right) d r=\frac{1}{\left(\alpha_{n}^{2}-\alpha_{m}^{2}\right)}\left\{\alpha_{m} r m_{0}\left(\alpha_{n} r\right) m_{-1}\left(\alpha_{m} r\right)+\right. \\
& \left.+\left(-\alpha_{n} r\right) m_{-1}\left(\alpha_{n} r\right) m_{0}\left(\alpha_{m} r\right)\right\}\left.\right|_{R} ^{R}\left\{\alpha_{m}\left[R m_{0}\left(\alpha_{n} R\right) m_{-1}\left(\alpha_{m} R\right)\right]-\alpha_{n}\left[R m_{0}\left(\alpha_{n} R\right) \cdot\right.\right. \\
& =\frac{1}{\left(\alpha_{n}^{2}-\alpha_{m}^{2}\right)}\left\{\alpha_{-1}\left(\alpha_{m} R\right)\right]-\alpha_{n}\left[R m_{-1}\left(\alpha_{n} R\right) m_{0}\left(\alpha_{m} R\right)\right]+\alpha_{n}\left[\alpha M_{-1}\left(\alpha_{n} R\right)\right. \\
& \left.\left.=m_{0}\left(\alpha_{m} R\right)\right]\right\}
\end{aligned}
$$


Nicting that

$$
m_{n}\left(\alpha_{n} R\right)=(-1)^{n} m_{n}\left(d_{n} R\right)
$$

\&. $\left.m_{1}\left(\alpha_{n} X\right)=J_{1}\left(\alpha_{n} R\right)-\frac{J_{1}\left(\alpha_{n} R\right)}{N_{1}\left(\alpha_{n} R\right)} N_{1} \alpha_{n} R\right)=0$ obtain,

$I=\frac{1}{\left(\alpha_{n}^{2}-\alpha_{m}^{2}\right)}\left\{\alpha_{m}\left[-R m_{0}\left(\alpha_{n} R\right) M_{1}\left(\alpha_{m} R\right)\right]+\alpha_{n}\left[R m_{1}\left(\alpha_{n} R\right) m_{0}\left(\alpha_{m} R\right)\right]\right\}$ $=\frac{1}{\left(\alpha_{n}^{2}-\alpha_{m}^{2}\right)}\left\{R\left[\alpha_{n} m_{1}\left(\alpha_{n} R\right) m_{0}\left(\alpha_{m} R\right)-\alpha_{m} m_{0}\left(\alpha_{n} R\right) m_{1}\left(\alpha_{m} R\right)\right]\right\}$ Finelly note that

- $M_{0}\left(\alpha_{n} R\right)=J_{0}\left(\alpha_{n} R\right)-\frac{I_{1}\left(\alpha_{n} R\right)}{N_{1}\left(\alpha_{n} R\right)} N_{0}\left(\alpha_{n} R\right)=0$

Thus, because of $E_{q_{n}}(1)$

$$
I=0 \text { for } m \neq n
$$

For m-n:

$$
\begin{aligned}
I & =\int_{R}^{R} r m_{0}^{2}\left(\alpha_{r} r\right) d r=\left.\frac{1}{2} r^{2}\left[m_{0}^{2}\left(\alpha_{n} r\right)+m_{1}^{2}\left(\alpha_{n} r\right)\right]\right|_{R} ^{R} \\
& =\frac{1}{2}\left\{B^{2}\left[m_{0}^{2}\left(\alpha_{n} R\right)+m_{1}^{2}\left(\alpha_{n} R\right)\right]-R^{2}\left[m_{0}^{2}\left(\alpha_{n} R\right)+m_{1}^{2}\left(\alpha_{n} R\right)\right]\right\}
\end{aligned}
$$

where

$$
m_{1}\left(\alpha_{n} r\right)=-\frac{d n_{i}\left(x_{n r}\right)}{d\left(\alpha_{n r}\right)}=J_{1}\left(\alpha_{n r}\right)-\frac{J_{1}\left(\alpha_{n} R\right)}{N_{1}\left(\alpha_{n} R\right)} N_{1}\left(\alpha_{n} r\right)
$$

Thins, using $E_{q n}(3)$,

$$
\begin{aligned}
& \int_{R}^{x} r M_{0}\left(\alpha_{n} r\right) d r=A_{n} \cosh \left(\alpha_{n} \frac{w}{2}\right) I_{(n=m)}{ }^{+} 0 \\
& A_{n}=\frac{\int_{R}^{\alpha} r m_{0}\left(\alpha_{n} r\right) d r}{\cosh \left(\alpha_{n} \frac{\alpha}{2}\right) I_{(n=m)}} \quad \begin{array}{l}
n_{1} \\
\text { alt other terms }
\end{array}
\end{aligned}
$$


$423 a$

Now,

$$
\begin{aligned}
\int_{R}^{R} r m_{0}\left(\alpha_{n} r\right) d r & =-\left.\frac{1}{\alpha_{n}^{2}}\left(\alpha_{n} r\right) m_{1}\left(\alpha_{n} r\right)\right|_{R} ^{R} \\
& =\frac{1}{\alpha_{n}}\left[R M_{1}\left(\alpha_{n}(\alpha)-R M_{1}\left(\alpha_{n} R\right)\right] .\right.
\end{aligned}
$$

So

$$
A_{n}=\frac{\frac{1}{\alpha_{n}}\left[\alpha m_{1}\left(\alpha_{n} \alpha\right)-R m_{1}\left(\alpha_{n} R\right)\right]}{\cosh \left(\alpha_{n} \cdot \frac{w}{2}\right) \cdot I_{n=m}}
$$

where $I_{n=m}$ is given by $E_{q n} \cdot(4)$

$$
\& \theta(r, z)=\sum_{n=0}^{\infty} A_{n} \cosh \left(\alpha_{n} z\right) M_{0}\left(\alpha_{n} r\right) \quad\left(\epsilon_{q n} 2\right)
$$

(C)

Rate of void growth is obtained by "volume balance"

$$
\begin{aligned}
\frac{d}{d t}\left(\frac{4}{3} \pi R^{3}\right) & =2 \Omega \int_{0}^{w / 2} J(z) d z \\
& \left.4 \pi R^{2} \frac{d R}{d t}=2 \Omega \int_{0}^{w / 2}(2 \pi R)\left\{D_{v g b} \frac{\partial C v}{d r}\right)_{R} d z\right\} \\
\therefore \quad \frac{d R}{d t}= & \frac{D_{u g} b \Omega C_{r}^{e q}}{R}\left[\exp \left(\frac{\sigma r}{k T}\right)-\exp \left(\frac{2 \gamma}{R} \frac{\Omega}{k T}\right)\right] \int_{0}^{w / 2}\left(\frac{\partial t}{\partial r}\right)_{R} d z
\end{aligned}
$$

Since $\sigma \Omega<k T \& \frac{2 \gamma \Omega}{R}<k T$ after nucleation,

we can write:

$$
\begin{aligned}
& \text { can write: } \\
& \frac{d R}{d t} \cong \frac{D_{r g b} \Omega}{R k T}\left(\sigma-\frac{2 \beta^{1}}{R}\right) \int_{0}^{w / 2}\left(\frac{\partial \theta}{\partial r}\right)_{R} d z
\end{aligned}
$$

Since $\left.\frac{\partial \theta}{\partial r}\right)_{R}=-\sum_{n=0}^{\infty} A_{n} \cosh \left(\alpha_{n} z\right) \alpha_{n} m_{1}\left(\alpha_{n} R\right)$

$$
\therefore \frac{d K}{d t}=-\frac{D_{g b}}{R k T} \Omega\left(\sigma-\frac{2 \gamma}{R}\right) \sum_{n=0}^{\infty} A_{n} \sinh \left(\frac{\alpha_{n} w}{2}\right) m_{1}\left(\alpha_{n} R\right)
$$


426

Problem 18.11

$$
\therefore=\sigma_{a} \times \frac{1}{\left(\begin{array}{c}
\text { fraction of initial grain boundary area } \\
\text { not occupied by bibles }
\end{array}\right)}
$$

where

$$
\begin{aligned}
& \sigma_{a}=\text { applied stress. } \\
& \therefore \sigma=\sigma_{a} \times\left(\frac{1}{1-f_{b}}\right)
\end{aligned}
$$

where.

$f_{b}=$ fraction of grain boundary occupied by bubbles.

Now,

$$
f_{b} \cong\left(\frac{R}{R}\right)^{2}
$$

$\therefore \quad \sigma \cong \frac{\sigma_{a}}{1-\left(\frac{R}{R}\right)^{2}} \ll$ use in $E_{q n} 18.85$ 
Problem 18.12

$$
\begin{aligned}
& \text { Major } \mathrm{He}^{4} \text { production reactions in Type } 304 \mathrm{~s} . \mathrm{s} \\
& \frac{\text { Thermal flux }}{B^{10}(n, \alpha)} \quad \frac{\text { Fast }}{B^{10}(n, \alpha)} \\
& \left\{\begin{array}{l}
N_{i}^{58}+n \rightarrow N_{i}^{59}+\gamma \\
N_{i}^{59}+n \rightarrow \mathrm{Fe}^{56}+H_{e}^{4}
\end{array}\right\} \quad N_{i}(n, \alpha) \\
& \mathrm{Fe}(n, \alpha) \\
& \operatorname{Cr}(n, \infty)
\end{aligned}
$$

let

$$
\begin{aligned}
& N=\text { number density of atoms } \\
& \phi_{T}=\text { thermal flux } \\
& \phi_{F}=\text { fast flux }
\end{aligned}
$$

Atom balance differential equations:

$$
\begin{aligned}
& \frac{d N_{f e}}{d t}=-N_{F_{e}} \sigma_{F}^{F_{e}} \phi_{F} \\
& \frac{d N_{c r}}{d t}=-N_{c r} \sigma_{F}^{c r} \phi_{F} \\
& \frac{d N_{B^{10}}}{d t}=-N_{B^{\prime 0}} \sigma_{T}^{B^{10}} \phi_{T}-N_{B^{10}} \sigma_{F}{B^{10}} \phi_{F} \\
& =-N_{B^{\prime o}}\left(\sigma_{T}^{B^{\prime 0}} \phi_{T}+\sigma_{F} B^{10} \phi_{F}\right)
\end{aligned}
$$

For $N_{i}$ we can decouple fast \& thermal components if we assume only small fraction of $N$ : will be consumed $\left(N_{N_{i}}(t) \cong N_{N_{i}^{0}}\right)$. ie. Write separate equations for fast \& thermal He production, then add to obtain total He production by $\mathrm{Ni}_{i}$. Thus, for fast component

$$
\begin{aligned}
\frac{d N_{N_{i}}^{(F)}}{d t} & =-N_{N_{i}} \sigma_{F}^{N_{i}} \phi_{F} \\
& \cong-N_{N_{i}}^{(F)} \sigma_{F}^{N_{i}} \phi_{F}
\end{aligned}
$$

where

$$
N_{N_{i}}^{(F)}=\text { number of } N_{i} \text { atums if } \phi_{r}=0
$$


$\&$ for thermal component of $N_{i}^{s 8} \frac{\text { atoms }}{\mathrm{cm}^{3}}$ if $\phi_{F}=0$

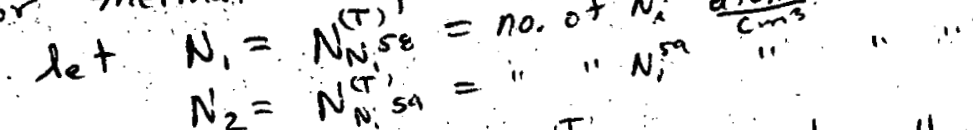

$N_{2}=N_{N}^{\left(s_{1}\right.}=N_{\text {He(N) }}^{T}=$ number He atoms $\mathrm{km}^{3}$ produced

by thermal necutrons on nickel.

$$
\begin{aligned}
& \frac{d N_{1}}{d t}=-N_{1} \sigma_{T}^{N_{i}^{s 8}} \phi_{T} \\
& \frac{d N_{2}}{d t}=-\frac{d N_{1}}{d t}-N_{2} \sigma_{T}^{N_{i}^{s q}} \phi_{T}
\end{aligned}
$$

Equations $1,2,3,4$ have solutions

$$
\begin{aligned}
& N_{F e}=N_{F e}^{0} e^{-\sigma_{F}^{F e} \phi_{F} t} \\
& N_{c r}=N_{c r}^{o} e^{-\sigma_{F}^{c r} \phi_{F} t}
\end{aligned}
$$

$N_{B^{10}}=N_{B^{10}}^{0} e^{-\left(\sigma_{T}^{B^{i *}} \phi_{T}+\sigma_{F}^{B^{10}} \phi_{F}\right) t}$

$N_{N_{i}}^{(F)}=N_{N_{i}}^{(F)} e^{-\sigma_{F}^{N i} \phi_{F} t}$

Equations 5,6 may be combined:

$$
\frac{d N_{2}}{d t}=N_{1} \sigma_{T}^{N_{1}^{s e}} \phi_{T}-N_{2} \sigma_{T}^{N_{T}^{N q}} \phi_{T}
$$

Equation (5) may be solved \&substitated into (11):

$$
\begin{aligned}
& N_{1}=N_{1}^{0} e^{-\sigma_{T}^{N_{s}^{s e}} \phi_{T} t} \\
& \frac{d N_{2}}{d t}=N_{1}^{0} \sigma_{T}^{N_{1}^{s t}} \phi_{T} e^{-\sigma_{T}^{N_{1}^{s t}} \phi_{T} t}-N_{2} \sigma_{T}^{N_{1}^{s t}} \phi_{T}
\end{aligned}
$$

This equation appears often in discussion of compind decay (see eg Foster \& wirght PP 49-50) and has solution: 


$$
\begin{aligned}
& N_{2}=\left(\frac{\lambda_{1}}{\lambda_{2}-\lambda_{1}}\right) N_{1}^{0}\left(e^{-\lambda_{1} t}-e^{-\lambda_{2} t}\right)+N_{2}^{0} e^{-\lambda_{2} t} \\
& \text { where } \lambda_{1}=\sigma_{T}^{58} \phi_{T} \\
& \lambda_{2}=\sigma_{T}^{59} \phi_{T} .
\end{aligned}
$$

$$
\begin{aligned}
& \text { Now, } \\
& N_{1}+N_{2}+N_{3}=N_{1}^{O}+N_{2}^{0} \\
& \therefore N_{3}=N_{1}^{0}+N_{2}^{0}-N_{1}-N_{2}
\end{aligned}
$$

Similarly, for equations $7,8,9,10$, the number of He atoms $/ \mathrm{cm}^{3}$ from $X(n, x)$ reactions $=$ the number of $X$ atoms/can"buint" $=N_{x}^{0}-N_{x}(t)$

Thus,

$$
\begin{aligned}
& N_{H e}=N_{F e}^{0}\left(i-e^{-\sigma_{F}^{F_{C}} \phi_{F} t}\right)+N_{e r}^{0}\left(1-e^{-\sigma_{F}^{\sigma_{r} \phi_{F} t}}\right)+
\end{aligned}
$$

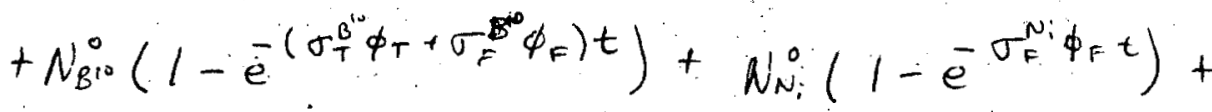

$$
\begin{aligned}
& +\left[N_{N_{i}^{s 8}}^{0}\left(1-e^{-\sigma_{T}^{N_{T}^{58} \phi_{T} t}}\right)+N_{N^{54}}^{0}\left(1-e^{-\sigma_{T}^{N_{T}^{54}} \phi_{T} t}\right)+\right. \\
& +\left(\left(\frac{-\lambda_{1}}{\lambda_{2}-\lambda_{1}}\right) N_{N_{1}}^{0}\left(e^{-\lambda_{1} t}-e^{-\lambda_{2} t}\right)\right]
\end{aligned}
$$

Now,

$$
\begin{aligned}
& \begin{array}{l}
\phi_{F}=10^{15} \mathrm{n} / \mathrm{cm}^{2}-\operatorname{sic} \\
\phi_{T}=10^{13} \quad t=3.15 \times 10^{7} \mathrm{sec} .
\end{array} \\
& \phi_{T}=10^{F e}=0.23 \times 10^{27} \mathrm{~cm}^{2} \rightarrow t \lambda \mathrm{cm}^{2} \mathrm{sec} \sigma_{F}^{F e} \phi_{F} t=724 \times 10^{-6} \\
& \sigma_{F}^{c r}=02 \times 10^{-27} \mathrm{~cm}^{2} \longrightarrow t \lambda^{c r}=\sigma_{F}^{c r} \phi_{F} t=6.3 \times 10^{-6} \\
& \sigma_{F}^{N}=42 \times 10^{-27} \mathrm{~cm}^{2} \quad \longrightarrow t \lambda \lambda^{N}=\sigma_{F}^{N} \phi_{F} t \quad 1.32 \times 10^{-4} \\
& \sigma_{F}^{B^{10}}=623 \times 10^{27} \mathrm{~cm}^{2} \rightarrow \rightarrow \lambda_{F}^{B^{10}}=\sigma_{F} B^{10}=t=196 \times 10^{-2} \\
& \sigma_{T} B^{10}=3840 \times 10^{-24} \mathrm{~cm}^{2} \quad \rightarrow t \lambda \quad \rightarrow \lambda_{T} \sigma_{T}^{10} \phi t=121 \\
& \sigma_{T} u^{5}=4.4 \times 10^{-24} \mathrm{~cm}^{2} \quad \rightarrow t \lambda_{1}=1.39 \times 10^{-3} \\
& \sigma_{T}^{N 59}=13.0 \times 10^{-34} \mathrm{~cm}^{2} \rightarrow t \lambda_{2}=41 \times 10^{-3}
\end{aligned}
$$


430

Also,

$$
N_{i}^{0}=f_{i} \cdot \frac{P_{S S}}{A_{i}} \times 6.02 \times 10^{23} \frac{a \operatorname{tornt} t}{m+c} \times I_{i}
$$

where

$f_{i}=$ weight fraction of element $i=\left(\right.$ weight $\left.\eta_{i}\right) / 100$

$\rho_{\text {ss }}=$ mass density of stainless steel $\cong 8.32 \mathrm{gm} / \mathrm{cm}$

$A_{i}=$ atomic weight of element' $i$

$I_{i}=$ natural isotope fraction of isotope $i$

Therefore,

$$
\begin{aligned}
& N_{F_{e}}^{c}=\left(\frac{70}{100}\right) \cdot \frac{8.32}{55.85} \times 6.02 \times 10^{23}=6.28 \times 10^{22}\left[\frac{a+c o n}{c m} c^{2}\right] \\
& N_{c r}^{c}=\left(\frac{19}{100}\right) \cdot \frac{8.32}{520} \times 6.02 \times 10^{22}=1.83 \times 10^{22} \\
& N_{B^{10}}^{0}=\left(\frac{10005}{100}\right) \cdot \frac{8.32}{10.0^{1}} \times 6.02 \times 10^{23} \times 0.196=4.90 \times 10^{17} \\
& N_{N_{i}}^{0}=\left(\frac{9}{100}\right) \cdot \frac{8.32}{58.71} 6.02 \times 10^{23}=\quad 7.68 \times 10^{21} \\
& N_{i}^{0}=\left(\frac{9}{100}\right) \cdot \frac{8.32}{58.71} 6.02 \times 10^{23} \times 0.679=5.21 \times 10^{21} \\
& N_{N}^{0} 5^{-9}=\left(\frac{9}{100}\right) \cdot \frac{8.32}{59} 6.02 \times 10^{23} \times 0= \\
& N_{m_{n}}=\left(\frac{0.8}{100}\right) \cdot \frac{8.32}{84.94} 6.02 \times 10^{23}= \\
& 7.29 \times 10^{9-0} \\
& N_{S_{i}}^{0}=\left(\frac{0.5}{100}\right) \cdot \frac{8.32}{28.09} 6.02 \times 10^{23}=892 \times 10^{20} \\
& N_{m_{0}}=\left(\frac{0.2}{100}\right) \cdot \frac{8.32}{95.74} 6.02 \times 10^{23}=\quad 1.04 \times 10^{20} \\
& N_{c}^{i}=\left(\frac{0.06}{100}\right) \cdot \frac{8.32}{12.01} 6.02 \times 10^{23}=\quad 2.50 \times 10^{20} \\
& N^{0} \cong \sum N_{i}^{0} \times\left(-\frac{100 \%}{99.5605 \%}\right) \cong 9.08 \times 10^{22} \times \frac{100}{19.560 .5} \\
& \cong 9.12 \times 10^{22} / \mathrm{cm}^{3}
\end{aligned}
$$

where

$$
N^{0}=\text { initial number of atoms in } 1 \mathrm{~cm}^{3} \mathrm{sis}
$$

Q where factor $\sim\left(\frac{100}{99.5005}\right)$ compensates for "minor elements" not curbed in sum by assuming they are of same "average atomic mass" as "major elements". 
431

Approximating,

$$
e^{-\lambda t} \cong 1-\lambda t+\frac{(\lambda t)^{2}}{2}-\cdots . \quad \text { for }|\lambda t|<<1
$$

obtain, using $E_{q n}(/ 6)$ :

$$
\begin{aligned}
& N_{\text {He }} \cong\left(6.28 \times 10^{22}\right)\left(7.24 \times 10^{-6}\right)+ \\
& +\left(1.8 .3 \times 10^{22}\right)\left(6.3 \times 10^{-6}\right)+\left(4.90 \times 10^{(2)}\right)\left(1-e^{-\left(1.21+1.96 \times 10^{-2}\right)}\right)+ \\
& +\left(7.68 \times 10^{21}\right)\left(1.32 \times 10^{-4}\right)+\left(5.21 \times 10^{21}\right)\left(1-1+\lambda_{1} t-\frac{\left.a_{1} t\right)^{2}}{2}+\right. \\
& \left.+\left(\frac{-\lambda_{1}}{\lambda_{2}-\lambda_{1}}\right)\left(1-\lambda_{1} t+\frac{\left(\lambda_{1} t\right)^{2}}{2}-1+\lambda_{2} t-\frac{\left(\lambda_{2} t\right)^{2}}{2}\right)\right] \\
& \cong 4.5 .5 \times 10^{17}+1.15 \times 10^{17}+\left(4.90 \times 10^{17}\right)(1-0.242)+ \\
& +\left(1.01 \times 10^{18}\right)+\left(5.2 \times 10^{\prime \prime}\right)\left[\frac{\left[\left(\lambda_{1} t\right)\left(\lambda_{2} t\right)\right.}{2}\right] \\
& \therefore N_{H e} \cong 1.95 \times 10^{18} \mathrm{~cm}^{3} \quad 2.85 \times 10^{-6} \\
& \therefore \quad \frac{N_{H e}}{N^{6}} \times 10^{6}=\frac{1.95 \times 10^{18}}{9.12 \times 10^{22}} \times 10^{6} \cong 21.4 \mathrm{ppm} \mathrm{He}
\end{aligned}
$$

Note: Thermal nickel term makes only small addition to Nu e relative to cither. terms \& may be neglected in less exacting. analysis. 
432

Problem 18.13

$$
\sigma=\frac{1}{2} \sigma_{\text {crit }}=\frac{1}{2}\left(\frac{4}{3 \sqrt{3}} \frac{\gamma}{R_{0}}\right)=\frac{2}{3 \sqrt{3}} \cdot \frac{\gamma}{R_{0}}
$$

where $\sigma_{\text {crit }}$ is given by $E_{q n} 18.98$

Substitute for $\sigma$ in Eq n 18.97:

$$
\frac{2}{3 \sqrt{3}} \frac{\gamma}{R_{0}}=\frac{2 \gamma}{R}\left(1-\frac{R_{0}^{2}}{R^{2}}\right)
$$

or

$$
R^{3}-R_{0} 3 \sqrt{3} R^{2}+3 \sqrt{3} R_{0}^{3}=0 \text {, where } R_{0}=1000 \AA \text {. }
$$

The above cubic equation in $R$ may be solved numerically or analytically (by general cubic formula) to yield real $>0$ root:

$$
\begin{aligned}
& R=\sqrt{3}\left(1+\left(2 \cos 20^{\circ}\right)\right) R_{0} \cong 4.987 R_{0} \\
& \therefore R \cong 4987 \AA
\end{aligned}
$$

When 2 bubbles coalesce, the stress-free radius, $R_{0}$,' is (from Egg. 18.44)

$$
\left.\begin{array}{l}
R_{0}^{2} \propto m \\
R_{0}^{\prime 2} \propto 2 m
\end{array}\right\} \Rightarrow R_{0}^{\prime}=\sqrt{2} R_{0}
$$

The stress remains the same \& applying Eq. 18.97 to the coalesced bubble of radius $R^{\prime}$, obtain.

$$
\sigma=\frac{2 \gamma}{3 \sqrt{3} R_{0}}=\frac{2 \gamma}{R^{\prime}}\left(1-\frac{R_{0}^{\prime 2}}{R^{\prime 2}}\right)=\frac{2 \gamma}{R^{\prime}}\left(1-2 \cdot \frac{R_{0}^{2}}{R^{\prime 2}}\right)
$$

or

$$
R^{\prime 3}-R_{0} 3 \sqrt{3} \cdot R^{2}+6 \sqrt{3} R_{0}^{3}=0 ; R_{0}=1000 \AA
$$

Numerical or analytical solution yields

$$
\begin{aligned}
R^{\prime} & =\sqrt{3} R_{0} \\
& \cong 1732 \AA
\end{aligned}
$$

Note for this example, $R^{\prime}<R$ 
433

Problem 18.14

(A) Equilibrium conditions (no stress):

at bubble surface

$$
P=\frac{2 \gamma}{\rho}
$$

at intersection with grain boundary,

$$
\gamma_{g b}=2 \gamma \cos \theta
$$

(2)

ideal gas law
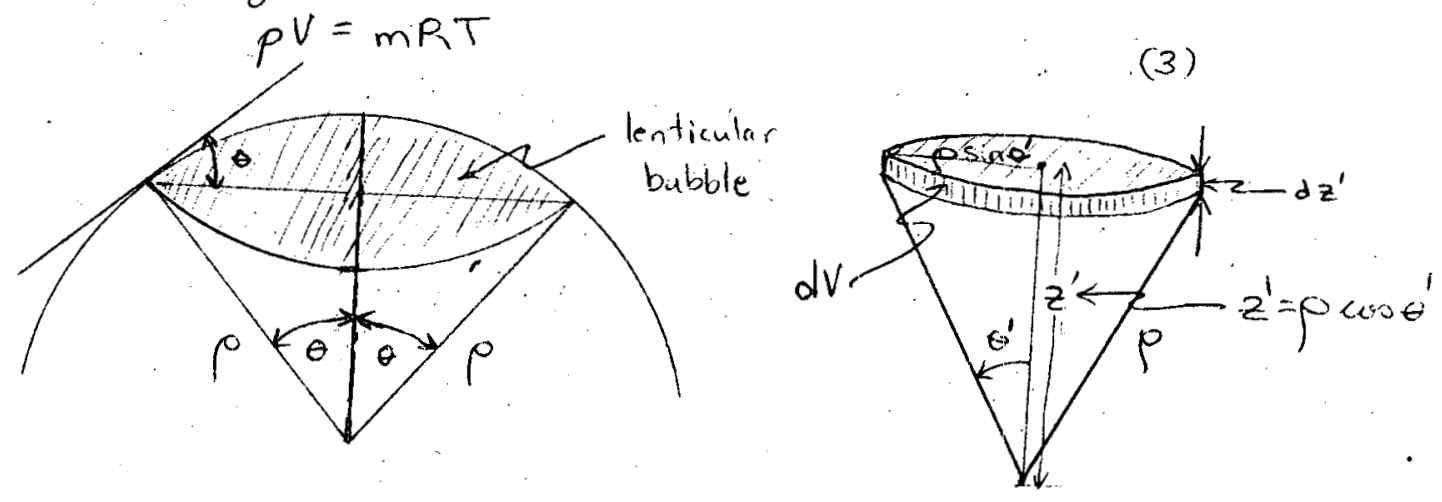

To find volume of top half of lenticular bubble, integrate $d V=\pi\left(p \sin \theta^{\prime}\right)^{2} d z^{\prime}$ from $z^{\prime}=\rho \cos \theta$ to $z^{\prime}=\rho:$

$$
\begin{aligned}
& \frac{1}{2} V=\int_{p=0}^{p} \pi \rho^{2} \sin ^{2} \theta^{\prime} d z^{\prime} \quad ; \quad \operatorname{lt}\left\{\begin{array}{l}
\mu=\cos \theta \\
\mu^{\prime}=\cos \theta^{\prime}
\end{array}\right. \\
& =\int_{\Gamma \mu}^{\rho} \pi \rho^{2} \cdot\left(1-\mu^{\prime 2}\right) d z^{\prime} \\
& =\pi \rho^{2} \int_{\rho \mu}^{p}\left(1-\frac{z^{2}}{p^{2}}\right) d z^{\prime} \\
& =\pi \rho^{3}\left[(1-\mu)-\frac{\left.\left(1-\mu^{3}\right)\right]}{3}=\frac{\pi \rho^{3}}{3}\left(3-3 \mu-1+\mu^{3}\right) .\right. \\
& \therefore V=\frac{2 \pi \rho^{3}}{3}(1-\mu)^{2}(2+\mu) \\
& (4)
\end{aligned}
$$


434

Eliminating p from (1)\&(3) obtain,

$$
\frac{2 y}{\rho}=\frac{m k T}{v}
$$

Using (4),

$$
\begin{aligned}
& \frac{2 \gamma}{\rho}=\frac{3 m k T}{2 \pi \rho^{3}(1-\mu)^{2}(2+\mu)} \\
& \therefore \quad \rho=\left[\frac{3 m k T}{4 \pi \gamma(1-\mu)^{2}(2+\mu)}\right]^{1 / 2} \\
& \sum \text { when } \gamma_{g b}=0, \mu=0 \text {, (s) reduces to } E_{q 13} 18.94 \text {. }
\end{aligned}
$$

(B)

With tensile stress (hydrostatic), Eq (1) becomes

$$
p+\sigma=\frac{2 \gamma}{\rho}
$$

where $\rho=$ new radius of curvature.

$$
\begin{aligned}
& \sum q_{n}(3) \&(4) \text { imply } \\
& p=\frac{m k T}{V}=\frac{3 m k T}{2 \pi p^{3}(1-\mu)^{2}(2+\mu)} \quad \text { ( } \mu \text { unchanged) } \\
& \therefore \quad \sigma=\frac{2 \gamma}{\rho}-\frac{3 m k T}{2 \pi p^{3}(1-\mu)^{2}(2+\mu)}
\end{aligned}
$$

If we plot $\sigma(p)$ for fixed $m, \mu$, we obtain curves similar to those in fig :18.41.

$$
\sigma=\frac{2 \gamma}{\rho}-\frac{a}{\rho^{3}}
$$

(Ga)

where $a=\frac{3 m k T}{2 \pi(1-\mu)^{2}(2+\mu)}$

(c)

Critical stress condition!.

$$
\frac{d \sigma}{d \rho}=0
$$


435

$$
\begin{aligned}
& \frac{d \sigma}{d \rho}=-\frac{2 \gamma}{\rho^{2}}+\frac{3 a}{\rho^{4}}=0 . \\
& \therefore \rho=\sqrt{\frac{3 a}{2 \gamma}} \quad \text { at } \sigma=\sigma_{\text {crit. }} .
\end{aligned}
$$

substitute into (Ga):

$$
\sigma_{\text {crit }}=2 \gamma \sqrt{\frac{2 \gamma}{3 a}}-a\left(\frac{2 \gamma}{3 a}\right)^{3 / 2}
$$

or

$$
\sigma_{c r i t}=\frac{2}{3} \frac{(2 \gamma)^{3 / 2}}{\sqrt{3 a}}
$$

Now, for spherical bubble,

$$
\mu=0 ; a=a^{*}=\frac{3 m k T}{4 \pi}
$$

Thus.

$$
\sigma_{\text {crit }}^{*}=\frac{2}{3} \frac{(2 \gamma)^{3 / 2}}{\sqrt{3 a^{*}}}
$$

$\therefore \frac{\sigma_{c r}+}{\sigma_{c_{r i}}{ }^{*}}=\sqrt{\frac{a^{*}}{a}}=\frac{(1-\mu) \sqrt{2+\mu}}{\sqrt{2}}$

When $\frac{\gamma_{g b}}{\gamma}=0.4, \mu=0.2$ (using En 2)

Thus

$$
\begin{array}{r}
\frac{\sigma_{c n t}}{\sigma_{c r} t^{*}} \cong 0.84 \ll 16 \% \text { reduction } \\
\text { of critical stress }
\end{array}
$$

See G.W Lewthwaite, I. Null Mater. 42, 235(1972) for additional details. 
436

Prablem 18.15

$\left(E_{g n} \quad 18.103\right)$

(1)

$\left(E_{8 n} 18.98\right)$ (2)

- $\quad M=\frac{4}{3} \pi R_{0 \text { cr }}^{2}\left(\frac{2 \gamma}{k T}\right) N$

Substituting (1) \&(2) into (3) obtain:

$$
\begin{aligned}
& M=\frac{4}{3} \pi(0.77)^{2} \frac{\gamma^{\prime 2}}{\sigma^{2}} \frac{2 \gamma^{2}}{k T} \frac{\pi^{2}}{\epsilon_{f}^{2} 12 d^{3}}
\end{aligned}
$$

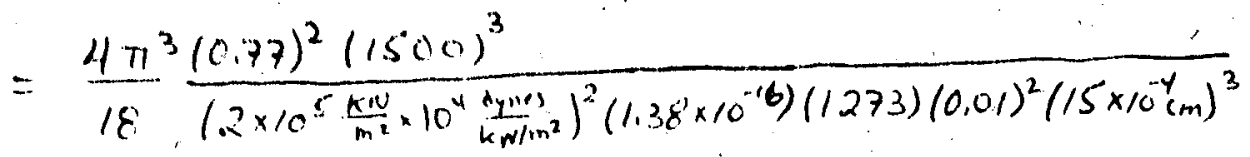

$$
\begin{aligned}
& \cong 5.81 \times 10^{16} / \mathrm{cm}^{2}
\end{aligned}
$$

fraction He $=\frac{M}{N_{S}}=\frac{M}{(1 / \Omega)}=\frac{M a_{0}^{3}}{4}$

for fcc steel, $a_{0} \cong 3.52 \times 10^{-8} \mathrm{sm}$

$$
\begin{aligned}
\therefore \quad \text { ppon He } & =\frac{\left(5.81 \times 10^{16}\right)}{4}\left(3.52 \times 10^{-8}\right)^{3} \times 10^{6} \\
& =0.63 \mathrm{ppm}
\end{aligned}
$$


437

Problein 18:16

(A)

Assuming cubic shape for grain.

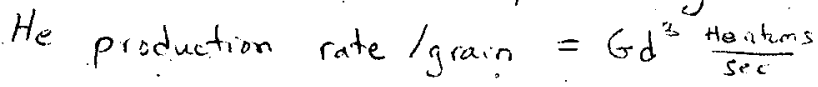

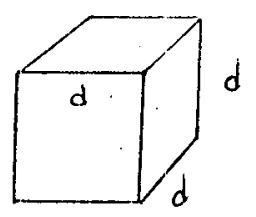

Number of bubble sites per grain $=\frac{\mathrm{Ngb} 6 d^{2}}{2}$

$\therefore$ rate of He atom capture/site $\equiv Q=\frac{6 d^{3}}{3 d^{2}} \quad$ by 2 grains.

(B)

$$
Q=\frac{G d}{3 N_{j b}} \quad \frac{\text { He atoms }}{\text { sitersec }} \text {. }
$$

Since bubble is at equilibrium for $t \leq t_{c}$,

$$
\sigma+p-\frac{2 \gamma}{R}=0
$$

By ideal gas láw,

$$
P\left(\frac{4}{3} \pi R^{3}\right)=m k T
$$

where $m=Q t=$ He atoms in the bubble at

Combining the preceding 3 equations;

$$
\sigma+\left[\frac{Q k T}{\frac{4}{3} \pi}\right] \frac{t}{R^{3}}-\frac{2 \sigma}{R}=0
$$

This equation is equivalent to Eqn 1897 \& a plot of the formula would look like Fig. 18.41 except that the parameter would be $t$, not ko.

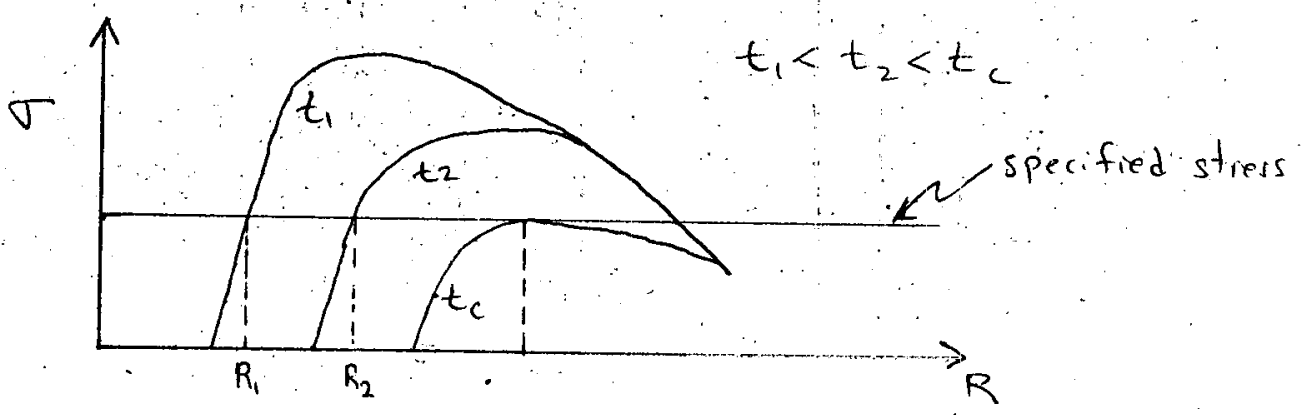


438

The specified stress intersects each curve at a unique value of $R$, so that the curve $R(t)$ for $t<t_{c}$ can be obtained. When the stress line is tangent to the peak of the curve, $t=t_{c}$.

Af this point,

$$
\left.\frac{d \sigma}{d R}\right)_{R c}=0
$$

But the equilibrium condition must also hold at te:

$$
\sigma+\left[\frac{C_{\alpha} K T}{\frac{4}{3} \pi}\right] \frac{t_{c}}{R_{c}^{2}}-\frac{2 \gamma}{R_{c}}=0
$$

Combining above 2 equations yields:

$$
t_{c}=\frac{4 \pi}{3}\left(\frac{2 \gamma}{k T}\right) \frac{1}{Q}\left(\frac{4}{3 \sqrt{3}} \frac{\gamma}{\sigma}\right)^{2}
$$

The bubble radius at te mas also be determined:

$$
R_{c}=\frac{4}{3} \frac{\gamma}{\sigma}
$$

(C)

For $t>t_{c}$, growth is given by $E_{q 1}$ 18.102, with $p=p(R, t)$ known. $i e^{\prime}$

$$
\begin{aligned}
& \frac{d R}{d t}=\frac{W D_{g b} \Omega}{\sigma^{2} k T}\left[\sigma-\frac{2 \gamma}{R}+\left(\frac{Q k T}{\frac{4}{3} \pi}\right) \frac{t}{R^{3}}\right] \frac{(R / R)^{2}-1}{2 \ln \left(\frac{R}{R}\right)-1+\left(\frac{R}{R}\right)^{2}}
\end{aligned}
$$

The rupture time is obtained by numerical integration of the above formula, starting at $R=R_{c}$ ot $t=t_{c}$ \& stopping at $t=t_{R}$ at which. $R=R_{F}=\sqrt{\frac{\pi}{4}} x$. Note that $E_{q} 18.88$ does not apply in this case because $(d R / d t)$ is not separable in $R \& t$. 


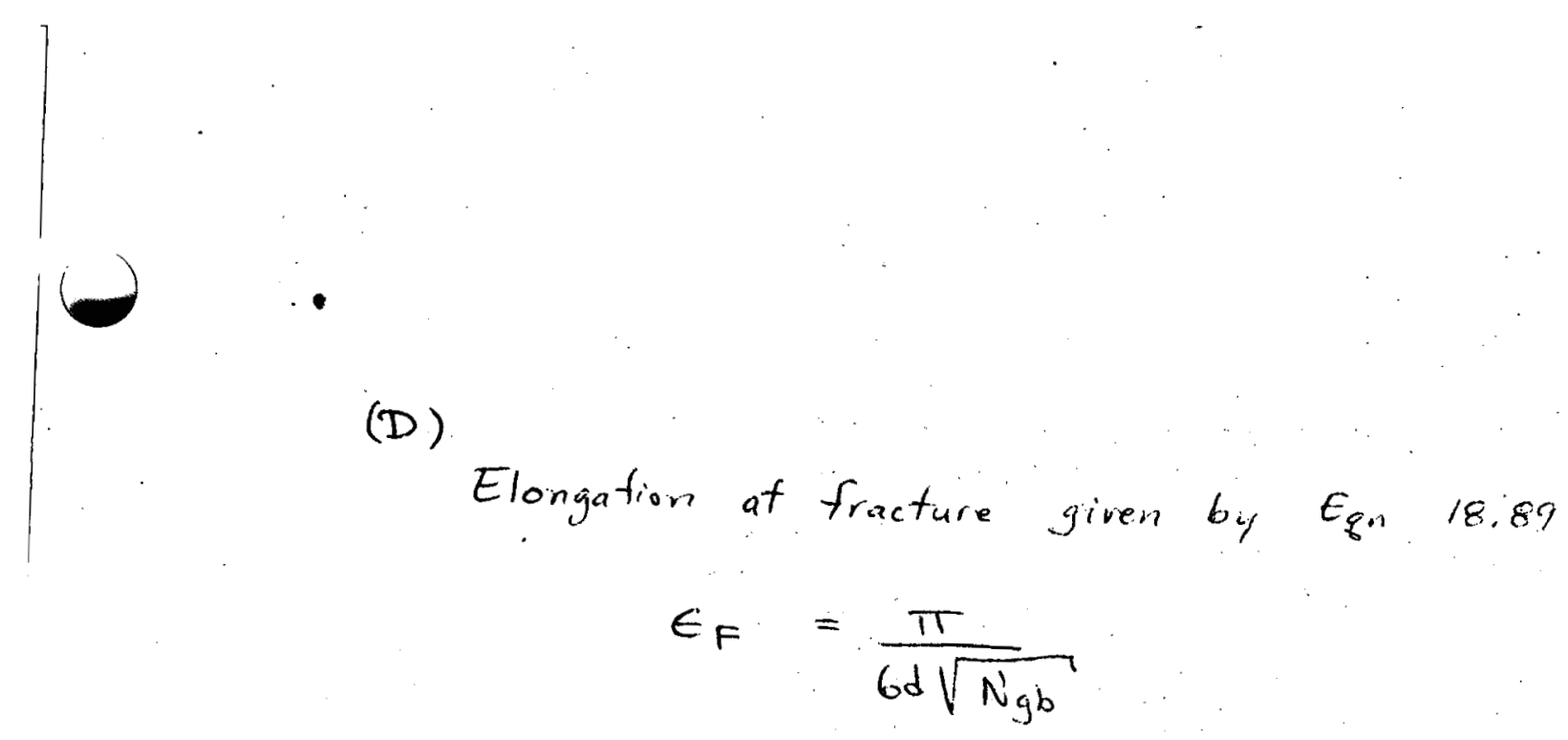

(D)

$$
\begin{aligned}
& \text { Elongation at fracture given by } \epsilon_{q n} 18.89 \\
& \qquad \epsilon_{F}=\frac{\pi}{6 d \sqrt{N_{g b}}}
\end{aligned}
$$


440

Problem 18.17

Assuming steady state creep,

$$
t_{R}=\frac{\epsilon_{F}}{\dot{\epsilon}}
$$

Also,

$$
\epsilon=A \sigma^{n}
$$

(from $\left.E_{80}, 8.46\right)$

where. $A, n$ can be obtained from Fig 10.29 Additionally, from Fig 18.30 .

$$
\epsilon==B(\phi t)^{m}
$$

where B \&.m can be obtained from Fig 18.30

Thus,

$$
t_{R}=\frac{B(\phi t)^{m}}{A \sigma^{n}}
$$

Using hoop stress -Fission gas pressure relation,

$$
\sigma=\frac{e D}{2 t_{c 1}}
$$$$
\text { ( } \left.E_{q n} 18.84\right)
$$

\& from statement of problem

$$
\begin{gathered}
P=c t \\
\therefore \quad t_{R}=\frac{B(\phi t)^{m}}{A}\left(\frac{2 t_{c l}}{c t D}\right)^{n}
\end{gathered}
$$

Using life fraction approach, (Eq 18.10t), obtain:

$$
\sum_{k=1}^{t_{F} / \Delta t} \frac{\Delta t}{t_{R}(k \Delta t)}=1 \Rightarrow \int_{1}^{t \in / \Delta t} \frac{\Delta t d k}{t_{R}(k \Delta t)}=1
$$

where $t_{F}$ is divided into equal intervals of $\Delta t$ Let

$$
k \Delta t=t \quad \& \therefore d k=\frac{d t}{\Delta t}
$$


Thus,

441

$$
\begin{aligned}
& \int_{1}^{t_{F / \Delta t}} \frac{\Delta t d k}{t_{R}(k \Delta t)}=\int_{0}^{t_{F}} \frac{d t}{t_{R}(t)}=1 \\
0 \quad & \int_{0}^{t_{F}} d t \frac{\left(\frac{C D}{2 t_{C l}}\right)^{n} \frac{t^{n}}{B \phi^{m} t^{m}}=1}{t_{F}} \int_{0}^{t^{n-m}} d t=\left(\frac{2 t_{C l}}{C D}\right)^{n} \frac{B \phi^{m}}{A} \\
& \left.\frac{t^{n-m+1}}{(n-m+1)}\right|_{0} ^{t_{F}}=\left(\frac{2 t_{c l}}{C D}\right)^{n} \frac{B \phi^{m}}{A} \\
\text { or } \quad & t_{F}=\left[\left(\frac{2 t_{C l}}{C D}\right)^{n} \frac{B \phi^{m}}{A}(n-m+1)\right] \frac{1}{n-m+1}
\end{aligned}
$$

valid for $n-m+1>0$

For $n-m+1 \leq 0, t_{F}=\infty$ 
442

PROBLEM 19.1

Since $\frac{\Delta V}{V}=\frac{\text { volume } 7 \text { voids }}{\text { volume of metal }}$

The number of vacancies in the voids is $\frac{(\Delta V / v)}{\Omega}$ per unit volume of metal.

The total number of vacancy created at penance It is, $D \Sigma_{s} \Phi t$ (per unit volume of metal)

$\therefore$ Fraction of vacancies created which one wi voids $=\frac{(\Delta V / v)}{\nu \Sigma_{s} \Omega \Phi}$

At a fleece of $5 \times 10^{22}$ Fig 19.4 chows that the swilling is $\sim 37$, Take $P=30, \Sigma_{3}=0.2 \mathrm{~cm}^{-1}$

$$
\begin{aligned}
\Omega & =12 \dot{A}^{3} \\
\Rightarrow & \frac{(\Delta V / V)}{\nu \Sigma_{3} \Omega \Phi t}=\frac{3 \times 10^{-2}}{(30) \times(0.2) \times\left(1.2 \times 10^{-33}\right)\left(5 \times 10^{22}\right)} \\
& =0.8 \%
\end{aligned}
$$


443

PROBLEM 19.2

$E_{q}(19-43)$ is; $\frac{h(m)}{h(m+1)}=\frac{N^{2 q}(m)}{N^{e q}(m+1)}+\frac{\beta_{i}}{\beta_{v}}=F(m)$

then $\frac{h(m+1)}{h(m)}=[F(m)]^{-1}$

Joking log of hath rides, $\ln \left[\frac{h(m+1)}{h(m)}\right]=-\ln F(m)$

Summing from $m^{\prime}=0$ to $m^{\prime}=m-1$

$$
\sum_{m^{\prime}=0}^{m-1} \ln \left[\frac{h\left(m^{\prime}+1\right)}{h\left(m^{\prime}\right)}\right]=\ln \prod_{m^{\prime}=0}^{m-1} \frac{h\left(m^{\prime}+1\right)}{h\left(m^{\prime}\right)}=\ln \frac{h(m)}{h(0)}=-\sum_{m^{\prime}=0}^{m-1} \ln F\left(m^{\prime}\right)
$$

From $E_{\dot{q}}(19-36)$ it is seen that;

$$
\lim _{m \rightarrow 0} \frac{N^{2 q}(m)}{N^{2 q}(m+1)}=\lim _{m \rightarrow 0} \frac{1}{s_{v}} \exp \left[\frac{2}{3} \equiv m^{-1 / 3}\right] \rightarrow \infty
$$

However $\frac{\beta_{i}}{\beta_{v}} \leq 1$.

thus $\lim _{m \rightarrow 0} \frac{h(m+1)}{h(m)}=\frac{N^{e f}(m+1)}{N^{e}(m)}$

clearly, $h(0)$ cm be chen as $N^{e q}(0)=N_{s}$

$$
\text { So: } \ln \left[\frac{h(m)}{N_{s}}\right]=-\sum_{m^{\prime}=0}^{m-1} \ln \left[\frac{N^{2 q}\left(m^{\prime}\right)}{N^{2 q}\left(m^{\prime}+1\right)}+\frac{\beta_{i}}{\beta_{v}}\right]
$$

Which is eq $(19.44)$ 
444

PROBLEM 19.3

Classical Nucleation Theory.

$$
\begin{aligned}
& h(m)=N^{0 .}(m)=N \text { s exp }\left[m \ln s_{v}-\xi m^{2 / 3}\right] \\
& \text { where } \xi=\left(36 \pi \Omega^{2}\right)^{1 / 3} \frac{\gamma}{k T}
\end{aligned}
$$

$$
I=\frac{\beta_{r}\left(m_{c}\right)}{\int_{0}^{\infty} \frac{d m}{N^{q}(m)}} \quad E_{q}(19.47)
$$

Let $f(m)=m \ln s_{v}-\xi m^{2 / 3}$

$f$ lias a maximum value at $m_{c}=\left(\frac{2 \xi}{3 \ln s_{1}}\right)^{3}$. which is che critical cluster size Expand $f(m)$ aleut mo:

$$
\begin{aligned}
& f(m)=f\left(m_{c}\right)+\left(\frac{d f / j^{0}}{d m}\right)_{m_{c}}\left(m-m_{c}\right)+\frac{1}{2}\left(\frac{d^{2} f}{d m^{2}}\right) m_{c}\left(m-m_{c}\right)^{2}+\cdots \\
& \frac{d f}{d m}=\ln s_{v}-\frac{2}{3} \xi m^{-\frac{1}{3}} \\
& \frac{d^{2} f}{d m^{2}}=\frac{2}{9} m^{-\frac{4}{3}} \\
& \left(\frac{d^{2} f}{d m^{2}}\right)_{m_{c}}=\frac{2}{9} \xi\left(\frac{3 \ln s_{v}}{2 \xi}\right)^{4}=\frac{9}{8} \frac{\left(\ln s_{v}\right)^{4}}{\xi^{3}} \\
& f\left(m_{c}\right)=\left(\frac{2 \xi}{3 \ln s_{v}}\right)^{3} \ln s_{v}-\xi\left(\frac{2 \xi}{3 \ln s_{v}}\right)^{2} \\
& \left.=\left(\frac{2}{3}\right)^{3} \frac{\xi^{3}}{\left(\ln s_{v}\right)^{2}}-\frac{2}{3}\right)^{2} \frac{\xi^{3}}{\left(\ln s_{v}\right)^{2}}=-\frac{\xi^{3}}{\left(\ln s_{v}\right)^{2}} \\
& N^{2}(m)=N_{s} \exp \left[f\left(m_{c}\right)+\frac{\left(\ln s_{v}\right)^{4}}{3^{3}}\left(m-m_{c}\right)^{3}\right]
\end{aligned}
$$

But $N_{s} e^{f\left(m_{c}\right)}=N^{\alpha q}\left(m_{c}\right)$ 
445

$$
\begin{aligned}
& \Rightarrow-N^{e q}(m)=N^{e q}\left(m_{c}\right) \exp \left[\frac{q}{1 t} \frac{\left(\ln s_{v}\right)^{4}}{\xi^{3}}\left(m-m_{c}\right)^{2}\right] \\
& I=\frac{\beta_{r}\left(m_{c}\right) N^{2 q}\left(m_{c}\right)}{\int_{1}^{\infty} e^{-k\left(m-m_{c}\right)^{2}} d m} ; k=\frac{q}{16} \frac{\left(\ln s_{r}\right)^{4}}{\xi^{3}}
\end{aligned}
$$

Let $t=m-m_{c}$

when $m=1 \quad t=1-m_{c}=-\left(m_{c}-1\right)$

when $m=\infty$

$$
\begin{aligned}
& \int_{1}^{\infty} e^{-k\left(m-m_{c}\right)^{2}} d m^{\prime}=\int_{-\left(m_{c}-1\right)}^{\infty} e^{-k t^{2}} d t=\int_{-\left(m_{c}-1\right)}^{0} e^{-k t^{2}} d t \\
& +\int_{0}^{\infty} e^{-k t^{2}} d t \\
& \text { But }\left(m_{c}-1\right)>1, \int_{-\infty}^{0} e^{-k t^{2}} d t=\int_{0}^{\infty} e^{-k t^{2}} d t \\
& \Rightarrow \int_{1}^{\infty} e^{-k\left(m-m_{c}\right)^{2}} d m=2 \int_{0}^{\infty} e^{-k t^{2}} d t=\frac{2}{\sqrt{k}} \int_{0}^{\infty} e^{-\omega^{2}} d \omega \\
& =\frac{2}{\sqrt{k}} \cdot \frac{\sqrt{\pi}}{2}-\left(\frac{\pi}{k}\right)^{1 / 2}=\sqrt{\pi} \frac{4}{3} \times \frac{\pi^{3 / 2}}{\left(\ln s_{v}\right)^{2}}
\end{aligned}
$$

where: $\omega=\sqrt{k} t$

$$
\begin{aligned}
& \Rightarrow I=\frac{3}{4 \sqrt{\pi}} \frac{\left(\ln s_{v}\right)^{2}}{3^{3 / 2}} \beta_{v}\left(m_{c}\right) N^{e q}\left(m_{c}\right) \\
& N^{e q}\left(m_{c}\right)=N_{s} \exp \left[m_{c} \ln s_{v}-\xi m_{c}^{3 / 3}\right] \\
& =N_{s} \exp \left[\left(\frac{2 \xi}{3 \ln s_{v}}\right)^{3} \ln s_{v}-\xi\left(\frac{3 \xi^{3}}{3 \ln s_{v}}\right)^{2}\right] \\
& \Rightarrow N^{2 q}\left(m_{c}\right)=N_{s} \exp \left[-\frac{4}{27} \frac{\xi^{3}}{(\ln s)^{2}}\right]
\end{aligned}
$$


$4+46$

In a gas - i quid system, Ns is intirperted as the total number of molecule in the system. In the present cause, $N_{s}$ is the total number is lattice sites.

$$
\begin{aligned}
& \beta_{v}\left(m_{c}\right)=4 \pi R_{c} D_{v} C_{v} \text { (diffusion limited) } \\
& c_{v}=s_{v} c_{v}^{e v} \\
& m_{c}=\frac{\left(4 / 3 \pi R_{c}^{3}\right)}{\Omega} \\
& R_{c}=\left(\frac{3 \Omega}{4 \pi}\right)^{1 / 3} m_{c}^{1 / 3}=\left(\frac{3 \Omega}{4 \pi}\right)^{1 / 3}\left(\frac{23}{3 \ln 5 v}\right) \\
& \beta_{v}\left(m_{c}\right)=(4 \pi)^{2 / 3}(3 \Omega)^{1 / 4} \frac{33}{3} \frac{s_{v}}{\ln s_{v}} D_{v} C_{v}^{q} \\
& I=\frac{3}{2 \sqrt{4 \pi}} \frac{\left(\ln S_{j}\right)^{2}}{\xi^{3 / 2}}(4 \pi)^{2 / 3}\left(3 \Omega_{1}\right)^{1 / 3} \frac{\sum \xi}{3} \frac{S_{v}}{\ln 5 v} D_{v} C_{v}^{i t} x \\
& N_{s} \exp \left[-\frac{4}{27} \frac{3^{3}}{(\ln 5 v)^{2}}\right] \\
& \Rightarrow I=\frac{(4 \pi)^{1 / 6}(32)^{1 / 3}}{\xi^{1 / 2}} \text { Ns } D_{v} C_{v}^{\operatorname{sg}} S_{v} \ln S_{v} \exp \left[-\frac{4}{27} \frac{\xi^{3}}{(\ln 5 v)^{3}}\right]
\end{aligned}
$$


447

PROBLEM .19.4

$E_{q}(19.45)$ is $\frac{d(N / n)}{d m}=-\frac{I}{\beta_{V}(m) n(m)}$

which implies; $\int_{1}^{(N / h)} d\left(\frac{N}{h}\right)=-I \int_{1}^{m} \frac{d m^{\prime}}{\beta r\left(m^{\prime}\right) h\left(m^{\prime}\right)}$

Since $n\left(m^{\prime}\right)$ teas a chap minimmin at $m^{\prime}=m_{c}$, the main contribution to the integrals comes from the

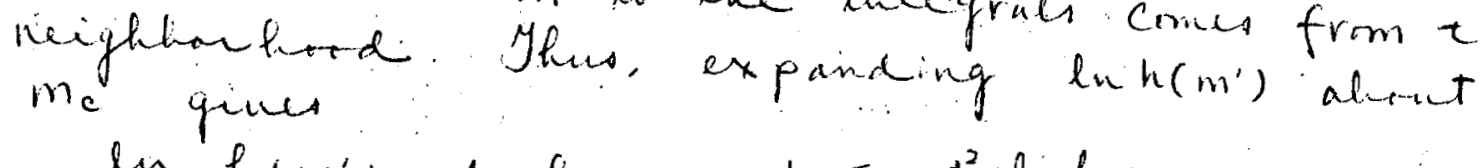

$$
\begin{aligned}
\ln h\left(m^{\prime}\right) & =\ln h\left(m_{c}\right)+\frac{1}{2}\left[\frac{d^{2} \ln h}{d m^{2}}\right]_{m_{c}}\left(m^{\prime}-m_{c}\right)^{2} . \\
& =\ln h\left(m_{c}\right)+h\left(m^{\prime}-m_{c}\right)^{2}
\end{aligned}
$$

where $k=\frac{1}{2}\left[\frac{d^{2} \ln h}{d m^{2}}\right]_{m_{c}}$

or $k\left(m^{\prime}\right)=\hbar\left(m_{c}\right) e^{k\left(m^{\prime}-m_{c}\right)^{2}}$

Siketituting into the about integral smith $\beta_{v}(m) \cong \beta_{0}\left(m_{c}\right)$, We chain;

$$
\frac{N(m)}{h(m)}-1=-\frac{I}{\beta_{0}\left(m_{c}\right) h\left(m_{c}\right)} \cdot \int_{1}^{m} e^{-k\left(m^{\prime}-m_{c}\right)^{2}} d m^{\prime}
$$

From eq $(19.47)$ and $(19.48)$ it is clear that,

$$
\left[\frac{I}{\beta_{1}\left(m_{c}\right) k\left(m_{c}\right)}\right]^{-1}=\int_{1}^{\infty} e^{-k\left(m^{\prime}-m_{c}\right)^{2} d m^{\prime \prime}}
$$

So, $\frac{N(m)}{h(m)}=1-\frac{\int_{1}^{m} e^{-k\left(m-m_{c}\right)^{2}} d m^{\prime}}{\int_{1}^{\infty} e^{-k\left(m^{\prime}-m_{c}\right)^{2}} d m^{\prime}}$

Let $Q(m)=\int_{1}^{m} e^{-k\left(m^{\prime}-m_{c}\right)^{2}} d m^{\prime}$ 
448

let $x=\sqrt{k}\left(m^{\prime}-m_{c}\right)$

$d x=\sqrt{k} d m^{\prime} \quad d m^{\prime}=\frac{1}{\sqrt{k}} d x$

when $m^{\prime}=1$

$$
x=-\sqrt{k}\left(m_{c}-1\right)
$$$$
x=\sqrt{k}\left(i n-m_{c}\right)
$$

$$
\begin{aligned}
& m^{\prime}=m \\
& Q(m)=\frac{1}{\sqrt{k}} \int_{-\sqrt{k}\left(m_{c}-1\right)}^{\sqrt{k}\left(m-m_{c}\right)} e^{-x^{2}} d x=\frac{1}{\sqrt{k}}\left[\int_{0}^{\sqrt{k}\left(m_{c}-1\right)} e^{-x^{2}} d x+\int_{0}^{\sqrt{k}\left(m-m_{c}\right)} e^{-x^{2}} d y\right]
\end{aligned}
$$

But $\operatorname{erf}(Y)=\frac{2}{\sqrt{\pi}} \int_{0}^{Y} e^{-x^{2}} d x$

$$
\begin{aligned}
& Q(m)=\frac{\sqrt{\pi}}{2 \sqrt{k}}\left\{\operatorname{erf}\left[\sqrt{k}\left(m-m_{c}\right)\right]+\operatorname{erf}\left[\sqrt{k}\left(m_{c}-1\right)\right]\right\} \\
& Q(\infty)=\frac{\sqrt{\pi}}{2 \sqrt{k}}\left\{1+\operatorname{erfc}\left[\sqrt{k}\left(m_{c}-1\right)\right]\right\}
\end{aligned}
$$

assume $\sqrt{k}\left(m_{c}-1\right)>3$, terf $\left[\sqrt{k}\left(m_{c}-1\right)\right] \rightarrow 1$.

$$
\begin{aligned}
& Q(\infty)=\left(\frac{\pi}{k}\right)^{1 / 2} \\
& \Rightarrow \frac{Q(m)}{Q(\alpha)}=\frac{1}{2}\left\{1+k f\left[\sqrt{l}\left(m-m_{c}\right)\right]\right\} \\
& \therefore \frac{N(m)}{h(m)}=1-\frac{Q(m)}{Q(\infty)} \\
& \frac{N(m)}{h(m)}=1-\frac{1}{2}\left\{1+\operatorname{krf}\left[\sqrt{k}\left(m-m_{c}\right)\right]\right\} \\
& \Rightarrow N(m)=\frac{1}{2} h(m) \operatorname{erfc}\left[\sqrt{k}\left(m-m_{c}\right)\right]
\end{aligned}
$$


449

PRCBLEM 19.5

Point defect calance at heginnig of irraciation.

$$
\begin{aligned}
& N=0 \\
& \int_{e}=0 \\
& f_{N}=f_{d}=10^{3} \cos ^{2} \\
& \left.\begin{array}{l}
\nu=30\left(E_{q}(19.94)\right) \\
\Sigma_{0}=0.2 \ldots-1
\end{array}\right\} \nu \Sigma_{0} I=30 \times 0.2 \times 10^{14}
\end{aligned}
$$

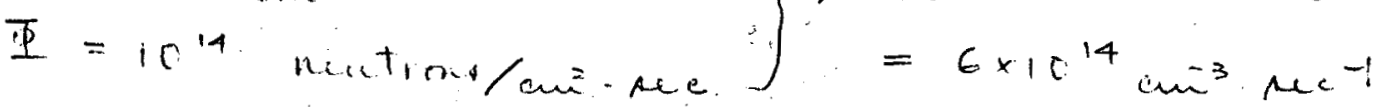

$$
\begin{aligned}
& Z_{v} \cong \frac{2 \pi}{\ln \left(\frac{R}{R_{t}}\right)}=\frac{63}{\ln \left(\frac{18+2}{3}\right)}=c .98 \\
& Z_{i}=1.02 z_{v} \cong 1 . c_{1}
\end{aligned}
$$

Dislocation capture radius $R$

$$
\begin{aligned}
& \left(\pi R^{2}\right) \rho_{d}=1 \\
& R=\frac{1}{\sqrt{\pi} \cdot 9 d}=1.8 \times 10^{-5} \mathrm{~cm}=1.8 \times 10^{3} \AA
\end{aligned}
$$

Since $D_{i}>D_{t}$

$$
\text { kir }=\frac{z_{i r} D_{i} \Omega}{a_{c}^{2}} \quad[\operatorname{eg}(13,42)]
$$

For izon in $=12 A^{3}$

$$
a_{0}{ }^{2} \approx \Omega^{2 / 3}=5: 24 \dot{A}^{2}
$$

For intertitial $D_{k}=a_{r}^{2} D \exp \left(-\varepsilon_{i}^{*} / k T\right)$

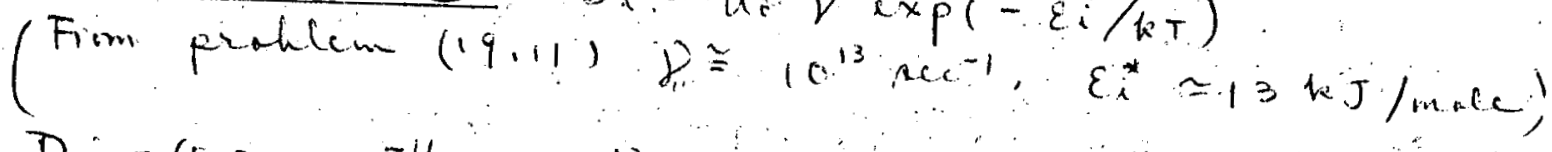$$
D_{i}=\left(5.24 \times 10^{-16}\right) \times\left(10^{13}\right) \operatorname{sxp}\left(-\frac{13}{8.31 \times 0.773}\right)=t .9 \times 10^{4} \frac{\mathrm{cm}^{2}}{\mathrm{AcC}}
$$$$
k i v=\left(10^{2}\right)\left(6.9 \times 10^{-4}\right) \times \frac{12}{5.24} \times 10^{-8}=1.6 \times 10^{-4} \mathrm{~cm}^{3} / \mathrm{se}
$$

For Vacancig $D_{r}=a_{i}^{2} P \operatorname{cxp}\left(-\varepsilon_{v}^{*} / k T\right)$ 
450

From problem $19.11, \quad \varepsilon_{v}^{*}=125 \mathrm{~kJ} / \mathrm{mal}$

$$
\begin{aligned}
& \therefore \quad D_{v}=\left(5.24 \times 10^{-16}\right) \times 10^{13} \exp \left(-\frac{125}{8.31 \times 0.773}\right)=1.85 \times 10^{11} \frac{\mathrm{cm}^{2}}{\mathrm{eec}^{2}} \\
& \text { A. Equation ( } 19.136 \text { ) and (19.137) (neguet } c_{v}^{8} \text { ) } \\
& \nu \Sigma_{3} \Phi=Z_{v} \int_{N} D_{v} C_{v}+k_{i v} C_{i} C_{V} \ldots \ldots-\cdots(1) \\
& \nu \Sigma_{s} \Phi=z_{i} \rho_{N} D_{i} C_{i}+k_{i v} C_{i} C_{v} \ldots \ldots(2) \\
& \frac{C_{r} \Sigma_{s} I}{\int_{d}}=z_{v}\left(D_{v} C_{v}\right)+\left(\frac{K_{i} v}{\int_{d} D_{i} D_{v}}\right)\left(D_{i} C_{i}\right)\left(D_{v} C_{v}\right)-(i a) \\
& \frac{\nu \Sigma_{3} \Phi}{\rho_{d}}=z_{i}\left(D_{i} C_{i}\right)+\left(\frac{R_{i} v}{\int_{d} D_{i} D_{v}}\right)\left(D_{i} C_{i}\right)\left(D_{v} C_{v}\right) \cdots(2 n) \\
& \frac{\nu \Sigma_{3} \Phi}{\rho_{\alpha}}=\frac{.6 \times 10^{14}}{10^{4}}=6 \times 10^{5} \mathrm{em}^{-1} \mathrm{mc}^{-1}
\end{aligned}
$$

$$
\frac{k i v}{\rho_{d} D_{i} D_{v}}=\frac{1.6 \times 10^{-9}}{\left(10^{4}\right)\left(6.9 \times 10^{-4}\right)\left(1.85 \times 10^{-11}\right)}=1.25 \times 10^{-4} \mathrm{em- \mu c}
$$

Sinteract (Ia) fron (za)

$$
Z_{v}\left(D_{v} C_{v}\right)=Z_{i}\left(D_{i} C_{i}\right) \ldots \ldots \text { (3) }
$$

$$
\begin{aligned}
& 6 \times 10^{5}=0.98\left(D_{v} C_{v}\right)+\left(1.25 \times 10^{-4}\right)\left(\frac{c 9.3}{{ }^{1}}\right)\left(D_{v} C_{v}\right)^{2} \\
& \Rightarrow 1.25 \times 10^{-4}\left(D_{v} C_{v}\right)^{2}+\left(D_{v} C_{v}\right)-6 \times 10^{5}=0 \\
& \text { soluc tie alure equation } D_{v} C_{v}=6.54 \times 10^{4} \\
& \Rightarrow C_{v}=\frac{6.54 \times 10^{4}}{.1 .85 \times 10^{-11}}=3.53 \times 10^{15} \\
& C_{v}^{8 f}=\frac{1}{2} \exp \left(-\frac{16}{8.31 \times 0.773}\right)=1.27 \times 10^{12}
\end{aligned}
$$


451

$$
\begin{aligned}
& S_{v}=\frac{C_{v}}{C_{v}^{29}}=\frac{3.53 \times 10^{15}}{1.27 \times 10^{12}}=2780 \\
& D_{i} C_{i}=(0.98)\left(6.54 \times 10^{4}\right)=6.41 \times 10^{4} \\
& C_{i}=\frac{6.41 \times 10^{4}}{6.9 \times 10^{-4}}=9.3 \times 10^{7} \\
& C_{v}^{2 q}=\frac{1}{\Omega} \exp \left(-\frac{420^{-1}}{8.31 \times .77^{3}}\right)=3.35 \times 10^{-6} \\
& S_{i}=\frac{C_{i}}{C_{i}^{21}}=\frac{9.3 \times 10^{7}}{3.35 \times 10^{-6}}=2.8 \times 10^{13}
\end{aligned}
$$

B. From Equations $(19.136)$ and 119.137$)$ the ratio the ratio of the terms representing vacancy and interstitial alcioption lin voids. (the first term on the right) is

$$
\left(\frac{\beta_{i}}{\beta_{v}}\right)_{\text {void }}=\frac{4 \pi R N D_{i} C_{i}}{4 \pi R N D_{V}\left[C_{v}-C_{v}^{e q} \exp \left(\frac{\partial \gamma \Omega}{R R^{2}}\right)\right]}
$$

Since $\frac{C_{v}}{C_{v}^{2}} \gg 1$, and the surface tension term is $\sim$ unity

$$
\left(\frac{\beta_{i}}{\beta_{v}}\right)_{\text {veld }}=\frac{D_{i} C_{i}}{D_{v} c_{v}}=\frac{z_{v}}{Z_{i}}=0.98
$$

For dislocation, the lexis on right of equation (1) and ( 2 ) gives

$$
\left(\frac{\beta_{v}}{\beta_{i}}\right)_{\text {dire }}=\frac{z_{v} \rho_{N} D_{v} C_{v}}{z_{i} \rho_{N} D_{i} C_{i}}=\frac{z_{v} D_{v} C_{v}}{z_{i} D_{i} C_{i}}=1
$$

The ratio is vastly unity because in gen (ie he fore voids have hin Nucleated): the 
452

dislocations must abash vacancies and interstitial $\therefore$ at the same rate.

c. Void nucleation

From Fig $(19.10)$, For $S_{V}=2780 . \quad T=773^{\circ} \mathrm{K}$

interpolation yields $I_{\text {void }} \cong 10^{7}$ voids/ans-pec

at startup $\left(\frac{\beta_{v}}{\beta_{i}}\right)_{\text {dial }} \cong 1 \quad I_{\operatorname{loop}}=0$

D. if the steed contained a concentration $N p$ of incoherent precipitate particles of radius RP a term $4 \pi R_{p} N_{P} D_{V} C_{V}$ would be added to the right hand rich of eq (1) and $a$ comparable term $4 \pi R_{p} N_{p} D_{i} C_{i} t_{0}$ eq (2) and of (3) would be replaced by

0. 
453

$$
\begin{aligned}
& \text { For } x=0.1 \\
& \left(\frac{\beta_{i}}{\beta_{i}}\right)_{\text {vi dd }}=0.98[1+(0.02)(0.1)]=0.982 \\
& \left(\frac{\beta_{v}}{\beta_{i}}\right)_{\text {aldol }}=1-(0.02)(0.1)=0.998 .
\end{aligned}
$$

The void nucleation rate is NOT significantly to 0.982 . However the loop nucleation rate is given by $(19,92)$

$$
\begin{aligned}
I_{\text {loop }} & =\left(2 \times 10^{32}\right)\left(2 \times 10^{-3}\right)\left(2.8 \times 10^{13}\right)^{3} \exp \left(-\frac{11.50}{8.31 \times .733}\right) \\
& =2 \times 10^{-3} \frac{\text { mule }}{\mathrm{Cn}^{3}-\text { rec }}
\end{aligned}
$$

which is tile negligibly small. 
454

i)

PROBLEM 19.6

Let $P$ le the helium production rate From Fig $(18: 40)$ the production rate for a fluency of $10^{15}$ is.

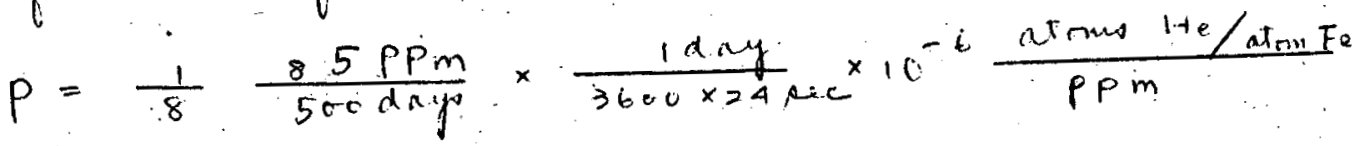

$$
\begin{aligned}
& \times 0.85 \times 10^{23} \text { atoms } \mathrm{Fe} / \mathrm{cm}^{3} \text {. } \\
& =2 \times 10^{10} \frac{\text { atrmis He }}{\operatorname{an}^{3}-\operatorname{Rec}}
\end{aligned}
$$

The $P$ replaces $T_{x \in} \dot{F}$ in fission gas table mulention. in $\mathrm{UO}_{2}$.

$$
k_{11}=\frac{z_{11} \Omega D_{H e}}{a^{2}} \quad\left[E_{q}(13.44)\right]
$$

where $k_{11}$ is the He -He interaction rate constant

$$
\begin{aligned}
& z_{11} \simeq 84 \\
& \Omega=12 \times 10^{-24 \mathrm{~cm}^{3}} \\
& a=3 \dot{A}_{12 \Omega} D_{H_{e}} a^{2}, z_{12}=12 \\
& k_{12}=\frac{z_{12}}{9 \times 10^{-1 t}}=1.1 \times 10^{-22} \mathrm{~cm}^{3} / \mathrm{sec} \\
& k_{11}=\frac{(84)\left(12 \times 10^{-16}\right)}{k_{12}}=2.7 \times 10^{-23} \mathrm{~cm} / \mathrm{scc} \\
& \left(\frac{P}{3 k_{11}}\right)\left(\frac{k_{2}}{2 b}\right)^{2}=\frac{2 \times 10^{10}}{3\left(1.1 \times 10^{-32}\right)}\left[\frac{2.7 \times 10^{-33}}{2 \times 10^{-6}}\right]^{2}
\end{aligned}
$$

According to Fig $13=13.1 \times 10^{-2}$ rededion-dominded limit is applicable, and $C_{c}$ is given le

$$
\text { Eq }(13 \cdot 142) \quad C_{c}=\left(\frac{2 b Y H_{2} \dot{F}}{3 k_{11}}\right)^{1 / 3}
$$


455

$$
\begin{aligned}
& \Rightarrow C_{c}=\left[\frac{(2)\left(10^{-6}\right)\left(2 \times 10^{10}\right)}{(3)\left(1.1 \times 10^{-22}\right)\left(2.7 \times 10^{-3}\right)}\right]^{1 / 3}=1.65 \times 10^{16} \text { atimes He } \\
& C_{>c}=\left(\frac{1.1 \times 10^{-32}}{2 \times 10^{-6}}\right)^{1 / 3}\left(\frac{2 \times 10^{10}}{3 \times 2.7 \times 10^{-23}}\right)^{2 / 3} \\
& =1.5 \times 10^{16} \frac{\text { diatous }}{a m^{3}} \\
& t_{c}=\frac{1.25 \times 10^{16}+2\left(1.5 \times 10^{16}\right)}{2 \times 10^{10}}=2.3 \times 10^{6} \mathrm{ecc}
\end{aligned}
$$

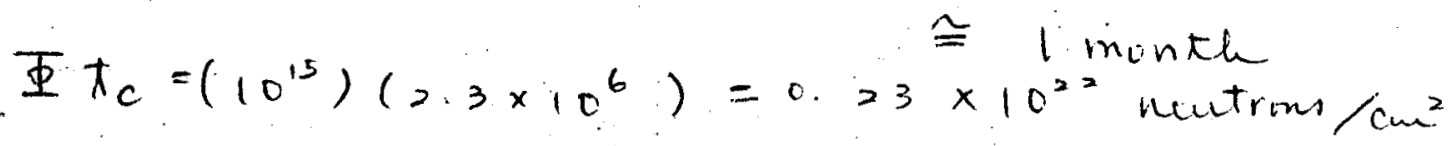

He labble incleation is complete at abrest $1 / 4$ of the fluence requide for void ruchetion 
456

PROBLEM 19.7

Neglecting vacancies in di-interetitial balance

$$
\begin{aligned}
& i+i \frac{R_{1} f_{2}}{R_{1 r}} i=\ldots . . . . .(1) \\
& i+i_{2} \frac{R_{2} t_{2}}{R_{2 r}} i_{3} \ldots \ldots(2) \\
& R_{1 f}=\beta_{i}(1) C_{i} \quad \ldots \ldots-\ldots \ldots \text { (3) } \\
& R_{1 r}=\alpha_{i}(2) N_{2}+2 b N_{2} \ldots(4)
\end{aligned}
$$

where $2 b N_{2}$ is recolution tirm. thene is 2 in this term, hecruse there are 2 intentitial per di-intentitial

$N_{2}$. concentration of di-intersticials.

acuing rention (1) in aquilibiom, becruce of low intenaction rate of (2)

$$
\begin{aligned}
& \beta_{i}(1) C_{i}=\left[x_{i}(2)+2 b\right] N_{2} \\
\therefore \quad & N_{2}=\frac{\beta_{i}(1) c_{i}}{\alpha_{i}(2)+2 b}
\end{aligned}
$$

llaig $(19.69)$ for $\beta_{i}(1)$ and $(19.72)$ foi $\alpha_{i 12}$

$$
N_{2}=\frac{\frac{g_{1 i} \Omega D_{i} C_{i}{ }^{2}}{a_{i}{ }^{2}}}{\frac{z_{1 i} D_{i}}{a_{0}{ }^{2}} e^{-B / k T}+2 b}=\frac{\Omega \cdot e^{B / k T} C_{i}{ }^{2}}{\left.1+\frac{2 a_{0}^{2} b}{z_{1 i} D_{i}} e^{B / k T}--(5)\right)}
$$

The tri-intertitial larlance hecomes

$$
\begin{aligned}
& \text { tri - iterititial linlanie } \\
& I_{2}=I_{3}-\ldots-\ldots(6)
\end{aligned}
$$

$E_{q}(19.85)$ is $I_{2}=\beta_{i}(2) N_{2}-\beta_{v}(3) N_{3}-3 b N_{3}--(7)$

$$
\begin{aligned}
& \left.=\beta_{i}(2) N_{2}-\beta_{1}(3) N_{3}-\uparrow_{1} \uparrow_{3} i^{\text {resil }} \rightarrow-i+i_{2}\right) \\
& \left(i_{2}+i_{3} \rightarrow i_{3}\right)\left(i_{3}+v-i_{2}\right)\left(i_{3} \stackrel{ }{\longrightarrow}\right.
\end{aligned}
$$


457

and

$$
\begin{aligned}
& I_{3}=\beta_{i}(3) N_{3}-\beta_{1}(4) N_{4}-4 b N_{4} \ldots \ldots(8) \\
& \left(i_{3}+i \rightarrow i_{4}\right) \quad\left(i_{4}+v \rightarrow i_{3}\right)\left(i_{4} \stackrel{r e s e l}{\longrightarrow} i+i_{3}\right) \\
& \left(\beta_{i}(3)+\beta_{V}(3)+3 b\right) N_{3}=\beta_{i}(2) N_{2}+\left(\beta_{V}(4)+4 b\right) N_{4} \ldots \text { (9) } \\
& \left(\beta_{i}(4)+\beta_{V}(4)+4 b\right) N_{4}=\beta_{i}(3) N_{3}+\left(\beta_{V}(5)+5 b\right) N_{5}--(10)
\end{aligned}
$$

Now

$$
\begin{aligned}
& \beta_{i}(n)=z_{n i} \frac{\Omega}{a_{0}^{2}} D_{i} C_{i} \ldots-\ldots(11 a) \\
& \beta_{v}(n)=z_{n v} \frac{\Omega}{a_{0}^{2}} D_{v} C_{v} \ldots \ldots(11 b)
\end{aligned}
$$

Aatume $z_{n i}=z_{n r}=10 \mathrm{n}$, ie the comlinatorial. numles is piepartional to the inumber of pint defecta in the clinter

$\operatorname{Let} \frac{\beta_{i}(2)}{\beta_{V}(2)}=\frac{\beta_{i}(3)}{\beta_{V}(3)}=\frac{\beta_{i}(4)}{\beta_{V}(4)}=\frac{\beta_{i}}{\beta_{V}}=\frac{D_{i} C_{i}}{D_{V} C_{V}}--(12)$

- couctiant determined from point defect balance

And

$$
\frac{n b}{\beta_{v}(n)}=\frac{n b}{10 n \frac{\Omega}{a_{0}^{2}} D_{V} c_{V}}=\frac{a_{0}^{2} b}{10 \Omega D_{V} c_{V}}=y \text {, a conctint }- \text { (13) }
$$

Diviae ef (9) hy $\beta(3)$ and eq (10) lny (4)

then

$$
\begin{aligned}
& \left(1+\frac{\beta_{i}}{\beta_{v}}+q\right) N_{3}=\frac{2}{3} \beta_{i} N_{2}+\frac{4}{3}(1+q) N_{4}--(14) \\
& \left(1+\frac{\beta_{i}}{\beta_{v}}+q\right) N_{4}=\frac{3 \beta_{i}}{4 \beta_{v}} N_{3}+\frac{5}{4}(1+f) N_{5} \cdots(15)
\end{aligned}
$$


458

In general.

$$
\left(1+\frac{\beta_{i}}{\beta_{v}}+q\right) n \cdot N_{n}=\frac{\beta_{i}(n-1)}{\beta_{v}} N_{n}+(1+q)(n+1) N_{n+1}-(16)
$$

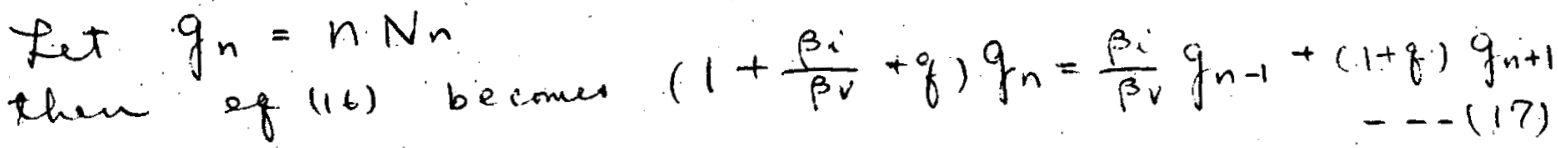

$--(17)$

Eq (IT) is a record order difference eq there. should be 2 elution.

Where $q=0$ and $\beta_{i} / \beta_{v}=1$. the two Ablution ane.

$$
\begin{aligned}
& g_{n}^{(1)}=k_{1} \quad\left(N_{n}=k_{1} / n\right) \\
& g_{n}^{(p)}=K_{2} n\left(N_{n}=K_{2}\right)
\end{aligned}
$$

The solution $g_{n}=K_{1}$, also Antiffies eq (17). We true NOT been able to find the second pollution. However, We: chalk assume that this second solution gives No which increases with $n$, and to can he discarded. Therefore from the fist solution $\left(g_{n}=k,\right)$ We kame $n N_{n}=$ constant $=2 N_{2}$

$$
\text { or } \quad N_{3}=\frac{2 N_{2}}{3}-\ldots-\cdots(18)
$$

the $(18)$ in $(7)$

$$
\begin{gathered}
I_{2}=I_{\text {lamp }}=\beta_{i}(2) N_{2}-2 / 3\left[\beta_{v}(3)+3 b\right] N_{2} \\
\text { But } 2 / 3 \beta_{v}(3)=\beta_{v}(2), \frac{2 b}{\left.\beta_{v i}\right)}=q \\
I_{\operatorname{cog} p}=\beta_{i}(2) N_{2}-\beta_{v}(2)(1+q) N_{2} \\
\therefore=\left[1-\frac{\beta_{v}}{\beta_{i}}(1+q)\right] \cdot \beta_{i}(2) N_{2}
\end{gathered}
$$


459

dubctitute of (5), (13) and $(19.75)$

$$
I_{\text {loop }}=\left[1-\frac{\beta_{v}}{\beta_{i}}\left(1+\frac{a_{i}^{2} b}{10 \Omega D_{v c v}}\right)\right] \frac{I_{k+p}^{*}}{\left[1+\frac{2 a_{c}^{2} b}{\partial_{1 i} D_{i}} e^{8 / k T}\right]^{0}}-(19)
$$

Whare $I_{\text {Roop }}^{*}=\frac{z_{2 i} \Omega^{2} D_{i} C_{i}{ }^{3}}{a_{0}{ }^{2}} \exp (B / k T)$ Eq $(19.77)$

When $b \rightarrow 0$ ef $(19)$ reduces to eq $(19.90)$ 
460

PROBLEM 19.8

Volume contraction

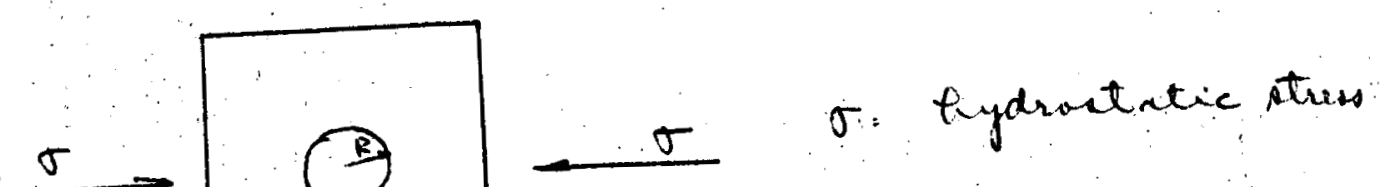

Free energy of the Ayer-

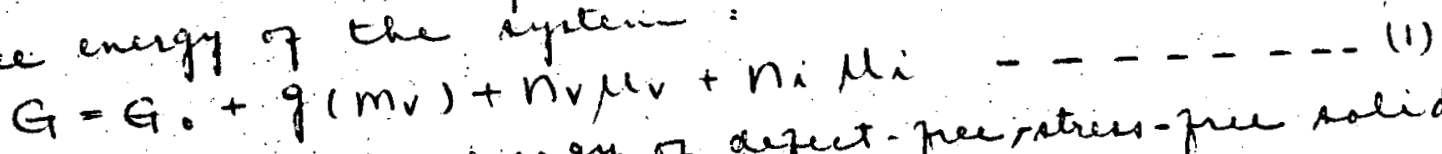

- where $G_{0}$ is pres energy of defect-peerstres-free solid $g(m$,$) is the reversible wale required$ to create a void containing mv vacancies agamic external procure $\sigma$ with constant internal gas precise $P$ in the crusty.

$$
\begin{aligned}
& g\left(m_{v}\right)=\epsilon\left(m_{v}\right)+\Delta V_{\text {exr }} \sigma-\Delta V_{\text {void }} \rho \ldots \ldots(2) \\
& \Delta V_{\text {ext }}=\Delta V_{\text {Via }}=m_{v} \Omega \ldots-\ldots \text { - (3) } \\
& \epsilon\left(m_{v}\right)=4 \pi R^{2} \gamma-\ldots-\ldots-14 \\
& m_{v}=\frac{4 / 3 \pi R^{3}}{\sqrt{2}}-\cdots \cdots-\ldots \text { (5) } \\
& G=G_{0}+\epsilon\left(m_{v}\right)+m_{v} \Omega(\sigma-p)+n_{v} \mu_{v}+n_{i} \mu_{i} \\
& \delta G=\frac{d \epsilon}{a m_{v}} \delta m_{v}+\Omega(\sigma-p) \delta m_{v}+\mu_{v} \delta n_{v}+\mu_{i} \delta n_{i}=0 \text {. } \\
& \delta m_{v}=\delta n_{i}-\delta n_{v}
\end{aligned}
$$




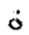

461

$$
\begin{aligned}
& \delta G=\left[\frac{a \epsilon}{d m_{v}}+2(\sigma-p)\right]\left(\delta n_{i}-j n_{v}\right)+\mu_{v} \delta n_{v}+\mu_{i} \delta n_{i}=0 \\
& \frac{d \epsilon}{d m_{v}}=\frac{d \epsilon}{d R} \frac{d R}{d m_{v}}=(8 \pi R \gamma)\left(\frac{\Omega}{4 \pi R^{2}}\right)=\left(\frac{2 \gamma}{R}\right) \Omega \\
& \left.\left(\frac{\lambda \gamma}{R}-p+\sigma\right) \Omega+\mu i=i \quad-\ldots\right) \\
& \text { But } \left.\mu_{i}=-\mu_{v} \quad \cdots-\ldots-\ldots\right)
\end{aligned}
$$

Chemical patential of vacancit.

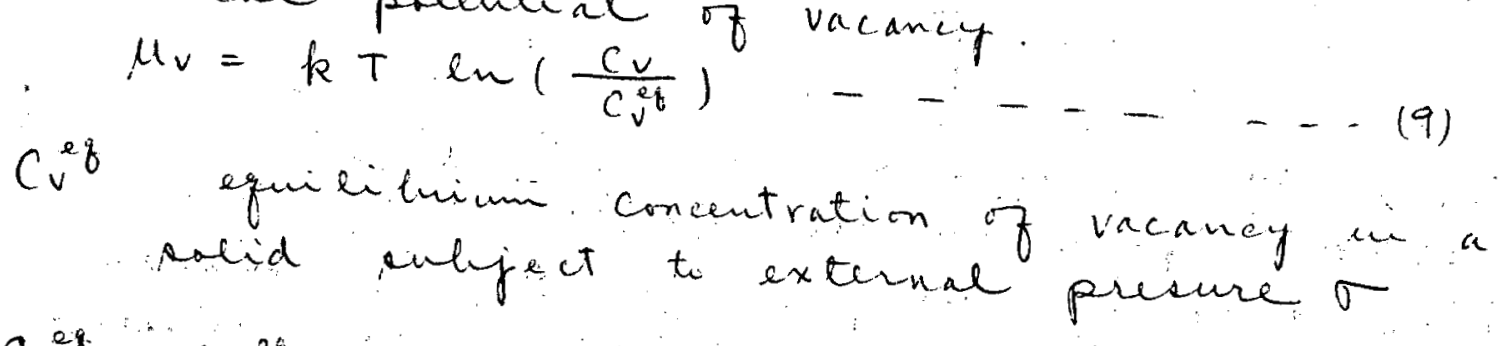

$$
E_{v}^{e q}=\left(C_{v}^{q}\right)_{0} \exp \left(-\frac{\sigma \tau}{h T}\right)----(10)
$$

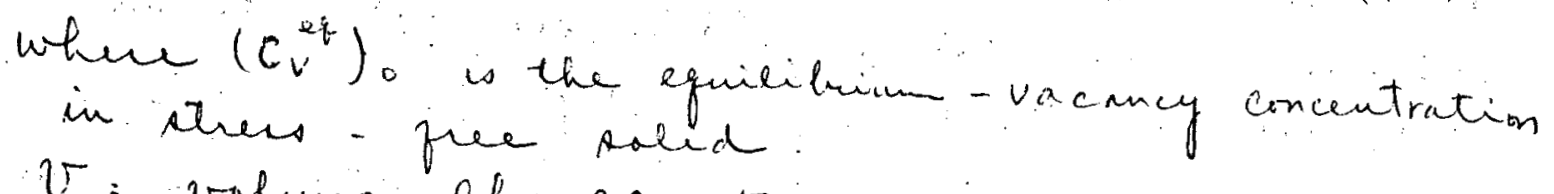

$2 T=$ repuine change of Aalid when an atom move from interion to rungace

1) Volume 7 calid ixitially $=V$

2) Remone an. atom to surifie, NOT allomg

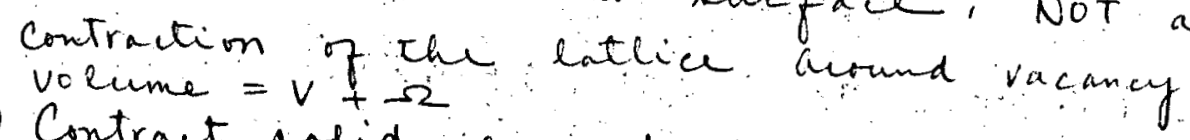

$$
\begin{aligned}
& \text { Volume }=v+\Omega 2-\psi_{V}
\end{aligned}
$$

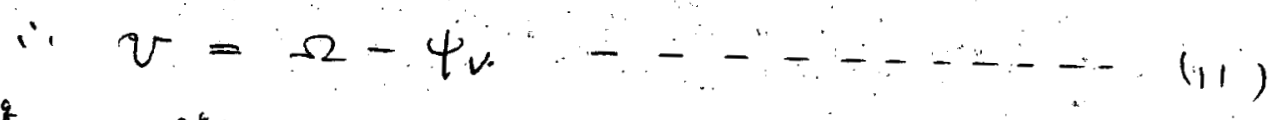

$$
\begin{aligned}
& C_{v}^{e q}=\left(C_{v}^{e q}\right)_{0} \cdot \exp \left[-\frac{T\left(\Omega-\varphi_{v}\right)}{k T}\right]-(12)
\end{aligned}
$$


462

Sulutitute (12) into (9)

$$
\mu_{v}=k T \ln \left(\frac{C_{v}}{C_{v}^{2 t}}\right)=k T \ln \left[\frac{C_{v}}{\left(C_{v}\right)_{v}}\right]+k T \ln \left[\frac{\left(C_{v}^{2 t}\right)_{0}}{C_{v}^{p q}}\right]
$$

But, $\ln \left[\frac{\left(C_{v}^{e q}\right)_{0}}{C_{v}^{\varepsilon^{j}}}\right]=\frac{\sigma\left(\Omega-\Psi_{v}\right)}{k T}$

$$
\therefore \mu_{v}=k T \ln \left[\frac{C_{v}}{\left(C_{v}^{0}\right)_{0}}\right]+\sigma\left(\Omega-\psi_{v}\right)--(13)
$$

Insert (13) into (6)

$$
\begin{aligned}
& \left(\frac{\partial \gamma}{R}-p\right) \Omega+\sigma \Omega=k T \ln \left[\frac{C_{v}}{\left(C_{v}^{q}\right)_{0}}\right]+\sigma \Omega-\sigma \psi_{v} \\
& C_{v}=\left(C_{v}^{q}\right)_{0} \exp \left[\frac{(? \gamma / R-p) \Omega+\sigma \psi_{v}}{k T}\right]
\end{aligned}
$$

This is the equiliburim vacancy concentration in the solid adjacent to a void of radius $R$, with internal gas premise $P$ and in a hydrostatic (compressive) dues field $\sigma$ 
463

PROBLEM 19.9

$A$. The diffucion of is

$$
D_{v} \nabla^{2} C_{v}+G^{\prime \prime}=k^{2} D_{v} C_{v}
$$

where $k^{2}=z_{v} \rho_{d}+4 \pi R N+4 \pi R_{p} N_{p}$

$$
\text { and } G^{\prime}=\nu \Sigma_{s} \phi-k_{i v} C_{i} C_{V}
$$

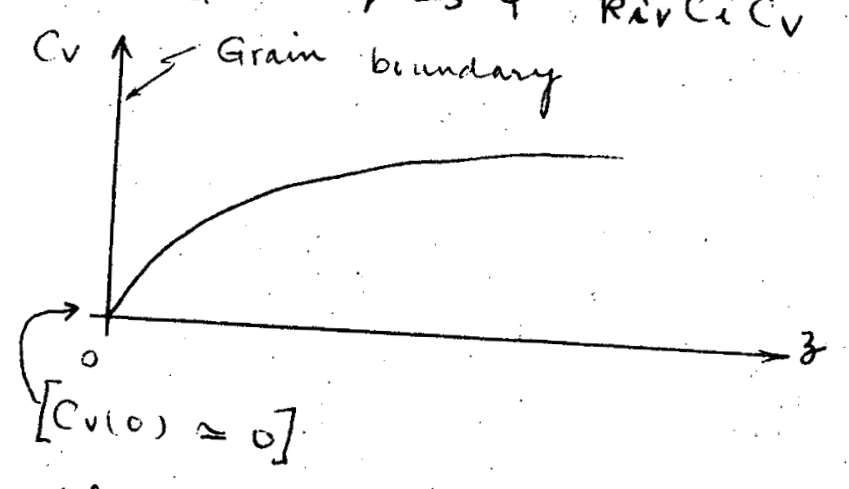

Undur the arsumption of remi-infinite medium,

$$
\frac{d^{2} c_{v}}{d z^{2}}-k^{2} c_{v}=-\frac{G^{\prime}}{D_{v}}=-G
$$

B.C $\quad$ 1) $C_{v}(0)=0$

$\Rightarrow C_{v}(\infty)$ is finile

$\Rightarrow$ the polution is $C_{v}(z)=A e^{-k z}+B E^{k z}+\frac{G}{k^{2}}$

$\begin{array}{ll}\left.C_{v} \infty\right) \text { is finic } & \Rightarrow B=0 \\ C_{v}(0)=0 \quad \Rightarrow \quad A+\frac{\theta^{2}}{k^{2}}=0 & \Rightarrow A=-\frac{G}{k^{2}}\end{array}$

$C_{v}(z)=\frac{G}{k^{2}}\left(1-e^{-k z}\right)$

Ro $J_{V}=-D_{V}\left(\frac{d C_{v}}{d z}\right)_{z}=-\frac{D_{V} G}{k}$ 
B.

Total ahsouption hy grain boundary $=4 \pi\left(\frac{d}{2}\right)^{2} J_{v}$ $Q_{V}^{g b}=\frac{4 \pi\left(\frac{d}{2}\right)^{2} J_{V}}{\frac{4}{3} \pi\left(\frac{d}{2}\right)^{3}}=\frac{6 J_{V}}{d}=\frac{6 D V G}{k \cdot d} \frac{\text { vacancies }}{\mathrm{am}^{3}-\mu i}$.

Since $Q_{v}^{g b}=k_{g b}^{2} D_{r} C_{v}(\infty)$

$$
=k g b^{2} D_{v} \cdot \frac{G}{k^{2}}=\frac{6 D_{v} G}{k d}
$$

Thui $k g^{2}=\frac{6 k}{d}$ the abouc equation is che graim boundary
sink efficiency for uke in paint defect
balance 
465

$$
\begin{aligned}
& \frac{\text { PROBLEM } 19.10}{\text { From Eq }(19.139)=} \\
& \frac{\dot{R}^{2}}{\dot{R}_{0}}=F(\eta)+\frac{\dot{R}_{e}}{\dot{R}_{v}} \\
& \text { Eq }(19,142)= \\
& F(\eta)=\frac{2}{\eta}[\sqrt{1+\eta}-1] \\
& \eta=\frac{4 z_{i v} D \sum_{s} \Phi \Sigma_{2}}{D_{v} a_{0}^{2}\left(z_{v} \rho_{d}\right)^{2}} \ldots \ldots \text { (3) }
\end{aligned}
$$

-

$$
\begin{aligned}
& D_{v}=10^{3} a_{r}^{2} \exp \left(-\varepsilon_{v}^{*} / k T\right) \\
& a_{0} \simeq \Omega^{1 / 3} ; z_{v} \simeq 1, \quad \Omega \simeq \| \AA^{3} \text { (For Mo): } \\
& \eta=\frac{4 \times(30)(100)(0.3)\left(10^{14}\right)}{10^{13}\left(2.2 \times 10^{-8}\right)\left(10^{10}\right)^{2}} \exp \left(\frac{190}{8.31\left(T_{\mathrm{m} / 10^{3}}\right)} \frac{1}{T / T_{m}}\right) \\
& =1.6 \times 10^{-8} \exp \left[\frac{7.88}{T / T_{m}}\right] \ldots(5)
\end{aligned}
$$

From Eq $(19.140)$ inch $N-N_{p}=0$

$$
\dot{R}_{0}=\frac{P \Sigma_{s} \Phi\left(z_{i}-z_{v}\right) \Omega}{R z_{v} z_{i} f_{d}}
$$

From $E_{q}(19.144)\left(R_{p}=\rho_{L}=0, \quad \rho_{N}=\rho_{d}\right)$

$$
\dot{R}_{e}=-\frac{D_{v} C_{v}^{e q} \Omega z_{v} \rho_{d}\left(\frac{z r}{R} \frac{\Omega}{k T}\right)}{R z_{v} \rho_{d}}
$$

Fro, (t) and (7)

$$
\frac{\dot{R}_{e}}{\dot{R}_{0}}=-\frac{D_{v} C_{v}^{v}-\Omega\left(\frac{2 r}{k T}\right) z_{i} z_{v} \rho_{d}}{R \nu \Sigma_{s} \Phi\left(z_{i}-z_{v}\right)}
$$


466

where $\frac{k T}{2 \gamma} \cong 1 \dot{A}^{2}$

$$
\begin{aligned}
& D_{v} C_{v}^{v} \Omega=10^{13} a_{0}^{2} \exp \left(-\frac{\varepsilon_{v}^{*}}{k T}\right) \exp \left(-\frac{\varepsilon_{v}}{k T}\right) \\
& =10^{13} \Omega^{2 / 3} \exp \left(-\frac{\varepsilon^{*}+\varepsilon_{V}}{k T}\right) \\
& \frac{R_{e}}{\dot{R}_{0}}=-\frac{\left(10^{13}\right)\left(11^{3 / 3} \times 10^{-16}\right)(1.02)\left(10^{10}\right) \exp \left[-\frac{190+190}{(8.31)\left(T m / 10^{3}\right)} \frac{1}{(T / T m)}\right]}{\left(10^{-16}\right)\left(300 \times 10^{-8}\right)(100)(0.3)\left(10^{14}\right)(0.02)} \\
& \frac{\dot{R}_{e}}{\dot{R}_{e}}=->8 \times 10^{15} \exp \left(-\frac{15.8}{T / T_{m}}\right) \\
& \begin{array}{l|c|c|c|c|}
T / T_{m} & 7 & F(7) & R_{e} / R_{0} & K / R_{0} \\
\hline 0.1 & 2.7 \times 10^{26} & 1.22 \times 10^{-13} & -7 \times 10^{-54} & 1.22 \times 10^{-13} \\
0.2 & 2.1 \times 10^{9} & 4.4 \times 10^{-5} & -1.4 \times 10^{-19} & 4.4 \times 10^{-5} \\
0.3 & 4.09 \times 10^{3} & 0.031 & -38 \times 10^{-8} & 0.031 \\
0.35 & 95.9 & 0.184 & -7 \times 10^{-5} & 0.184 \\
0.4 & 5.75 & 0.56 & -0.020 & 0.54 \\
0.41 & 3.56 & 0.64 & -0.051 & 0.59 \\
0.42 & 2.25 & 0.80 & -0.129 & 0.67 \\
0.43 & 1.46 & 0.78 & -0.309 & 0.47 \\
0.44 & 0.96 & 0.33 & -0.711 & 0.12 \\
0.45 & 0.64 & 0.88 & -1.06 & -0.18
\end{array}
\end{aligned}
$$

From the alone table peak raid curdling if or Mo should he ar $\frac{T}{T_{m}}=0.42$, ie at $2945^{\circ} \mathrm{C}$ 

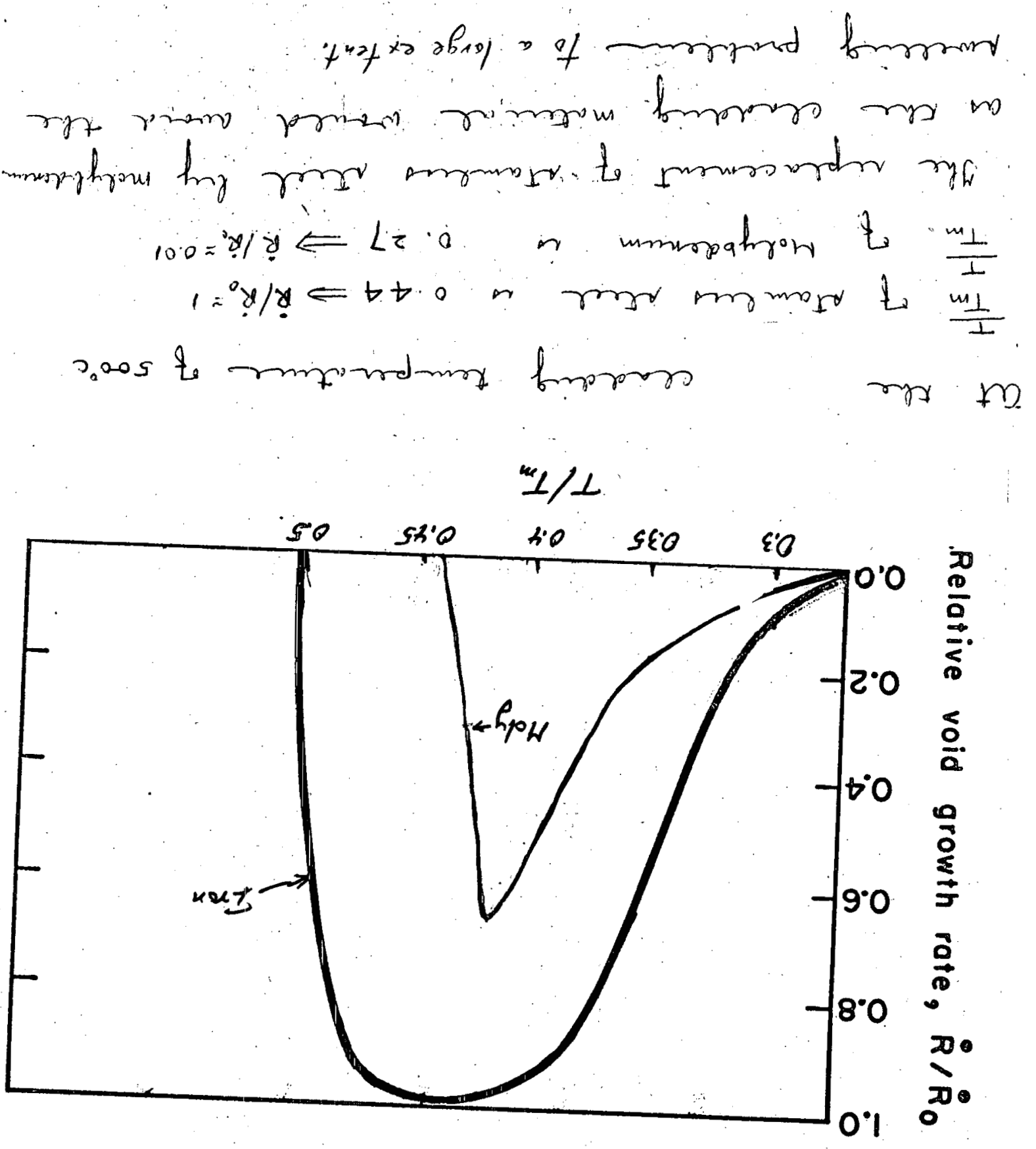
468

PROBLEM 19.11

$$
\frac{\text { ROBLE } 19.11}{D_{v} C_{v}^{2 q}}=\frac{e^{-\left(\varepsilon_{v}^{* q}+\varepsilon_{v}\right) / k T}}{e^{-\left(\varepsilon_{i}^{*}+\varepsilon_{i}\right) / k T}}=\exp \left[\frac{\left(\varepsilon_{i}^{*}+\varepsilon_{i}\right)-\left(\varepsilon_{v}^{*}+\varepsilon_{v}\right)}{k T}\right] .
$$$$
\varepsilon_{i}^{*}+\varepsilon_{i}=433 \mathrm{~kJ} / \mathrm{mali}
$$$$
\varepsilon_{v}^{*}+\varepsilon_{v}=285 \mathrm{~kJ} / \text { mole }
$$

$$
\text { at } T=773^{\circ} \mathrm{K}
$$

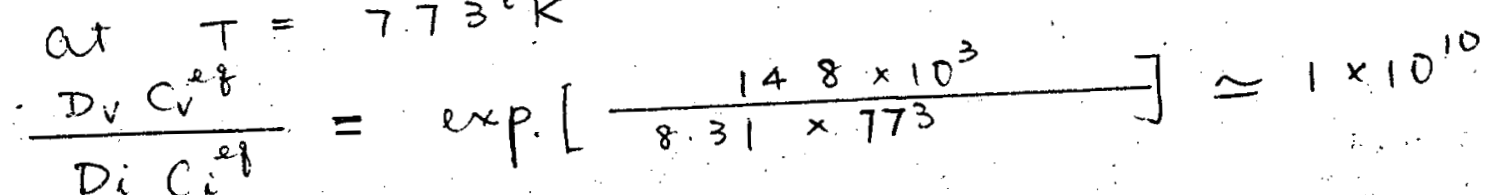

Since $\frac{D_{r} C_{r}^{e q}}{D_{i} C_{i}^{e q}}$ is large, $\gamma^{*}$ is tenge compared

to unity and the solution would be located in the region of Fig 19.17 wick $\theta i \simeq 0, \theta i r 1$ In this ane, Yo from eq $(19.130)$ his the limiting hchouion (axtumig $a_{0} / R_{p}<1$ ):

and from $E_{q}(19.131): Y_{v}=\frac{D_{i} C_{i}^{2 q}}{D_{v} C_{v}^{2 q}} \simeq 10^{-10}$

$\therefore$ Vacancy alcioption by coherent precipitates is negligible at thermal equidibiom 
469

PROBLEM 19.12

In the absence of thermal emersion, Eq (19.95)

becomes:

$$
Q_{v}^{v i d}=4 \pi R N D_{v} C_{v} w_{v} \ldots \ldots \text { (1) }
$$

and for interstitial:

$$
Q^{\text {void }}=4 \pi R N D_{i} C_{i} w_{i} \ldots \ldots . . . .(2)
$$

Point defect balance becomes $\left(E_{q}(19,136)\right.$ and Eq (19.130) neglect recombination and coherent precipitates)

$$
\begin{aligned}
& \nu \Sigma_{5} \Phi=4 \pi R N D_{v} C_{v} \omega_{v}+z_{v} \rho_{a} D_{v} C_{v}--(3) \\
& \nu \Sigma_{s} \Phi=4 \pi R N D_{i} C_{i} \omega_{i}+z_{i} \int_{d} D_{i} D_{i} C_{i} \ldots-(4)
\end{aligned}
$$

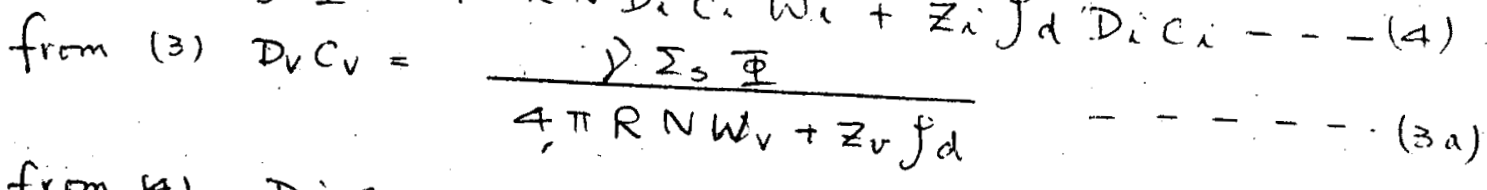

$$
\begin{aligned}
& \text { from (4) } D_{i} C_{i}=\frac{D \Sigma_{3} \Phi}{4 \pi R N W_{i}+Z_{i} f_{d}}-\ldots-(4 a)
\end{aligned}
$$

The void growth low, Eq (19:i38) becomes (with void lias factors included):

$$
\dot{R}_{0}=\frac{\Omega}{R}\left(D_{v} C_{v} w_{v}-D_{i} C_{i} w_{i}\right) \ldots(-\ldots)
$$

Clang (Ba) and $(4 a)$ in (5)

$$
\begin{aligned}
& \dot{R}_{c}=\frac{P \Sigma_{s} \Phi \Omega}{R}\left[\frac{W_{v}}{4 \pi R N W_{v}+Z_{v} \rho_{d}}-\frac{W_{i}}{4 R W_{i}}\right. \\
& =\frac{P \sum_{s} \Omega\left[4 \pi R \Delta W_{i} W_{v}+\rho_{d} z_{i} W_{v}-4 \pi R N W_{v} W_{i}-\rho_{d} z_{v} W_{i}\right]}{R\left(4 \pi R N W_{v}+z_{v} \rho_{d}\right)\left(4 \pi R N W_{i}+z_{i} \rho_{d}\right.} \\
& =\frac{\nu \Sigma_{s} \Phi \Omega \rho_{d}\left(z_{i} W_{v}-z_{v} W_{i}\right)}{R\left(4 \pi R N W_{v}+z_{v} \rho_{d}\right)\left(4 \pi R W_{i}+z_{i} \rho_{d}\right)}
\end{aligned}
$$


470

In denominator, let $W_{v}=W_{i}, Z_{i}=Z_{v}$ (NOT in numeration, though)

$$
\begin{aligned}
& \dot{R}_{0}=\frac{p \Sigma_{s} \Phi \Omega \rho_{d} W_{v}\left(z_{i}-z_{v} \frac{W_{i}}{W_{v}}\right)}{R\left(4 \pi N W_{v}+z_{v} \rho \rho_{d}\right)^{2}} \\
& =\frac{\nu \Sigma_{s} \Phi \Omega \rho_{d} W_{v}\left(z_{i}-z_{v} \frac{W_{i}}{\left.W_{v}\right)}\right.}{R Z_{v}^{2} \rho_{d}^{2}\left[1+\frac{4 \pi R N W_{v}}{z_{v} \rho_{d}}\right]^{2}} \times \frac{4 \pi R N}{4 \pi R N} \\
& \dot{R}_{0}=\frac{\nu \Sigma_{S} \Phi \Omega\left(Z_{i}-z_{v} \frac{W_{i}}{W_{v}}\right)}{4 \pi R^{2} N Z_{v}} \times \frac{4 \pi R N W_{v} / z_{v} \rho d}{\left[1+\frac{4 \pi R N W_{v}}{Z_{v} \rho d}\right]^{2}} \\
& \text { Let } X=\frac{4 \pi R N W_{V}}{Z_{V} \rho d}\left(\text { Replace } E_{q}(19.146)\right) \\
& \Rightarrow \dot{R}_{i}=\frac{\nu \Sigma_{s} \Phi \Omega}{4 \pi R^{2} N}\left(\frac{z_{i}-z_{v} \frac{\omega_{i}}{\omega_{v}}}{z_{v}}\right) \frac{x}{(1+x)^{2}} \cdots-(8)
\end{aligned}
$$




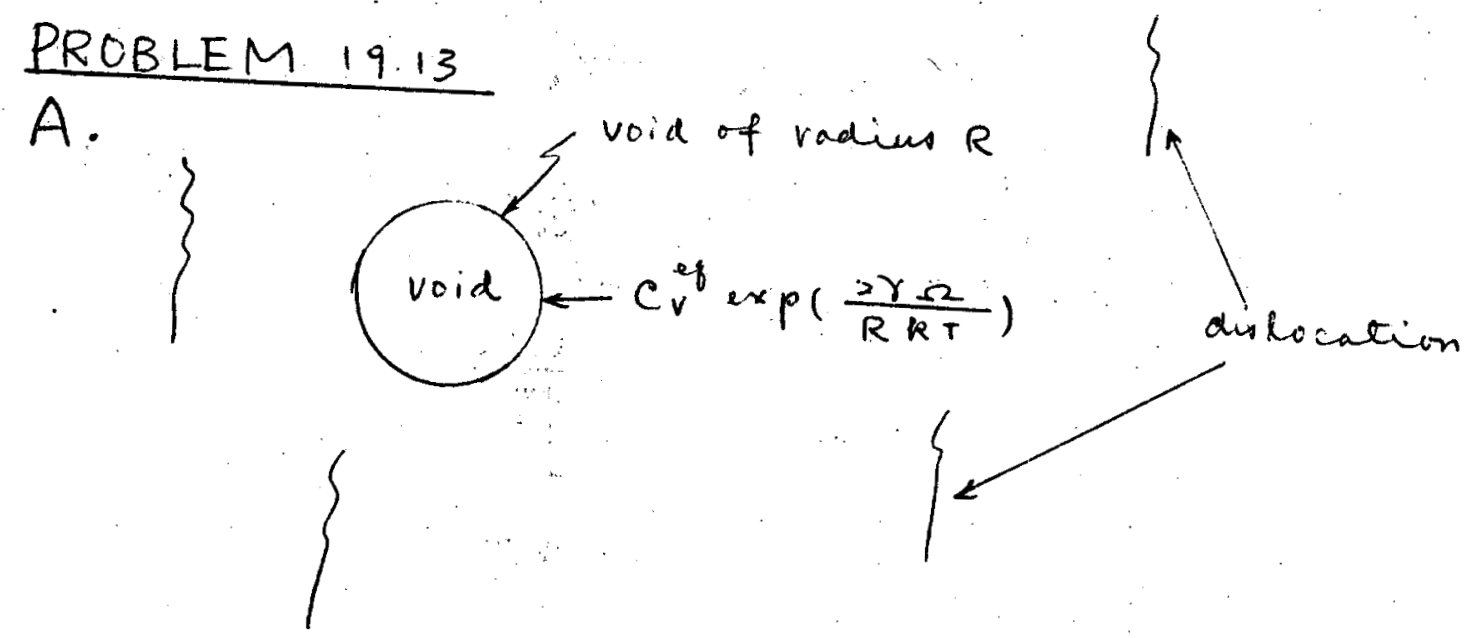

Qvid

From Eq $(19.95)$

$$
Q_{v}^{\text {vide }}=4 \pi R N D_{v}\left[C_{v}^{\text {eq }} \exp \left(\frac{2 \gamma \Omega}{R k T}\right)-C_{V}\right] \ldots
$$

- $Q_{v}^{d}=\operatorname{sink}$

$Q_{v}^{d}=z_{v} D_{v} \rho_{d}\left(C_{v}-C_{v}{ }^{2 q}\right) \ldots \ldots$ dislecations, From $E_{f}(19.101)$

Pure annealing $Q_{v}^{d}=Q_{v}^{\text {veid } \ldots . . . . ~(3) ~}$

or

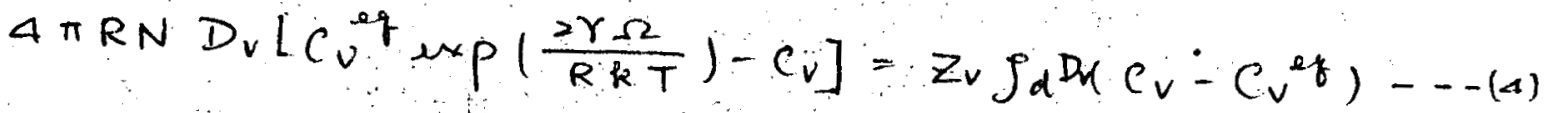

Solve (4) for $C_{v}$

$$
C_{v}=C_{v}^{e f}\left[\frac{1+\frac{Z_{v} \rho_{d}}{4 \pi R N}+\frac{2 \gamma \Omega}{R k T}}{1+\frac{z_{v} \rho_{d}}{4 \pi R N}}\right] \cdots-(5)
$$

Sulctitute into ii)

$$
Q_{v}^{\text {vid }}=4 \pi R N D_{v} C_{v} f \frac{\frac{2 \gamma \Omega}{R k T}}{1+\frac{4 \pi R N}{Z_{V} \rho_{d}}}--(6)
$$


472

Qvid/N : rate of lie of vacancies from a single void

$\left(Q_{v}^{v o i d} / N\right) \Omega$ rate of volume lois from void

$$
\frac{d}{d t}\left(\frac{4}{3} \pi R^{3}\right)=-\frac{Q_{v}^{\text {void }}}{N} \Omega
$$

$$
\begin{aligned}
& \text { Whig (6) } \\
& \frac{d R}{d t}=-\frac{2 \gamma D_{v} C_{v}^{2 q} \Omega^{2}}{R^{2} k T} \frac{1}{1+\frac{4 \pi R N}{Z_{v} \rho d}}-\cdots(7) \\
& \text { Integrate (7) } \\
& \int_{R_{0}}^{R} R^{2}\left(1+\frac{4 \pi N}{Z_{v} \rho d} R\right) d R=-\frac{2 \gamma D_{v} C_{v}^{2 q} \Omega^{2}}{k T} t \\
& R_{0}^{3}-R^{3}+\frac{3}{4}\left(\frac{4 \pi N}{Z_{v} \rho d}\right)\left(R_{0}^{4}-R^{4}\right)=3 D_{v} C_{v}^{2 q} \Omega^{2}\left(\frac{2 \gamma}{k T}\right) t-(8)
\end{aligned}
$$

When $\frac{4 \pi N}{Z_{V} \rho_{d}} \longrightarrow 0$, Recons solution of problem

$$
(18.1 a)
$$

B. Only vacancy concentration at void. Surface is changed

$$
\begin{aligned}
& \frac{d}{d t}\left(\frac{4}{3} \pi R^{3}\right)=-\frac{Q_{v}^{\text {id }} \Omega}{N}=-4 \pi R D_{v} \Omega\left[C_{v}^{\text {eq }} \exp \left(\frac{-P+\frac{2 r}{R} \Omega}{k \cdot T}\right)\right. \\
& \left.-C_{v}^{e q}\right] \\
& \text { But } P\left(\frac{4}{3} \pi R^{3}\right)=j k T \\
& \because \frac{d R}{d t}=-\frac{D_{v} C_{v}^{2 r} \Omega}{R}\left[\frac{2 \gamma}{R}-\frac{j k T}{\frac{4}{3} \pi R^{3}}\right] \frac{\Omega}{k T}
\end{aligned}
$$




$$
\begin{aligned}
& \frac{d R}{d t}=-D_{v} C_{v}^{2} \Omega^{2}\left(\frac{\partial \gamma}{k T}\right) \frac{1}{R^{2}}\left[1-\frac{j}{\frac{a}{3} \pi}\left(\frac{k T}{2 \gamma}\right) \frac{1}{R^{3}}\right] \\
& \text { Let } a=\frac{j \cdot k T}{4 / 3 \pi k \gamma} \\
& \therefore \frac{R^{2} d R}{1-a / R^{2}}=-D_{v} C_{v}^{8 q} \Omega^{2}\left(\frac{2 \gamma}{k T}\right) d t \\
& \int_{R}^{R_{v}} \frac{R^{2} d R}{1-a / R^{2}}=D_{v} C_{v}^{q} \Omega^{2}\left(\frac{\partial \gamma}{k T}\right) \cdot t
\end{aligned}
$$


474

PROBLEM 19.14

(A).

the ares per atom is $(\Omega)^{2 / 3}=a_{0}^{2}$ aria per lop is $\pi R_{l}^{2}$

$m$ : rumbler of atoms in a loop$$
m=\frac{\pi R_{e}{ }^{2}}{a_{0}{ }^{2}}
$$$$
\frac{d m}{d t}=\left(J_{i}-J_{v}\right)=\pi R_{l}=\frac{d}{d t}\left(\frac{\pi R_{i}^{2}}{a_{0}^{2}}\right)
$$$$
=\frac{2 \pi R_{l}}{a_{0}^{2}} \frac{d R_{l}}{d t}
$$$$
\therefore \frac{d R_{l}}{d t}=a_{0}^{2}\left[z_{i} D_{i}\left(C_{i}-C_{i}^{l}\right)-z_{v} D_{v}\left(C_{v}-C_{v}^{l}\right)\right]
$$
(B). Setting $C_{v}^{q q}=k_{i v}=N_{p}=0$, in $E_{q}(19.136)$ and
(19.137) gives

$$
\begin{aligned}
& D \Sigma_{S} \Phi=\left[4 \pi R N+z_{V}\left(\rho_{N}+\rho_{L}\right)\right] C_{V} D_{V} \\
& \gamma \Sigma_{S} \Phi=\left[4 \pi R N+z_{i}\left(\rho_{N}+\rho_{L}\right)\right] C_{i} D_{i}
\end{aligned}
$$

Solving for $C_{i}, C_{V}$

$$
\begin{aligned}
& C_{v}=\frac{P \Sigma_{3} \Phi}{D_{V}\left[4 \pi R N+z_{V}\left(\rho_{N}+\rho_{L}\right)\right]} \\
& C_{i}=\frac{P \Sigma_{3} \Phi}{D_{i}\left[4 \pi R N+z_{V}\left(\rho_{N}+\rho_{L}\right)\right]}
\end{aligned}
$$

Inert $C_{V}, C_{i}$ into the loop grow ch low of pout (A), and Let $\rho_{d}=\rho_{N}+\rho_{L}$

$$
\therefore \frac{d R_{l}}{d t}=a_{0}^{2} \nabla \Sigma_{s} \Phi\left[\frac{z_{i}}{4 \pi R N+Z_{i} \rho_{l}}-\frac{Z_{v}}{4 \pi R N+Z_{0} \rho_{l}}\right]
$$


475

(c)

$$
\begin{aligned}
& \rho_{d}=\rho_{d}^{0}+2 \pi R_{R} N_{l} \\
& \frac{d \rho_{d}}{d t}=2 \pi N_{l} \frac{d R_{l}}{d t}
\end{aligned}
$$

Whe recult of part (B) for $\frac{d R_{l}}{d t}$

$$
\begin{aligned}
\therefore \frac{d \rho_{d}}{d t} & =2 \pi N l a_{0}^{2} \nu \Sigma_{s} \Phi\left[\frac{z_{i}}{4 \pi R N+z_{i} \rho_{d}}-\frac{z_{v}}{4 \pi R N+z_{v} \rho_{d}}\right] \\
& \text { if } z_{i} \simeq z_{v} \\
\frac{d \rho_{d}}{d t} & =\frac{2 \pi N_{l} \frac{\Omega}{a_{0}} \nu \Sigma_{s} \Phi}{4 \pi R N+z_{v} \rho_{d}}\left(z_{i}-z_{v}\right)
\end{aligned}
$$

(D) $E_{q} \cdot(19.140)$ can he written as$$
R \frac{d R}{d t}=\frac{\left(\nu \Sigma_{s} \Phi \Omega\right)\left(\frac{z_{i}-z_{v}}{z_{v}}\right) z_{v} \rho_{d}}{\left(4 \pi R N+z_{v} \rho_{d}\right)^{2}} \text {, since } N_{p}=0 \text { and }
$$

Let $x=\frac{4 \pi R N}{z_{v i}} . \quad Z_{i} \simeq Z_{v}$

- dimencionles time

thein equation $(190140)$ hecomes

$$
\frac{d x}{d \theta}=\frac{Y}{x(x+Y)^{2}}
$$

Aisumig $\rho_{d} \cong$ contant $\Rightarrow Y \cong$ constant

or $\int_{X(0)}^{X(\theta)}\left(x^{3}+2 Y x^{2}+Y^{2} x\right) d x=\int_{0}^{\theta} Y d \theta$ 
476

$$
\therefore \quad Y \theta=\frac{1}{4}\left(x^{4}-x_{0}^{4}\right)+\frac{2}{3} Y\left(x^{3}-x_{0}^{3}\right)+\frac{1}{2} Y^{2}\left(x^{2}-x_{0}^{2}\right)--(1)
$$

where $x_{0}=\frac{4 \pi R_{c} N}{Z_{v} \rho_{d}^{0}}$

$$
\begin{aligned}
Y & =\frac{\rho_{d}\left(t_{0}\right)}{\rho_{d}{ }^{\circ}}=\frac{\rho_{d}^{0}+2 \pi R_{l c} N_{l}}{\rho_{d}} \\
& =1+\frac{2 \pi R_{l c} N_{l}}{\rho_{i}}
\end{aligned}
$$

From (1) $x(\theta)$ con be valued

(E) From pout (C)

$$
\begin{aligned}
& \frac{d \rho_{d}}{d t}=\frac{2 \pi N i \Omega \nu \Sigma_{s} \Phi}{\left(4 \pi R N+z_{0} \rho_{d}\right) a_{0}}\left(z_{i}-z_{v}\right) \ldots(2) \\
& \text { Let } x=\frac{4 \pi R N}{z \cdot \rho a^{\circ}} \\
& Y=\frac{Z_{v} \rho_{d}}{z_{v} \rho_{a}^{0}} \\
& \theta=\frac{(4 \pi N)^{2}}{\left(Z_{V} \rho_{a}^{0}\right)^{3}}\left(8 \sum_{3}\right)\left(\frac{Z_{i}-z_{V}}{Z_{r}}\right)\left(t-t_{0}\right) \\
& \text { and } A=\frac{N_{l} Z_{v}{ }^{3} \rho_{d}}{8 \pi a_{0} N^{2}}=a \text { constant }
\end{aligned}
$$

then eq (2) becomes

$$
\frac{d y}{d \theta}=\frac{A}{x+Y}
$$

at time to, the number of vacancies in the void nucleic is equal to the number of interstitial in the em ln y loops

$$
\begin{aligned}
& \frac{\pi R_{l e}^{2} N_{c}}{a_{0}{ }^{2}}=\frac{4 / 3 \pi R_{c}{ }^{3} N}{R} \\
& \therefore R_{R}=\left[\frac{4}{3} R_{c}{ }^{3} \frac{N_{0}{ }^{2}}{N_{l}}\right]^{1 / 2}
\end{aligned}
$$


477

$$
\begin{aligned}
& \rho_{d}(0)=\rho_{d}+2 \pi R_{l c} N e \\
& \text { given } R_{c}=10 \dot{A}=10^{-7} \mathrm{~cm} \\
& N=10^{14} \text { voids } / \mathrm{cm}^{3} \\
& \begin{array}{l}
N_{l}=10^{15} \text { loops } / \mathrm{cm}^{3} \\
\rho_{d}^{0}=10^{9}
\end{array} \\
& \begin{array}{l}
\rho_{d}^{0}=109 \mathrm{~cm}^{-2} \\
\Omega=12 \mathrm{~A}^{3}
\end{array} \\
& X_{0}=\frac{4 \pi R_{c} N}{z_{V} \rho_{d^{0}}}=0.126 \\
& R_{l e}=\left[\frac{4}{3} R_{c}^{3} \frac{N}{N_{l}} \frac{a_{0}^{2}}{\Omega}\right]^{1 / 2}=0.76 \times 10^{-7} \mathrm{~cm} \\
& Y(0)=1+\frac{2 \pi R_{l c} N_{k}}{\rho_{a}^{0}}=148 \\
& A=\frac{N_{e} z_{v}{ }^{3} \rho_{i}^{0}}{8 \pi a_{0} N^{2}}=1.73 \times 10^{2}
\end{aligned}
$$

Now. solve the dipperintial equations numerically

$$
\left\{\begin{array}{l}
\frac{d x}{d \theta}=\frac{Y}{X(X+Y)^{2}} \\
\frac{d Y}{d \theta}=\frac{A}{X+Y}
\end{array}\right.
$$

with the initial conditions

$$
\begin{aligned}
& X_{0}=0.12 .6 \\
& Y_{0}=Y(0)=1.48 \\
& X_{i+1}
\end{aligned}
$$

and $X_{i+1}=X_{i}+\frac{Y_{i}(\Delta \theta)}{X_{i}\left(X_{i}+Y_{i}\right)^{2}}$.

$$
\begin{aligned}
& Y_{i+1}=Y_{i}+\frac{1(2 \cdot 3(\Delta \theta)}{X_{i}+Y_{i}} \\
& \Delta \theta=0=1
\end{aligned}
$$

With $\Delta \theta=001$ the ringlet are plotted 
478

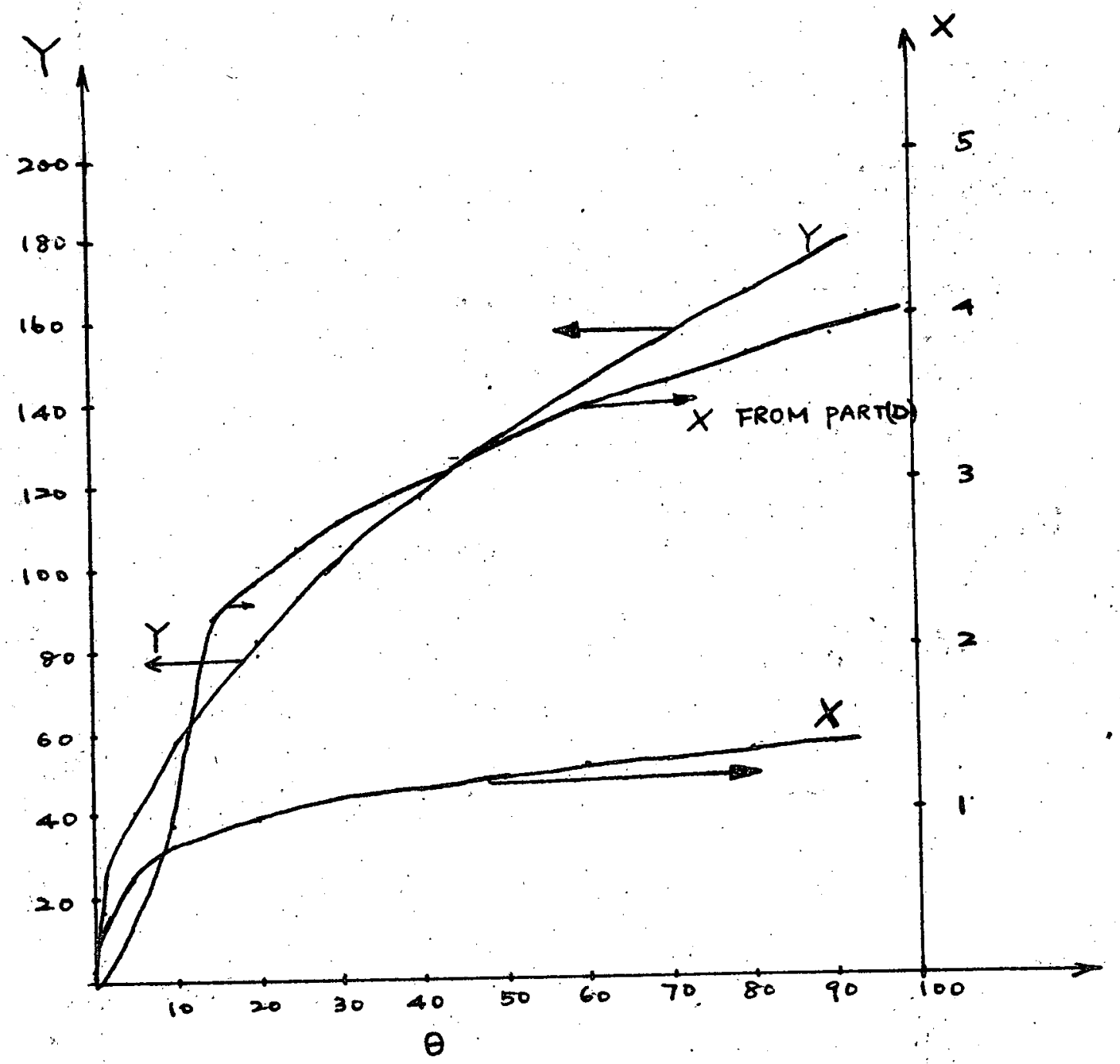

(F)

at a quince $5 \times 10^{22}, \theta=91$.

From the alcove plat, $x=1.418$. when $\theta=91$

$$
\begin{aligned}
& x=1.418 \Rightarrow R=112 \AA \\
& \frac{\Delta V}{V}=\left(\frac{4}{3} \pi R^{3}\right) \times N=0.67
\end{aligned}
$$

when $Y$ is ass used constant at $Y_{0}=1.48$, the foot show that $X=4$ at $\theta=91$. or, $R=316 \mathrm{~A}$. In this cause, $\Delta V / v=1.35 \%$. The increased

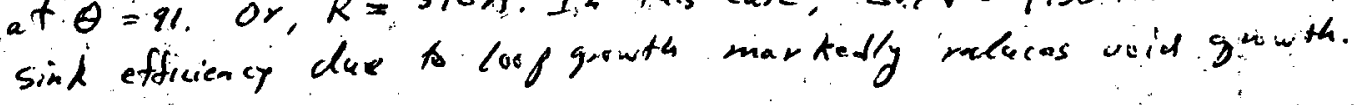


479 concentration of point defects:

$$
\begin{aligned}
& \dot{R}=\frac{A}{R}-\Omega\left(D_{r} C_{V}-D_{i} C_{i}\right)=\text { Constant }_{A}-(1) \\
& \text { (2) }
\end{aligned}
$$

(A.) For a uniform initial distribution, the

$$
R^{2}=R_{0}^{2}+2 A\left(t-t_{0}\right)
$$

and the impelling at time $t>t_{0}$ is:

$$
\begin{aligned}
& \left(\frac{\Delta V}{V}\right)_{t}=\left(\frac{4}{3} \pi \cdot R^{3}\right) N=\frac{4}{3} \pi\left[R_{0}^{2}+2 A\left(t-t_{0}\right)\right]^{3 / 2} N \\
& \text { For } R_{0}^{2}<<A\left(t-t_{0}\right) .
\end{aligned}
$$

(B.) General initial distribution $N_{0}\left(R_{0}\right)$ and distribution at time t $N(R, t)$

$$
N=\text { combatant }=\int_{0}^{\infty} N\left(R_{0}\right) d R_{0}=\int_{0}^{\infty} N(R, t) d R
$$

by grow eh law (3) $R$ o and $R$ are netted by

$$
\begin{aligned}
& R_{0}(R, t)=\left[R^{2}-A\left(t-t_{0}\right)\right]^{1 / 2}--(5) \\
& (R, t) \text { is what the }
\end{aligned}
$$

$R_{0}(R, t)$ is what the radius of void mut have been at to of radius at $t$ is $R$ 
480

$$
N(R, t)=N_{0}\left(R_{0}\right)\left|\frac{\partial R_{0}}{\partial R}\right| \ldots-\ldots(6)
$$

From (5) $\left|\frac{d R_{0}}{d R}\right|_{t}=\frac{R}{\left[R^{2}-2 A\left(\pi-\pi_{0}\right)\right]^{1 / 2}}$

$$
\therefore N(R, t)=\frac{R N_{0}\left[R_{0}\left(R, t_{0}\right)\right]}{\left[R^{2}->A\left(*-t_{0}\right)\right]^{1 / 2}} \cdots(7)
$$

$R_{0}(R, t)$ is given by eg (5)

$\therefore$ swelling $\left(\frac{\Delta V}{V}\right)_{t}=\int_{0}^{\infty} \frac{4}{3} \pi R^{3} N(R \cdot t) d R---(8)$

Use (7) in (8)

$$
\frac{\Delta V}{V}=\frac{4 \pi}{3} \int_{0}^{\infty} \frac{R^{4} N \cdot[R \cdot(R \cdot \pi)]}{\left[R^{2}-2 A\left(t-\pi_{0}\right)\right]^{1 / 2}} d R \ldots(9)
$$

(c) If the initial distribution is

$$
\begin{aligned}
& N_{0}\left[R_{0}(R . t)\right]=\delta\left[R_{0}(R . t)-R_{0}\right] N \\
&=\delta\left[\sqrt{R^{2}-2 A\left(t-t_{0}\right)}-R_{0}\right] N \\
& \frac{\Delta V}{V}=\frac{4}{3} \pi N \int_{0}^{\infty} \frac{R^{4} \delta\left[\sqrt{R^{2}-2 A\left(t-\pi_{0}\right)}-R_{0}\right]}{\left[R-2 A\left(\pi-\pi_{0}\right)\right]^{1 / 2}} d R
\end{aligned}
$$

$$
\begin{aligned}
& \text { Let } u^{2}=R^{2}-2 A\left(t-t_{0}\right) \\
& R^{4} d R=\frac{1}{2} R^{3} d R^{2}=u\left[u^{2}+2 A\left(t-t_{0}\right)\right]^{3 / 2} d u \\
& d R^{2}=>u d u \\
& \quad R^{3}=\left[u^{2}+2 A\left(\pi-t_{0}\right)\right]^{3 / 2} \\
& \frac{\Delta v}{v}=\frac{4}{3} \pi N \int_{0}^{\infty} \frac{u\left[u^{2}+2 A\left(t-t_{0}\right)\right]^{3 / 2} g\left(u-R_{0}\right) d u}{u}=\frac{4 \pi}{3} N\left[R_{0}^{2}+2 A\left(t-t_{0}\right)\right]
\end{aligned}
$$

neglecting $Q_{0}^{2}$, this is the result of bart $(A)$. 
481

PROBLEM $199^{\circ} 16$

(A.)

$$
\begin{aligned}
& \mathcal{L}=R \theta \ldots(1) \\
& A=\frac{R^{2}}{2}(\theta-\sin \theta) \ldots(x)
\end{aligned}
$$

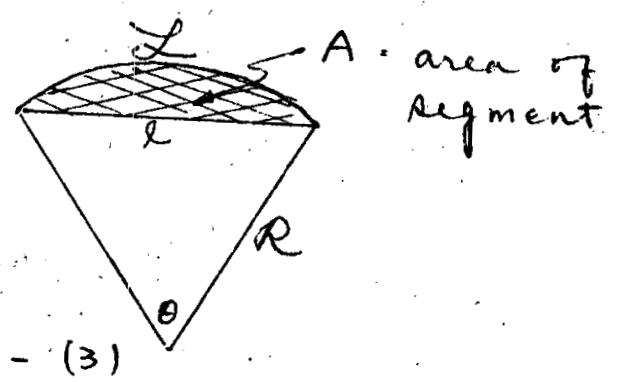

From (3) $R=\frac{l / 2}{\sin \frac{8}{2}} \cdots \cdots-(4)$

Insert (4) into (2)

$$
A=\frac{l^{2}}{8}\left(\frac{\theta-\sin \theta}{\sin ^{2} \theta / 2}\right)=\frac{l^{2}}{4}\left(\frac{\theta-\sin \theta}{1-\cos \theta}\right)
$$

lnient (4) into (1.)

$$
\mathscr{L}=\frac{l}{2}\left(\frac{\theta}{\sin \frac{\theta}{2}}\right)
$$

$$
\begin{aligned}
& \frac{d \mathcal{L}}{d \theta}=\frac{l}{2}\left[\frac{\sin (\theta / 2)-\left(\frac{\theta}{2}\right) \cos \left(\frac{\theta}{2}\right)}{\sin ^{2} \theta / 2}\right] \\
& \frac{d \mathcal{L}}{d A}=\frac{d \mathcal{L} / d \theta}{d \theta / d A}=\frac{(\theta / 2)[\sin \theta / 2-\theta / 2 \cos (\theta / 2)](1-\cos \theta)^{2}}{(l / 2)^{2} \sin ^{2} \theta /(1-\cos \theta)^{2}-(\theta-\sin \theta) \sin \theta} \\
& =\frac{\sin \theta / 2}{(2 / 2)} \quad \sin ^{2}(\theta / 2)
\end{aligned}
$$$$
=\frac{\sin \theta / 2}{(\theta / 2)}\left[4 \times \frac{\sin (\theta / 2)-(\theta / 2) \sin (\theta / 2) \cos (\theta / 2)}{(1-\cos \theta)^{2}-(\theta-\sin \theta) \sin \theta}\right]
$$$$
=\frac{1}{R}\left[4 \times \frac{\left.\frac{1}{2}(1-\cos \theta)-1 \frac{\theta}{2}\right) \frac{1}{2} \sin \theta}{1-2 \cos \theta+\cos ^{2} \theta-\theta \sin \theta+\sin ^{2} \theta}\right]
$$

$$
\begin{aligned}
& =\frac{1}{R} 2 \times \frac{1-\cos \theta-\frac{1}{2} \theta \sin }{2-2 \cos \theta-\theta \sin \theta} \\
& =\frac{1}{R} \frac{1-\cos \theta-\frac{1}{2} \theta \min \theta}{1-\cos \theta-\frac{1}{2} \theta \min \theta}=\frac{1}{R} \times 1=\frac{1}{R}
\end{aligned}
$$


482

$\therefore \frac{d L}{d A}=\frac{1}{R}$ for ale $\theta$

(B.)

$$
\begin{aligned}
& A=\frac{1}{2} R^{2}(\theta-\sin \theta) \\
& R=\frac{2 / 2}{\sin (\theta / 2)} \\
& \text { Let } q=\frac{1}{R}
\end{aligned}
$$

$$
\begin{aligned}
A & =\frac{1}{2} \frac{\theta-\sin \theta}{g^{2}} \\
& =\frac{l^{2}}{\theta} \frac{\theta-\sin \theta}{\sin ^{2} \theta / 2} \\
\frac{d A}{d q} & =\frac{d A}{d \theta} \frac{d \theta}{d q}=\frac{d A / d \theta}{d q / d \theta} \\
\frac{d A}{d \theta} & =\frac{l^{2}}{4}\left[\frac{1-\cos \theta-\frac{1}{2} \theta \min \theta}{2 \sin (\theta / 2)}\right] \\
\frac{d q}{d \theta} & =\frac{1}{(l / 2)} \frac{1}{2} \cos \left(\frac{\theta}{2}\right) \\
\frac{d A}{d(1 / R)} & =\frac{l^{3}}{8} \cdot \frac{1-\cos \theta-\frac{1}{2} \theta \sin \theta}{\sin ^{4}\left(\frac{\theta}{2}\right) \cos (\theta / 2)}
\end{aligned}
$$

Eliminate $\theta_{\ell}$ in the above equation big uni

$$
R=\frac{l / 2}{\sin \left(\frac{\theta}{2}\right)}
$$

Whin $\theta \rightarrow 0$$$
\cos \theta \cong 1-\frac{\theta^{2}}{2}+\frac{\theta^{4}}{4 !}
$$$$
1-\cos \theta=\frac{\theta^{2}}{2}-\frac{\theta^{4}}{4 !}
$$$$
\frac{1}{2} \theta \sin \theta=\frac{1}{2} \theta^{2}-\frac{\theta^{4}}{2 \times 31}
$$$$
1-\cos \theta-\frac{1}{2} \theta \sin \theta=\frac{\theta^{2}}{2}-\frac{\theta^{4}}{4 !}-\frac{\theta^{2}}{2}+\frac{\theta^{4}}{2 \times 3 !}=\theta^{4}\left(\frac{1}{2 \times 31}-\frac{1}{4 !}\right)
$$$$
=\frac{\theta^{4}}{4 !}
$$ 
483

$$
\begin{aligned}
& \sin ^{4}\left(\frac{\theta}{2}\right) \cong \frac{\theta^{4}}{16} \\
& \therefore \frac{a A}{d\left(\frac{1}{R}\right)} \cong \frac{l^{3}}{8} \times \frac{16}{4 !}=\frac{l^{3}}{12}
\end{aligned}
$$

When $0 \rightarrow \pi$

$$
\begin{aligned}
& \cos \theta \rightarrow-1, \operatorname{ain} \theta \rightarrow 0 \\
& \sin \left(\frac{\theta}{2}\right) \rightarrow 1, \cos \left(\frac{\theta}{3}\right) \rightarrow 0 \\
& \frac{d A}{d(1 / 0)} \rightarrow \infty
\end{aligned}
$$

(c) Evaluation: of $\left(\frac{\partial \mathscr{Z}}{\partial A}\right)_{R}$ for end connected to veid.

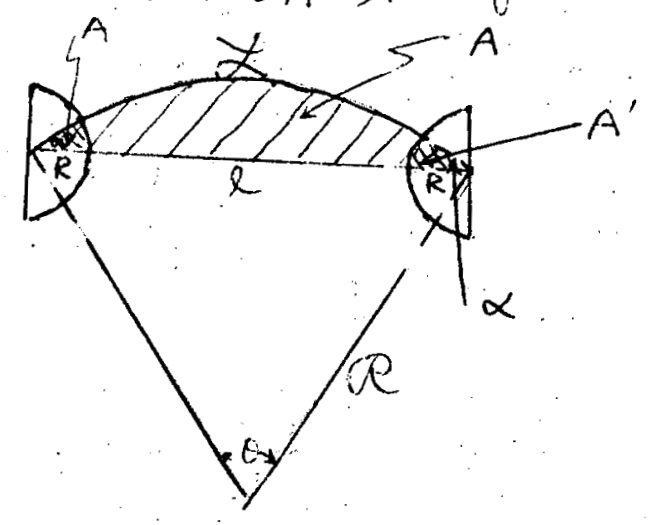

$$
A+>A^{\prime}=\frac{1}{2}: R^{2}(\theta-\operatorname{anc} \theta)
$$

arume lengti of line in wid $\simeq R$

$$
\begin{aligned}
R & =\frac{R / 2}{R(\theta / 2)} \cdots \cdots(3) \\
\alpha & =\frac{\theta}{2} \\
A^{\prime} & =\left(\pi R^{2}\right) \frac{\alpha}{2 \pi}=\frac{R^{2} \theta}{4}-\cdots(4) \\
\text { (4) in (1) } A & =\frac{1}{2} R^{2}(\theta-\sin \theta)-\frac{1}{2} R^{2} \theta \cdots(5)
\end{aligned}
$$


484

Invert (3) inte (2) $\mathcal{L}=\frac{A}{2}\left[\frac{\theta}{\sin \left(\frac{\theta}{2}\right)}\right]-2 R \cdots(6)$

$$
\left(\frac{\partial \mathcal{L}}{\partial \theta}\right)_{R}=\frac{l}{2}\left\{\frac{\sin (\theta / 2)-(\theta / 2) \cos (\theta / 2)}{\sin ^{2}(\theta / 2)}\right\}=\frac{l}{2}\left\{\frac{1-\cos \theta-\frac{1}{2} \theta \sin \theta}{\partial \sin ^{3}(\theta / 2)}\right\}-(7)
$$

$$
\begin{aligned}
& A=\frac{l^{2}}{4}\left(\frac{\theta-\sin \theta}{1-\cos \theta}\right)-\frac{1}{2} R^{2} \theta \ldots(8) \\
& \left(\frac{\partial A}{\partial \theta}\right)_{R}=\frac{l^{2}}{4}\left[1-\frac{(\theta-\sin \theta) \sin \theta}{(1-\cos \theta)^{2}}\right] \cdots \frac{1}{2} R^{2} \\
& =\frac{l^{2}}{4}\left[\frac{1-\cos \theta-\frac{1}{2} \theta \sin \theta}{2 \operatorname{Rin}^{4}\left(\frac{1}{2}\right)}\right] \cdot \frac{1}{2} R^{2} \\
& \left.\frac{\partial \mathcal{L}}{\partial A}\right)_{R}=\frac{(\partial \mathscr{X} / \partial \theta)_{R}}{(\partial / \partial \dot{\theta})_{R}} \\
& =\frac{\frac{l}{2}\left\{\frac{1-\cos \theta-\frac{1}{2} \theta \sin \theta}{2 \sin ^{3}(\theta)}\right\}}{\left(\frac{l}{2}\right)^{2}\left\{\frac{1-\cos \theta-\frac{1}{2} \theta \sin \theta}{2 \sin ^{4}(\theta / 2)}\right\}-\frac{1}{2} R^{2}} \\
& =\frac{\sin (\theta / 2)}{l / 2}\left\{\frac{1}{1-\frac{\frac{1}{2} R^{2}}{(l / 2)^{2}} \frac{\sin ^{4}\left(\frac{\theta}{2}\right)}{1-\cos \theta-\frac{1}{2} \theta \sin \theta}}\right\} \\
& =\frac{1}{R} \cdot\left\{\frac{1}{1-\frac{\frac{1}{2} R^{2}}{\left(\frac{d}{2}\right)^{2}} \times \frac{2 \sin ^{4}\left(\frac{\theta}{2}\right)}{1-\cos \theta-\frac{1}{2} \theta \sin \theta}}\right\} \\
& =\frac{1}{R} \frac{}{f(R, l, R)}
\end{aligned}
$$$$
\left(\frac{2 \mathscr{L}}{\partial A}\right)_{R}=\frac{1}{R} \frac{1}{f(R, i, R)}
$$ 
485

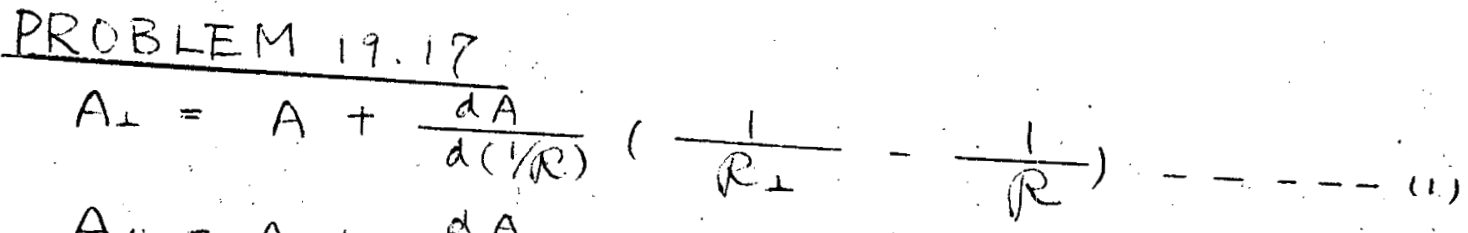$$
A_{11}=A+\frac{d A}{d(k)}\left(\frac{1}{R_{11}}-\frac{1}{R}\right) \ldots(2)
$$$$
C_{i}^{\prime}=C_{i}+\delta C_{i} \ldots \ldots \ldots-\ldots-\ldots(3)
$$

where $\frac{d A}{d(1 / P)}=P$

$$
\begin{aligned}
& \text { (1) } \times \frac{1}{3}+(2) \times \frac{2}{3} \\
& \frac{1}{3} A_{+}+\frac{2}{3} A_{\|}=A+\frac{1}{3} P\left(\frac{1}{R_{2}}-\frac{1}{R}\right)+\frac{2}{3} P\left(\frac{1}{R_{11}}-\frac{1}{R}\right) \\
& C_{i}+\delta+\frac{b f_{d} A}{l \Omega}+\frac{b y d P}{l \Omega}\left[\frac{1}{3}\left(\frac{1}{R_{1}}-\frac{1}{R}\right)+\frac{2}{3}\left(\frac{1}{R_{i}}-\frac{1}{R}\right)\right] \\
& =\left[\frac{\partial_{v} \Sigma_{s} \Phi}{k_{i v}}+\frac{4 \pi}{1.5} \frac{\sum_{s} \Phi_{1} R_{0}^{5}}{\Omega^{2} D_{i}}\right] \frac{1}{C_{i}+\delta} \\
& =\left[\frac{\nu_{V} \Sigma_{j} \Phi}{k_{i v}}+\frac{4 \pi}{15} \frac{\Sigma_{j} \Phi R_{0}^{3}}{\Omega^{3} D_{i}}\right] \frac{1}{C_{i}\left(1+\frac{s}{C_{i}}\right)} \\
& \simeq\left[\frac{\partial_{V} \Sigma_{i} \Phi}{R_{i v}}+\frac{4 \pi}{15} \frac{\Sigma_{i} \Phi R_{0}}{\Omega^{2} D_{i}}\right] \frac{1}{C_{i}}\left(1-\frac{\delta}{C_{i}}\right)
\end{aligned}
$$

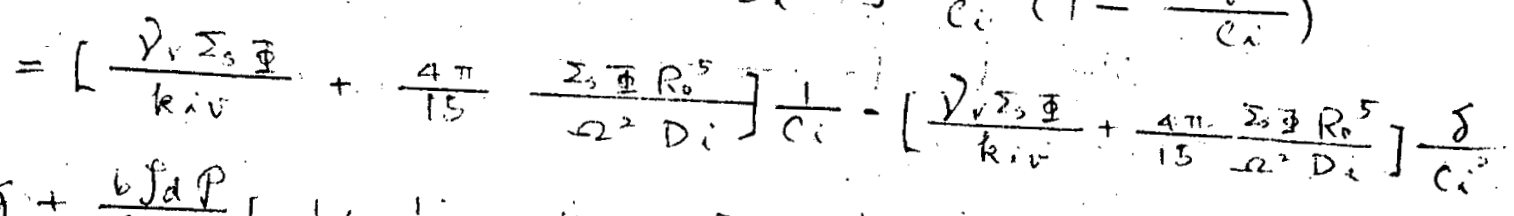

$$
\begin{aligned}
& \Rightarrow J+\frac{b \int_{d P}}{l \Omega}\left[\frac{1}{3}\left(\frac{1}{R_{2}}-\frac{1}{R}\right)+\frac{2}{3}\left(\frac{1}{R_{11}}-\frac{1}{R}\right)\right] \\
& =\left[\frac{\gamma_{r} \Sigma_{3} \Phi}{k_{i}}+\frac{4 \pi}{15} \frac{\Sigma_{s} \Phi R_{0}{ }^{5}}{\Omega^{2} D_{i}}\right] \frac{\delta}{C_{i}{ }^{2}} \ldots(4) \\
& \ln \left(\frac{C_{i}^{\prime}}{C_{i}^{2} i}\right)=\ln \left(\frac{C_{i}+g}{C_{i}^{2+}}\right)=\ln \left[\frac{C_{i}}{C_{i}^{+{ }^{2} t}}\left(1+\frac{\delta}{C_{i}}\right)\right] \\
& =\ln \left(\frac{\dot{C}_{i}}{C_{i}^{2}}\right)+\ln \left(1+\frac{\delta}{C_{i}}\right) \cong \ln \left(\frac{C_{i}}{C_{i}^{j}}\right)+\frac{\delta}{C_{i}}
\end{aligned}
$$


$48^{8} 6$

$$
\begin{aligned}
& \frac{T}{b R_{\perp}}-\sigma=\frac{k T}{\Omega} \ln \left(\frac{c_{i}}{c_{i}^{2} f}\right)+\frac{k T}{\Omega} \frac{\delta}{c_{i}} \\
& \frac{T_{d}}{b R_{\perp}}-\sigma=\frac{T_{d}}{b R}+\frac{k T}{\Omega} \frac{\delta}{c_{i}} \\
& \frac{T_{d}}{b}\left(\frac{1}{R_{\perp}}-\frac{1}{R}\right)=\sigma+\frac{k T}{\Omega} \frac{\delta}{c_{i}}- \\
& \frac{\tau_{d}}{b}\left(\frac{1}{R_{1}}-\frac{1}{R}\right)=\frac{k T}{\Omega} \frac{\delta}{c_{i}}--
\end{aligned}
$$

Ributitite (5) and (6) into (4)

$$
\begin{aligned}
& \delta+\frac{b f_{d} \rho}{l \Omega} \frac{b}{\tau_{d}}\left[\frac{1}{3}\left(\sigma+\frac{k T}{\Omega} \frac{\delta}{c_{i}}\right)+\frac{2}{3}\left(\frac{k T}{\Omega} \frac{\delta}{c_{i}}\right)\right] \\
& =\left[\frac{P_{v} \Sigma_{s} \Phi}{k_{i v}}+\frac{4 \pi}{15} \frac{\Sigma_{s} \Phi R_{0}{ }^{5}}{\Omega^{2} D_{i}}\right] \frac{\delta}{C_{i}^{2}} \\
& j+\frac{b^{2} \rho_{d} P}{l \Omega \tau_{d}}\left[\frac{1}{3} \sigma+\frac{k T}{\Omega} \frac{\delta}{c_{i}}\right]=\left[\frac{\gamma_{v} \Sigma_{s} \Phi}{k_{i v}}+\frac{4 \pi}{15} \frac{\Sigma_{3} \Phi R_{0}^{5}}{\Omega^{2} D_{i}}\right] \frac{\delta}{C_{i}{ }^{2}}
\end{aligned}
$$

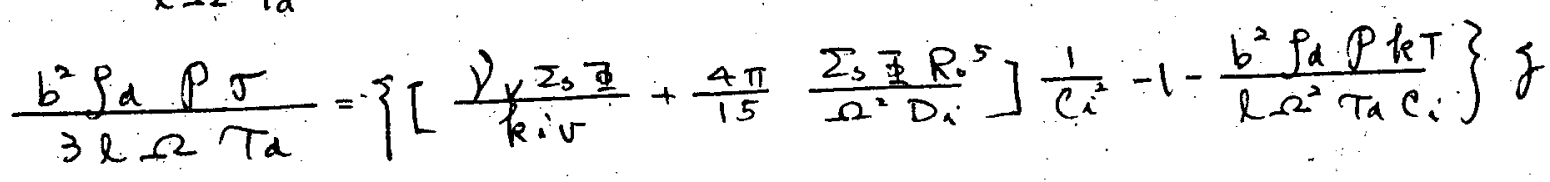

$$
\begin{aligned}
& \therefore \dot{j}=\frac{b^{2} \rho_{d} \rho \sigma}{3 l \Omega T_{d}\left\{\left[\frac{P_{v \Sigma_{s} \Phi}}{k_{i v}}+\frac{4 \pi}{15} \frac{\Sigma_{s} \Phi R_{i}^{5}}{\Omega^{2} D_{i}}\right] \frac{1}{C_{i}^{2}}-1-\frac{b^{2} \rho_{d} P_{k T}}{l \Omega^{2} \tau_{d} C_{i}}\right\}}
\end{aligned}
$$

Sulictilnte (7)" into (5) and rolve for $\frac{1}{R_{\perp}}-\frac{1}{R}$, then into (1) to get $A_{\perp}-A$ 
487

PROBLEM 19.18

number of uncallipard aistes in (m,dim) per PKA $=N(m) a_{m}=\frac{\nu}{\ln p} \frac{\alpha m}{m^{2}}$

Rale 7 formation of uncallapsed disks in $(m, d i n) / \frac{\text { s }}{4}$

$$
=\left(\frac{p}{\ln p}\right) \Sigma \text { 里 } \frac{d m}{m^{2}}
$$

Velume increace

uncollapsed disk 7 sige $m=m \Omega$

$$
\begin{aligned}
& \frac{d\left(\frac{\Delta v}{l}\right)}{d t}=\int_{1}^{m_{c}} \frac{\nu}{\ln \nu} \sum_{s} \Phi m \Omega \frac{d m}{m^{2}}=\Sigma_{s} \Phi-2\left(\frac{\gamma}{\ln \gamma}\right) \ln n \\
& \therefore \frac{\Delta V}{V}=\Sigma_{3} \Phi \Omega\left(\frac{P}{2 n \nu}\right) 2 n m_{c} \times T \\
& =\Omega \Sigma_{s}(\Phi t)\left(\frac{\nu}{\ln \nu}\right) \ln m_{c} \\
& \Omega=12 \times 10^{-24} \mathrm{~cm}^{3} . \\
& \Sigma_{5}=0.2 \mathrm{~cm}^{-1} \\
& \text { 里t }=10^{20} \text { neutrons/ } \mathrm{cm}^{2} \\
& m_{c} \simeq 200 \\
& \dot{\nu}=500 \text {. } \\
& \therefore \frac{\Delta V}{V}=\left(12 \times 0^{2-4}\right) \times(02)\left(10^{30}\right)\left(\frac{5+0}{2 n-500}\right) \ln 200 \\
& \simeq 10 \%
\end{aligned}
$$


$488^{\circ}$

PROBLEM 19.19

(A.) Let $\Omega\left[D_{r} C_{v}-D_{i} C_{i}\right]=A(t)$, then $E_{q}(19.175)$ (with $R_{c} \approx 0$ ) may he written as :

$$
\frac{1}{2} R^{2}=\int_{\tau}^{t} A\left(t^{\prime}\right) d t^{\prime}
$$

Take derivative with respect to $T$. at constant $t$

$$
\begin{aligned}
& \frac{\partial}{\partial T}\left(\frac{1}{\partial} R^{2}\right)=R\left(\frac{\partial R}{\partial T}\right)_{t}=\frac{R}{\left(\frac{\partial T}{\partial R}\right)_{t}} \\
& \frac{\partial}{\partial T} \int_{T}^{K} A\left(t^{\prime}\right) d t^{\prime}=-A(\tau) \\
& \therefore\left(\frac{\partial T}{\partial R}\right)_{\star}=-\frac{R}{A(\tau)}
\end{aligned}
$$

$\sin E_{q}(19173)$

$$
N(R \cdot t)=\frac{R I_{\text {nude }}[T(R+)]}{\Omega\left\{D _ { v } \left(C_{v}[T(R+t)]-D_{i}\left(i^{i}[T(R \cdot t)]\right\}\right.\right.}
$$

It is assumed that Co and Ci are conditions. and $I_{\text {mane }}=$ construe

$$
N(R, t)=\left\{\begin{array}{cc}
\frac{R I_{\text {null }}}{\Omega\left(D_{v} C_{v}-D_{i} C_{i}\right)} & \text { for } 0 \leq R \leq\left[2 \Omega\left(D_{i} C_{v}-D_{i}\left(C_{i}\right)\right]^{1 / 2} \sqrt{\pi}\right. \\
0 & \text { for } R>\left[2 \Omega\left(D_{v} C_{v}-D_{i}\left(C_{i}\right)\right]^{1 / 2} \sqrt{\pi}\right.
\end{array}\right.
$$

(B.)

$$
\begin{aligned}
& A=\Omega\left(D_{v} C_{V}-D_{i} C_{i}\right) \\
& \frac{\Delta V}{V}=\int_{0}^{R_{\max }} \frac{4}{3} \pi R^{3} N\left(R_{\cdot} t\right) d R
\end{aligned}
$$


489

$$
\begin{aligned}
& N(R)=\frac{R I_{\text {nucl }}}{A} \text {, up to } R_{\text {mix }}=\sqrt{2 A t} \\
& \frac{\Delta V}{V}=\frac{4}{3} \pi \frac{I_{\text {nucl }}}{A} \int_{0}^{\sqrt{2 A T}} R^{4} d R \\
& =\frac{4 \pi \times 2^{5 / 2}}{15} I_{\text {nuel }} A^{3 / 2} t^{5 / 2} \\
& =\frac{2^{9 / 2}}{15} \pi I_{\text {nucl }} \Omega^{3 / 2}\left(D_{v}\left(v-D_{i} C_{i}\right)^{3 / 2}+t^{5 / 2}\right.
\end{aligned}
$$

- Mhis model neglects the cinks of vacancies and interctitials inctrodaced ly thic void themsilues and by the Cut contant flinx 1 里:

$$
\frac{\Delta V}{V} \sim(\Phi t)^{5 / 2}
$$


490

CHAPTER 20

PROBLEM 20. 1

A.

Balance over dice dx in one ley of loop of Fig 20.7

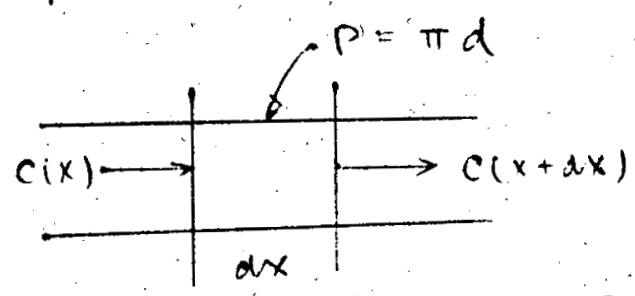

Cross sectional wren $A=\pi \frac{d^{2}}{4}$

$$
\begin{aligned}
& \text { Input }=v c A \\
& \text { output }=v\left(c+\frac{d c}{d x} d x\right) A \\
& \therefore \text { Net input from wall }=v \frac{d c}{d x} d x \cdot A \\
& \quad=K d(C \text { ant }-C) P d x \\
& \therefore \frac{d c}{d \cdot x}=\frac{K d(C \text { ant }-c) P}{v A}=\frac{4 K a}{d v}\left(C_{\text {ant }}-C\right)
\end{aligned}
$$

which is equation (>0.12)

$$
\begin{aligned}
& \text { Let } \eta=\frac{x}{L} \\
& \frac{d c}{d r}=\frac{4 K a L}{v^{2}}(c \text { ant }-c) \\
& \text { Let } \frac{4 K a L}{v d}=\gamma \\
& \frac{d c}{d \eta}+\gamma c=r c_{\text {ant }} \\
& \text { Integrand } C=A e^{-\gamma(\gamma}+C \text { ant } \\
& \text { when }-\bar{C}=0, C=C(0)=A+C \text { ant } \\
& \therefore A=C(0)-C_{A A T}
\end{aligned}
$$


491

aT $H_{0} \lg$

$$
\frac{C_{\text {nt }}^{n}-C_{(\eta)}}{C_{\text {nat }}^{n}-C_{(0)}}=e^{-\gamma_{h} T} \ldots \ldots 11
$$

(1) is equation (20.14a) in the text at cold leg

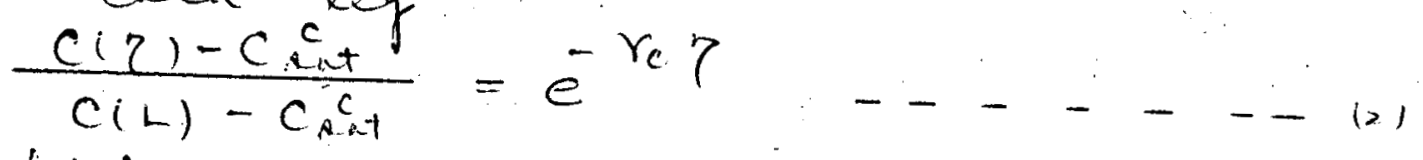

- which is equation $(20.14 b)$ in the text

B. at $x=L$

$$
\text { From (1) } \frac{C_{\text {nut }}^{h}-C(L)}{C_{\text {nit }}^{h}-C(0)}=e^{-\gamma_{h}} \cong 1-\gamma_{h}-\ldots(1)
$$

at $x>2 L$

From $121 \frac{C(0)-C_{\text {Lat }}^{c}}{C(1)-C_{\text {ant }}^{c}}=e^{-\gamma_{c}} \equiv 1-\gamma_{c} \ldots \ldots(4)$

Let $\beta=\frac{C_{x i t}^{c}}{C_{i n t}^{n}}<1$

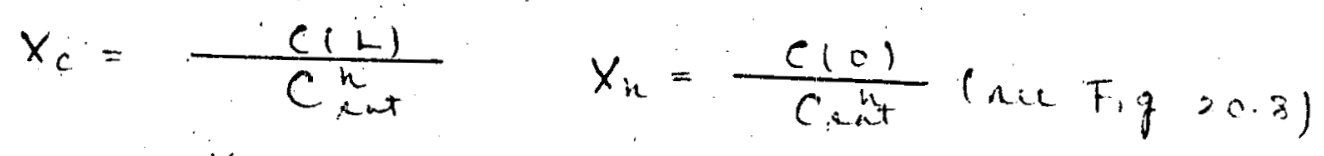

From (3) $\quad \frac{1-x_{c}}{1-x_{n}}=1-\gamma_{n}$

$$
\text { or } x_{c}=\gamma_{n}+\left(1-r_{n}\right) x_{n}-(5)
$$

From (4) $\frac{x_{h}-\beta}{x_{c}-\beta}=1-\gamma_{c}$

or $\quad x_{n}=\left(1-\gamma_{c}\right) x_{c}+\gamma_{c}$

driving (5) and (t)

$$
\left.x_{n}=\frac{\left.\beta \gamma_{c}+1-\gamma_{0}\right) \gamma_{n}}{1-\left(1-\gamma_{c}\right)\left(1-\gamma_{n}\right)}-17\right)
$$

as $\beta \rightarrow 1$. $x_{n} \rightarrow 1$ and as $\gamma_{n} \rightarrow 0 \quad x_{n \rightarrow 1}$

$$
X_{0}=\frac{\gamma_{n}+\beta \gamma_{c}\left(1-\gamma_{n}\right)}{1-\left(1-\gamma_{c}\right)\left(1-\gamma_{n}\right)} \cdots+\cdots 1
$$


492

a. $-1 \quad x_{c} \rightarrow 1$ and $a_{A} \gamma_{c} \rightarrow 0 \quad x_{c} \rightarrow 1$

C: Expond to firet onder in $\gamma_{c}$ dencminator. $7(7)$ :

$$
\begin{aligned}
& 1-\left(1-\gamma_{c}\right)\left(1-\gamma_{n}\right)=1-\left[1-\left(\gamma_{c}+\gamma_{n}\right)+\gamma_{c} r_{n}\right] \\
& =\gamma_{c}+r_{n}-\gamma_{c} \gamma_{n}=\gamma_{n}+\left(1-\gamma_{n}\right) \gamma_{c} \\
& =\gamma_{n}\left[1+\left(\frac{1-\gamma_{n}}{\gamma_{n}}\right) \gamma_{c}\right]
\end{aligned}
$$

$$
\begin{aligned}
& \text { Numerator of equation }(7) \\
& \beta \gamma_{c}+\left(1-\gamma_{c}\right) \gamma_{n}=\gamma_{n}\left[1-\gamma_{c}+\frac{\beta}{\gamma_{n}} \gamma_{c}\right] \\
& =\gamma_{n}\left[1+\left(\frac{\beta-\gamma_{n}}{\gamma_{n}}\right) \gamma_{c}\right] \\
& \quad x_{k}=\frac{1+\left(\frac{\beta-\gamma_{n}}{\gamma_{n}}\right) \gamma_{c}}{1+\left(\frac{1-\gamma_{n}}{\gamma_{n}}\right) \gamma_{c}}---
\end{aligned}
$$

For $r_{0} \rightarrow$ (9) becomes

$$
\begin{aligned}
x_{n} \rightarrow & 1-\left[\frac{1-\gamma_{n}}{\gamma_{n}}-\frac{\beta-\gamma_{n}}{\gamma_{n}}\right] \gamma_{c} \\
& 1-\left(\frac{1-\gamma_{n}-\beta+\gamma_{n}}{\gamma_{n}}\right) \gamma_{c} \\
x_{n}=1-(1-\beta) \frac{\gamma_{c}}{\gamma_{n}} & 1-\gamma_{c} \\
1-x_{n} & =(1-\beta) \frac{\gamma_{n}}{\gamma_{n}}
\end{aligned}
$$

Uang (10) in 15$)$

$$
\begin{aligned}
& x_{c}=x_{n}+\left(1-\gamma_{n}\right)\left[1-\frac{\gamma_{c}}{\gamma_{n}}(1-\beta)\right] \\
& \text { or } x_{c}=1-\left(\frac{1-\gamma_{n}}{r_{n}}\right)(1-\beta) \gamma_{c}-\frac{1}{j}-(11)
\end{aligned}
$$

eati of remoinl of metal from tot ley = rate 7 metal dipintion in cald leg

$$
=\text { few rate } x[C(L)-C(0)] \text { gmosec }
$$


493

flow rate $=V A \mathrm{~cm}^{3} / \mathrm{sec}$ rate of metal trampout $=V A[C(L)-C(0)]$ $=v A C_{p a t}^{n}\left(x_{c}-x_{n}\right)$

ling (ic) and (ii)

rate of metal transient $=V A C_{\text {sat }}^{h} \gamma_{c}(1-\beta)$

$$
\begin{aligned}
& =v \frac{\pi d^{2}}{4}\left[C_{\text {Rat }}^{n}-C_{\text {Ant }}^{c}\right]\left(\frac{4 K_{d c} L}{v d}\right) \\
& =\pi d L K_{d c}\left(C_{\text {int }}^{h}-C_{\text {Rat }}^{c}\right)
\end{aligned}
$$

This is just the deposition rate over entire cold leg (area' $\pi d L$ ) with a bring force equal to the patination concutration difference between the that and cold leg if $C_{\text {nat }}^{c} \ll C_{\text {ant }}^{n}$, transport rate is independent of. $T_{h}-T_{c}$. 
794

PROBLEM 20.2

Type 316 sated contain 0.3 wt $\%$ dillon ( $X_{\text {si }} \cong 0.006$ atom fraction).

The reaction is

$\mathrm{Na}=\mathrm{O}$ (ablution in $\mathrm{Na})+\frac{1}{3} S_{i}$ (solution in rede)

$$
=\frac{1}{3} \mathrm{Na}_{2} \mathrm{SiO}_{3}(\mathrm{~s})+\frac{4}{3} \mathrm{Na}(\mathrm{l}) \ldots(1)
$$

Since the silicon form ideal solution in serer, the activity of silicon in sated is equal to. its atom fraction $X_{\text {si }}$, the law of mast is

$$
\frac{1}{a_{0}^{*} x_{3 i}^{1 / 3}}=\exp \left(-\frac{\Delta G_{1}^{0}}{R T}\right) \cdots(2)
$$

where $a_{0}^{*}$ : critical oxygen activity for $\mathrm{NaSiO}_{3}$ precipitation

$\Delta G_{1}{ }^{\circ}$. standard At ate que energy change

$$
\begin{aligned}
& 2 \mathrm{Na}(l)+\frac{1}{2} \mathrm{O}_{2}(g)=\mathrm{Na}_{2} \mathrm{O}(\mathrm{s})--(3) \\
& \Delta G_{f \mathrm{Na}, \mathrm{C}}^{0}=-39 \tau+118\left(\mathrm{~T} / 10^{\mathrm{s}}\right) \mathrm{kJ} / \text { Equation }(=0.16)
\end{aligned}
$$

Formation of $\mathrm{Na}, \mathrm{SiO}_{3}$ from the elements:

$$
\begin{aligned}
& \frac{2}{3} \mathrm{Na}(l)+\frac{1}{3} S_{i}(3)+\frac{1}{2} C_{2}(g)=\frac{1}{3} \mathrm{Na}_{2} S_{i} \mathrm{O}_{3}(5) \ldots(4) \\
& \Delta G_{f \mathrm{Na}_{2} \mathrm{SiC}}^{0}=-5+1+9.5\left(\mathrm{~T} / 10^{3}\right) \mathrm{KT} / \mathrm{malc} \\
& \Delta G_{1}^{0}=\Delta G_{f N A=S i C_{3}}^{0}-\Delta G_{f N a O O}^{0}=-114-23\left(T / 10^{\circ}\right) \\
& \text { at } T=700^{\circ} \mathrm{C}=973^{\circ} \mathrm{K} \mathrm{C} \quad \Delta G_{1}^{\circ}=-136^{\circ} .4 \mathrm{~kJ} / \mathrm{molcc}
\end{aligned}
$$


495

$$
\begin{aligned}
a_{0}^{*} & =x_{3 i}^{-\frac{1}{3}} \exp \left(\Delta G_{i} / R T\right) \\
& =(0.006)^{1 / 3} \exp [-136.4 /(8.314)(0.973)] \\
& =2.6 \times 10^{-7}
\end{aligned}
$$

From. Eq (20.18), $C_{\text {Rat }}=5680 \mathrm{pPm} @ 700^{\circ} \mathrm{C}$

$\therefore$ The critical oxygen concentration in sodium

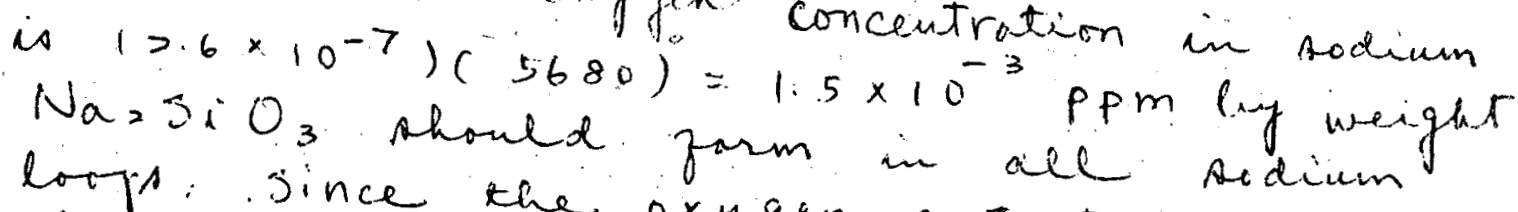
tor pi. Since the oxygen content is greater 
496

PROBLEM 20.3 IRON surface

Sodium

$$
C_{0}=0 x y g e n \text { concentration }
$$
in bulk Aodinin

Let $\operatorname{Cint}(0)=$ interface concentration of 0 in sodium

Diffusion rate of oxygen to surface firm - - $(1)$ bilk podium $=k_{d}\left(C_{D}-C_{\text {int }}(0)\right) \mathrm{gms} \% \mathrm{~cm}^{2}-\mu \mathrm{c}$

The reaction proceeds at the interface. according to Eq $(>0.24)$ as long as $C_{\text {int }}(0)$ is greater them the critical oxygen concentration

$$
a_{0}^{*}=\exp \left(\Delta G^{0} / R_{T}\right)-\ldots-\cdots(2)
$$

Whet $\Delta G^{\circ}$ is the standard free energy of the ration given by equation 120.23). In this problem, $\Delta G^{\circ}$ is assumed to be more negative than. the value calculated in the text., so that $a_{0}^{*}$ is ales small. The stand and. oxygen concentration is given by.

$$
C_{0}^{*}=a_{0}^{*} C_{\text {ant }}(0)
$$

where $($ out 10$)$ is the actuation concentration of 
497

oxygen in Na $\left(E_{q}(20,181)\right.$

The rate of this reaction

$N_{a}=O$ (solution in $N_{a}$ at interface) $+\frac{1}{3} \mathrm{Fe}(\mathrm{s})$

$$
=\frac{1}{3} \mathrm{FeO} \cdot\left(\mathrm{Na}_{2} \mathrm{O}\right)=(\mathrm{s})+\frac{2}{3} \cdot \mathrm{Na}(\mathrm{l})
$$

is proportional to the difference between $C_{\text {int }}(0)$ and $C_{0}^{*}$.

The rate of reaction of $\mathrm{Na}_{2} \mathrm{O}$ with iron $=\operatorname{kr}\left(C_{\text {int }}(0)-C_{0}^{*}\right)$

Since the diffusion and reaction steps are in series, We may equate (1) and (4) and

$$
\begin{aligned}
& \operatorname{kd}\left(C_{0}-C_{\text {int }}(0)\right)=k_{R}\left(C_{\text {int }}(0)-C_{0}^{*}\right) \ldots(5) \\
& \text { or } C_{\text {int }}(0)=\frac{\left(\frac{k d}{k_{R}}\right) C_{0}+C_{0}^{*}}{1+\frac{k_{d}}{k_{R}}}-\ldots(6)
\end{aligned}
$$

Sulsticiting (6) into (1) gives the oxygen transport rate to the ruizace: Rate of oxygen transit $=K d\left(C_{0}-C_{0}^{*}\right) \ldots(7)$

Where $K_{d}$ is the overall transfer coefficient including the chemical reaction tip represented

$$
\frac{1}{k_{d}}=\frac{1}{k_{a}}+\frac{1}{k_{R}}-\ldots-(8)
$$

Eg (7) gives the rite of oxygen transport to the surface To get che corrasion rate: 
498

each gram atom of 0 removes $1 / 3$ gram atom of $F_{e}$ therefore

$$
\dot{m}=\frac{1}{3} \times \frac{56}{16} \times \text { oxygen transport rate in } \frac{\text { gmo }}{\operatorname{con}^{2}-\mu_{c}}-(-9)
$$

Using (7) and (8) in (9)

$$
\dot{m}=\frac{56}{48}\left[\frac{1}{\frac{1}{k d}+\frac{1}{k_{R}}}\right]\left(C_{0}-C_{0}^{*}\right) \frac{g m \cdot F_{e}}{c^{2}-\mu_{c}}-f(10)
$$

No corrosion occurs if $C_{0} \leqslant C_{0}^{*}$ In this calculation based upon $\mathcal{F}(20.25)$ in the text $C_{0}^{*}$ was. estimated to be $130 \mathrm{ppm}$ \& $450^{\circ} \mathrm{C}$ and $1650 \mathrm{ppm}$ (a) $700^{\circ} \mathrm{C}$ generally, $C_{0} \leq 5 \mathrm{PPm}$. However, if $\Delta G_{f i n f e r i z e}$ is mare negative than indicated big $E_{q}(20.21): \Delta G^{\circ}$ of equation $(20.23)$ is aldo more negative Hence, equations (2) and (3) show that $C_{0}$ * becomes smaller if $\left.C_{\text {ant }}\right)$ is less than $\sim 1$ ppm Curation hin formation of sodium ferrite could occur and it rate would be given by equation (10) 


\section{9}

PROBLEM 20.4

$\Omega=12 \AA^{3}=12 \times 10^{-24} \mathrm{~cm}^{3}$

$(3 \Omega)^{1 / 3}=\left(36 \times 10^{-34}\right)^{1 / 3}=3.3 \times 10^{-8} \mathrm{~cm}$

$\gamma=100$ dyres/an (interfacial terision)

$\frac{r}{k T}=10^{3}$ dyne $\left(.8 \times 10^{3}\right)=1.1 \times 10^{-13}$ dyne. $\mathrm{an}$

kT $\frac{10^{2} \text { dyne } / \mathrm{cm}}{1.1 \times 10^{-13} \text { dyre }-\mathrm{cm}}=10^{15} \mathrm{~cm}^{-2}$

$\left(36 \pi \Omega^{2}\right)^{1 / 3}=(4 \pi)^{1 / 3}(3 \Omega)^{2 / 3}=2.5 \times 10^{-15} \mathrm{~cm}^{2}$
$\xi=2.5$

$(4 \pi)^{1 / 6} \frac{(3 \Omega)^{1 / 3}}{3^{1 / 2}}=\frac{1.5 \times 3.3 \times 10^{-8}}{\sqrt{2.5}}=3 \times 10^{-8} \mathrm{am}$

In mideation formule, $N_{s}=$ Iron atoms $/ c \cdot c$

$\simeq c=S C_{\text {Aat (Fe) }}^{\text {tat }}$ (i.e the number of nuileation rites = number of
atoms in solution)
$\therefore I=3 \times 10^{-8} D\left(C_{\text {nat }}^{\text {nat }} 5\right)^{2} \ln 5 \exp \left[-\frac{4}{27} \frac{\xi^{3}}{(\ln .5)^{2}}\right]$
$S=\frac{\left.C_{\text {Ant }}^{\text {not }}\right]}{S}$ $S=\frac{C_{\text {nat }}^{\operatorname{not}}\left(F_{e}\right)}{C_{\text {Cat }}^{\text {Cold }}}$

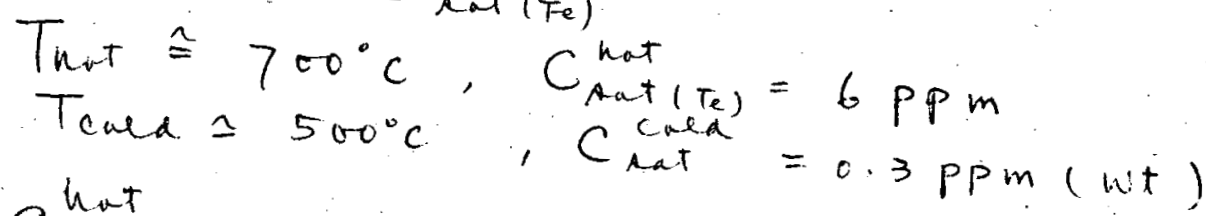

$\left.C_{\text {nat }}^{\text {hat }} F_{e}\right)=3 \times 10^{-7} \frac{g m_{c} F_{e}}{g m N_{a}} \times 0.8 \frac{g m N_{n}}{m^{2}} \times \frac{g m a t o m}{5 t g m F_{e}}$

$\times 6.02 \times 10^{23} \frac{\text { atoms } F_{e}}{\text { gmatom } F_{e}}=3 \times 10^{15} \frac{\text { atomo } F_{\varepsilon}}{a^{3}}$

Whin the fot leg ralution in cabled to $500^{\circ} \mathrm{C}$, Initial $S=\frac{6}{0.3}=20, \ln s=3$ 
500

$\left(C_{\text {nat }} S\right)^{2}=\left[\left(3 \times 10^{15}\right) \cdot(20)\right]^{2}=3.6 \times 10^{33} \mathrm{~cm}^{-6}$ the diffusivity of iron in poblimm is

$$
\begin{aligned}
& \frac{4}{27} \frac{\xi^{3}}{(\ln 5)^{2}}=\frac{4}{27} \times \frac{(2.5)^{3}}{(3)^{2}}=0.26 \\
& e^{-0.76} \simeq 1
\end{aligned}
$$

The nucleation rate is

$$
I=\left(3 \times 10^{-8}\right)\left(1 \times 10^{-5}\right)\left(3.6 \times 10^{33}\right)\left(.3 \times 10^{1}\right)
$$

the size of the critical rielcus is

$$
m_{c}=\left(\frac{2 \xi}{3 \ln 5}\right)^{3}=\left(\frac{5}{9}\right)^{3}<1
$$

This means that classical ideation theory is probably NOT valid 
501

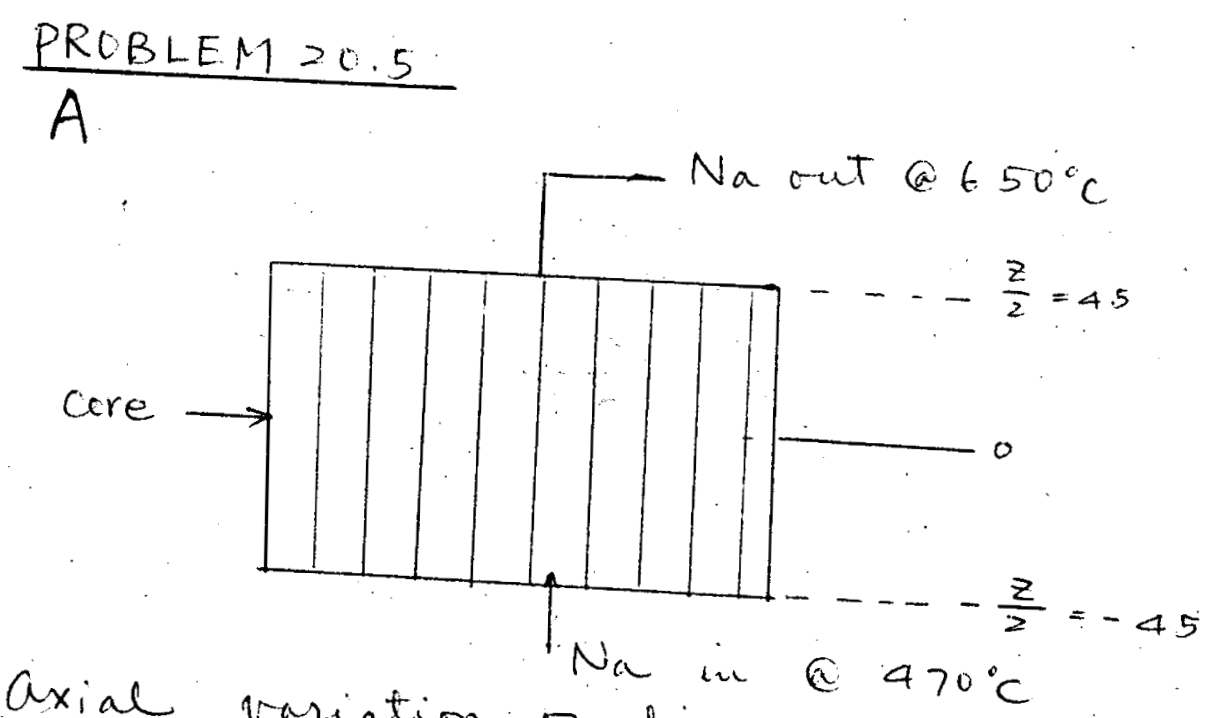

axial variation of linear power

$$
P(z)=P_{0} \cdot \cos \left(\frac{\pi z}{z^{\prime}}\right)
$$

hire $z^{\prime}$ is déturnined by the axial porte perking factor

$$
\begin{aligned}
& \quad \frac{P(z=0)}{P\left(z=\frac{z}{2}\right)}=1.8=\frac{1}{\cos \left(\frac{\pi z}{2 z^{\prime}}\right)} \\
& \therefore \frac{\pi z}{2 z^{\prime}}=\cos ^{-1}\left(\frac{1}{1.8}\right)=0.98 \\
& P(z)=P_{0} \cos \left(\frac{\pi z}{z^{\prime}} \frac{z}{z}\right)=P_{0} \cos \left(1.86 \frac{z}{z}\right)--11
\end{aligned}
$$

Neglecting power generation in blanket:

$$
\begin{aligned}
& \text { Total } \text { rod power }^{z / 2}=\int_{-\frac{z}{2}}^{z} p(z) d z=P_{0} \int_{-\frac{z}{2}}^{z / 2} \cos \left(1.9 t \frac{z}{z}\right) \\
& =\frac{2 z P_{0}}{1.96}
\end{aligned}
$$

also

$$
\text { Total rod power }=\frac{\text { reactor electrical power }}{\text { thermal efficiency } x \text { number of rod }}
$$


502

$$
=\frac{10^{9} \mathrm{wath}_{\mathrm{s}}}{0.4 \times 10^{5}}=2.5 \times 10^{4} \text { watts }
$$

For $2 z=90 \mathrm{~cm}$

$$
P_{0}=\frac{2.5 \times 10^{4} \times 1.96}{90}=544 \mathrm{wnts} / \mathrm{am}
$$

Heat Baldence in sodina:

$W$ : Aodiun flow/rod

$C_{p}=$ rodium keat coppicity

$$
\begin{aligned}
& p=w c_{p} \frac{d T}{d z}=P_{0} \cos \left(1.96 \frac{z}{z}\right) \\
& \text { Integrating } T_{T(z)}-T_{\text {inket }}=\left(\frac{P_{0}}{\omega c_{p}}\right) \int_{-\frac{z}{2}}^{z} \cos \left(1 . q t \frac{z}{z}\right) d z \\
& T_{\text {N. }}(3)-T_{\text {incet }}=\frac{P_{0}}{W C_{p}} \frac{z}{1.96}\left[\sin \left(1.96 \frac{z}{z}\right)+\operatorname{Ain}(0.98)\right]
\end{aligned}
$$

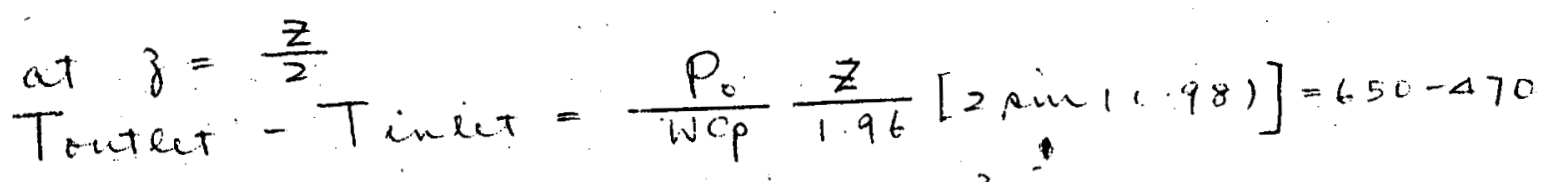

$$
\begin{aligned}
& \therefore T_{N a}(z)=470+180\left[\frac{\sin \left(1.9+\frac{3}{7}\right)+0.83}{1.66}\right]-\cdots(2)
\end{aligned}
$$

$T c e=$ temperatiure of gutride of claddigh

hNa = adium fiem teat trousfer coefficient Heat flux Mhough clad wall

$$
=\frac{P}{2 \pi R_{a}}=h_{\mathrm{Na}}\left(T_{C e}-T_{\mathrm{Na}}\right)
$$

$R_{a}$ : quel nod radius $=0.135 \mathrm{~cm}$ (Talde 10.2)

$$
\begin{aligned}
\therefore T_{C L} & =T_{N a}+\frac{P}{2 \pi R_{a} h N a} \\
T_{C L} & =T_{N}+\frac{P_{0}}{2 \pi R_{a} h N a} \cos \left(1.96 \frac{z}{z}\right)
\end{aligned}
$$


503

From Table 10, $h_{\mathrm{Na}} \cong 12 \frac{w_{a} \text { tts }}{\mathrm{cm}^{2} \cdot{ }^{\circ} \mathrm{C}}$

$$
\begin{aligned}
T_{C l}= & T_{N a}+\frac{544}{(6.28)(0.315)(12)} \cos \left(1.96 \frac{z}{z}\right) \\
= & T_{N a}+23^{\circ} \mathrm{C} \cos \left(1.96 \frac{3}{z}\right) \\
\therefore \operatorname{Ta}(z)= & 470+\left(80\left[\frac{\sin (1.963 / z)+083}{1.66}\right]\right. \\
& +23 \cos \left(1.96 \frac{3}{z}\right)-\ldots-\ldots
\end{aligned}
$$

Ciuzacion rate from eq $(20.55)$

$$
\begin{aligned}
& C_{0}=5 \mathrm{ppm} \\
& R=8.31 \mathrm{~J} / \text { imace }{ }^{5} \mathrm{~K} .
\end{aligned}
$$

neglect $x / d$

$$
\begin{aligned}
\therefore \dot{m} & =\left(1.1 \times 10^{8}\right)(.5)^{1.3} \exp \left(-\frac{146}{8 \cdot 31 T C / 10^{3}}\right) \\
& \dot{m}=8.9 \times 10^{8} \mathrm{exp}\left(-\frac{[? .57}{\left(T C L / 10^{3}\right)}\right]---(4)
\end{aligned}
$$

Carraion in fuelid pations of core

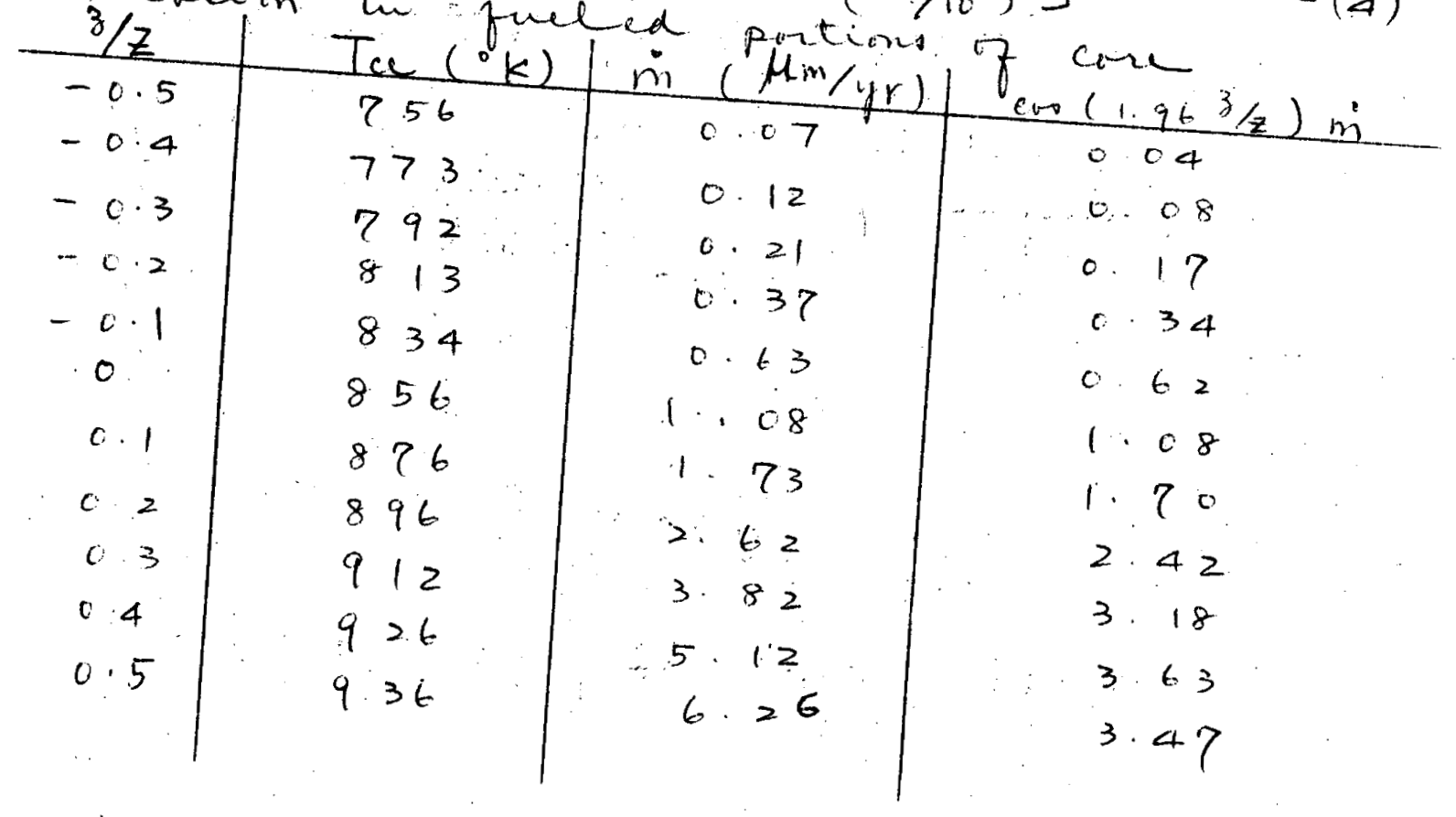


504

Total core carsacion rate

$$
\begin{aligned}
& \dot{m}\left(\frac{g m s}{a^{2} \cdot y r}\right)=\dot{m}\left(\frac{\mu m}{y^{r}}\right) \frac{c m}{10^{4} \mu m} \times 7 \cdot 9 \frac{g m s}{c_{m}^{3}} \\
& \therefore \dot{m}\left(\frac{g m o}{c m^{2} \cdot y r}\right)=\dot{m}\left(\frac{\mu m}{y r}\right) \times \eta 9 \times 10^{-4}
\end{aligned}
$$

Metal las rate from one fuel rod consider a slice of thick ere dz rate if metal pres in dz

$$
\begin{aligned}
& =\dot{m}\left(\frac{g m a}{m^{2} \cdot y r}\right)=\pi R_{a} d z=\int_{-z / 2}^{z / 2} \dot{m}(z) 2 \pi R_{a} d z \\
& \text { metal lois rate fro rod } \\
& =2 \pi R_{a} z \int_{-1 / 2}^{1 / 2} \dot{m}(z / z) d(z / z) \\
& =(628)(0.315)(90) \int_{-1 / 2}^{1 / 2} \dot{m}(z / z) d(z / z)
\end{aligned}
$$$$
=178 \cos ^{2} \times\left[\frac{0.1(0.7)}{2}+0.1(0.12)\right]
$$$$
+0.1 \times(5 \cdot 12)+\frac{0.1(626)}{2}
$$$$
=1 \tau 8 \mathrm{~cm}^{2} \times 1.88
$$

$\therefore$ metal bess rate from fuel rod il fueled portion

$$
\begin{aligned}
\text { melon }) & =\left(7.9 \times 10^{-4}\right)(178)(1.88) \\
& =0.27 \mathrm{gmo} / \mathrm{gr}
\end{aligned}
$$

The top axial he nat $(30 \mathrm{~cm})$ so at the water sodium temperature of $650^{\circ} \mathrm{C}$ for which the carricion rate.

$$
\dot{m}=89 \times 10^{+8} \exp \left(-\frac{17.57}{0.923}\right)=4.81 \mu \mathrm{m} / \mathrm{yr}
$$


505

The ared is $(6.28)(0.315)(30)=59.3 \mathrm{~cm}^{2}$ metal laes rate from upper axinl heared pection is $(4.81)\left(7.9 \times 10^{-4}\right)(59.3)=0.23 \mathrm{gms} / \mathrm{gr}$ $\therefore$ Tatal metal eas rate per lod

$$
=0.50 \text { gins } / \mathrm{gr}
$$

For 40 years iperation at a $25 \%$ load factor the tatal metal lass $=(0.50) \mathrm{gms} / \mathrm{gr} \times 30 \mathrm{gr}$ $\times 10^{5}$ rods $=1.5 \times 10^{6} \mathrm{gmos}$.

B $\mathrm{Fe}^{54}(\mathrm{n} \cdot \mathrm{P}) \mathrm{Mn}^{54}$

[The reaction $M_{n}{ }^{55^{\circ}}(n: 2 n) M_{n}{ }^{54}$ is negligible] at time $t$ actinity in tigpe 3045.5 cladrigy$$
\frac{d M_{n}^{54}}{d T}=\sigma_{e f f} N_{F e}^{54} \Phi-\lambda N_{M_{n}}^{54}
$$

$\lambda$ decay contant of $M_{n-54}=\frac{0.693}{t / 2}=81 \mathrm{Yr}^{-1}$

I total fact flix at the pration in fiustion

$$
\begin{aligned}
\mathrm{N}_{\mathrm{Fe}}^{54}= & \left(0.85 \times 10^{23}\right)(0.85)(0.058) \\
& (\text { metac atom }
\end{aligned}
$$

$$
=32 \times 10^{21} \frac{\text { atomo } F_{2}^{54}}{\cos ^{3} \cdot \text { Nted }}
$$

$\sigma_{\text {eff }}=$ effective crocs rection for rention in neution Apectinu $=16 \mathrm{mb}$ 
506

$M_{n}^{54}$ release due to the $F_{e}^{54}(n \cdot p) M_{n}^{54}$ reaction in the cladding, Chider a slice dz of cladding when the corrosion rate os in and the fat flux is $\Phi$. In time at the amount of steel lint by corrosion is $\left(2 \pi R_{a} d z\right) \dot{m} d t$ gino. She $M_{n}^{5}$ content of the steel is given by $e_{1}$ (1) and the number of Mn atoms lust in at from the slice is.

$$
(2 \pi R a d z) \dot{m} \text { at } \times \frac{N_{M n}^{54}}{S_{x} \text { den }}
$$

or the ratio of $M_{n}^{54}$ recency from the soutine que pin is

$$
\frac{2 \pi R_{n}}{\rho} \sigma_{\text {eff }} N_{F e}^{54}\left(1-e^{-\lambda}\right) \int_{-z / 2}^{z^{\prime} / 2} \text { 主 }(z) \dot{m}(z) d z
$$

where the integral is extended into the

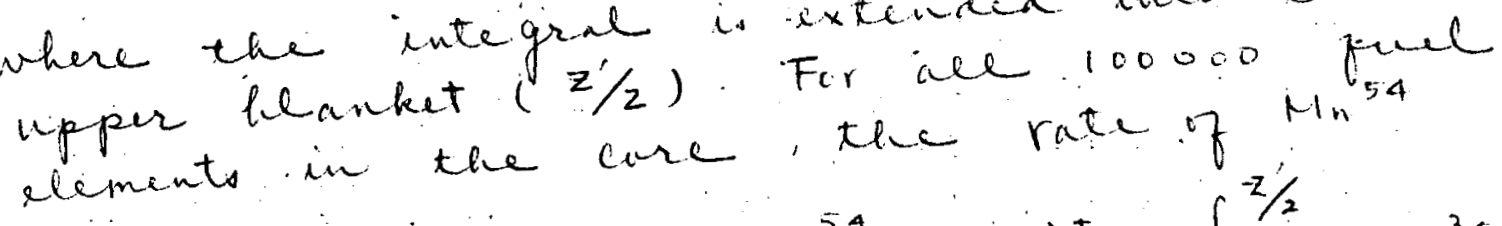
relive is ${ }^{5}(t)=10^{5} \frac{2 \pi R a}{\rho} \frac{\tau_{e+1} N_{F e}^{54}}{\lambda}\left(1-e^{-\lambda t}\right) \int_{0} \int_{-z / 2}^{-z / 2} \cos \left(1 \cdot 16 \frac{z}{2}\right) x$

when the flux shape follow the linear power axial distribution avid in addition, extends into the axial hairnets. From pint $A \cdot z^{\prime} / z=\frac{\pi}{1 \cdot 9 t}$ and $Q(t)$ is

$$
\begin{aligned}
& \left.Q(t)=10^{-4}\left(10^{5} 2 \pi R_{a} z\right) \frac{\left(\sigma_{e}+N_{f e}^{54}\right)}{\lambda}\left(1-e^{-\lambda t}\right) \Phi_{0} x\right)+\dot{m}_{B l} \int_{\frac{1}{2}}^{0.80} \cos \left(1.96 \frac{z}{z}\right) d(z / z) \\
& {\left[\int_{\frac{1}{2}}^{1 / 2} \cos (1.96 z / z) \dot{m}\left(\frac{z}{2}\right) d(z / z)\right.}
\end{aligned}
$$


507

where $\dot{m}$ is now expressed in $\mu \mathrm{m} / \mathrm{gr}$ $\dot{m}(z / z)$ in fueled region from Table in

Assume ale $M_{n}^{54}$ is retained in ald trap

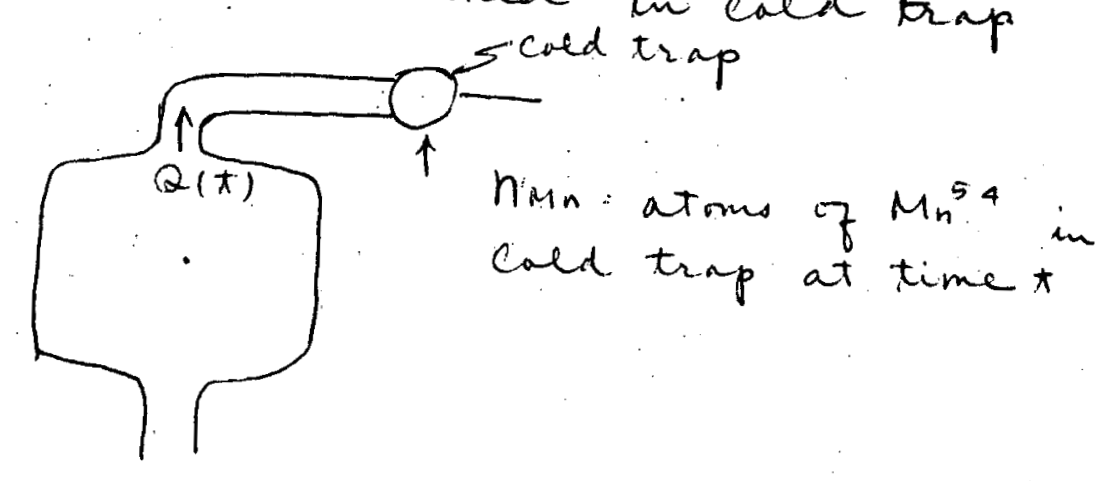

$\mathrm{Mn}^{54}$ balance on cold trap

Input $=Q(t)$ atoms $M_{n}^{54} / y r$

$$
\begin{aligned}
& \text { decay }=\lambda n_{\text {min }} \text { aton } M_{n}^{54 / g r} \\
& \quad \frac{d n_{M_{n}}}{d t}=Q(t)-\lambda_{M_{n}} n_{M_{n}}
\end{aligned}
$$

Now write $Q(k)$ as $Q(t)=B\left(1-e^{-\lambda \pi}\right)$

where

$$
\begin{aligned}
& B=10^{-4}\left(10^{5} 2 \pi R_{a} z\right) \frac{\operatorname{reff} N_{\mathrm{Fe}}^{54}}{\lambda} \Phi_{0}\left[\int_{-1 / 2}^{1 / 3} \cos (1.96 z / z) \dot{m} d(z / z)\right. \\
& \left.+\dot{m}_{B l} \int_{1 / 2}^{0} \cos (-196 z / z) d(3 / z)\right] \\
& \frac{a n_{M n}}{d t}=B\left(1-e^{-\lambda t}\right)-\lambda n_{M n}
\end{aligned}
$$

Integrating

$$
\left.n_{M_{n}}=C^{-\lambda t}+B e^{-x t} \int e^{x t}(1) e^{-\lambda t}\right) d t
$$


508

$$
=C e^{-\lambda t}+\frac{B}{\lambda}\left(1-\lambda t e^{-\lambda t}\right)
$$

Evaluate $C$ from condition $n_{\text {min }}(0)=0$

$$
\begin{aligned}
& \Rightarrow C=-B / \lambda \\
& \therefore n_{M n}=\frac{B}{\lambda}\left[1-\lambda t e^{-x t}-e^{-\lambda t}\right]=\frac{B}{\lambda}\left[1-(1+\lambda t) e^{-\lambda t}\right]
\end{aligned}
$$

or with $a_{M n}=$ activity of $M_{n}^{54}$ in cold trip

$$
a_{m_{n}}=B\left[1-(1+\lambda t) e^{-\lambda t}\right] \ldots-\cdots(2)
$$

Evaluation of $B$ :

internal area of fueled section of cone

$$
\begin{aligned}
& \text { internal area of fueled section of cole } \\
& =10^{5} 2 \pi R_{a} Z=\left(10^{5}\right)(6.28)(0.315)(90)=1.78 \times 10^{7} \mathrm{~cm}^{2} \\
& \sigma_{\text {eff }} N_{F R}^{54}=\left(16 \times 10^{-27}\right)\left(3.2 \times 10^{21}\right)=5.12 \times 10^{-5} \mathrm{~cm}^{-1} \\
& \Phi_{0}=7 \times 10^{15} \mathrm{~cm}^{-2}-\mathrm{pec}^{-1}
\end{aligned}
$$$$
\lambda=0.81 \mathrm{Yr}^{-1}=2.58 \times 10^{-8} \mathrm{sec}^{-1}
$$

From part $A$ table

$$
\begin{aligned}
& \text { From } \operatorname{pant} A \\
& \int_{-\frac{1}{2}}^{1 / 2} \cos (1.96 \mathrm{~g} / z) \dot{m} d\left(\frac{z}{z}\right)=0.1\left(\frac{0.04}{2}+0.08+\cdots+\frac{3.47}{2}\right) \\
& =1.50 \mu \mathrm{m} / \mathrm{yr} \\
& \dot{m}_{B R} \int_{1 / 2}^{0.80} \cos (1.96 \mathrm{z} / z) d(3 / z)=(0.0865)(4.81) \\
& =0.42 \mu \mathrm{m} / \mathrm{gr} \\
& \therefore B=\left(10^{-4}\right)\left(1.78 \times 10^{7}\right)\left(\frac{5.12 \times 10^{-5}}{2.58 \times 10^{-8}}\right)\left(7 \times 10^{15}\right)(1.50+0.42) \\
& =4.75 \times 10^{22} \mathrm{yr}^{-1}
\end{aligned}
$$


509

Converting to Ciries

$$
\begin{aligned}
& B=\frac{4.7 .5 \times 10^{32} \mathrm{yr}^{1}}{3.14 \times 10^{7} \frac{\mathrm{sec}}{\mathrm{gr}} \times 3.7 \times 10^{10} \frac{\mathrm{dio} / \mathrm{Mc}}{\mathrm{Ci}}}=4.1 \times 10^{4} \mathrm{Ci} \\
& \begin{array}{l}
\text { at } \dot{t}=1 y r \quad \lambda t=0.81 \\
1-(1+\lambda t) e^{-\lambda t}=1-1.81 e^{-0.81}=0.195
\end{array} \\
& a_{M n}=\left(4.1 \times 10^{4}\right)(0.195)=0.8 \times 10^{4} \mathrm{Ci}
\end{aligned}
$$


510

PROBLEM 20.6

Subutituting Eq $(20.59)$ into Eq (20.63)

$$
\ln K_{H}=\ln \left(C_{\text {Aat }}(H)-\Delta G_{f}^{0} \mathrm{NiOH} / R_{T}\right.
$$

$$
\begin{aligned}
& \text { or } \begin{aligned}
\Delta G_{f \mathrm{NaOH}} & =R \frac{T}{10^{3}}\left[\ln C_{\text {ANt }}(H)-\ln K_{H}\right] \ldots \\
& =R\left(T / 10^{3}\right)\left[\left(14-\frac{55}{R\left(T / 10^{3}\right)}\right)-\left(5 \cdot 3-\frac{2 \cdot 3}{R\left(T / 10^{3}\right)}\right)\right]
\end{aligned} \\
& \Delta G_{f \mathrm{NAOH}}^{0}=-52.7+8 . T R\left(T / 10^{3}\right) \\
& \Delta G_{f \mathrm{NaOH}}=-52.7+.72(\mathrm{~T} / 10 \mathrm{c}) \mathrm{kJ} / \mathrm{mole}
\end{aligned}
$$

which is $\sim 6$ Karl/inace different from volue from equation (20.58) at 1000 ' $\mathrm{K}$ 
511

PROBLEM 20.7

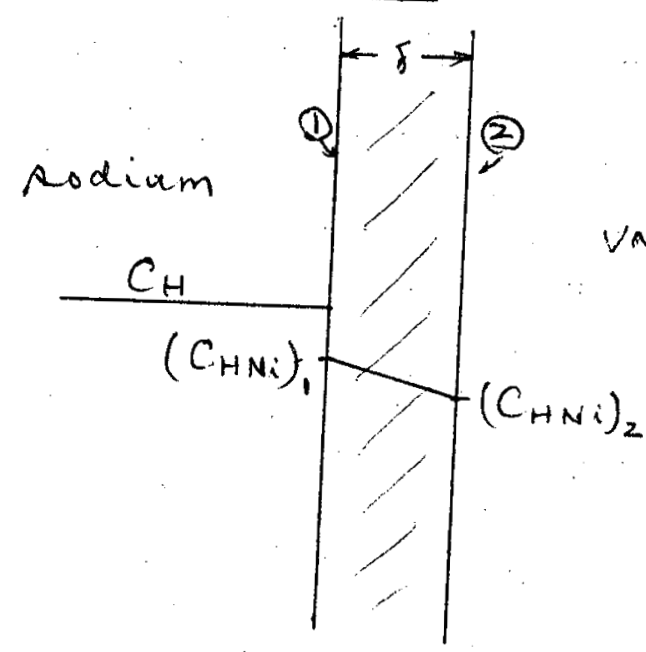

vacuum chanter

$$
\mathrm{PH}_{2}
$$

pump

Hydrogen equilibrates between the sodium and the exposed face of the nickel:

$H($ volution in $N a)=H\left(\right.$ solution in $N_{i}$ at $\left.D\right)$ Let $P_{H_{2}}^{\text {eq }}$ he the Hydrogen presume in equilibrium with concentration $C_{H}$ in odium.

From eq $(20.62)$

$$
\left(P_{H_{2}}^{e q}\right)^{1 / 2}=\frac{C_{H}}{K H N N} \cdot-\cdots+\cdots,
$$

where $K_{H N a}$ is the Sieverti law construct for Eyolugen in sodium

Sickert's frow for tifelrogin in nickel is

$$
\begin{aligned}
& K_{H N_{i}}=\frac{\left(C_{H N i}\right)_{1}}{\left(P_{H>}^{2}\right)^{1 / 2}}=\frac{\left(C_{H N_{i}}\right)_{1}}{\left(C_{H} / K_{H N}\right)} \cdot \frac{p P m}{a m_{m} / 2} \\
& \text { or }\left(C_{H N_{i}}\right)_{1}=\left(\frac{K_{H N i}}{K_{H N a}}\right) C_{H}-\ldots-(2)
\end{aligned}
$$


$5 / 2$

AT the sprite face of the Niclect membrane $\mathrm{PH}_{2}$ is in equilishium with $\left(\mathrm{CHNi}_{\mathrm{N}}\right)_{2}$

$$
\left(C_{H N i}\right)_{2}=K_{H N i} P_{H_{2}}^{1 / 2}
$$

The flax of $H$ atoms through the mericherane

is

$$
J=A B \frac{D_{H N i}}{\gamma}\left[\left(C_{H N i}\right)_{1}-\left(C_{H N_{i}}\right)_{2}\right], \frac{\text { atoms } H}{\text { ReC }}
$$

Where $g$ is inembane thickness

A surface area of mencluane

Cling (2) and (3)

$$
B=6.02 \times 10^{23} \rho_{\mathrm{Ni}} \times 10^{-6} \frac{\text { atoms } \mathrm{H} / \mathrm{mm}^{3} \mathrm{Ni}}{\mathrm{PPm}}
$$

$\Rightarrow \quad \int_{N i}$ dencity of nickel

$$
J=A B \frac{D_{H N i}}{\delta}\left[\left(\frac{K_{H N i}}{K_{H N N}}\right) C_{H}-K_{H N i} P_{H_{2}}^{1 / 2}\right] \ldots(4)
$$

The pumping speer 5 of a vacuum pump is related ti the molecular through pint $Q$ (molecules/Aec) ling the ideal gas low

$$
P S=Q R T \text {. }
$$

where $T_{0}=$ temperature of pump operation $=300^{\circ} \mathrm{K}$

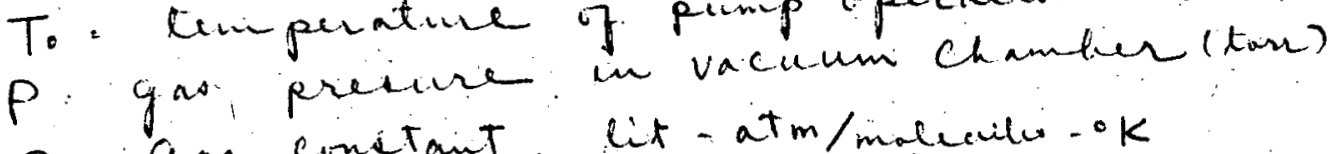
$R$ : gas constant, lit-atm/maliculio-oK

For the present case $P=P_{H_{2}}$

$$
Q=\frac{1}{2} J\left(\text { fen x of } H_{2}=\frac{1}{2} \text { fen of } H\right)
$$

$$
\therefore J=\frac{2 P_{H 2} S}{R T_{0}} \cdots \cdots(5)
$$




$$
1
$$$$
513
$$

Equate (4) and (5)

$$
P_{H_{2}}=\underbrace{\left[\frac{R T_{0} A B D_{H N i} K_{H N i}}{2 \delta S}\right]}_{\text {Tonctant }}\left[\frac{C_{H}}{K_{H N a}}-P_{H_{2}}^{1 / 2}\right]--(6)
$$

If the conctant in ef (6) is finown measurement of $\dot{P}_{H_{2}}$ in the dynamic mode determines $C_{H}$ liy ef $(t)$.

$$
\left(\frac{C_{H}}{K_{H N a}}\right)^{2}=P_{H_{2}}=P_{H_{2}^{2}}^{2} \text { which irepresents the }
$$

equiliturim mide of operation

Note. Yhe curreit mearused lig the ion puop is proportional to the gas presure. 
514

$\frac{\text { PROBLEM } 20.8}{\text { at } 370^{\circ} \mathrm{C}\left(643{ }^{\circ} \mathrm{K}\right)}$

From equation $(20.18)$ or Fig 20.9 :

$$
\begin{aligned}
C_{\text {dat }}(0) & =\exp \left[14: 4-\frac{46.5}{(8.31)(0.643)}\right]=298 \mathrm{ppm} \\
a_{0} & =\frac{C_{0}}{C_{\text {ant }}(0)}=\frac{5}{298}=0.017 \\
& =\text { oxygen e activity }
\end{aligned}
$$

From eq $(20: 16)$

$$
\Delta G_{f N=0}^{0}=-397+118(0.643)=-321 \mathrm{~kJ} / \mathrm{male}
$$

with air as the reference clement

$$
\mathrm{PO}_{2}=0.21 \cdot \mathrm{atm} \text {. }
$$

From eq $(20.67)$

$$
\begin{array}{r}
\Delta \varepsilon=\frac{(8.31)(0.643)}{(4)(96.48)}[\ln (0.21)-2 \ln (0.017) \\
\left.-2 \times \frac{(-321)}{(8.31)(0.643)}\right]
\end{array}
$$

$$
\Delta \varepsilon=1.75 \text { volts. }
$$


515

PROBLEM 20.9 A. Interms of Sieverti low, coustant for the

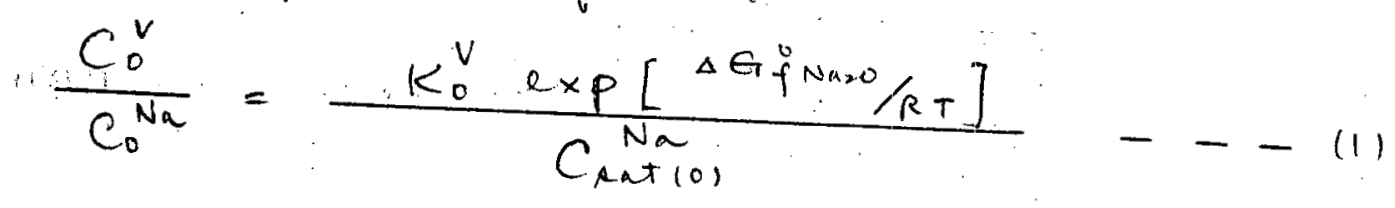

at $750^{\circ} \mathrm{C}$

$$
\begin{aligned}
& \text { Eq }(20.16) \rightarrow \Delta G_{f i N 20}^{0}=-397+118(1.023)=-276 \mathrm{~kJ} / \mathrm{mele} \\
& \text { B.Eq }(20.18) \rightarrow \quad C_{R a t(5)}^{N}=\exp \left[14.4-\frac{46.5}{(8.31)(1.023)}\right] \\
& =7560 \mathrm{ppm} \text { ly wt }
\end{aligned}
$$

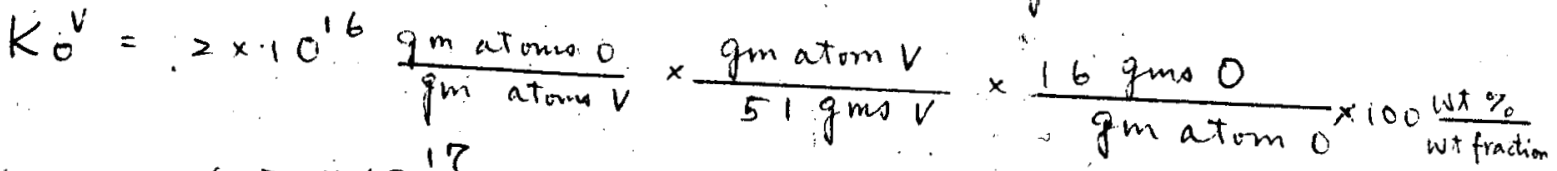

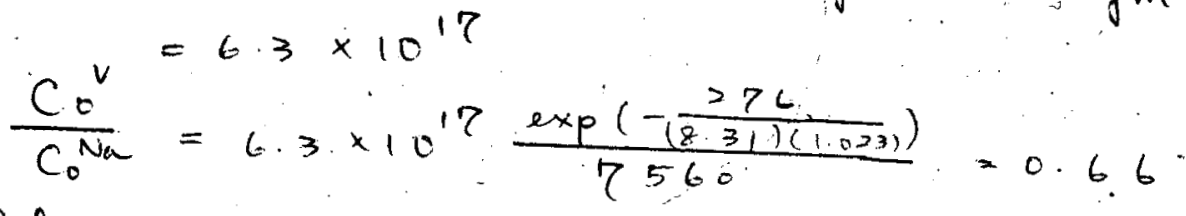

Alout a fretor of 2 laver tham straight lime pution of Fig 20:26

$$
B \cdot\left(C_{0}^{N^{n}}\right)_{\text {MAX }}=(0.66)(2.3)=1.5 \mathrm{ppm}
$$

c. fractional eiros in $\left(C_{0}^{N \sim}\right)_{M A x}=$ fraitional erim

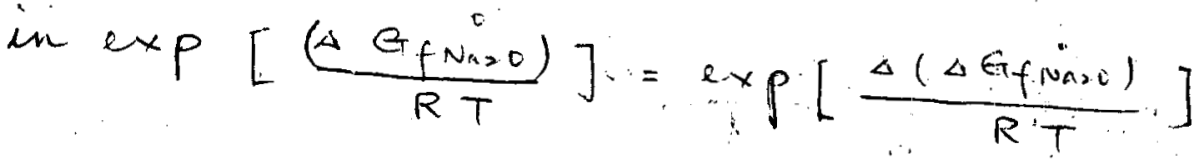

$$
\begin{aligned}
& =\exp \left(\frac{10}{8.31 \times 1.023}\right)=3.2 \text {. }
\end{aligned}
$$$$
\text { i.e } 320 \%
$$

C: 
$5 / 6$

PROBLEM 20.10

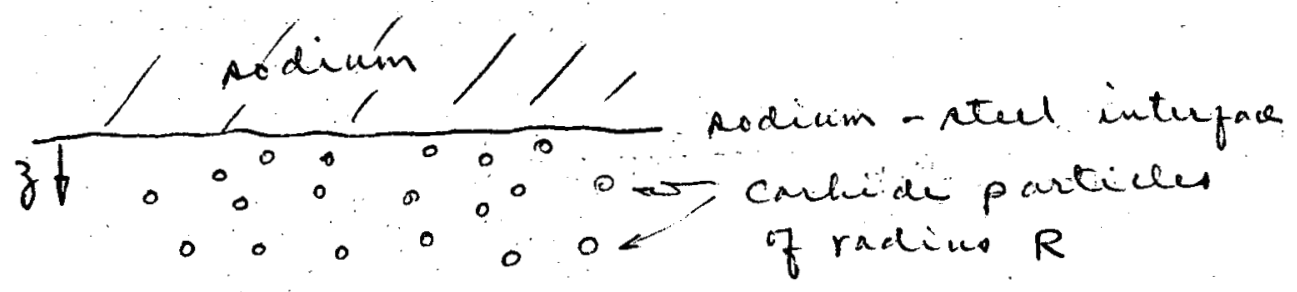

$a_{c}^{N a}$ activity of colon in sodium (specified)

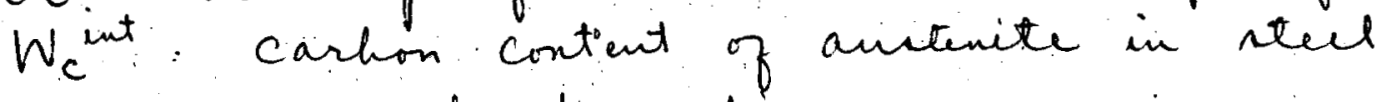
exposed to sodium

Boundary condition at $z=0$ ( the sediment peel interface) (Omit $T$ in functional dependence if $\left.r_{c}\right)$

From eq $(20.97)$
$a_{c}=W_{c}^{\text {int }} \gamma_{c}\left[W_{c r}^{\text {int }}, W_{N i}^{\text {int }}\right] \ldots \ldots$ (1)

From (20.105) at the inter face

$$
W_{N i}^{\text {int }}=W_{N i}^{0}\left(\frac{x-W_{c r}^{0}}{x-W_{c r}^{i \omega r}}\right) \quad-\ldots-\text { (2) }
$$

Wee in (")

$$
\left.a_{c}^{N_{a}}=F \mid W_{c r}^{i n t}, W_{N i}^{*}\left(\frac{x-W_{c i}^{c}}{x-W_{c r}^{\text {int }}}\right), W_{c}^{i n t}\right\}
$$

solve for wert

$$
\begin{aligned}
& w_{c r}^{\text {int }}=F^{-1}\left(w_{c}^{\text {int }}, X, W_{r}^{0}, W_{v i}^{0}, a_{c}\right) \cdots(3) \\
& \text { Fem (20.139) } f^{\text {int }}=\frac{\rho_{e} / \rho_{05}}{1: 0.58}\left(\frac{R_{\text {int }}}{R}\right)^{3}-\ldots \text { (5) }
\end{aligned}
$$

Where $R_{\text {int }}=$ radius of Carbide particles in steal expand to sodium 
517

U given ln (20.133) in terms of $N$

Combine (4) and (5) and equate to (3)

$W_{c}^{\text {int }}$. Can be solved as a function of $R_{\text {int }}$ $--(6)$

From (>0.136) applied to reel at sodium -steel inter tace:

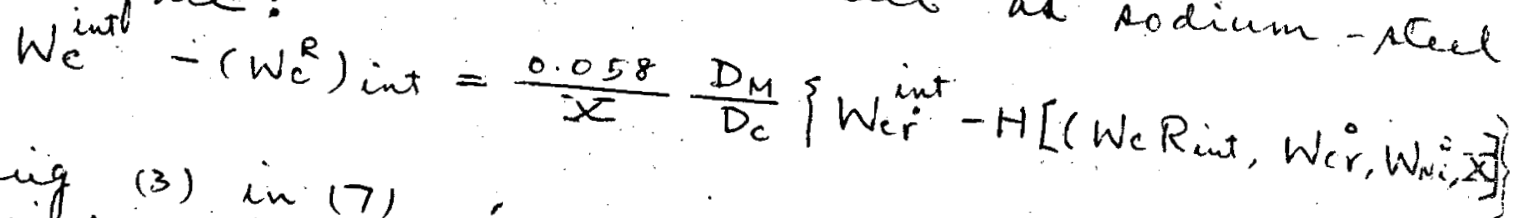

Haig (3) in (7),

$$
\begin{aligned}
& W_{c}^{\text {un }}-\left(W_{c}^{R}\right) \text { int }=\frac{0.058}{X} \frac{D_{M}}{D_{c}}\left\{F^{-1}\left(W_{c}^{\text {int }}, \bar{X}, W_{c r}^{0}, W_{N_{i}}^{0}, a_{c}\right)\right. \\
& \left.\left.-H L\left(W_{c}^{R}\right)_{\text {int }} ; W_{c r}^{0}, W_{N_{i}}^{0}, \bar{X}\right]\right\} \cdots-\cdots(8)
\end{aligned}
$$

Solve $\left(W_{c}^{R}\right)$ int as a function of $W_{c}^{\text {cit }}$ from (8)

Combine (6) and (8), $\Rightarrow$ have $\left(W_{c}^{k}\right)_{\text {int }}$ as function of $R_{i \text {. }}$.

From (20.140)
$\quad \frac{d R_{\text {int }}}{d t}=\left(\frac{1.058}{0.058}\right) \frac{\rho_{s s}}{\rho_{c}} \frac{D_{c}}{R_{\text {int }}}\left[W_{c}^{\text {int }}-\left(W_{c}^{R}\right)_{\text {int }}\right]--(9)$ ling (6) far $W_{c}^{\text {int }}$ aid $\left.(6)+18\right)$ for $\left(W_{c}^{R}\right)$ int,
$d t$

Salve (9) for $R_{\text {int }}(*)$ : Substitute into 161 , gives $W_{c}^{i n t}(t)$, the time dependent quantity Eq (20.138) boundmig condition $W_{c}(0, t)$ for 
518

Bocinalary condition at $z=0$ (bulk reel) here, we neglect diffusion because all concentration gid dent vane. The concentration of carton in the austenite is governed on dy by precipitation kinetics of diculued carbon to the carbide particles. From Eq (20.138) with the concentration gradient omitted:

$$
\frac{d}{d t}\left[\left(1-f_{\infty}\right) W_{c \infty}\right]=-4 \pi R_{\infty} D_{c} N\left(W_{c \infty}-W_{c}^{R}\right)-(10)
$$

where $R_{\infty}=$ Radium of Carbide particles for from sodium - steel interface.

$$
\begin{aligned}
& \frac{d R_{\infty}}{d t}=\left(\frac{1.058}{0.058}\right)\left(\frac{\rho_{s s}}{\rho_{c}}\right) \frac{D_{c}}{R_{\infty}}\left(W_{c \infty}-W_{c \infty}^{R}\right)--(11) \\
& F_{r m}(>0.139) \quad f_{\infty}=\frac{\left(\rho_{c} / \rho_{3 ;}\right)}{1.058}\left(\frac{R_{\infty}}{R}\right)^{3} \ldots-(12) \\
& \text { From }(20.137) \\
& \frac{D_{C}}{D_{M}} \frac{-\bar{X}}{0.058}\left(W_{c \infty}-W_{c}^{R} \infty\right)=\frac{W_{c r}^{0}-f_{\infty} X}{1-f_{\infty}}-H\left(W_{c \infty}^{R}, W_{c r}^{c}, W_{N i}^{0}, \bar{x}\right)
\end{aligned}
$$

Solve for Wow (t) and $R_{\Delta}(*)$ by integrating $(10)$ and (II) with $f_{\infty}$ and $W_{c}^{R}$ given $k_{\text {-y }}$ (12) and (13) The initial condition for this integration are

$$
\begin{aligned}
& W_{c \infty}(0)=\left(W_{c}^{\top}\right)_{\text {fab }}-\ldots(14) \\
& R_{\infty}(0)=0 \quad \ldots-\ldots(15)
\end{aligned}
$$

which assumes that ale carbon in the $a_{1}$ - fabricated stele is present in supersaturated solution in the austenite: 
519

PROBLEM 20.11

A.

Equivalence of the two formulations of the diffusion equations [ie $E_{q A}(20.123)$ and $E_{q}(20.128)$ ] $c a n$ be seen from the ald th equation $(20.140)$

$$
D_{c}\left(w_{c}=w_{c}^{R}\right)=\frac{\rho_{c}}{\rho_{s s}} \frac{0.058}{1.058} R \frac{d R}{d t}
$$

Substitute this into $(20: 138)$$$
\text { or } \frac{\left.W_{c}(1-f)\right]}{\partial t}+4 \pi N \frac{\rho_{c}}{\rho_{s s}} \frac{0.058}{1.058} R^{2} \frac{d R}{d t}=D_{c} \frac{\partial^{2} W_{c}}{t^{2}}
$$

$\frac{\partial\left[W_{c}(1-f)\right]}{\partial t}+4 \pi N \frac{\rho_{c}}{\rho_{35}} \frac{0.058}{1.058} \frac{1}{3} \frac{d R^{3}}{d t}=D_{c} \frac{\partial^{3} W_{c}}{\partial z^{2}}$

Remove $\frac{\partial}{\partial t}$

$$
\frac{\partial}{\partial t}\left[W_{c}(i-f)+\left(\frac{4}{3} \pi R^{3}\right) N \frac{\rho_{c}}{\rho_{s}} \frac{0.058}{1.058}\right]=D_{c} \frac{\partial^{2} W_{c}}{\partial z^{2}}
$$

But from $(20.133), \frac{4}{3} \pi R^{3} N=\left(\frac{R}{R}\right)^{3}$ and the second term on the right is $0.058 \mathrm{f}$ thy Eq $(20.139)$

$$
\therefore \frac{\partial}{\partial t}\left[W_{c}(1-f)+0.058 f\right]=D_{c} \frac{\partial^{2} W_{c}}{\partial z^{2}}
$$

$B_{y}$ eq $(20.109) W_{c}^{\top}=W_{c}(1-f)+0.058 f$

$$
\therefore \frac{\partial W_{c}^{\top}}{\partial t}=D_{c} \frac{\partial^{2} W_{c}}{\partial z^{2}} \text { Which is Eq }(20.123)
$$

B

If $D_{i}$ is very lunge in Eg $(20.134)$, but $J_{i}$ is finite their $W_{i}=W_{i}^{R}$ (Baric requirement of the equilihriming intel

But. $D_{c}$ not infinite in $E_{q}(20,123)$ 
520

PROBLEM 20.12

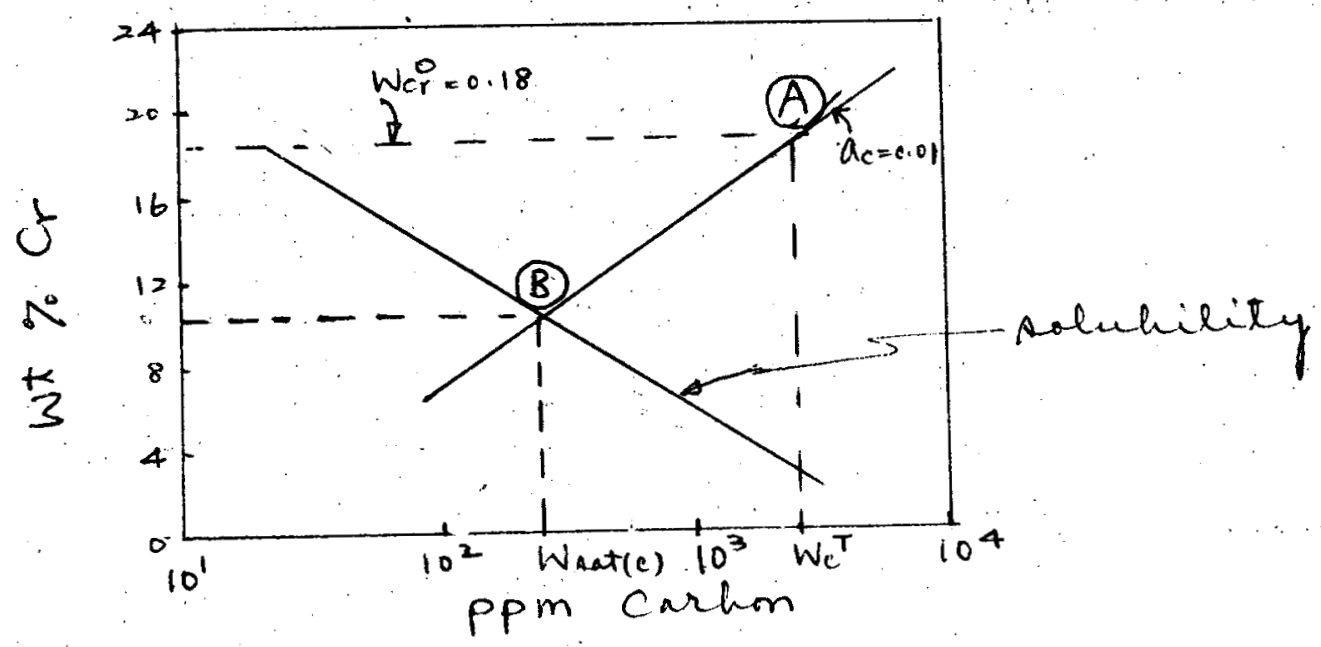

The specifications $a_{c}=0.01, W_{c r}=0.18$ fix the point (A) on the plot, the carton concentration of $A$ is

$$
\omega_{c}^{\top}=1.1 \times 10^{-3}(1100 \mathrm{ppm})
$$

B Since lith phase of the steel (austenite and carbide) have the same carbon activity, the composition of the austenite phase is given by moving $a_{c}=0.01$ isoactinity line from (A) to the solubility line at point (B) at (B)

$$
\begin{aligned}
& W_{\text {ant }}(\mathrm{c})=3 \times 10^{-4}=300 \\
& W_{c r}=0.11=11 \%
\end{aligned}
$$


521

C. Eliminatiog $f$ from $E_{q}(20.103)$ and (20.109)

$$
\frac{W_{c}^{T}-W_{\text {ant }}}{0.05 .8-W_{\text {Aat }}}=\frac{W_{C r}^{0}-W_{C r}}{x-W_{C r}}
$$

or

$$
X=W_{c r}+\left(\frac{0.058-W_{\text {aat }}(c)}{W_{c}^{\top}-W_{\text {nat }}}\right)\left(W_{c r}^{0}-W_{c r}\right)
$$

Uning the reult of (A) and (B)

$$
x=5.16
$$

D.

Since $x=\left(\frac{C_{r}}{C_{r}^{\prime}+F_{e}}\right)_{\text {corlide }}$, it cannot

be $>1$. Herefore lither Eqs $(20.113)$ $\because(20.101)$ or lath are incarrect representation of the equilierium hehomiar of carbon in staiselus stees. 
522

PROBLEM 20.13

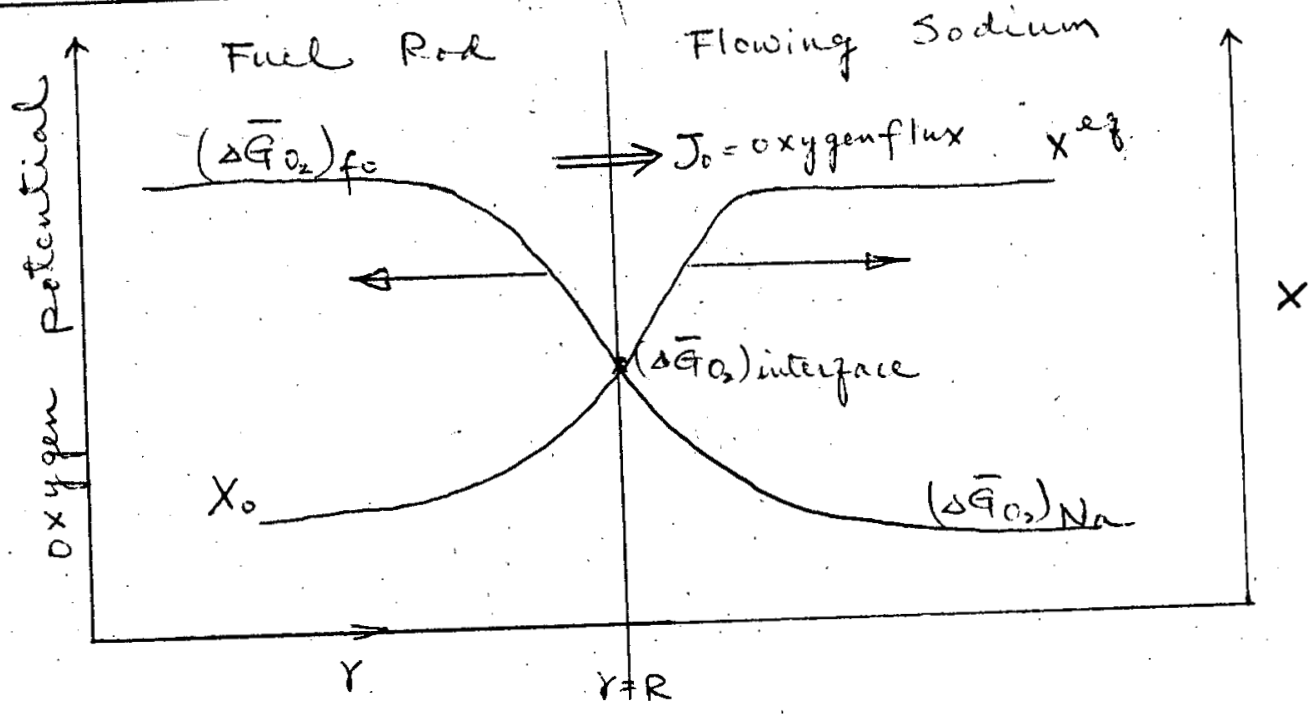

The diffucion equation in fucl

$$
\begin{aligned}
& \frac{\partial x}{\partial t}=D_{f} \frac{1}{r} \frac{\partial}{\partial r}\left(r \frac{\lambda \cdot x}{\partial r}\right)-\ldots-\cdots(1) \\
& \text { I. C. } x(r .0)=x_{0} \quad-\quad-\quad---(2) \\
& \text { B.C. } 1\left(\frac{\partial x}{\partial y}\right)_{0}=0
\end{aligned}
$$

equilikim at interface is maintaincil

$$
\text { ‥e. } \left.{ }^{\text {equint }} C_{0}^{\text {int }}=52-750 \times \text { int }---14\right)
$$

The oxygen fent into rodium

$$
J_{0}=k_{d} \frac{\rho_{N}}{16 \times 10^{6}}\left[C_{\text {int }}-C_{0}\right]=D_{0}^{f} \frac{\rho_{f}}{2 \eta^{\circ}}\left(\frac{\partial x}{\partial r}\right)_{R}, \frac{g_{\text {matimo }}}{C_{\text {in }}^{2} \text { arc }}
$$

where $\left(\Delta \bar{G}_{0_{2}}\right) f$ has hecome equal to $\left(\Delta \bar{G}_{0}\right) \mathrm{Na}$ wruck he $x_{\text {eq }}$ given $l_{y}=C_{0}=52-250 \times \mathrm{eq}-(.5)$ or $\frac{750 k d \int_{N a}}{16 \times 10^{6}}\left(x_{i q q}-x_{i n t}\right)=D_{0}^{f} \frac{\rho_{f}}{270}\left(\frac{\partial x}{\partial r}\right)_{R}--(6)$ (6) is the recond $B \cdot C$ Let $\theta=\frac{x^{2}-x-x_{0}}{\left.x^{2}-x_{0}-\cdots\right)}$ 
523

$$
\begin{aligned}
& \eta=\frac{Y}{R}+--\cdots-(8) \\
& \tau=\frac{D_{0} f t}{R^{2}}-\cdots-\cdots-(9) \\
& \frac{\partial \theta}{\partial \tau}=\frac{1}{\eta} \frac{\partial}{\partial \eta}\left(\tau \frac{\partial \theta}{\partial \eta}\right) \ldots(10) \\
& \text { IfC. } \quad \theta(\eta \cdot 0)=1 \ldots \ldots(11) \\
& \text { BiC } \quad\left(\frac{\partial \theta}{\partial \eta}\right)_{\eta=0}=0 \ldots \ldots \text { (12) } \\
& \frac{\eta 50 k_{d} \rho_{N_{n}}}{16 \times 10^{6}}\left[x_{e f}-x_{\text {int }}\right]=\frac{D_{t}^{f} \rho_{f}}{2 \eta 0 R}\left(\frac{\partial x}{\partial \eta}\right)_{R} \\
& =-\frac{D_{0}^{f} \rho_{f}}{2 \eta 0 R}\left(x_{q}-x_{0}\right)\left(\frac{\partial \theta}{\partial \eta}\right)_{\eta-1} \\
& \text { But } \frac{x_{\text {eq }}-x_{\text {int }}}{x_{\text {eq }}-x_{0}}=\theta(1 \cdot \tau) \\
& A \theta(1 \cdot \tau)+\left(\frac{\partial \theta}{2 \eta}\right)_{\eta=1}=0-\ldots(13) \\
& \text { where } \frac{1}{A}=\frac{D_{0}^{f} 16 \times 10^{6}}{750 \times 270 \mathrm{Rkd}} \frac{\rho_{f}}{\rho_{\mathrm{Na}}} \ldots(13)
\end{aligned}
$$
solution of $(10)-(13)$ in Callow and Jaeger
"Conduction of Heat in Solid" Ind edition
$P .202$ P. 202

$$
\left.\theta=\sum_{n=1}^{\infty} e^{-\beta_{n}^{2} \tau} \frac{2 A J_{0}\left(\beta_{n} \eta\right)}{\left(\beta_{n}^{2}+A^{2}\right) J_{0}\left(\beta_{n}\right)} \cdots-+15\right)
$$

where Bn's are the rats of $\beta J_{1}(\beta)=A J_{0}(\beta) \cdots(16)$ For the parasitic specified

$$
A=\frac{(250)(270)(0.3)(0.75)}{\left(4 \times 10^{-13}\right)\left(16 \times 10^{6}\right)(2)}=3.6 \times 10^{9}
$$

$\therefore$ the roots $\beta_{n} \ll A($ sec $P 393$ if condition and Jaeger) and (15) reduce to (lng (16) too)

$$
\theta=2 \sum_{n=1}^{\infty} e^{-\beta_{n}^{2} \tau} \frac{J_{0}\left(\beta_{n} \eta\right)}{\beta_{n} J_{1}\left(\beta_{n}\right)}---(1 \eta)
$$


s.

524

Which is the solution for $x_{\text {of }}=x_{\text {int }}$ (ice when $A \rightarrow \infty$ Eq (13) reduces to $\theta(1 . \tau)=0$ ) the solution (17) is plated on Fig $>4$ of Carclius and Jaeger

For $\theta(0 . T)=0.5, \tau=0.2$

from (9) $\quad t=\frac{(0.2)(0.3)^{2}}{4 \times 10^{-13}}=4.5 \times 10^{10} \mathrm{Aec}$

$=1433 \mathrm{yrs}$

However, this enlentation neglect the strong temperature dependence of $D_{0}{ }^{+}$, which word make oxygen diffusion much moose rapid in the center of the fuel than assumed here. For addition. $D_{0}^{f}$ is NOT Renown for hyportochiometric oxide fuel 
525

PROBLEM 20.14

$T_{1}-$ fuel particle

mass $m: T_{1}=3500^{\circ} \mathrm{K}$ initially.

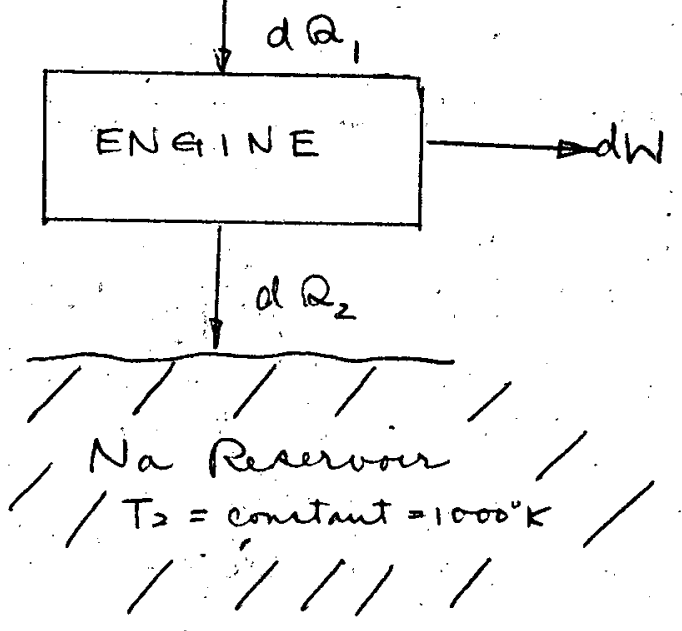

Carnot efficiency $\frac{d W}{d Q_{1}}=\frac{T_{1}-T_{2}}{T_{1}}$

$$
\begin{aligned}
& d Q_{1}=-m C_{p} d T_{1} \\
& \frac{1}{m C_{p}} \frac{d W}{d T_{1}}=-\frac{T_{1}-T_{2}}{T_{1}}=-1-\frac{T_{2}}{T_{1}} \\
& \frac{W}{m C_{p}}=-\int_{T_{1}}^{T_{2}}\left(1-\frac{T_{2}}{T_{1}}\right) d T_{1}^{\prime}-\int_{T_{2}}^{T_{1}}\left(1-\frac{T_{2}}{T_{1}}\right) d T_{1} \\
& \frac{W}{m C_{p}}=\left(T_{1}-T_{2}\right)-T_{2} \ln \left(\frac{T_{1}}{T_{2}}\right)
\end{aligned}
$$

$Q_{1}$. Total energy last ln particle

$$
\begin{aligned}
\eta=\frac{W}{Q_{1}} & =1-\frac{T_{2}}{T_{1}-T_{2}} \ln \left(\frac{T_{1}}{T_{2}}\right)=1-\frac{100}{1500} \ln 2.5 \\
& =0.99=3970
\end{aligned}
$$


526

CHAPTER 21

Problem 21.1

(A)

Neglecting swelling \& creep, $E_{q}(2127)$ becomes, when applied to the entire cylinder

$$
u(r)=c_{2} r+\alpha\left(\frac{1+\nu}{1-\nu}\right) \frac{1}{r} \int_{0}^{r} T r^{\prime} d r^{\prime}
$$

and thus,

$$
\frac{d u}{d r}=c_{2}+\alpha\left(\frac{1+\nu}{1-\nu}\right)\left\{T(r)-\frac{1}{r^{2}} \int_{0}^{r} T r^{\prime} d r\right\}, \quad(\mid b)
$$

where we have used foundry condition $u(r)$ bounded at $r=0 \rightarrow C_{1}=0$

$\therefore \frac{d u}{d r}+\frac{u}{r}=2 c_{2}+\alpha\left(\frac{1+\nu}{1-\nu}\right) T(r)$

(Ic)

Eq (21.23) becomes:

$$
\begin{aligned}
& \sigma_{r}(r)=\frac{E}{1+\nu}\left\{C_{2}+\alpha\left(\frac{1+\nu}{1-\nu}\right)\left[T(r)-\frac{1}{r^{2}} \int_{0}^{r} T r^{\prime} d r^{\prime}\right]+\right. \\
& \left.+\frac{\nu}{1-2 \nu}\left[2 C_{2}+\alpha\left(\frac{1+\nu}{1-\nu}\right) T(r)+\epsilon_{z}-3 \alpha T(r)\right]-\alpha T\right\}
\end{aligned}
$$

Combining coefficients of $T(r)$, we find they sum to 0 :

$$
\sigma_{r}(r)=\frac{E}{1+\nu}\left\{\frac{C_{2}}{1-2 \nu}-\alpha\left(\frac{1+\nu}{1-\nu}\right) \frac{1}{r^{2}} \int_{0}^{r} T r^{\prime} d r^{\prime}+\frac{\nu \epsilon_{z}}{1-2 \nu}\right\}
$$

No radial stress component at $r=R$ implies

$$
\frac{C_{2}}{1-2 \nu}+\frac{\nu \epsilon_{z}}{1-2 \nu}=\alpha\left(\frac{1+\nu}{1-\nu}\right) \frac{1}{R^{2}} \int_{0}^{R} T r \cdot d r^{\prime}
$$

or, $\sigma_{r}$ becomes

$$
\sigma_{r}(r)=\frac{\alpha E}{1-\nu}\left\{\frac{1}{R^{2}} \int_{0}^{R} T r^{\prime} d r^{\prime}-\frac{1}{r^{2}} \int_{0}^{r} T r^{\prime} d r^{\prime}\right\}
$$


527

$E_{q} \cdot(21.24)$ becimes:

$$
\begin{aligned}
& \sigma_{\theta}(r)=\frac{E}{1+\nu}\left\{c_{2}+\alpha\left(\frac{1+\nu}{1-\nu}\right) \frac{1}{r^{2}} \int_{0}^{r} T r^{\prime} d r^{\prime}+\right. \\
& \left.+\frac{\nu}{1-2 \nu}\left[2 c_{2}+\alpha\left(\frac{1+\nu}{1-\nu}\right) \pi(r)+\epsilon_{z}-3 \alpha T\right]-\alpha T\right\}
\end{aligned}
$$

$I_{n}$ this case, the coefficient of $T$ is $-\chi\left(\frac{1+\nu}{1-\nu}\right)$. Using $C_{2}$ as determined abive, Eq (3):

$$
\sigma_{\theta}(r)=\frac{\alpha E}{1-\nu}\left\{\frac{1}{R^{2}} \int_{0}^{R} T r^{\prime} d r^{\prime}+\frac{1}{r^{2}} \int_{0}^{r} T r^{\prime} d r^{\prime}-T\right\}
$$

Assume no axial restaint, ie

$$
\int_{0}^{R} 2 \pi r \sigma_{z} d r=0
$$

$E_{q}(21.25)$ becomes using $E_{q}(1 C)$

$$
\begin{aligned}
\sigma_{z}= & \frac{E}{1+\nu}\left\{\epsilon_{z}+\frac{\nu}{1-2 \nu}\left[2 c_{2}+\alpha\left(\frac{1+\nu}{1-\nu}\right) T+\epsilon_{z}-3 \alpha T\right]+\right. \\
& +(-\alpha T)\}
\end{aligned}
$$

or

$$
\sigma_{z}=\frac{E}{1+\nu}\left\{\frac{2 \nu}{1-2 \nu} c_{2}+\frac{1-\nu}{1-2 \nu} \epsilon_{z}-\alpha\left(\frac{1+\nu}{1-\nu}\right)+\right\}
$$

Apply $\frac{2}{R^{2}} \int_{0}^{R} r d r$ to this equation

$$
\therefore \quad 0=\frac{E}{1+\nu}\left\{\frac{2 \nu}{1-2 \nu} C_{2}+\frac{1-\nu}{1+2 \nu} \epsilon_{z}-2 \alpha\left(\frac{1+\nu}{1-\nu}\right) \frac{1}{R^{2}} \int_{0}^{R} \operatorname{Tr} d r\right\}
$$

Combine Eq's (7) \& (8)

$$
\therefore \quad \sigma_{z}=\frac{2 \alpha E}{1-\nu}\left\{\frac{1}{R^{2}} \int_{0}^{R} T r d r-\frac{1}{2} T\right\}
$$


528

Parabolic temperature profile:

$$
\begin{aligned}
T & =T_{s}+\left(T_{0}-T_{S}\right)\left(1-\frac{r^{2}}{R^{2}}\right) \\
& =T_{0}-\Delta T r^{2} / R^{2}
\end{aligned}
$$

where $\Delta T \equiv T_{0}-T_{S}$

$$
\begin{aligned}
& \frac{1}{r^{2}} \int_{0}^{r} T r^{\prime} d r^{\prime}=\frac{1}{2} T_{0}-\frac{1}{4} \Delta T \frac{r^{2}}{R^{2}} \\
& \frac{1}{R^{2}} \int_{0}^{R} T r d r=\frac{1}{2} T_{0}-\frac{1}{4} \Delta T
\end{aligned}
$$

Substitute $E_{q}^{\prime}$ s (10)\&(12) into (5)

$$
\sigma_{\theta}=-\frac{\alpha E}{1-\nu} \frac{\Delta T}{4}\left[1-3\left(\frac{r}{R}\right)^{2}\right]
$$

Use $E_{q}(15.212)$

$$
\Delta T=\frac{P}{4 \pi \bar{K}}
$$

which yields $E_{q}(21.49)$ :

$$
\sigma_{0}=-\frac{\alpha E \phi}{16 \pi(1-\nu) \bar{k}}\left[1-3\left(\frac{r}{R}\right)^{2}\right]
$$

Substitute Eq (10)\&(12) into" $(9)$,

$$
\sigma_{z}=-\frac{\alpha E}{1-2} \cdot \frac{\Delta T}{2}\left[1-2\left(\frac{\dot{r}}{R}\right)^{2}\right]
$$

(B)

For a hollow cylinder, the analog of $E_{q}(5)$ is:

$$
\sigma_{\theta}(r)=\frac{\alpha E}{1-\nu}\left\{\frac{1+\left(r_{0} / r\right)^{2}}{R^{2}-r_{0}^{2}} \int_{r_{0}}^{R} T r^{\prime} d r^{\prime}+\frac{1}{r^{2}} \int_{r_{0}}^{r} T r^{\prime} d r^{\prime}-T\right\} \quad(17)
$$


529

The temperature distribution in the fuel is given by solution of $E_{q}(10.49)$, subject to

$$
\begin{aligned}
& E_{8 n}(10.50) \&(10.51): \\
& T-T_{s}=\frac{1}{4} \frac{H R^{2}}{K}\left\{\left(1-\frac{r^{2}}{R^{2}}\right)+2\left(\frac{r_{0}}{R}\right)^{2} \ln \left(\frac{r}{R}\right)\right\}
\end{aligned}
$$

Linear Power is:

$$
\begin{aligned}
& P=\pi R^{2}\left(1-\frac{\dot{r}_{0}{ }^{2}}{R^{2}}\right) H \\
& E_{q}(18) \&(19) \quad \rightarrow \\
& T=T_{s}+\frac{P}{4 \pi k} \frac{1}{1-\left(r_{0} / R\right)^{2}}\left\{\left(1-\frac{r^{2}}{R^{2}}\right)+2\left(\frac{r_{0}}{R}\right)^{2} \ln \left(\frac{r}{R}\right)\right\}(20) \\
& \int_{r_{0}}^{r} T r^{\prime} d r^{\prime}=\frac{\rho}{4 \pi k} \frac{R^{2}}{\left(1-\frac{r_{0}^{2}}{R^{2}}\right)}\left\{\frac{1}{2}\left[\left(\frac{r}{R}\right)^{2}-\left(\frac{r_{0}}{R}\right)^{2}\right]-\frac{1}{4}\left[\left(\frac{r}{R}\right)^{4}-\left(\frac{r_{0}}{R}\right)^{4}\right]+\right. \\
& \left.+\left(\frac{r_{0}}{R}\right)^{2}\left[\frac{r^{2}}{R^{2}} \ln \left(\frac{r}{R}\right)-\left(\frac{r_{0}}{R}\right)^{2} \ln \left(\frac{r_{0}}{R}\right)\right]\right\}+ \\
& +T_{S} \frac{1}{2} R^{2}\left[\left(\frac{r}{R}\right)^{2}-\left(\frac{r g}{R}\right)^{2}\right]
\end{aligned}
$$

Finally, substitute $E_{q}(20) \&(21)$ into (17), noting that the $T_{s}$ terms cancel, and obtain:

$$
\begin{aligned}
\sigma_{\theta}(r)= & \frac{\alpha E P}{4 \pi(1-\nu) k} \frac{1}{1-\eta_{0}^{2}}\left\{\frac { 1 + ( \eta _ { 0 } / ) ^ { 2 } } { 1 - \eta _ { 0 } ^ { 2 } } \left[\frac{1}{2}\left(1-\eta_{0}^{2}\right)+\right.\right. \\
& \left.-\frac{1}{4}\left(1-\eta_{0}^{2}\right)-\eta_{0}^{4} \ln \eta_{0}\right]+\frac{1}{\eta^{2}}\left[\frac{1}{2}\left(\eta^{2}-\eta_{0}^{2}\right)+\right. \\
& \left.-\frac{1}{4}\left(\eta^{4}-\eta_{0}^{4}\right)+\eta_{0}^{2}\left(\eta^{2} \ln \eta-\eta_{0}^{2} \ln \eta_{0}\right)\right]+ \\
& \left.-\left[1-\eta^{2}+2 \eta_{0}^{2} \ln \eta\right]\right\}
\end{aligned}
$$

where $\eta \equiv \frac{r}{R}, \quad m_{0} \equiv \frac{r_{0}}{R}$ 
530

For $\eta_{0} \rightarrow 0, E_{q}(22)$ reduces to

$$
\sigma_{\theta}(r)=\frac{\alpha E P}{4 \pi(1-\nu) k}\left(\frac{3}{4} \eta^{2}-\frac{1}{4}\right)
$$

T this is eq (15)

(c)

$$
\begin{aligned}
\frac{\alpha E P}{4 \pi(1-\nu) k} & =\frac{10^{-5}\left(1.75 \times 10^{8}\right)(500)}{4 \pi(0.68)(0.03)} \\
& \cong 3.4 \times 10^{6} \mathrm{kn} / \mathrm{m}^{2}
\end{aligned}
$$

Radius of central void is given by

$$
\begin{aligned}
& 0.97\left(R^{2}-r_{0}^{2}\right)=0.85 R^{2} \\
& \rightarrow m_{0}=0.35
\end{aligned}
$$


C

Problem 21.1 (c)

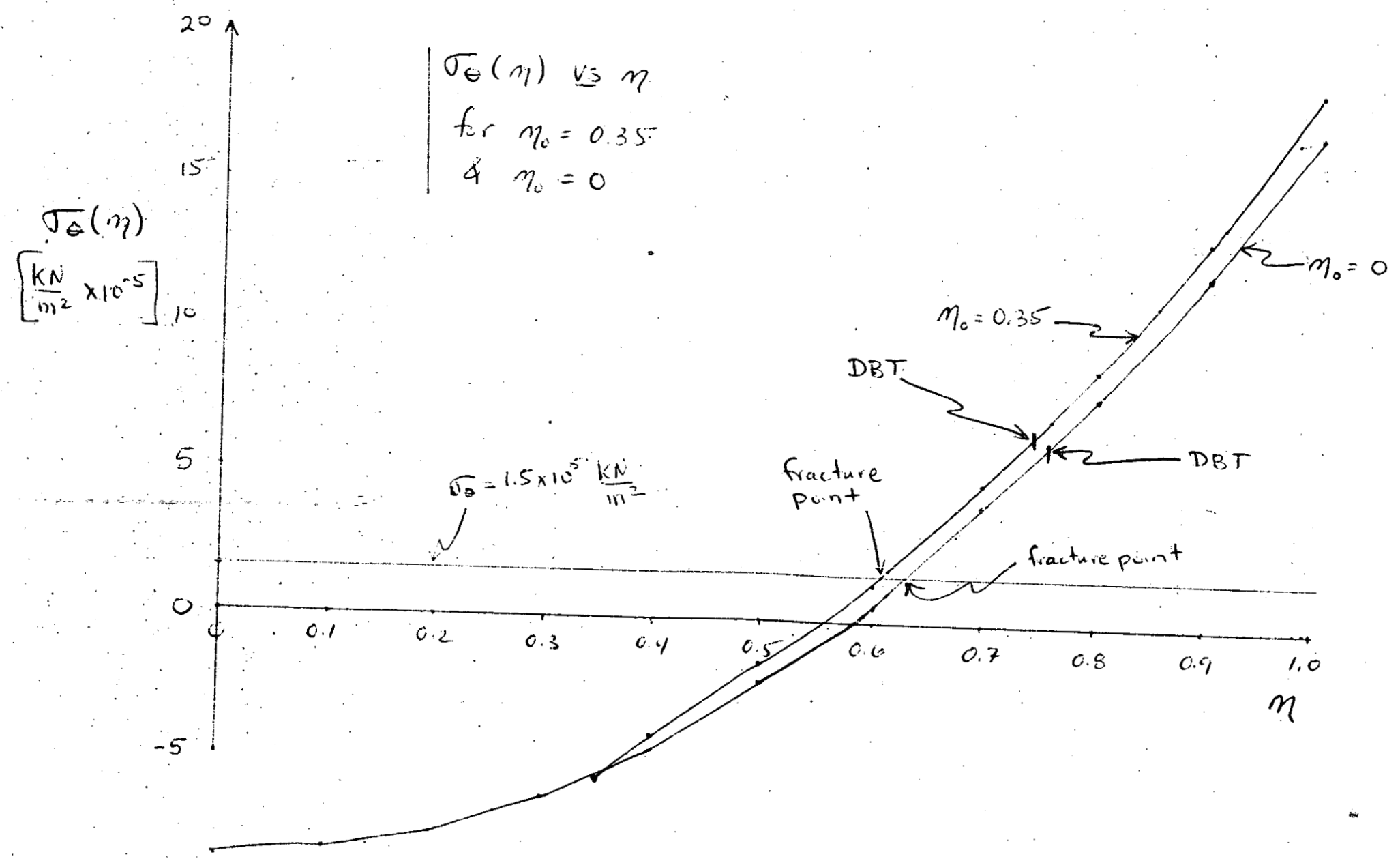


532

Problem 21.2

(A)

In the thin wall approximation,

$$
T \cong T_{i}-\frac{\Delta T_{c} x}{t_{c}}
$$

where $T_{i}=$ inside cladding temperature

$$
x_{i}=\text { distance from inside of cladding }
$$

In Eq (17) of Prob 21.1 (B)

$$
\begin{gathered}
\frac{1+\left(r_{0} / r\right)^{2}}{R^{2}-r_{0}^{2}}=\frac{2}{\left(R+r_{0}\right)\left(R-r_{0}\right)} \cong \frac{1}{R t_{c}} \\
\int_{r_{c}}^{r} T^{\prime} d r^{\prime} \cong R \int_{0}^{r} T d x^{\prime}=R\left[T_{i} x-\frac{1}{2} \frac{\Delta T_{c}}{t_{c}} x^{2}\right] \\
\sigma_{\theta}(x)=\frac{\alpha E}{1-\nu}\left\{\frac{1}{t_{c}}\left[T_{i} t_{c}-\frac{1}{2} \frac{\Delta T_{c}}{t_{c}} t_{c}^{2}\right]+\frac{1}{R}\left[T_{i}+\right.\right. \\
\left.\left.-\frac{1}{2} \frac{\Delta T_{c}}{t_{c}} x^{2}\right]-T_{i}+\frac{\Delta T_{c} x}{t_{c}}\right\}
\end{gathered}
$$

Neglect terms in: $X / R$ by thin-wall approximation,

$$
\sigma_{\theta}(x) \cong-\left(\frac{\alpha E}{1-\nu}\right) \frac{\Delta T_{c}}{2}\left(1-\frac{2 x}{l_{c}}\right)
$$

Maximum "occurs at outside $\left(x / t_{c}=1\right)$ of clad:

$$
\left(\sigma_{\theta}\right)_{\max }=\frac{\alpha E \Delta T_{C}}{2(1-\nu)}
$$


533

(B)

Heat flux thru cladding $=\frac{P}{2 \pi R}=\frac{k \Delta T_{c}}{t_{c}}$

Using above relation in (2)

$$
\left(\sigma_{0}\right)_{\max }=\frac{\alpha E P t_{c}}{4 \pi(1-\nu) R K}
$$

for $\left(\sigma_{\theta}\right)_{\text {max }}=\sigma_{y}$

$$
\begin{aligned}
& P_{\text {yield }}=\frac{4 \pi(1-\nu) R K \sigma_{y}}{\alpha E t_{c}} \\
& =\frac{4 \pi(0.68)(0.32+0.038)(0.24)\left(2.1 \times 10^{5}\right)}{\left(1.2 \times 10^{-5}\right)\left(1.4 \times 10^{8}\right)(0.038)}
\end{aligned}
$$

(c)

$P_{\text {yield }} \cong 2400^{\text {watts }} / \mathrm{cm}$

From prob' $18.6(A)$ :

$$
\sigma_{\theta}=\frac{P_{\text {plenum }} R}{t_{c}}
$$

for $\sigma_{\theta}=\sigma_{y}$,

$$
\begin{aligned}
\left(P_{P}\right)_{\text {yielding }}=\frac{{ }^{\circ} \sigma_{y} t_{c}}{R} & =\frac{\left(2.1 \times 10^{5}\right)(0.038)}{0.32} \\
& \cong 2.5 \times 10^{4} \frac{\mathrm{kN}}{\mathrm{m}^{2}} \\
& \cong 246 \mathrm{~atm}
\end{aligned}
$$

(D)

$$
\sigma_{\theta}=\left(\frac{500}{2400}+\frac{50}{246}\right)\left(2.1 \times 10^{5}\right) \cong 8.6 \times 10^{4} \cdot \frac{\mathrm{kN}}{\mathrm{m}^{2}}
$$

From Fig $18: 12, T=7270^{\circ} \mathrm{C}$ biaxial sties s $=8.6 \times 10+\mathrm{kN} / \mathrm{m}^{2}$

$$
\therefore t_{R} \cong .600 \text { hrs. }
$$


(E)

$$
534
$$

Adding $E_{q}(3) \&(4)$;

$$
\begin{aligned}
& \left(\sigma_{\theta}\right)_{\max }=\left[\frac{\alpha E P}{4 \pi(1-\nu) R K}\right] t_{c}+\frac{P_{p} R}{t_{c}} \\
& \text { or } \\
& \left(\sigma_{\theta}\right)_{\max }=a t_{c}+\frac{b}{t_{c}}
\end{aligned}
$$

At minimum of $\left(\sigma_{0}\right)$ inas

$$
\begin{aligned}
& \frac{d\left(\sigma_{0}\right)_{\text {miv }}}{d \cdot t_{c}}=0=a-\frac{b}{\left(t_{c}\right)^{2}} \\
& \therefore\left(t_{c}\right)_{\text {opt }}=\sqrt{\frac{b}{a}}
\end{aligned}
$$

$$
\begin{aligned}
\left(t_{c}\right)_{\Delta \rho t} & =\left[\frac{4 \pi(1-\nu) R^{2} k P_{p}}{\alpha E \rho}\right]^{1 / 2} \\
& =\left[\frac{4 \pi(0.68)(0.32)^{2}(0.24)\left(5.1 \times 10^{3}\right)}{\left(1.2 \times 10^{-5}\right)\left(1.4 \times 10^{8}\right)(500)}\right]^{1 / 2} \\
\left(t_{c}\right)_{\text {ogt }} & \cong 0.036 \mathrm{~cm}
\end{aligned}
$$


535

Problem 21.3

From prob $18.6(A)$

$$
\begin{aligned}
& \sigma_{a}=\frac{P_{p} R}{t_{c}} \\
& \sigma_{z}=\frac{P_{p} R}{2 t_{c}} \\
& \sigma_{r} \simeq 0
\end{aligned}
$$

From $(21.16)$ \& the creep law:

$$
\begin{aligned}
& \dot{\epsilon}_{\theta}^{c}=B\left(\sigma^{*}\right)^{n-1}\left[\sigma_{\theta}-\frac{1}{2} \sigma_{z}\right] \\
& \varepsilon_{q}(21.18) \rightarrow \\
& \sigma^{*}=\frac{1}{\sqrt{2}}\left[\sigma_{\theta}^{2}+\sigma_{z}^{2}+\left(\sigma_{\theta}-\sigma_{z}\right)^{2}\right]^{1 / 2} \\
& \text { From } \epsilon_{q}(1) \pm(2)
\end{aligned}
$$

From $E_{q}(1) \in(2)$ obtain,

$$
\begin{aligned}
& \sigma_{z}=\frac{1}{2} \sigma_{\theta} \\
& \therefore \sigma^{*}=\frac{1}{\sqrt{2}}\left[\sigma_{\theta}^{2}+\frac{1}{4} \sigma_{\theta}^{2}+\frac{1}{4} \sigma_{\theta}^{2}\right]^{1 / 2}=\frac{\sqrt{3}}{2} \sigma_{\theta} \\
& E_{q}(3) \text { becomes, }
\end{aligned}
$$

$$
\dot{\epsilon}_{\theta}^{c}=B\left(\frac{\sqrt{3}}{2}\right)^{n-1} \sigma_{\theta}^{n-1} \frac{3}{4} \sigma_{\theta}
$$

and at time $t$,

$$
\left(\frac{\Delta R}{R}\right)_{\text {creep }}=\dot{\epsilon}_{\theta}^{c} t=\left(\frac{3}{4}\right)^{\frac{n+1}{2}} B\left(\frac{P_{p} R}{t_{c}}\right)^{n} t
$$

Elastic deformation:

$$
\epsilon_{\theta}=\frac{u}{r} \cdot \frac{\text { thin }}{w, 11} \rightarrow \frac{\Delta R}{R}
$$

Combining E gs (22) \&(23) of Prob 18.6(c):

$$
\left(\frac{A R}{R}\right)_{\text {elastic }}=\frac{P_{P} R}{2 E t_{c}}(1-2 \nu)
$$


536

Problem 21.4
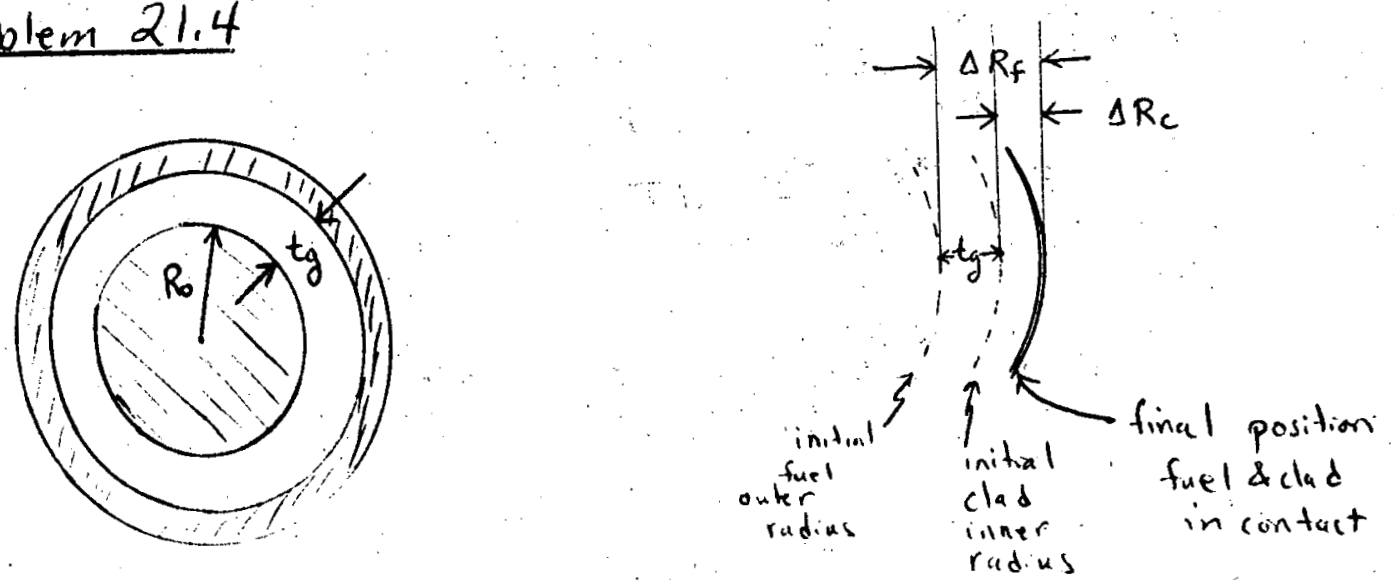

$$
\begin{aligned}
& \frac{\Delta R_{f}}{R_{0}}=\frac{1}{3} \alpha_{f}\left(T-T_{0}\right) \quad \text { note } \alpha=\frac{\text { volumetric cue }}{\text { of thermal expansion. }} \\
& \frac{\Delta R_{c}}{R_{0}}=\frac{1}{3} \alpha_{c}\left(T-T_{0}\right) \\
& \text { at } T=T^{*} ; \Delta R_{F}-\Delta R_{c}=t_{g}=\frac{1}{3}\left(\alpha_{f}-\alpha_{c}\right)\left(T^{*}-T_{0}\right) R_{0} \\
& \therefore T^{*}=T_{0}+\frac{3 t g}{\left(\alpha_{f}-\alpha_{c}\right) R_{0}}
\end{aligned}
$$

From thermodynamics,

$$
\begin{aligned}
& \left.\left.d P=\frac{\partial P}{\partial T}\right)_{V} d T+\frac{\partial P}{\partial V}\right)_{T} d V \\
& \left.\frac{\partial P}{\partial T}\right)_{v}=\frac{\alpha_{F}}{\beta_{f}} \quad, \frac{1}{\beta_{f}}=b_{u} / k \text { modulus of fie } \\
& \left.\frac{\partial P}{\partial V}\right)_{T}=-\frac{1}{\beta_{f} V}
\end{aligned}
$$

Now, adV is due to thermal expansion of clad for $T>T^{*}$, (by assumption) $d v=\alpha_{c} v d T$ 


$$
537
$$

$$
\begin{aligned}
\therefore \quad d p & =\frac{\alpha_{f}}{\beta_{f}} d T-\frac{\alpha_{c}}{\beta_{f}} d T \\
& =\frac{\alpha_{f}-\alpha_{c}}{\beta_{f}} d T \\
a r & =\frac{\left(\alpha_{f}-\alpha_{c}\right)}{\beta_{f}}\left(T-T^{*}\right)
\end{aligned}
$$


538

Problem 21.5

At $T=T^{*}$, the fuel contacts the cladding. For $T>T^{*}$, interfacial pressure is generated.

Let,

$$
\Delta T \equiv T-T^{*}
$$

The displacement equation is given by $E_{q}(2126)$. with $\epsilon_{i}^{c}=\epsilon^{s}=0$ Since $T \neq$ function of $r$, we have

$$
\frac{d}{d r}\left[\begin{array}{ll}
\frac{1}{r} & \frac{d(r u)}{d r}
\end{array}\right]=0
$$

Integrate:

$$
u=c_{1} r-\frac{c_{2}}{r}
$$

Because $u$ is bounded at $r=0, c_{2}=0 \&$

$$
\therefore u=C_{1} r
$$

Boundary condition at $u=R$ is $u=0$, so that

$$
C_{1}=0
$$

But, we also require

$$
\sigma_{r}(R)=-P_{f c}
$$

With $u=\epsilon^{s}=\epsilon^{c}=0$, Equ's $(21.23)+(21.25)$ become:

$$
\begin{aligned}
& \sigma_{r}=\frac{E}{1+\nu}\left\{\frac{\nu}{1-2 \nu} \epsilon_{z}-\frac{1+\nu}{1-2 \nu} \times T\right\} \\
& \sigma_{z}=\frac{E}{1+\nu}\left\{\frac{1-\nu}{1-2 \nu} \epsilon_{z}-\frac{1+\nu}{1-2 \nu} \times T\right\}
\end{aligned}
$$

Neither $\sigma_{r}$ nor $\sigma_{z}$ depend upon $r$, so $\sigma_{r}$ in $E_{q}(4)$ is equal to - P $f_{c}$ at all $r$. 
539

(i)

Complete cixial restraint $\epsilon_{z}=0$

Use $\epsilon_{z}=0$ \& $\sigma_{r}=-p_{f c}$ in $E_{q}(4)$ to obtain

$$
P_{f c}=\frac{E_{\alpha} T}{1-2 \nu}
$$

According to $E_{q}$ (A.28) of Appendix to Che, the reciprocal of the compressibility of the fuel (ie.. its bulk modulus) is:

$$
\frac{1}{3 k}=\frac{1-2 \nu}{\epsilon}
$$

So that $E_{f}(4)$ reduces to the solution to problem 21.4(B), which was obtained from purely thermodynamic considerations.

(ii) No axial restraint condition is

$$
\int_{0}^{R} \sigma_{z} r d r=0
$$

but force $\sigma_{z} \neq$ functicic of $r$. This condition is equivalent to $\sigma_{z}=0$. Therefore, $\epsilon_{z}$ is determined from $E_{q}$ (S) as.

$$
\epsilon_{z}=\frac{1+\nu}{1-\nu} \alpha T
$$

Substituting $E_{q}$ (7) into (4) \& setting $\sigma_{r}=-p_{+c}$ as before yields:

$$
P_{f c}=\frac{E_{x} T}{1-\nu}
$$

For the case et complete loss of fuel strength ( $E=c$, $\left.\gamma=1_{2}\right) ; \quad p_{f c}=0$ \& the fuel expands in the clad like a heated liquid in an open tube. 
540

Problem 21.6

(A)

Tempirature Distibution

Region (1)

$$
\frac{1}{r^{2}} \frac{d}{d r}\left(r^{2} \frac{d T}{d r}\right)=-\frac{H}{k_{1}}
$$

Region 0

$$
\frac{1}{r^{2}} \frac{d}{d r}\left(r^{2} \frac{d T_{2}}{d r}\right)=0
$$

Boundry conditions: lassume fuel-clad theormal resistances negligible)

$$
\begin{aligned}
& T_{1}(0)=\text { buinded } \\
& T_{1}(R)=T_{2}(R) \\
& \left.\left.K_{1} \frac{d T_{1}}{d r}\right)_{R}=K_{2} \frac{d T_{2}}{d r}\right)_{R} \\
& T_{2}(\infty)=0
\end{aligned}
$$

The solutions of (1) $4(2)$ subject to (3), (4), (5), \&(6) are!

$$
\begin{aligned}
& T_{1}(r)=\frac{H}{6 k_{1}}\left(R^{2}-r^{2}\right)+\frac{R^{2} H}{3 k_{2}} \\
& T_{2}(r)=\left[\frac{\left(\frac{4}{3} \pi R^{3}\right) H}{4 \pi k_{2} r}\right]=\frac{R^{3} H}{3 k_{2} r}
\end{aligned}
$$

(B)

The differential equations of radial stress are (with spherical symetry):

Region (1): $\frac{1}{r^{4}} \frac{d}{d r}\left(r^{4} \frac{d \sigma_{r}}{d r}\right)=-\frac{2 E_{1} x_{1}}{1-\nu_{1}} \frac{1}{r} \frac{d T_{1}}{d r}$

(9)

$\operatorname{Regin}(2): \frac{1}{r^{4}} \frac{d}{d r}\left(r^{4} \frac{d J_{r_{2}}}{d r}\right)=-\frac{2 E_{2} \cdot x_{2}}{1-\nu_{2}} \frac{1}{r} \frac{d T_{2}}{d r} \quad$ (10) 
(17) $\quad \perp x+((\theta 0+1) x-\theta)=\theta$

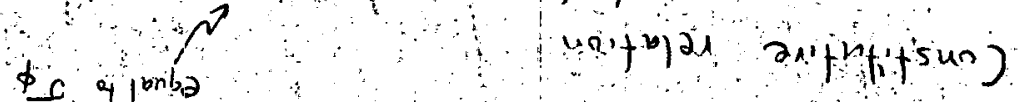

$\left(0_{2}\right)$

$\frac{1}{n}=\theta$

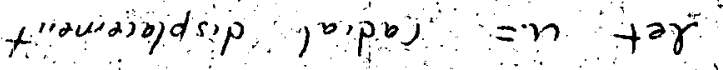

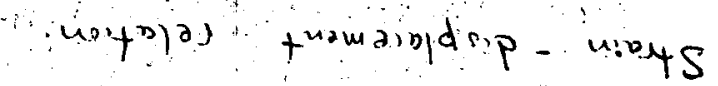

(b)

$$
\frac{p}{\rho P}+\frac{z}{T}+10=0
$$

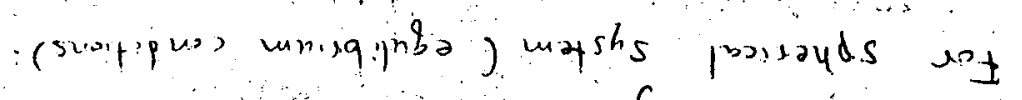

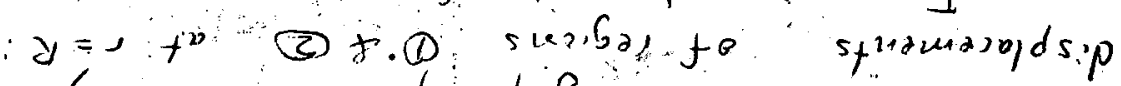

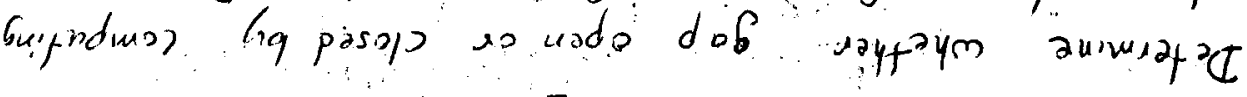

(a)

$$
\frac{\left({ }^{2} x-1\right)^{2} x \varepsilon}{H^{2} x^{2} \exists}={ }^{2} x
$$

(t)

$$
\left(\frac{\varepsilon}{y}-\frac{1}{T}\right)_{\varepsilon} y^{2} y-\frac{\varepsilon d}{\varepsilon y} d y-=(1)^{2}-1 .
$$

(91)

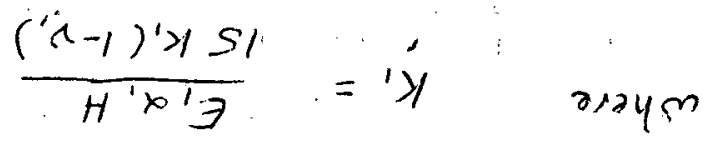

(s)

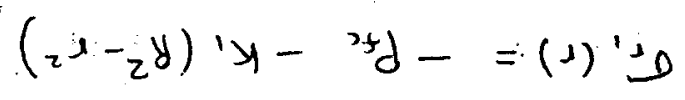

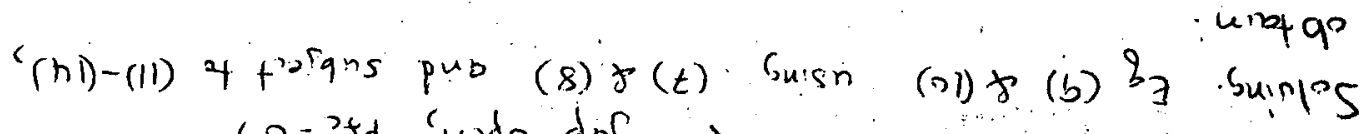
$(0=79$ cuado dof $f:)$

(b)

$$
3 f d-=(y)^{2} D
$$

(8)

$$
\begin{aligned}
{ }^{2} d- & =(y)^{1} D \\
0 & =(\infty)^{2} D
\end{aligned}
$$

(ii)

$$
\text { papursq }=(0) \text { D }
$$

seetpuns hipunog 
542

Combine $E_{q}(19)$, (20), (21) \&obtain ur) in terms ot $\sigma r \&$ $\frac{d \sigma r}{d r}$, ie:

$$
u=\frac{r}{E}\left\{(1-\nu)\left(\sigma_{r}+\frac{1}{2} r \frac{d \sigma_{r}}{d r}\right)-\nu \sigma_{r}\right\}+\chi T_{r}
$$

Now insert. $E_{q}(7),(8), \&(15),(17)$ into (22) \& evaluate $u_{1}(R) \& u_{2}(R)$ :

Assume gap open:

set $P_{t c}=0$. If $u_{2}(R)>u_{1}(R)$ assumption is che If not, must assume gap closed set $u_{1}(R)=u_{2}(R)$ \& solve for $\mathrm{FFC}$.

In sphere

$$
\begin{aligned}
& u_{1}=\frac{r}{E_{1}}\left\{\left(1-\nu_{1}\right)\left[-P_{f c}-k_{1}\left(R^{2}-r^{2}\right)+k_{1} r^{2}\right]-\nu_{1}\left(-P_{t c}-k_{1}\left(R^{2} \cdot r^{2}\right)\right)\right\}+ \\
& \quad+\frac{\alpha_{1} H}{6 k_{1}}\left(R^{2}-r^{2}\right)+\frac{\alpha_{1} R^{2} H_{r}}{3 k_{2}}
\end{aligned}
$$

$$
\begin{aligned}
& \text { at } r=R \\
& u_{1}(R)=\frac{R}{E_{1}}\left\{\left(1-\nu_{1}\right)\left(-p_{f_{c}}+k_{1} R^{2}\right)+\nu_{1} p_{f_{c}}\right\}+\frac{\alpha_{1} R^{3} H}{3 k_{2}} \\
& \therefore u_{1}(R)=\frac{R}{E_{1}}\left\{\left(1-\nu_{1}\right) k_{1} R^{2}-\left(1-2 v_{1}\right) p_{f_{c}}\right\}+\frac{\alpha_{1} R^{3} H}{3 k_{2}}
\end{aligned}
$$

Outside sphere:

$$
\begin{aligned}
u_{2} & =\frac{r}{E_{2}}\left\{( 1 - \nu _ { 2 } ) \left[-P_{f_{c}} \frac{R^{3}}{r^{3}}-k_{2} R^{3}\left(\frac{1}{r}-\frac{R^{2}}{r^{3}}\right)+\frac{3 P_{c} \frac{R^{3}}{2}+}{r^{3}}+\right.\right. \\
& \left.\left.-\frac{k_{2} R^{3}}{2}\left(-\frac{1}{r}+3 \frac{R^{2}}{r^{3}}\right)\right]+\nu\left[P_{f_{c}} \frac{R^{3}}{r^{3}}+k_{2} R^{3}\left(\frac{1}{r}-\frac{R^{2}}{r^{3}}\right)\right]\right\}+ \\
& +\frac{\left(\frac{4}{3} \pi R\right) \times H r}{4 \pi k_{2} r}
\end{aligned}
$$


543

at $r=R$ :

$$
\therefore \quad u_{2}(R)=\frac{R}{E_{2}}\left\{\frac{1}{2}\left(1+\nu_{2}\right) P_{f_{C}}-\left(1-\nu_{2}\right) k_{2} R^{2}\right\}+\frac{x_{2} R^{3} H}{3 k_{2}}
$$

If gap ujen $p_{f c}=0$

from (23).

$$
\begin{aligned}
& u_{1}(R)=\frac{\left(1-\nu_{1}\right) k_{1} R^{3}}{E_{1}}+\frac{\alpha_{1} R^{3} H}{3 k_{2}}=\frac{\alpha_{1} R^{3} H}{3}\left(\frac{1}{5 k_{1}}+\frac{1}{k_{2}}\right) \\
& \text { from (22): } \\
& u_{2}(R)=\frac{-\left(1-\nu_{2}\right) k_{2} R^{3}}{E_{2}}+\frac{\alpha R^{3} H}{3 k_{2}}=0 \\
& u_{1}(R)>u_{2}(R) \Rightarrow \therefore \text { gap dojed } \\
& \text { Set } u_{1}(R)=u_{2}(R) \text { from (23)\&(24): }
\end{aligned}
$$$$
\frac{R}{E_{1}}\left[\left(1-\nu_{1}\right) k_{1} R^{2}-\left(1-2 \nu_{1}\right) P_{f_{c}}\right]+\frac{x_{1} R^{3} H}{3 k_{2}}=
$$

$$
\begin{aligned}
& =\frac{R}{E_{2}}\left[\frac{1}{2}\left(1+\nu_{2}\right) P_{f_{c}}-\left(1-\nu_{2}\right) k_{2} R^{2}\right]+\frac{\alpha_{2} R^{3} H}{3 k_{2}} \\
& \frac{\left(1-\nu_{1}\right) k_{1} R^{2}}{E_{1}}+\frac{\left(1-\nu_{2}\right) k_{2} R^{2}}{E_{2}}+\frac{\left(\alpha_{1}-\alpha_{2}\right) R^{2} H}{3 k_{2}}= \\
& =\left[\frac{\left(1-2 \nu_{1}\right)}{E_{1}}+\frac{\left(1+\nu_{2}\right)}{2 E_{2}}\right] P_{f_{C}}
\end{aligned}
$$




$$
\frac{\alpha_{1} H R^{2}}{15 k_{1}}+\frac{\alpha_{2} H R^{2}}{3 k_{2}}+\frac{\left(\alpha_{1}-\alpha_{2}\right) H R^{2}}{3 k_{2}}=\left[\frac{1-2 \nu_{1}}{E_{1}}+\frac{1+\nu_{2}}{2 \epsilon_{2}}\right] P_{f_{c}}
$$

Thus

$$
P_{x_{c}}=\frac{\frac{\alpha_{1} H R^{2}}{3}\left(\frac{1}{5 k_{1}}+\frac{1}{k_{2}}\right)}{\frac{1-2 \gamma_{1}}{E_{1}}+\frac{1+\nu_{2}}{2 E_{2}}}
$$

(25) 
545

Problem 21.7.

For the $\angle M F B R$ core considered in problem 20.5, the radial reduction of the linear power is neglected and the axial dependence of $P$ is given by

$$
\gamma(y)=544 \cos \left(1.96 \frac{y}{z}\right) \quad \omega / \mathrm{cm}
$$

where $z=$ core height $(-z / 2 \leq 3 \leq z / 2)$ With $\mathrm{Na}$ inlet $\&$ outlet temperatures of $470^{\circ} \mathrm{C}$ \& $650^{\circ} \mathrm{C}$, respectively, and a $N_{a}$ heat transfer coefficient of $12 . \omega / \mathrm{cm}^{2}-{ }^{\circ} \mathrm{C}$, the temperature of the outside of the cladding is:

$$
\begin{aligned}
T_{c_{0}}=470+180 & \left\{\frac{\sin (1.963 / z)+0.83}{1.66}\right\}+ \\
& +23 \cos (1.963 / z)
\end{aligned}
$$

In the thin will approximation, heat flux through the cladding is:

$$
\frac{p}{2 \pi R_{c}}=\frac{k_{c} \Delta T_{c}}{t_{c}}
$$

where $\Delta T_{c} \equiv$ temperature drop thru the cladding The mid-wall (ie average) cladding temperature is:

$$
\overline{T_{c}}=T_{c_{0}}+\frac{1}{2} \Delta T_{c}=T_{c_{0}}+\frac{\gamma t_{c}}{4 \pi R_{c} k_{c}}
$$

Using $E_{f}(1)$ \& the cladding property data.

$$
\begin{gathered}
\bar{T}_{0}=T_{c_{0}}+22 \cos (1.96 \mathrm{~m} / \mathrm{z}) \\
\eta
\end{gathered}
$$

see table 
546

(B)

Use $E_{q}(10,56)$ to find initial power density of fuel :

$$
H_{0}=\left[3,07 \times 10^{-i} q_{0} \sigma_{F} N_{t_{0}} \Phi_{c}\right]
$$

where

$$
\begin{aligned}
& q_{0}=0.15 \\
& \sigma_{f}=2 \text { barns } \\
& n_{f 0}^{\prime} \cong 2.2 \times 10^{22} \text { atoms } / \mathrm{cm}^{3}
\end{aligned}
$$

(90\% dinge thel'mitrallu)

the linear pier is

$$
\begin{aligned}
\theta & =\pi R_{c}^{2} H_{0} \\
& =\pi R_{c}^{2}\left(307 \times 10^{-11}\right) q_{0} N_{t_{0}} \sigma_{f} \Phi_{0}
\end{aligned}
$$

The linear power $P$ is held constant through irradiation. Despite restructuring \& burnup, the product ( $\pi R_{c}^{2} q_{0} N_{f_{0}}$ ), which is the number of $P_{u}$ atoms per cm. of fuel, remains constant if the breeding ratio is unity. Therefore, the fast flax is. also independent of irradiation time

$$
\begin{aligned}
\Phi & =\frac{\gamma}{\left(3.07 \times 10^{-11}\right) \pi R_{c}^{2} z N_{f o} \sigma_{. f}} \\
& =\frac{544 \cos (1.96 \mathrm{~m} / z)}{\left.\left(3.07 \times 10^{-11}\right)(3.14)(0.315)^{2}(0.15)\left(2.2 \times 10^{-2.2}\right) 12 \times 10^{-16}\right)} \\
\therefore \Phi(3) & \cong 8.6 \times 10^{1.5} \cos (1.96 \mathrm{~g} / z) \frac{\text { neutrons }}{\left(\mathrm{m}^{2} \mathrm{sec}\right.}
\end{aligned}
$$

see table 
547

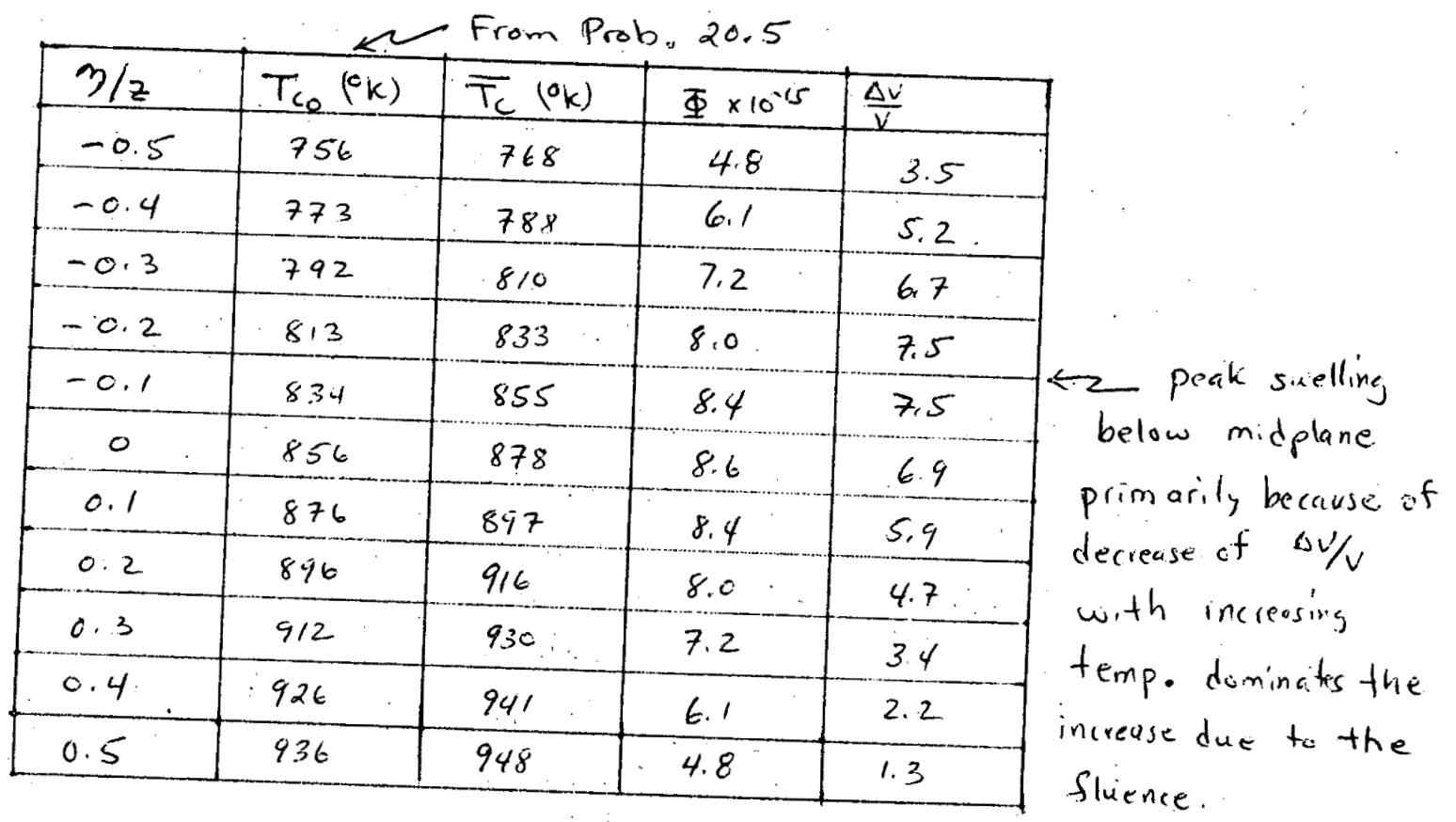

(c)

$$
\begin{aligned}
& \begin{array}{l}
\text { From } E_{q}(19.120) \overbrace{}^{\Delta v}(\%)=\left(10 \cdot \frac{\Phi}{\Phi_{0}}\right)^{g(0)} F(T)=\Phi_{0} t \times 10^{22}\left(\frac{\Phi}{\Phi_{0}}\right)
\end{array} \\
& g(\theta)=2.05-\frac{27}{\theta}+\frac{7 \theta}{\theta^{2}}, \theta=T-623 \\
& \therefore F(T)=(T-40) \times 10^{-10} \exp \left\{32.6-\frac{5100}{T}-0.015 T\right\}
\end{aligned}
$$


Problem 21.8

(1) (2)
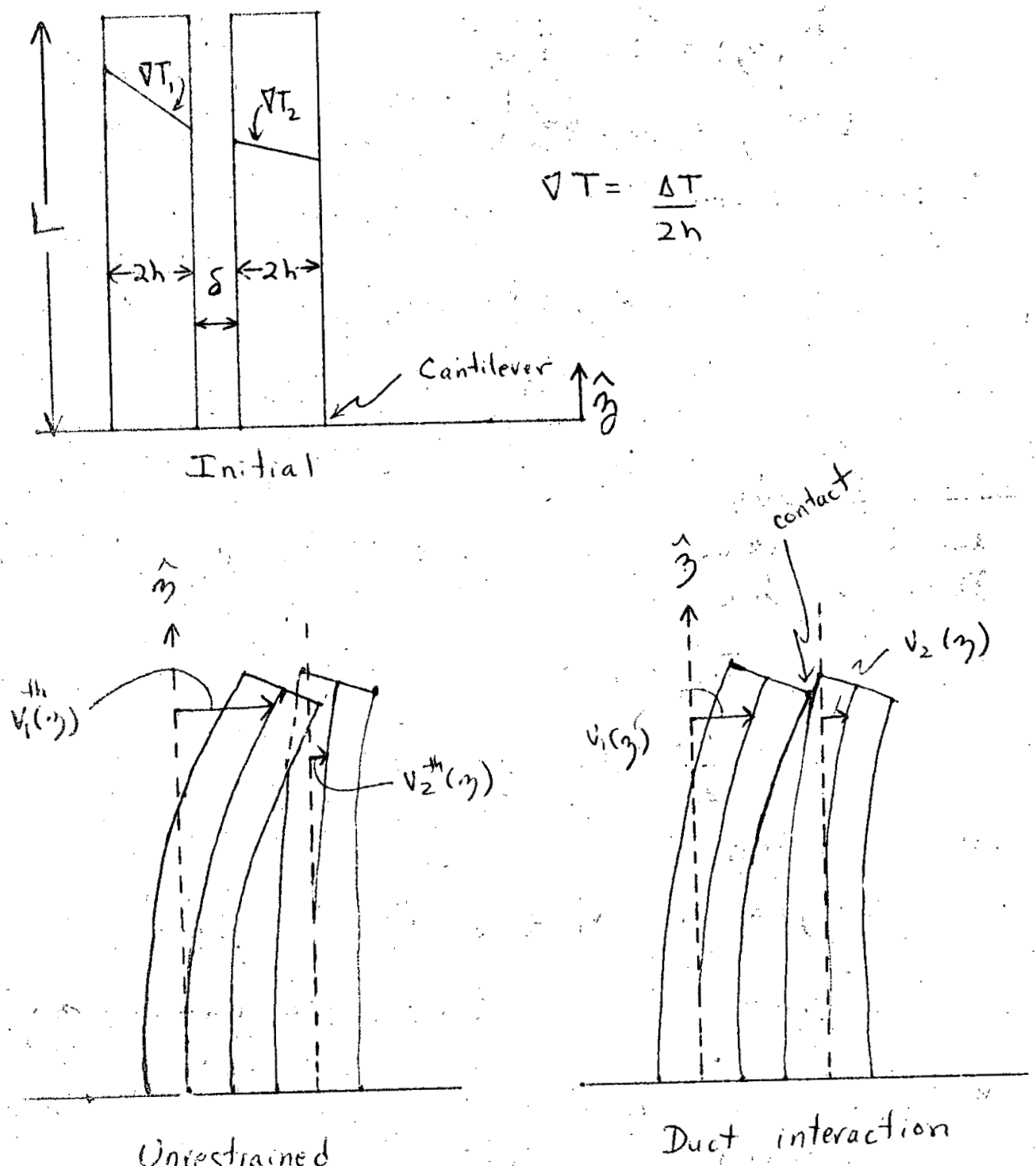

Uniestrained 
549

(A) Unrestrained thermal bowing:

from $E_{q}(2168)$

$$
\begin{aligned}
& V_{1}^{\text {th }}(\eta)=\frac{1}{2} \times \nabla T_{1} \eta^{2} \\
& v_{i}^{\text {th }}(\eta)=\frac{1}{2} \times \nabla T_{2} \eta^{2}
\end{aligned}
$$

Elements touch if $v_{1}^{\text {th }}(L)-v_{2}^{\text {th }}(L) \geq \delta$

or

$$
\begin{aligned}
& \frac{1}{2} \alpha\left(\nabla T_{1}-\nabla T_{2}\right) L^{2} \geq \delta \\
& \nabla T_{1}-\nabla T_{2} \geq \frac{2 \delta}{\alpha L^{2}}
\end{aligned}
$$

(B) Wines touching

let $F=$ interaction force (negative on (1), pristine on (2) Deflection due to $F$ is:

$$
\begin{aligned}
& v_{1}^{m}=-\frac{E_{2}^{2}}{2 E I}\left(L-\frac{3}{3}\right) \\
& v_{2}^{m}=\frac{F_{3}^{2}}{2 E I}\left(L-\frac{3}{3}\right)
\end{aligned}
$$

Total deflections:

$$
\begin{aligned}
& v_{1}(\eta)=v_{1}^{\text {th }}+v_{1}^{m}=\frac{1}{2} \alpha \cdot \nabla T_{1} \eta^{2}-\frac{F_{3}^{2}}{2 E I}\left(L-\frac{\eta}{3}\right) \\
& v_{2}(\eta)=v_{2}^{\text {th }}+v_{2}^{m}=\frac{1}{2} \times \nabla T_{2} \eta^{2}+\frac{F_{3}^{2}}{2 E I}\left(L-\frac{3}{3}\right)
\end{aligned}
$$

When just touching at top

or

$$
V_{1}(L)=V_{2}(L)+\delta
$$$$
\frac{1}{2} \alpha\left(\nabla T_{1}-\nabla T_{2}\right) L^{2}-\frac{2 F L^{3}}{3 E I}=\delta
$$

$$
\therefore F=\frac{3 E I}{4 L^{3}}\left\{\alpha L^{2}\left(\nabla T_{1}-\nabla T_{2}\right)-2 \delta\right\}
$$


550

Problem 21.9

a) In Eq 21.79 , neglect $\Delta \epsilon^{s}$ and set $F=K V(L, t)$

$$
\text { set } \quad F=k v(L, t)
$$

Integrating, (noting that $\Delta T \neq \Delta T(z)$ )

$$
v(z, t)=\frac{\alpha \Delta T}{4 h} z^{2}-\frac{K\left[v(L, t)+C \Phi E \int_{0}^{t} v(L, t) d t^{\prime}\right]}{2 E I}\left(L-\frac{z}{3}\right) z^{2}
$$

$$
\begin{aligned}
& v(z, t)=\frac{\alpha \Delta T}{4 l_{1}} L^{2}-\frac{K}{2 E I}\left[v(L, t)+c \Phi E \int_{0}^{t} v\left(L, t^{\prime}\right) d t^{\prime}\right] \cdot \frac{2}{3} L^{3}
\end{aligned}
$$

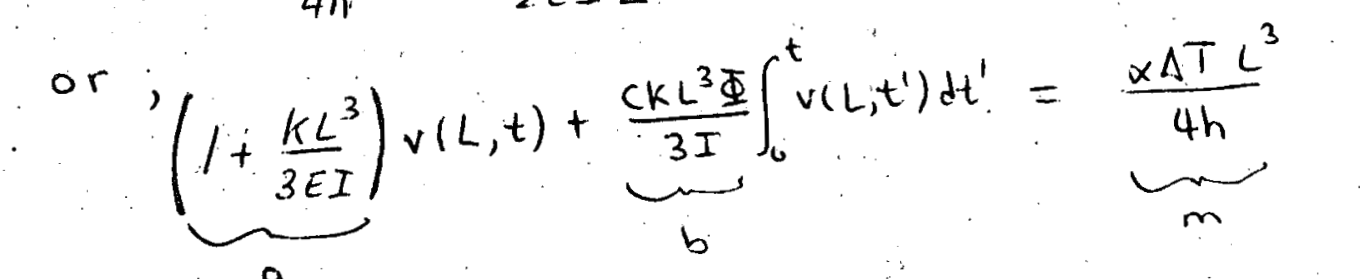

Take derivative with respect to $t$, obtain

$$
\begin{aligned}
& a \frac{d v}{d t}+b v=0 \\
& \text { I.C: } \quad v v(L, 0)=\mathrm{m} / a \quad(\text { from integral equip) } \\
& \text { Solution: } v(L, t)=(m / a) \cdot e^{-\left(\frac{b}{a} \cdot t\right)} \\
& \quad v(L, t)=\left[\frac{\frac{\alpha \Delta T L^{2}}{4 h}}{\left(1+\frac{k L^{3}}{3 E I}\right)}\right] \exp \left[-\frac{\frac{c k L^{3} I}{3 I}}{\left(1+\frac{K L^{3}}{3 E I}\right)} \cdot t\right]
\end{aligned}
$$


Thus, deflection profile is:

$v(z, t)=\frac{\alpha \Delta T}{4 h} z^{2}-\frac{k}{2 E I} \cdot \frac{m}{a}\left[e^{-\frac{b}{a} t}+\frac{C \Phi E}{b / a}\left(1-e^{-\frac{b}{a} t}\right)\right]\left(L-\frac{z}{3}\right) z^{2}$

b) When $\Delta T=0$ at $t=t^{*}$.

$v^{*}\left(z, t^{*}\right)=-\frac{k}{2 E I} \frac{m}{a}\left[e^{-\frac{b}{a} t^{*}}+\frac{c \Phi E}{b / a}\left(1-e^{-\frac{b}{a} t^{*}}\right)\right]\left(L-\frac{z}{3}\right) z^{2}$

Note: The flux term is retained because it applies to $0 \leq t \leq t^{*}$.

When spring is removed as well, the first term in. the brackets also disappears, and:

$f^{*}\left(z, t^{*}\right)=-\frac{k}{2 I} \frac{m}{b} c \Phi\left(1-e^{-\frac{b}{a} t^{*}}\right)\left(L-\frac{z}{3}\right) z^{2}$

\section{NOTice}

This book was prepared as an account of work sponsored by the United States and Development Admer United States nor the United States Energy Hesearch contractors, subcontractors or their en any of their employees, nor any of their implied, or assumes any or their employees, makes any warranty, express or completenese or us a responsibility for the accuracy disclosed, or represents that its use would not infring privetely owned rights

*U.S. GOVERNMENI PRINTING OFFICE, 1976 - 648-488/10280 - Region 4 